\section{Pacific Northwest}

National Laboratory

Operated by Battelle for the

U.S. Department of Energy

\title{
Assessment of the 296-S-21 Stack Sampling Probe Location
}

\author{
J. A. Glissmeyer
}

September 2006

Prepared for the U.S. Department of Energy under Contract DE-AC05-76RL01830 


\title{
DISCLAIMER
}

This report was prepared as an account of work sponsored by an agency of the United States Government. Neither the United States Government nor any agency thereof, nor Battelle Memorial Institute, nor any of their employees, makes any warranty, express or implied, or assumes any legal liability or responsibility for the accuracy, completeness, or usefulness of any information, apparatus, product, or process disclosed, or represents that its use would not infringe privately owned rights. Reference herein to any specific commercial product, process, or service by trade name, trademark, manufacturer, or otherwise does not necessarily constitute or imply its endorsement, recommendation, or favoring by the United States Government or any agency thereof, or Battelle Memorial Institute. The views and opinions of authors expressed herein do not necessarily state or reflect those of the United States Government or any agency thereof.

\author{
PACIFIC NORTHWEST NATIONAL LABORATORY \\ operated by \\ BATTELLE \\ for the \\ UNITED STATES DEPARTMENT OF ENERGY \\ under Contract DE-ACO5-76RL01830
}

Printed in the United States of America
Available to DOE and DOE contractors from the Office of Scientific and Technical Information, P.O. Box 62, Oak Ridge, TN 37831-0062; ph: (865) 576-8401 fax: (865) 5765728
email: reports@adonis.osti.gov




\title{
ASSESSMENT OF THE 296-S-21 STACK SAMPLING PROBE LOCATION
}

\author{
J. A. Glissmeyer
}

September 2006

Prepared for CH2MHill under a Related Services Agreement with the U.S. Department of Energy

under Contract DE-AC05-76RL01830

Pacific Northwest National Laboratory

Richland, Washington 99352 


\begin{abstract}
Tests were performed to assess the suitability of the location of the air sampling probe on the 296-S-21 stack according to the criteria of ANSI N13.1-1999, Sampling and Monitoring Releases of Airborne Radioactive Substances from the Stacks and Ducts of Nuclear Facilities. Pacific Northwest National Laboratory conducted most tests on a 3.67:1 scale model of the stack. CH2MHill also performed some limited confirmatory tests on the actual stack. The tests assessed the capability of the air-monitoring probe to extract a sample representative of the effluent stream. The tests were conducted for the practical combinations of operating fans and addressed:
\end{abstract}

1. Angular Flow-The purpose is to determine whether the velocity vector is aligned with the sampling nozzle. The average yaw angle relative to the nozzle axis should not be more than $20^{\circ}$. The measured values ranged from 5 to 10 degrees on the scale model and 10 to 12 degrees on the actual stack.

2. Uniform Air Velocity-The gas momentum across the stack cross section where the sample is extracted should be well mixed or uniform. The uniformity is expressed as the variability of the measurements about the mean, the coefficient of variance (COV). The lower the COV value, the more uniform the velocity. The acceptance criterion is that the COV of the air velocity must be $\leq 20 \%$ across the center two-thirds of the area of the stack. At the location simulating the sampling probe, the measured values ranged form 4 to $11 \%$, which are within the criterion.

To confirm the validity of the scale model results, air velocity uniformity measurements were made both on the actual stack and on the scale model at the test ports 1.5 stack diameters upstream of the sampling probe. The results ranged from 6 to $8 \% \mathrm{COV}$ on the actual stack and 10 to $13 \% \mathrm{COV}$ on the scale model. The average difference for the eight runs was $4.8 \% \mathrm{COV}$, which is within the validation criterion. The fact that the scale model results were slightly higher than the actual stack suggests that the other test results on the scale model are conservative relative to the actual stack.

3. Uniform Concentration of Tracer Gases-A uniform contaminant concentration in the sampling plane enables the extraction of samples that represent the true concentration. This was first tested using a tracer gas to represent gaseous effluents. The fan is a good mixer, so injecting the tracer downstream of the fans provides worst-case results. The acceptance criteria are that 1) the COV of the measured tracer gas concentration is $\leq 20 \%$ across the center two-thirds of the sampling plane and 2) at no point in the sampling plane does the concentration vary from the mean by $>30 \%$. The results on the scale model at the point simulating the sampling probe ranged from 0.3 to $6 \% \mathrm{COV}$, and the maximum single point deviation from the mean was $-10 \%$.

4. Uniform Concentration of Tracer Particles-Uniformity in contaminant concentration at the sampling probe was further demonstrated using tracer particles large enough to exhibit inertial effects. Particles of $10-\mu \mathrm{m}$ aerodynamic diameter were used. The acceptance criterion is that the COV of particle concentration is $\leq 20 \%$ across the center two-thirds of the sampling plane. The scale model results ranged form 2 to $9 \%$.

Based on these tests, the location of the air sampling probe on the 296-S-21 stack meets the requirements of the ANSI/HPS N13.1-1999 standard. 



\section{ACKNOWLEDGMENTS}

The author would like to express his appreciation to James G. Droppo and Brad G. Fritz of

Pacific Northwest National Laboratory for performing most of the testing required for the work described in this report. Wayne Cosby formatted and edited the report. 



\section{CONTENTS}

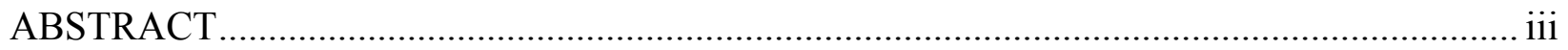

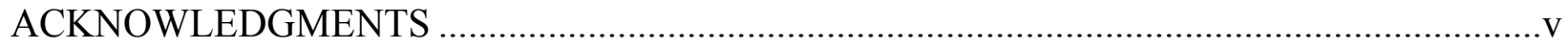

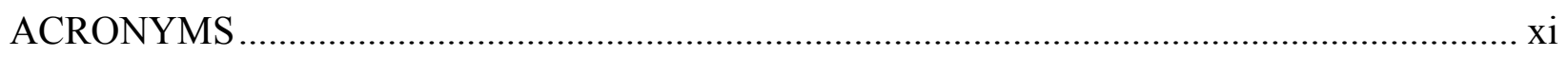

1.0 INTRODUCTION: QUALIFICATION OF HV-C2 AIR MONITORING LOCATION....1.1

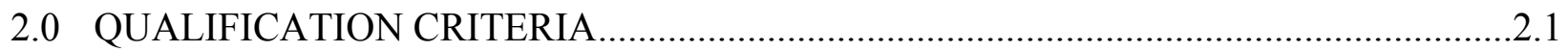

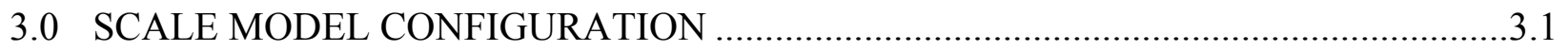

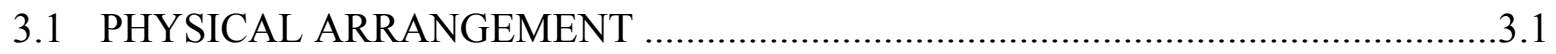

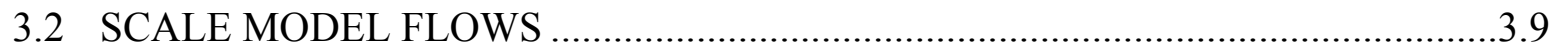

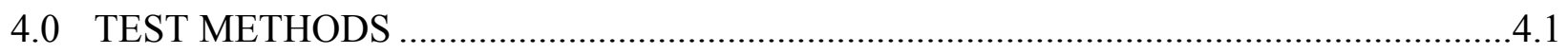

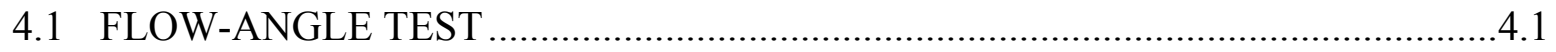

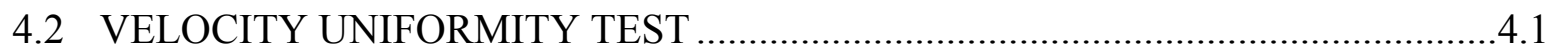

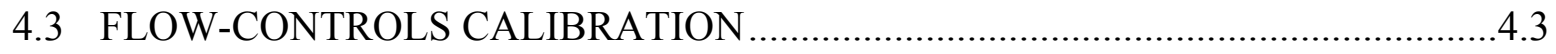

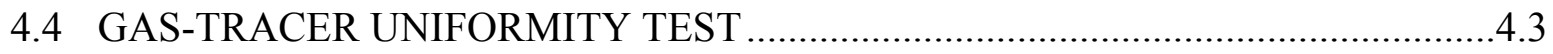

4.5 PARTICLE-TRACER UNIFORMITY TEST …....................................................

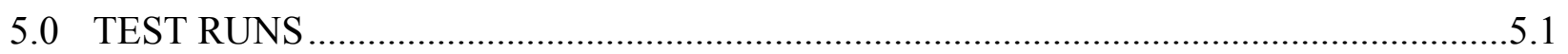

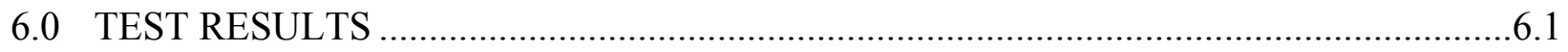

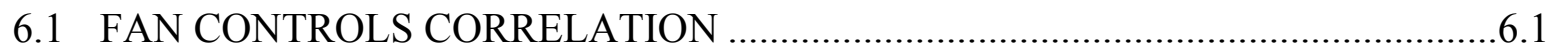

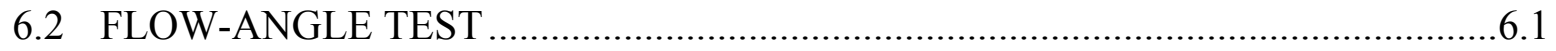

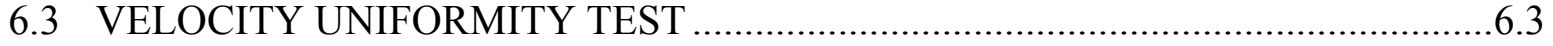

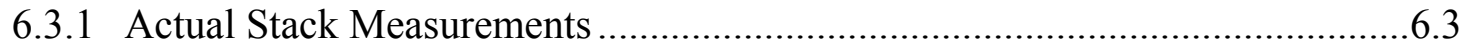

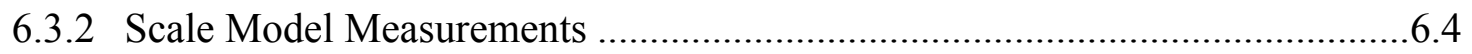

6.3.3 Scale Model Validation Measurements................................................................6.5

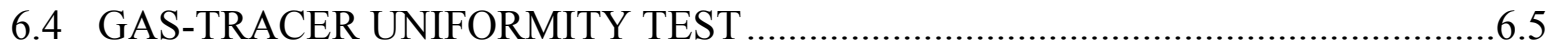

6.5 PARTICLE TRACER UNIFORMITY TEST …………........................................

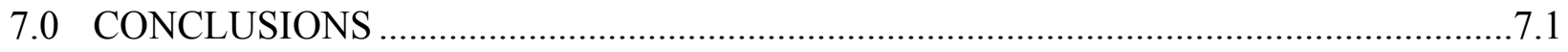

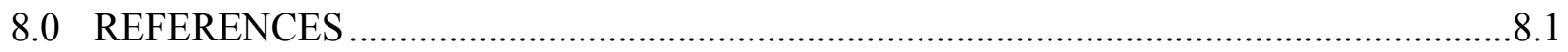

APPENDIX A: FLOW CALIBRATION PROCEDURE ……...............................................

APPENDIX B: FLOW CALIBRATION DATA SHEETS .......................................................... 
APPENDIX C: VELOCITY UNIFORMITY PROCEDURE ................................................ 1 APPENDIX D: VELOCITY UNIFORMITY DATA SHEETS ............................................ 1 APPENDIX E: FLOW ANGLE PROCEDURE................................................................ E. 1 APPENDIX F: FLOW ANGLE DATA SHEETS .............................................................. F.1 APPENDIX G: TRACER GAS UNIFORMITY PROCEDURE .............................................. APPENDIX H: TRACER GAS UNIFORMITY DATA SHEETS ........................................1 APPENDIX I: PARTICLE TRACER UNIFORMITY PROCEDURE.................................... I.1 APPENDIX J: PARTICLE TRACER UNIFORMITY DATA SHEETS ..................................... APPENDIX K: FLOW ANGLE AND VELOCITY UNIFORMITY DATA FOR THE ACTUAL STACK ............................................................................................. 


\section{FIGURES}

3.1. Layout of Actual Stack as Viewed from Southeast of the Stack .........................................1

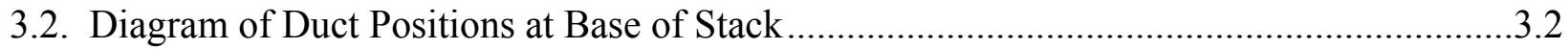

3.3. Plan and End Views of Scale Model............................................................................. 3.3

3.4. Side View of Test Section.........................................................................................

3.5. View of Scale Model Showing (1. to r.) Fans 1 to 4, HEPA Filters, and Injection Ports .....3.5

3.6. Duct from Fan 1 to the Base of the Stack and the Aerosol Tracer Injection Setup...............6

3.7. Base of Stack and Connection of Discharge Ducts from Fans 2, 3, and 4 (1. to r.) .............3.7

3.8. Side of Stack Showing Test Ports and the Transition to the Stack...................................

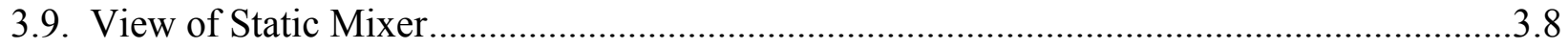

3.10. View Down Stack Towards Static Mixer ...............................................................

4.1. Flow Angle Measurement Apparatus ...................................................................... 4.2

4.2. View of Type-S Pitot Tube Inserted into Model Stack.................................................4.2

4.3. Electronic Manometer and Pitot Tube Inserted in Stack ............................................4.3

4.4. Tracer Gas Sampling Probe Inserted in Stack ..........................................................4.4

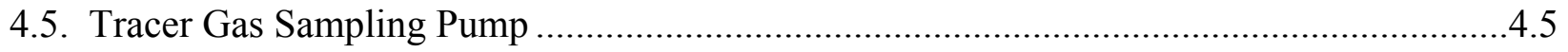

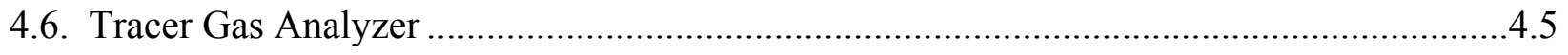

4.7. Particle Counter Connected to Probe ..........................................................................

6.1. Plot of Scale Model Flow Angle Results with Fans 3 and 4 Operating (Run FA-9)...........6.2

6.2. Plot of Flow Angle Measurements on Actual Stack, Run 4, Fans 3, 4...........................6.3

6.3. Velocity Profile at Actual Stack with Fans 1, 2, and 3 (Run 1).....................................6.4

6.4. Scale Model Velocity Profile with Fans 1, 2, and 3 Operating (Run VT-6) .....................6.5

6.5. Plot of Gas-Tracer Results for Injection Port 1 and Fans 1, 2, and 3 (Run GT-1) .............6.7

6.6. Plot of Particle Tracer Concentration for Run PT-1 .................................................6.8 


\section{TABLES}

3.1. Key Dimensions of Actual and Scale-Model Stacks .....................................................

3.2 Summary of Flow Parameters of Actual Stack and Scale Model at Port 4 .......................3.9

5.1. Minimum Test Runs to be Performed on Scale Model..................................................5.1

6.1. Flowrates Measured at Test Port 4 During Control Correlation......................................6.1

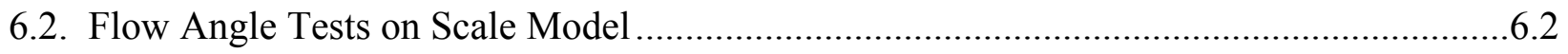

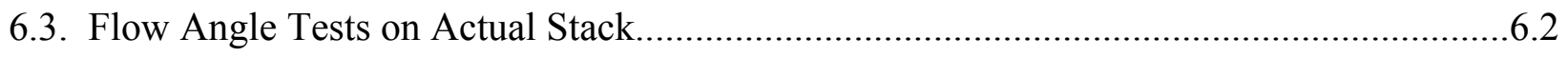

6.4. Velocity Uniformity Tests on Actual Stack ............................................................6.3

6.5. Velocity Uniformity Tests on Scale Model at Air Sampling Probe Location ....................6.4

6.6. Scale Model Velocity Uniformity Tests Simulating Actual Stack Tests..........................6.5

6.7. Summarized Results of Gas-Tracer Uniformity Tests at Test Port 4 .............................6.6

6.8. Results of Gas-Tracer Uniformity Tests at Other Test Ports ........................................6.7

6.9. Summarized Particle Tracer Uniformity Tests .......................................................6.8

7.1. Conclusions on Air Sampling System Tests ........................................................... 
ACRONYMS

AD aerodynamic diameter

$\mathrm{COV} \quad$ coefficient of variance

EPA U.S. Environmental Protection Agency

HEPA high-efficiency particulate air

OPC optical particle counter

Q/D ratio of flowrate (Q) to stack diameter (D) 


\subsection{INTRODUCTION: QUALIFICATION OF HV-C2 AIR MONITORING LOCATION}

This series of tests documents an assessment of how the current air-monitoring system for the 296-S-21 Stack meets the applicable regulatory criteria regarding the placement of the airsampling probe. Pacific Northwest National Laboratory conducted the tests on a scale model of the stack. CH2MHill staff also conducted some confirmatory tests at the actual 291-S-21 stack. The standard governing the performance of the tests, test methods, and acceptance criteria is ANSI/HPS N13.1-1999, Sampling and Monitoring Releases of Airborne Radioactive Substances From the Stack and Ducts of Nuclear Facilities. 


\subsection{QUALIFICATION CRITERIA}

The qualification criteria for an air-monitoring probe location are as follows:

1. Angular Flow-Sampling nozzles are usually aligned with the axis of the stack. If the air travels up the stack in cyclonic fashion, the air velocity vector approaching the nozzle could be misaligned with the sampling nozzles enough to impair the extraction of particles.

Consequently, the flow angle is measured in the stack at the elevation of the sampling nozzle. The average flow angle must not deviate from the axis of the sampling nozzle by more than $20^{\circ}$.

2. Uniform Air Velocity-The gas momentum across the stack cross section where the sample is extracted should be well mixed or uniform. Consequently, the velocity is measured at several points in the stack at the elevation of the sampling nozzle. The uniformity is expressed as the variability of the measurements about the mean. This is expressed using the coefficient of variance (COV), which is the standard deviation divided by the mean and expressed as a percentage. The lower the COV value, the more uniform the velocity. The acceptance criterion is that the COV of the air velocity must be $\leq 20 \%$ across the center twothirds of the area of the stack.

3. Uniform Concentration of Tracer Gases-A uniform contaminant concentration in the sampling plane enables the extraction of samples that represent the true concentration. This is first tested using a tracer gas to represent gaseous effluents. The fan is a good mixer, so injecting the tracer downstream of the fan provides worst-case results. The acceptance criteria are that 1) the COV of the measured tracer gas concentration is $\leq 20 \%$ across the center two-thirds of the sampling plane, and 2) at no point in the sampling plane does the concentration vary from the mean by $>30 \%$.

4. Uniform Concentration of Tracer Particles-Uniformity in contaminant concentration at the sampling probe is further demonstrated using tracer particles large enough to exhibit inertial effects. Particles of $10-\mu \mathrm{m}$ aerodynamic diameter (AD) are used by default unless it is known that larger particles are present in the airstream. The acceptance criterion is that the $\mathrm{COV}$ of particle concentration is $\leq 20 \%$ across the center two-thirds of the sampling plane.

The tests to assess compliance with Criteria 1 to 4 can be performed on the actual stack or with a scale model. The ANSI/HPS N13.1-1999 standard sets acceptance criteria for the use of a scale model as a substitute for the actual stack.

- The scale model and its sampling location must be geometrically similar to the actual stack.

- The product of the model's mean air velocity times the hydraulic diameter will be within a factor of six of the actual stack.

- The Reynolds number for the prototype and model stacks must $>10,000$. 
The scale model results are considered valid if:

- The velocity profile in the actual stack meets the uniformity criterion.

- The velocity uniformity COV for the actual and model stacks agree within 5\% COV. 


\subsection{SCALE MODEL CONFIGURATION}

This section describes the configuration for the scale model and the flows in the scale model.

\subsection{PHYSICAL ARRANGEMENT}

Four fans are available to power the exhaust air in the 296-S-21 stack, three electric (EF1, EF2, and EF3) fans and one diesel powered (EF4). Normally, only the three electric fans are used. The diesel fan is used as a backup in case of a loss of electric power, during which time operations inside the 222-S laboratory cease. It is also used in conjunction with any one of the electric fans during maintenance of one of those fans.

Figure 3.1 shows the stack with the ducts from each fan feeding the bottom of the stack. Three of the four ducts are shown, and the fourth is behind the others. A static mixer is located at the top of the square-round transition. The sampling point is close to the top of the stack. The fan output runs about 29,000, 23,500, 26,500, and 50,000 cfm for fans EF1, EF2, EF3, and EF4, respectively.

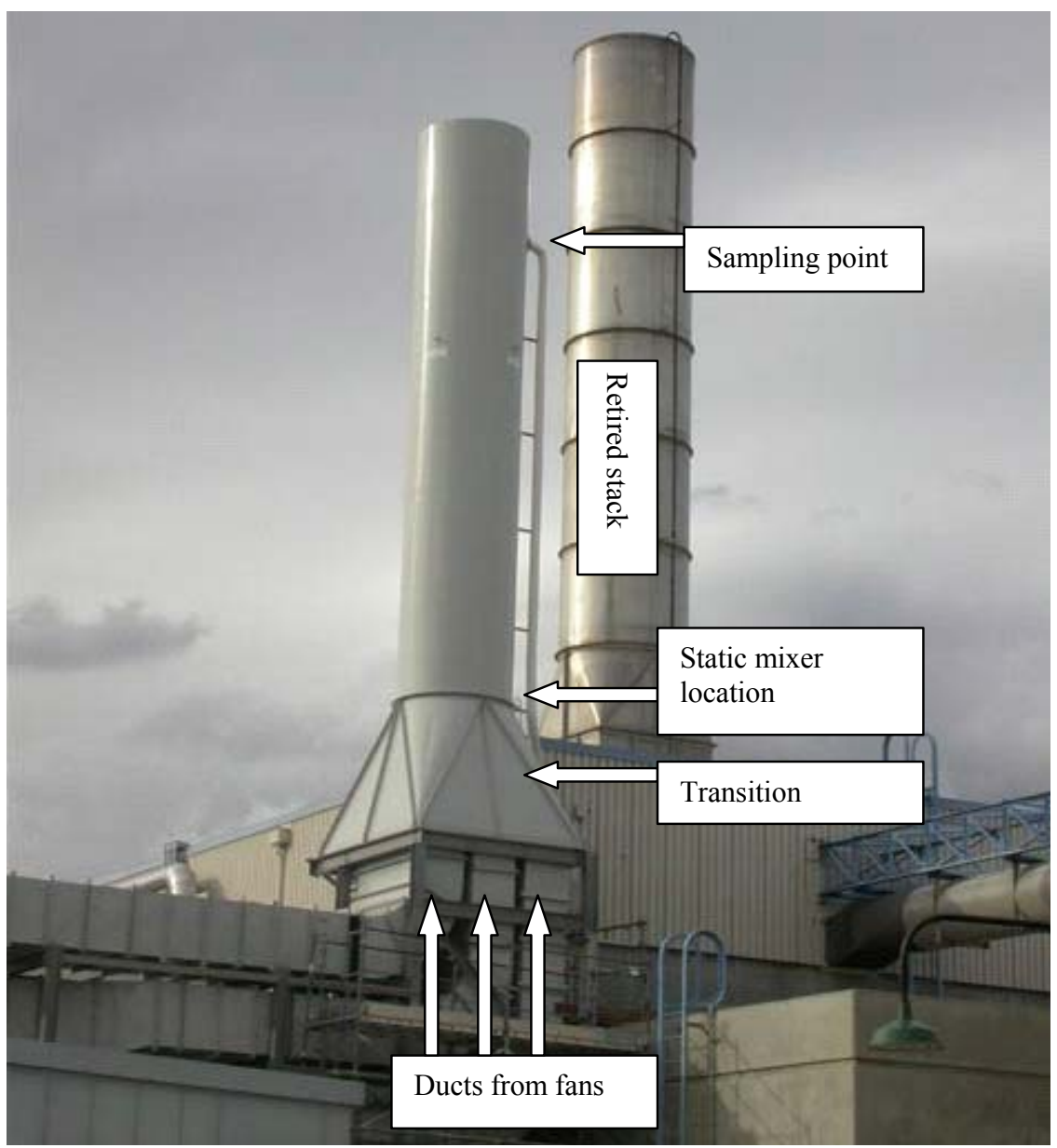

Figure 3.1. Layout of Actual Stack as Viewed from Southeast of the Stack 
Figure 3.2 is a crude representation of the base of the stack proper where the four ducts discharge into the stack. Note that the output of each fan enters the stack base at a different position instead of through a common manifold arrangement.

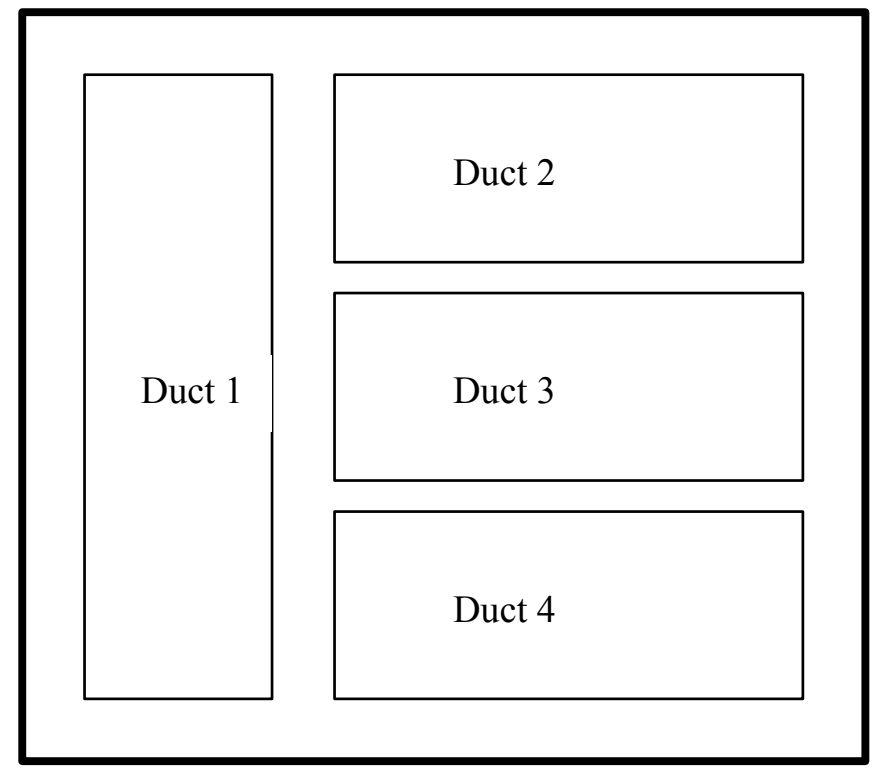

Figure 3.2. Diagram of Duct Positions at Base of Stack

Assuming that the flowrate from any one fan varies only within a small range $( \pm 20 \%)$, the only potential cause for changes in mixing would be which combination of fans is in use. The tests documented in this report will assess whether that mixing occurs for each different combination of fans. There are four possible combinations of fans when the lab is operating-Fans 1,2, and 3 run most of the time, and when Fan 4 is used, it is run with any one of the others. Potentially, if there is a release of airborne contaminants, it could be entering the stack from more than one fan at a time; however, it is assumed that a release via one duct at a time would be the worst case for mixing.

Figure 3.3 shows the plan and end views of the scale model. For convenience in testing, the stack was modeled as if it were tipped on its side in the easterly direction. This does not affect the test results.

It was necessary to only model the stack from the final bends in the four ducts entering the stack to the top of the stack. It was recognized that regardless of the mixing that occurs between the fans and the base of the transition, the discharge from each fan does not mix with that of the other fans until the transition and static mixer are reached. The mixing of the fan discharges occurs entirely between the base of the transition and the sampling point. The shape of the last bends into the transition base was also retained to preserve whatever flow-angle effects are caused by these bends. The distance from the transition base to the tracer injection points was also scaled from the same distances on the actual stack to the injection points that would have been used had the tests been done on the actual stack. 
Figure 3.4 shows a side view of the test section with the test-port pairs numbered left to right. Most tests were done using Test Port 4 because that simulates the location of the sampling probe. Some scale model validity tests were also done at Test Port 3 to simulate the test ports

$\mathrm{CH} 2 \mathrm{MHill}$ used for flow angle and velocity uniformity determinations on the actual stack. Test Ports 1 and 2 were available for supplementary tests at different distances from the static mixer if that became necessary.

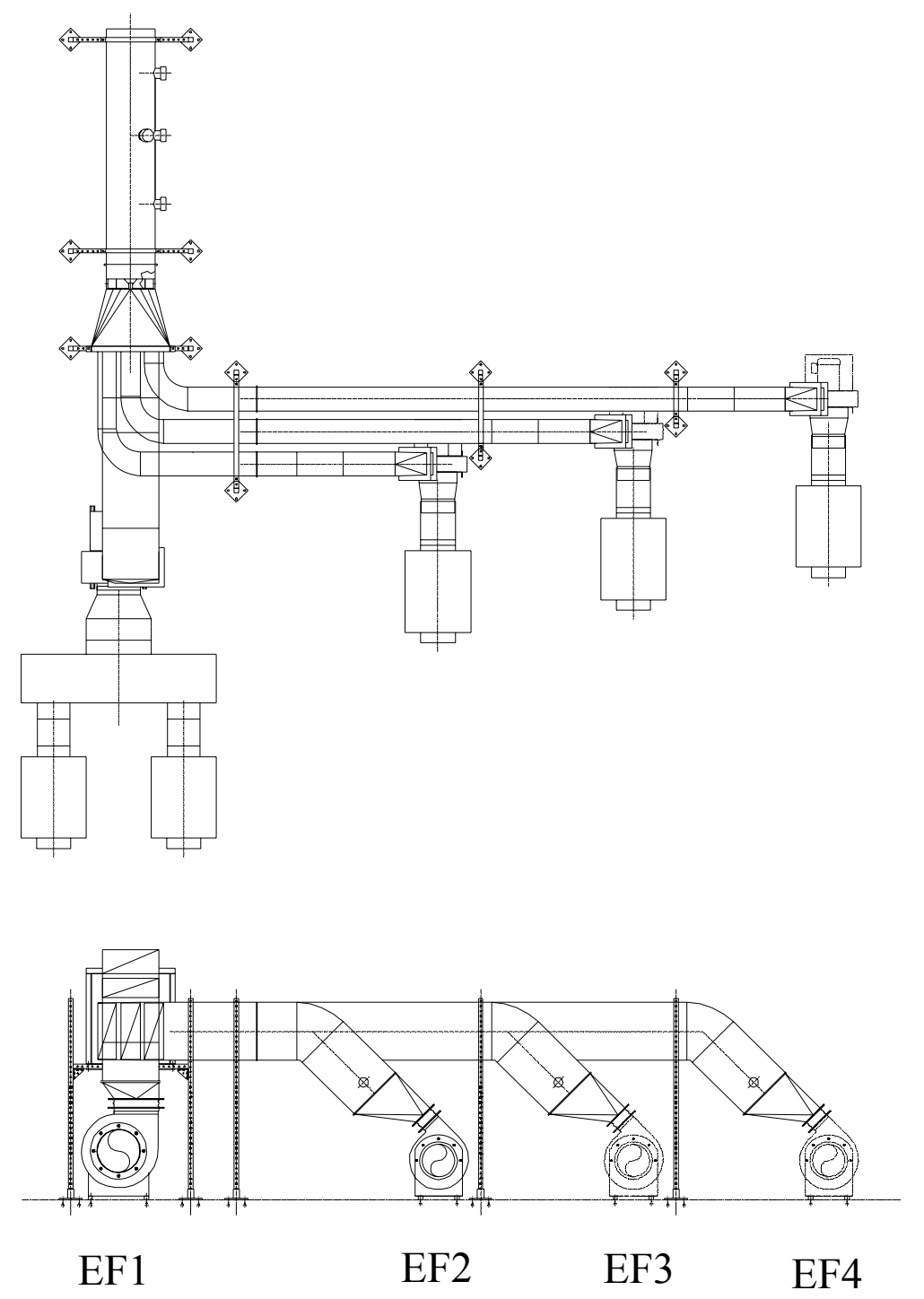

Figure 3.3. Plan and End Views of Scale Model 


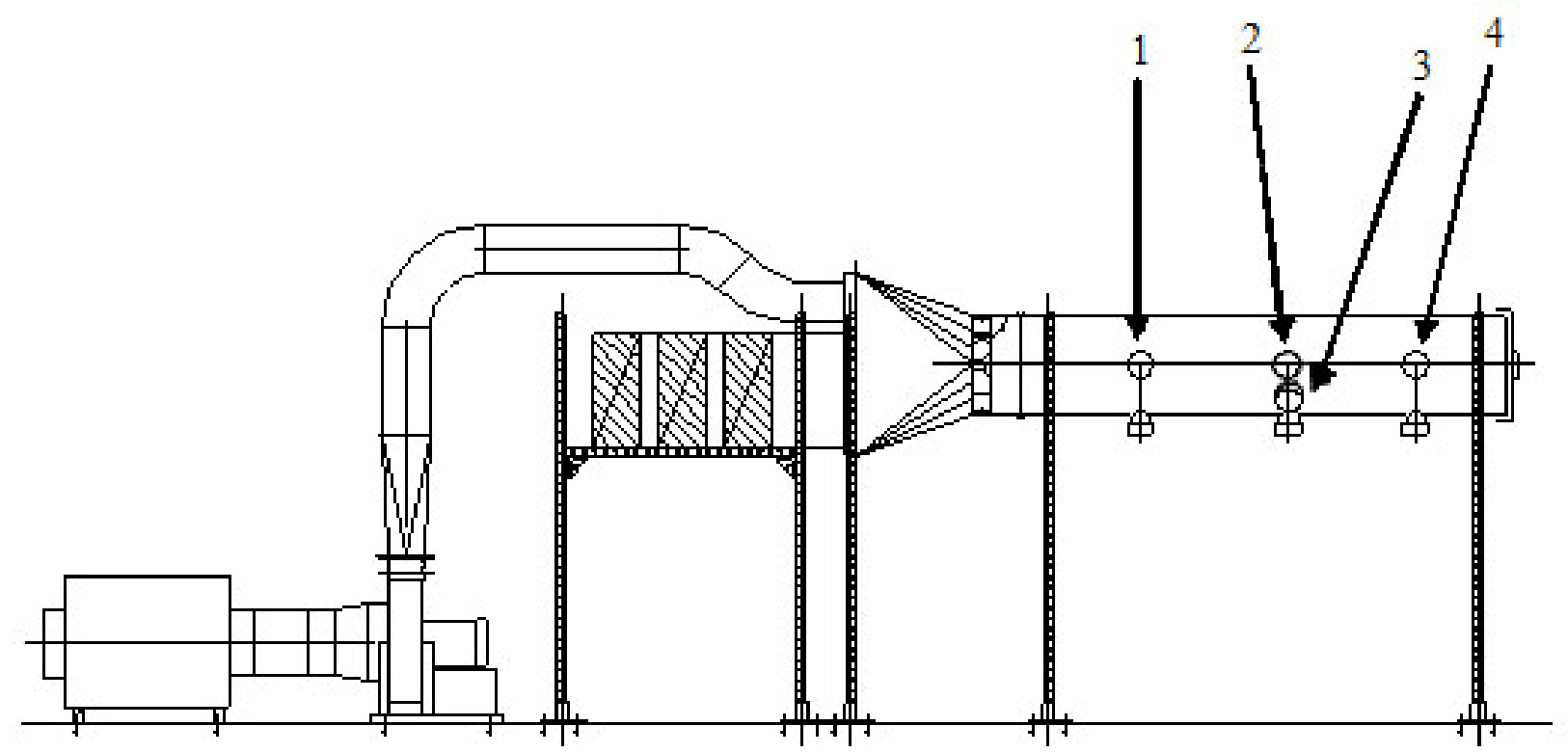

Figure 3.4. Side View of Test Section

Table 3.1 lists some key dimensions of the actual and as-built scale model stack.

Table 3.1. Key Dimensions of Actual and Scale-Model Stacks

\begin{tabular}{|c|c|c|}
\hline Key dimensions & Actual Stack & Scale Model \\
\hline End of mixer to end of stack & 342.4 in. (5.2 D) & 92.625 in. $(5.1 \mathrm{D})$ \\
\hline End of mixer to Test Port 1 & N.A. & 27.375 in. (1.5 D) \\
\hline $\begin{array}{l}\text { End of mixer to velocity test ports } \\
\text { (Test Port 3) }\end{array}$ & 198 in. $(3.0 \mathrm{D})$ & 54.625 in. $(3.0 \mathrm{D})$ \\
\hline $\begin{array}{l}\text { End of mixer to probe location } \\
\text { (Test Port 4) }\end{array}$ & 282.4 in. (4.3 D) & 77.75 in. (4.3 D) \\
\hline Stack diameter & 66 in. & $\begin{array}{c}18 \text { in. at Test Ports } 1,2, \& 3 \\
17-7 / 8 \text { in. at Test Port } 4\end{array}$ \\
\hline Transition length & 84.5 in. & 33 in. \\
\hline Overall transition base $\mathrm{H} \times \mathrm{W}$ & 122-in. $\mathrm{H} \times 106.5$-in $\mathrm{W}$ & 33.25-in. $\mathrm{H} \times 29$-in. $\mathrm{W}$ \\
\hline $\begin{array}{l}\text { Fan duct cross sections at } \\
\text { transition base } \mathrm{W} \times \mathrm{H}\end{array}$ & 76-in. $\mathrm{W} \times 26$-in. $\mathrm{H}$ & 20.75-in. W × 7-in. H \\
\hline $\begin{array}{l}\text { Fan duct cross sections at } \\
\text { injection ports }\end{array}$ & 78-in. $\mathrm{W} \times$ 32-in. $\mathrm{H}$ & 21.25-in. $\mathrm{W} \times 8.75$-in $\mathrm{H}$ \\
\hline \multicolumn{3}{|l|}{$\begin{array}{l}\text { Distance from transition base to } \\
\text { tracer injection port }\end{array}$} \\
\hline Fan 1 & $20.5 \mathrm{ft}$ & $5.1 \mathrm{ft}$ \\
\hline Fan 2 & $32.5 \mathrm{ft}$ & $8.4 \mathrm{ft}$ \\
\hline Fan 3 & $39.8 \mathrm{ft}$ & $9.9 \mathrm{ft}$ \\
\hline Fan 4 & $>98 \mathrm{ft}$ & $21.5 \mathrm{ft}$ \\
\hline
\end{tabular}


Figure 3.5 to Figure 3.10 show views of the scale model. Figure 3.5 shows the scale model from the side of the fans and ducts and the high-efficiency particulate air (HEPA) filters used at the fan inlets. Figure 3.6 shows the model from the side showing how the discharge from Fan-1 connects with the base of the stack. Figure 3.7 shows the ducts from Fans 2, 3, and 4 connecting to the base of the stack. Figure 3.8 shows the side of the stack and the transition from the base to the stack. The static mixer is located where the transition meets the stack. Figure 3.9 shows the static mixer before installation, and Figure 3.10 is a view down the stack looking at the static mixer.

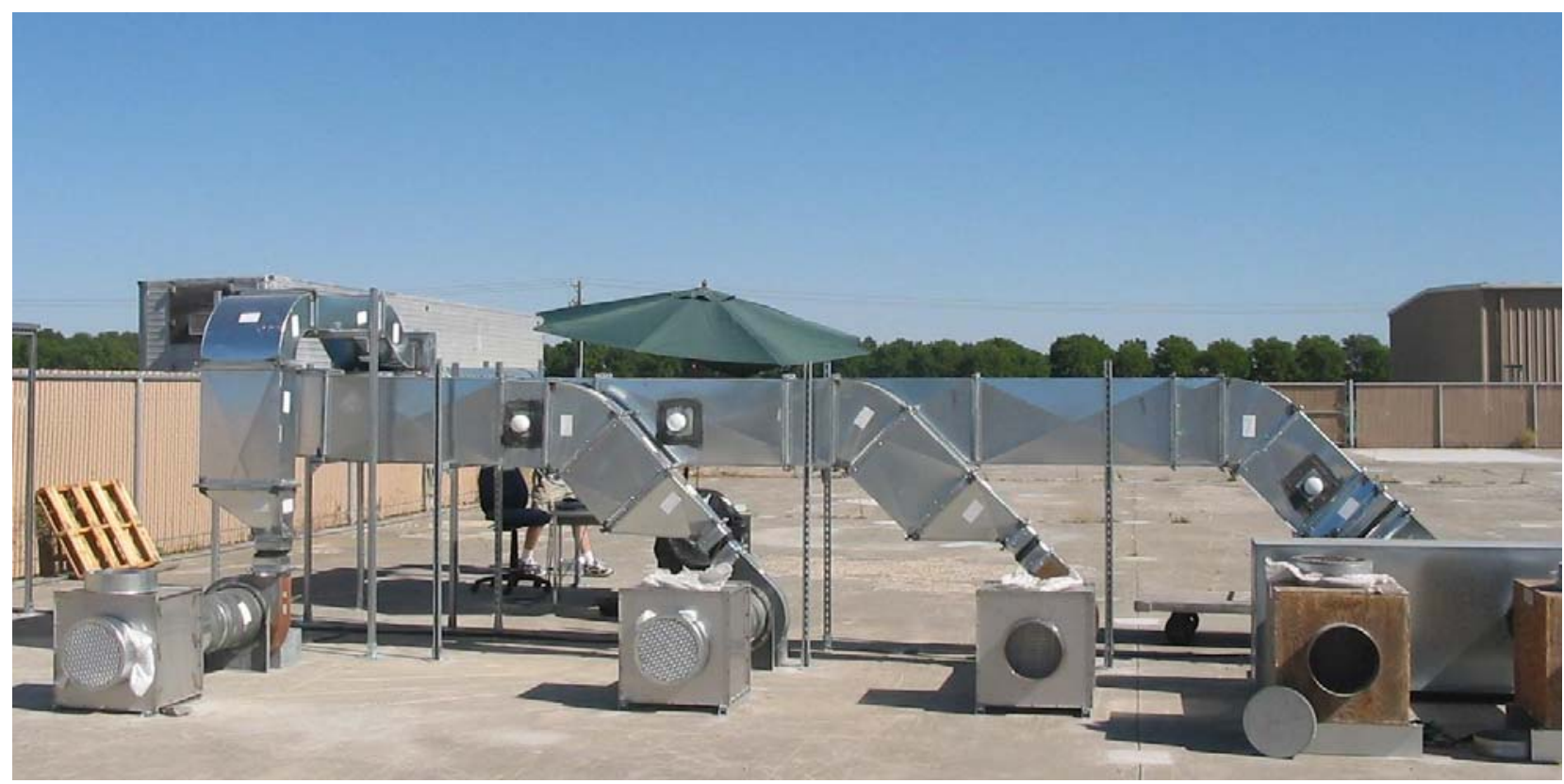

Figure 3.5. View of Scale Model Showing (l. to r.) Fans 1 to 4, HEPA Filters, and Injection Ports 


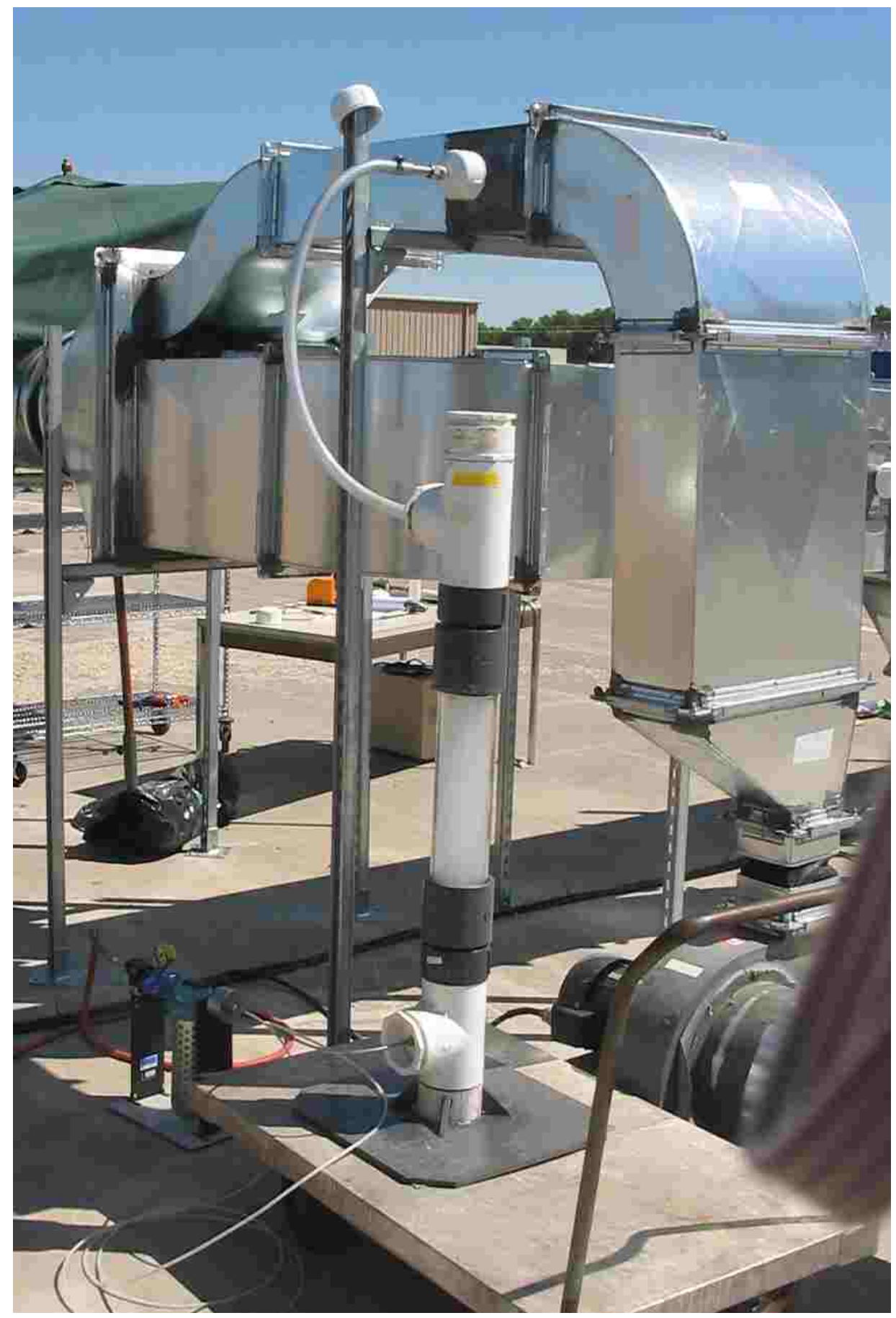

Figure 3.6. Duct from Fan 1 to the Base of the Stack and the Aerosol Tracer Injection Setup 


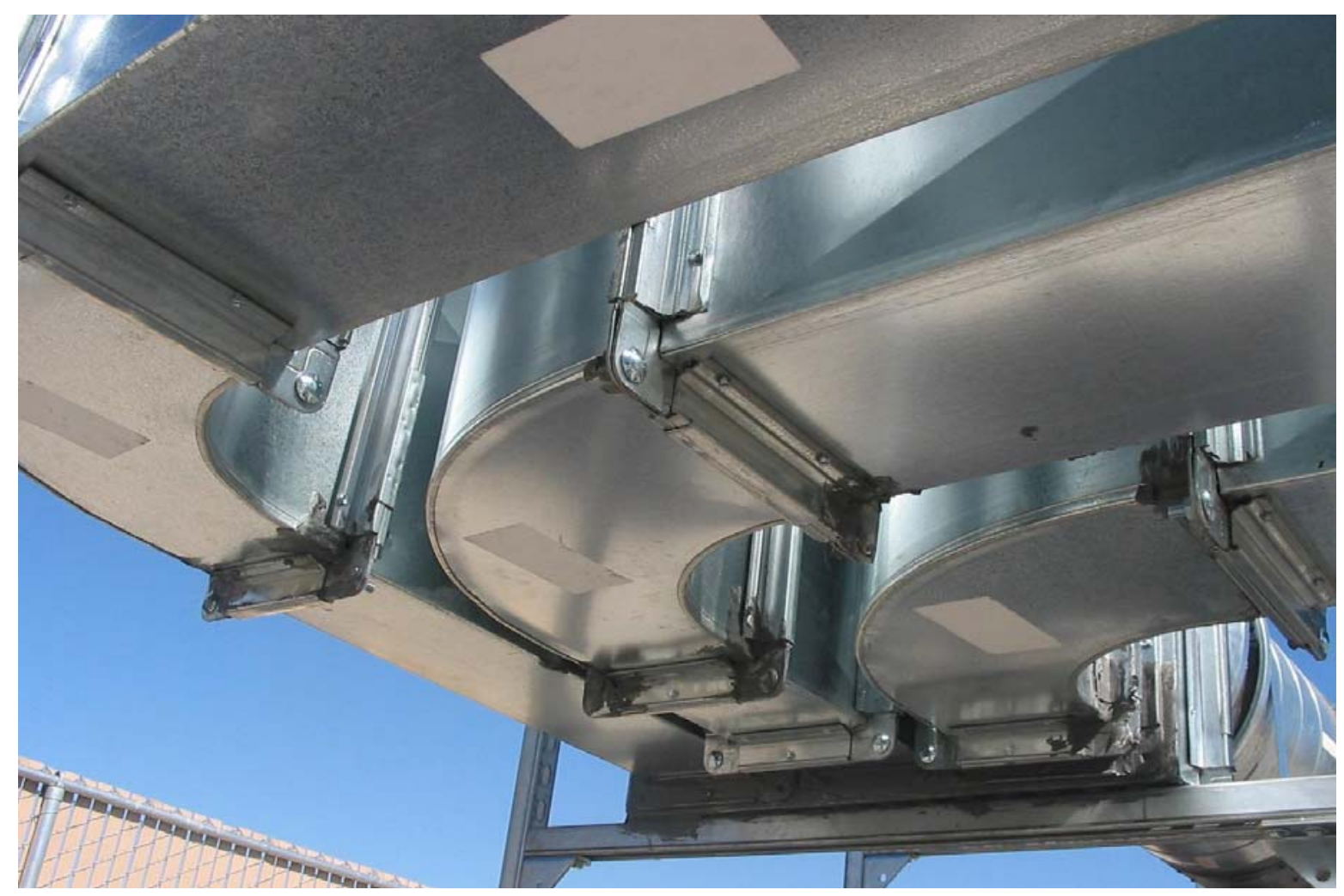

Figure 3.7. Base of Stack and Connection of Discharge Ducts from Fans 2, 3, and 4 (l. to r.)

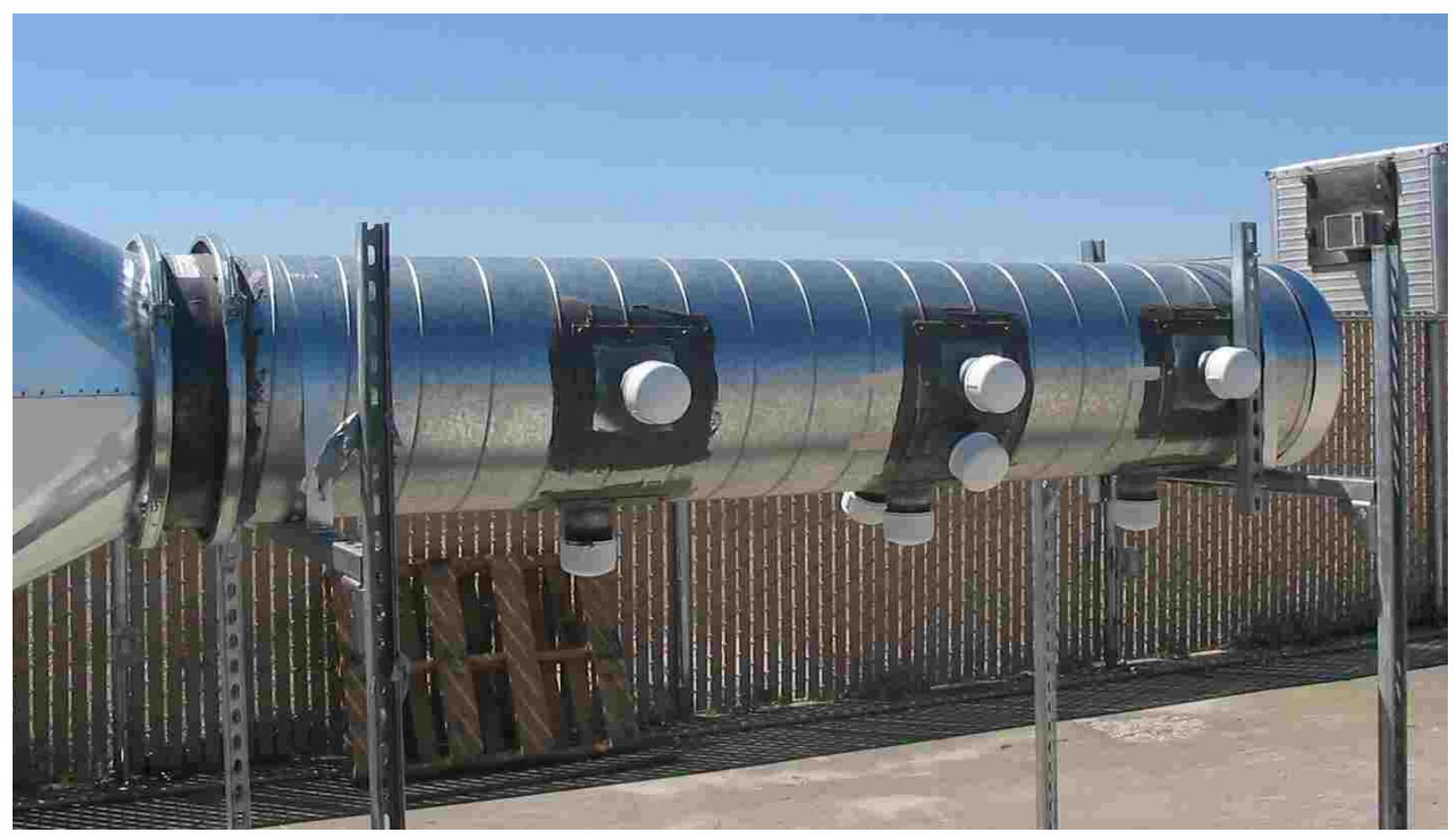

Figure 3.8. Side of Stack Showing Test Ports and the Transition to the Stack 


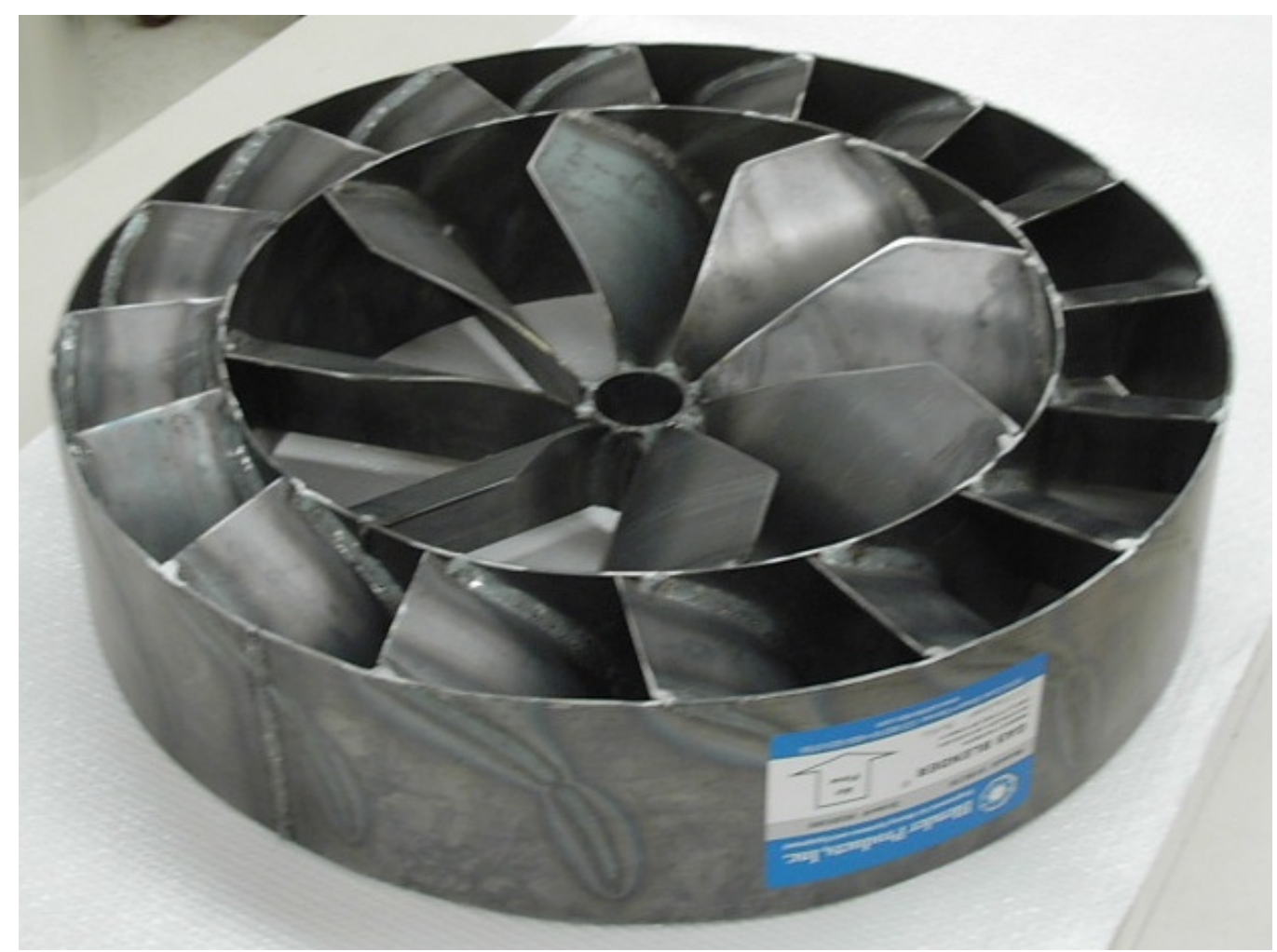

Figure 3.9. View of Static Mixer

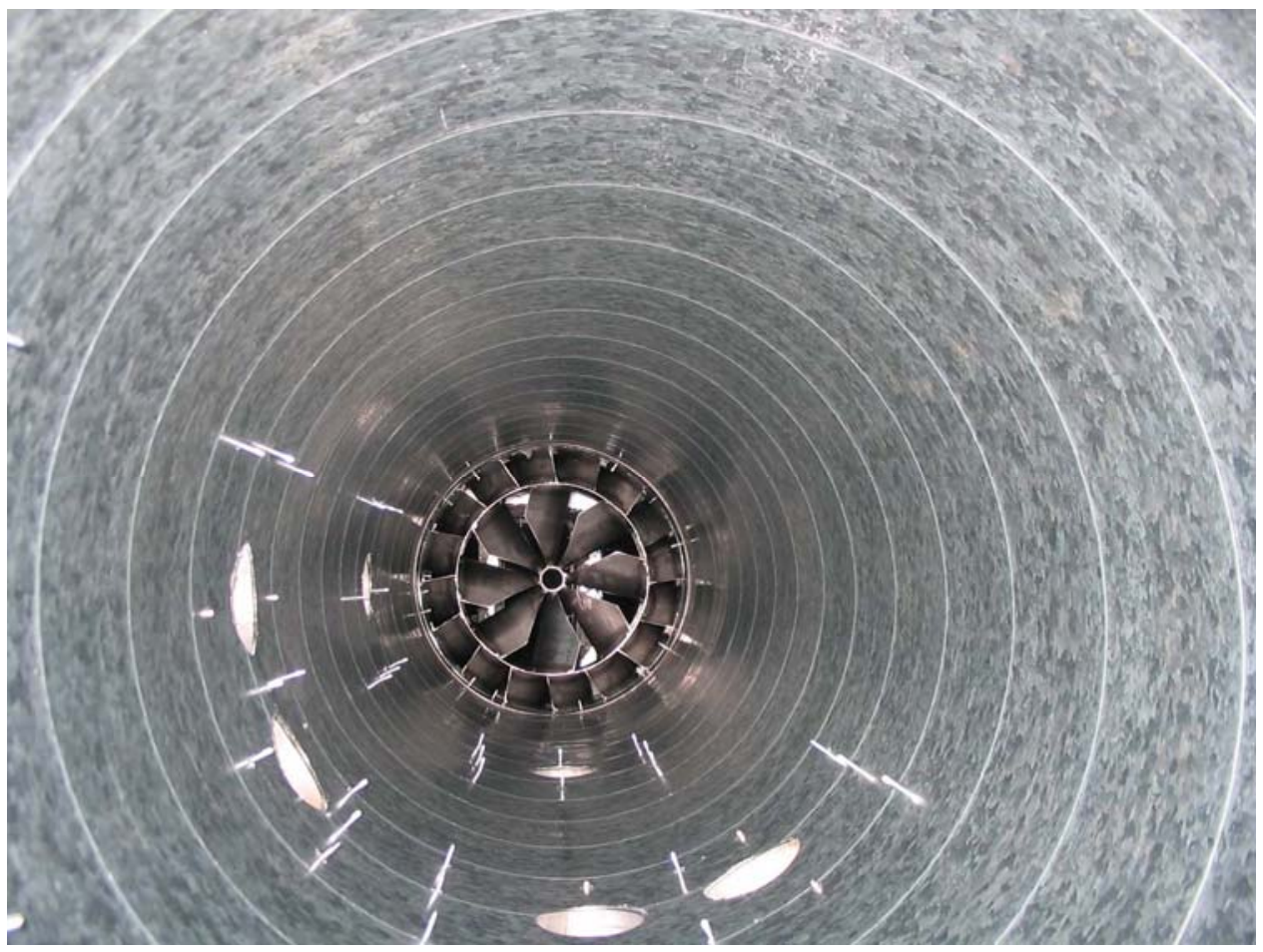

Figure 3.10. View Down Stack Towards Static Mixer 


\subsection{SCALE MODEL FLOWS}

For the scale model results to apply to the actual stack, the ANSI N13.1-1999 standard requires that the scale model's product of mean velocity $\times$ hydraulic diameter be within a factor of six of the actual stack. For stacks with a circular cross section, this is equivalent to requiring that the ratio of flowrate to stack diameter (Q/D) be within a factor of six of the actual stack. The standard also requires that the Reynolds number for the prototype and model stacks must $>10,000$.

Table 3.2 summarizes the prototype and scale model flow parameters that satisfy the scaling requirements, assuming that the scale model stack has a $17--^{7} / 8$-in. diameter that was measured at Port 4. CH2MHill provided the data for the actual stack. (The diameter measured at the other ports was 18 -in.) The flowrate and velocity values for the scale model are treated as minimum target values. These are helpful for selecting fans with appropriate capacity. Higher values can be used. It was prudent to choose equipment with higher values in mind to verify that the minima are achieved.

Table 3.2 Summary of Flow Parameters of Actual Stack and Scale Model at Port 4

\begin{tabular}{|l|c|c|c|c|c|c|}
\hline & \multicolumn{2}{|c|}{ Air Flow cfm } & \multicolumn{2}{c|}{ Air Velocity fpm } & \multicolumn{2}{c|}{ Reynolds Number } \\
\hline $\begin{array}{l}\text { Fan } \\
\text { Combinations }\end{array}$ & $\begin{array}{c}\text { Actual } \\
\text { Stack }\end{array}$ & $\begin{array}{c}\text { Scale Model } \\
\text { Min. }\end{array}$ & $\begin{array}{c}\text { Actual } \\
\text { Stack }\end{array}$ & $\begin{array}{c}\text { Scale Model } \\
\text { Min. }\end{array}$ & $\begin{array}{c}\text { Actual } \\
\text { Stack }\end{array}$ & $\begin{array}{c}\text { Scale Model } \\
\text { Min. }\end{array}$ \\
\hline 1 & 29000 & 1309 & 1221 & 751 & $7.1 \mathrm{E}+05$ & $1.2 \mathrm{E}+05$ \\
\hline 2 & 23500 & 1061 & 989 & 609 & $5.7 \mathrm{E}+05$ & $9.5 \mathrm{E}+04$ \\
\hline 3 & 26500 & 1196 & 1115 & 686 & $6.5 \mathrm{E}+05$ & $1.1 \mathrm{E}+05$ \\
\hline 4 & 50000 & 1743 & 2105 & 1295 & $1.2 \mathrm{E}+06$ & $2.0 \mathrm{E}+05$ \\
\hline $1,2,3$ & 79000 & 3566 & 3325 & 2046 & $1.9 \mathrm{E}+06$ & $3.2 \mathrm{E}+05$ \\
\hline 1,4 & 79000 & 3566 & 3325 & 2046 & $1.9 \mathrm{E}+06$ & $3.2 \mathrm{E}+05$ \\
\hline 2,4 & 73500 & 3318 & 3094 & 1904 & $1.8 \mathrm{E}+06$ & $3.0 \mathrm{E}+05$ \\
\hline 3,4 & 76500 & 3453 & 3220 & 1981 & $1.9 \mathrm{E}+06$ & $3.1 \mathrm{E}+05$ \\
\hline
\end{tabular}

The air temperature in the operating stack did not need to be simulated because it had a negligible effect on the scale of turbulence produced by the geometry of the system. However, the air temperature in the model was maintained above $55^{\circ} \mathrm{F}$ so that the tracer measurement instrumentation was within its operating range and provided reliable data. 


\subsection{TEST METHODS}

This section describes the flow-angle and velocity uniformity tests, the flow-controls calibration, and the gas-tracer and particle-tracer uniformity tests.

\subsection{FLOW-ANGLE TEST}

The air velocity vector approaching the sample nozzle should be aligned with the axis of the nozzle, within an acceptable angle, so sample extraction performance is not degraded. The test method is based on 40 CFR 60, Appendix A, Method 1, Section 2.4, "Verification of the Absence of Cyclonic Flow."

The term "flow angle" is the angle between the air velocity vector and the axis of the sampling nozzle. The flow angle was measured at a grid of points in a cross section of the stack at the scaled elevation of the actual sampling probe. The grid was an array of points in an x-pattern in the cross section of the duct. One line of points was aligned in the same direction as the existing actual sampling probe. The other line will be perpendicular to that. The number and distance between measurement points was based on the U.S. Environmental Protection Agency (EPA) procedure 40 CFR 60, Appendix A, Method 1.

Measurements were made using a type-S pitot tube attached by flexible tubes to a slant-tube manometer, and an angle-indicating device was attached to the pitot tube as shown in Figure 4.1. Figure 4.2 shows the view down the stack towards the type-S pitot tube at Port 3 Northeast. Procedure EMS-JAG-05, "Test to Determine Flow Angle at the Elevation of a Sampler Probe," provides the general procedure to determine the mean flow angle. A copy of the procedure is in Appendix E.

\subsection{VELOCITY UNIFORMITY TEST}

The uniformity of air velocity where the air sample is being extracted verifies that the air momentum in the stack is well mixed. To determine uniformity, air velocity was measured at the same grid of points used for the angular flow test. The method used was based on 40 CFR 60, Appendix A, Method 1.

The air velocity was measured three times at each grid point, and each measurement was recorded. The measurements at each grid point are averaged to determine the average velocity at each grid point. The values for each grid point in the center $2 / 3$ of the stack are used to calculate the mean and standard deviation of velocity for the sampling location. The percent relative standard deviation (also called the COV) was calculated as 100 times the standard deviation divided by the mean. This value should be less than or equal to $20 \%$.

The equipment used included a standard pitot tube (Prandtl type) and a calibrated electronic manometer and air velocity meter. Figure 4.3 shows the setup. Procedure EMS-JAG-04, "Test to Determine Uniformity of Gas Velocity at the Elevation of a Sampler Probe," shown in Appendix C, was used for this test. 


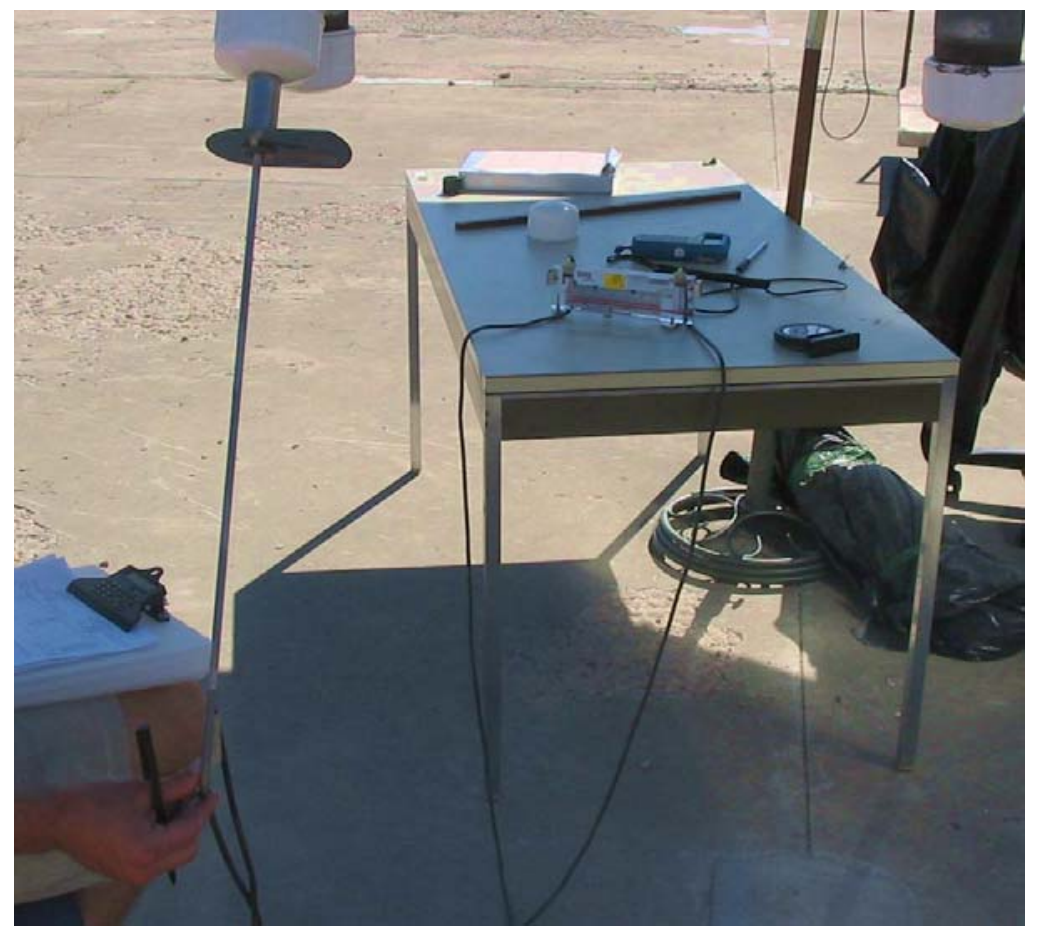

Figure 4.1. Flow Angle Measurement Apparatus

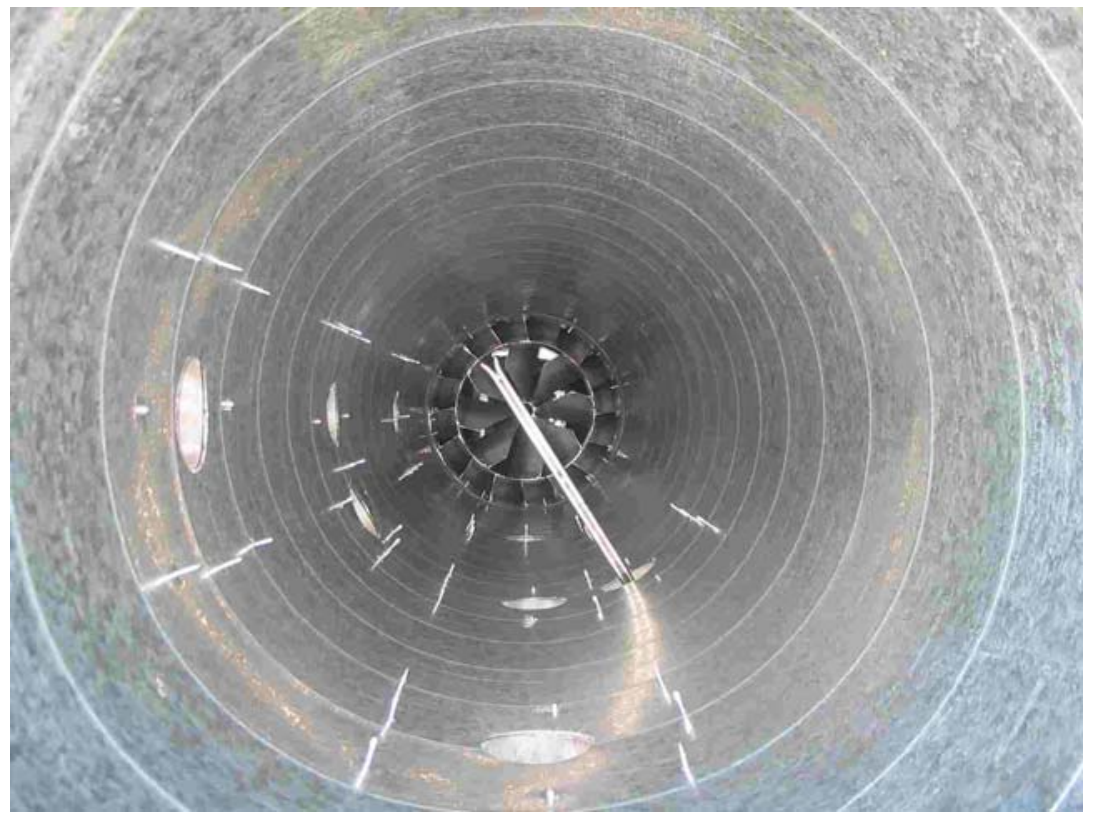

Figure 4.2. View of Type-S Pitot Tube Inserted into Model Stack 


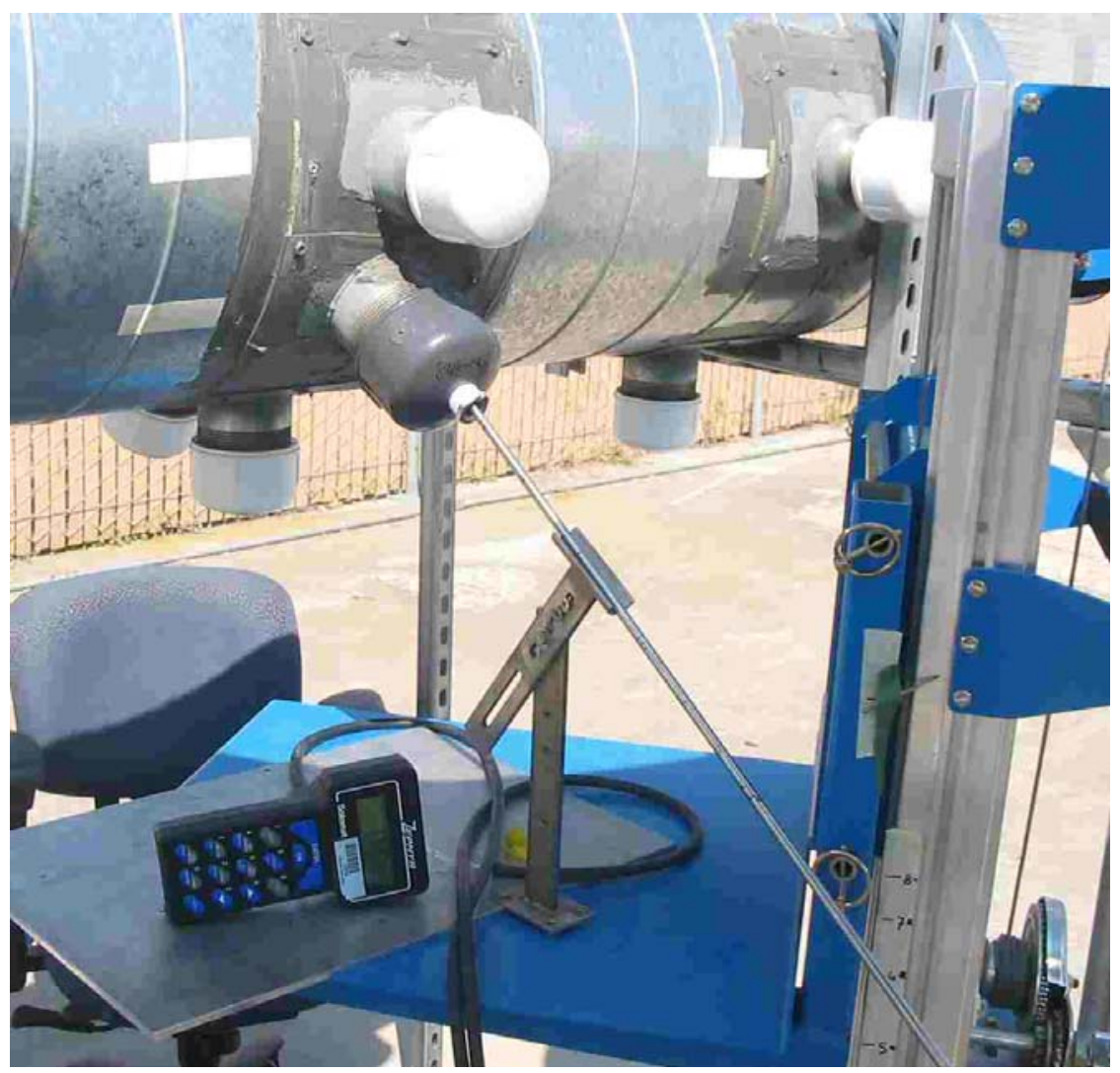

Figure 4.3. Electronic Manometer and Pitot Tube Inserted in Stack

\subsection{FLOW-CONTROLS CALIBRATION}

The first test performed with the scale model was to obtain an approximate calibration of the fan flow controls. Each fan has its own speed control, which is a variable frequency drive with settings from 0 to $60 \mathrm{~Hz}$. The first step was to measure the flowrate of each fan separately with the speed control set at $30 \mathrm{~Hz}$. Then a point with average velocity is identified. A calibrated air velocity meter is used to take readings at that point while the speed settings are varied from 5 to $60 \mathrm{~Hz}$. Procedure EMS-JAG-03, "Test to Calibrate Ventilation Flow Controller," shown in Appendix A, was used for this preliminary test. The results provide the approximate settings to achieve the flowrate desired for the balance of the tests.

\subsection{GAS-TRACER UNIFORMITY TEST}

The gaseous contaminant concentration uniformity was demonstrated using sulfur hexafluoride as a tracer gas. The tracer gas was injected into the air downstream of a fan along the centerline of the duct. One injection location at a time was tested. Once the injection point yielding the worst case results was identified, additional tests were done where the tracer was injected along the centerline of the duct and at four points near the corners or edge of the duct.

For each injection position, the tracer concentration was measured at the sampling location using the same measurement grid used for the other tests. The tracer concentration was measured three 
times at each grid point, and each measurement was recorded. The measurements at each grid point are averaged to determine the average concentration at each grid point. The values for each grid point in the center $2 / 3$ of the stack are used to calculate the mean and standard deviation of concentration for the sampling location. The percent COV is calculated as 100 times the standard deviation divided by the mean. This value should be less than or equal to $20 \%$. The average concentration values for all grid points were also compared to the mean to determine if the concentration at any point deviates from the mean by more than $30 \%$.

The tracer gas concentration was measured with an Innova AirTech Instruments A/S (Ballerup, Denmark) Model 1302 photoacoustic gas analyzer. Because the result is the standard deviation of the readings divided by the mean, the calibration bias is unimportant to the test results.

However, the analyzer response was checked using calibration standards before conducting the test series to verify that the instrument responds adequately to changes in concentration. If the indicated concentration was within $20 \%$ of the standard, the response was acceptable.

The measurements were made at Test Port 4 because they simulate the position of the sampling probe. Figure 4.4 to Figure 4.6 show the tracer gas sampling probe, the sampling pump, and the gas analyzer, respectively. Procedure EMS-JAG-01, "Test to Determine Uniformity of a Tracer Gas at a Sampler Probe," shown in Appendix G, was used for this test.

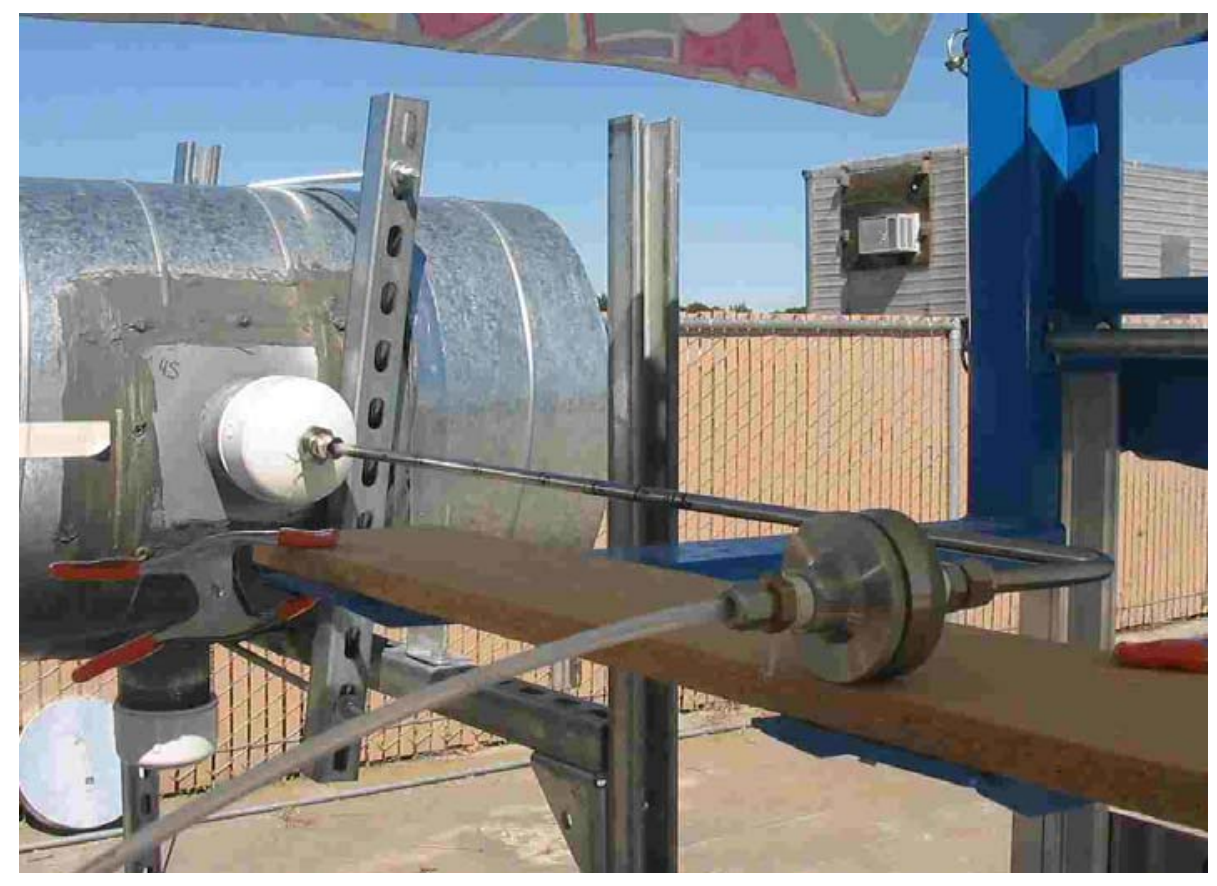

Figure 4.4. Tracer Gas Sampling Probe Inserted in Stack 


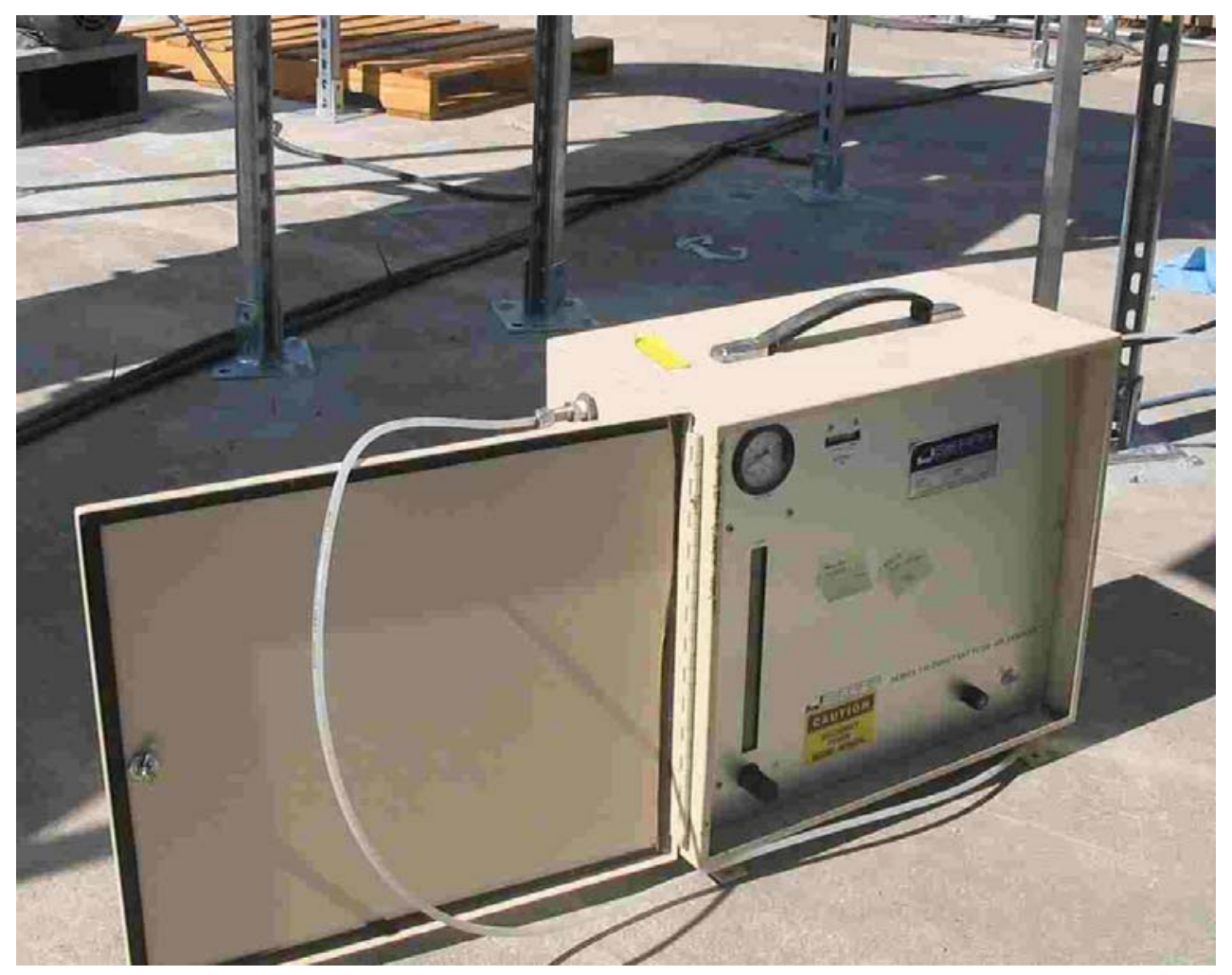

Figure 4.5. Tracer Gas Sampling Pump

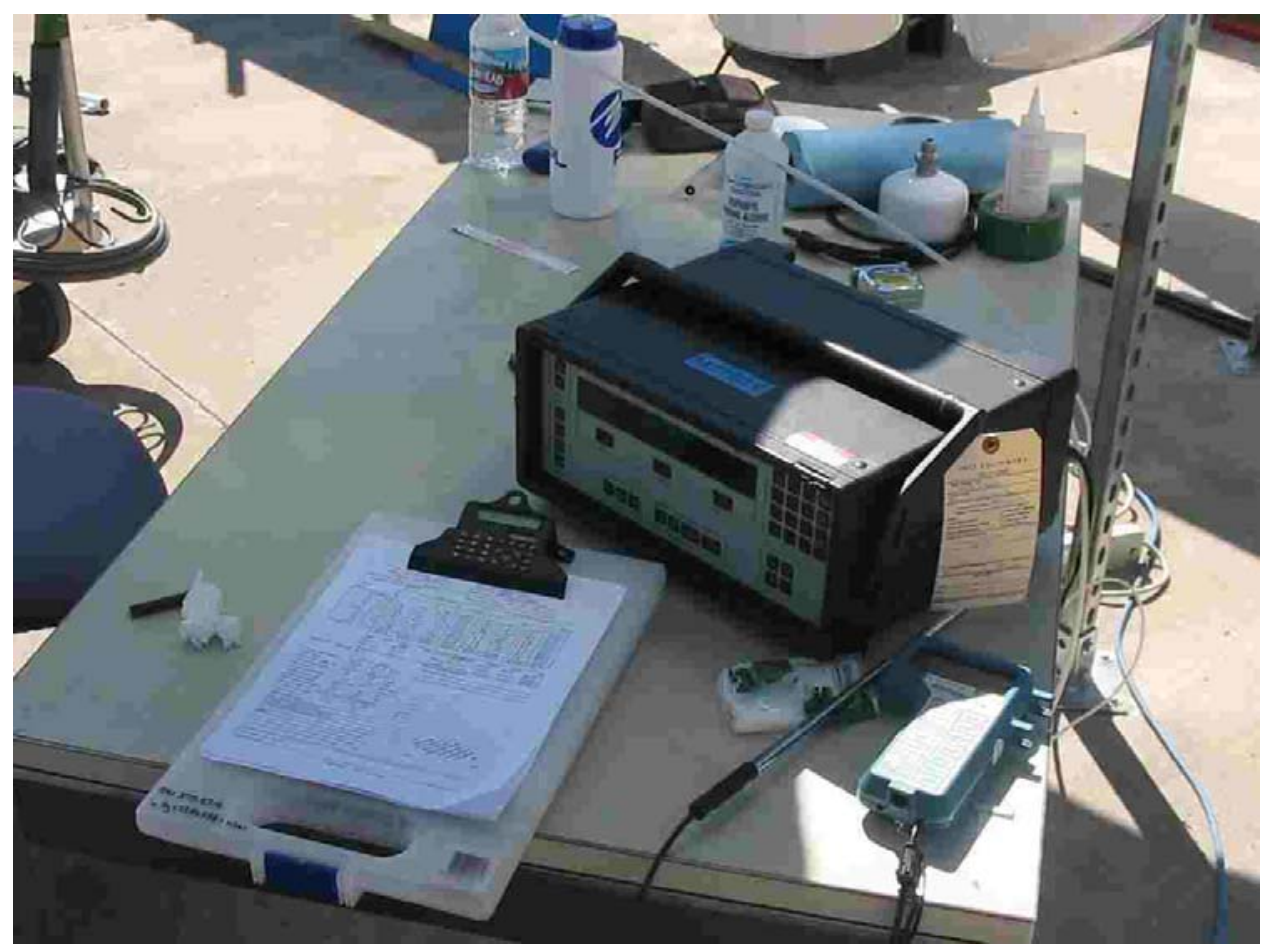

Figure 4.6. Tracer Gas Analyzer 


\subsection{PARTICLE-TRACER UNIFORMITY TEST}

The test for uniformity of tracer particles is similar to the test for uniformity of tracer gases. The general approach is to inject a polydisperse aerosol into the test stack downstream of a fan. The concentration of the particles is then measured at the sampling grid points with a calibrated optical particle counter (OPC, Met-One Model A2408, Grants Pass, Oregon). A simple probe was used to extract the sample and deliver it to the OPC. Figure 4.7 shows the sampling probe and OPC located at Test Port 4.

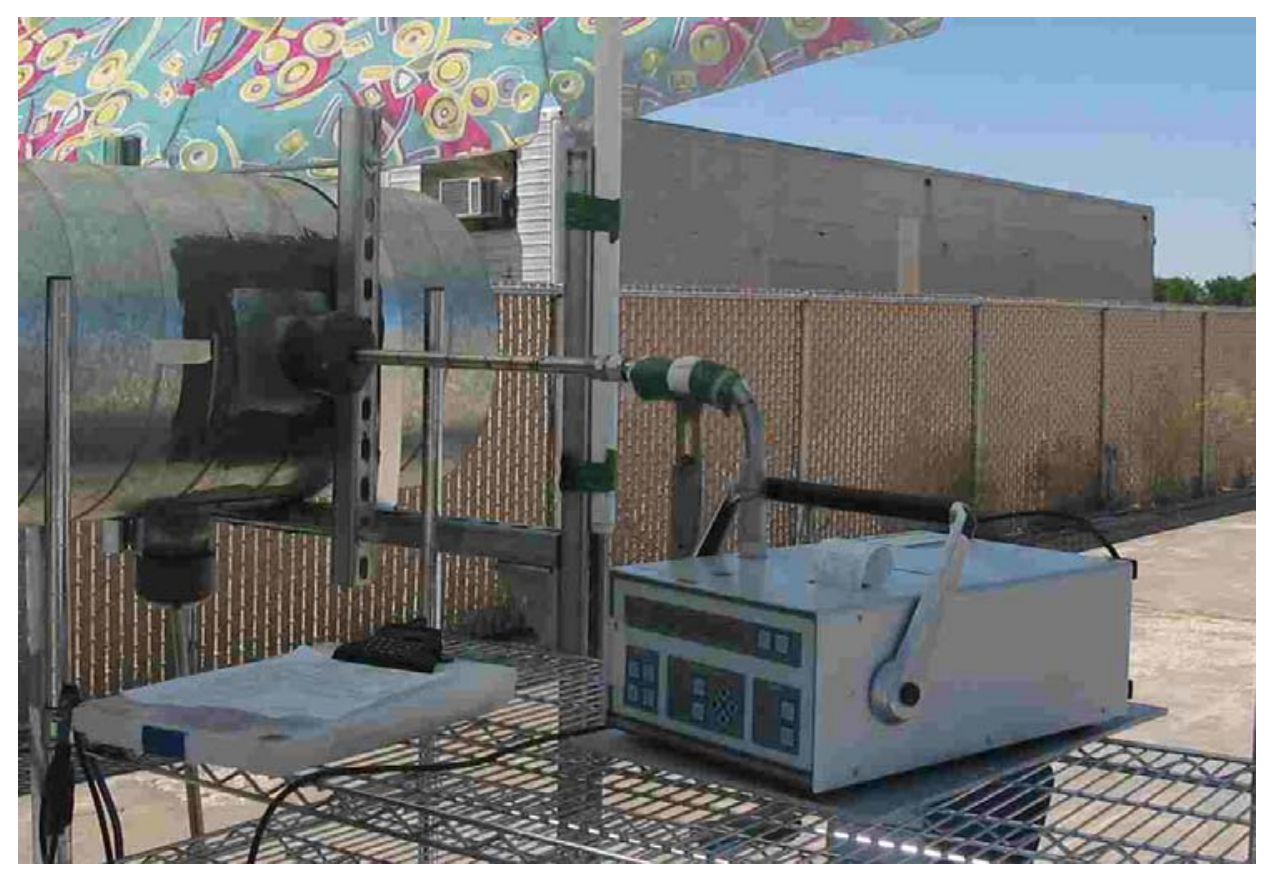

Figure 4.7. Particle Counter Connected to Probe

The tracer aerosol was vacuum pump oil (Fisherbrand $19^{\mathrm{TM}}$ ). It is drawn into a compressed-airdriven spray nozzle and is housed in the chamber shown in Figure 3.6. The chamber provides a means for injecting the particles into the airflow through a probe.

The tracer injection ports were the same as for the gaseous tracer; however, only the centerline injection point was used. The layout of measurement points was the same as for all of the other tests.

The OPCs sort the number of particles into six size channels. Each concentration reading was the count of particles in the 9 - to $11-\mu \mathrm{m}$ channel. The readings were recorded on a data sheet. Three readings were taken at each point and averaged. The coefficient of variance of the average concentration readings at all points was calculated, and the result was compared to the acceptance criterion for uniformity. The particle mixing was acceptable if the COV of the tracer particles of $10-\mu \mathrm{m} \mathrm{AD}$ was less than or equal to $20 \%$ across the center two-thirds of the sampling plane. 
Procedure EMS-JAG-02, "Test to Determine Uniformity of a Tracer Aerosol at a Sampler Probe," shown in Appendix I, was used for this test. 


\subsection{TEST RUNS}

Table 5.1 lists the test matrix where one of each test type was conducted for each fan (and potential source of contamination) combination. There are 10 such combinations.

Only a single test run was required for a given combination for the flow angle, velocity uniformity, and particle-tracer uniformity measurements. However, to completely qualify a monitoring location, ANSI/HPS N13.1-1999 requires that the gas tracer be injected at five positions in the injection cross section, one at a time, along the centerline, and four positions around the perimeter or in the corners of the duct. This could result in five test runs for the gastracer injection, or about 50 gas-tracer runs to cover all of the combinations.

To reduce the time to perform the gas-tracer tests, we performed one gas-tracer injection per combination. After the combination with the worst case of results was identified, then the additional corner injection tests were conducted for that one configuration.

Additional flow angle and velocity uniformity tests were performed using Test Port 3 to compare with the same measurements made on the actual stack. Also, additional flow-measurement tests were performed over the range of fan control settings to obtain an approximate calibration of the fan control setting versus flowrate.

Table 5.1. Minimum Test Runs to be Performed on Scale Model

\begin{tabular}{|c|c|c|c|c|c|}
\hline \multirow[b]{2}{*}{$\begin{array}{c}\text { Fan Where Tracer } \\
\text { Was Injected }\end{array}$} & \multirow[b]{2}{*}{ Other Operating Fans } & \multicolumn{4}{|c|}{ Estimated Number of Test Runs } \\
\hline & & $\begin{array}{l}\text { Flow } \\
\text { Angle }\end{array}$ & Velocity & $\begin{array}{c}\text { Gas } \\
\text { Tracer }\end{array}$ & $\begin{array}{c}\text { Particle } \\
\text { Tracer }\end{array}$ \\
\hline \multicolumn{2}{|c|}{ Same as used on actual stack for validation } & 2 & 2 & & \\
\hline 1 & 2,3 & 1 & 1 & 1 & 1 \\
\hline 2 & 1,3 & 0 & 0 & 1 & 1 \\
\hline 3 & 1,2 & 0 & 0 & 1 & 1 \\
\hline 1 & 4 & 1 & 1 & 1 & 1 \\
\hline 2 & 4 & 1 & 1 & 1 & 1 \\
\hline 3 & 4 & 1 & 1 & 1 & 1 \\
\hline 4 & 1 & 0 & 0 & 1 & 1 \\
\hline 4 & 2 & 0 & 0 & 1 & 1 \\
\hline 4 & 3 & 0 & 0 & 1 & 1 \\
\hline 4 & none & 1 & 1 & 1 & 1 \\
\hline \multicolumn{2}{|c|}{ Worst case gas-tracer combination } & 0 & 0 & 5 & 0 \\
\hline Total & & 7 & 7 & 15 & 10 \\
\hline
\end{tabular}




\subsection{TEST RESULTS}

The results will be presented by each type of test.

\subsection{FAN CONTROLS CORRELATION}

All of the measurements made for this test were done using Test Port 4. The half speed measurements were done prior to any of the other scale model tests. Table 6.1 lists the results. These measurements were repeated later at the speed settings used for the tracer tests, and those results are included in Table 6.1. The flowrates were measured using the same procedure as for the velocity uniformity test. The observed uniformity COV result was also calculated for these tests for comparison with the velocity uniformity tests. The measurements of velocity as a function of control setting are shown in Appendix B.

Table 6.1. Flowrates Measured at Test Port 4 During Control Correlation

\begin{tabular}{|c|c|c|c|c|}
\hline Operating Fans & Run No. & Flowrate cfm & $\begin{array}{c}\text { \% COV with Center- } \\
\text { Point }\end{array}$ & $\begin{array}{c}\text { \% COV Without } \\
\text { Center-Point }\end{array}$ \\
\hline 1-half speed & VT-4 & 751 & 5.4 & - \\
\hline 1-56 Hz & VT-17 & 4.5 & 4.5 \\
\hline 2- half speed & VT-3 & 1411 & 4.3 & - \\
\hline 2-44 Hz & VT-18 & 4.5 & - \\
\hline 3-half speed & VT-2 & 1218 & 4.4 & 8.0 \\
\hline 3-50 Hz & VT-19 & 827 & 7.6 & - \\
\hline 4-half speed & VT-1 & 1401 & 9.2 & 11.0 \\
\hline 4-52 Hz & VT-20 & 1329 & 10.6 & \\
\hline
\end{tabular}

Because of inefficiencies in the scale-model fans, the target flowrates were not always achieved in the other tests, even though settings based on the control calibration indicated that the targets should be met. This did not turn out to be very consequential because the test results are more dependent on the geometry than the flowrate. Table 6.1 shows that there was no significant difference in velocity uniformity when the fans were at half speed versus the settings usually used in the tests.

\subsection{FLOW-ANGLE TEST}

Table 6.2 lists the results for the flow-angle tests performed on the scale model. Tests were done for all fan combinations at Test Port 4 and selected combinations for the other test ports. Figure 6.1 shows a chart of typical results from the scale model.

CH2MHill also tested the actual stack using ports equivalent to the scale model's Test Port 3. Table 6.3 shows the results. Figure 6.2 shows a plot of typical results from these tests. In all cases, the results at the actual stack and with the scale model meet the criterion that the average absolute angle be less than $20^{\circ}$. Appendix F provides the data sheets. 
Table 6.2. Flow Angle Tests on Scale Model

\begin{tabular}{|c|c|c|c|}
\hline Test Port & Operating Fans & Run No. & Avg. Absolute Angle \\
\hline 4 & $1,2,3$ & FA-1 & 5.2 \\
\hline 4 & 1,4 & FA-2 & 6.4 \\
\hline 4 & 2,4 & FA-3 & 5.6 \\
\hline 4 & 3,4 & FA-4 & 6.6 \\
\hline 4 & 4 & FA-5 & 6.8 \\
\hline 4 & 4 & FA-6 & 7.3 \\
\hline 1 & $1,2,3$ & FA-7 & 10.3 \\
\hline 3 & $1,2,3$ & FA-8 & 7.3 \\
\hline 3 & 3,4 & FA-9 & 7.8 \\
\hline
\end{tabular}

Table 6.3. Flow Angle Tests on Actual Stack

\begin{tabular}{|c|c|c|c|}
\hline Test Port & Operating Fans & Run No. & Avg. Absolute Angle \\
\hline 3 equivalent & $1,2,3$ & 1 & 12.4 \\
\hline 3 equivalent & 1,4 & 2 & 10.5 \\
\hline 3 equivalent & 2,4 & 3 & 10.8 \\
\hline 3 equivalent & 3,4 & 4 & 9.9 \\
\hline
\end{tabular}
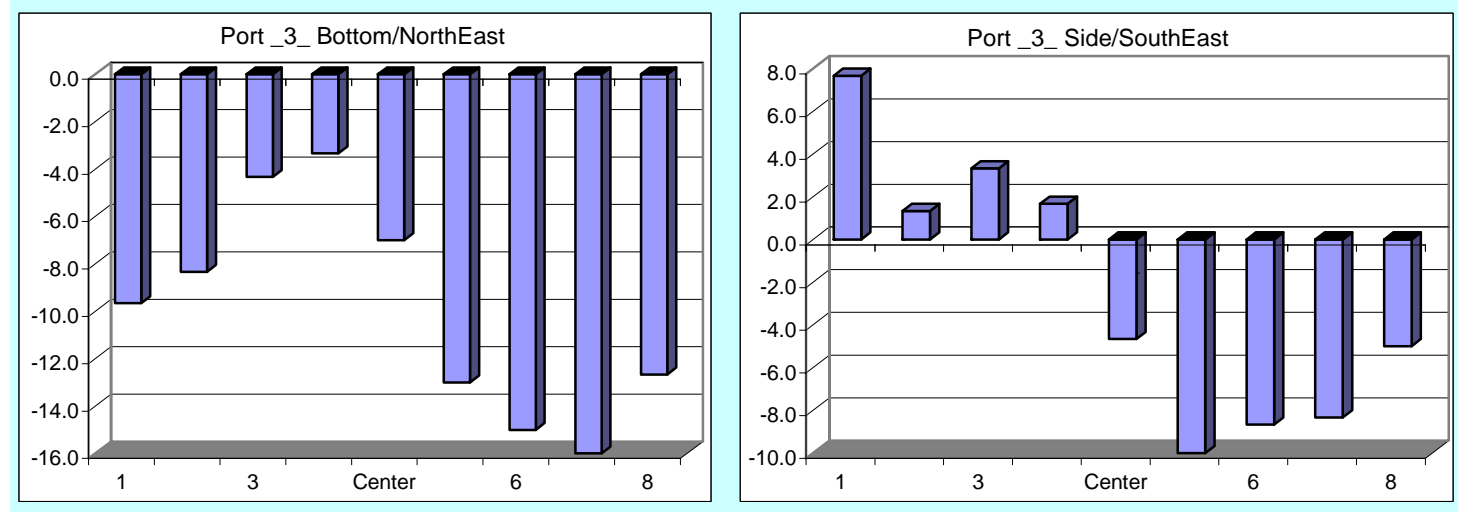

Figure 6.1. Plot of Scale Model Flow Angle Results with Fans 3 and 4 Operating (Run FA-9) 

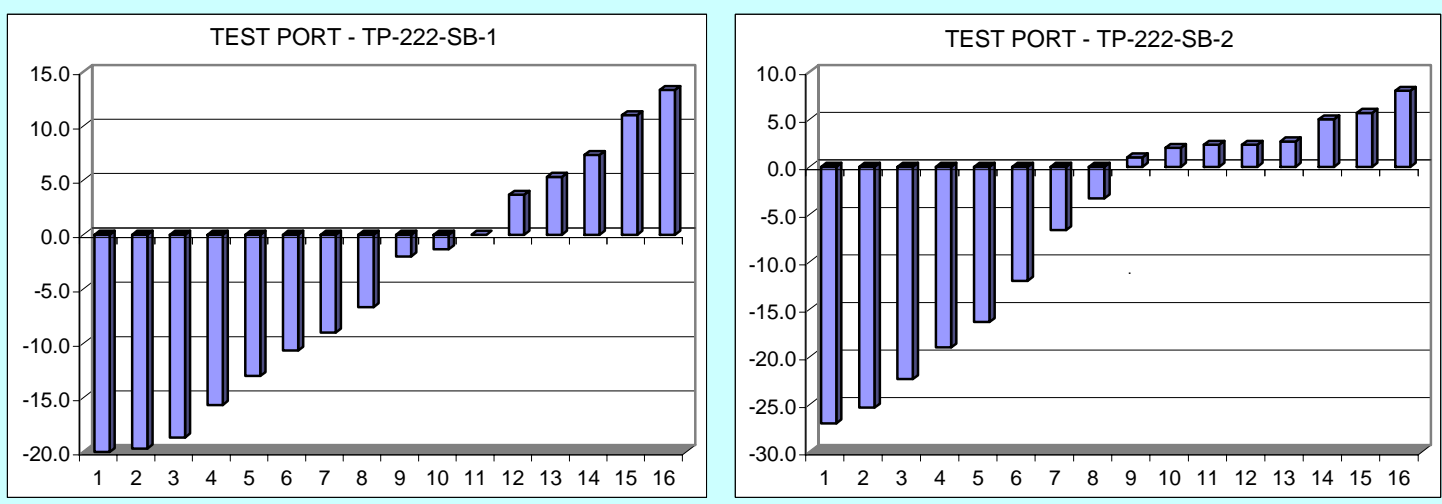

Figure 6.2. Plot of Flow Angle Measurements on Actual Stack, Run 4, Fans 3, 4

\subsection{VELOCITY UNIFORMITY TEST}

\subsubsection{Actual Stack Measurements}

CH2MHill provided velocity uniformity data taken on the actual 296-S-21 stack at an elevation equivalent to the Test Port 3 on the scale model. A 16-point measurement grid per the EPA method was used, and no centerpoint readings were taken. Appendix D shows the results in detail. The \% COV is calculated for the center two-thirds area of the stack, and Table 6.4 summarizes the values. The $<20 \%$ COV criterion is met in these tests.

The COV values in Table 6.4 are those that the scale model results for Test Port 3 should match within 5\% COV units. Figure 6.3 shows a sample plot for when Fans 1, 2, and 3 are operational. Note the artifact of the static mixer where low velocity is found in the middle of the stack in addition to the outer edges. One factor to be aware of is that the values taken at the first couple of points may be biased by the large yaw angles measured at those points as shown in Figure 6.2. However, even if those possible are affected, they do not enter into the calculation of the $\% \mathrm{COV}$ in the center two-thirds of the cross sectional area.

Table 6.4. Velocity Uniformity Tests on Actual Stack

\begin{tabular}{|c|c|c|c|c|}
\hline Operating Fans & Run No. & Flowrate cfm & $\begin{array}{c}\text { Center 2/3 area \% COV } \\
\text { w/o Center-Point }\end{array}$ & Meets COV Criterion \\
\hline $1,2,3$ & 1 & 81331 & 7.2 & Yes \\
\hline 1,4 & 2 & 78668 & 6.3 & Yes \\
\hline 2,4 & 3 & 76964 & 7.9 & Yes \\
\hline 3,4 & 4 & 76983 & 6.7 & Yes \\
\hline
\end{tabular}




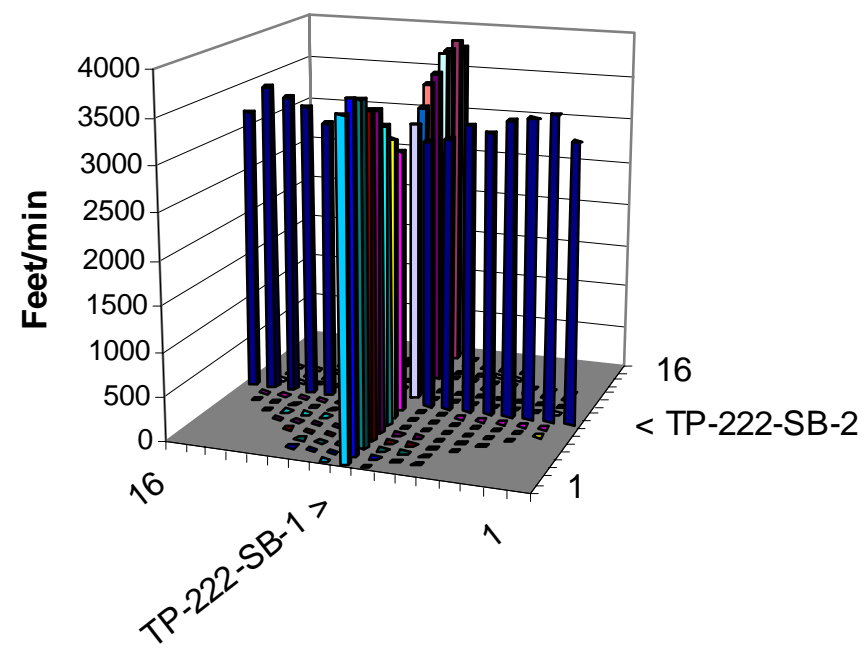

Figure 6.3. Velocity Profile at Actual Stack with Fans 1, 2, and 3 (Run 1)

\subsubsection{Scale Model Measurements}

Several test runs were performed on the scale model using an 8-point traverse grid with the addition of the centerpoint. Generally, the addition of the centerpoint has little impact on the COV results and provides information on whether something interesting happens at the center. Appendix D provides the detailed results for each test. Table 6.5 summarizes the results for the various fan combinations. The tests done at the Test Port 4 simulate the elevation of the sampling probe.

For the 8-point traverse grid, the center two-thirds area excludes the measurement points nearest the wall. The \% COV was calculated for the center two-thirds area both with and without considering the centerpoint measurements. In all cases, the uniformity criterion is met for the location simulating the sampling probe. In addition, one test performed at Test Port 1 shows that the velocity is sufficiently non-uniform to prohibit air sampling that close to the static mixer.

Table 6.5. Velocity Uniformity Tests on Scale Model at Air Sampling Probe Location

\begin{tabular}{|c|c|c|c|c|c|c|}
\hline Test Port & $\begin{array}{c}\text { Operating } \\
\text { Fans }\end{array}$ & Run No. & $\begin{array}{c}\text { Flowrate } \\
\text { cfm }\end{array}$ & $\begin{array}{c}\text { Center 2/3 Area } \\
\text { \% COV with } \\
\text { Center-Point }\end{array}$ & $\begin{array}{c}\text { Center 2/3 Area } \\
\text { \% COV w/o Center- } \\
\text { Point }\end{array}$ & $\begin{array}{c}\text { Meets COV } \\
\text { Criterion }\end{array}$ \\
\hline 1 & $1,2,3$ & VT-7 & 3515 & 72.3 & 42.8 & No \\
\hline 4 & $1,2,3$ & VT-6 & 3443 & 4.8 & 3.7 & Yes \\
\hline 4 & 1,4 & VT-8 & 3298 & 6.1 & 5.9 & Yes \\
\hline 4 & 2,4 & VT-9 & 3029 & 5.2 & 5.3 & Yes \\
\hline 4 & 3,4 & VT-10 & 3146 & 6.8 & 6.4 & Yes \\
\hline 4 & 4 & VT-11 & 2324 & 10.8 & 11.1 & Yes \\
\hline
\end{tabular}


Figure 6.4 shows the plot of the velocity profile at Test Port 4 with Fans 1, 2, and 3 operating.

\subsubsection{Scale Model Validation Measurements}

Several velocity uniformity test runs were also performed at Test Port 3 that simulate the test elevation on the actual stack where measurements were made. Appendix D shows the detailed results from each test, and Table 6.6 summarizes those results. The results where the centerpoint is omitted must be used for comparison against the measurements on the actual stack.

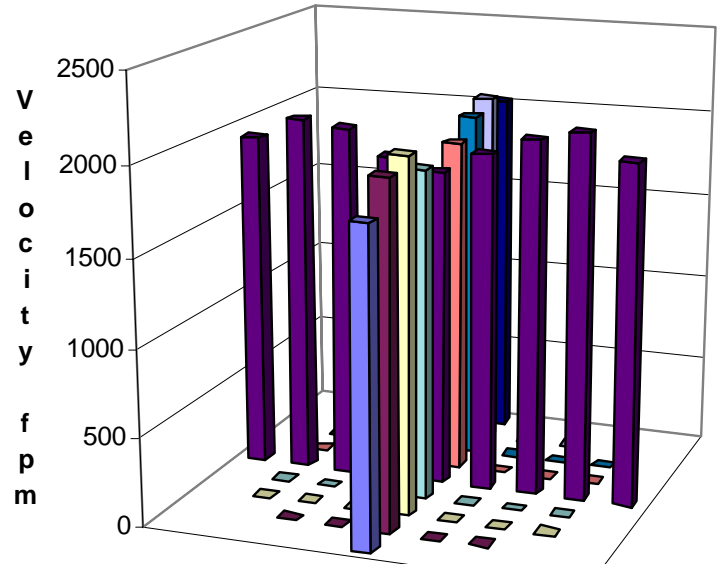

Figure 6.4. Scale Model Velocity Profile with Fans 1, 2, and 3 Operating (Run VT-6)

The difference in the center two-thirds area COV between the actual stack and scale model were calculated, and the individual run results were somewhat mixed with some meeting the $<5 \%$ COV criterion and some slightly exceeding that criterion. In the case of Run VT-12 where Fans 3 and 4 were running, that configuration was repeated twice more using means to more accurately relocate the pitot tube each time. It was then repeated a fourth time with a 16-point traverse, like the actual stack. These modifications to the technique reduced the difference to a $\%$ COV value to within the 5\% criterion. A 16-point traverse was then conducted to repeat Run VT-15 where Fans 1 and 4 were operating. In that case, the difference increased. Overall, the average difference of all the runs in Table 6.6 was $4.8 \% \mathrm{COV}$ and was within the criterion.

Table 6.6. Scale Model Velocity Uniformity Tests Simulating Actual Stack Tests

\begin{tabular}{|c|c|c|c|c|c|c|c|}
\hline $\begin{array}{l}\text { Test } \\
\text { Port }\end{array}$ & $\begin{array}{l}\text { Operating } \\
\text { fans }\end{array}$ & $\begin{array}{l}\text { Run } \\
\text { No. }\end{array}$ & $\begin{array}{c}\text { Flowrate } \\
\text { cfm }\end{array}$ & $\begin{array}{c}\text { Center } 2 / 3 \text { area } \% \\
\text { COV with center- } \\
\text { point }\end{array}$ & $\begin{array}{c}\text { Center } 2 / 3 \text { area } \\
\% \mathrm{COV} \text { w/o } \\
\text { center-point }\end{array}$ & $\begin{array}{l}\text { Actual } \\
\text { stack }\end{array}$ & $\begin{array}{c}\text { Model (-) } \\
\text { Actual } \\
\text { COV }\end{array}$ \\
\hline 3 & $1,2,3$ & VT-5 & 3544 & 13.9 & 10.5 & 7.2 & 3.3 \\
\hline \multirow{2}{*}{3} & \multirow{2}{*}{1,4} & VT-15 & 3721 & 13.9 & 11.4 & \multirow{2}{*}{6.3} & 5.1 \\
\hline & & VT-21 & 3826 & - & 12.4 & & 6.1 \\
\hline 3 & 2,4 & VT-14 & 3435 & 13.4 & 11.0 & 7.9 & 3.1 \\
\hline \multirow{4}{*}{3} & \multirow{4}{*}{3,4} & VT-12 & 3372 & 14.7 & 12.7 & \multirow{4}{*}{6.7} & 6.0 \\
\hline & & VT-13 & 3540 & 14.2 & 11.6 & & 4.9 \\
\hline & & VT-16 & 3665 & 14.7 & 12.1 & & 5.4 \\
\hline & & VT-22 & 3773 & - & 11.3 & & 4.6 \\
\hline
\end{tabular}

\subsection{GAS-TRACER UNIFORMITY TEST}

Several gas-tracer uniformity tests were performed on the scale model at the location corresponding to the elevation of the sampling probe. Test Port 4 is 4.3 stack diameters downstream of the static mixer. Appendix $\mathrm{H}$ provides the detailed results for each run. Table 6.7 summarizes these results. The \% COV was calculated for the measured gas 
concentration at the points in the center two-thirds area of the stack. The percent deviation from the mean concentration was also calculated for any point in the measurement grid.

The tracer was initially injected on the centerline on the injection ports for the fan combinations. The worst case from these tests was when the tracer was injected downstream of Fan 1 when Fans 1, 2, and 3 were used. Additional tests were performed for this configuration with tracer injected in the center of the duct and within $25 \%$ of a hydraulic diameter from the corners.

Figure 6.5 shows a sample plot of results from a gas-tracer test.

Table 6.7. Summarized Results of Gas-Tracer Uniformity Tests at Test Port 4

\begin{tabular}{|c|c|c|c|c|}
\hline $\begin{array}{c}\text { Fan Where Tracer Is } \\
\text { Injected }\end{array}$ & Other Operating Fans & Run No. & $\begin{array}{c}\text { Center 2/3 \% } \\
\text { COV }\end{array}$ & $\begin{array}{c}\text { \% Deviation } \\
\text { from Mean }\end{array}$ \\
\hline 1 center & 2,3 & GT-1 & 4.7 & 8.2 \\
\hline 1 center & 2,3 & GT-11 & 5.2 & 9.4 \\
\hline 1 northwest & 2,3 & GT-13 & 4.2 & -10.2 \\
\hline 1 northeast & 2,3 & GT-12 & 4.8 & -10 \\
\hline 1 southwest & 2,3 & GT-14 & 5.9 & 9.6 \\
\hline 1 southeast & 2,3 & GT-15 & 4.9 & 8.4 \\
\hline 2 center & 1,3 & GT-10 & 3.8 & -6.7 \\
\hline 3 center & 1,2 & GT-9 & 3.3 & -8.3 \\
\hline 1 center & 4 & GT-2 & 3.6 & 7.5 \\
\hline 2 center & 4 & GT-3 & 2.4 & -5.9 \\
\hline 3 center & 4 & GT-4 & 3.2 & -6.8 \\
\hline 4 center & 1 & GT-6 & 1.9 & -4.4 \\
\hline 4 center & 2 & GT-7 & 0.9 & 2.0 \\
\hline 4 center & 3 & GT-5 & 1.8 & 2.6 \\
\hline 4 center & GT- -8 & 0.3 & 0.7 \\
\hline
\end{tabular}

The $\%$ COV results ranged from 0.3 to 5.9. The maximum deviation from the mean concentration observed during a run ranged from 0.7 to -10.2. Therefore, the acceptance criteria were easily met, namely that 1 ) the COV of the measured tracer gas concentration is $\leq 20 \%$ across the center two-thirds of the sampling plane, and 2) at no point in the sampling plane does the concentration vary from the mean by $>30 \%$. The static mixer seems to perform its mixing function by the time the air reaches the sampling point.

While not directly pertaining to meeting performance criteria, two additional tests were performed at Test Ports 1 and 2 to investigate how proximity to the static mixer could effect the mixing of the gas tracer. The results are listed in Table 6.8. These results show that, in this case, a sampling location as close as 3.0 stack diameters to the mixer would also have been acceptable. In contrast, at only 1.5 stack diameters distance, the criterion governing deviation from the mean would not have been met. 
Table 6.8. Results of Gas-Tracer Uniformity Tests at Other Test Ports

\begin{tabular}{|c|c|c|c|c|c|}
\hline $\begin{array}{c}\text { Fan Where } \\
\text { Tracer Is Injected }\end{array}$ & $\begin{array}{c}\text { Other } \\
\text { Operating } \\
\text { Fans }\end{array}$ & $\begin{array}{c}\text { Test ports and } \\
\text { number of stack } \\
\text { diameters from static } \\
\text { mixer }\end{array}$ & Run No. & $\begin{array}{c}\text { Center 2/3 \% } \\
\text { COV }\end{array}$ & $\begin{array}{c}\text { \% Deviation } \\
\text { from Mean }\end{array}$ \\
\hline 1 center & 2,3 & $2(3.0 \mathrm{D})$ & GT-16 & 5.8 & -9.6 \\
\hline 1 center & 2,3 & $1(1.5 \mathrm{D})$ & GT-17 & 13.3 & 35.8 \\
\hline
\end{tabular}

\subsection{PARTICLE TRACER UNIFORMITY TEST}

Several runs of the particle tracer uniformity test were performed with measurements made at Test Port 4, simulating the location of the sampling probe. The tracer aerosol was injected along the duct centerlines downstream of the fans with the various combinations of operating fans. Appendix J gives the detailed results for each run, and Table 6.9 summarizes those results. The $\%$ COV was calculated for the 9- to $11-\mu \mathrm{m}$ diameter particle concentration at the measurement points in the center two-thirds area of the stack.

The output of the aerosol generator was

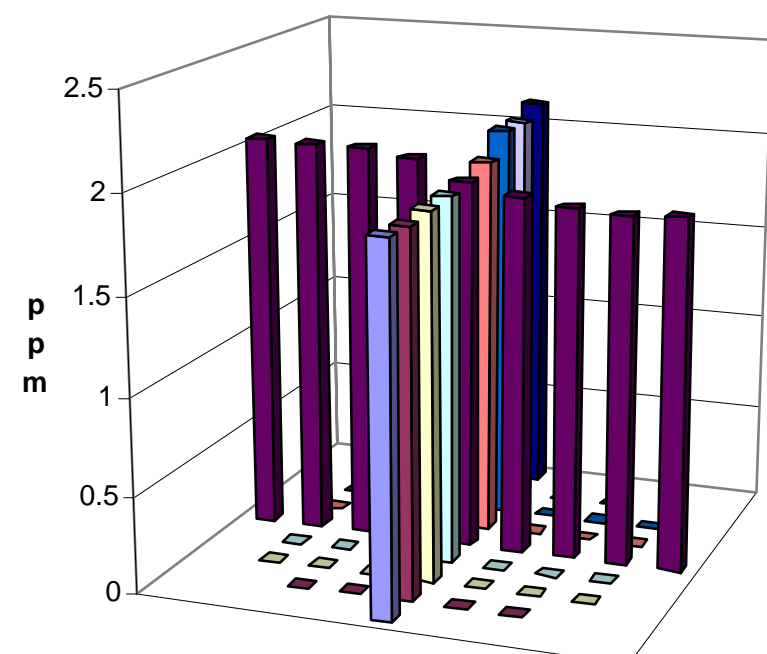

Figure 6.5. Plot of Gas-Tracer Results for Injection Port 1 and Fans 1, 2, and 3 (Run GT-1) varying with time and affecting the results (Runs PT-6, 7 and 9) in some tests. Concentration readings were normalized to equalize the centerpoint values to remove that effect. Table 6.9 shows the COV values both with and without normalization. Cases where the time variation occurred are apparent when the COV values are compared. The runs were completed in the two cases with the most temporal significant effect. Examining these cases (Runs PT 7 and 11, and Runs PT 9, 12, and 13) shows that the normalization method was valid because where the temporal variation did not occur, both the normalized and un-normalized COVs were more consistent.

Figure 6.6 shows the bar chart particle concentration measurements for Run PT-1. The acceptance criteria were easily met, namely that the COV of the measured tracer particles is $\leq 20 \%$ across the center two-thirds of the sampling plane. 
Table 6.9. Summarized Particle Tracer Uniformity Tests

\begin{tabular}{|c|c|c|l|l|l|}
\hline $\begin{array}{c}\text { Fan Where } \\
\text { Tracer Is } \\
\text { Injected }\end{array}$ & $\begin{array}{c}\text { Other } \\
\text { Operating } \\
\text { Fans }\end{array}$ & $\begin{array}{c}\text { Number } \\
\text { Runs }\end{array}$ & \multicolumn{1}{|c|}{ Run No. } & $\begin{array}{c}\text { Un-normalized \% } \\
\text { COV }\end{array}$ & $\begin{array}{c}\text { Normalized } \\
\text { \% COV }\end{array}$ \\
\hline 1 & 2,3 & 2 & PT-1, 14 & $8.8,11.0$ & $6.5,9.0$ \\
\hline 2 & 1,3 & 1 & PT-3 & 5.6 & 5.0 \\
\hline 3 & 1,2 & 1 & PT-4 & 6.7 & 3.4 \\
\hline 1 & 4 & 1 & PT-2 & 9.9 & 7.0 \\
\hline 2 & 4 & 1 & PT-6 & 18.1 & 4.0 \\
\hline 3 & 4 & 1 & PT-5 & 5.7 & 5.2 \\
\hline 4 & 1 & 2 & PT-7, 11 & $27.3,6.7$ & $3.6,4.3$ \\
\hline 4 & 2 & 1 & PT-8 & 6.4 & 3.5 \\
\hline 4 & 3 & 3 & PT-9, 12, 13 & $21.6,4.0,3.6$ & $2.0,3.2,3.4$ \\
\hline 4 & none & 1 & PT-10 & 4.8 & 3.9 \\
\hline
\end{tabular}

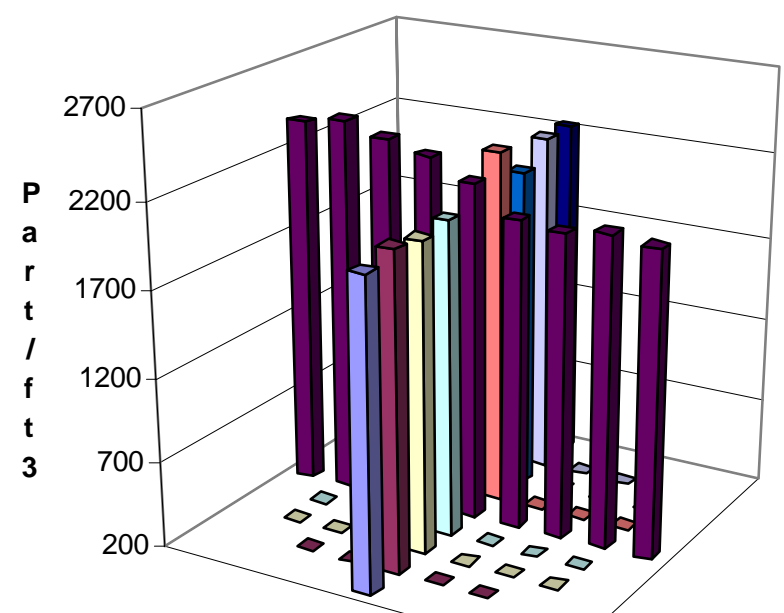

Figure 6.6. Plot of Particle Tracer Concentration for Run PT-1 


\subsection{CONCLUSIONS}

Tests were performed to assess the suitability of the location of the air sampling probe on the 296-S-21 stack according to the criteria of ANSI N13.1-1999, Sampling and Monitoring Releases of Airborne Radioactive Substances From the Stacks and Ducts of Nuclear Facilities. A scale model of the stack was used for most of the tests. As required, $\mathrm{CH} 2 \mathrm{MHill}$ performed some confirmatory tests on the actual stack, and the results compared well with that of the scale model. The tests demonstrated that the location for the air-sampling probe meets all performance criteria for air sampling systems at nuclear facilities. The tests on the scale model were shown to apply to the actual stack as indicated by the comparison of velocity uniformity results. Table 7.1 summarizes the results and conclusions for these tests.

Table 7.1. Conclusions on Air Sampling System Tests

\begin{tabular}{|l|l|c|c|}
\hline \multicolumn{1}{|c|}{ Test } & \multicolumn{1}{|c|}{ Criteria } & Range of results & Meets \\
\hline Flow angle on scale model stack & $<20^{\circ}$ & $5^{\circ}-10^{\circ}$ & Yes \\
\hline Flow angle on actual stack & $<20^{\circ}$ & $10^{\circ}-12^{\circ}$ & Yes \\
\hline $\begin{array}{l}\text { Velocity uniformity on scale } \\
\text { model stack }\end{array}$ & $\mathrm{COV} \leq 20 \%$ & $\begin{array}{c}4-11 \% \mathrm{COV} \text { at probe } \\
10-13 \% \text { at } 1.5 \mathrm{D} \\
\text { upstream of probe }\end{array}$ & Yes \\
\hline $\begin{array}{l}\text { Velocity uniformity on actual } \\
\text { stack }\end{array}$ & $\mathrm{COV} \leq 20 \%$ & $\begin{array}{c}6-8 \% \text { at } 1.5 \mathrm{D} \text { upstream } \\
\text { of probe }\end{array}$ & Yes \\
\hline Velocity uniformity comparison, & Agree within $+/-5 \% \mathrm{COV}$ & $\begin{array}{c}3.3-6.1 \%(4.8 \% \text { on } \\
\text { average })\end{array}$ & Yes \\
\hline $\begin{array}{l}\text { Gas-tracer uniformity on scale } \\
\text { model stack }\end{array}$ & $\begin{array}{l}\mathrm{COV} \leq 20 \% \text { in center } 2 / 3 \text { of } \\
\text { stack } \\
\leq 30 \% \text { maximum deviation } \\
\text { from mean }\end{array}$ & $\begin{array}{c}0.3-6 \% \mathrm{COV} \\
-10 \% \text { deviation from mean }\end{array}$ & Yes \\
\hline $\begin{array}{l}\text { Particle tracer uniformity on scale } \\
\text { model stack. } \\
\text { Time-normalized results. }\end{array}$ & $\begin{array}{l}\mathrm{COV} \leq 20 \% \text { in center } 2 / 3 \text { of } \\
\text { stack }\end{array}$ & $2-9 \% \mathrm{COV}$ & Yes \\
\hline
\end{tabular}




\subsection{REFERENCES}

40 CFR 60, Appendix A, Method 1. "Method 1 - Sample and Velocity Traverses for Stationary Sources." U.S. Environmental Protection Agency, Code of Federal Regulations, as amended

American National Standards Institute (ANSI). 1999. Sampling and Monitoring Releases of Airborne Radioactive Substances From the Stacks and Ducts of Nuclear Facilities. ANSI/HPS N13.1 - 1999, American National Standards Institute, New York. 


\section{Appendix A}

Flow Calibration Procedure 
APPENDIX A: FLOW CALIBRATION PROCEDURE

\begin{tabular}{|c|c|c|c|}
\hline \multicolumn{4}{|l|}{ PNNL Operating Procedure } \\
\hline $\begin{array}{l}\text { Title: Test to Calibrate } \\
\text { Ventilation Flow } \\
\text { Controller }\end{array}$ & $\begin{array}{l}\text { Org. Code: } \\
\text { Procedure No.: } \\
\text { Rev. No.: }\end{array}$ & $\begin{array}{l}\text { D7E } \\
\text { EMS }\end{array}$ & \\
\hline Work Location: General & Effective Date: & \multicolumn{2}{|c|}{ April 28, 2006} \\
\hline Author: John A. Glissmeyer & Supersedes Date: & \multicolumn{2}{|c|}{ November 18, 1998} \\
\hline $\begin{array}{l}\text { Identified Hazards: } \\
\square \text { Radiological } \\
\square \text { Hazardous Materials } \\
\text { 囚 Physical Hazards } \\
\square \text { Hazardous Environment } \\
\square \text { Other: }\end{array}$ & \multicolumn{3}{|c|}{$\begin{array}{c}\text { Identified Use Category: } \\
\square \text { Mandatory Use } \\
\square \text { Continuous Use } \\
\text { 冈 Reference Use } \\
\square \text { Information Use }\end{array}$} \\
\hline \multicolumn{4}{|c|}{ Are One-Time Modifications Allowed? $\square$ Yes $\quad$ No } \\
\hline Person Signing & \multicolumn{2}{|c|}{ Signature } & Date \\
\hline \multicolumn{4}{|l|}{$\begin{array}{l}\text { Technical review: } \\
\text { J. Matthew Barnett }\end{array}$} \\
\hline \multicolumn{4}{|l|}{$\begin{array}{l}\text { Project Manager: } \\
\text { John Glissmeyer }\end{array}$} \\
\hline \multicolumn{4}{|l|}{$\begin{array}{l}\text { Line Manager: } \\
\text { James Droppo }\end{array}$} \\
\hline $\begin{array}{l}\text { Quality Engineer: } \\
\text { Barry L. Sachs }\end{array}$ & & & \\
\hline
\end{tabular}




\subsection{Purpose}

The performance of new stack sampling systems must be shown to satisfy the requirements of 40 CFR 61, Subpart H, "National Emission Standards for Emissions of Radionuclides Other than Radon from Department of Energy Facilities." This regulation governs portions of the design and implementation of effluent air sampling. The stack sampler performance is adequately characterized when potential contaminants in the effluent are of a uniform concentration at the sampling location (plane) and line losses are within acceptable limits. This procedure is used when needed to facilitate testing these characteristics. This procedure is a means to quickly correlate flow control device settings with the ventilation flowrate in a stack or duct. This correlation is determined prior to other tests of the stack monitoring system. This correlation makes it easier to set the flow control to achieve repeatable values of flowrate. Other procedures that may follow address flow angle, uniformity of gas velocity, and uniformity of gas and aerosol contaminants.

\subsection{Applicability}

This procedure can be used for tests on actual or scale model stacks and ducts to determine the correlation between stack flow control settings and the measured stack flowrate. The tests are applicable within the following constraints:

- The flowrate range covered in the test includes the target flowrate range outlined in the applicable test plan.

- The operating limits of the air velocity measurement device used.

This procedure may need to be repeated if there are significant changes made in the ventilation system or loading of the ventilation filters during the conduct of the overall test plan.

\subsection{Prerequisites and Conditions}

Conditions and concerns that must be satisfied prior to performing this procedure are listed below:

- The job-hazards analysis for the work area must be followed.

- Special training may be required to access the test ports.

- The flow control device must be installed and means available for its adjustment.

- Air velocity measurement equipment must be within calibration.

- The Test Instruction must be read and understood.

- This procedure must be read and understood.

\subsection{Precautions and Limitations}

Access to the test ports may require the use of ladders, scaffolding or manlifts, which may necessitate special training for sampling personnel and any observers. The training requirements will be indicated in the job hazard analysis. 


\subsection{Equipment Used for Measurements}

The following are essential items of equipment:

- Calibrated electronic air velocity sensor; or a pitot tube compliant with acceptable design and a calibrated slant tube or electronic manometer to read the pitot tube differential pressure,

- To provide information about the test conditions, commercial grade sensors for stack temperature, barometric pressure, static pressure, and humidity provide acceptable information. Likewise, data from a nearby meteorology or facility station is acceptable.

- Platform, ladders, or manlifts as needed to access the test ports,

- Fittings to limit leakage around the velocity sensor and to stabilize it sufficiently so it can be re-positioned for repeatability.

\subsection{Work Instructions for Setup, Measurements, and Data Reduction}

Job specific instructions given in the Test Instruction, illustrated in Exhibit A, will provide specific details and operating parameters necessary to perform this procedure.

\subsection{Preliminary Steps}

6.1.1 Verify that the interior dimensions of the stack or duct at the measurement location agree with those used in calculating the grid of measurement points given in the Test Instruction or data form. The measurement location should be approximately the same as the air sampling nozzle inlet.

Note. The grid of velocity measurement points is calculated in accordance with 40 CFR 60, Appendix A, Method 1. A center point is also added.

6.1.2 Provide essential supplies at the sampling location. (velocity sensor or pitot tube, manometer, connecting tubing, fittings to adapt pitot tube to the test ports, marking pens, data forms, writing and pitot tube supporting platforms).

6.1.3 Verify that the flow control device is capable of the flow control settings given in the Test Instruction, particularly that setting to be used for the detailed velocity traverse.

6.1.4 Prepare a spreadsheet for calculating results and plotting data. See the illustration in Exhibit B. Label the columns of traverse data by the 
direction of the traverse. For example, if the first reading is closest to the east port, and the last reading is closest to the west port, then label the traverse east-west. Print blank copies of the spreadsheet as data forms for hand recording measurements for later transfer to the spreadsheet.

6.1.5 Mark the pitot tube or velocity sensor for each point in the measurement grid. Use a permanent marker so the inlet can be placed at each successive measurement point.

6.1.6 Obtain barometric pressure, stack static pressure, stack air temperature, and stack air relative humidity information for the flow measurement location.

6.1.7 If a pitot tube is used, attach the manometer to the pitot tube. Insert the pitot tube or velocity sensor in the stack and seal the opening around it.

\subsection{Flow Measurement}

6.2.1 Set the flow controller as instructed for the detailed velocity traverse.

6.2.2 Verify that the directional orientations and the numbered sample positions are consistent with the data form.

6.2.3 Measure and record, on the data form, the velocity reading at each measurement point in succession. If the readout device has an averaging feature, record the average of a series of several readings.

6.2.4 Repeat Step 6.2.3 two more times for a total of three measurements at each point.

6.2.5 Calculate the average air velocity at each grid point and then for the entire grid, by hand or using the spreadsheet, and identify the point(s) where the velocity most nearly equals the average.

- Review the data forms for completeness.

- Sign and date the data forms attesting to having completed the data collection portion of the procedure. 


\subsection{Estimated Flow at Other Settings}

6.3.1 Prepare a data form for recording average air velocity measured over the range of flow control settings. (See Exhibit C.)

6.3.2 Place the velocity sensor or pitot tube at the point of average velocity as determined in Step 6.2.5.

6.3.3 Record the velocity reading for each flow controller setting specified in the Test Instruction.

6.3.4 Repeat Step 6.3.3 two more times for a total of three replicate measurements at each flow setting.

6.3.5 Review the data forms for completeness.

6.3.6 Sign and date the data forms attesting to having completed this portion of the procedure.

\subsection{Calculations}

6.4.1 Perform the following calculations using a spreadsheet as illustrated in Attachment B

6.4.2 Calculate the mean velocity at each flow control setting

6.4.3 Calculate the cross sectional area of the stack or duct where the flow measurements were taken

6.4.4 Calculate the approximate flowrate at each flow control setting

6.4.5 Plot the measured velocity and flow versus flow control setting as illustrated in Attachment B

6.4.6 Have the data transfers and calculations reviewed and verified, including those done in step 6.2.5. The reviewer should sign the finished data forms.

\subsection{Records}

7.1 Transfer the original data forms and calculations to the records custodian as project records 


\section{Exhibits/Attachments}

Exhibit A - Typical Test Instruction

\begin{tabular}{|c|c|c|}
\hline \multicolumn{3}{|c|}{ Test Instruction } \\
\hline $\begin{array}{l}\text { Project: Model Stack } \\
\text { Calibration } 28361\end{array}$ & Date: December 25, 2006 & Work Package: K83017 \\
\hline \multicolumn{3}{|c|}{ Tests: Calibration of Ventilation Flow Controller for Model Full-Scale Stack } \\
\hline \multicolumn{3}{|l|}{ Staff: David Maughan } \\
\hline \multicolumn{3}{|c|}{$\begin{array}{l}\text { Operating Manual for Solomat Zephyr } \\
\text { Procedure EMS-JAG-03 Test to Calibrate Ventilation Flow Controller. }\end{array}$} \\
\hline \multicolumn{3}{|c|}{$\begin{array}{l}\text { Full-Scale Model Stack, Fan and Fan Speed Controller located in ELF Bldg. } \\
\text { Solomat Zephyr and pitot tube }\end{array}$} \\
\hline \multicolumn{3}{|c|}{$\begin{array}{l}\text { Safety Considerations: } \\
\text { Review and observe the applicable Workshop Job Hazard Analysis for the project }\end{array}$} \\
\hline $\begin{array}{l}\text { Instructions: } \\
\text { 1. Assemble the } \\
\text { elevation of the sampl } \\
2 . \quad \text { Layout the me } \\
\text { stack wall: } 0.5,0.66,1 \\
3 . \quad \text { Measure the ve } \\
\text { measurement thrice. } \\
4 . \quad \text { Record data on } \\
5 . \quad \text { Identify point } \\
6 . \quad \text { Mount pitot tu } \\
\text { over the } 5 \text { - } 60 \text { Hz ran } \\
7 . \quad \text { Record and pld } \\
6 .\end{array}$ & $\begin{array}{l}\text { ent for the flow controller c } \\
\text { be } \\
\text { ent points with the followin } \\
04,3.16,4.28,5.10,5.66,5 \\
\text { at each point with the flow } \\
\text { ty data forms } \\
\text { age velocity } \\
\text { at point and measure veloci } \\
\text { ata } \\
\text { nting fixtures and retain ass }\end{array}$ & $\begin{array}{l}\text { bration test at the ports at the } \\
\text { listances from the inside of the } \\
\text { inches } \\
\text { atroller set at } 30 \mathrm{~Hz} \text {. Repeat each }\end{array}$ \\
\hline \multicolumn{3}{|c|}{ Desired Completion Date: } \\
\hline \multicolumn{3}{|l|}{ Approvals: } \\
\hline Test completed by: & & Date: \\
\hline
\end{tabular}




\section{Exhibit B - Typical Velocity Traverse Data Form \\ VELOCITY TRAVERSE DATA FORM}

Site Model

Date Oct. 16, 2001

Testers Glissmeyer and Maughan Stack Dia.

Stack X-Area Elevation

Distance to disturbance

Velocity units $\mathrm{ft} / \mathrm{min}$
Run No. VT-1

Fan Configuration 4-fan: EF1, EF4, EF5, EF7

Fan Setting $51 \mathrm{~Hz}$

Stack Temp

Start/End Time 1432-1530 hours

Center $2 / 3$ from 21.34

Points in Center $2 / 3 \longdiv { 2 }$ to: $\underline{7}$

Data Files: NA

\begin{tabular}{|c|c|c|c|c|c|c|c|c|c|}
\hline \multirow{2}{*}{\multicolumn{2}{|c|}{$\begin{array}{l}\text { Traverse--> } \\
\text { Trial ----> }\end{array}$}} & \multicolumn{4}{|c|}{ West } & \multicolumn{4}{|c|}{ North } \\
\hline & & 1 & 2 & 3 & Mean & 1 & 2 & 3 & Mean \\
\hline CorrectLabel & Depth, in. & \multicolumn{4}{|c|}{ Velocity } & \multicolumn{4}{|c|}{ Velocity } \\
\hline 1 & 0.75 & 2044 & 1990 & 1967 & 2000.3 & 1603 & 1611 & 1734 & 1649.3 \\
\hline 2 & 2.47 & 2058 & 2067 & 2069 & 2064.7 & 1768 & 1877 & 1825 & 1823.3 \\
\hline 3 & 4.56 & 2036 & 2067 & 1941 & 2014.7 & 1849 & 1830 & 1859 & 1846.0 \\
\hline 4 & 7.59 & 1892 & 1965 & 1855 & 1904.0 & 1811 & 1723 & 1823 & 1785.7 \\
\hline Center & 11.75 & 1868 & 1808 & 1793 & 1823.0 & 1783 & 1892 & 1769 & 1814.7 \\
\hline 5 & 15.91 & 1982 & 1875 & 1892 & 1916.3 & 1997 & 1998 & 2070 & 2021.7 \\
\hline 6 & 18.94 & 1972 & 2016 & 2009 & 1999.0 & 2193 & 2220 & 2157 & 2190.0 \\
\hline 7 & 21.03 & 2096 & 2181 & 2099 & 2125.3 & 2241 & 2235 & 2258 & 2244.7 \\
\hline 8 & 22.75 & 1960 & 1978 & 1987 & 1975.0 & 2193 & 2132 & 2174 & 2166.3 \\
\hline Averages --- & $--->$ & 1989.8 & 1994.1 & 1956.9 & 1980.3 & 1937.6 & 1946.4 & 1963.2 & 1949.1 \\
\hline
\end{tabular}

\begin{tabular}{|c|c|c|c|c|c|c|}
\hline AII & $\mathrm{ft} / \mathrm{min}$ & Dev. from mean & Center 2/3 & West & North & All \\
\hline Mean & 1964.7 & & Mean & 1978.1 & 1960.9 & 1969.5 \\
\hline Min Point & 1649.3 & $-16.1 \%$ & Std. Dev. & 103.5 & 191.9 & 148.4 \\
\hline Max Point & 2244.7 & $14.3 \%$ & cov as $\%$ & 5.2 & 9.8 & 7.5 \\
\hline
\end{tabular}

Flow w/o C-Pt Vel Avg w/o C-Pt

Stack temp

Equipment temp Ambient temp

Stack static

Ambient pressure

Total Stack pressure

Ambient humidity

\begin{tabular}{|c|c|c|}
\multicolumn{1}{c|}{ Start } & Finish & \multicolumn{1}{c}{} \\
\hline 72.5 & 70.8 & $\mathrm{~F}$ \\
\hline 71.6 & 75 & $\mathrm{~F}$ \\
\hline 70 & 70.7 & $\mathrm{~F}$ \\
\hline 0.5 & 0.52 & mbars \\
\hline 986.3 & 985.8 & mbars \\
\hline 986.8 & 986.3 & mbars \\
\hline $38 \%$ & $36 \%$ & $\mathrm{RH}$ \\
\hline
\end{tabular}

Notes:

Initial trial run to evaluate stack and instrument configuration.

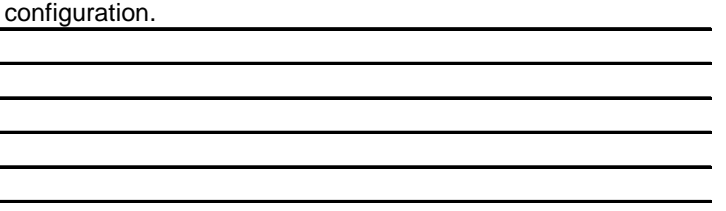

\section{Instuments Used:}

Pitot \#5, 36-in. standard

Solmat Zephyr SN 12951472 Cal. Due 7/26/02

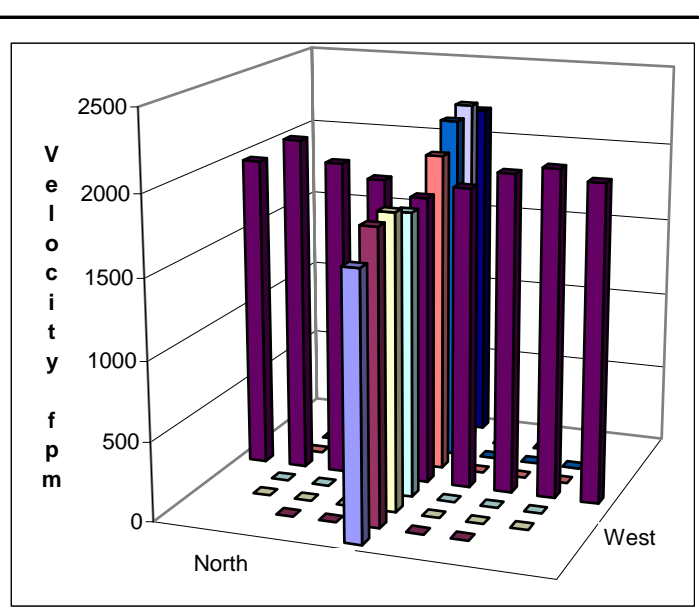

Signature signifies compliance with

Signature verifying data and calculations:

Procedure EMS-JAG-4

Signature/date 


\section{Exhibit C - Typical Velocity vs. Flow Controller Setting Data Form VELOCITY vs. FREQUENCY DATA FORM}

\begin{tabular}{|c|c|c|c|c|c|c|c|}
\hline & \multirow{2}{*}{$\begin{array}{l}\text { Site } \\
\text { Date }\end{array}$} & \multicolumn{2}{|c|}{ Model } & \multicolumn{4}{|c|}{ Run No. VT-1 } \\
\hline & & & \multirow{2}{*}{\multicolumn{2}{|c|}{$\begin{array}{l}\text { Stack Temp } \\
\text { Stack RH\% }\end{array}$}} & 75 & $\operatorname{deg} \mathrm{F}$ \\
\hline & \multicolumn{2}{|c|}{ Glissmeyer } & & & & \multicolumn{2}{|c|}{$35 \%$} \\
\hline & \multicolumn{3}{|c|}{ Stack Dia. 18} & \multirow{3}{*}{\multicolumn{2}{|c|}{$\begin{array}{r}\text { Baro Press } \\
\text { Fan Configuration }\end{array}$}} & 1000 & mbar \\
\hline \multirow{2}{*}{\multicolumn{4}{|c|}{$\begin{array}{r}\text { Stack X-Area } \frac{254.5}{\text { Elevation }} \\
100 \text { in. }\end{array}$}} & & & \multicolumn{2}{|c|}{ Fan 2} \\
\hline & & & & \multirow{2}{*}{\multicolumn{2}{|c|}{$\begin{array}{l}\text { Start/End Time } \\
\text { Reference point fron }\end{array}$}} & \multicolumn{2}{|c|}{$1430 / 1532$} \\
\hline El. above & sturbance & & inches & & & $n$ velocity & est VT \\
\hline \multicolumn{4}{|c|}{ Velocity Readings, units = } & & & & \\
\hline & & & & & $\begin{array}{r}\text { Target } \\
\mathrm{cfm}\end{array}$ & $\begin{array}{r}\text { Target } \\
\mathrm{fpm}\end{array}$ & $\begin{array}{r}\text { Estmtd } \\
\mathrm{Hz}\end{array}$ \\
\hline & & & & & 5800 & 1926 & 50 \\
\hline & \multirow{2}{*}{\multicolumn{3}{|c|}{ fpm }} & & 5900 & 1960 & 52 \\
\hline $\mathrm{Hz}$ & & & & Mean & StDev & 2 StDev & cfm \\
\hline 5 & 122 & 100 & 112 & 111.33 & 11.02 & 22.03 & 196.74 \\
\hline 10 & 230 & 252 & 236 & 239.33 & 11.37 & 22.74 & 422.94 \\
\hline 15 & 350 & 349 & 360 & 353.00 & 6.08 & 12.17 & 623.80 \\
\hline 20 & 450 & 452 & 445 & 449.00 & 3.61 & 7.21 & 793.45 \\
\hline 25 & 550 & 560 & 555 & 555.00 & 5.00 & 10.00 & 980.77 \\
\hline 30 & 650 & 655 & 652 & 652.33 & 2.52 & 5.03 & 1152.77 \\
\hline 35 & 750 & 745 & 758 & 751.00 & 6.56 & 13.11 & 1327.13 \\
\hline 40 & 850 & 846 & 845 & 847.00 & 2.65 & 5.29 & 1496.77 \\
\hline 45 & 950 & 956 & 940 & 948.67 & 8.08 & 16.17 & 1676.43 \\
\hline 50 & 1050 & 1043 & 1062 & 1051.67 & 9.61 & 19.22 & 1858.45 \\
\hline 55 & 1150 & 1152 & 1135 & \begin{tabular}{|l|}
1145.67 \\
\end{tabular} & 9.29 & 18.58 & 2024.56 \\
\hline 60 & 1250 & 1235 & 1248 & \begin{tabular}{|l|}
1244.33 \\
\end{tabular} & 8.14 & 16.29 & 2198.92 \\
\hline
\end{tabular}

Run No. VT-1

Instuments Used:

Cal Exp. Date:

Solmat Zephyr SN 12951472

$7 / 26 / 2006$

Pitot \#5, 36-in. standard

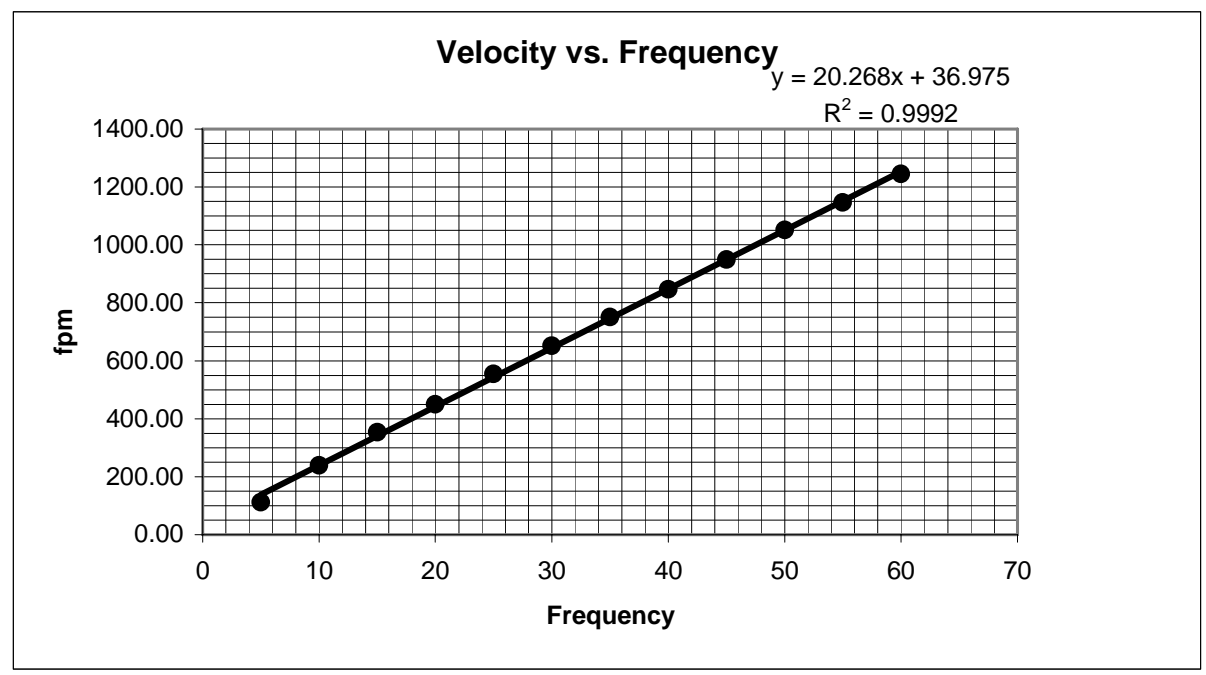

Signature signifies compliance with Procedure EMS-JAG-3

Signature verifying data and calculations:

Signature/date 


\section{Appendix B}

\section{Flow Calibration Data Sheets}




\section{APPENDIX B: FLOW CALIBRATION DATA SHEETS}

\begin{tabular}{|c|c|c|c|c|c|c|c|c|c|}
\hline & & & DCITY & JERSE D & DATA FORM & & & & \\
\hline & Site & 296-S-21 Mo & & & Run No. & VT-1 & & & \\
\hline & Date & $7 / 12 / 2006$ & & Fan C & onfiguration & EF4 & no prefilter & & \\
\hline & Testers & Brad Fritz \& & ohn Glissme & & Fan Setting & 30 & $\mathrm{~Hz}$ & & \\
\hline & Stack Dia. & $177 / 8$ & & & Stack Temp & 79.5 & $\operatorname{deg} F$ & & \\
\hline & tack X-Area & 250.9 & & Sta & rt/End Time & $10: 20 / 11: 17$ & & & \\
\hline & Elevation & N.A. & & Cen & ter $2 / 3$ from & 1.64 & to: & 16.23 & \\
\hline Distance to & disturbance & $773 / 4$ & nches & Points in & Center $2 / 3$ & 2 & to: & 7 & \\
\hline & elocity units & $\mathrm{ft} / \mathrm{min}$ & & & Data Files: & $\mathrm{NA}$ & & & \\
\hline Traverse--> & & & Port_4_Bo & tom/East & & & Port_4_s & Side/South & \\
\hline Trial ----> & & 1 & 2 & 3 & Mean & 1 & 2 & 3 & Mean \\
\hline Point & Depth, in. & & Veloc & & & & & ocity & \\
\hline 1 & 0.58 & 589 & 630 & 619 & 612.7 & 741 & 721 & 669 & 710.3 \\
\hline 2 & 1.89 & 689 & 692 & 675 & 685.3 & 773 & 754 & 759 & 762.0 \\
\hline 3 & 3.49 & 688 & 702 & 682 & 690.7 & 754 & 752 & 726 & 744.0 \\
\hline 4 & 5.81 & 691 & 695 & 647 & 677.7 & 720 & 697 & 700 & 705.7 \\
\hline Center & 9.00 & 747 & 689 & 743 & 726.3 & 715 & 724 & 687 & 708.7 \\
\hline 5 & 12.19 & 779 & 824 & 759 & 787.3 & 751 & 772 & 785 & 769.3 \\
\hline 6 & 14.51 & 844 & 839 & 844 & 842.3 & 859 & 874 & 836 & 856.3 \\
\hline 7 & 16.11 & 877 & 835 & 869 & 860.3 & 845 & 896 & 876 & 872.3 \\
\hline 8 & 17.42 & 805 & 846 & 806 & 819.0 & 793 & 858 & 768 & 806.3 \\
\hline verages ---- & ----- & 745.4 & 750.2 & 738.2 & 744.6 & 772.3 & 783.1 & 756.2 & 70.6 \\
\hline
\end{tabular}

\begin{tabular}{|ll}
\hline AlI & $\mathrm{ft} / \mathrm{min}$ \\
Mean & 757.6 \\
Min Point & 612.7 \\
Max Point & 872.3 \\
\hline
\end{tabular}

Flow w/o C-Pt Vel Avg w/o C-Pt

Stack temp Equipment temp Ambient temp Stack static Ambient pressure Total Stack pressure Ambient humidity

\begin{tabular}{|c|c|c|}
\hline \multicolumn{1}{|c|}{ Start } & Finish & \\
\hline 78 & 81 & $F$ \\
\cline { 1 - 2 } N.A. & N.A. & F \\
\hline 77 & 80 & F \\
\hline 0.00 & 0.00 & mbars \\
\hline 29.4 & 29.4 & in. Hg \\
\cline { 1 - 2 } 996.3 & 996.3 & mbars \\
\cline { 1 - 2 } $36 \%$ & $36 \%$ & RH \\
\hline
\end{tabular}

Notes: Horizontal diam. 17-7/8 in. Vertical diam. 17-7/8 in.

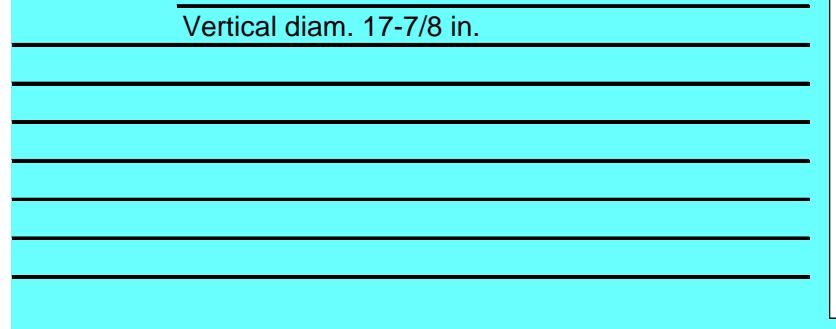
Dev. from mean Center 2/3 Mean
$-19.1 \%$ Std. Dev. $15.1 \%$ COV as $\%$

$\begin{array}{r}\text { East } \\ 752.9 \\ 77.0 \\ 10.2 \\ \hline\end{array}$

\begin{tabular}{rr} 
South & All \\
\hline 774.0 & 763.5 \\
66.4 & 69.9 \\
8.6 & $\mathbf{9 . 2}$ \\
\hline
\end{tabular}

Instuments Used:

Temp > TSI 8360 SN $209060 \mathrm{Cal}$ due 9/27/06

Vel >> Solomat Zephyr SN 12951472 Cal due 8/29/06 Pitot \# 5 -- 36" Standard Pitot Dwyer

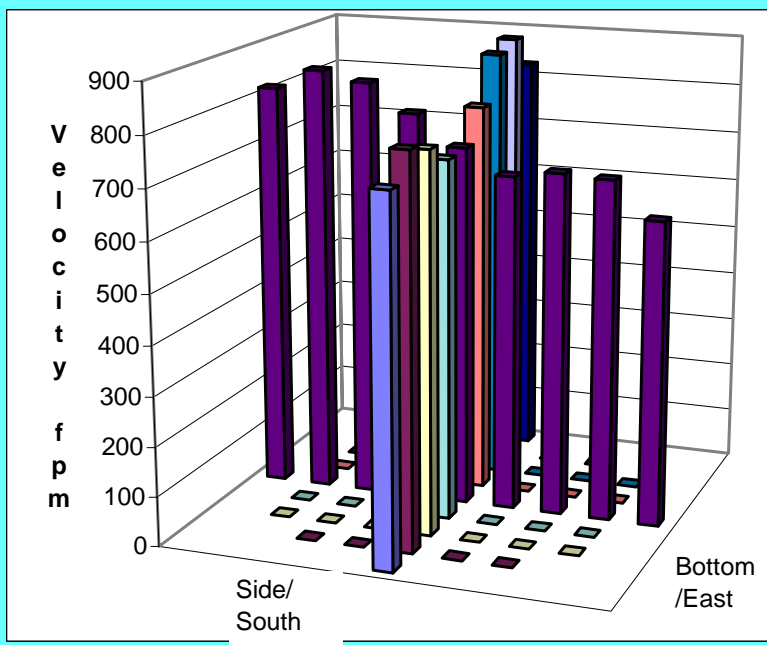

Signature signifies compliance with Procedure EMS-JAG-4 Signature/date
Signature verifying data and calculations:

Signature on file copy 


\section{VELOCITY TRAVERSE DATA FORM}

Site 296-S-21 Model

Date 7/12/2006

Testers Brad Fritz \& John Glissmeyer Stack Dia.

Stack X-Area $177 / 8$ in

Elevation N.A.

Distance to disturbance

Velocity units $\underline{\mathrm{ft} / \mathrm{min}}$
Run No. VT-2

Fan Configuration EF3

Fan Setting $\mathbf{3 0}$

Stack Temp

Start/End Time 11:30/12:15

Center $2 / 3$ from

Points in Center $2 / 3$

Data Files: NA

\begin{tabular}{|c|c|c|c|c|c|c|c|c|c|}
\hline \multirow{2}{*}{\multicolumn{2}{|c|}{$\begin{array}{l}\text { Traverse--> } \\
\text { Trial ----> }\end{array}$}} & \multicolumn{4}{|c|}{ Port_4__Bottom/East } & \multicolumn{4}{|c|}{ Port_4_Side/South } \\
\hline & & 1 & 2 & 3 & Mean & 1 & 2 & 3 & Mean \\
\hline Point & Depth, in. & \multicolumn{4}{|c|}{ Velocity } & \multicolumn{4}{|c|}{ Velocity } \\
\hline 1 & 0.58 & 433 & 408 & 374 & 405.0 & 383 & 420 & 480 & 427.7 \\
\hline 2 & 1.89 & 440 & 448 & 451 & 446.3 & 480 & 460 & 479 & 473.0 \\
\hline 3 & 3.49 & 454 & 438 & 454 & 448.7 & 481 & 476 & 455 & 470.7 \\
\hline 4 & 5.81 & 432 & 460 & 424 & 438.7 & 444 & 443 & 447 & 444.7 \\
\hline Center & 9.00 & 459 & 465 & 450 & 458.0 & 443 & 481 & 456 & 460.0 \\
\hline 5 & 12.19 & 504 & 504 & 455 & 487.7 & 497 & 481 & 505 & 494.3 \\
\hline 6 & 14.51 & 514 & 513 & 492 & 506.3 & 487 & 521 & 550 & 519.3 \\
\hline 7 & 16.11 & 551 & 523 & 507 & 527.0 & 548 & 524 & 505 & 525.7 \\
\hline 8 & 17.42 & 491 & 485 & 490 & 488.7 & 515 & 521 & 439 & 491.7 \\
\hline Averages ---- & $----->$ & 475.3 & 471.6 & 455.2 & 467.4 & 475.3 & 480.8 & 479.6 & 478.6 \\
\hline
\end{tabular}

\begin{tabular}{|lrr|lrrr|}
\hline All & ft/min & Dev. from mean & Center 2/3 & East & South & All \\
Mean & 473.0 & & Mean & 473.2 & 484.0 & 478.6 \\
Min Point & 405.0 & $-14.4 \%$ & Std. Dev. & 34.0 & 30.3 & 31.5 \\
Max Point & 527.0 & $11.4 \%$ & COV as $\%$ & 7.2 & 6.3 & $\mathbf{6 . 6}$ \\
\hline
\end{tabular}

Flow w/o C-Pt Vel Avg w/o C-Pt

827 acfm

$475 \mathrm{fpm}$

Stack temp

Equipment temp

Ambient temp

Stack static

Ambient pressure

Total Stack pressure

Ambient humidity

\begin{tabular}{|c|c|c|}
\multicolumn{1}{c|}{ Start } & Finish & \multicolumn{1}{l}{} \\
\hline 86 & 84 & $F$ \\
\cline { 1 - 2 } N.A. & N.A. & F \\
\hline 82 & 83 & F \\
\hline 0.00 & 0.00 & mbars \\
\hline 29.42 & 29.42 & in. Hg \\
\hline 996.3 & 996.3 & mbars \\
\cline { 1 - 2 } $36 \%$ & $31 \%$ & RH \\
\hline
\end{tabular}

Notes: Horizontal diam. 17-7/8 in. Vertical diam. 17-7/8 in.

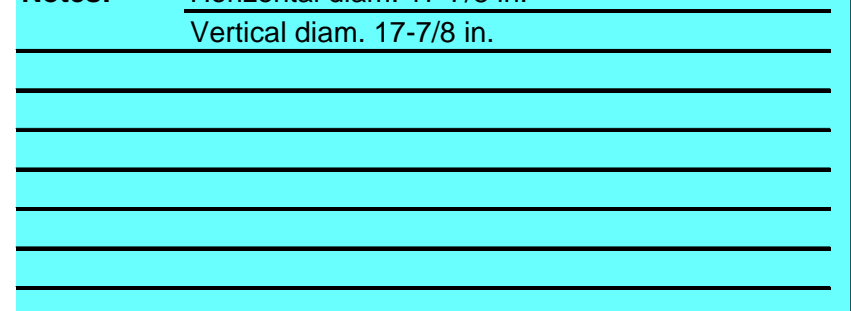

Instuments Used:

Temp >> TSI 8360 SN 209060 Cal due 9/27/06 Vel $>>$ Solomat Zephyr SN 12951472 Cal due 8/29/06 Pitot \# 5 -- 36" Standard Pitot Dwyer

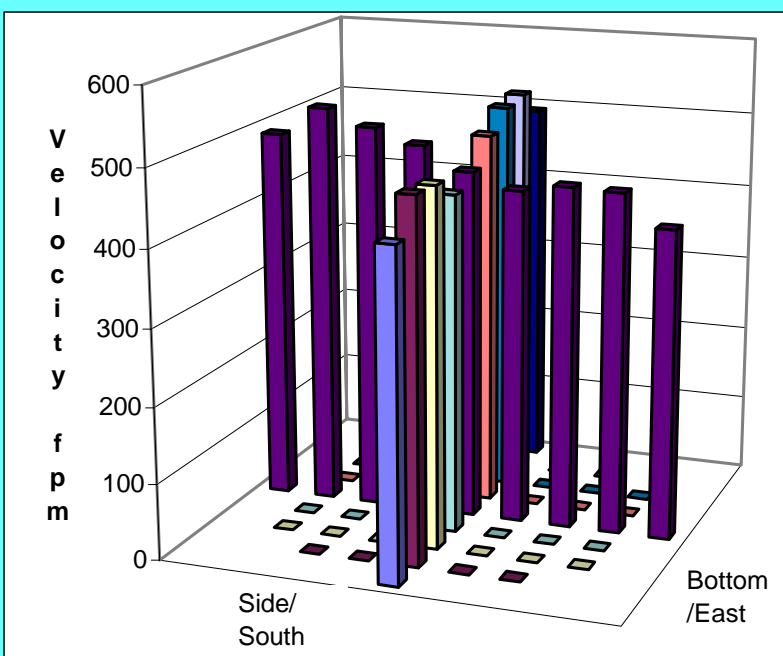

Signature signifies compliance with Procedure EMS-JAG-4 Signature/date
Signature verifying data and calculations:

Signature on file copy 


\section{VELOCITY TRAVERSE DATA FORM}

Site 296-S-21 Model

Date 7/12/2006

Testers Brad Fritz

Stack Dia.

Stack X-Area

$177 / 8$ in

Elevation N.A.

Distance to disturbance

Velocity units $\mathrm{ft} / \mathrm{min}$
Run No. VT-3

Fan Configuration EF2

Fan Setting $\mathbf{3 0}$

Stack Temp

Start/End Time 1300/1350

Center $2 / 3$ from

Points in Center $2 / 3$

Data Files: $\mathrm{NA}$

\begin{tabular}{|c|c|c|c|c|c|c|c|c|c|}
\hline \multirow{2}{*}{$\begin{array}{l}\text { Traverse--> } \\
\text { Trial ----> }\end{array}$} & & \multicolumn{4}{|c|}{ Port_4_Bottom/East } & \multicolumn{4}{|c|}{ Port_4_Side/South } \\
\hline & & 1 & 2 & 3 & Mean & 1 & 2 & 3 & Mean \\
\hline Point & Depth, in. & \multicolumn{4}{|c|}{ Velocity } & \multicolumn{4}{|c|}{ Velocity } \\
\hline 1 & 0.58 & 413 & 418 & 428 & 419.7 & 471 & 460 & 439 & 456.7 \\
\hline 2 & 1.89 & 467 & 479 & 461 & 469.0 & 497 & 463 & 496 & 485.3 \\
\hline 3 & 3.49 & 475 & 492 & 460 & 475.7 & 441 & 485 & 452 & 459.3 \\
\hline 4 & 5.81 & 467 & 455 & 466 & 462.7 & 457 & 433 & 445 & 445.0 \\
\hline Center & 9.00 & 451 & 431 & 431 & 437.7 & 466 & 438 & 444 & 449.3 \\
\hline 5 & 12.19 & 463 & 449 & 448 & 453.3 & 469 & 461 & 454 & 461.3 \\
\hline 6 & 14.51 & 484 & 516 & 454 & 484.7 & 470 & 475 & 499 & 481.3 \\
\hline 7 & 16.11 & 516 & 519 & 500 & 511.7 & 452 & 509 & 500 & 487.0 \\
\hline 8 & 17.42 & 505 & 504 & 494 & 501.0 & 408 & 459 & 450 & 439.0 \\
\hline Averages -- & 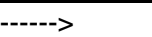 & 471.2 & 473.7 & 460.2 & 468.4 & 459.0 & 464.8 & 464.3 & 462.7 \\
\hline
\end{tabular}

\begin{tabular}{|lrr|lrrr|}
\hline All & ft/min & Dev. from mean & Center 2/3 & East & South & All \\
Mean & 465.5 & & Mean & 470.7 & 467.0 & 468.8 \\
Min Point & 419.7 & $-9.9 \%$ & Std. Dev. & 23.7 & 17.5 & 20.1 \\
Max Point & 511.7 & $9.9 \%$ & Cov as \% & 5.0 & 3.7 & $\mathbf{4 . 3}$ \\
\hline
\end{tabular}

Flow w/o C-Pt Vel Avg w/o C-Pt

816 acfm

$468 \mathrm{fpm}$

Stack temp

Equipment temp

Ambient temp

Stack static

Ambient pressure

Total Stack pressure

Ambient humidity

\begin{tabular}{|c|c|c|}
\multicolumn{1}{c|}{ Start } & Finish & \multicolumn{2}{l}{} \\
\hline 85.6 & 89.4 & $F$ \\
\cline { 1 - 2 } N.A. & N.A. & F \\
\hline 84 & 89 & F \\
\hline 0.00 & 0.00 & mbars \\
\hline 29.42 & 29.41 & in. Hg \\
\hline 996.0 & 996.0 & mbars \\
\cline { 1 - 2 } $31 \%$ & $29 \%$ & RH \\
\hline
\end{tabular}

Instuments Used:

Temp >> TSI 8360 SN $209060 \mathrm{Cal}$ due 9/27/06 Vel >> Solomat Zephyr SN 12951472 Cal due 8/29/06 Pitot \# 5 -- 36" Standard Pitot Dwyer

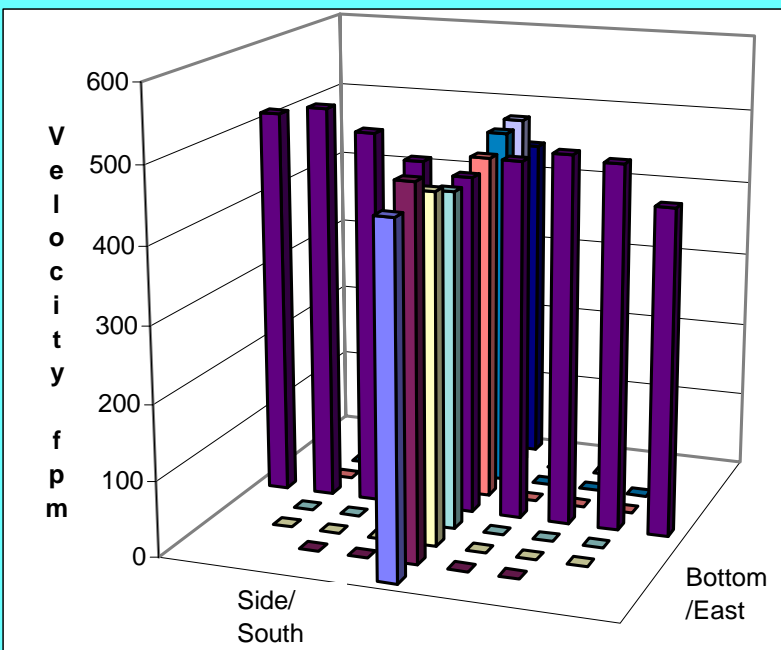

Signature signifies compliance with

Procedure EMS-JAG-4

Signature/date
Signature verifying data and calculations:

Signature on file copy 


\section{VELOCITY TRAVERSE DATA FORM}

Site 296-S-21 Model

Date 7/12/2006

Testers Brad Fritz

Stack Dia.

Stack X-Area

$177 / 8$ in

Elevation N.A.

Distance to disturbance

Velocity units $\mathrm{ft} / \mathrm{min}$
Run No. VT-4

Fan Configuration EF1

Fan Setting $\mathbf{3 0}$

Stack Temp

Start/End Time 1400/1450

Center 2/3 from

Points in Center $2 / 3$

Data Files: NA

\begin{tabular}{|c|c|c|c|c|c|c|c|c|c|}
\hline \multirow{2}{*}{$\begin{array}{l}\text { Traverse--> } \\
\text { Trial ----> }\end{array}$} & & \multicolumn{4}{|c|}{ Port_4_Bottom/East } & \multicolumn{4}{|c|}{ Port_4_Side/South } \\
\hline & & 1 & 2 & 3 & Mean & 1 & 2 & 3 & Mean \\
\hline Point & Depth, in. & \multicolumn{4}{|c|}{ Velocity } & \multicolumn{4}{|c|}{ Velocity } \\
\hline 1 & 0.58 & 415 & 422 & 387 & 408.0 & 419 & 401 & 435 & 418.3 \\
\hline 2 & 1.89 & 482 & 470 & 480 & 477.3 & 439 & 447 & 450 & 445.3 \\
\hline 3 & 3.49 & 466 & 460 & 492 & 472.7 & 428 & 441 & 434 & 434.3 \\
\hline 4 & 5.81 & 448 & 429 & 451 & 442.7 & 396 & 401 & 406 & 401.0 \\
\hline Center & 9.00 & 412 & 411 & 392 & 405.0 & 398 & 406 & 418 & 407.3 \\
\hline 5 & 12.19 & 410 & 439 & 421 & 423.3 & 378 & 434 & 416 & 409.3 \\
\hline 6 & 14.51 & 425 & 431 & 430 & 428.7 & 429 & 394 & 434 & 419.0 \\
\hline 7 & 16.11 & 414 & 433 & 442 & 429.7 & 455 & 435 & 431 & 440.3 \\
\hline 8 & 17.42 & 410 & 416 & 413 & 413.0 & 441 & 432 & 415 & 429.3 \\
\hline Averages --- & 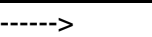 & 431.3 & 434.6 & 434.2 & 433.4 & 420.3 & 4212 & 426.6 & 422.7 \\
\hline
\end{tabular}

\begin{tabular}{|lrr|lrrr|}
\hline All & ft/min & Dev. from mean & Center 2/3 & East & South & All \\
Mean & 428.0 & & Mean & 439.9 & 422.4 & 431.1 \\
Min Point & 401.0 & $-6.3 \%$ & Std. Dev. & 26.5 & 17.6 & 23.4 \\
Max Point & 477.3 & $11.5 \%$ & Cov as \% & 6.0 & 4.2 & $\mathbf{5 . 4}$ \\
\hline
\end{tabular}

Flow w/o C-Pt Vel Avg w/o C-Pt

$751 \mathrm{acfm}$

$431 \mathrm{fpm}$

Stack temp

Equipment temp

Ambient temp

Stack static

Ambient pressure

Total Stack pressure

Ambient humidity

\begin{tabular}{|c|c|l}
\multicolumn{1}{|c|}{ Start } & Finish & \\
\hline 89.4 & 89.6 & $\mathrm{~F}$ \\
\cline { 1 - 2 } N.A. & N.A. & $\mathrm{F}$ \\
\hline 88.7 & 89.1 & $\mathrm{~F}$ \\
\hline 0.00 & 0.00 & mbars \\
\cline { 1 - 2 } 29.41 & 29.41 & in. Hg \\
\cline { 1 - 2 } 996.0 & 996.0 & mbars \\
\cline { 1 - 2 } $31 \%$ & $31 \%$ & $\mathrm{RH}$ \\
\hline
\end{tabular}

Notes: Horizontal diam. 17-7/8 in. Vertical diam. 17-7/8 in.

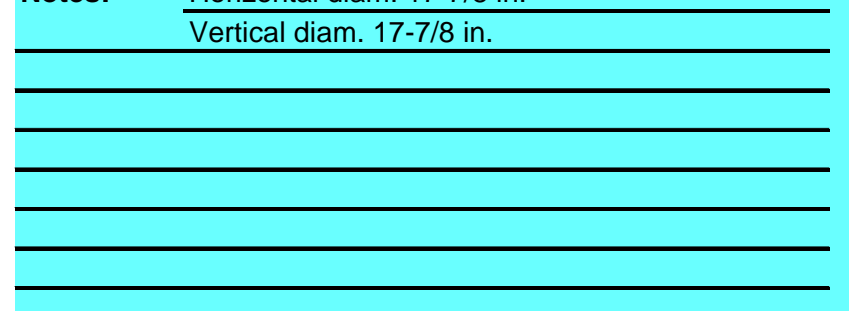

Instuments Used:

Temp >> TSI 8360 SN 209060 Cal due 9/27/06 Vel $>>$ Solomat Zephyr SN 12951472 Cal due 8/29/06 Pitot \# 5 -- 36" Standard Pitot Dwyer

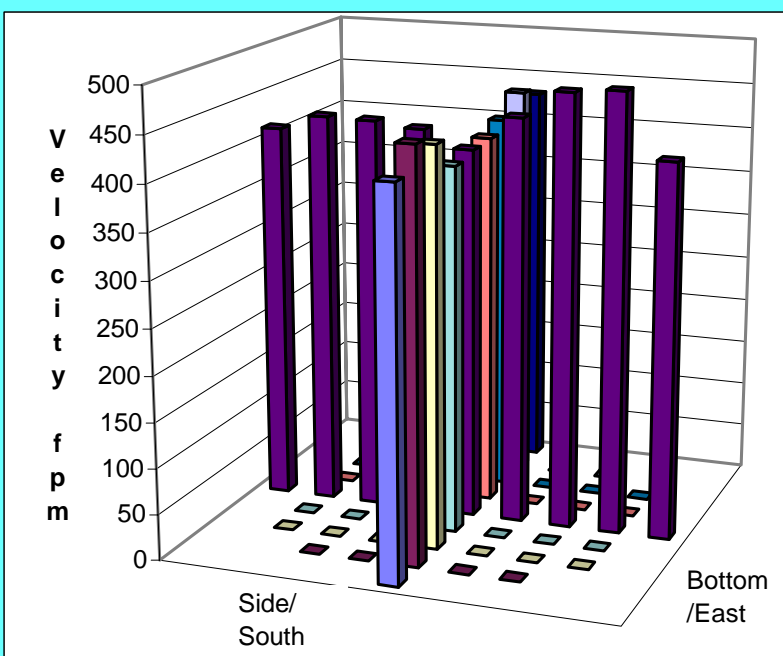

Signature signifies compliance with Procedure EMS-JAG-4 Signature/date
Signature verifying data and calculations:

Signature on file copy 


\section{VELOCITY vS. FREQUENCY DATA FORM}

\begin{aligned} Site & 296-S-21 Scale Model \\ Date & \multicolumn{2}{c}{$7 / 13 / 2006$} \\ Tester & \multicolumn{1}{c}{ BG Fritz } \\ Stack Dia. & 17.875 in. \\ Stack X-Area & 250.9 in2 \\ Elevation & Forts \# 4 \end{aligned}

Site 296-S-21 Scale Model Date

El. above disturbance $\overline{77.75 \quad \text { inches }}$

Velocity Readings, units $=\quad \mathrm{fpm}$

\begin{tabular}{rcc} 
Run No. & VF-1 & \\
\cline { 3 - 3 } Stack Temp & 89.2 & deg F \\
\cline { 2 - 3 } Stack RH\% & $34 \%$ & $\%$ RH \\
\cline { 2 - 3 } Baro Press & 1003 & $\mathrm{mbar}$ \\
\cline { 2 - 3 } Configuration & $\# 1-$ no pre-filter \\
\cline { 2 - 3 } & $11: 25$ to $12: 00$
\end{tabular}

Start/End Time $11: 25$ to $12: 00$

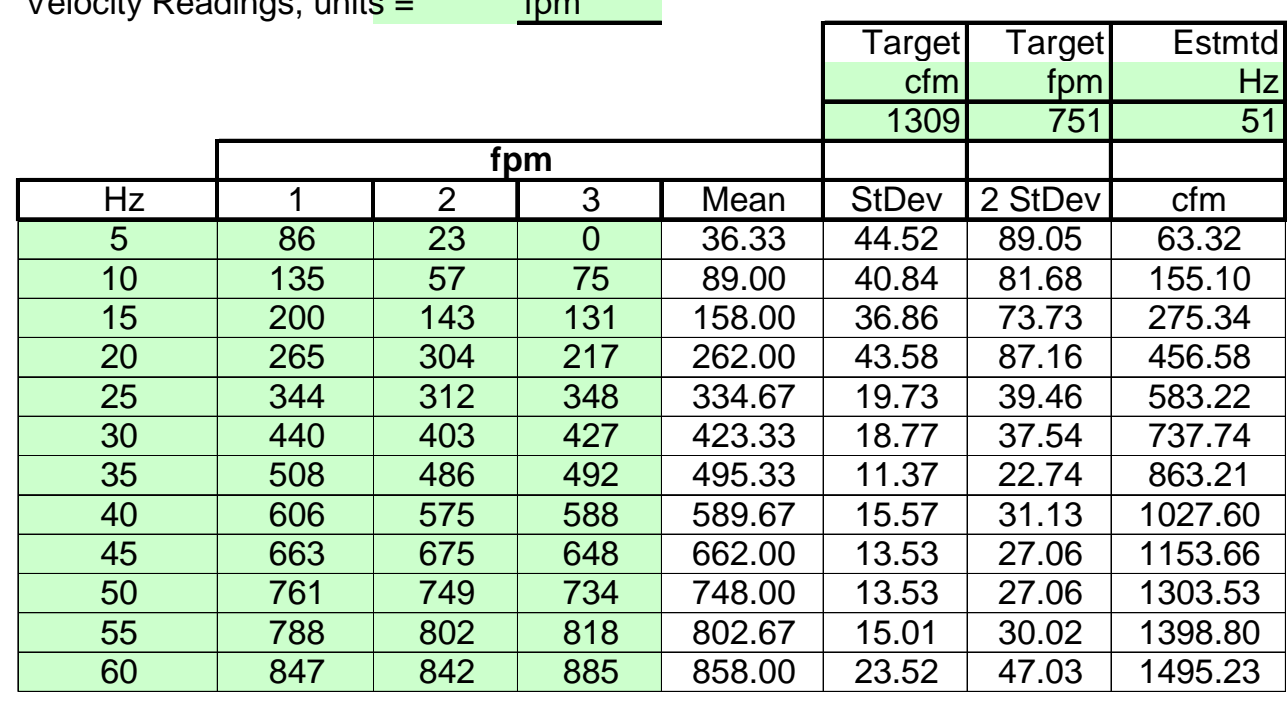

Instuments Used:

Solmat Zephyr SN 12951472

Pitot \# 5 Dwyer Std. 36-in

Temp >> TSI 8360 SN 209060

Cal Exp. Date:

N.A. 9/27/2006

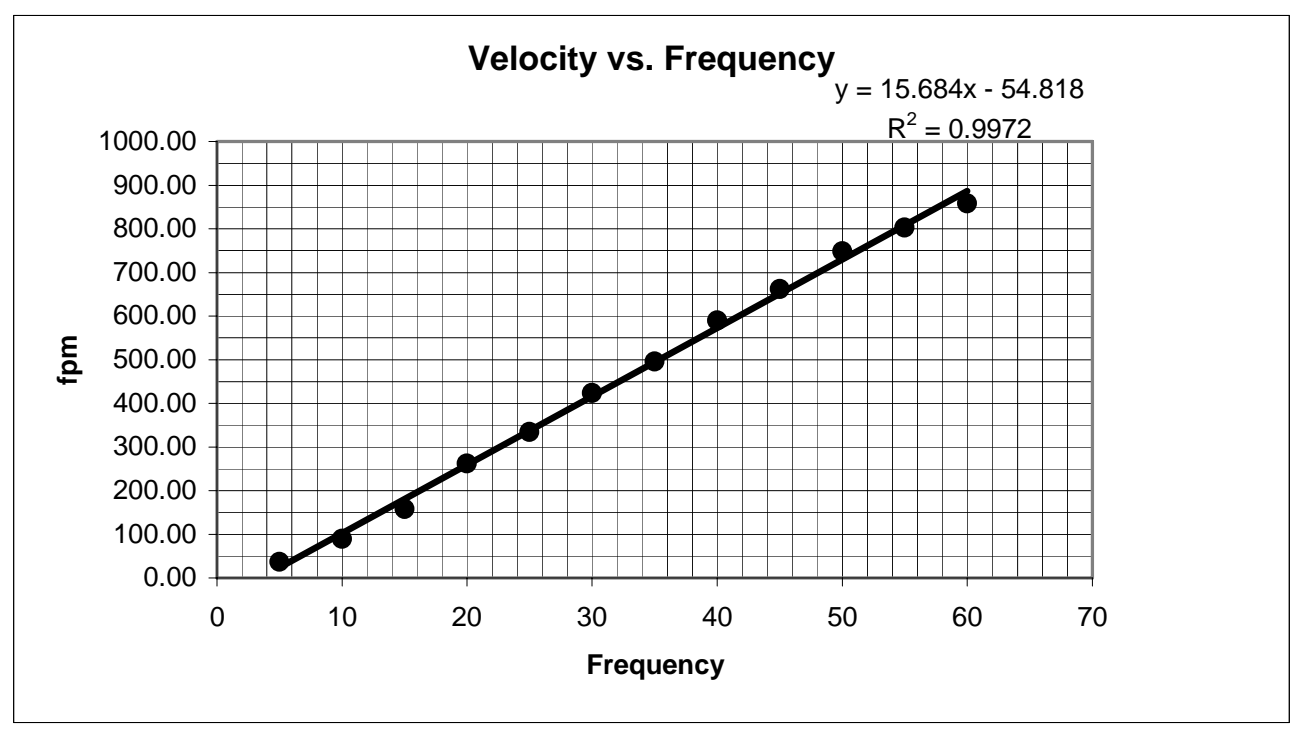

Signature signifies compliance with

Procedure EMS-JAG-3

Signature/date
Signature verifying data and calculations:

Signature on file 


\section{VELOCITY VS. FREQUENCY DATA FORM}

\section{Site 296-S-21 Scale Mode Date \\ Tester $7 / 13 / 2006$ \\ Stack Dia. 17.875 BG Fritz \\ Stack X-Area 250.9 \\ Elevation Ports \# 4 \\ in. \\ in2}

El. above disturbance $\overline{77.75}$

Velocity Readings, units $=$ inches fpm

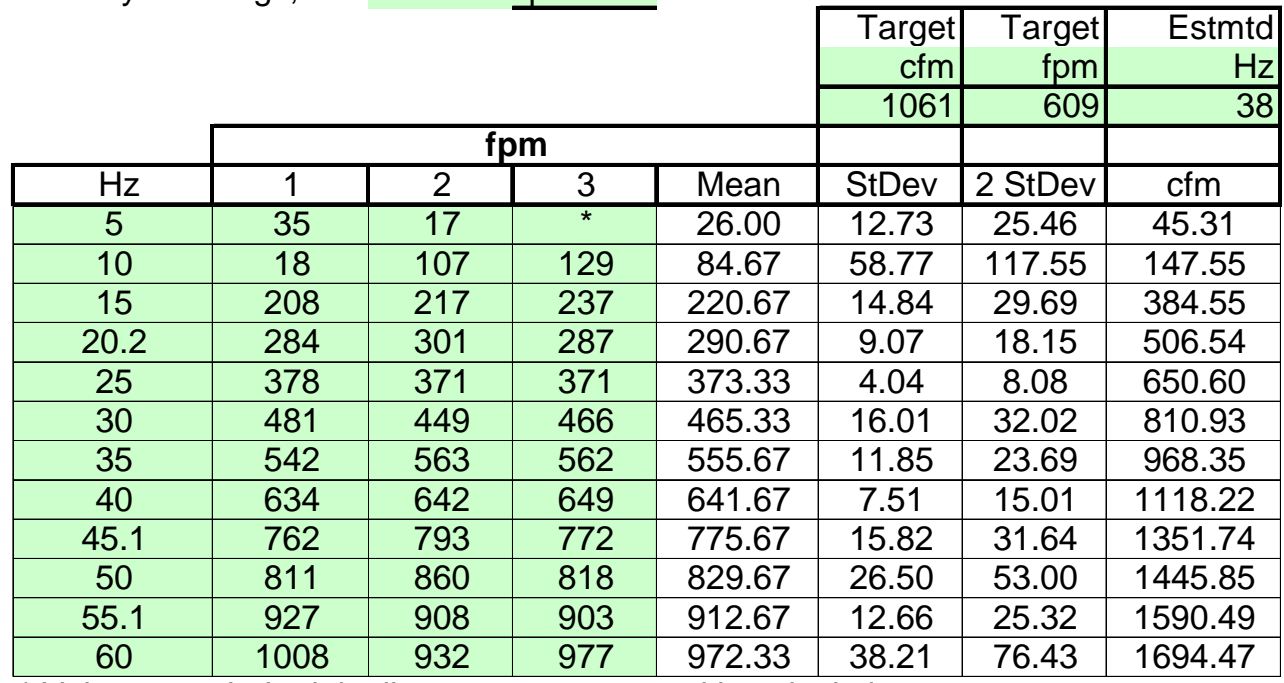

* Value recorded originally as -2 was not used in calculation.

Instuments Used:

Run No. VF-2

Stack Temp $89 \quad \operatorname{deg} \mathrm{F}$

Stack $\mathrm{RH} \% 30 \% \quad \% \mathrm{RH}$

Baro Press 1003 mbar

Fan Configuration \#2 no pre-filter

Start/End Time 1400 to 1425

Solmat Zephyr SN 12951472

Pitot \# 5 Dwyer Std. 36-in

Temp >> TSI 8360 SN 209060

Cal Exp. Date:

$8 / 29 / 2006$

N.A.

9/27/2006

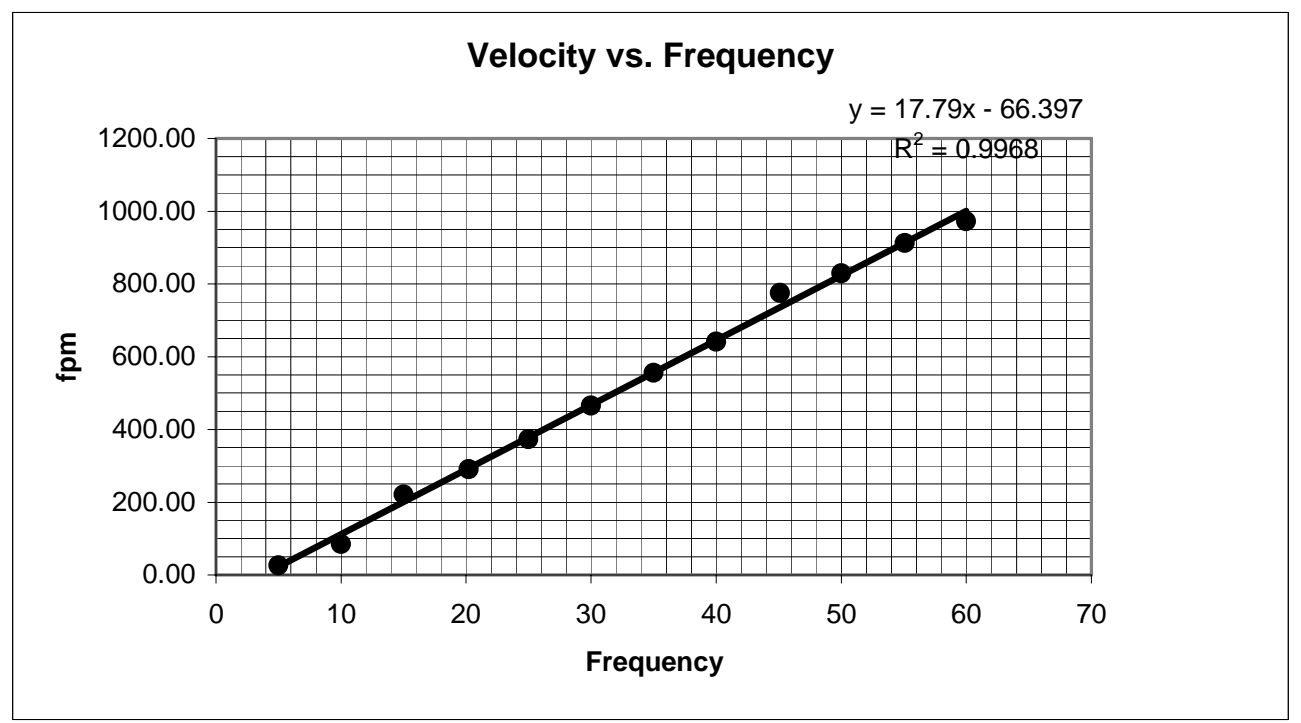

Signature signifies compliance with

Procedure EMS-JAG-3

Signature/date
Signature verifying data and calculations:

Signature on file 


\section{VELOCITY vS. FREQUENCY DATA FORM}

\begin{tabular}{|c|c|c|c|c|c|c|}
\hline Site & 296-S-21 & Scale M & Run No. & VF-3 & & \\
\hline Date & $7 / 13 /$ & 2006 & Stack Temp & 90.7 & $\operatorname{deg} F$ & \\
\hline Tester & $\overline{B G}$ & -ritz & Stack RH\% & $34 \%$ & $\% \mathrm{RH}$ & \\
\hline Stack Dia. & 17.875 & in. & Baro Press & 1003 & mbar & \\
\hline Stack X-Area & 250.9 & in2 & Fan Configuration & Fan 3 & pre-filter & \\
\hline Elevation & Ports \# 4 & & Start/End Time & 125 & 1325 & \\
\hline e disturbance & 77.75 & inches & Reference point fror & veloci & est VT & Port South 4-2 \\
\hline Readings, units & & $\mathrm{ppm}$ & & & & \\
\hline
\end{tabular}

El. above disturbance $\overline{77.75 \quad \text { inches }}$ Reference point from velocity test VT : Port South 4-2 Velocity Readings, units $=\quad \mathrm{fpm}$

\begin{tabular}{|c|c|c|c|c|c|c|c|}
\hline & & & & \multirow{4}{*}{$\begin{array}{r}\text { Target } \\
\text { cfm } \\
1196 \\
\end{array}$} & \multirow{3}{*}{$\begin{array}{r}\text { Target } \\
\text { fpm } \\
\end{array}$} & \multirow{3}{*}{$\begin{array}{r}\text { Estmtd } \\
\mathrm{Hz} \\
\end{array}$} \\
\hline & & & & & & & \\
\hline & & & & & & & \\
\hline & & & & & & 686 & 43 \\
\hline & \multicolumn{4}{|c|}{ fpm } & & & \\
\hline $\mathrm{Hz}$ & 1 & 2 & 3 & Mean & StDev & 2 StDev & $\mathrm{cfm}$ \\
\hline 5 & N.A. & 49 & 92 & 70.50 & 30.41 & 60.81 & 122.86 \\
\hline 10 & 142 & 68 & 104 & 104.67 & 37.00 & 74.01 & 182.40 \\
\hline 15 & 182 & 189 & 177 & 182.67 & 6.03 & 12.06 & 318.33 \\
\hline 20 & 277 & 280 & 297 & 284.67 & 10.79 & 21.57 & 496.09 \\
\hline 25 & 390 & 368 & 400 & 386.00 & 16.37 & 32.74 & 672.68 \\
\hline 30.1 & 475 & 471 & 467 & 471.00 & 4.00 & 8.00 & 820.81 \\
\hline 35 & 565 & 594 & 534 & 564.33 & 30.01 & 60.01 & 983.46 \\
\hline 40.4 & 643 & 662 & 612 & 639.00 & 25.24 & 50.48 & 1113.58 \\
\hline 45 & 702 & 735 & 708 & 715.00 & 17.58 & 35.16 & 1246.02 \\
\hline 50 & 845 & 788 & 799 & 810.67 & 30.24 & 60.48 & 1412.74 \\
\hline 54.9 & 865 & 867 & 907 & 879.67 & 23.69 & 47.38 & 1532.98 \\
\hline 60 & 962 & 921 & 966 & 949.67 & 24.91 & 49.81 & 1654.97 \\
\hline
\end{tabular}

Note -- $81 \mathrm{fpm}$ appears to be the minimum detectable velocity for the Zephyr.

The instrument's readings are biased by the contribution of many zero readings. ambient temperature was 85 deg. F.

Instuments Used:

Solmat Zephyr SN 12951472

Pitot \# 5 Dwyer Std. 36-in

Temp >> TSI 8360 SN 209060
Cal Exp. Date:

$8 / 29 / 2006$

N.A. 9/27/2006

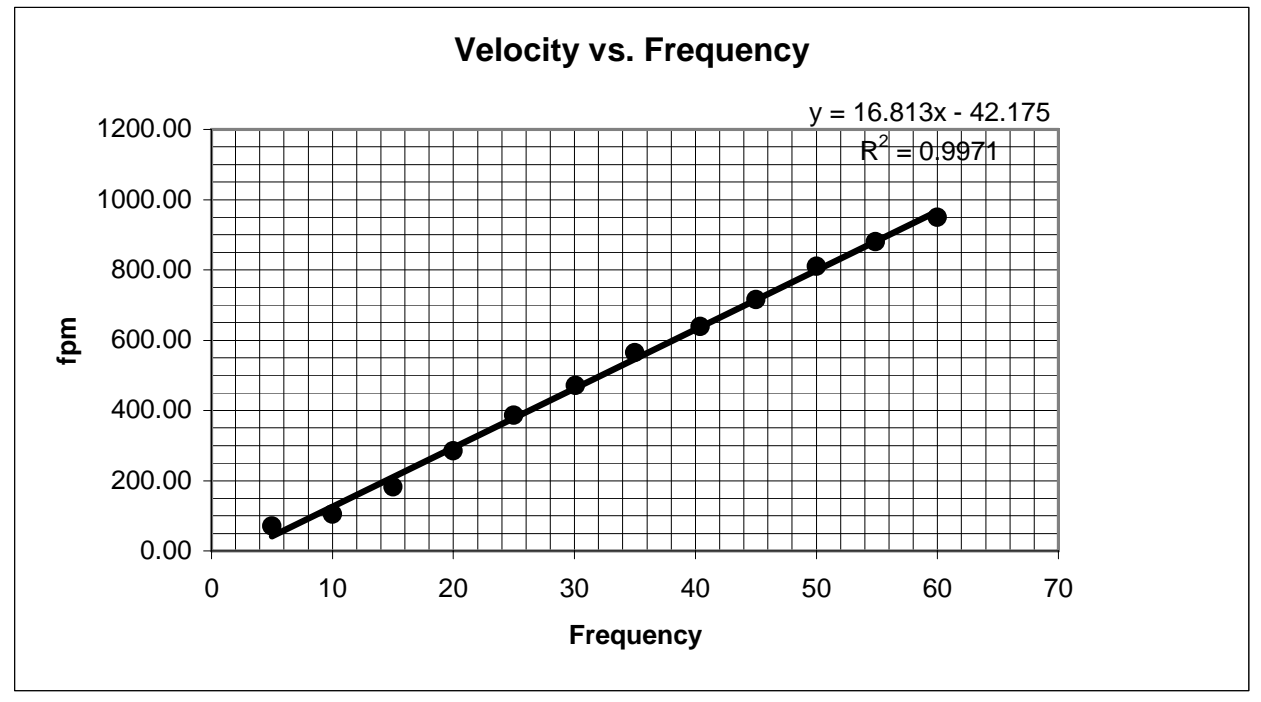

Signature signifies compliance with

Procedure EMS-JAG-3

Signature/date
Signature verifying data and calculations:

Signature on file

B.7 


\section{VELOCITY vS. FREQUENCY DATA FORM}

\section{Site 296-S-21 Scale Model Date \\ Tester $7 / 13 / 2006$ \\ Stack Dia. 17.875 BG Fritz \\ Stack X-Area 250.9 \\ Elevation Ports \# 4 \\ in. \\ in2}

El. above disturbance $\overline{77.75}$

Velocity Readings, units = inches fpm (1)

\begin{tabular}{|c|c|c|c|c|c|c|c|}
\hline & $\begin{array}{r}\text { Target } \\
\mathrm{cfm}\end{array}$ & $\begin{array}{r}\text { Target } \\
\mathrm{fpm} \\
\end{array}$ & \multirow[t]{2}{*}{$\begin{array}{r}\text { Estmtd } \\
\mathrm{Hz} \\
45\end{array}$} \\
\hline & & & & & 1743 & 1295 & \\
\hline & \multicolumn{4}{|c|}{ fpm } & & & \\
\hline $\mathrm{Hz}$ & 1 & 2 & 3 & Mean & StDev & $2 \mathrm{StDev}$ & cfm \\
\hline 5 & N.A. & N.A. & N.A. & \#DIV/O! & \#DIV/O! & \#DIV/0! & \#DIV/O! \\
\hline 10 & 95 & 125 & 133 & 117.67 & 20.03 & 40.07 & 205.06 \\
\hline 15 & 311 & 307 & 318 & 312.00 & 5.57 & 11.14 & 543.72 \\
\hline 20 & 493 & 508 & 503 & 501.33 & 7.64 & 15.28 & 873.67 \\
\hline 25.1 & 656 & 581 & 646 & 627.67 & 40.72 & 81.45 & 1093.83 \\
\hline 30 & 806 & 807 & 805 & 806.00 & 1.00 & 2.00 & 1404.61 \\
\hline 35 & 980 & 938 & 961 & 959.67 & 21.03 & 42.06 & 1672.40 \\
\hline 40 & 1143 & 1139 & 1092 & 1124.67 & 28.36 & 56.72 & 1959.94 \\
\hline 45.1 & 1302 & 1303 & 1312 & 1305.67 & 5.51 & 11.02 & 2275.37 \\
\hline 50 & 1461 & 1488 & 1460 & 1469.67 & 15.89 & 31.77 & 2561.17 \\
\hline 55 & 1651 & 1685 & 1636 & 1657.33 & 25.11 & 50.21 & 2888.21 \\
\hline 60 & 1716 & 1757 & 1788 & 1753.67 & 36.12 & 72.23 & 3056.09 \\
\hline
\end{tabular}

Note -- Values less than $81 \mathrm{fpm}$ were not recorded.

Instuments Used:

Run No. VF-4

Stack Temp $89 \quad$ deg F

Stack $\mathrm{RH} \% 30 \% \quad \% \mathrm{RH}$

Baro Press 1003 mbar

Fan Configuration fan 4 no pre-filter Start/End Time 1330 to 1350

Reference point from velocity test VT : port south 4- 5

Solmat Zephyr SN 12951472

Pitot \# 5 Dwyer Std. 36-in

Temp >> TSI 8360 SN 209060

Cal Exp. Date:

$\frac{8 / 29 / 2006}{\text { N.A. }}$

9/27/2006

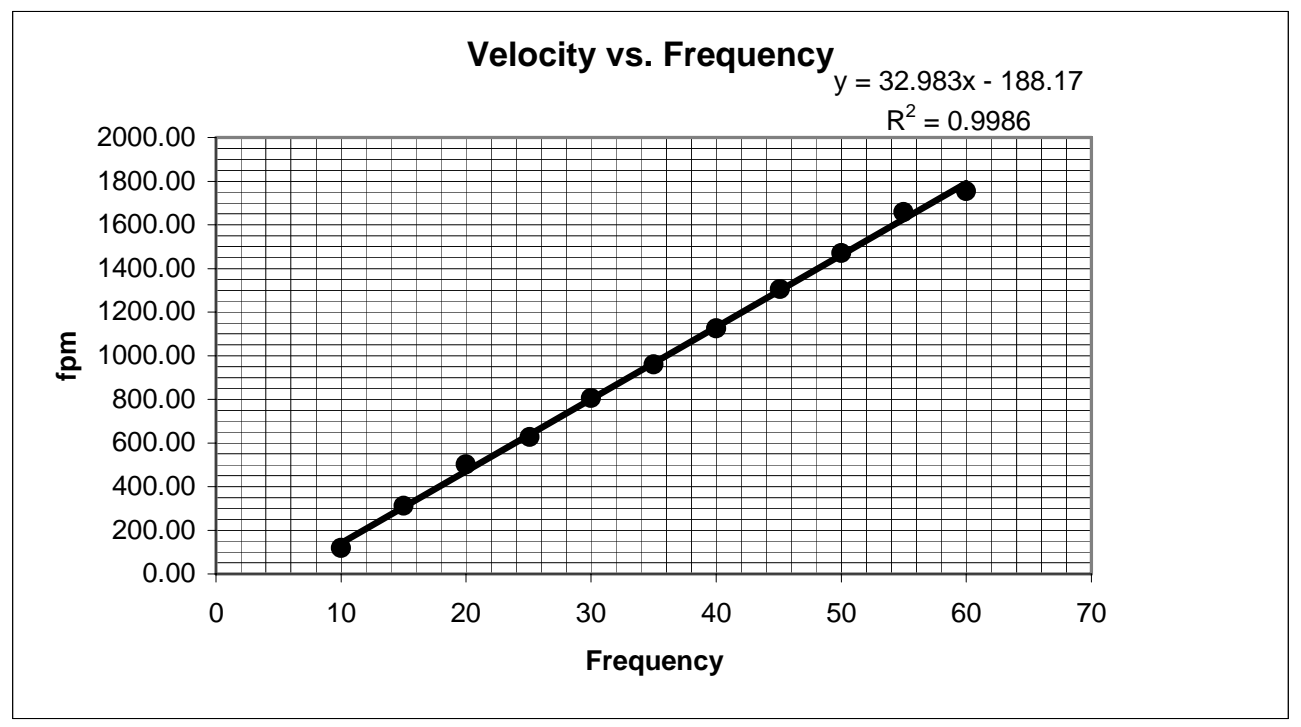

Signature signifies compliance with

Procedure EMS-JAG-3

Signature/date
Signature verifying data and calculations:

Signature on file 


\section{VELOCITY TRAVERSE DATA FORM}

Site 296-S-21 Model

Date 8/7/2006

Testers BG Fritz

Stack Dia.

Stack X-Area

$177 / 8$ in.

Elevation N.A.

Distance to disturbance

Velocity units $\mathrm{ft} / \mathrm{min}$
Run No. VT-17

Fan Configuration 1

Fan Setting 56

Stack Temp

Start/End Time 14:30 to 15:30

Center 2/3 from

Points in Center $2 / 3$

Data Files: NA

\begin{tabular}{|c|c|c|c|c|c|c|c|c|c|}
\hline \multirow{2}{*}{\multicolumn{2}{|c|}{$\begin{array}{l}\text { Traverse--> } \\
\text { Trial ----> }\end{array}$}} & \multicolumn{4}{|c|}{ Port_4_Bottom/East } & \multicolumn{4}{|c|}{ Port_4_Side/South } \\
\hline & & 1 & 2 & 3 & Mean & 1 & 2 & 3 & Mean \\
\hline Point & Depth, in. & \multicolumn{4}{|c|}{ Velocity } & \multicolumn{4}{|c|}{ Velocity } \\
\hline 1 & $9 / 16$ & 790 & 813 & 774 & 792.3 & 747 & 740 & 764 & 750.3 \\
\hline 2 & $114 / 16$ & 853 & 899 & 917 & 889.7 & 793 & 849 & 787 & 809.7 \\
\hline 3 & $38 / 16$ & 908 & 862 & 848 & 872.7 & 847 & 855 & 815 & 839.0 \\
\hline 4 & $513 / 16$ & 860 & 804 & 826 & 830.0 & 794 & 743 & 770 & 769.0 \\
\hline Center & 9 & 804 & 766 & 764 & 778.0 & 784 & 808 & 761 & 784.3 \\
\hline 5 & $123 / 16$ & 780 & 780 & 753 & 771.0 & 824 & 805 & 801 & 810.0 \\
\hline 6 & $148 / 16$ & 820 & 788 & 808 & 805.3 & 815 & 834 & 804 & 817.7 \\
\hline 7 & $16 \quad 1 / 16$ & 800 & 803 & 775 & 792.7 & 842 & 841 & 843 & 842.0 \\
\hline 8 & $176 / 16$ & 772 & 772 & 763 & 769.0 & 813 & 810 & 751 & 791.3 \\
\hline Averages --- & $----->>$ & 820.8 & 809.7 & 803.1 & 811.2 & 806.6 & 809.4 & 788.4 & 801.5 \\
\hline
\end{tabular}

\begin{tabular}{|lrr|lrrr|}
\hline All & $\underline{\mathrm{ft} / \mathrm{min}}$ & Dev. from mean & Center 2/3 & $\underline{\text { East }}$ & $\underline{\text { South }}$ & $\underline{\text { All }}$ \\
\cline { 2 - 3 } Mean & 806.3 & & Mean & 819.9 & 810.2 & 815.1 \\
Min Point & 750.3 & $-6.9 \%$ & Std. Dev. & 46.3 & 26.7 & 36.6 \\
Max Point & 889.7 & $10.3 \%$ & COV as \% & 5.6 & 3.3 & $\mathbf{4 . 5}$ \\
\hline
\end{tabular}

Flow w/o C-Pt Vel Avg w/o C-Pt 1411 acfm 809 fpm

Stack temp Equipment temp Ambient temp Stack static Ambient pressure Total Stack pressure Ambient humidity

\begin{tabular}{|c|c|l}
\multicolumn{1}{|c|}{ Start } & Finish & \\
\hline 105.0 & 105.0 & $\mathrm{~F}$ \\
\hline- & - & $\mathrm{F}$ \\
\hline 101 & 99 & $\mathrm{~F}$ \\
\hline 0.00 & 0.00 & mbars \\
\hline 29.36 & 29.33 & in Hg \\
\hline 994 & 993 & $\mathrm{mbars}$ \\
\hline $24 \%$ & $23 \%$ & $\mathrm{RH}$ \\
\hline
\end{tabular}

Instuments Used:

Temp >> TSI 8360 SN $209060 \mathrm{Cal}$ due 9/27/06 Vel $>>$ Solomat Zephyr SN 12951472 Cal due 8/29/06 Pitot \# 5 -- 36" Standard Pitot Dwyer

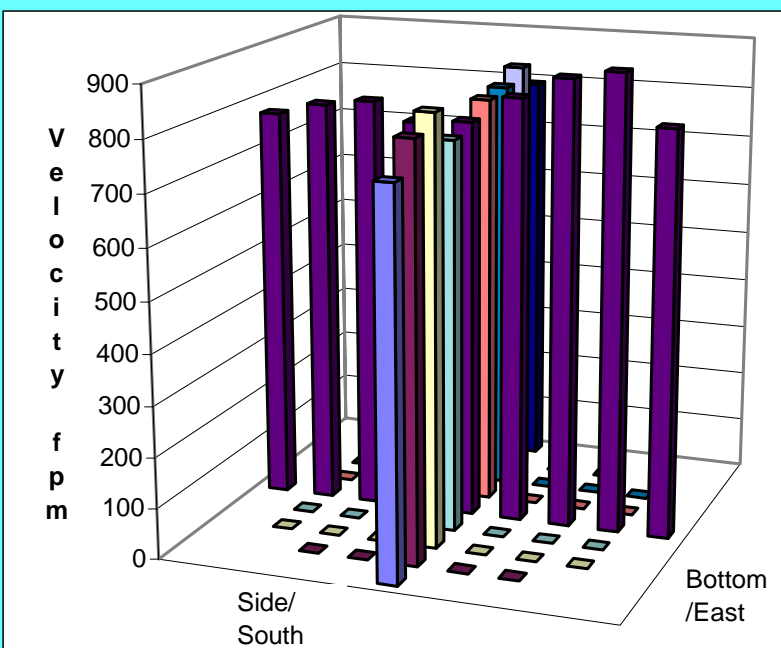

Signature signifies compliance with Procedure EMS-JAG-4 Signature/date
Signature verifying data and calculations:

Signature on file copy 


\section{VELOCITY TRAVERSE DATA FORM}

Site 296-S-21 Model

Date 8/7/2006

Testers BG Fritz

Stack Dia.

Stack X-Area

$177 / 8$ in

Elevation N.A.

Distance to disturbance

Velocity units $\mathrm{ft} / \mathrm{min}$
Run No. VT-17 No centerpoint

Fan Configuration 1 no prefilter

Fan Setting 56

Stack Temp

Start/End Time 14:30 to $15: 30$

Center $2 / 3$ from

Points in Center $2 / 3$

Data Files: NA

\begin{tabular}{|c|c|c|c|c|c|c|c|c|c|}
\hline \multirow{2}{*}{$\begin{array}{l}\text { Traverse--> } \\
\text { Trial ----> }\end{array}$} & & \multicolumn{4}{|c|}{ Port_4__Bottom/East } & \multicolumn{4}{|c|}{ Port_4_Side/South } \\
\hline & & 1 & 2 & 3 & Mean & 1 & 2 & 3 & Mean \\
\hline Point & Depth, in. & \multicolumn{4}{|c|}{ Velocity } & \multicolumn{4}{|c|}{ Velocity } \\
\hline 1 & $9 / 16$ & 790 & 813 & 774 & 792.3 & 747 & 740 & 764 & 750.3 \\
\hline 2 & $114 / 16$ & 853 & 899 & 917 & 889.7 & 793 & 849 & 787 & 809.7 \\
\hline 3 & $38 / 16$ & 908 & 862 & 848 & 872.7 & 847 & 855 & 815 & 839.0 \\
\hline 4 & $513 / 16$ & 860 & 804 & 826 & 830.0 & 794 & 743 & 770 & 769.0 \\
\hline Center & 9 & 804 & 766 & 764 & N.A. & 784 & 808 & 761 & N.A. \\
\hline 5 & $123 / 16$ & 780 & 780 & 753 & 771.0 & 824 & 805 & 801 & 810.0 \\
\hline 6 & $148 / 16$ & 820 & 788 & 808 & 805.3 & 815 & 834 & 804 & 817.7 \\
\hline 7 & $16 \quad 1 / 16$ & 800 & 803 & 775 & 792.7 & 842 & 841 & 843 & 842.0 \\
\hline 8 & $176 / 16$ & 772 & 772 & 763 & 769.0 & 813 & 810 & 751 & 791.3 \\
\hline Averages --- & $-\cdots>$ & 820.8 & 809.7 & 803.1 & 815.3 & 806.6 & 809.4 & 788.4 & 803.6 \\
\hline
\end{tabular}

\begin{tabular}{|lrr|lrrr|}
\hline All & $\underline{\mathrm{ft} / \mathrm{min}}$ & Dev. from mean & Center 2/3 & $\underline{\text { East }}$ & $\underline{\text { South }}$ & $\underline{\text { All }}$ \\
\cline { 2 - 3 } & 809.5 & & Mean & 826.9 & 814.6 & 820.7 \\
Min Point & 750.3 & $-7.3 \%$ & Std. Dev. & 46.5 & 26.4 & 36.6 \\
Max Point & 889.7 & $9.9 \%$ & Cov as \% & 5.6 & 3.2 & $\mathbf{4 . 5}$ \\
\hline
\end{tabular}

Flow w/o C-Pt Vel Avg w/o C-Pt 1411 acfm 809 fpm

Stack temp Equipment temp Ambient temp Stack static Ambient pressure Total Stack pressure Ambient humidity

\begin{tabular}{|c|c|l}
\multicolumn{1}{|c|}{ Start } & Finish & \\
\hline 105.0 & 105.0 & $\mathrm{~F}$ \\
\hline- & - & $\mathrm{F}$ \\
\hline 101 & 99 & $\mathrm{~F}$ \\
\hline 0.00 & 0.00 & mbars \\
\hline 29.36 & 29.33 & in Hg \\
\hline 994 & 993 & $\mathrm{mbars}$ \\
\hline $24 \%$ & $23 \%$ & $\mathrm{RH}$ \\
\hline
\end{tabular}

Instuments Used:

Temp >> TSI 8360 SN $209060 \mathrm{Cal}$ due 9/27/06 Vel $>>$ Solomat Zephyr SN 12951472 Cal due 8/29/06 Pitot \# 5 -- 36" Standard Pitot Dwyer

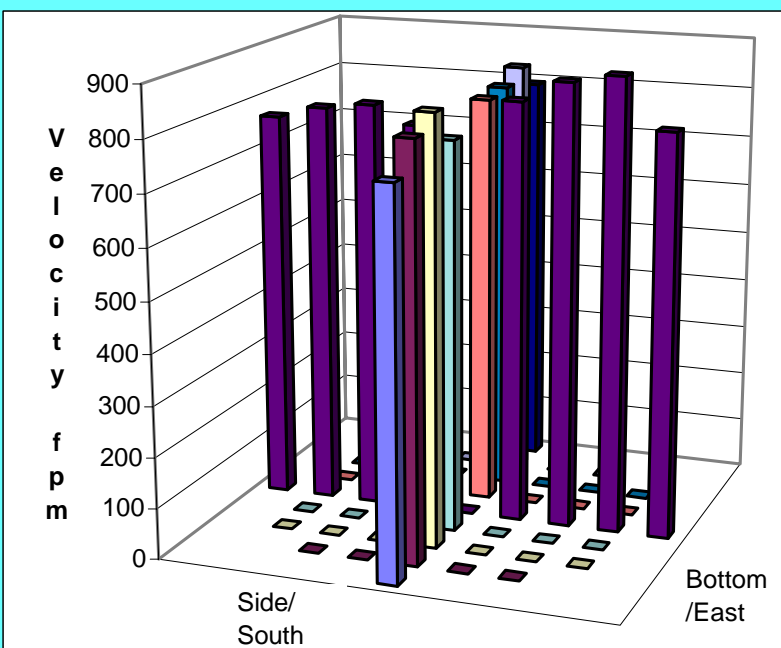

Signature signifies compliance with Procedure EMS-JAG-4 Signature/date
Signature verifying data and calculations:

Signature on file copy 


\section{VELOCITY TRAVERSE DATA FORM}

Site 296-S-21 Model

Date 8/8/2006

Testers BG Fritz

Stack Dia.

Stack X-Area

$177 / 8$ in.

Elevation N.A.

Distance to disturbance

Velocity units $\underline{\mathrm{ft} / \mathrm{min}}$
Run No. VT-18

Fan Configuration 2

Fan Setting 44

Stack Temp

Start/End Time 10:00 to 10:35

Center 2/3 from

Points in Center 2/3

Data Files: NA

\begin{tabular}{|c|c|c|c|c|c|c|c|c|c|}
\hline \multirow{2}{*}{\multicolumn{2}{|c|}{$\begin{array}{l}\text { Traverse--> } \\
\text { Trial ----> }\end{array}$}} & \multicolumn{4}{|c|}{ Port_4_Bottom/East } & \multicolumn{4}{|c|}{ Port_4_Side/South } \\
\hline & & 1 & 2 & tha & Mean & 1 & 2 & & Mean \\
\hline Point & Depth, in. & \multicolumn{4}{|c|}{ Velocity } & \multicolumn{4}{|c|}{ Velocity } \\
\hline 1 & $9 / 16$ & 639 & 680 & & 659.5 & 658 & 689 & & 673.5 \\
\hline 2 & $114 / 16$ & 701 & 677 & & 689.0 & 719 & 748 & & 733.5 \\
\hline 3 & $38 / 16$ & 698 & 704 & & 701.0 & 682 & 710 & & 696.0 \\
\hline 4 & $513 / 16$ & 656 & 675 & & 665.5 & 613 & 663 & & 638.0 \\
\hline Center & 9 & 678 & 659 & & 668.5 & 672 & 700 & & 686.0 \\
\hline 5 & $123 / 16$ & 683 & 687 & & 685.0 & 691 & 702 & & 696.5 \\
\hline 6 & $148 / 16$ & 732 & 729 & & 730.5 & 753 & 715 & & 734.0 \\
\hline 7 & $161 / 16$ & 753 & 742 & & 747.5 & 707 & 730 & & 718.5 \\
\hline 8 & $176 / 16$ & 678 & 717 & & 697.5 & 722 & 705 & & 713.5 \\
\hline Averages ---. & $-\cdots>$ & 690.9 & 696.7 & \#DIV/O! & 693.8 & 690.8 & 706.9 & \#DIV/O! & 698.8 \\
\hline
\end{tabular}

\begin{tabular}{|lrr|lrrr|}
\hline All & $\underline{\mathrm{ft} / \mathrm{min}}$ & Dev. from mean & Center 2/3 & East & $\underline{\text { South }}$ & $\underline{\text { All }}$ \\
Mean & 696.3 & & Mean & 698.1 & 700.4 & 699.3 \\
Min Point & 638.0 & $-8.4 \%$ & Std. Dev. & 30.8 & 33.4 & 30.9 \\
Max Point & 747.5 & $7.4 \%$ & COV as \% & 4.4 & 4.8 & $\mathbf{4 . 4}$ \\
\hline
\end{tabular}

Flow w/o C-Pt Vel Avg w/o C-Pt 1218 acfm $699 \mathrm{fpm}$

Stack temp Equipment temp Ambient temp Stack static Ambient pressure Total Stack pressure Ambient humidity

\begin{tabular}{|c|c|l}
\multicolumn{1}{|c|}{ Start } & Finish & \\
\hline 90.0 & 86.0 & $\mathrm{~F}$ \\
\hline- & - & $\mathrm{F}$ \\
\hline 89 & 84 & $\mathrm{~F}$ \\
\hline 0.00 & 0.00 & mbars \\
\cline { 1 - 2 } 29.492 & 29.509 & in Hg \\
\hline 999 & 999 & mbars \\
\hline $32 \%$ & $37 \%$ & $\mathrm{RH}$ \\
\hline
\end{tabular}

Notes: $\quad$ Based on VT-17, 2 traverses were considered enough for these supplemental tests. There was not a significant change in the velocity profile of VT-17 when analyzed using 2 or 3 traverses.

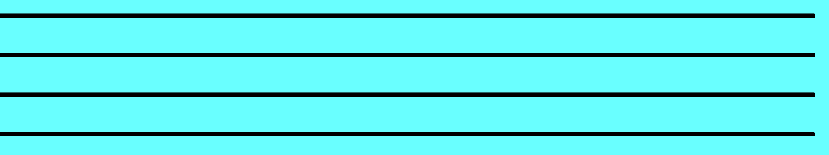

Instuments Used:

Temp >> TSI 8360 SN 209060 Cal due 9/27/06 Vel >> Solomat Zephyr SN 12951472 Cal due 8/29/06 Pitot \# 5 -- 36" Standard Pitot Dwyer

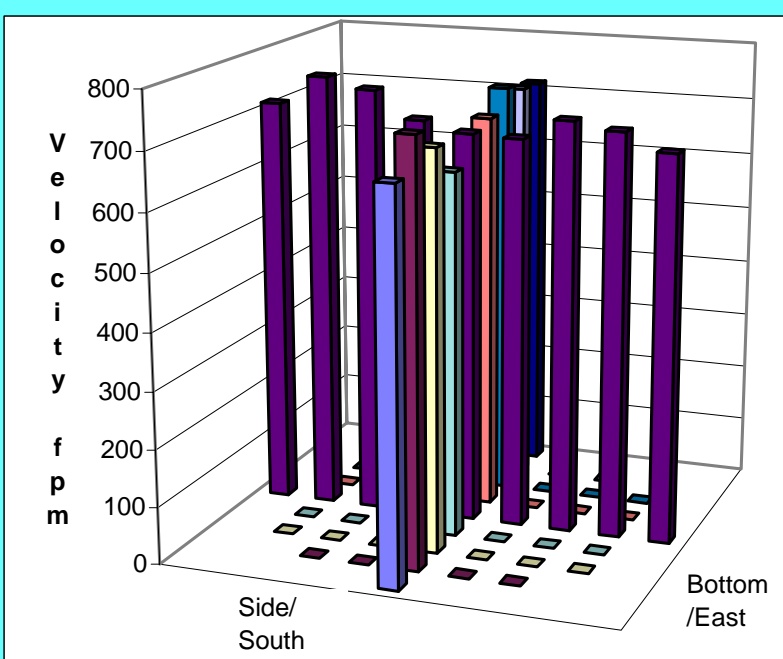

Signature signifies compliance with Procedure EMS-JAG-4 Signature/date
Signature verifying data and calculations:

Signature on file copy 


\section{VELOCITY TRAVERSE DATA FORM}

Site 296-S-21 Model

Date 8/8/2006

Testers BG Fritz

Stack Dia.

Stack X-Area

$177 / 8$ in

Elevation N.A.

Distance to disturbance

Velocity units $\mathrm{ft} / \mathrm{min}$
Run No. VT-18 No centerpoint

Fan Configuration 2 no prefilter

Fan Setting 44

Stack Temp

Start/End Time 10:00 to 10:35

Center $2 / 3$ from

Points in Center 2/3

Data Files: NA

\begin{tabular}{|c|c|c|c|c|c|c|c|c|c|}
\hline \multirow{2}{*}{\multicolumn{2}{|c|}{$\begin{array}{l}\text { Traverse--> } \\
\text { Trial ----> }\end{array}$}} & \multicolumn{4}{|c|}{ Port_4__Bottom/East } & \multicolumn{4}{|c|}{ Port_4_Side/South } \\
\hline & & 1 & 2 & & Mean & 1 & 2 & & Mean \\
\hline Point & Depth, in. & \multicolumn{4}{|c|}{ Velocity } & \multicolumn{4}{|c|}{ Velocity } \\
\hline 1 & $9 / 16$ & 639 & 680 & & 659.5 & 658 & 689 & & 673.5 \\
\hline 2 & $114 / 16$ & 701 & 677 & & 689.0 & 719 & 748 & & 733.5 \\
\hline 3 & $38 / 16$ & 698 & 704 & & 701.0 & 682 & 710 & & 696.0 \\
\hline 4 & $513 / 16$ & 656 & 675 & & 665.5 & 613 & 663 & & 638.0 \\
\hline Center & 9 & 678 & 659 & & N.A. & 672 & 700 & & N.A. \\
\hline 5 & $123 / 16$ & 683 & 687 & & 685.0 & 691 & 702 & & 696.5 \\
\hline 6 & $148 / 16$ & 732 & 729 & & 730.5 & 753 & 715 & & 734.0 \\
\hline 7 & $161 / 16$ & 753 & 742 & & 747.5 & 707 & 730 & & 718.5 \\
\hline 8 & $176 / 16$ & 678 & 717 & & 697.5 & 722 & 705 & & 713.5 \\
\hline Averages --- & $-\cdots>$ & 690.9 & 696.7 & \#DIV/O! & 696.9 & 690.8 & 706.9 & \#DIV/O! & 700.4 \\
\hline
\end{tabular}

\begin{tabular}{|lrr|lrrr|}
\hline All & ft/min & Dev. from mean & Center 2/3 & East & South & All \\
Mean & 698.7 & & Mean & 703.1 & 702.8 & 702.9 \\
Min Point & 638.0 & $-8.7 \%$ & Std. Dev. & 30.6 & 35.9 & 31.8 \\
Max Point & 747.5 & $7.0 \%$ & COV as \% & 4.3 & 5.1 & $\mathbf{4 . 5}$ \\
\hline
\end{tabular}

Flow w/o C-Pt Vel Avg w/o C-Pt $1218 \mathrm{acfm}$ 699 fpm

Stack temp Equipment temp Ambient temp Stack static Ambient pressure Total Stack pressure Ambient humidity

\begin{tabular}{|c|c|l}
\multicolumn{1}{|c|}{ Start } & Finish & \\
\hline 90.0 & 86.0 & $\mathrm{~F}$ \\
\hline- & - & $\mathrm{F}$ \\
\hline 89 & 84 & $\mathrm{~F}$ \\
\hline 0.00 & 0.00 & mbars \\
\cline { 1 - 2 } 29.492 & 29.509 & in Hg \\
\hline 999 & 999 & mbars \\
\hline $32 \%$ & $37 \%$ & $\mathrm{RH}$ \\
\hline
\end{tabular}

Notes: $\quad$ Based on VT-17, 2 traverses were considered enough for these supplemental tests. There was not a significant change in the velocity profile of VT-17 when analyzed using 2 or 3 traverses.

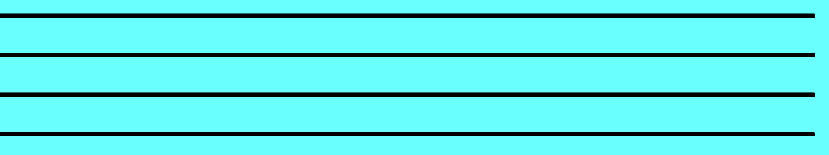

Instuments Used:

Temp >> TSI 8360 SN 209060 Cal due 9/27/06 Vel >> Solomat Zephyr SN 12951472 Cal due 8/29/06 Pitot \# 5 -- 36" Standard Pitot Dwyer

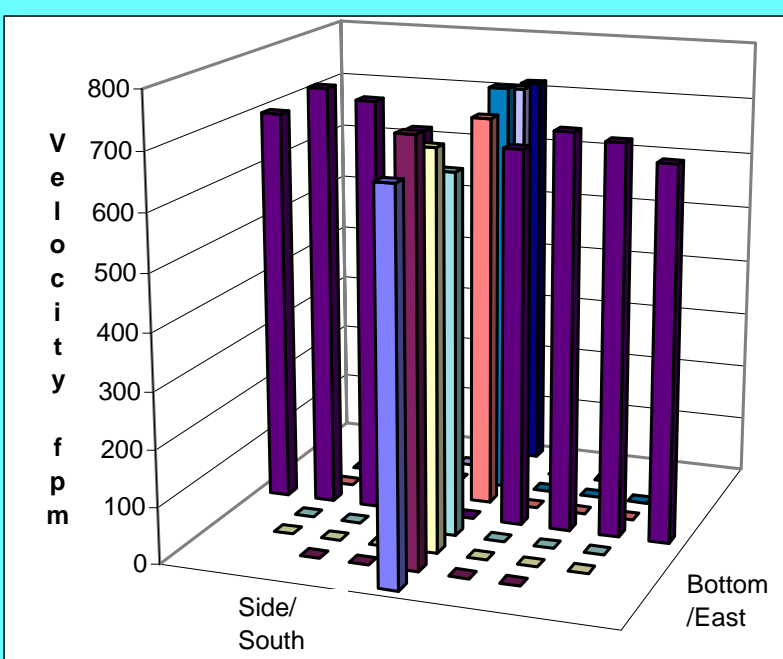

Signature signifies compliance with Procedure EMS-JAG-4 Signature/date
Signature verifying data and calculations:

Signature on file copy 


\section{VELOCITY TRAVERSE DATA FORM}

Site 296-S-21 Model

Date 8/8/2006

Testers BG Fritz

Stack Dia.

Stack X-Area

$177 / 8$ in

Elevation N.A.

Distance to disturbance

Velocity units $\underline{\mathrm{ft} / \mathrm{min}}$
Run No. VT-19

Fan Configuration 3

Fan Setting $\mathbf{5 0}$

Stack Temp

Start/End Time 11:15 to 11:40

Center $2 / 3$ from

Points in Center $2 / 3$

Data Files: NA

\begin{tabular}{|c|c|c|c|c|c|c|c|c|c|}
\hline \multirow{2}{*}{\multicolumn{2}{|c|}{$\begin{array}{l}\text { Traverse--> } \\
\text { Trial ----> }\end{array}$}} & \multicolumn{4}{|c|}{ Port_4_Bottom/East } & \multicolumn{4}{|c|}{ Port_4_Side/South } \\
\hline & & 1 & 2 & tha & Mean & 1 & 2 & & Mean \\
\hline Point & Depth, in. & \multicolumn{4}{|c|}{ Velocity } & \multicolumn{4}{|c|}{ Velocity } \\
\hline 1 & $9 / 16$ & 701 & 702 & & 701.5 & 715 & 720 & & 717.5 \\
\hline 2 & $114 / 16$ & 726 & 737 & & 731.5 & 779 & 796 & & 787.5 \\
\hline 3 & $38 / 16$ & 759 & 755 & & 757.0 & 778 & 803 & & 790.5 \\
\hline 4 & $513 / 16$ & 757 & 716 & & 736.5 & 734 & 780 & & 757.0 \\
\hline Center & 9 & 775 & 763 & & 769.0 & 774 & 758 & & 766.0 \\
\hline 5 & $123 / 16$ & 827 & 821 & & 824.0 & 830 & 808 & & 819.0 \\
\hline 6 & $148 / 16$ & 896 & 869 & & 882.5 & 931 & 899 & & 915.0 \\
\hline 7 & $161 / 16$ & 864 & 875 & & 869.5 & 922 & 889 & & 905.5 \\
\hline 8 & $176 / 16$ & 827 & 822 & & 824.5 & 852 & 829 & & 840.5 \\
\hline Averages -- & $\overline{--->>}$ & 792.4 & 784.4 & \#DIV/O! & 788.4 & 812.8 & 809.1 & \#DIV/O! & 810.9 \\
\hline
\end{tabular}

\begin{tabular}{|lrr|lrrr|}
\hline All & $\underline{\mathrm{ft} / \mathrm{min}}$ & Dev. from mean & Center 2/3 & East & $\underline{\text { South }}$ & $\underline{\text { All }}$ \\
\cline { 2 - 3 } Mean & 799.7 & & Mean & 795.7 & 820.1 & 807.9 \\
Min Point & 701.5 & $-12.3 \%$ & Std. Dev. & 62.7 & 64.7 & 62.5 \\
Max Point & 915.0 & $14.4 \%$ & COV as \% & 7.9 & 7.9 & $\mathbf{7 . 7}$ \\
\hline
\end{tabular}

Flow w/o C-Pt Vel Avg w/o C-Pt 1401 acfm 804 fpm

Stack temp Equipment temp Ambient temp Stack static Ambient pressure Total Stack pressure Ambient humidity

\begin{tabular}{|c|c|c|}
\hline Start & Finish & \\
\hline 86.0 & 86.0 & $\mathrm{~F}$ \\
\hline- & - & $\mathrm{F}$ \\
\hline 84 & 86 & $\mathrm{~F}$ \\
\hline 0.00 & 0.00 & mbars \\
\hline 29.509 & 29.51 & in $\mathrm{Hg}$ \\
\hline 999 & 999 & mbars \\
\hline $32 \%$ & $35 \%$ & $\mathrm{RH}$ \\
\hline
\end{tabular}

Notes: $\quad$ Only 2 traverses done per port. See note on data sheet VT-18.
Instuments Used:

Temp >> TSI 8360 SN $209060 \mathrm{Cal}$ due 9/27/06 Vel >> Solomat Zephyr SN 12951472 Cal due 8/29/06 Pitot \# 5 -- 36" Standard Pitot Dwyer

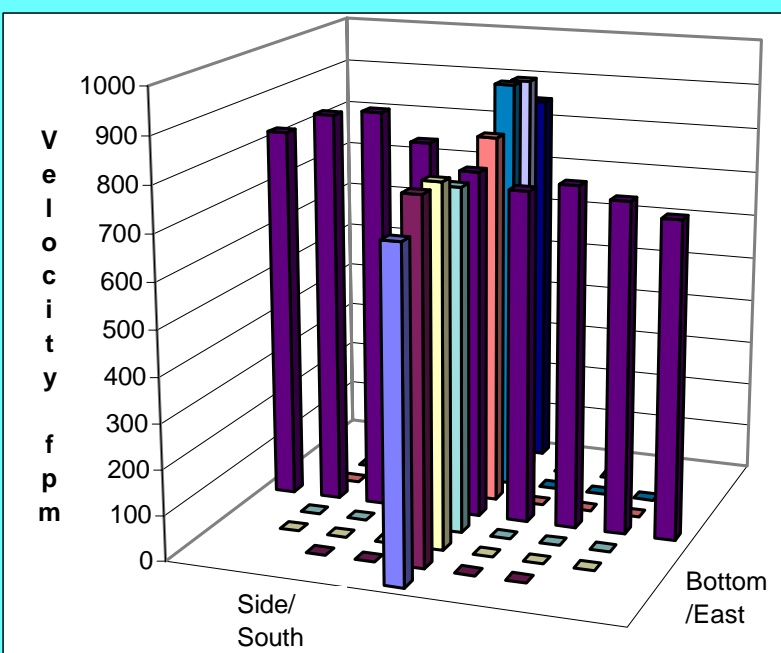

Signature signifies compliance with Procedure EMS-JAG-4 Signature/date
Signature verifying data and calculations:

Signature on file copy 


\section{VELOCITY TRAVERSE DATA FORM}

Site 296-S-21 Model

Date 8/8/2006

Testers BG Fritz

Stack Dia.

Stack X-Area

$177 / 8$ in

Elevation N.A.

Distance to disturbance

Velocity units $\mathrm{ft} / \mathrm{min}$
Run No. VT-19 No centerpoint

Fan Configuration 3 no prefilter

Fan Setting $\mathbf{5 0}$

Stack Temp

Start/End Time 11:15 to $11: 40$

Center $2 / 3$ from

Points in Center $2 / 3$

Data Files: NA

\begin{tabular}{|c|c|c|c|c|c|c|c|c|c|}
\hline \multirow{2}{*}{\multicolumn{2}{|c|}{$\begin{array}{l}\text { Traverse--> } \\
\text { Trial ----> }\end{array}$}} & \multicolumn{4}{|c|}{ Port_4__Bottom/East } & \multicolumn{4}{|c|}{ Port_4_Side/South } \\
\hline & & 1 & 2 & & Mean & 1 & 2 & & Mean \\
\hline Point & Depth, in. & \multicolumn{4}{|c|}{ Velocity } & \multicolumn{4}{|c|}{ Velocity } \\
\hline 1 & $9 / 16$ & 701 & 702 & & 701.5 & 715 & 720 & & 717.5 \\
\hline 2 & $114 / 16$ & 726 & 737 & & 731.5 & 779 & 796 & & 787.5 \\
\hline 3 & $38 / 16$ & 759 & 755 & & 757.0 & 778 & 803 & & 790.5 \\
\hline 4 & $513 / 16$ & 757 & 716 & & 736.5 & 734 & 780 & & 757.0 \\
\hline Center & 9 & 775 & 763 & & N.A. & 774 & 758 & & N.A. \\
\hline 5 & $123 / 16$ & 827 & 821 & & 824.0 & 830 & 808 & & 819.0 \\
\hline 6 & $148 / 16$ & 896 & 869 & & 882.5 & 931 & 899 & & 915.0 \\
\hline 7 & $161 / 16$ & 864 & 875 & & 869.5 & 922 & 889 & & 905.5 \\
\hline 8 & $176 / 16$ & 827 & 822 & & 824.5 & 852 & 829 & & 840.5 \\
\hline Averages --- & $-\cdots>$ & 792.4 & 784.4 & \#DIV/O! & 790.9 & 812.8 & 809.1 & \#DIV/O! & 816.6 \\
\hline
\end{tabular}

\begin{tabular}{|lrr|lrrr|}
\hline All & ft/min & Dev. from mean & Center 2/3 & East & $\underline{\text { South }}$ & $\underline{\text { All }}$ \\
\cline { 2 - 3 } Mean & 803.7 & & Mean & 800.2 & 829.1 & 814.6 \\
Min Point & 701.5 & $-12.7 \%$ & Std. Dev. & 67.5 & 65.9 & 65.4 \\
Max Point & 915.0 & $13.8 \%$ & Cov as \% & 8.4 & 8.0 & $\mathbf{8 . 0}$ \\
\hline
\end{tabular}

Flow w/o C-Pt Vel Avg w/o C-Pt 1401 acfm $804 \mathrm{fpm}$

Stack temp Equipment temp Ambient temp Stack static Ambient pressure Total Stack pressure Ambient humidity

\begin{tabular}{|c|c|c|}
\hline Start & Finish & \\
\hline 86.0 & 86.0 & $F$ \\
\hline - & - & $F$ \\
\hline 84 & 86 & $F$ \\
\hline 0.00 & 0.00 & mbars \\
\hline 29.509 & 29.51 & in $\mathrm{Hg}$ \\
\hline 999 & 999 & mbars \\
\hline $32 \%$ & $35 \%$ & $\mathrm{RH}$ \\
\hline
\end{tabular}

Notes: $\quad$ Only 2 traverses done per port. See note on data sheet VT-18.
Instuments Used:

Temp >> TSI 8360 SN $209060 \mathrm{Cal}$ due 9/27/06 Vel >> Solomat Zephyr SN 12951472 Cal due 8/29/06 Pitot \# 5 -- 36" Standard Pitot Dwyer

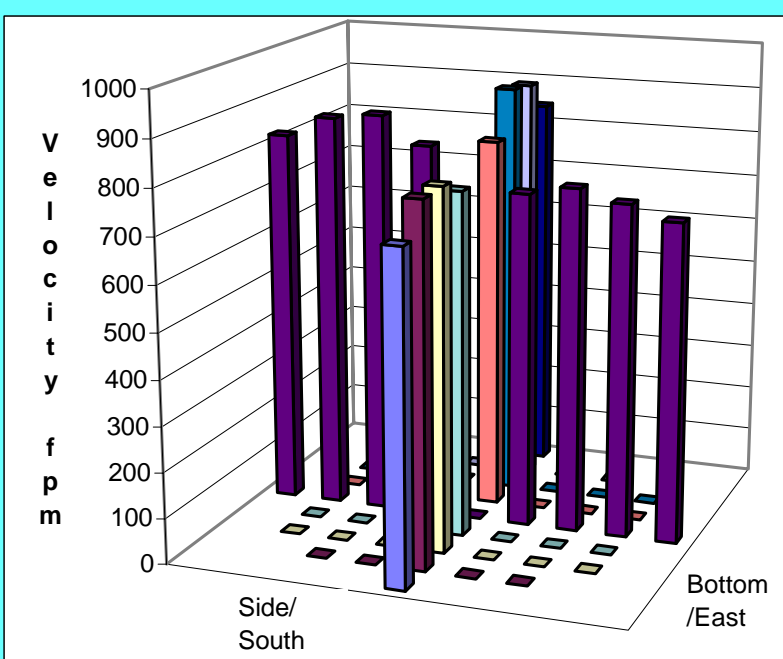

Signature signifies compliance with Procedure EMS-JAG-4 Signature/date
Signature verifying data and calculations:

Signature on file copy 


\section{VELOCITY TRAVERSE DATA FORM}

Site 296-S-21 Model

Date 8/8/2006

Testers BG Fritz

Stack Dia.

Stack X-Area

$177 / 8$ in

Elevation N.A.

Distance to disturbance

Velocity units $\mathrm{ft} / \mathrm{min}$
Run No. VT-20

Fan Configuration 4

Fan Setting $\mathbf{5 2}$

Stack Temp

Start/End Time 11:50 to 12:30

Center $2 / 3$ from

Points in Center $2 / 3$

Data Files: NA

\begin{tabular}{|c|c|c|c|c|c|c|c|c|c|}
\hline \multirow{2}{*}{$\begin{array}{l}\text { Traverse--> } \\
\text { Trial ----> }\end{array}$} & & \multicolumn{4}{|c|}{ Port_4_Bottom/East } & \multicolumn{4}{|c|}{ Port_4_Side/South } \\
\hline & & 1 & 2 & & Mean & 1 & 2 & 3 & Mean \\
\hline Point & Depth, in. & \multicolumn{4}{|c|}{ Velocity } & \multicolumn{4}{|c|}{ Velocity } \\
\hline 1 & $9 / 16$ & 1136 & 1167 & & 1151.5 & 1342 & 1359 & & 1350.5 \\
\hline 2 & $114 / 16$ & 1265 & 1276 & & 1270.5 & 1423 & 1421 & & 1422.0 \\
\hline 3 & $38 / 16$ & 1221 & 1288 & & 1254.5 & 1439 & 1431 & & 1435.0 \\
\hline 4 & $513 / 16$ & 1267 & 1226 & & 1246.5 & 1369 & 1371 & & 1370.0 \\
\hline Center & 9 & 1354 & 1378 & & 1366.0 & 1338 & 1365 & & 1351.5 \\
\hline 5 & $12 \quad 3 / 16$ & 1502 & 1573 & & 1537.5 & 1542 & 1509 & & 1525.5 \\
\hline 6 & $148 / 16$ & 1573 & 1599 & & 1586.0 & 1684 & 1697 & & 1690.5 \\
\hline 7 & $16 \quad 1 / 16$ & 1584 & 1601 & & 1592.5 & 1684 & 1715 & & 1699.5 \\
\hline 8 & $176 / 16$ & 1436 & 1415 & & 1425.5 & 1580 & 1658 & & 1619.0 \\
\hline Averages --- & $--->$ & 1370.9 & 1391.4 & \#DIV/O! & 1381.2 & 1489.0 & 1502.9 & \#DIV/0! & 1495.9 \\
\hline
\end{tabular}

\begin{tabular}{|lrr|lrrr|}
\hline All & $\underline{\mathrm{ft} / \mathrm{min}}$ & Dev. from mean & Center 2/3 & $\underline{\text { East }}$ & $\underline{\text { North }}$ & $\underline{\text { All }}$ \\
Mean & 1438.6 & & Mean & 1407.6 & 1499.1 & 1453.4 \\
Min Point & 1151.5 & $-20.0 \%$ & Std. Dev. & 159.6 & 144.9 & 154.0 \\
Max Point & 1699.5 & $18.1 \%$ & Cov as \% & 11.3 & 9.7 & $\mathbf{1 0 . 6}$ \\
\hline
\end{tabular}

Flow w/o C-Pt

2524 acfm

Vel Avg w/o C-Pt $1449 \mathrm{fpm}$

Stack temp

Equipment temp

Ambient temp

Stack static

Ambient pressure

Total Stack pressure

Ambient humidity

\begin{tabular}{|c|c|l|}
\multicolumn{1}{c|}{ Start } & Finish & \\
\hline 86.0 & 78.0 & $F$ \\
\hline- & - & $F$ \\
\hline 86 & 78 & $F$ \\
\hline 0.00 & 0.00 & mbars \\
\cline { 1 - 2 } 29.509 & 29.51 & in Hg \\
\hline 999 & 999 & mbars \\
\hline $35 \%$ & $53 \%$ & $\mathrm{RH}$ \\
\hline
\end{tabular}

Notes: $\quad$ Only 2 traverses done per port. See note on data sheet VT-18.
Instuments Used:

Temp $\gg$ > TSI 8360 SN $209060 \mathrm{Cal}$ due 9/27/06 Vel >> Solomat Zephyr SN 12951472 Cal due 8/29/06 Pitot \# 5 -- 36" Standard Pitot Dwyer

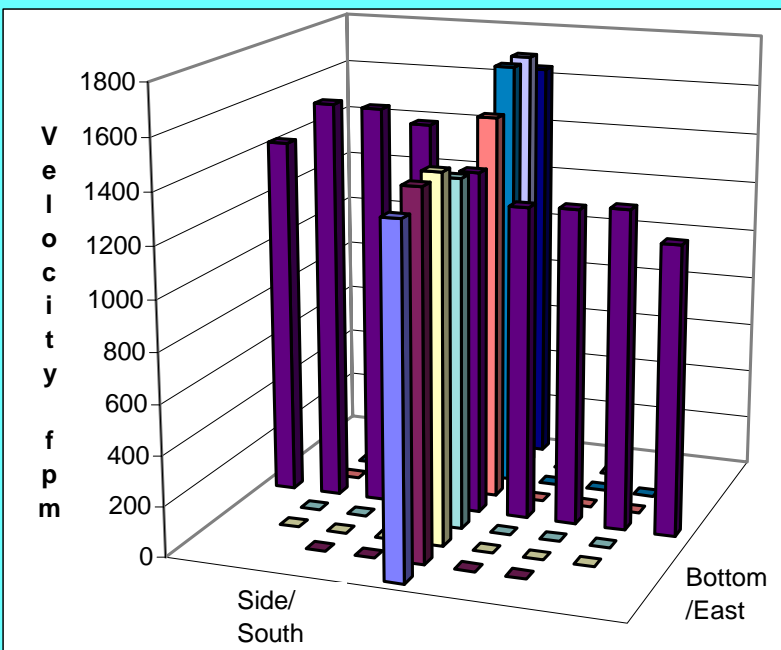

Signature signifies compliance with Procedure EMS-JAG-4

Signature/date
Signature verifying data and calculations:

Signature on file copy 


\section{VELOCITY TRAVERSE DATA FORM}

Site 296-S-21 Model

Date 8/8/2006

Testers BG Fritz

Stack Dia.

Stack X-Area

$177 / 8$ in

Elevation N.A.

Distance to disturbance

Velocity units $\mathrm{ft} / \mathrm{min}$
Run No. VT-20 No centerpoint

Fan Configuration 4

Fan Setting 52

Stack Temp

Start/End Time 11:50 to 12:30

Center $2 / 3$ from

Points in Center 2/3

Data Files: NA

no prefilter

$\mathrm{Hz}$

$773 / 4$ inches

(1/

\begin{tabular}{|c|c|c|c|c|c|c|c|c|c|}
\hline \multirow{2}{*}{\multicolumn{2}{|c|}{$\begin{array}{l}\text { Traverse--> } \\
\text { Trial ----> }\end{array}$}} & \multicolumn{4}{|c|}{ Port_4_Bottom/East } & \multicolumn{4}{|c|}{ Port_4_Side/South } \\
\hline & & 1 & 2 & & Mean & 1 & 2 & & Mean \\
\hline Point & Depth, in. & \multicolumn{4}{|c|}{ Velocity } & \multicolumn{4}{|c|}{ Velocity } \\
\hline 1 & $9 / 16$ & 1136 & 1167 & & 1151.5 & 1342 & 1359 & & 1350.5 \\
\hline 2 & $114 / 16$ & 1265 & 1276 & & 1270.5 & 1423 & 1421 & & 1422.0 \\
\hline 3 & $38 / 16$ & 1221 & 1288 & & 1254.5 & 1439 & 1431 & & 1435.0 \\
\hline 4 & $513 / 16$ & 1267 & 1226 & & 1246.5 & 1369 & 1371 & & 1370.0 \\
\hline Center & 9 & 1354 & 1378 & & N.A. & 1338 & 1365 & & N.A. \\
\hline 5 & $123 / 16$ & 1502 & 1573 & & 1537.5 & 1542 & 1509 & & 1525.5 \\
\hline 6 & $148 / 16$ & 1573 & 1599 & & 1586.0 & 1684 & 1697 & & 1690.5 \\
\hline 7 & $16 \quad 1 / 16$ & 1584 & 1601 & & 1592.5 & 1684 & 1715 & & 1699.5 \\
\hline 8 & $176 / 16$ & 1436 & 1415 & & 1425.5 & 1580 & 1658 & & 1619.0 \\
\hline Averages --- & $---->$ & 1370.9 & 1391.4 & \#DIV/O! & 1383.1 & 1489.0 & 1502.9 & \#DIV/0! & 1514.0 \\
\hline
\end{tabular}

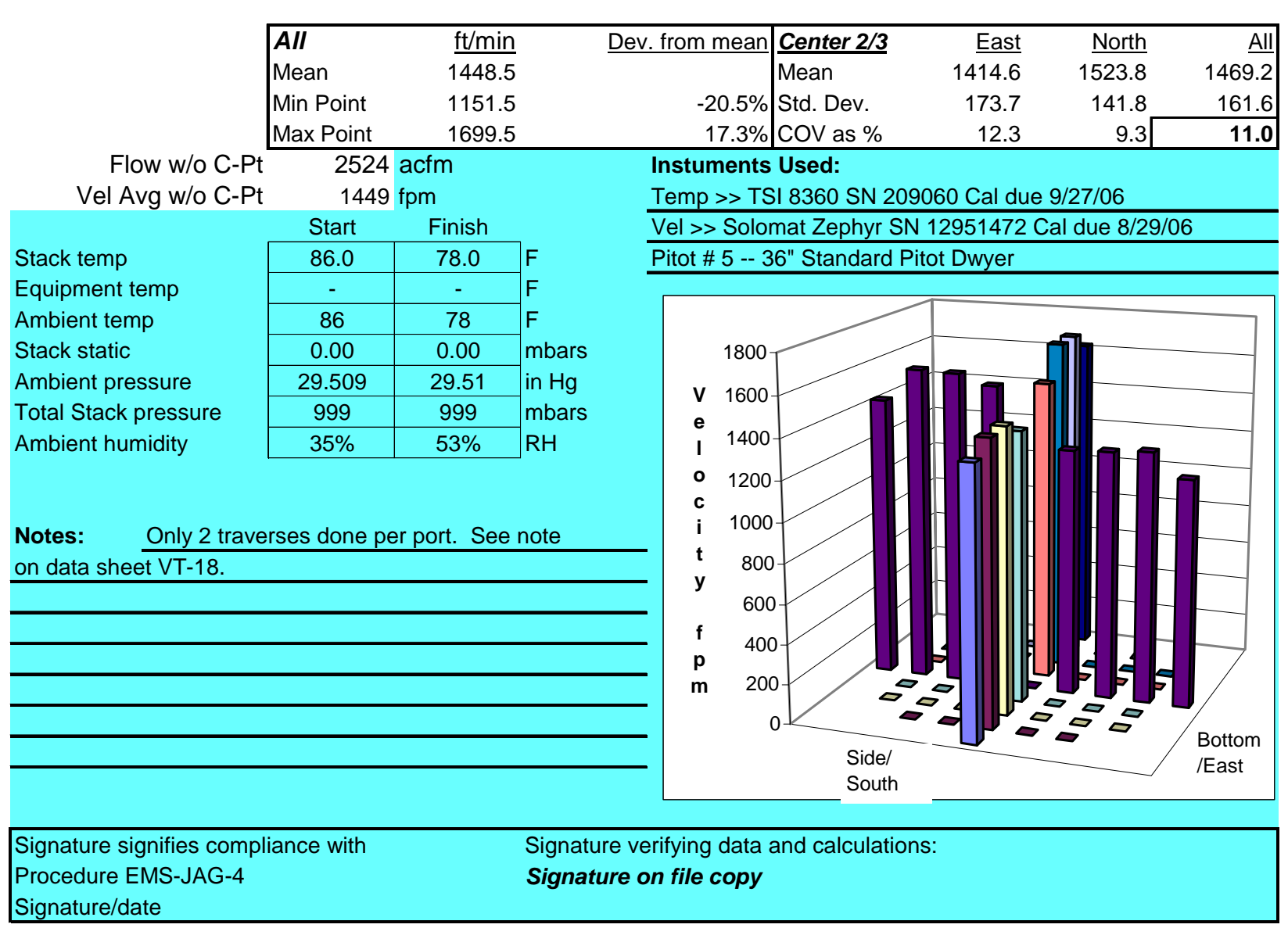


Appendix C

\section{Velocity Uniformity Procedure}


APPENDIX C: VELOCITY UNIFORMITY PROCEDURE

\begin{tabular}{|c|c|c|}
\hline \multicolumn{3}{|l|}{ PNNL Operating Procedure } \\
\hline $\begin{array}{l}\text { Title: Test to Determine } \\
\text { Uniformity of Gas } \\
\text { Velocity at the Elevation } \\
\text { of a Sampler Probe }\end{array}$ & $\begin{array}{l}\text { Org. Code: } \\
\text { Procedure No.: } \\
\text { Rev. No.: }\end{array}$ & $\begin{array}{l}\text { D7E74 } \\
\text { EMS-JAG-04 } \\
\qquad 1\end{array}$ \\
\hline Work Location: General & Effective Date: & April 28, 2006 \\
\hline Author: John A. Glissmeyer & Supersedes Date: & November 24, 1998 \\
\hline $\begin{array}{l}\text { Identified Hazards: } \\
\square \text { Radiological } \\
\square \text { Hazardous Materials } \\
\text { 冈 Physical Hazards } \\
\square \text { Hazardous Environment } \\
\square \text { Other: }\end{array}$ & \multicolumn{2}{|c|}{$\begin{array}{l}\text { Identified Use Category: } \\
\square \text { Mandatory Use } \\
\square \text { Continuous Use } \\
\text { 冈 Reference Use } \\
\square \text { Information Use }\end{array}$} \\
\hline
\end{tabular}

Are One-Time Modifications Allowed? $\square$ Yes $\bigotimes$ No

\begin{tabular}{|c|c|c|}
\hline Person Signing & Signature & Date \\
\hline $\begin{array}{c}\text { Technical review: } \\
\text { J. Matthew Barnett }\end{array}$ & & \\
\hline $\begin{array}{c}\text { Project Manager: } \\
\text { John Glissmeyer }\end{array}$ & \\
\hline $\begin{array}{c}\text { Line Manager: } \\
\text { James Droppo }\end{array}$ & \\
\hline $\begin{array}{c}\text { Quality Engineer: } \\
\text { Barry L. Sachs }\end{array}$ & & \\
\hline
\end{tabular}




\subsection{Purpose}

The performance of new stack sampling systems must be shown to satisfy the requirements of 40 CFR 61, Subpart H, "National Emission Standards for Emissions of Radionuclides Other than Radon from Department of Energy Facilities." This regulation governs portions of the design and implementation of effluent air sampling. The stack sampler performance is adequately characterized when potential contaminants in the effluent are of a uniform concentration at the sampling plane and line losses are within acceptable limits. (The sampling plane is the cross section of the stack or duct where the sampling nozzle inlet is located.) Uniformity of contaminant concentration is unlikely where the gas velocity throughout the sampling plane is significantly non-uniform. This procedure provides the means to determine the uniformity of gas velocity, and is performed prior to measurements of contaminant uniformity. This procedure is performed after the range of gas flow conditions are established. Other procedures that usually follow address flow angle, and uniformity of gas and aerosol contaminants.

\subsection{Applicability}

This procedure can be used for tests on actual or scale model stacks and ducts to determine the uniformity of air velocity throughout the sampling plane. The results also provide a detailed determination of the flowrate at the ventilation control settings used for the procedure. The tests are applicable within the following constraints:

- The operating limits of the air velocity measurement device used are observed.

- The air velocity sensor element does not occupy more than a few percent of the cross sectional area in the sampling plane.

This procedure may need to be repeated if there are significant changes made in the configuration of the ventilation system during the conduct of the remainder of the test plan. If the system under test operates within a limited range of airflow that does not change more than \pm $25 \%$, then this procedure is usually conducted once at the middle of the range. If the flow may vary more, then the procedure is performed at least at the extremes of flow.

\subsection{Prerequisites and Conditions}

Conditions and concerns that must be satisfied prior to performing this procedure are listed below:

- The job-hazards analysis for the work area must be prepared and followed.

- Safety glasses, hard toed or substantial shoes may be required in the work areas.

- Special training may be required to access the test ports of the stack.

- The flow control device must be installed and means available for its adjustment.

- Air velocity measurement equipment must be within calibration.

- The Test Instruction must be read and understood.

- This procedure must be read and understood. 


\subsection{Precautions and Limitations}

Access to the test ports may require the use of ladders, scaffolding or manlifts, which may necessitate special training for sampling personnel and any observers. The training requirements will be indicated in the job hazard analysis.

\subsection{Equipment Used for Measurements}

The following are essential items of equipment:

- Air velocity measurement apparatus, which may consist of a calibrated slant tube or electronic manometer, pitot tube, or an electronic sensor;

- Platform, ladders, or manlifts as needed to access the test ports;

- Fittings to limit leakage around the velocity sensor and to stabilize the sensor so it can be repositioned precisely.

- To provide information about the test conditions, commercial grade sensors for stack temperature, barometric pressure, static pressure, and humidity provide acceptable information. Likewise, data from a nearby meteorology or facility station is acceptable.

Further details on specific equipment for the job are provided in the Test Instruction. The air velocity instrumentation may be either the types used in 40 CFR 60, Appendix A, Method 2, or other measurement device for discrete points, such as a rotating vane or thermal anemometer. The user must be aware that different devices may give readings in terms of different gas conditions.

\subsection{Work Instructions for Setup, Measurements, and Calculations}

Job specific instructions given in the Test Instruction, illustrated in Exhibit B, will provide details and operating parameters necessary to perform this procedure.

\subsection{Preparing for Measurements:}

6.1.1 Verify that the interior dimensions of the stack or duct at the sampling plane agree with those used in calculating the grid of measurement points given in the Test Instruction or data sheet.

6.1.2 Provide essential supplies at the sampling location (velocity measuring instrumentation, fittings to adapt the sensor to the test ports, marking pens, data sheets, writing and sensor supporting platforms).

6.1.3 Verify that the ventilation flow control device is capable of the flow control settings given in the Test Instruction. 
6.1.4 Prepare a spreadsheet for calculating results and plotting data. See the illustration in Exhibit A. Label the columns of traverse data by the direction of the traverse. For example, if the first reading is closest to the east port, and the last reading is closest to the west port, then label the traverse east-west. Print blank copies of the spreadsheet as data forms for hand recording measurements for later transfer to the spreadsheet.

Note. For example, if the first reading is closest to the east port, and the last reading is closest to the west port, then label the traverse east-west. Also the first point is the one closest to the port.

Note. The grid of velocity measurement points is calculated in accordance with 40 CFR 60, Appendix A, Method 1. A centerpoint is included as a common reference and for graphical purposes. The layout design divides the area of the sampling plane so that each point represents approximately an enual_cized area

6.1.5 Mark the velocity sensor body to indicate the insertion depth for each point in the measurement grid.

6.1.6 Obtain barometric pressure, relative humidity, and stack or duct temperature and static pressure if needed to convert the velocity sensor readings to velocity units.

6.1.7 Insert the velocity sensor in the stack or duct and seal the opening around it. The seal does not have to be air tight.

\subsection{Velocity Uniformity Measurement}

6.2.1 Set the ventilation flow controller per the Test Instruction.

6.2.2 Verify that the directional orientations and the numbered measurement positions are consistent with the data sheet.

6.2.3 Measure and record, on the data sheet, the velocity or pressure reading at each measurement point in succession. If the readout device has an averaging feature, record the average of a series of several readings.

6.2.4 Repeat Step 6.2.3 two more times for a total of three measurements at each point.

6.2.5 Calculate the average air velocity for each measurement point. 
6.2.6 Review the data form for completeness.

6.2.7 Sign and date the data form indicating completion of the data collection portion of the procedure.

\subsection{Calculations}

6.3.1 Transfer the data to the spreadsheet created in Step 6.1.4.

6.3.2 Calculate the overall average and standard deviation of the velocity.

6.3.3 Calculate the coefficient of variance (COV, 100 times the standard deviation divided by the mean) using the average velocity for all points in the inner two-thirds of the cross section area (including the centerpoint).

6.3.4 Compare the observed COV for each run to the acceptance criterion. The acceptance criterion for the $\mathrm{COV}$ is $\bigcirc 20 \%$ for the inner two-thirds of the stack diameter.

6.3.5 Have the data transfers and calculations reviewed and verified. The reviewer should sign the printed data form.

\subsection{Records}

7.1 Transfer the original data forms and calculations to the records custodian as project records 


\section{Exhibits/Attachments}

\section{Exhibit A - Typical Velocity Traverse Data Form \\ VELOCITY TRAVERSE DATA FORM}

\begin{tabular}{|c|c|}
\hline \multicolumn{2}{|c|}{ Site Model } \\
\hline Date & Oct. 16, 2001 \\
\hline Testers & $\overline{\text { Glissmeyer and Maughan }}$ \\
\hline Stack Dia. & 23.5 in. \\
\hline Stack X-Area & 433.7 in. 2 \\
\hline Elevation & \\
\hline
\end{tabular}

Velocity units $\underline{\mathrm{ft} / \mathrm{min}}$
Run No. VT-1

Fan Configuration 4-fan: EF1, EF4, EF5, EF7

Fan Setting $\mathbf{5 1 \mathbf { ~ H z }}$

Stack Temp

Start/End Time 1432-1530 hours

Center 2/3 from

Points in Center 2/3

Data Files: NA

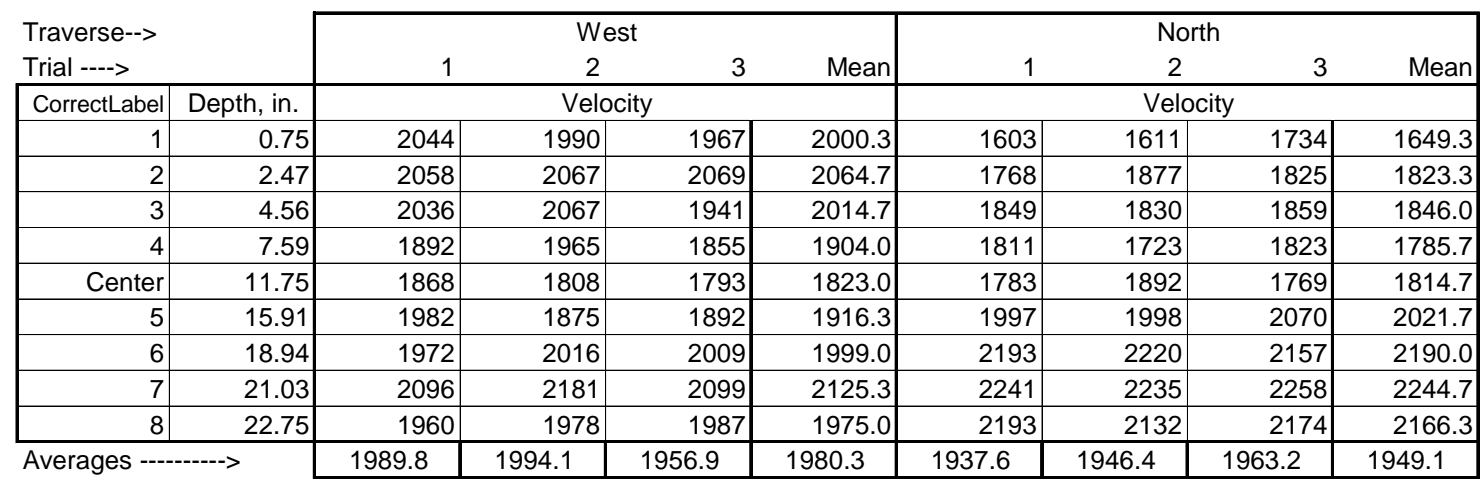

Averages -------->

\begin{tabular}{|llr|lrrr|}
\hline All & $\underline{\mathrm{ft} / \mathrm{min}}$ & Dev. from mean & Center 2/3 & $\underline{\text { West }}$ & $\underline{\text { North }}$ & All \\
Mean & 1964.7 & & Mean & 1978.1 & 1960.9 & 1969.5 \\
Min Point & 1649.3 & $-16.1 \%$ & Std. Dev. & 103.5 & 191.9 & 148.4 \\
Max Point & 2244.7 & $14.3 \%$ & COV as \% & 5.2 & 9.8 & 7.5 \\
\hline
\end{tabular}

Flow w/o C-Pt $5973 \mathrm{acfm}$ Vel Avg w/o C-Pt 1983 fpm

Stack temp Equipment temp Ambient temp Stack static Ambient pressure Total Stack pressure Ambient humidity

\begin{tabular}{|c|c|l}
\multicolumn{1}{c|}{ Start } & Finish & \\
\hline 72.5 & 70.8 & $\mathrm{~F}$ \\
\hline 71.6 & 75 & $\mathrm{~F}$ \\
\hline 70 & 70.7 & $\mathrm{~F}$ \\
\hline 0.5 & 0.52 & mbars \\
\hline 986.3 & 985.8 & mbars \\
\hline 986.8 & 986.3 & mbars \\
\hline $38 \%$ & $36 \%$ & $\mathrm{RH}$ \\
\hline
\end{tabular}

Notes:

Initial trial run to evaluate stack and instrument configuration.

\section{Instuments Used:}

Pitot \#5, 36-in. standard

Solmat Zephyr SN 12951472 Cal. Due 7/26/02

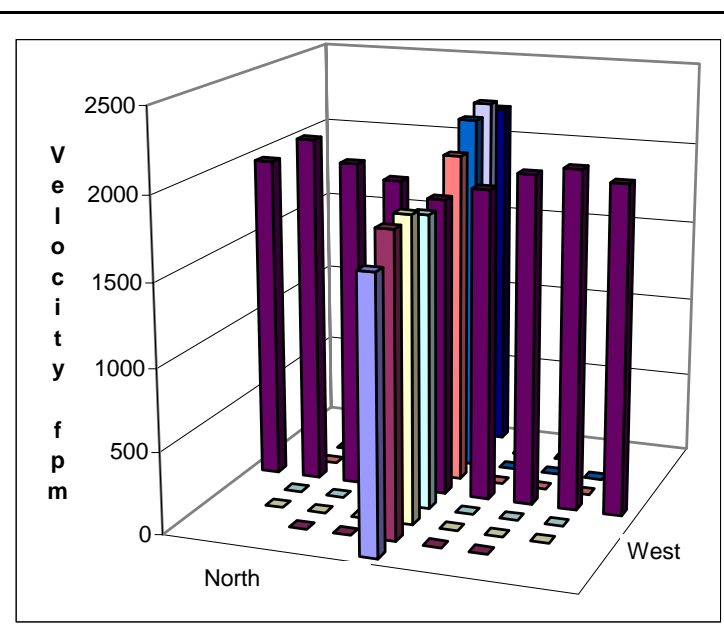

Signature signifies compliance with

Procedure EMS-JAG-4

Signature verifying data and calculations:

Signature/date

\section{Exhibit B -Typical Test Instruction}




\section{Test Instruction}

\begin{tabular}{|l|l|l|}
\hline Project: Model Stack & Date: August 19, 2007 & Work Package: K83017
\end{tabular}

Tests: Velocity Uniformity High Flow in Model Stack

Staff: David Maughan

Reference Procedures:

1. Operating Manual for Solomat Zephyr

2. Test to Determine Uniformity of Gas Velocity at the Elevation of a Sampler Probe, Procedure EMS-JAG-04

Equipment:

1. Model Stack, Fan and Fan Speed Controller located in 305 Bldg.

2. Solomat Zephyr and pitot tube

\section{Safety Considerations:}

Review and observe the applicable Job Hazard Analysis for the project

Instructions:

1. Assemble the equipment for the velocity uniformity test at the ports at the elevation of the sampling probe

2. Layout the measurement points with the following distances from the inside of the stack wall: $0.5,0.66,1.23,2.04,3.16,4.28,5.10,5.66,5.83$ inches.

3. Measure the velocity at each point at the high $(400 \mathrm{cfm})$ extreme of stack flow. Repeat each measurement twice.

4. Record data on velocity data sheets

5. Diagram mounting fixtures and retain assembly for subsequent tests

Desired Completion Date: $12 / 5 / 07$

Approvals:

John Glissmeyer, project manager

Test completed by:
Date

Date: 
Appendix D

Velocity Uniformity Data Sheets 


\section{APPENDIX D: VELOCITY UNIFORMITY DATA SHEETS}

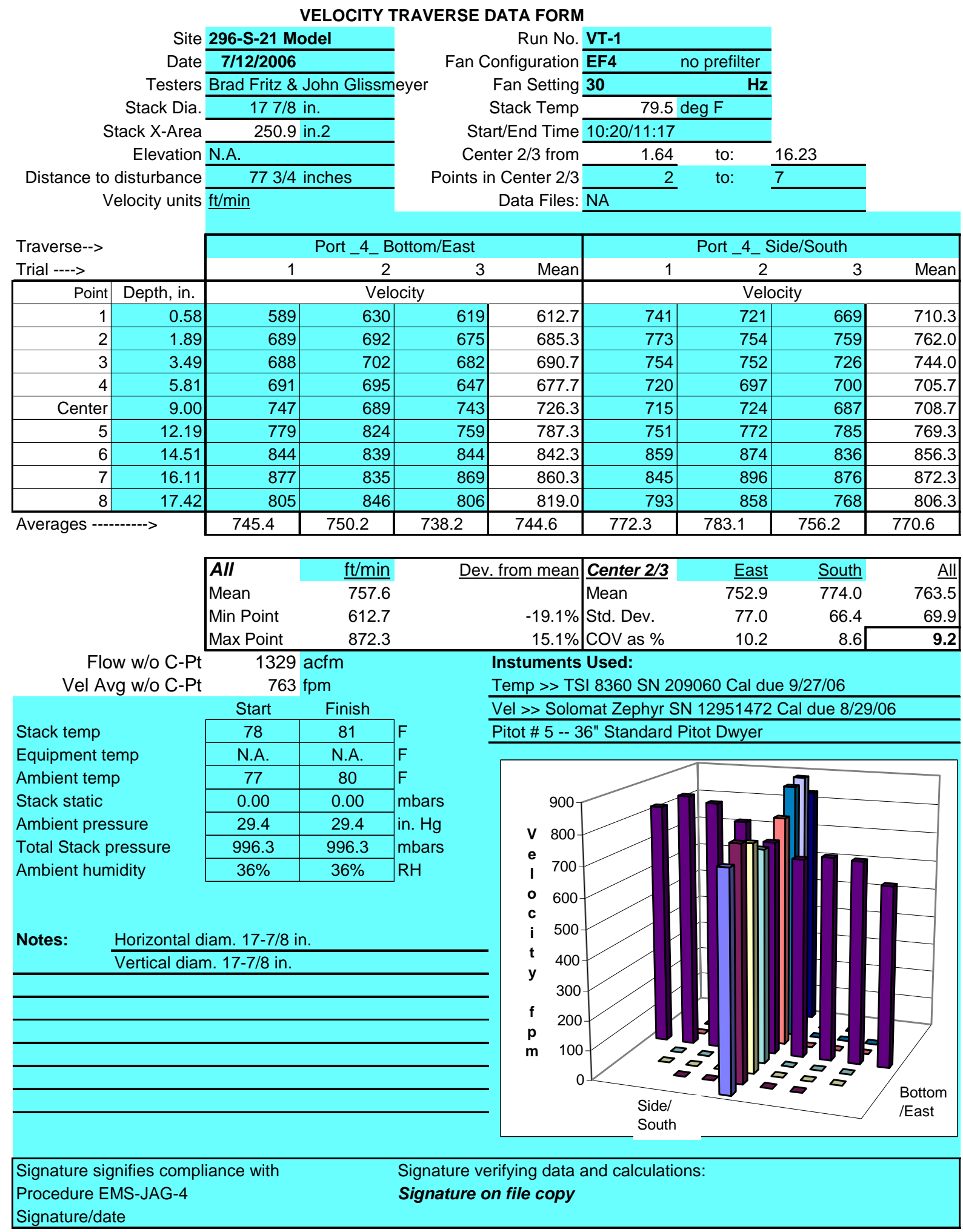




\section{VELOCITY TRAVERSE DATA FORM}

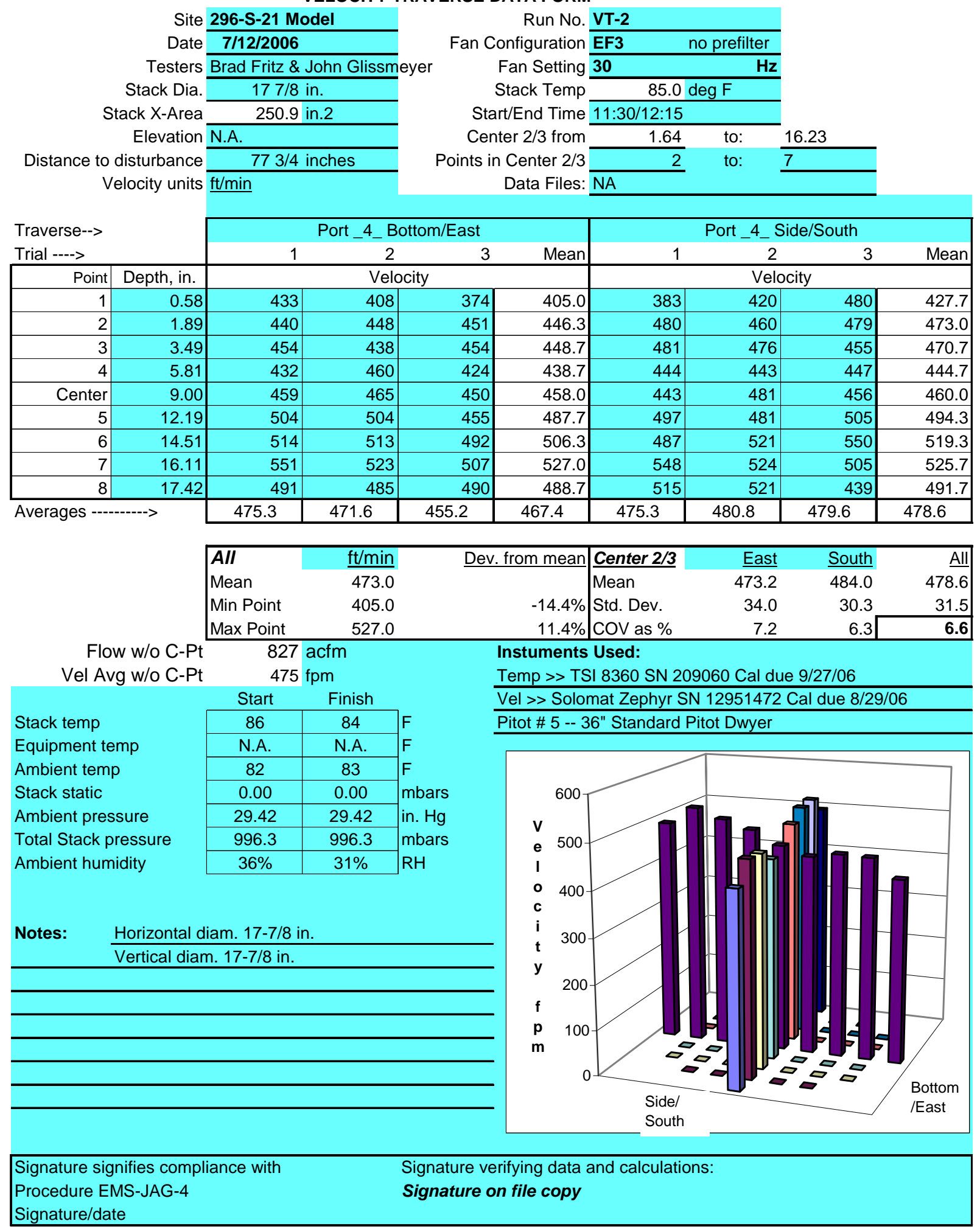




\section{VELOCITY TRAVERSE DATA FORM}

Site 296-S-21 Model Date 7/12/2006

Testers Brad Fritz

Stack Dia.

Stack X-Area

$177 / 8$ in

Elevation N.A.

Distance to disturbance

Velocity units $\underline{\mathrm{ft} / \mathrm{min}}$
Run No. VT-3

Fan Configuration EF2

Fan Setting $\mathbf{3 0}$

Stack Temp

Start/End Time 1300/1350

Center $2 / 3$ from

Points in Center 2/3

Data Files: NA

\begin{tabular}{|c|c|c|c|c|c|c|c|c|c|}
\hline \multirow{2}{*}{\multicolumn{2}{|c|}{$\begin{array}{l}\text { Traverse--> } \\
\text { Trial ----> }\end{array}$}} & \multicolumn{4}{|c|}{ Port_4_Bottom/East } & \multicolumn{4}{|c|}{ Port_4_Side/South } \\
\hline & & 1 & 2 & 3 & Mean & 1 & 2 & 3 & Mean \\
\hline Point & Depth, in. & \multicolumn{4}{|c|}{ Velocity } & \multicolumn{4}{|c|}{ Velocity } \\
\hline 1 & 0.58 & 413 & 418 & 428 & 419.7 & 471 & 460 & 439 & 456.7 \\
\hline 2 & 1.89 & 467 & 479 & 461 & 469.0 & 497 & 463 & 496 & 485.3 \\
\hline 3 & 3.49 & 475 & 492 & 460 & 475.7 & 441 & 485 & 452 & 459.3 \\
\hline 4 & 5.81 & 467 & 455 & 466 & 462.7 & 457 & 433 & 445 & 445.0 \\
\hline Center & 9.00 & 451 & 431 & 431 & 437.7 & 466 & 438 & 444 & 449.3 \\
\hline 5 & 12.19 & 463 & 449 & 448 & 453.3 & 469 & 461 & 454 & 461.3 \\
\hline 6 & 14.51 & 484 & 516 & 454 & 484.7 & 470 & 475 & 499 & 481.3 \\
\hline 7 & 16.11 & 516 & 519 & 500 & 511.7 & 452 & 509 & 500 & 487.0 \\
\hline 8 & 17.42 & 505 & 504 & 494 & 501.0 & 408 & 459 & 450 & 439.0 \\
\hline Averages --- & 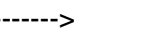 & 471.2 & 473.7 & 460.2 & 468.4 & 459.0 & 464.8 & 464.3 & 462.7 \\
\hline
\end{tabular}

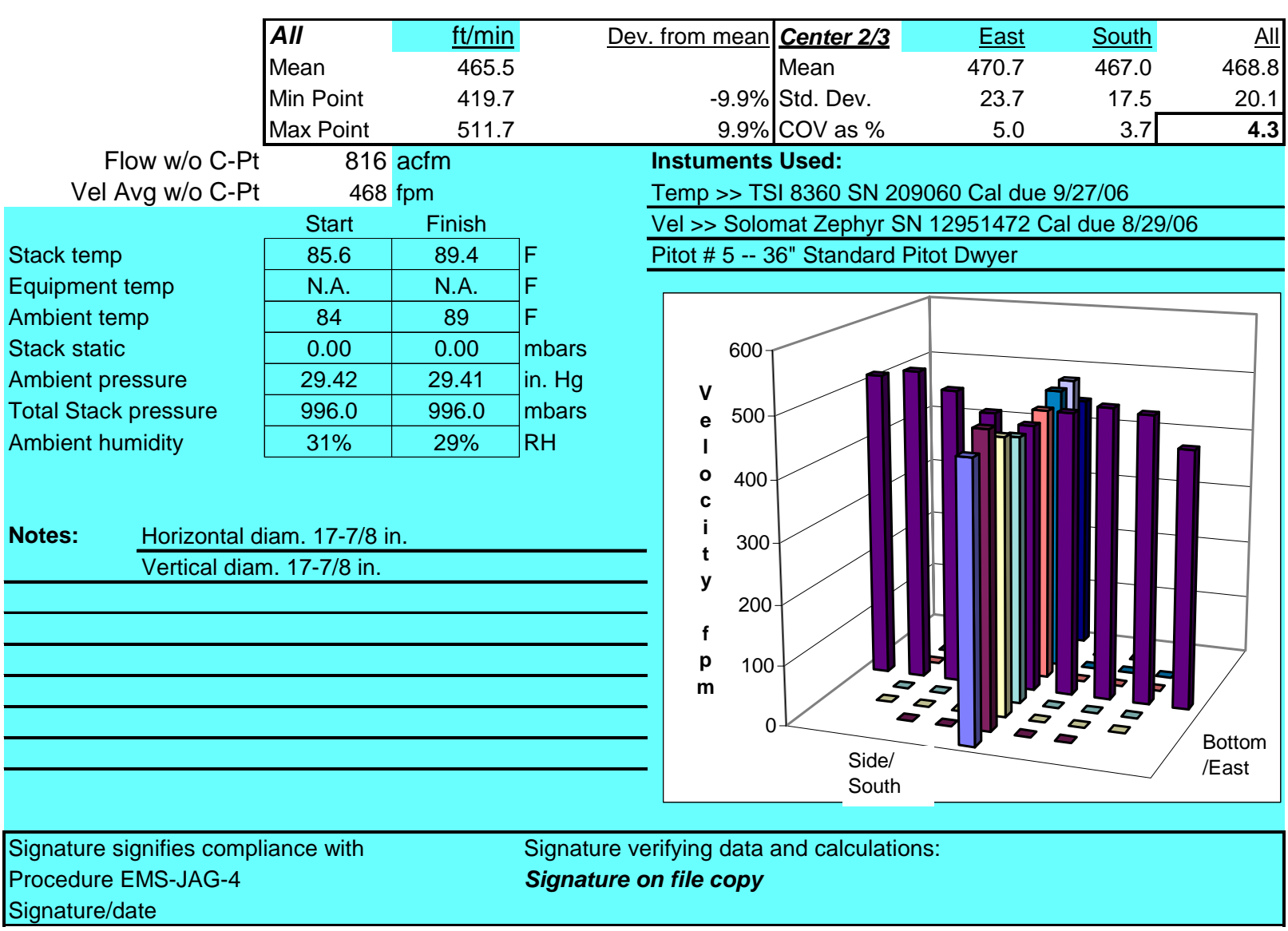




\section{VELOCITY TRAVERSE DATA FORM}

Site 296-S-21 Model

Date 7/12/2006

Testers Brad Fritz

Stack Dia.

Stack X-Area

$177 / 8$ in

Elevation N.A.

Distance to disturbance

Velocity units $\mathrm{ft} / \mathrm{min}$
Run No. VT-4

Fan Configuration EF1

Fan Setting $\mathbf{3 0}$

Stack Temp

Start/End Time 1400/1450

Center 2/3 from

Points in Center $2 / 3$

Data Files: NA

\begin{tabular}{|c|c|c|c|c|c|c|c|c|c|}
\hline \multirow{2}{*}{$\begin{array}{l}\text { Traverse--> } \\
\text { Trial ----> }\end{array}$} & & \multicolumn{4}{|c|}{ Port_4_Bottom/East } & \multicolumn{4}{|c|}{ Port_4_Side/South } \\
\hline & & 1 & 2 & 3 & Mean & 1 & 2 & 3 & Mean \\
\hline Point & Depth, in. & \multicolumn{4}{|c|}{ Velocity } & \multicolumn{4}{|c|}{ Velocity } \\
\hline 1 & 0.58 & 415 & 422 & 387 & 408.0 & 419 & 401 & 435 & 418.3 \\
\hline 2 & 1.89 & 482 & 470 & 480 & 477.3 & 439 & 447 & 450 & 445.3 \\
\hline 3 & 3.49 & 466 & 460 & 492 & 472.7 & 428 & 441 & 434 & 434.3 \\
\hline 4 & 5.81 & 448 & 429 & 451 & 442.7 & 396 & 401 & 406 & 401.0 \\
\hline Center & 9.00 & 412 & 411 & 392 & 405.0 & 398 & 406 & 418 & 407.3 \\
\hline 5 & 12.19 & 410 & 439 & 421 & 423.3 & 378 & 434 & 416 & 409.3 \\
\hline 6 & 14.51 & 425 & 431 & 430 & 428.7 & 429 & 394 & 434 & 419.0 \\
\hline 7 & 16.11 & 414 & 433 & 442 & 429.7 & 455 & 435 & 431 & 440.3 \\
\hline 8 & 17.42 & 410 & 416 & 413 & 413.0 & 441 & 432 & 415 & 429.3 \\
\hline Averages --- & 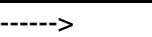 & 431.3 & 434.6 & 434.2 & 433.4 & 420.3 & 4212 & 426.6 & 422.7 \\
\hline
\end{tabular}

\begin{tabular}{|lrr|lrrr|}
\hline All & ft/min & Dev. from mean & Center 2/3 & East & South & All \\
Mean & 428.0 & & Mean & 439.9 & 422.4 & 431.1 \\
Min Point & 401.0 & $-6.3 \%$ & Std. Dev. & 26.5 & 17.6 & 23.4 \\
Max Point & 477.3 & $11.5 \%$ & Cov as \% & 6.0 & 4.2 & $\mathbf{5 . 4}$ \\
\hline
\end{tabular}

Flow w/o C-Pt Vel Avg w/o C-Pt

$751 \mathrm{acfm}$

$431 \mathrm{fpm}$

Stack temp

Equipment temp

Ambient temp

Stack static

Ambient pressure

Total Stack pressure

Ambient humidity

\begin{tabular}{|c|c|l}
\multicolumn{1}{|c|}{ Start } & Finish & \\
\hline 89.4 & 89.6 & $\mathrm{~F}$ \\
\cline { 1 - 2 } N.A. & N.A. & $\mathrm{F}$ \\
\hline 88.7 & 89.1 & $\mathrm{~F}$ \\
\hline 0.00 & 0.00 & mbars \\
\cline { 1 - 2 } 29.41 & 29.41 & in. Hg \\
\cline { 1 - 2 } 996.0 & 996.0 & mbars \\
\cline { 1 - 2 } $31 \%$ & $31 \%$ & $\mathrm{RH}$ \\
\hline
\end{tabular}

Notes: Horizontal diam. 17-7/8 in. Vertical diam. 17-7/8 in.

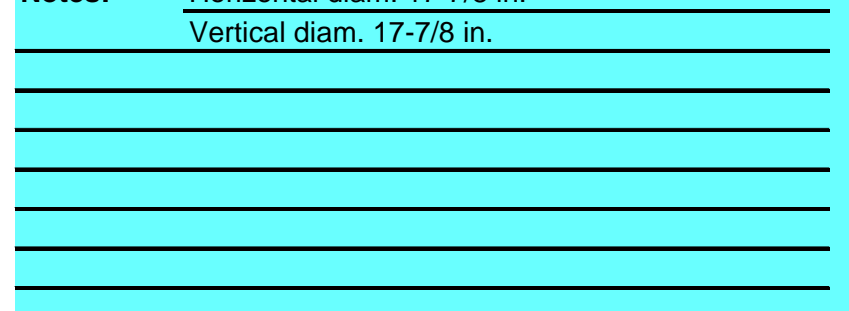

Instuments Used:

Temp >> TSI 8360 SN 209060 Cal due 9/27/06 Vel $>>$ Solomat Zephyr SN 12951472 Cal due 8/29/06 Pitot \# 5 -- 36" Standard Pitot Dwyer

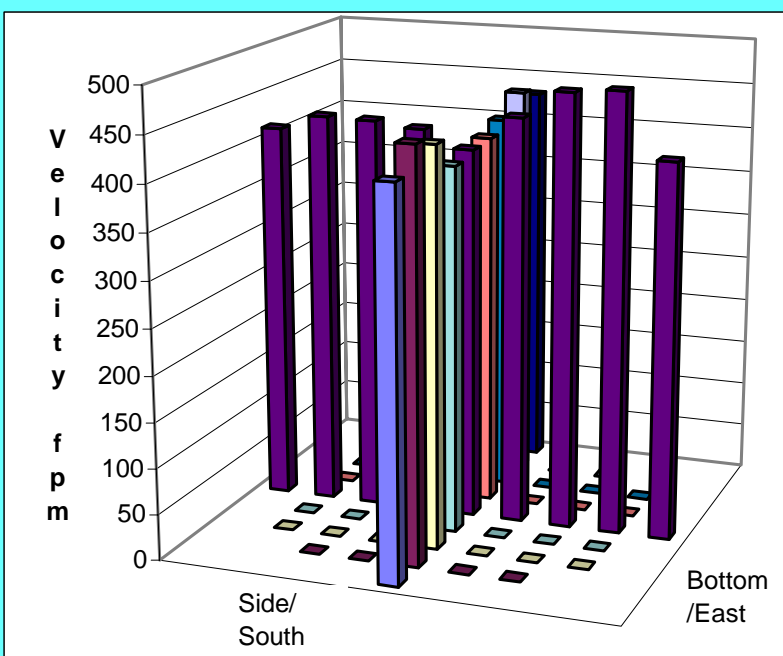

Signature signifies compliance with Procedure EMS-JAG-4 Signature/date
Signature verifying data and calculations:

Signature on file copy 


\section{VELOCITY TRAVERSE DATA FORM}

Site 296-S-21 Model

Date 7/14/2006

Testers JGD \& JAG

Stack Dia.

Stack X-Area

Elevation N.A.

Distance to disturbance

Velocity units $\underline{\mathrm{ft} / \mathrm{min}}$
Run No. VT-5

Fan Configuration 1, 2, 3

Fan Setting 53, 40, $45 \quad \mathrm{~Hz}$

Stack Temp $\frac{96.5 \mathrm{deg} F}{1437 / 1545}$

Start/End Time 1437/1545

Center $2 / 3$ from

Points in Center $2 / 3$ Data Files: NA

\begin{tabular}{|c|c|c|c|c|c|c|c|c|c|}
\hline \multirow{2}{*}{\multicolumn{2}{|c|}{$\begin{array}{l}\text { Traverse--> } \\
\text { Trial ----> }\end{array}$}} & \multicolumn{4}{|c|}{ Port_3_Bottom/NorthEast } & \multicolumn{4}{|c|}{ Port_3_Side/SouthEast } \\
\hline & & 1 & 2 & 3 & Mean & 1 & 2 & 3 & Mean \\
\hline Point & Depth, in. & \multicolumn{4}{|c|}{ Velocity } & \multicolumn{4}{|c|}{ Velocity } \\
\hline 1 & $9 / 16$ & 2211 & 2354 & 2331 & 2298.7 & 1999 & 2064 & 1986 & 2016.3 \\
\hline 2 & $114 / 16$ & 2253 & 2223 & 2242 & 2239.3 & 2155 & 2060 & 2089 & 2101.3 \\
\hline 3 & $38 / 16$ & 2086 & 2068 & 2075 & 2076.3 & 1949 & 1998 & 1986 & 1977.7 \\
\hline 4 & $513 / 16$ & 1720 & 1760 & 1795 & 1758.3 & 1561 & 1618 & 1670 & 1616.3 \\
\hline Center & 9 & 1510 & 1480 & 1476 & 1488.7 & 1468 & 1413 & 1381 & 1420.7 \\
\hline 5 & $123 / 16$ & 1732 & 1703 & 1738 & 1724.3 & 1747 & 1589 & 1716 & 1684.0 \\
\hline 6 & $148 / 16$ & 2004 & 2016 & 2001 & 2007.0 & 1989 & 1976 & 2067 & 2010.7 \\
\hline 7 & $16 \quad 1 / 16$ & 2142 & 2078 & 2159 & 2126.3 & 2125 & 2212 & 2042 & 2126.3 \\
\hline 8 & $176 / 16$ & 2141 & 2106 & 2151 & 2132.7 & 2229 & 2123 & 2232 & 2194.7 \\
\hline verages ---. & $--->$ & 1977.7 & 1976.4 & 1996.4 & 1983.5 & 1913.6 & 1894.8 & 1907.7 & 1905.3 \\
\hline
\end{tabular}

\begin{tabular}{|c|c|c|c|c|c|c|}
\hline$A I I$ & $\mathrm{ft} / \mathrm{min}$ & Dev. from mean & Center 2/3 & NorthEast & SouthEast & All \\
\hline Mean & 1944.4 & & Mean & 1917.2 & 1848.1 & 1882.7 \\
\hline Min Point & 1420.7 & $-26.9 \%$ & Std. Dev. & 266.8 & 273.3 & 261.9 \\
\hline Max Point & 2298.7 & $18.2 \%$ & cov as $\%$ & 13.9 & 14.8 & 13.9 \\
\hline
\end{tabular}

Flow w/o C-Pt Vel Avg w/o C-Pt 3544 acfm $2006 \mathrm{fpm}$

Stack temp Equipment temp Ambient temp Stack static Ambient pressure Total Stack pressure Ambient humidity

\begin{tabular}{|c|c|l}
\multicolumn{1}{|c|}{ Start } & Finish & \\
\hline 101.0 & 102.0 & $\mathrm{~F}$ \\
\hline 83 & 89 & $\mathrm{~F}$ \\
\hline 95 & 98 & $\mathrm{~F}$ \\
\hline 0.00 & 0.00 & mbars \\
\hline 29.48 & 29.48 & in Hg \\
\hline 998.0 & 998.0 & mbars \\
\hline $17 \%$ & $16 \%$ & $\mathrm{RH}$ \\
\hline
\end{tabular}

Notes: Horizontal diameter 18 in. Vertical diameter 18 in
Instuments Used:

Temp >> TSI 8360 SN $209060 \mathrm{Cal}$ due 9/27/06 Vel $>>$ Solomat Zephyr SN 12951472 Cal due 8/29/06 Pitot \# 5 -- 36" Standard Pitot Dwyer

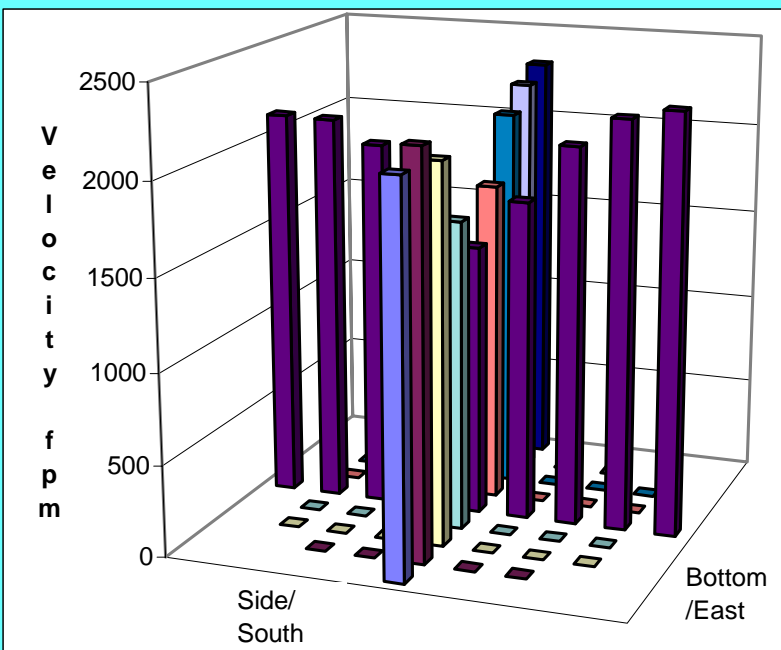

Signature signifies compliance with Procedure EMS-JAG-4 Signature/date
Signature verifying data and calculations:

Signature on file copy 


\section{VELOCITY TRAVERSE DATA FORM}

Site 296-S-21 Model

Date 7/14/2006

Testers JGD \& JAG

Stack Dia.

Stack X-Area

Elevation N.A.

Distance to disturbance

Velocity units $\mathrm{ft} / \mathrm{min}$
Run No. VT-5 No C.P.

Fan Configuration 1, 2, 3 no prefilter

Fan Setting 53, 40, $45 \quad \mathbf{H z}$

Stack Temp $96.5 \operatorname{deg} F$

Start/End Time 1437/1545

Center $2 / 3$ from

Points in Center $2 / 3$

Data Files: NA

\begin{tabular}{|c|c|c|c|c|c|c|c|c|c|}
\hline \multirow{2}{*}{\multicolumn{2}{|c|}{$\begin{array}{l}\text { Traverse--> } \\
\text { Trial ----> }\end{array}$}} & \multicolumn{4}{|c|}{ Port_3_Bottom/NorthEast } & \multicolumn{4}{|c|}{ Port_3_Side/SouthEast } \\
\hline & & 1 & 2 & 3 & Mean & 1 & 2 & 3 & Mean \\
\hline Point & Depth, in. & \multicolumn{4}{|c|}{ Velocity } & \multicolumn{4}{|c|}{ Velocity } \\
\hline 1 & $9 / 16$ & 2211 & 2354 & 2331 & 2298.7 & 1999 & 2064 & 1986 & 2016.3 \\
\hline 2 & $114 / 16$ & 2253 & 2223 & 2242 & 2239.3 & 2155 & 2060 & 2089 & 2101.3 \\
\hline 3 & $38 / 16$ & 2086 & 2068 & 2075 & 2076.3 & 1949 & 1998 & 1986 & 1977.7 \\
\hline 4 & $513 / 16$ & 1720 & 1760 & 1795 & 1758.3 & 1561 & 1618 & 1670 & 1616.3 \\
\hline Center & 9 & 1510 & 1480 & 1476 & N.A. & 1468 & 1413 & 1381 & N.A. \\
\hline 5 & $12 \quad 3 / 16$ & 1732 & 1703 & 1738 & 1724.3 & 1747 & 1589 & 1716 & 1684.0 \\
\hline 6 & $148 / 16$ & 2004 & 2016 & 2001 & 2007.0 & 1989 & 1976 & 2067 & 2010.7 \\
\hline 7 & $16 \quad 1 / 16$ & 2142 & 2078 & 2159 & 2126.3 & 2125 & 2212 & 2042 & 2126.3 \\
\hline 8 & $176 / 16$ & 2141 & 2106 & 2151 & 2132.7 & 2229 & 2123 & 2232 & 2194.7 \\
\hline Averages --- & ------> & 1977.7 & 1976.4 & 1996.4 & 2045.4 & 1913.6 & 1894.8 & 1907.7 & 1965.9 \\
\hline
\end{tabular}

\begin{tabular}{|llr|lrrrr|}
\hline All & $\underline{\mathrm{ft} / \mathrm{min}}$ & Dev. from mean & Center 2/3 & & NorthEast & SouthEast & All \\
Mean & 2005.6 & & Mean & & 1988.6 & 1919.4 & 1954.0 \\
Min Point & 1616.3 & $-19.4 \%$ & Std. Dev. & 206.3 & 216.8 & 204.9 \\
Max Point & 2298.7 & $14.6 \%$ & COV as \% & 10.4 & 11.3 & $\mathbf{1 0 . 5}$ \\
\hline
\end{tabular}

Flow w/o C-Pt Vel Avg w/o C-Pt 3544 acfm $2006 \mathrm{fpm}$

Stack temp Equipment temp Ambient temp Stack static Ambient pressure Total Stack pressure Ambient humidity

\begin{tabular}{|c|c|l}
\multicolumn{1}{|c|}{ Start } & Finish & \multicolumn{1}{l}{} \\
\hline 101.0 & 102.0 & $\mathrm{~F}$ \\
\hline 83 & 89 & $\mathrm{~F}$ \\
\hline 95 & 98 & $\mathrm{~F}$ \\
\hline 0.00 & 0.00 & mbars \\
\hline 998.00 & 998.00 & in. Hg \\
\hline 998.0 & 998.0 & mbars \\
\hline $17 \%$ & $16 \%$ & $\mathrm{RH}$ \\
\hline
\end{tabular}

\section{Notes:}

Horizontal diameter 18 in.

Vertical diameter 18 in.

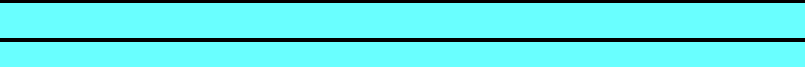

Instuments Used:

Temp >> TSI 8360 SN $209060 \mathrm{Cal}$ due 9/27/06 Vel >> Solomat Zephyr SN 12951472 Cal due 8/29/06 Pitot \# 5 -- 36" Standard Pitot Dwyer

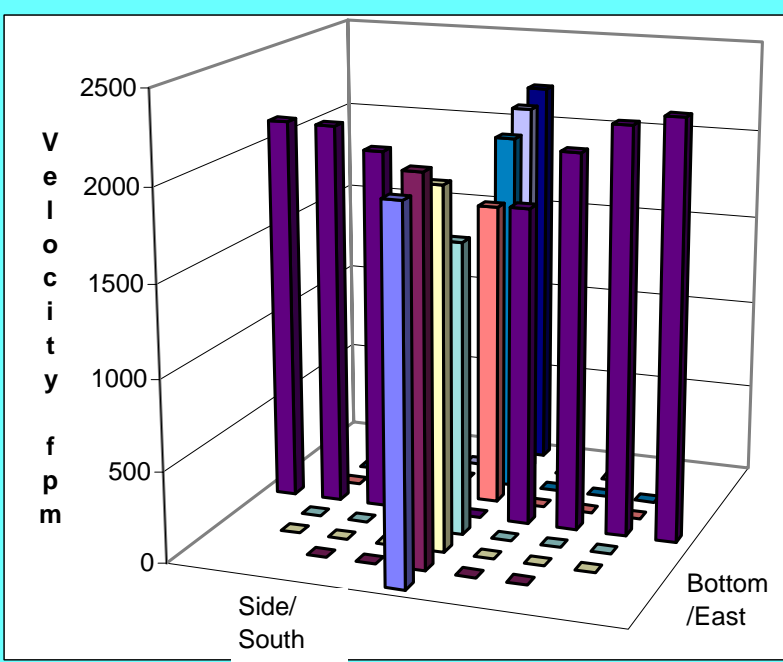

Signature signifies compliance with Procedure EMS-JAG-4 Signature/date
Signature verifying data and calculations:

Signature on file copy 


\section{VELOCITY TRAVERSE DATA FORM}

Site 296-S-21 Model

Date 7/15/2006

Testers Droppo \& Glissmeyer

Stack Dia. $177 / 8$ in.

Stack X-Area 250.9 in.2

Elevation N.A.

Distance to disturbance

Velocity units $\mathrm{ft} / \mathrm{min}$
Run No. VT-6

Fan Configuration 1, 2, 3

Fan Setting 54, 41, $46 \quad \mathrm{~Hz}$

Stack Temp $81.5 \operatorname{deg} F$

Start/End Time 1020/1130

Center 2/3 from

Points in Center 2/3 Data Files: NA

\begin{tabular}{|c|c|c|c|c|c|c|c|c|c|}
\hline \multirow{2}{*}{\multicolumn{2}{|c|}{$\begin{array}{l}\text { Traverse--> } \\
\text { Trial ----> }\end{array}$}} & \multicolumn{4}{|c|}{ Port_4_Bottom/East } & \multicolumn{4}{|c|}{ Port_4_Side/South } \\
\hline & & 1 & 2 & 3 & Mean & 1 & 2 & 3 & Mean \\
\hline Point & Depth, in. & \multicolumn{4}{|c|}{ Velocity } & \multicolumn{4}{|c|}{ Velocity } \\
\hline 1 & $9 / 16$ & 1942 & 1982 & 1938 & 1954.0 & 1664 & 1850 & 1820 & 1778.0 \\
\hline 2 & $114 / 16$ & 2044 & 2142 & 2105 & 2097.0 & 1973 & 1942 & 1967 & 1960.7 \\
\hline 3 & $38 / 16$ & 2036 & 2042 & 2042 & 2040.0 & 1985 & 2119 & 1956 & 2020.0 \\
\hline 4 & $513 / 16$ & 1939 & 1952 & 1926 & 1939.0 & 1874 & 1957 & 1828 & 1886.3 \\
\hline Center & 9 & 1770 & 1844 & 1837 & 1817.0 & 1818 & 1829 & 1785 & 1810.7 \\
\hline 5 & $123 / 16$ & 1838 & 1865 & 1941 & 1881.3 & 1919 & 1800 & 2040 & 1919.7 \\
\hline 6 & $148 / 16$ & 2001 & 2032 & 2044 & 2025.7 & 2016 & 1915 & 2128 & 2019.7 \\
\hline 7 & $161 / 16$ & 2024 & 2041 & 2115 & 2060.0 & 2070 & 2006 & 2154 & 2076.7 \\
\hline 8 & $176 / 16$ & 1962 & 1942 & 1910 & 1938.0 & 2069 & 1907 & 2065 & 2013.7 \\
\hline Averages --- & $\cdots$ & 1950.7 & 1982.4 & 1984.2 & 1972.4 & 1932.0 & 1925.0 & 1971.4 & 1942.8 \\
\hline
\end{tabular}

\begin{tabular}{|lrr|lrrr|}
\hline All & $\underline{\mathrm{ft} / \mathrm{min}}$ & Dev. from mean & Center 2/3 & $\underline{\text { East }}$ & $\underline{\text { South }}$ & $\underline{\text { All }}$ \\
Mean & 1957.6 & & Mean & 1980.0 & 1956.2 & 1968.1 \\
Min Point & 1778.0 & $-9.2 \%$ & Std. Dev. & 103.1 & 91.3 & 94.4 \\
Max Point & 2097.0 & $7.1 \%$ & COV as \% & 5.2 & 4.7 & $\mathbf{4 . 8}$ \\
\hline
\end{tabular}

Flow w/o C-Pt Vel Avg w/o C-Pt 1976 fpm

Stack temp Equipment temp Ambient temp Stack static Ambient pressure Total Stack pressure Ambient humidity

\begin{tabular}{|c|c|l}
\multicolumn{1}{|c|}{ Start } & Finish & \\
\hline 89.0 & 89.0 & $\mathrm{~F}$ \\
\hline 77 & 82 & $\mathrm{~F}$ \\
\hline 80 & 83 & $\mathrm{~F}$ \\
\hline 0.00 & 0.00 & mbars \\
\cline { 1 - 2 } $1003 \mathrm{mb}$ & 29.60 & in Hg \\
\hline 1003.0 & 1002.0 & $\mathrm{mbars}$ \\
\hline $33 \%$ & $29 \%$ & $\mathrm{RH}$ \\
\hline
\end{tabular}

Notes:

Horizontal diameter $17-7 / 8$ in

Vertical diameter 17-7/8 in.

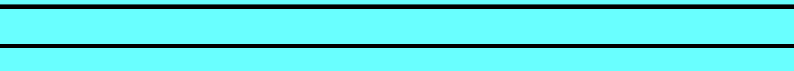

Instuments Used:

Temp >> TSI 8360 SN 209060 Cal due 9/27/06 Vel >> Solomat Zephyr SN 12951472 Cal due 8/29/06 Pitot \# 5 -- 36" Standard Pitot Dwyer

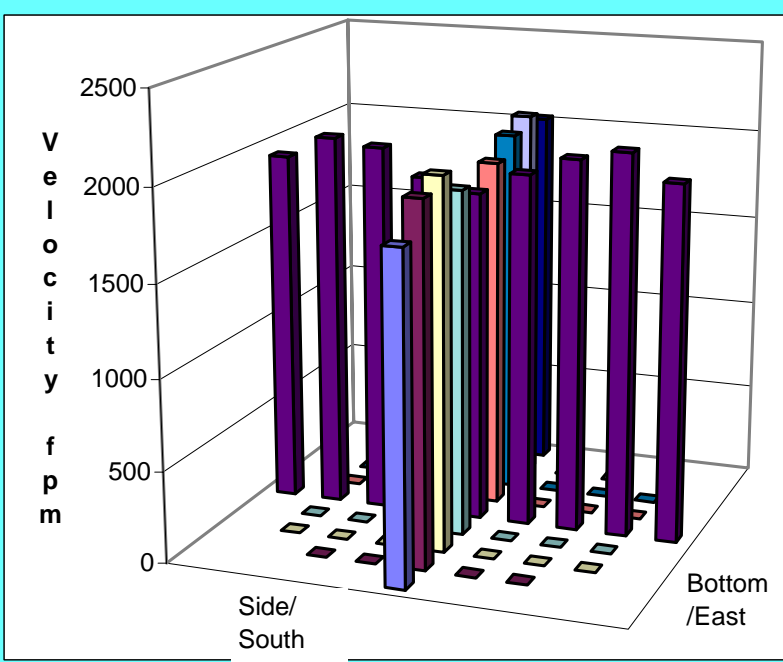

Signature signifies compliance with Procedure EMS-JAG-4 Signature/date
Signature verifying data and calculations:

Signature on file copy 


\section{VELOCITY TRAVERSE DATA FORM}

Site 296-S-21 Model

Date 7/15/2006

Testers Droppo \& Glissmeyer

Stack Dia. $177 / 8$ in.

Stack X-Area 250.9 in.2

Elevation N.A.

Distance to disturbance

Velocity units $\mathrm{ft} / \mathrm{min}$
Run No. VT-6

No C.P.

Fan Configuration 1, 2, 3 no prefilter

Fan Setting $\mathbf{5 4 , 4 1 , 4 6 \quad \mathrm { Hz }}$

Stack Temp

Start/End Time 1020/1130

Center $2 / 3$ from

Points in Center $2 / 3$

Data Files: NA

\begin{tabular}{|c|c|c|c|c|c|c|c|c|c|}
\hline \multirow{2}{*}{$\begin{array}{l}\text { Traverse--> } \\
\text { Trial ----> }\end{array}$} & & \multicolumn{4}{|c|}{ Port_4_Bottom/East } & \multicolumn{4}{|c|}{ Port_4_Side/South } \\
\hline & & 1 & 2 & 3 & Mean & 1 & 2 & 3 & Mean \\
\hline Point & Depth, in. & \multicolumn{4}{|c|}{ Velocity } & \multicolumn{4}{|c|}{ Velocity } \\
\hline 1 & $9 / 16$ & 1942 & 1982 & 1938 & 1954.0 & 1664 & 1850 & 1820 & 1778.0 \\
\hline 2 & $114 / 16$ & 2044 & 2142 & 2105 & 2097.0 & 1973 & 1942 & 1967 & 1960.7 \\
\hline 3 & $38 / 16$ & 2036 & 2042 & 2042 & 2040.0 & 1985 & 2119 & 1956 & 2020.0 \\
\hline 4 & $513 / 16$ & 1939 & 1952 & 1926 & 1939.0 & 1874 & 1957 & 1828 & 1886.3 \\
\hline Center & 9 & 1770 & 1844 & 1837 & N.A. & 1818 & 1829 & 1785 & N.A. \\
\hline 5 & $123 / 16$ & 1838 & 1865 & 1941 & 1881.3 & 1919 & 1800 & 2040 & 1919.7 \\
\hline 6 & $148 / 16$ & 2001 & 2032 & 2044 & 2025.7 & 2016 & 1915 & 2128 & 2019.7 \\
\hline 7 & $16 \quad 1 / 16$ & 2024 & 2041 & 2115 & 2060.0 & 2070 & 2006 & 2154 & 2076.7 \\
\hline 8 & $176 / 16$ & 1962 & 1942 & 1910 & 1938.0 & 2069 & 1907 & 2065 & 2013.7 \\
\hline Averages --- & $--->$ & 1950.7 & 1982.4 & 1984.2 & 1991.9 & 2.0 & 1925.0 & 1971.4 & 1959.3 \\
\hline
\end{tabular}

\begin{tabular}{|lrr|lrrr|}
\hline All & $\underline{\mathrm{ft} / \mathrm{min}}$ & Dev. from mean & Center 2/3 & East & $\underline{\text { South }}$ & $\underline{\text { All }}$ \\
Mean & 1975.6 & & Mean & 2007.2 & 1980.5 & 1993.8 \\
Min Point & 1778.0 & $-10.0 \%$ & Std. Dev. & 80.9 & 71.1 & 74.0 \\
Max Point & 2097.0 & $6.1 \%$ & COV as $\%$ & 4.0 & 3.6 & $\mathbf{3 . 7}$ \\
\hline
\end{tabular}

Flow w/o C-Pt Vel Avg w/o C-Pt 3443 acfm 1976 fpm

Stack temp Equipment temp Ambient temp Stack static Ambient pressure Total Stack pressure Ambient humidity

\begin{tabular}{|c|c|c|}
\hline Start & Finish & \\
\hline 89.0 & 89.0 & $F$ \\
\hline 77 & 82 & $F$ \\
\hline 80 & 83 & $F$ \\
\hline 0.00 & 0.00 & mbars \\
\hline $1003 \mathrm{mb}$ & 29.60 & in $\mathrm{Hg}$ \\
\hline 1003.0 & 1002.0 & mbars \\
\hline $33 \%$ & $29 \%$ & $\mathrm{RH}$ \\
\hline
\end{tabular}

Notes:

Horizontal diameter 17-7/8 in.

Vertical diameter 17-7/8 in.

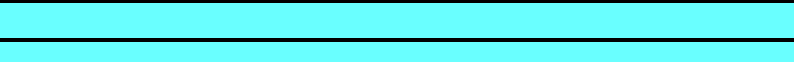

Instuments Used:

Temp $>>$ TSI 8360 SN $209060 \mathrm{Cal}$ due 9/27/06 Vel >> Solomat Zephyr SN 12951472 Cal due 8/29/06 Pitot \# 5 -- 36" Standard Pitot Dwyer

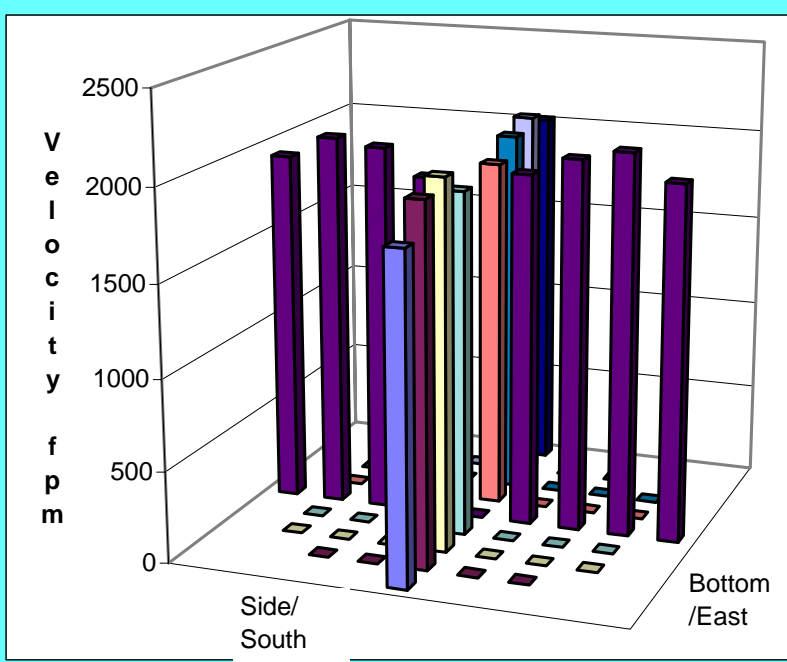

Signature signifies compliance with Procedure EMS-JAG-4 Signature/date
Signature verifying data and calculations:

Signature on file copy 


\section{VELOCITY TRAVERSE DATA FORM}

Site 296-S-21 Model

Date 7/15/2006

Testers Droppo \& Glissmeyer

Stack Dia. 18 in.

Stack X-Area 254.5 in. 2

Elevation N.A.

Distance to disturbance

Velocity units $\mathrm{ft} / \mathrm{min}$
Run No. VT-7

Fan Configuration 1, 2, 3

Fan Setting 54, 41, $46 \quad \mathrm{~Hz}$

Stack Temp

Start/End Time 1145/1250

Center $2 / 3$ from

Points in Center $2 / 3$ Data Files: NA

\begin{tabular}{|c|c|c|c|c|c|c|c|c|c|}
\hline \multirow{2}{*}{$\begin{array}{l}\text { Traverse--> } \\
\text { Trial ----> }\end{array}$} & & \multicolumn{4}{|c|}{ Port_1_Bottom/East } & \multicolumn{4}{|c|}{ Port_1_Side/South } \\
\hline & & 1 & 2 & 3 & Mean & 1 & 2 & 3 & Mean \\
\hline Point & Depth, in. & \multicolumn{4}{|c|}{ Velocity } & \multicolumn{4}{|c|}{ Velocity } \\
\hline 1 & $9 / 16$ & 2606 & 2582 & 2607 & 2598.3 & 2484 & 2573 & 2756 & 2604.3 \\
\hline 2 & $114 / 16$ & 2406 & 2400 & 2424 & 2410.0 & 2414 & 2495 & 2374 & 2427.7 \\
\hline 3 & $38 / 16$ & 2182 & 2186 & 1974 & 2114.0 & 2249 & 2207 & 2014 & 2156.7 \\
\hline 4 & $513 / 16$ & 514 & 93 & 580 & 395.7 & 700 & 899 & 759 & 786.0 \\
\hline Center & 9 & -429 & -476 & -678 & -527.7 & -760 & -126 & -56 & -314.0 \\
\hline 5 & $123 / 16$ & 1252 & 976 & 1359 & 1195.7 & 860 & 1173 & 516 & 849.7 \\
\hline 6 & $148 / 16$ & 2382 & 2305 & 2442 & 2376.3 & 2279 & 2371 & 2269 & 2306.3 \\
\hline 7 & $16 \quad 1 / 16$ & 2366 & 2388 & 2283 & 2345.7 & 2762 & 2549 & 2403 & 2571.3 \\
\hline 8 & $176 / 16$ & 2471 & 2321 & 2172 & 2321.3 & 2304 & 2375 & 2433 & 2370.7 \\
\hline verages ---. & $\cdots$ & 1750.0 & 1641.7 & 1684.8 & 1692.1 & 1699.1 & 1835.1 & 1718.7 & 1751.0 \\
\hline
\end{tabular}

\begin{tabular}{|lrr|lrrr|}
\hline All & $\underline{\mathrm{ft} / \mathrm{min}}$ & Dev. from mean & Center 2/3 & East & South & $\underline{\text { All }}$ \\
Mean & 1721.6 & & Mean & 1472.8 & 1540.5 & 1506.7 \\
Min Point & -527.7 & $-130.7 \%$ & Std. Dev. & 1162.4 & 1103.1 & 1089.3 \\
Max Point & 2604.3 & $51.3 \%$ & COV as $\%$ & 78.9 & 71.6 & $\mathbf{7 2 . 3}$ \\
\hline
\end{tabular}

Flow w/o C-Pt Vel Avg w/o C-Pt 3515 acfm 1989 fpm

Stack temp Equipment temp Ambient temp Stack static Ambient pressure Total Stack pressure Ambient humidity

\begin{tabular}{|c|c|l}
\hline Start & Finish & \\
\hline 89.0 & 91.0 & $F$ \\
\hline 82 & 83 & $F$ \\
\hline 83 & 86 & F \\
\hline-0.35 & -0.12 & mbars \\
\cline { 1 - 2 } 29.6 & 29.58 & in Hg \\
\hline 1002 & 1002 & mbars \\
\hline $29 \%$ & $29 \%$ & $\mathrm{RH}$ \\
\hline
\end{tabular}

Notes:

Horizontal diameter 18 in.

Vertical diameter 18 in.

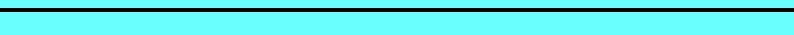

Instuments Used:

Temp >> TSI 8360 SN $209060 \mathrm{Cal}$ due 9/27/06 Vel >> Solomat Zephyr SN 12951472 Cal due 8/29/06 Pitot \# 5 -- 36" Standard Pitot Dwyer

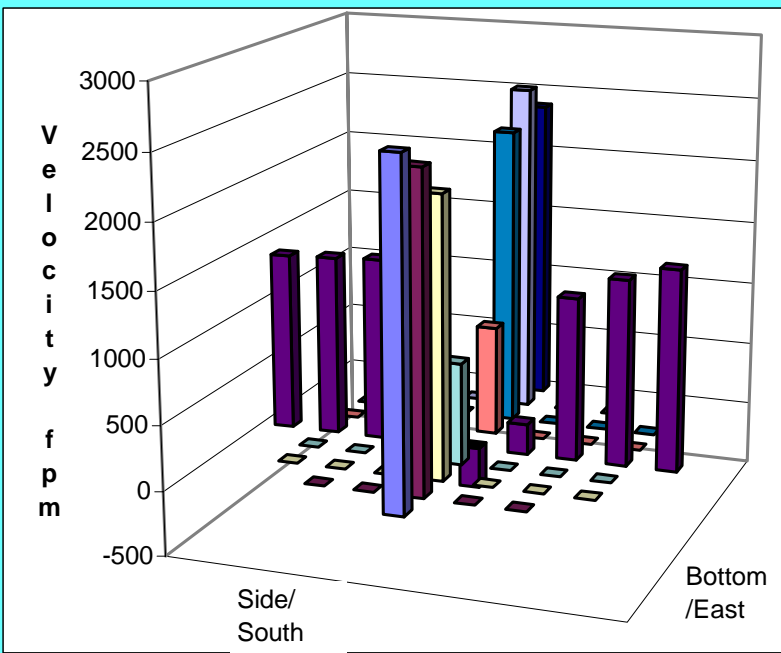

Signature signifies compliance with Procedure EMS-JAG-4 Signature/date
Signature verifying data and calculations:

Signature on file copy 


\section{VELOCITY TRAVERSE DATA FORM}

Site 296-S-21 Model

Date 7/15/2006

Testers Droppo \& Glissmeyer

Stack Dia. 18 in.

Stack X-Area 254.5 in. 2

Elevation N.A.

Distance to disturbance

Velocity units $\mathrm{ft} / \mathrm{min}$
Run No. VT-7

No C.P.

Fan Configuration 1, 2, 3 no prefilter

Fan Setting $\mathbf{5 4 , 4 1 , 4 6 \quad \mathrm { Hz }}$

Stack Temp $84.5 \mathrm{deg} F$

Start/End Time 1145/1250

Center $2 / 3$ from

Points in Center $2 / 3$

Data Files: NA

\begin{tabular}{|c|c|c|c|c|c|c|c|c|c|}
\hline & & \multirow{2}{*}{\multicolumn{4}{|c|}{ Port_1_Bottom/East }} & \multirow{2}{*}{\multicolumn{4}{|c|}{ Port_1_Side/South }} \\
\hline \multirow{2}{*}{\multicolumn{2}{|c|}{$\begin{array}{l}\text { Tra } \\
\text { Tria }\end{array}$}} & & & & & & & & \\
\hline & & \multirow[t]{2}{*}{1} & 2 & \multirow{2}{*}{\multicolumn{2}{|c|}{3}} & \multirow[t]{2}{*}{1} & 2 & 3 & Mean \\
\hline Point & Depth, in. & & Velocity & & & & \multicolumn{2}{|c|}{ Velocity } & \\
\hline 1 & $9 / 16$ & 2606 & 2582 & 2607 & 2598.3 & 2484 & 2573 & 2756 & 2604.3 \\
\hline 2 & $114 / 16$ & 2406 & 2400 & 2424 & 2410.0 & 2414 & 2495 & 2374 & 2427.7 \\
\hline 3 & $38 / 16$ & 2182 & 2186 & 1974 & 2114.0 & 2249 & 2207 & 2014 & 2156.7 \\
\hline 4 & $513 / 16$ & 514 & 93 & 580 & 395.7 & 700 & 899 & 759 & 786.0 \\
\hline Center & 9 & -429 & -476 & -678 & N.A. & -760 & -126 & -56 & N.A. \\
\hline 5 & $123 / 16$ & 1252 & 976 & 1359 & 1195.7 & 860 & 1173 & 516 & 849.7 \\
\hline 6 & $148 / 16$ & 2382 & 2305 & 2442 & 2376.3 & 2279 & 2371 & 2269 & 2306.3 \\
\hline 7 & $16 \quad 1 / 16$ & 2366 & 2388 & 2283 & 2345.7 & 2762 & 2549 & 2403 & 2571.3 \\
\hline 8 & $176 / 16$ & 2471 & 2321 & 2172 & 2321.3 & 2304 & 2375 & 2433 & 2370.7 \\
\hline \multicolumn{2}{|l|}{ Averages ----- } & 1750.0 & 1641.7 & 1684.8 & 1969.6 & 1699.1 & 1835.1 & 1718.7 & 2009.1 \\
\hline
\end{tabular}

\begin{tabular}{|lrr|lrrr|}
\hline All & $\underline{\mathrm{ft} / \mathrm{min}}$ & Dev. from mean & Center 2/3 & East & South & All \\
Mean & 1989.4 & & Mean & 1806.2 & 1849.6 & 1827.9 \\
Min Point & 395.7 & $-80.1 \%$ & Std. Dev. & 829.2 & 811.1 & 782.3 \\
Max Point & 2604.3 & $30.9 \%$ & COV as $\%$ & 45.9 & 43.9 & $\mathbf{4 2 . 8}$ \\
\hline
\end{tabular}

Flow w/o C-Pt Vel Avg w/o C-Pt

3515 acfm

1989 fpm

Stack temp

Equipment temp

Ambient temp

Stack static

Ambient pressure

Total Stack pressure

Ambient humidity

\begin{tabular}{|c|c|l}
\hline Start & Finish & \\
\hline 89.0 & 91.0 & $F$ \\
\hline 82 & 83 & F \\
\hline 83 & 86 & F \\
\hline-0.35 & -0.12 & mbars \\
\cline { 1 - 2 } 29.6 & 29.58 & in Hg \\
\hline 1002 & 1002 & mbars \\
\hline $29 \%$ & $29 \%$ & $\mathrm{RH}$ \\
\hline
\end{tabular}

Notes:

Horizontal diameter 18 in.

Vertical diameter 18 in.

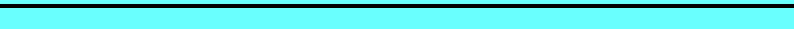

Instuments Used:

Temp >> TSI 8360 SN 209060 Cal due 9/27/06 Vel >> Solomat Zephyr SN 12951472 Cal due 8/29/06 Pitot \# 5 -- 36" Standard Pitot Dwyer

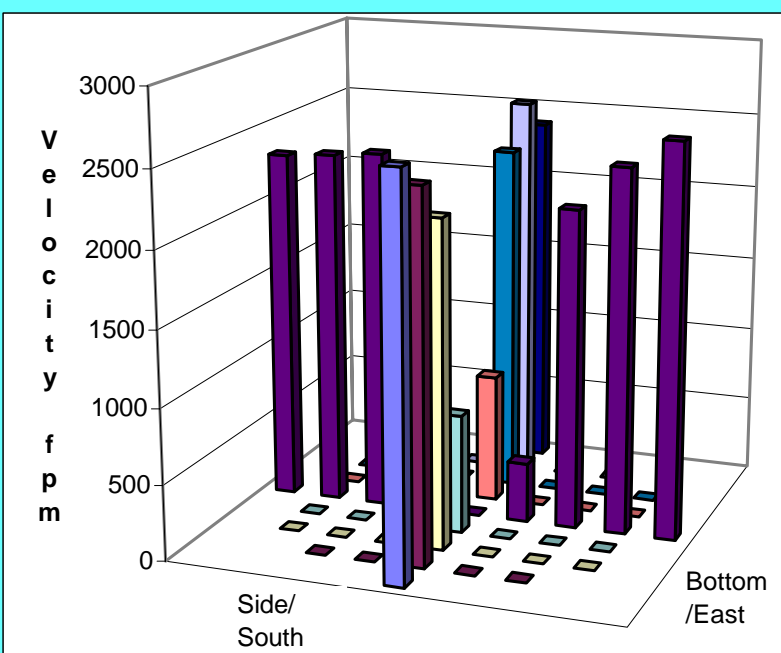

Signature signifies compliance with

Procedure EMS-JAG-4

Signature/date
Signature verifying data and calculations:

Signature on file copy 


\section{VELOCITY TRAVERSE DATA FORM}

Site 296-S-21 Model

Date 7/15/2006

Testers Droppo \& Glissmeyer

Stack Dia.

Stack X-Area

$177 / 8$ in

Elevation N.A.

Distance to disturbance

Velocity units $\mathrm{ft} / \mathrm{min}$
Run No. VT-8

Fan Configuration 1, 4

Fan Setting $\mathbf{5 4 , 4 8}$

Stack Temp

Start/End Time 1255/1330

Center 2/3 from

Points in Center $2 / 3$ Data Files: NA

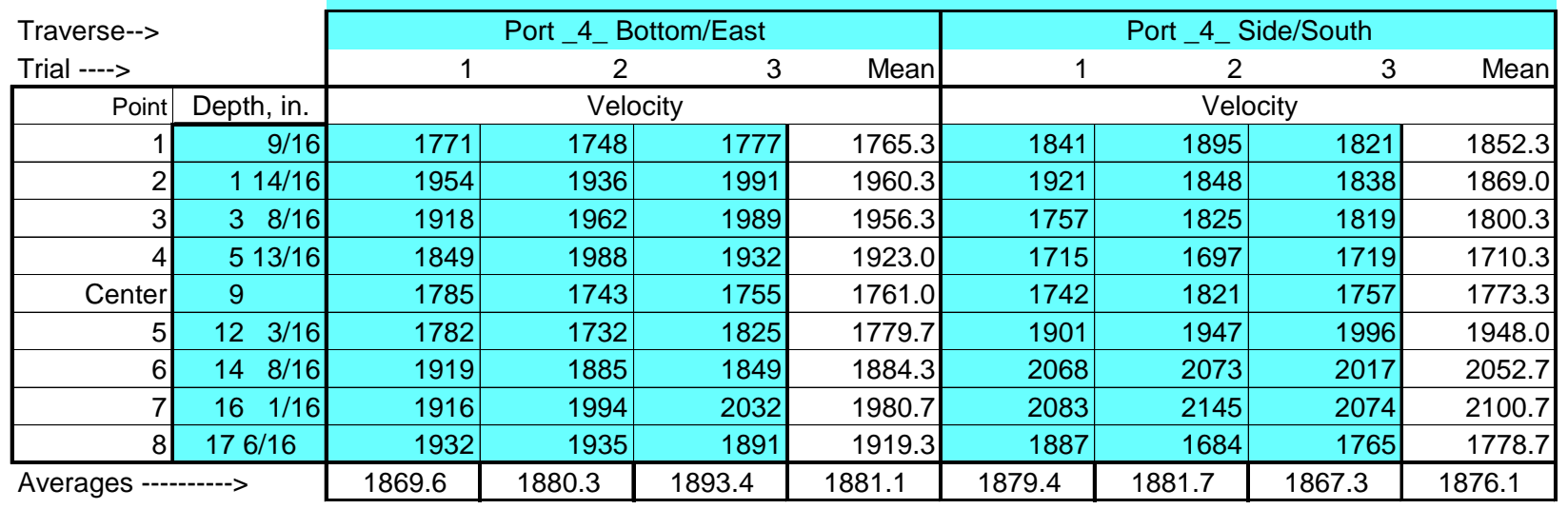

\begin{tabular}{|lrr|lrrr|}
\hline All & $\underline{\mathrm{ft} / \mathrm{min}}$ & Dev. from mean & Center 2/3 & East & $\underline{\text { South }}$ & $\underline{\text { All }}$ \\
Mean & 1878.6 & & Mean & 1892.2 & 1893.5 & 1892.8 \\
Min Point & 1710.3 & $-9.0 \%$ & Std. Dev. & 88.9 & 146.3 & 116.3 \\
Max Point & 2100.7 & $11.8 \%$ & COV as $\%$ & 4.7 & 7.7 & $\mathbf{6 . 1}$ \\
\hline
\end{tabular}

Flow w/o C-Pt 3298 acfm Vel Avg w/o C-Pt $1893 \mathrm{fpm}$

Stack temp Equipment temp Ambient temp Stack static Ambient pressure Total Stack pressure Ambient humidity

\begin{tabular}{|c|c|l}
\multicolumn{1}{|c|}{ Start } & Finish & \\
\hline 91.0 & 93.0 & $\mathrm{~F}$ \\
\hline 83 & 85 & $\mathrm{~F}$ \\
\hline 86 & 90 & $\mathrm{~F}$ \\
\hline 0.00 & 0.00 & mbars \\
\hline 29.58 & 29.57 & in Hg \\
\hline 1002 & 1002 & mbars \\
\hline $29 \%$ & $28 \%$ & $\mathrm{RH}$ \\
\hline
\end{tabular}

Notes:

Horizontal diameter $17-7 / 8$ in.

Vertical diameter 17-7/8 in.

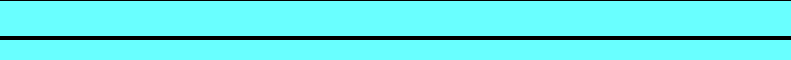

Instuments Used:

Temp >> TSI 8360 SN 209060 Cal due 9/27/06 Vel >> Solomat Zephyr SN 12951472 Cal due 8/29/06 Pitot \# 5 -- 36" Standard Pitot Dwyer

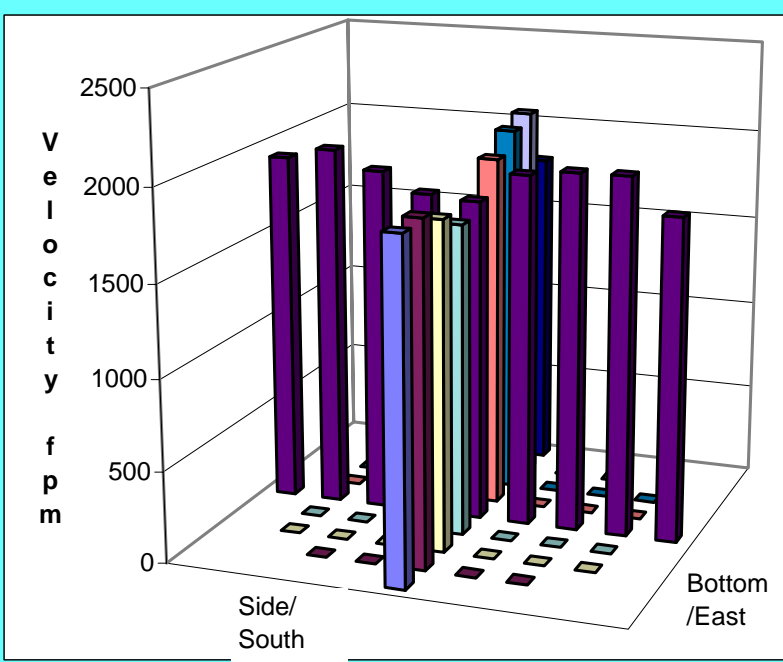

Signature signifies compliance with Procedure EMS-JAG-4 Signature/date
Signature verifying data and calculations:

Signature on file copy 


\section{VELOCITY TRAVERSE DATA FORM}

Site 296-S-21 Model

Date 7/15/2006

Testers Droppo \& Glissmeyer

Stack Dia. $177 / 8$ in.

Stack X-Area 250.9 in.2

Elevation N.A.

Distance to disturbance

Velocity units $\mathrm{ft} / \mathrm{min}$
Run No. VT-8 No C.P.

Fan Configuration $1,4 \quad$ no prefilter

Fan Setting $\mathbf{5 4 , 4 8 \quad ~} \mathbf{H z}$

Stack Temp

Start/End Time 1255/1330

Center 2/3 from

Points in Center 2/3 Data Files: $\mathrm{NA}$

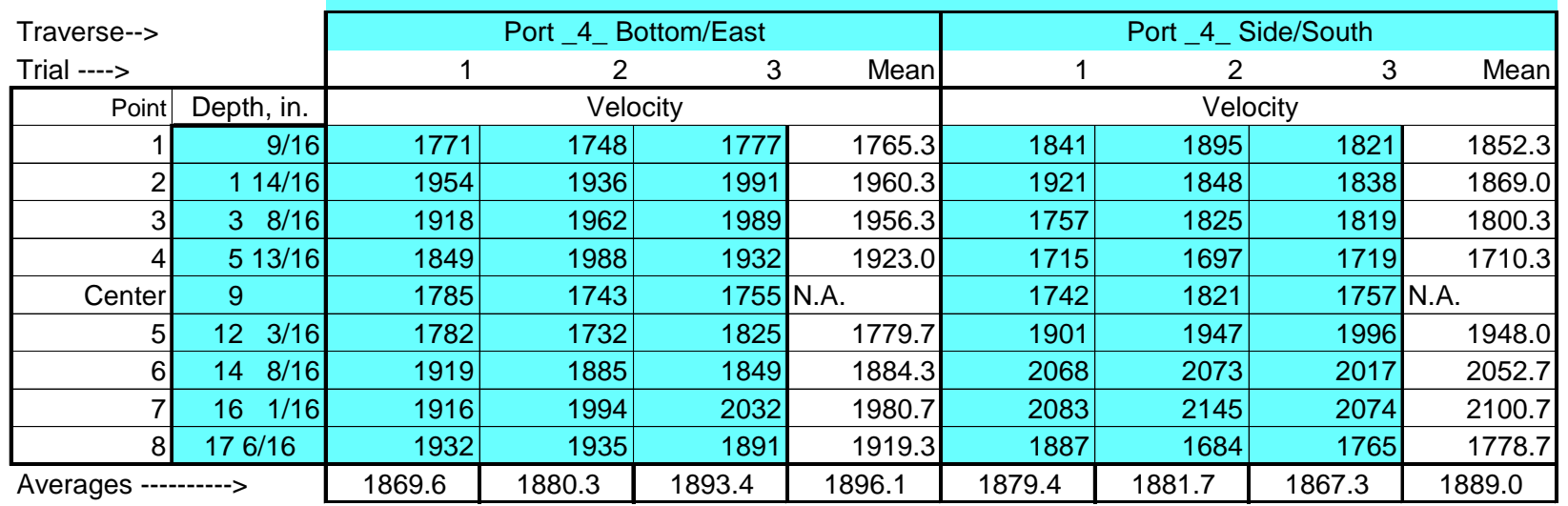

\begin{tabular}{|lrr|lrrr|}
\hline All & $\underline{\mathrm{ft} / \mathrm{min}}$ & Dev. from mean & Center 2/3 & East & $\underline{\text { South }}$ & $\underline{\text { All }}$ \\
Mean & 1892.6 & & Mean & 1914.1 & 1913.5 & 1913.8 \\
Min Point & 1710.3 & $-9.6 \%$ & Std. Dev. & 74.0 & 149.4 & 112.4 \\
Max Point & 2100.7 & $11.0 \%$ & COV as $\%$ & 3.9 & 7.8 & 5.9 \\
\hline
\end{tabular}

Flow w/o C-Pt 3298 acfm Vel Avg w/o C-Pt $1893 \mathrm{fpm}$

Stack temp Equipment temp Ambient temp Stack static Ambient pressure Total Stack pressure Ambient humidity

\begin{tabular}{|c|c|l}
\hline Start & Finish & \\
\hline 91.0 & 93.0 & $F$ \\
\hline 83 & 85 & $\mathrm{~F}$ \\
\hline 86 & 90 & $\mathrm{~F}$ \\
\hline 0.00 & 0.00 & mbars \\
\hline 29.58 & 29.57 & in Hg \\
\hline 1002 & 1002 & $\mathrm{mbars}$ \\
\hline $29 \%$ & $28 \%$ & $\mathrm{RH}$ \\
\hline
\end{tabular}

Notes:

Horizontal diameter $17-7 / 8$ in.

Vertical diameter 17-7/8 in.

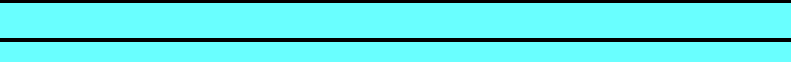

Instuments Used:

Temp >> TSI 8360 SN 209060 Cal due 9/27/06 Vel >> Solomat Zephyr SN 12951472 Cal due 8/29/06 Pitot \# 5 -- 36" Standard Pitot Dwyer

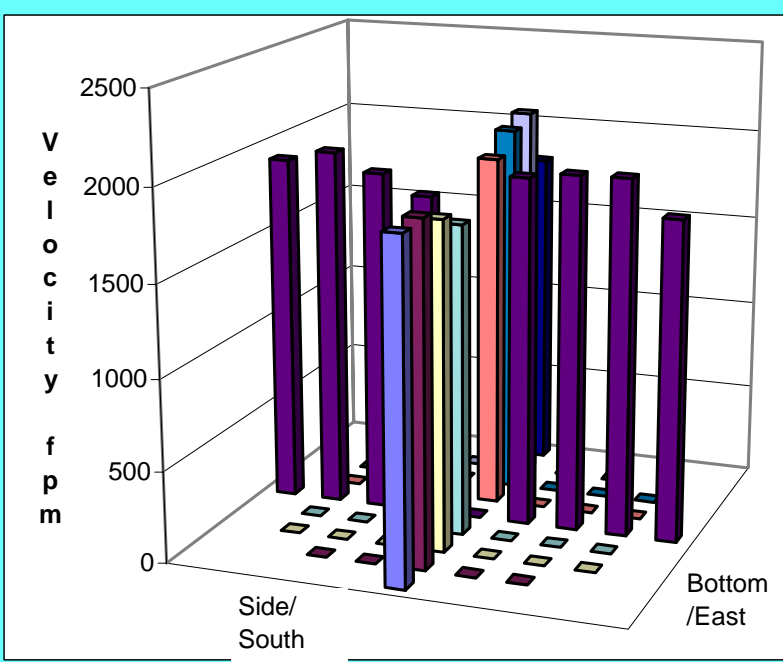

Signature signifies compliance with Procedure EMS-JAG-4 Signature/date
Signature verifying data and calculations:

Signature on file copy 


\section{VELOCITY TRAVERSE DATA FORM}

Site 296-S-21 Model

Date 7/17/2006

Testers Droppo \& Fritz

Stack Dia.

Stack X-Area

$177 / 8$ in

Elevation N.A.

Distance to disturbance

Velocity units $\mathrm{ft} / \mathrm{min}$
Run No. VT-9

Fan Configuration 2, 4

Fan Setting 41, 48

Stack Temp

Start/End Time 1045/1130

Center $2 / 3$ from

Points in Center $2 / 3$ Data Files: NA

\begin{tabular}{|c|c|c|c|c|c|c|c|c|c|}
\hline \multirow{2}{*}{\multicolumn{2}{|c|}{$\begin{array}{l}\text { Traverse--> } \\
\text { Trial ----> }\end{array}$}} & \multicolumn{4}{|c|}{ Port_4_Bottom/East } & \multicolumn{4}{|c|}{ Port_4_Side/South } \\
\hline & & 1 & 2 & 3 & Mean & 1 & 2 & 3 & Mean \\
\hline Point & Depth, in. & \multicolumn{4}{|c|}{ Velocity } & \multicolumn{4}{|c|}{ Velocity } \\
\hline 1 & $9 / 16$ & 1667 & 1720 & 1697 & 1694.7 & 1585 & 1653 & 1623 & 1620.3 \\
\hline 2 & $114 / 16$ & 1803 & 1701 & 1794 & 1766.0 & 1688 & 1716 & 1605 & 1669.7 \\
\hline 3 & $38 / 16$ & 1770 & 1856 & 1791 & 1805.7 & 1646 & 1623 & 1702 & 1657.0 \\
\hline 4 & $513 / 16$ & 1703 & 1710 & 1741 & 1718.0 & 1576 & 1572 & 1581 & 1576.3 \\
\hline Center & 9 & 1614 & 1703 & 1727 & 1681.3 & 1681 & 1592 & 1658 & 1643.7 \\
\hline 5 & $123 / 16$ & 1613 & 1751 & 1745 & 1703.0 & 1714 & 1808 & 1738 & 1753.3 \\
\hline 6 & $148 / 16$ & 1760 & 1804 & 1696 & 1753.3 & 1928 & 1938 & 1867 & 1911.0 \\
\hline 7 & $161 / 16$ & 1763 & 1762 & 1759 & 1761.3 & 1881 & 1899 & 1847 & 1875.7 \\
\hline 8 & $176 / 16$ & 1649 & 1693 & 1678 & 1673.3 & 1764 & 1941 & 1915 & 1873.3 \\
\hline Averages --- & $\cdots$ & 1704.7 & 1744.4 & 1736.4 & 1728.5 & 1718.1 & 1749.1 & 1726.2 & 1731.1 \\
\hline
\end{tabular}

\begin{tabular}{|lrr|lrrr|}
\hline All & $\underline{\mathrm{ft} / \mathrm{min}}$ & Dev. from mean & Center 2/3 & East & South & $\underline{\text { All }}$ \\
Mean & 1729.8 & & Mean & 1741.2 & 1726.7 & 1734.0 \\
Min Point & 1576.3 & $-8.9 \%$ & Std. Dev. & 42.6 & 125.5 & 90.4 \\
Max Point & 1911.0 & $10.5 \%$ & COV as $\%$ & 2.4 & 7.3 & $\mathbf{5 . 2}$ \\
\hline
\end{tabular}

Flow w/o C-Pt Vel Avg w/o C-Pt 3029 acfm $1738 \mathrm{fpm}$

Stack temp Equipment temp Ambient temp Stack static Ambient pressure Total Stack pressure Ambient humidity

\begin{tabular}{|c|c|l}
\multicolumn{1}{|c|}{ Start } & Finish & \\
\hline 87.4 & 88.7 & $\mathrm{~F}$ \\
\cline { 1 - 2 } N.A. & 84 & $\mathrm{~F}$ \\
\hline 86 & 86 & $\mathrm{~F}$ \\
\hline 0.00 & 0.00 & mbars \\
\hline 29.63 & 29.62 & in Hg \\
\hline 1003 & 1003 & mbars \\
\hline $30 \%$ & $27 \%$ & $\mathrm{RH}$ \\
\hline
\end{tabular}

Notes:

Horizontal diameter $17-7 / 8$ in.

Vertical diameter 17-7/8 in.

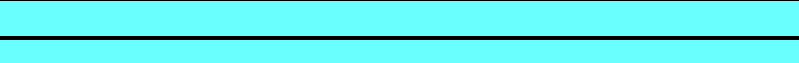

Instuments Used:

Temp >> TSI 8360 SN 209060 Cal due 9/27/06 Vel >> Solomat Zephyr SN 12951472 Cal due 8/29/06 Pitot \# 5 -- 36" Standard Pitot Dwyer

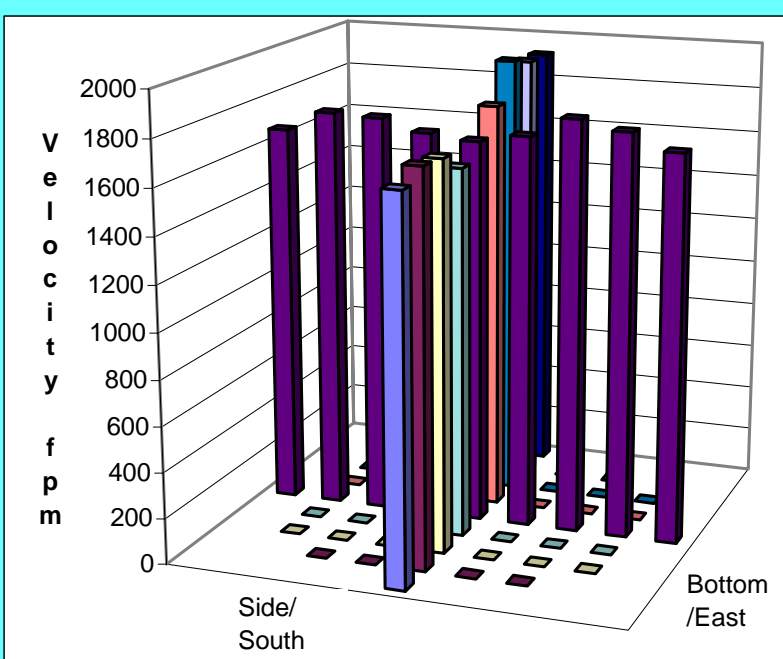

Signature signifies compliance with Procedure EMS-JAG-4 Signature/date
Signature verifying data and calculations:

Signature on file copy 


\section{VELOCITY TRAVERSE DATA FORM}

Site 296-S-21 Model

Date 7/17/2006

Testers Droppo \& Fritz

Stack Dia.

Stack X-Area

$177 / 8$ in

Elevation N.A.

Distance to disturbance

Velocity units $\underline{\mathrm{ft} / \mathrm{min}}$
Run No. VT-9 No C.P.

Fan Configuration 2,4 no prefilter

Fan Setting 41, $48 \quad \mathbf{H z}$

Stack Temp

Start/End Time 1045/1130

Center $2 / 3$ from

Points in Center 2/3 Data Files: NA

\begin{tabular}{|c|c|c|c|c|c|c|c|c|c|}
\hline \multirow{2}{*}{\multicolumn{2}{|c|}{$\begin{array}{l}\text { Traverse--> } \\
\text { Trial ----> }\end{array}$}} & \multicolumn{4}{|c|}{ Port_4_Bottom/East } & \multicolumn{4}{|c|}{ Port_4_Side/South } \\
\hline & & 1 & 2 & 3 & Mean & 1 & 2 & 3 & Mean \\
\hline Point & Depth, in. & \multicolumn{4}{|c|}{ Velocity } & \multicolumn{4}{|c|}{ Velocity } \\
\hline 1 & $9 / 16$ & 1667 & 1720 & 1697 & 1694.7 & 1585 & 1653 & 1623 & 1620.3 \\
\hline 2 & $114 / 16$ & 1803 & 1701 & 1794 & 1766.0 & 1688 & 1716 & 1605 & 1669.7 \\
\hline 3 & $38 / 16$ & 1770 & 1856 & 1791 & 1805.7 & 1646 & 1623 & 1702 & 1657.0 \\
\hline 4 & $513 / 16$ & 1703 & 1710 & 1741 & 1718.0 & 1576 & 1572 & 1581 & 1576.3 \\
\hline Center & 9 & 1614 & 1703 & 1727 & N.A. & 1681 & 1592 & 1658 & N.A. \\
\hline 5 & $123 / 16$ & 1613 & 1751 & 1745 & 1703.0 & 1714 & 1808 & 1738 & 1753.3 \\
\hline 6 & $148 / 16$ & 1760 & 1804 & 1696 & 1753.3 & 1928 & 1938 & 1867 & 1911.0 \\
\hline 7 & $16 \quad 1 / 16$ & 1763 & 1762 & 1759 & 1761.3 & 1881 & 1899 & 1847 & 1875.7 \\
\hline 8 & $176 / 16$ & 1649 & 1693 & 1678 & 1673.3 & 1764 & 1941 & 1915 & 1873.3 \\
\hline Averages ---- & $-->$ & 1704.7 & 1744.4 & 1736.4 & 1734.4 & 1718.1 & 1749.1 & 1726.2 & 1742.1 \\
\hline
\end{tabular}

\begin{tabular}{|lrr|lrrr|}
\hline All & $\underline{\mathrm{ft} / \mathrm{min}}$ & Dev. from mean & Center 2/3 & East & $\underline{\text { South }}$ & $\underline{\text { All }}$ \\
Mean & 1738.3 & & Mean & 1751.2 & 1740.5 & 1745.9 \\
Min Point & 1576.3 & $-9.3 \%$ & Std. Dev. & 36.7 & 131.5 & 92.2 \\
Max Point & 1911.0 & $9.9 \%$ & COV as \% & 2.1 & 7.6 & $\mathbf{5 . 3}$ \\
\hline
\end{tabular}

Flow w/o C-Pt Vel Avg w/o C-Pt 3029 acfm $1738 \mathrm{fpm}$

Stack temp Equipment temp Ambient temp Stack static Ambient pressure Total Stack pressure Ambient humidity

\begin{tabular}{|c|c|l}
\multicolumn{1}{|c|}{ Start } & Finish & \\
\hline 87.4 & 88.7 & $F$ \\
\cline { 1 - 2 } N.A. & 84 & F \\
\hline 86 & 86 & F \\
\hline 0.00 & 0.00 & mbars \\
\hline 29.63 & 29.62 & in Hg \\
\hline 1003 & 1003 & mbars \\
\hline $30 \%$ & $27 \%$ & RH \\
\hline
\end{tabular}

Notes:

Horizontal diameter $17-7 / 8$ in.

Vertical diameter 17-7/8 in.

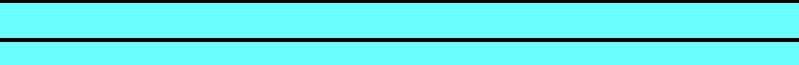

Instuments Used:

Temp $>>$ TSI 8360 SN $209060 \mathrm{Cal}$ due 9/27/06 Vel >> Solomat Zephyr SN 12951472 Cal due 8/29/06 Pitot \# 5 -- 36" Standard Pitot Dwyer

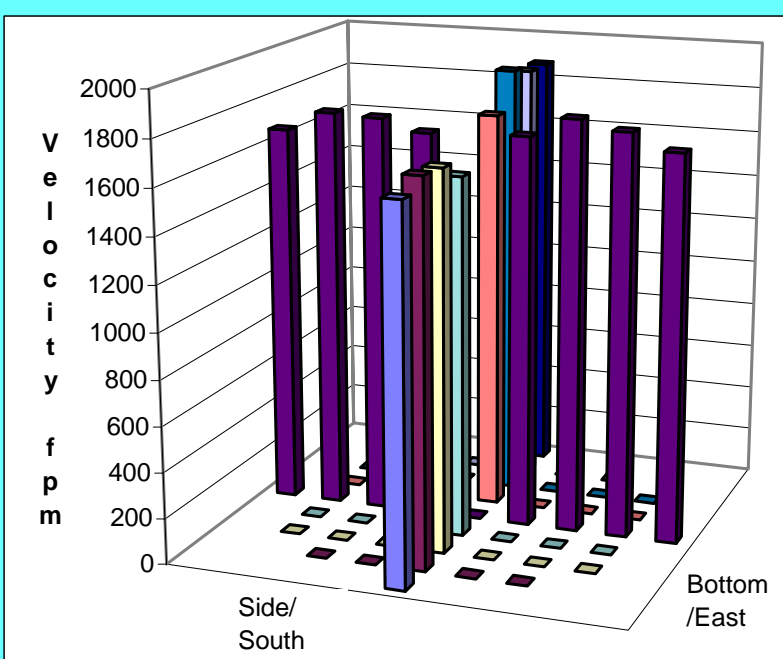

Signature signifies compliance with Procedure EMS-JAG-4 Signature/date
Signature verifying data and calculations:

Signature on file copy 


\section{VELOCITY TRAVERSE DATA FORM}

Site 296-S-21 Model

Date 7/17/2006

Testers BF \& JD

Stack Dia.

Stack X-Area

$177 / 8$ in.

Elevation N.A.

Distance to disturbance

Velocity units $\underline{\mathrm{ft} / \mathrm{min}}$
Run No. VT-10

Fan Configuration 3,4

Fan Setting 46, 48

Stack Temp

Start/End Time 12:45/13:18

Center $2 / 3$ from

Points in Center 2/3 Data Files: $\mathrm{NA}$

\begin{tabular}{|c|c|c|c|c|c|c|c|c|c|}
\hline \multirow{2}{*}{\multicolumn{2}{|c|}{$\begin{array}{l}\text { Traverse--> } \\
\text { Trial ----> }\end{array}$}} & \multicolumn{4}{|c|}{ Port_4_Bottom/East } & \multicolumn{4}{|c|}{ Port_4_Side/South } \\
\hline & & 1 & 2 & 3 & Mean & 1 & 2 & 3 & Mean \\
\hline Point & Depth, in. & \multicolumn{4}{|c|}{ Velocity } & \multicolumn{4}{|c|}{ Velocity } \\
\hline 1 & $9 / 16$ & 1773 & 1710 & 1758 & 1747.0 & 1627 & 1639 & 1598 & 1621.3 \\
\hline 2 & $114 / 16$ & 1954 & 1837 & 1871 & 1887.3 & 1817 & 1821 & 1761 & 1799.7 \\
\hline 3 & $38 / 16$ & 1760 & 1837 & 1825 & 1807.3 & 1838 & 1759 & 1709 & 1768.7 \\
\hline 4 & $513 / 16$ & 1558 & 1609 & 1707 & 1624.7 & 1560 & 1644 & 1595 & 1599.7 \\
\hline Center & 9 & 1606 & 1709 & 1691 & 1668.7 & 1596 & 1670 & 1685 & 1650.3 \\
\hline 5 & $123 / 16$ & 1815 & 1802 & 1730 & 1782.3 & 1774 & 1922 & 1756 & 1817.3 \\
\hline 6 & $148 / 16$ & 1914 & 1926 & 1892 & 1910.7 & 1951 & 1903 & 1962 & 1938.7 \\
\hline 7 & $16 \quad 1 / 16$ & 1914 & 1899 & 1898 & 1903.7 & 2042 & 1903 & 1992 & 1979.0 \\
\hline 8 & $176 / 16$ & 1743 & 1781 & 1721 & 1748.3 & 1961 & 1998 & 1891 & 1950.0 \\
\hline Averages ---- & $-->$ & 1781.9 & 1790.0 & 1788.1 & 1786.7 & 1796.2 & 1806.6 & 1772.1 & 1791.6 \\
\hline
\end{tabular}

\begin{tabular}{|lrr|lrrr|}
\hline All & $\underline{\mathrm{ft} / \mathrm{min}}$ & Dev. from mean & Center 2/3 & East & South & All \\
Mean & 1789.1 & & Mean & 1797.8 & 1793.3 & 1795.6 \\
Min Point & 1599.7 & $-10.6 \%$ & Std. Dev. & 114.7 & 138.3 & 122.1 \\
Max Point & 1979.0 & $10.6 \%$ & COV as $\%$ & 6.4 & 7.7 & $\mathbf{6 . 8}$ \\
\hline
\end{tabular}

Flow w/o C-Pt Vel Avg w/o C-Pt 3146 acfm $1805 \mathrm{fpm}$

Stack temp Equipment temp Ambient temp Stack static Ambient pressure Total Stack pressure Ambient humidity

\begin{tabular}{|c|c|l}
\hline Start & Finish & \\
\hline 93.6 & 94.2 & $F$ \\
\hline 76 & 83 & $F$ \\
\hline 93 & 93 & F \\
\hline 0.00 & 0.00 & mbars \\
\hline 29.59 & 29.58 & in Hg \\
\hline 1002 & 1002 & mbars \\
\hline $16 \%$ & $18 \%$ & $\mathrm{RH}$ \\
\hline
\end{tabular}

Instuments Used:

Temp >> TSI 8360 SN $209060 \mathrm{Cal}$ due 9/27/06 Vel >> Solomat Zephyr SN 12951472 Cal due 8/29/06 Pitot \# 5 -- 36" Standard Pitot Dwyer

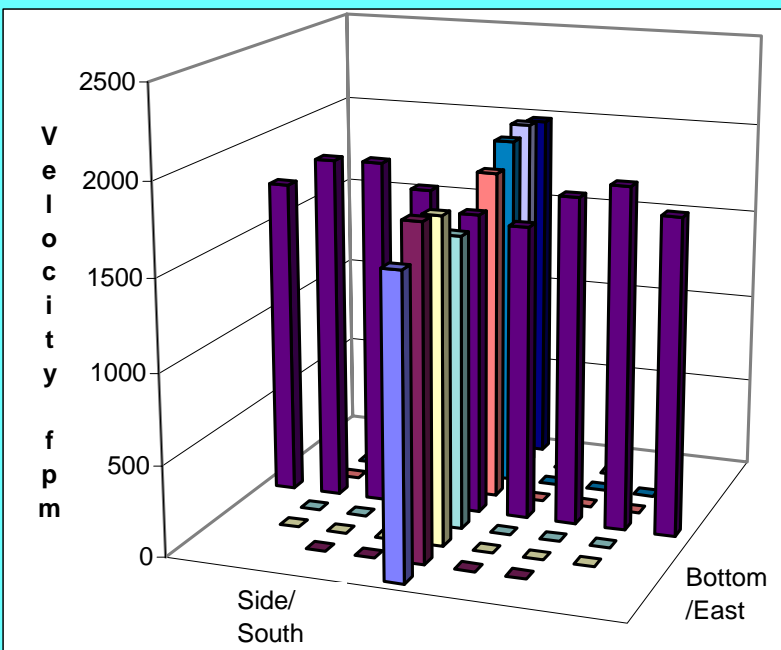

Signature signifies compliance with Procedure EMS-JAG-4 Signature/date
Signature verifying data and calculations:

Signature on file copy 


\section{VELOCITY TRAVERSE DATA FORM}

Site 296-S-21 Model

Date 7/17/2006

Testers BF \& JD

Stack Dia.

Stack X-Area

$177 / 8$ in.

Elevation N.A.

Distance to disturbance

Velocity units $\underline{\mathrm{ft} / \mathrm{min}}$
Run No. VT-10 No C.P.

Fan Configuration $3,4 \quad$ no prefilter

Fan Setting 46, $48 \quad \mathrm{~Hz}$

Stack Temp 93.6 deg F

Start/End Time 12:45/13:18

Center $2 / 3$ from

Points in Center $2 / 3$ Data Files: NA

\begin{tabular}{|c|c|c|c|c|c|c|c|c|c|}
\hline & & \multirow{2}{*}{\multicolumn{4}{|c|}{ Port_4_Bottom/East }} & \multirow{2}{*}{\multicolumn{4}{|c|}{ Port_4_Side/South }} \\
\hline \multirow{2}{*}{\multicolumn{2}{|c|}{$\begin{array}{l}\text { Tra } \\
\text { Tria }\end{array}$}} & & & & & & & & \\
\hline & & \multirow[t]{2}{*}{1} & --2 & \multirow[t]{2}{*}{3} & \multirow[t]{2}{*}{ Mean } & \multirow[t]{2}{*}{1} & 2 & 3 & Mean \\
\hline Point & Depth, in. & & Velocity & & & & \multicolumn{2}{|c|}{ Velocity } & \\
\hline 1 & $9 / 16$ & 1773 & 1710 & 1758 & 1747.0 & 1627 & 1639 & 1598 & 1621.3 \\
\hline 2 & $114 / 16$ & 1954 & 1837 & 1871 & 1887.3 & 1817 & 1821 & 1761 & 1799.7 \\
\hline 3 & $38 / 16$ & 1760 & 1837 & 1825 & 1807.3 & 1838 & 1759 & 1709 & 1768.7 \\
\hline 4 & $513 / 16$ & 1558 & 1609 & 1707 & 1624.7 & 1560 & 1644 & 1595 & 1599.7 \\
\hline Center & 9 & 1606 & 1709 & 1691 & N.A. & 1596 & 1670 & 1685 & N.A. \\
\hline 5 & $12 \quad 3 / 16$ & 1815 & 1802 & 1730 & 1782.3 & 1774 & 1922 & 1756 & 1817.3 \\
\hline 6 & $148 / 16$ & 1914 & 1926 & 1892 & 1910.7 & 1951 & 1903 & 1962 & 1938.7 \\
\hline 7 & $16 \quad 1 / 16$ & 1914 & 1899 & 1898 & 1903.7 & 2042 & 1903 & 1992 & 1979.0 \\
\hline 8 & $176 / 16$ & 1743 & 1781 & 1721 & 1748.3 & 1961 & 1998 & 1891 & 1950.0 \\
\hline \multicolumn{2}{|c|}{ Averages ----------> } & 1781.9 & 1790.0 & 1788.1 & 1801.4 & 1796.2 & 1806.6 & 1772.1 & 1809.3 \\
\hline
\end{tabular}

\begin{tabular}{|lrr|lrrr|}
\hline All & $\underline{\mathrm{ft} / \mathrm{min}}$ & Dev. from mean & Center 2/3 & East & South & All \\
Mean & 1805.4 & & Mean & 1819.3 & 1817.2 & 1818.3 \\
Min Point & 1599.7 & $-11.4 \%$ & Std. Dev. & 109.1 & 134.8 & 116.9 \\
Max Point & 1979.0 & $9.6 \%$ & COV as $\%$ & 6.0 & 7.4 & $\mathbf{6 . 4}$ \\
\hline
\end{tabular}

Flow w/o C-Pt Vel Avg w/o C-Pt 3146 acfm $1805 \mathrm{fpm}$

Stack temp Equipment temp Ambient temp Stack static Ambient pressure Total Stack pressure Ambient humidity

\begin{tabular}{|c|c|l}
\hline Start & Finish & \\
\hline 93.6 & 94.2 & $F$ \\
\hline 76 & 83 & $F$ \\
\hline 93 & 93 & F \\
\hline 0.00 & 0.00 & mbars \\
\hline 29.59 & 29.58 & in Hg \\
\hline 1002 & 1002 & mbars \\
\hline $16 \%$ & $18 \%$ & $\mathrm{RH}$ \\
\hline
\end{tabular}

Notes:

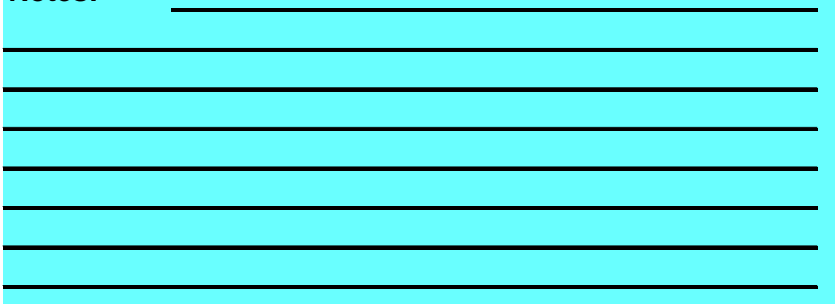

Instuments Used:

Temp >> TSI 8360 SN $209060 \mathrm{Cal}$ due 9/27/06 Vel >> Solomat Zephyr SN 12951472 Cal due 8/29/06 Pitot \# 5 -- 36" Standard Pitot Dwyer

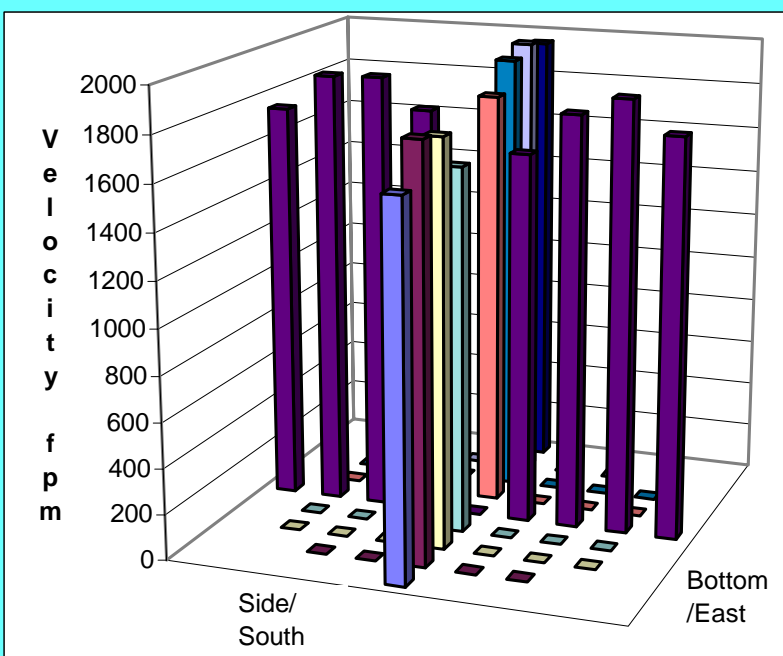

Signature signifies compliance with Procedure EMS-JAG-4 Signature/date
Signature verifying data and calculations:

Signature on file copy 


\section{VELOCITY TRAVERSE DATA FORM}

Site 296-S-21 Model

Date 7/17/2006

Testers BF \& JD

Stack Dia.

Stack X-Area

$177 / 8$ in.

Elevation N.A.

Distance to disturbance

Velocity units $\underline{\mathrm{ft} / \mathrm{min}}$
Run No. VT-11

Fan Configuration 4

Fan Setting $\mathbf{5 0}$

Stack Temp

Start/End Time 1325/1350

Center $2 / 3$ from

Points in Center $2 / 3$

Data Files: NA

\begin{tabular}{|c|c|c|c|c|c|c|c|c|c|}
\hline \multirow{2}{*}{\multicolumn{2}{|c|}{$\begin{array}{l}\text { Traverse--> } \\
\text { Trial ----> }\end{array}$}} & \multicolumn{4}{|c|}{ Port_4_Bottom/East } & \multicolumn{4}{|c|}{ Port_4_Side/South } \\
\hline & & 1 & 2 & 3 & Mean & 1 & 2 & 3 & Mean \\
\hline Point & Depth, in. & \multicolumn{4}{|c|}{ Velocity } & \multicolumn{4}{|c|}{ Velocity } \\
\hline 1 & $9 / 16$ & 1147 & 1109 & 1072 & 1109.3 & 1134 & 1177 & 1170 & 1160.3 \\
\hline 2 & $114 / 16$ & 1132 & 1221 & 1231 & 1194.7 & 1362 & 1384 & 1258 & 1334.7 \\
\hline 3 & $38 / 16$ & 1158 & 1107 & 1176 & 1147.0 & 1230 & 1284 & 1283 & 1265.7 \\
\hline 4 & $513 / 16$ & 1132 & 1151 & 1100 & 1127.7 & 1296 & 1243 & 1222 & 1253.7 \\
\hline Center & 9 & 1228 & 1266 & 1277 & 1257.0 & 1209 & 1203 & 1235 & 1215.7 \\
\hline 5 & $123 / 16$ & 1442 & 1452 & 1378 & 1424.0 & 1310 & 1345 & 1397 & 1350.7 \\
\hline 6 & $148 / 16$ & 1517 & 1528 & 1514 & 1519.7 & 1548 & 1534 & 1505 & 1529.0 \\
\hline 7 & $16 \quad 1 / 16$ & 1474 & 1543 & 1526 & 1514.3 & 1488 & 1567 & 1443 & 1499.3 \\
\hline 8 & $176 / 16$ & 1427 & 1415 & 1356 & 1399.3 & 1524 & 1540 & 1450 & 1504.7 \\
\hline Averages --- & $--->$ & 1295.2 & 1310.2 & 1292.2 & 1299.2 & 1344.6 & 1364.1 & 1329.2 & 1346.0 \\
\hline
\end{tabular}

\begin{tabular}{|lrr|lrrr|}
\hline All & $\underline{\mathrm{ft} / \mathrm{min}}$ & Dev. from mean & Center 2/3 & East & $\underline{\text { South }}$ & $\underline{\text { All }}$ \\
Mean & 1322.6 & & Mean & 1312.0 & 1349.8 & 1330.9 \\
Min Point & 1109.3 & $-16.1 \%$ & Std. Dev. & 170.6 & 121.8 & 143.7 \\
Max Point & 1529.0 & $15.6 \%$ & COV as $\%$ & 13.0 & 9.0 & $\mathbf{1 0 . 8}$ \\
\hline
\end{tabular}

Flow w/o C-Pt $2324 \mathrm{acfm}$ Vel Avg w/o C-Pt $1333 \mathrm{fpm}$

\begin{tabular}{|c|c|}
\hline Start & Finish \\
\hline 94.2 & 93.9 \\
\hline 84 & 87 \\
\hline 93 & 93 \\
\hline 0.00 & 0.00 \\
\hline 29.58 & 29.58 \\
\hline 1002 & 1002 \\
\hline $18 \%$ & $18 \%$ \\
\hline
\end{tabular}

Notes:

Stack temp

Equipment temp

Ambient temp

Stack static

Ambient pressure

Total Stack pressure

Ambient humidity

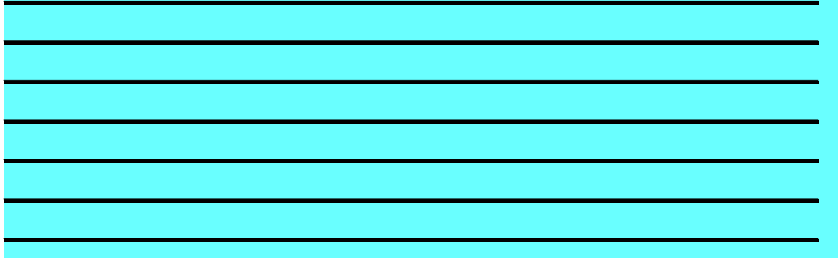

Instuments Used:

Temp $>>$ TSI 8360 SN $209060 \mathrm{Cal}$ due 9/27/06

Vel >> Solomat Zephyr SN 12951472 Cal due 8/29/06

Pitot \# 5 -- 36" Standard Pitot Dwyer

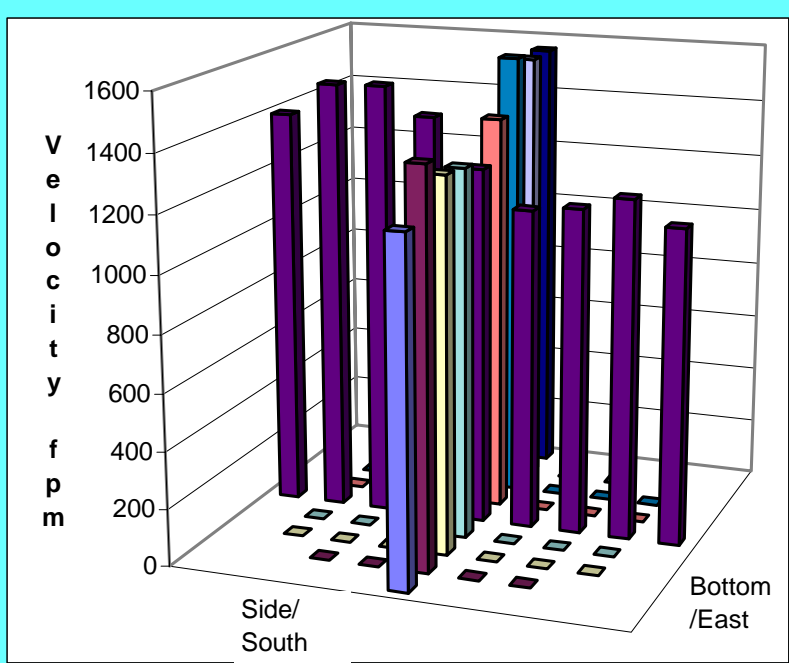

Signature signifies compliance with

Procedure EMS-JAG-4

Signature/date
Signature verifying data and calculations:

Signature on file copy 


\section{VELOCITY TRAVERSE DATA FORM}

Site 296-S-21 Model

Date 7/17/2006

Testers BF \& JD

Stack Dia.

Stack X-Area

$177 / 8$ in

Elevation N.A.

Distance to disturbance

Velocity units $\underline{\mathrm{ft} / \mathrm{min}}$
Run No. VT-11 No C.P.

Fan Configuration $4 \quad$ no prefilter

Fan Setting $\mathbf{5 0 \quad \mathbf { H z }}$

Stack Temp

Start/End Time 1325/1350

Center $2 / 3$ from

Points in Center 2/3

Data Files: NA

\begin{tabular}{|c|c|c|c|c|c|c|c|c|c|}
\hline \multirow{2}{*}{$\begin{array}{l}\text { Traverse--> } \\
\text { Trial ----> }\end{array}$} & & \multicolumn{4}{|c|}{ Port_4_Bottom/East } & \multicolumn{4}{|c|}{ Port_4_Side/South } \\
\hline & & 1 & 2 & 3 & Mean & 1 & 2 & 3 & Mean \\
\hline Point & Depth, in. & \multicolumn{4}{|c|}{ Velocity } & \multicolumn{4}{|c|}{ Velocity } \\
\hline 1 & 9/16 & 1147 & 1109 & 1072 & 1109.3 & 1134 & 1177 & 1170 & 1160.3 \\
\hline 2 & $114 / 16$ & 1132 & 1221 & 1231 & 1194.7 & 1362 & 1384 & 1258 & 1334.7 \\
\hline 3 & $38 / 16$ & 1158 & 1107 & 1176 & 1147.0 & 1230 & 1284 & 1283 & 1265.7 \\
\hline 4 & $513 / 16$ & 1132 & 1151 & 1100 & 1127.7 & 1296 & 1243 & 1222 & 1253.7 \\
\hline Center & 9 & 1228 & 1266 & 1277 & N.A. & 1209 & 1203 & 1235 & N.A. \\
\hline 5 & $123 / 16$ & 1442 & 1452 & 1378 & 1424.0 & 1310 & 1345 & 1397 & 1350.7 \\
\hline 6 & $148 / 16$ & 1517 & 1528 & 1514 & 1519.7 & 1548 & 1534 & 1505 & 1529.0 \\
\hline 7 & $16 \quad 1 / 16$ & 1474 & 1543 & 1526 & 1514.3 & 1488 & 1567 & 1443 & 1499.3 \\
\hline 8 & $176 / 16$ & 1427 & 1415 & 1356 & 1399.3 & 1524 & 1540 & 1450 & 1504.7 \\
\hline Averages ---- & $--->$ & 1295.2 & 1310.2 & 1292.2 & 1304.5 & 1344.6 & 1364.1 & 1329.2 & 1362.3 \\
\hline
\end{tabular}

\begin{tabular}{|llr|lrrr|}
\hline All & $\underline{\mathrm{ft} / \mathrm{min}}$ & Dev. from mean & Center 2/3 & East & $\underline{\text { South }}$ & $\underline{\text { All }}$ \\
Mean & 1333.4 & & Mean & 1321.2 & 1372.2 & 1346.7 \\
Min Point & 1109.3 & $-16.8 \%$ & Std. Dev. & 185.0 & 116.6 & 149.8 \\
Max Point & 1529.0 & $14.7 \%$ & COV as $\%$ & 14.0 & 8.5 & $\mathbf{1 1 . 1}$ \\
\hline
\end{tabular}

Flow w/o C-Pt 2324 acfm Vel Avg w/o C-Pt $1333 \mathrm{fpm}$

\begin{tabular}{|c|c|}
\hline Start & Finish \\
\hline 94.2 & 93.9 \\
\hline 84 & 87 \\
\hline 93 & 93 \\
\hline 0.00 & 0.00 \\
\hline 29.58 & 29.58 \\
\hline 1002 & 1002 \\
\hline $18 \%$ & $18 \%$ \\
\hline
\end{tabular}

Notes:

Stack temp

Equipment temp

Ambient temp

Stack static

Ambient pressure

Total Stack pressure

Ambient humidity

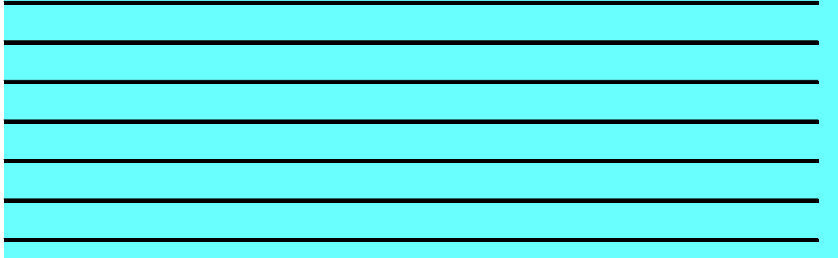

Instuments Used:

Temp >> TSI 8360 SN 209060 Cal due 9/27/06

Vel >> Solomat Zephyr SN 12951472 Cal due 8/29/06

Pitot \# 5 -- 36" Standard Pitot Dwyer

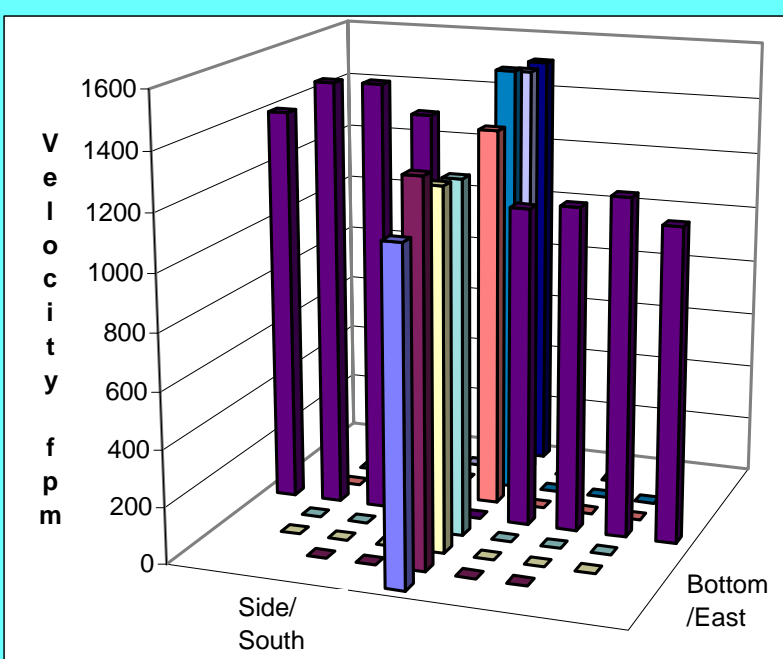

Signature signifies compliance with

Procedure EMS-JAG-4

Signature/date
Signature verifying data and calculations:

Signature on file copy 


\section{VELOCITY TRAVERSE DATA FORM}

Site 296-S-21 Model

Date 7/17/2006

Testers BF \& JD

Stack Dia.

Stack X-Area

Elevation N.A.

Distance to disturbance

Velocity units $\mathrm{ft} / \mathrm{min}$
Run No. VT-12

Fan Configuration 3,4

Fan Setting 48, 50

Stack Temp

Start/End Time 1400/1420

Center $2 / 3$ from

Points in Center $2 / 3$

Data Files: NA

\begin{tabular}{|c|c|c|c|c|c|c|c|c|c|}
\hline \multirow{2}{*}{\multicolumn{2}{|c|}{$\begin{array}{l}\text { Traverse--> } \\
\text { Trial ----> }\end{array}$}} & \multicolumn{4}{|c|}{ Port_3_No. East } & \multicolumn{4}{|c|}{ Port_3_So. East } \\
\hline & & 1 & 2 & 3 & Mean & 1 & 2 & 3 & Mean \\
\hline Point & Depth, in. & \multicolumn{4}{|c|}{ Velocity } & \multicolumn{4}{|c|}{ Velocity } \\
\hline 1 & $9 / 16$ & 2040 & 2159 & 2155 & 2118.0 & 1688 & 1911 & 1759 & 1786.0 \\
\hline 2 & $114 / 16$ & 2202 & 2186 & 2193 & 2193.7 & 1890 & 1868 & 1912 & 1890.0 \\
\hline 3 & $38 / 16$ & 1946 & 2116 & 2171 & 2077.7 & 1917 & 1783 & 1762 & 1820.7 \\
\hline 4 & $513 / 16$ & 1666 & 1728 & 1827 & 1740.3 & 1549 & 1405 & 1535 & 1496.3 \\
\hline Center & 9 & 1512 & 1435 & 1472 & 1473.0 & 1436 & 1427 & 1451 & 1438.0 \\
\hline 5 & $123 / 16$ & 1593 & 1584 & 1499 & 1558.7 & 1658 & 1559 & 1598 & 1605.0 \\
\hline 6 & $148 / 16$ & 1817 & 1972 & 1929 & 1906.0 & 1929 & 1890 & 1835 & 1884.7 \\
\hline 7 & $16 \quad 1 / 16$ & 2134 & 2041 & 2130 & 2101.7 & 2193 & 2145 & 2184 & 2174.0 \\
\hline 8 & $176 / 16$ & 2011 & 2045 & 2010 & 2022.0 & 2051 & 2165 & 2257 & 2157.7 \\
\hline Averages ---- & $-->$ & 1880.1 & 1918.4 & 1931.8 & 1910.1 & 1812.3 & 1794.8 & 1810.3 & 1805.8 \\
\hline
\end{tabular}

\begin{tabular}{|lrr|lrrrr|}
\hline All & $\underline{\mathrm{ft} / \mathrm{min}}$ & Dev. from mean & Center 2/3 & & NorthEast & SouthEast & All \\
Mean & 1858.0 & & Mean & 1864.4 & 1758.4 & 1811.4 \\
Min Point & 1438.0 & $-22.6 \%$ & Std. Dev. & 281.1 & 259.7 & 265.7 \\
Max Point & 2193.7 & $18.1 \%$ & COV as $\%$ & 15.1 & 14.8 & $\mathbf{1 4 . 7}$ \\
\hline
\end{tabular}

Flow w/o C-Pt Vel Avg w/o C-Pt 3372 acfm $1908 \mathrm{fpm}$

Stack temp Equipment temp Ambient temp Stack static Ambient pressure Total Stack pressure Ambient humidity

\begin{tabular}{|c|c|l}
\hline Start & Finish & \\
\hline 93.9 & 95.6 & $F$ \\
\hline 87 & 88 & $F$ \\
\hline 92 & 93 & F \\
\hline 0.00 & 0.00 & mbars \\
\hline 29.57 & 29.56 & in Hg \\
\hline 1002 & 1002 & mbars \\
\hline $18 \%$ & $16 \%$ & $\mathrm{RH}$ \\
\hline
\end{tabular}

Instuments Used:

Temp >> TSI 8360 SN $209060 \mathrm{Cal}$ due 9/27/06 Vel $>>$ Solomat Zephyr SN 12951472 Cal due 8/29/06 Pitot \# 5 -- 36" Standard Pitot Dwyer

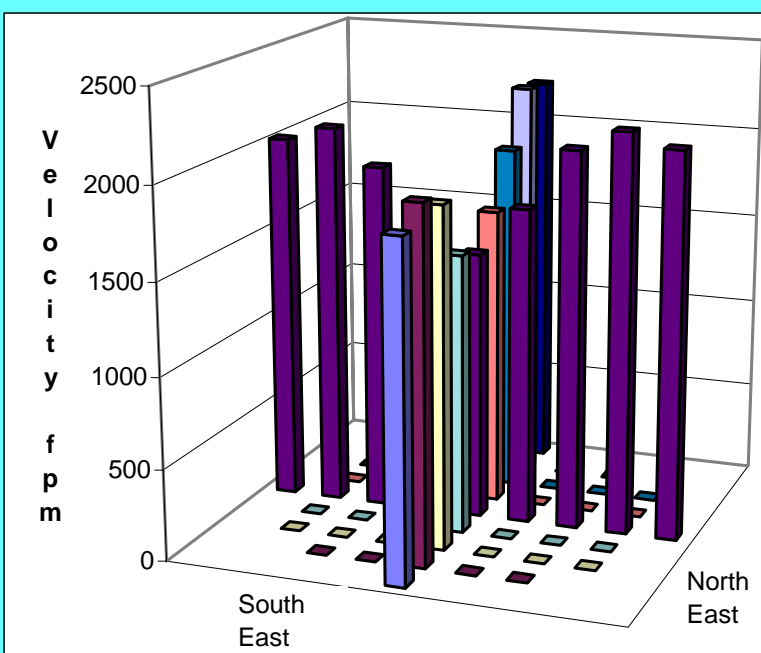

Signature signifies compliance with Procedure EMS-JAG-4 Signature/date
Signature verifying data and calculations:

Signature on file copy 


\section{VELOCITY TRAVERSE DATA FORM}

Site 296-S-21 Model

Date 7/17/2006

Testers BF \& JD

Stack Dia.

Stack X-Area

Elevation N.A.

Distance to disturbance

Velocity units $\mathrm{ft} / \mathrm{min}$
Run No. VT-12 No C. P.

Fan Configuration $\mathbf{3 , 4}$ no prefilter

Fan Setting 48, $50 \quad \mathrm{~Hz}$

Stack Temp $93.6 \mathrm{deg} F$

Start/End Time 1400/1420

Center $2 / 3$ from

Points in Center 2/3

Data Files: $\mathrm{NA}$

\begin{tabular}{|c|c|c|c|c|c|c|c|c|c|}
\hline \multirow{2}{*}{\multicolumn{2}{|c|}{$\begin{array}{l}\text { Traverse--> } \\
\text { Trial ----> }\end{array}$}} & \multicolumn{4}{|c|}{ Port_3_No. East } & \multicolumn{4}{|c|}{ Port_3_So. East } \\
\hline & & 1 & 2 & 3 & Mean & 1 & 2 & 3 & Mean \\
\hline Point & Depth, in. & \multicolumn{4}{|c|}{ Velocity } & \multicolumn{4}{|c|}{ Velocity } \\
\hline 1 & $9 / 16$ & 2040 & 2159 & 2155 & 2118.0 & 1688 & 1911 & 1759 & 1786.0 \\
\hline 2 & $114 / 16$ & 2202 & 2186 & 2193 & 2193.7 & 1890 & 1868 & 1912 & 1890.0 \\
\hline 3 & $38 / 16$ & 1946 & 2116 & 2171 & 2077.7 & 1917 & 1783 & 1762 & 1820.7 \\
\hline 4 & $513 / 16$ & 1666 & 1728 & 1827 & 1740.3 & 1549 & 1405 & 1535 & 1496.3 \\
\hline Center & 9 & 1512 & 1435 & 1472 & N.A. & 1436 & 1427 & 1451 & N.A. \\
\hline 5 & $123 / 16$ & 1593 & 1584 & 1499 & 1558.7 & 1658 & 1559 & 1598 & 1605.0 \\
\hline 6 & $148 / 16$ & 1817 & 1972 & 1929 & 1906.0 & 1929 & 1890 & 1835 & 1884.7 \\
\hline 7 & $16 \quad 1 / 16$ & 2134 & 2041 & 2130 & 2101.7 & 2193 & 2145 & 2184 & 2174.0 \\
\hline 8 & $176 / 16$ & 2011 & 2045 & 2010 & 2022.0 & 2051 & 2165 & 2257 & 2157.7 \\
\hline Averages ---- & $--->$ & 1880.1 & 1918.4 & 1931.8 & 1964.8 & 1812.3 & 1794.8 & 1810.3 & 1851.8 \\
\hline
\end{tabular}

\begin{tabular}{|llr|lrrrr|}
\hline All & $\underline{\mathrm{ft} / \mathrm{min}}$ & Dev. from mean & Center 2/3 & & NorthEast & SouthEast & All \\
Mean & 1908.3 & & Mean & & 1929.7 & 1811.8 & 1870.7 \\
Min Point & 1496.3 & $-21.6 \%$ & Std. Dev. & 243.0 & 238.7 & 237.8 \\
Max Point & 2193.7 & $15.0 \%$ & COV as $\%$ & 12.6 & 13.2 & $\mathbf{1 2 . 7}$ \\
\hline
\end{tabular}

Flow w/o C-Pt 3372 acfm Vel Avg w/o C-Pt $1908 \mathrm{fpm}$

\begin{tabular}{|c|c|}
\hline Start & Finish \\
\hline 93.9 & 95.6 \\
\hline 87 & 88 \\
\hline 92 & 93 \\
\hline 0.00 & 0.00 \\
\hline 29.57 & 29.56 \\
\hline 1002 & 1002 \\
\hline $18 \%$ & $16 \%$ \\
\hline
\end{tabular}

Notes:

Stack temp

Equipment temp

Ambient temp

Stack static

Ambient pressure

Total Stack pressure

Ambient humidity

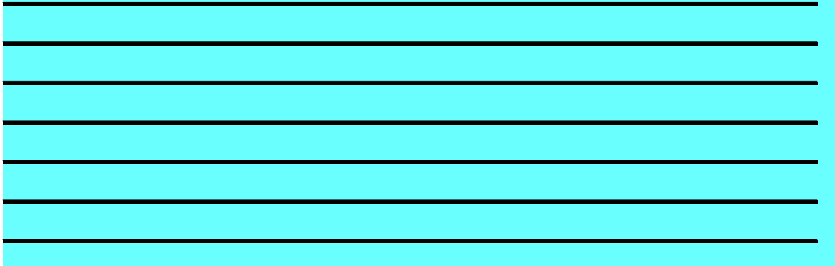

Instuments Used:

Temp >> TSI 8360 SN 209060 Cal due 9/27/06 Vel $>>$ Solomat Zephyr SN 12951472 Cal due 8/29/06 Pitot \# 5 -- 36" Standard Pitot Dwyer

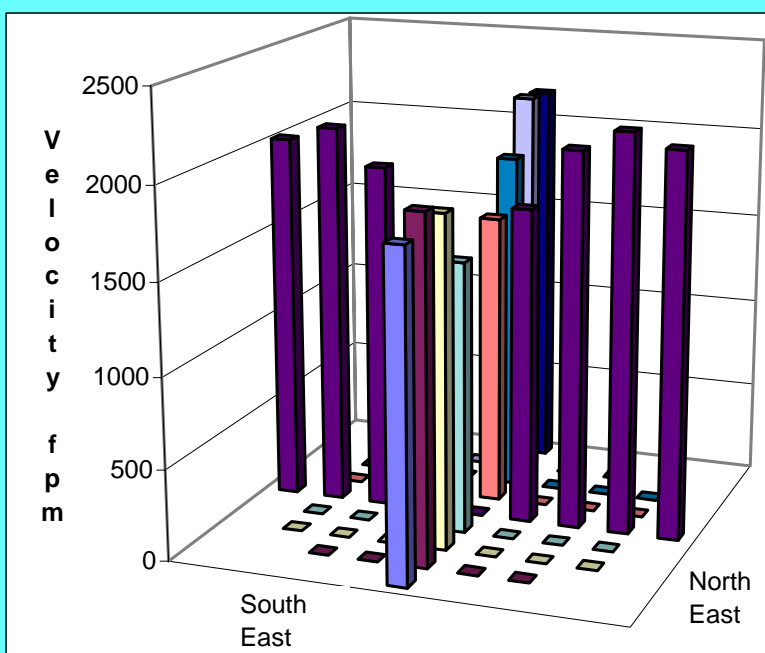

Signature signifies compliance with

Procedure EMS-JAG-4

Signature/date
Signature verifying data and calculations:

Signature on file copy 


\section{VELOCITY TRAVERSE DATA FORM}

Site 296-S-21 Model

Date 7/18/2006

Testers JAG \& BGF

Stack Dia.

Stack X-Area 18 in

Elevation N.A.

Distance to disturbance

Velocity units $\mathrm{ft} / \mathrm{min}$
Run No. VT-13

Fan Configuration 3,4 Fan Setting $\mathbf{5 0 , 5 2}$

Stack Temp

Start/End Time 1015/1105

Center $2 / 3$ from

Points in Center $2 / 3$ Data Files: $\mathrm{NA}$

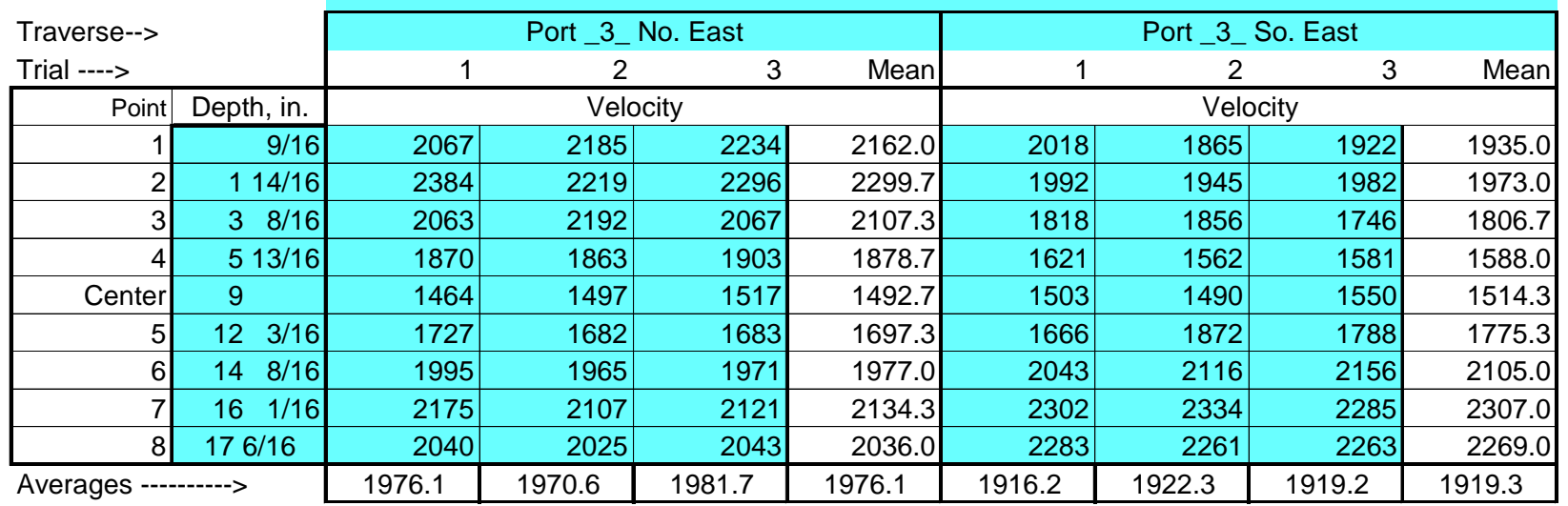

\begin{tabular}{|llr|lrrrr|}
\hline All & $\underline{\mathrm{ft} / \mathrm{min}}$ & Dev. from mean & Center 2/3 & & NorthEast & SouthEast & \multicolumn{1}{rl}{} \\
Mean & 1947.7 & & Mean & & 1941.0 & 1867.0 & 1904.0 \\
Min Point & 1492.7 & $-23.4 \%$ & Std. Dev. & 276.7 & 281.6 & 270.9 \\
Max Point & 2307.0 & $18.4 \%$ & COV as \% & 14.3 & 15.1 & $\mathbf{1 4 . 2}$ \\
\hline
\end{tabular}

Flow w/o C-Pt 3540 acfm Vel Avg w/o C-Pt $2003 \mathrm{fpm}$

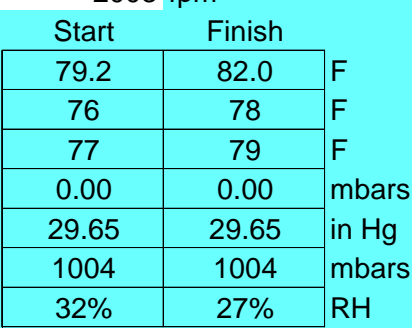

Notes:

Stack temp Equipment temp Ambient temp Stack static Ambient pressure Total Stack pressure Ambient humidity

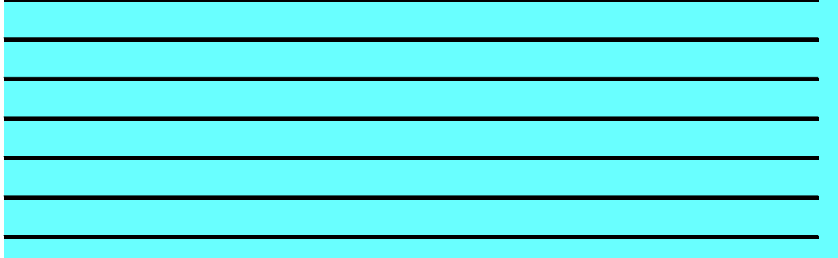

Instuments Used:

Temp >> TSI 8360 SN 209060 Cal due 9/27/06 Vel $>>$ Solomat Zephyr SN 12951472 Cal due 8/29/06 Pitot \# 5 -- 36" Standard Pitot Dwyer

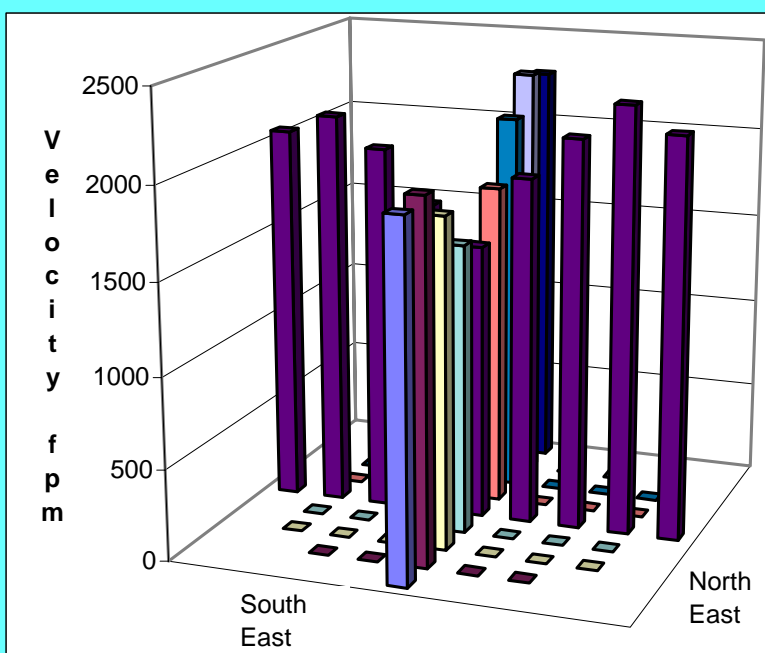

Signature signifies compliance with Procedure EMS-JAG-4 Signature/date
Signature verifying data and calculations:

Signature on file copy 


\section{VELOCITY TRAVERSE DATA FORM}

Site 296-S-21 Model

Date 7/18/2006

Testers JAG \& BGF

Stack Dia.

Stack X-Area

Elevation N.A.

Distance to disturbance

Velocity units $\mathrm{ft} / \mathrm{min}$
Run No. VT-13 No C.P.

Fan Configuration $\mathbf{3 , 4}$ no prefilter

Fan Setting $\mathbf{H z}$

Stack Temp

Start/End Time 1015/1105

Center $2 / 3$ from

Points in Center $2 / 3$

Data Files: $\mathrm{NA}$

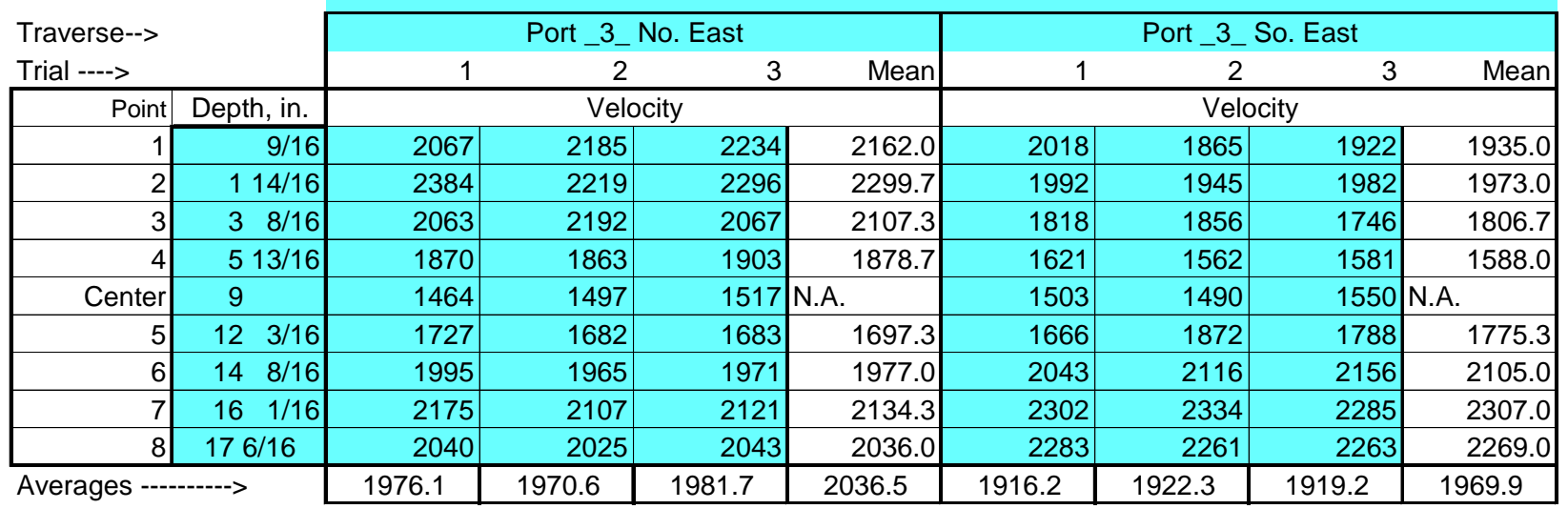

\begin{tabular}{|llr|lrrrr|}
\hline All & $\underline{\mathrm{ft} / \mathrm{min}}$ & Dev. from mean & Center 2/3 & & NorthEast & SouthEast & All \\
Mean & 2003.2 & & Mean & 2015.7 & 1925.8 & 1970.8 \\
Min Point & 1588.0 & $-20.7 \%$ & Std. Dev. & 212.0 & 257.1 & 229.5 \\
Max Point & 2307.0 & $15.2 \%$ & COV as \% & 10.5 & 13.4 & $\mathbf{1 1 . 6}$ \\
\hline
\end{tabular}

Flow w/o C-Pt 3540 acfm Vel Avg w/o C-Pt $2003 \mathrm{fpm}$

\begin{tabular}{|c|c|}
\hline Start & Finish \\
\hline 79.2 & 82.0 \\
\hline 76 & 78 \\
\hline 77 & 79 \\
\hline 0.00 & 0.00 \\
\hline 29.65 & 29.65 \\
\hline 1004 & 1004 \\
\hline $32 \%$ & $27 \%$ \\
\hline
\end{tabular}

Notes:

Stack temp Equipment temp Ambient temp Stack static Ambient pressure Total Stack pressure Ambient humidity

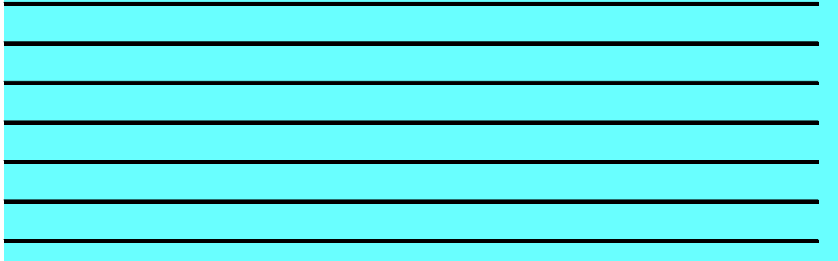

Instuments Used:

Temp >> TSI 8360 SN $209060 \mathrm{Cal}$ due 9/27/06 Vel >> Solomat Zephyr SN 12951472 Cal due 8/29/06 Pitot \# 5 -- 36" Standard Pitot Dwyer

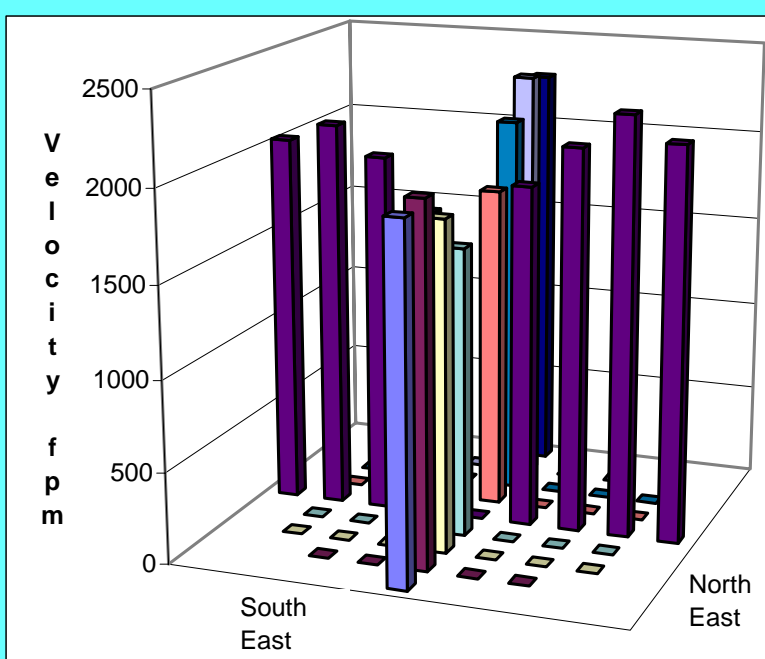

Signature signifies compliance with Procedure EMS-JAG-4 Signature/date
Signature verifying data and calculations:

Signature on file copy 


\section{VELOCITY TRAVERSE DATA FORM}

Site 296-S-21 Model

Date 7/18/2006

Testers JAG \& BGF

Stack Dia.

Stack X-Area 18 in

Elevation N.A.

Distance to disturbance

Velocity units $\mathrm{ft} / \mathrm{min}$
Run No. VT-14

Fan Configuration 2, 4

Fan Setting 45, 52

Stack Temp

Start/End Time 1112/1150

Center $2 / 3$ from

Points in Center $2 / 3$ Data Files: $\mathrm{NA}$

\begin{tabular}{|c|c|c|c|c|c|c|c|c|c|}
\hline \multirow{2}{*}{\multicolumn{2}{|c|}{$\begin{array}{l}\text { Traverse--> } \\
\text { Trial ----> }\end{array}$}} & \multicolumn{4}{|c|}{ Port_3_No. East } & \multicolumn{4}{|c|}{ Port_3_So. East } \\
\hline & & 1 & 2 & 3 & Mean & 1 & 2 & 3 & Mean \\
\hline Point & Depth, in. & \multicolumn{4}{|c|}{ Velocity } & \multicolumn{4}{|c|}{ Velocity } \\
\hline 1 & $9 / 16$ & 2173 & 2121 & 2129 & 2141.0 & 1895 & 1987 & 1871 & 1917.7 \\
\hline 2 & $114 / 16$ & 2172 & 2091 & 2132 & 2131.7 & 1919 & 1924 & 1982 & 1941.7 \\
\hline 3 & $38 / 16$ & 2056 & 2054 & 1992 & 2034.0 & 1761 & 1840 & 1830 & 1810.3 \\
\hline 4 & $513 / 16$ & 1764 & 1795 & 1673 & 1744.0 & 1508 & 1543 & 1632 & 1561.0 \\
\hline Center & 9 & 1450 & 1485 & 1436 & 1457.0 & 1471 & 1545 & 1526 & 1514.0 \\
\hline 5 & $12 \quad 3 / 16$ & 1509 & 1598 & 1603 & 1570.0 & 1783 & 1911 & 1833 & 1842.3 \\
\hline 6 & $148 / 16$ & 1924 & 1917 & 1936 & 1925.7 & 2138 & 2066 & 1997 & 2067.0 \\
\hline 7 & $16 \quad 1 / 16$ & 2049 & 2035 & 2086 & 2056.7 & 2241 & 2208 & 2192 & 2213.7 \\
\hline 8 & $176 / 16$ & 1954 & 1900 & 1987 & 1947.0 & 2196 & 2193 & 2195 & 2194.7 \\
\hline Averages --- & $----->$ & 1894.6 & 1888.4 & 1886.0 & 1889.7 & 1879.1 & 1913.0 & 1895.3 & 1895.8 \\
\hline
\end{tabular}

\begin{tabular}{|llr|lrrrr|}
\hline All & $\underline{\mathrm{ft} / \mathrm{min}}$ & Dev. from mean & Center 2/3 & & NorthEast & SouthEast & All \\
Mean & 1892.7 & & Mean & 1845.6 & 1850.0 & 1847.8 \\
Min Point & 1457.0 & $-23.0 \%$ & Std. Dev. & 260.0 & 253.6 & 246.7 \\
Max Point & 2213.7 & $17.0 \%$ & COV as $\%$ & 14.1 & 13.7 & $\mathbf{1 3 . 4}$ \\
\hline
\end{tabular}

Flow w/o C-Pt Vel Avg w/o C-Pt 3435 acfm $1944 \mathrm{fpm}$

Stack temp Equipment temp Ambient temp Stack static Ambient pressure Total Stack pressure Ambient humidity

\begin{tabular}{|c|c|l}
\hline Start & Finish & \\
\hline 82.0 & 84.0 & $F$ \\
\hline 78 & 79 & $F$ \\
\hline 79 & 79 & F \\
\hline 0.00 & 0.00 & mbars \\
\hline 29.65 & 29.64 & in Hg \\
\hline 1004 & 1004 & mbars \\
\hline $27 \%$ & $26 \%$ & $\mathrm{RH}$ \\
\hline
\end{tabular}

\section{Notes:}

Horizontal diameter 18 in.

Vertical diameter 18 in.

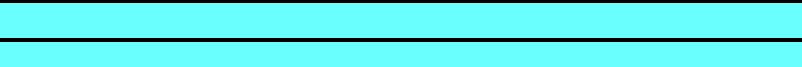

Instuments Used:

Temp >> TSI 8360 SN $209060 \mathrm{Cal}$ due 9/27/06 Vel >> Solomat Zephyr SN 12951472 Cal due 8/29/06 Pitot \# 5 -- 36" Standard Pitot Dwyer

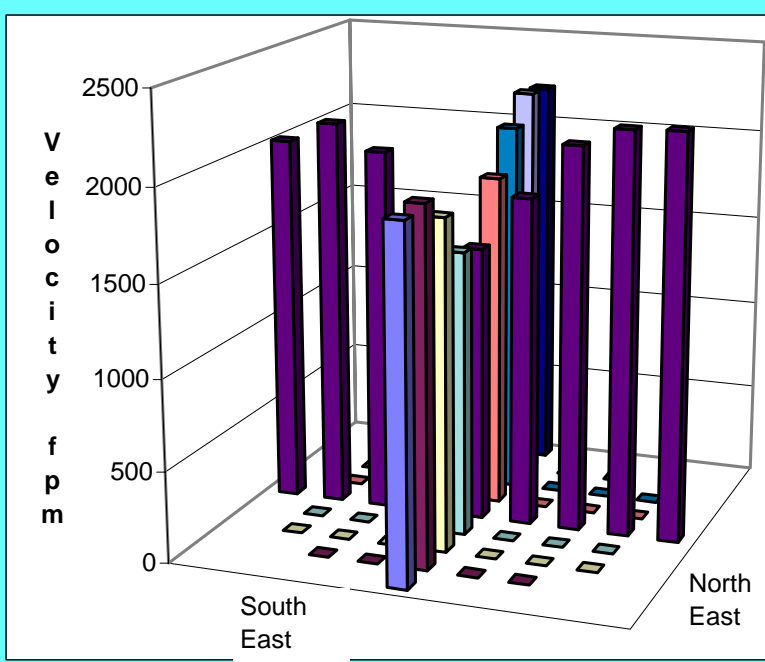

Signature signifies compliance with Procedure EMS-JAG-4 Signature/date
Signature verifying data and calculations:

Signature on file copy 


\section{VELOCITY TRAVERSE DATA FORM}

Site 296-S-21 Model

Date 7/18/2006

Testers JAG \& BGF

Stack Dia.

Stack X-Area

Elevation N.A.

Distance to disturbance

Velocity units $\mathrm{ft} / \mathrm{min}$
Run No. VT-14 No C.P.

Fan Configuration $2,4 \quad$ no prefilter

Fan Setting 45, $\mathbf{5 2} \mathrm{Hz}$

Stack Temp $93.6 \mathrm{deg} F$

Start/End Time 1112/1150

Center $2 / 3$ from

Points in Center $2 / 3$ Data Files: $\overline{N A}$

\begin{tabular}{|c|c|c|c|c|c|c|c|c|c|}
\hline \multirow{2}{*}{\multicolumn{2}{|c|}{$\begin{array}{l}\text { Traverse--> } \\
\text { Trial ----> }\end{array}$}} & \multicolumn{4}{|c|}{ Port_3_No. East } & \multicolumn{4}{|c|}{ Port_3_So. East } \\
\hline & & 1 & 2 & 3 & Mean & 1 & 2 & 3 & Mean \\
\hline Point & Depth, in. & \multicolumn{4}{|c|}{ Velocity } & \multicolumn{4}{|c|}{ Velocity } \\
\hline 1 & $9 / 16$ & 2173 & 2121 & 2129 & 2141.0 & 1895 & 1987 & 1871 & 1917.7 \\
\hline 2 & $114 / 16$ & 2172 & 2091 & 2132 & 2131.7 & 1919 & 1924 & 1982 & 1941.7 \\
\hline 3 & $38 / 16$ & 2056 & 2054 & 1992 & 2034.0 & 1761 & 1840 & 1830 & 1810.3 \\
\hline 4 & $513 / 16$ & 1764 & 1795 & 1673 & 1744.0 & 1508 & 1543 & 1632 & 1561.0 \\
\hline Center & 9 & 1450 & 1485 & 1436 & N.A. & 1471 & 1545 & 1526 & N.A. \\
\hline 5 & $123 / 16$ & 1509 & 1598 & 1603 & 1570.0 & 1783 & 1911 & 1833 & 1842.3 \\
\hline 6 & $148 / 16$ & 1924 & 1917 & 1936 & 1925.7 & 2138 & 2066 & 1997 & 2067.0 \\
\hline 7 & $16 \quad 1 / 16$ & 2049 & 2035 & 2086 & 2056.7 & 2241 & 2208 & 2192 & 2213.7 \\
\hline 8 & $176 / 16$ & 1954 & 1900 & 1987 & 1947.0 & 2196 & 2193 & 2195 & 2194.7 \\
\hline Averages ---- & $-->$ & 1894.6 & 1888.4 & 1886.0 & 1943.8 & 1879.1 & 1913.0 & 1895.3 & 1943.5 \\
\hline
\end{tabular}

\begin{tabular}{|lrr|lrrrr|}
\hline All & $\underline{\mathrm{ft} / \mathrm{min}}$ & Dev. from mean & Center 2/3 & & NorthEast & SouthEast & All \\
Mean & 1943.6 & & Mean & & 1910.3 & 1906.0 & 1908.2 \\
Min Point & 1561.0 & $-19.7 \%$ & Std. Dev. & 214.2 & 225.4 & 209.7 \\
Max Point & 2213.7 & $13.9 \%$ & COV as \% & 11.2 & 11.8 & $\mathbf{1 1 . 0}$ \\
\hline
\end{tabular}

Flow w/o C-Pt Vel Avg w/o C-Pt 3435 acfm $1944 \mathrm{fpm}$

Stack temp Equipment temp Ambient temp Stack static Ambient pressure Total Stack pressure Ambient humidity

\begin{tabular}{|c|c|l}
\hline Start & Finish & \\
\hline 82.0 & 84.0 & $F$ \\
\hline 78 & 79 & $F$ \\
\hline 79 & 79 & F \\
\hline 0.00 & 0.00 & mbars \\
\hline 29.65 & 29.64 & in Hg \\
\hline 1004 & 1004 & mbars \\
\hline $27 \%$ & $26 \%$ & $\mathrm{RH}$ \\
\hline
\end{tabular}

Notes:

Horizontal diameter 18 in.

Vertical diameter 18 in.

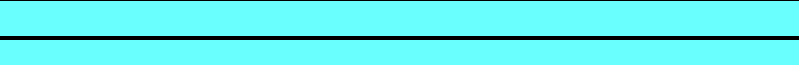

Instuments Used:

Temp $>>$ TSI 8360 SN $209060 \mathrm{Cal}$ due 9/27/06 Vel >> Solomat Zephyr SN 12951472 Cal due 8/29/06 Pitot \# 5 -- 36" Standard Pitot Dwyer

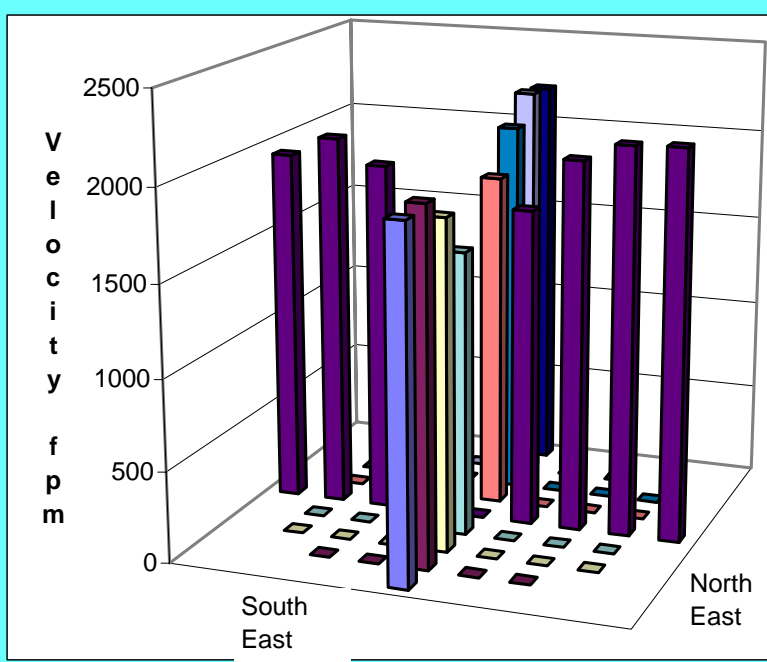

Signature signifies compliance with Procedure EMS-JAG-4 Signature/date
Signature verifying data and calculations:

Signature on file copy 


\section{VELOCITY TRAVERSE DATA FORM}

Site 296-S-21 Model

Date 8/7/2006

Testers JGD \& BGF

Stack Dia.

Stack X-Area

Elevation N.A.

Distance to disturbance

Velocity units $\mathrm{ft} / \mathrm{min}$
Run No. VT-15

Fan Configuration 1, 4

Fan Setting $\mathbf{5 6 , 5 2}$

Stack Temp

Start/End Time 1100/1200

Center $2 / 3$ from

Points in Center $2 / 3$ Data Files: NA

\begin{tabular}{|c|c|c|c|c|c|c|c|c|c|}
\hline \multirow{3}{*}{$\begin{array}{l}\text { Traverse--> } \\
\text { Trial ----> }\end{array}$} & & \multicolumn{8}{|c|}{$1 \mathrm{st}$} \\
\hline & & \multicolumn{4}{|c|}{ Port_3_No. East } & \multicolumn{4}{|c|}{ Port_3_So. East } \\
\hline & & 1 & 2 & 3 & Mean & 1 & $\overline{2}$ & 3 & Mean \\
\hline Point & Depth, in. & \multicolumn{4}{|c|}{ Velocity } & \multicolumn{4}{|c|}{ Velocity } \\
\hline 1 & $9 / 16$ & 2303 & 2253 & 2320 & 2292.0 & 2062 & 2120 & 2073 & 2085.0 \\
\hline 2 & $114 / 16$ & 2302 & 2340 & 2298 & 2313.3 & 2126 & 2133 & 2199 & 2152.7 \\
\hline 3 & $38 / 16$ & 2233 & 2242 & 2239 & 2238.0 & 2064 & 1999 & 2127 & 2063.3 \\
\hline 4 & $513 / 16$ & 1980 & 1996 & 2007 & 1994.3 & 1615 & 1690 & 1651 & 1652.0 \\
\hline Center & 9 & 1628 & 1591 & 1545 & 1588.0 & 1609 & 1604 & 1557 & 1590.0 \\
\hline 5 & $123 / 16$ & 1708 & 1631 & 1687 & 1675.3 & 1992 & 1926 & 1945 & 1954.3 \\
\hline 6 & $148 / 16$ & 1949 & 1974 & 1879 & 1934.0 & 2272 & 2233 & 2246 & 2250.3 \\
\hline 7 & $16 \quad 1 / 16$ & 2135 & 2204 & 2162 & 2167.0 & 2407 & 2385 & 2383 & 2391.7 \\
\hline 8 & $176 / 16$ & 2254 & 2351 & 2304 & 2303.0 & 2233 & 2303 & 2133 & 2223.0 \\
\hline Averages --- & $-\cdots--->$ & 2054.7 & 2064.7 & 2049.0 & 2056.1 & 2042.2 & 2043.7 & 2034.9 & 2040.3 \\
\hline
\end{tabular}

\begin{tabular}{|llr|lrrrr|}
\hline All & $\underline{\mathrm{ft} / \mathrm{min}}$ & Dev. from mean & Center 2/3 & & NorthEast & SouthEast & All \\
Mean & 2048.2 & & Mean & 1987.1 & 2007.8 & 1997.5 \\
Min Point & 1588.0 & $-22.5 \%$ & Std. Dev. & 277.2 & 298.4 & 276.9 \\
Max Point & 2391.7 & $16.8 \%$ & COV as \% & 14.0 & 14.9 & $\mathbf{1 3 . 9}$ \\
\hline
\end{tabular}

Flow w/o C-Pt Vel Avg w/o C-Pt 3721 acfm $2106 \mathrm{fpm}$

Stack temp Equipment temp Ambient temp Stack static Ambient pressure Total Stack pressure Ambient humidity

\begin{tabular}{|c|c|l}
\multicolumn{1}{|c|}{ Start } & Finish & \\
\hline 97.0 & 96.0 & $\mathrm{~F}$ \\
\hline 83 & 87 & $\mathrm{~F}$ \\
\hline 91 & 93 & $\mathrm{~F}$ \\
\hline 0.00 & 0.00 & mbars \\
\hline 29.42 & 29.40 & in Hg \\
\hline 996 & 996 & $\mathrm{mbars}$ \\
\hline $28 \%$ & $23 \%$ & $\mathrm{RH}$ \\
\hline
\end{tabular}

Notes:

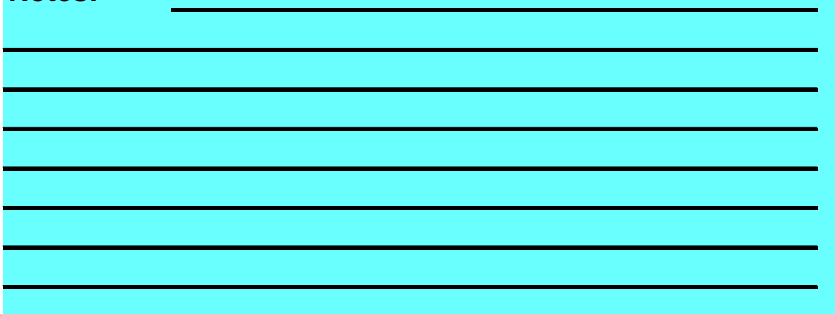

Instuments Used:

Temp >> TSI 8360 SN 209060 Cal due 9/27/06 Vel >> Solomat Zephyr SN 12951472 Cal due 8/29/06 Pitot \# 5 -- 36" Standard Pitot Dwyer

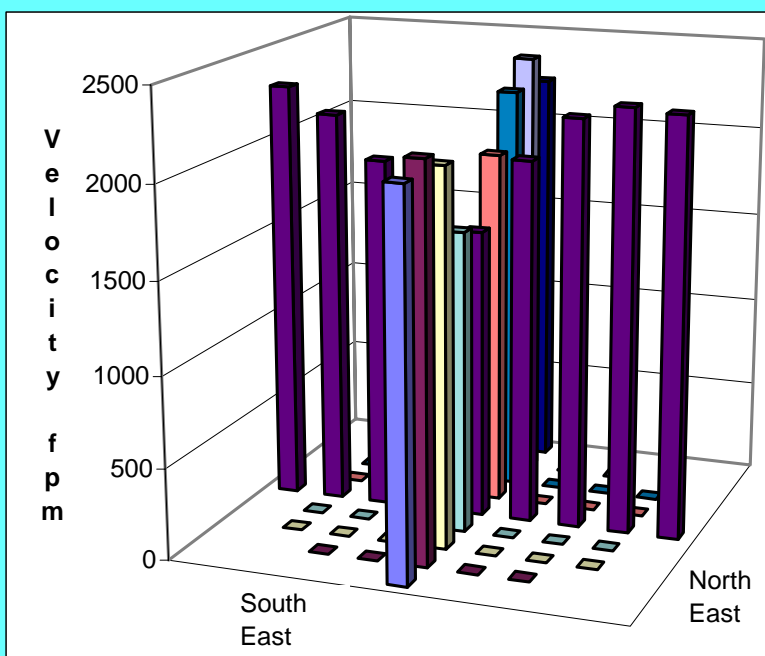

Signature signifies compliance with Procedure EMS-JAG-4 Signature/date
Signature verifying data and calculations:

Signature on file copy 


\section{VELOCITY TRAVERSE DATA FORM}

Site 296-S-21 Model

Date 8/7/2006

Testers JGD \& BGF

Stack Dia.

Stack X-Area

Elevation N.A.

Distance to disturbance

Velocity units $\mathrm{ft} / \mathrm{min}$
Run No. VT-15 No centerpoint

Fan Configuration 1, 4 no prefilter

Fan Setting 56, $52 \quad \mathrm{~Hz}$

Stack Temp

Start/End Time 1100/1200

Center 2/3 from

Points in Center $2 / 3$ Data Files: NA

\begin{tabular}{|c|c|c|c|c|c|c|c|c|c|}
\hline \multirow{3}{*}{$\begin{array}{l}\text { Traverse--> } \\
\text { Trial ----> }\end{array}$} & & \multicolumn{8}{|c|}{$1 \mathrm{st}$} \\
\hline & & \multicolumn{4}{|c|}{ Port_3_No. East } & \multicolumn{4}{|c|}{ Port_3_So. East } \\
\hline & & 1 & 2 & 3 & Mean & 1 & 2 & 3 & Mean \\
\hline Point & Depth, in. & \multicolumn{4}{|c|}{ Velocity } & \multicolumn{4}{|c|}{ Velocity } \\
\hline 1 & $9 / 16$ & 2303 & 2253 & 2320 & 2292.0 & 2062 & 2120 & 2073 & 2085.0 \\
\hline 2 & $114 / 16$ & 2302 & 2340 & 2298 & 2313.3 & 2126 & 2133 & 2199 & 2152.7 \\
\hline 3 & $38 / 16$ & 2233 & 2242 & 2239 & 2238.0 & 2064 & 1999 & 2127 & 2063.3 \\
\hline 4 & $513 / 16$ & 1980 & 1996 & 2007 & 1994.3 & 1615 & 1690 & 1651 & 1652.0 \\
\hline Center & 9 & 1628 & 1591 & 1545 & N.A. & 1609 & 1604 & 1557 & N.A. \\
\hline 5 & $12 \quad 3 / 16$ & 1708 & 1631 & 1687 & 1675.3 & 1992 & 1926 & 1945 & 1954.3 \\
\hline 6 & $14 \quad 8 / 16$ & 1949 & 1974 & 1879 & 1934.0 & 2272 & 2233 & 2246 & 2250.3 \\
\hline 7 & $16 \quad 1 / 16$ & 2135 & 2204 & 2162 & 2167.0 & 2407 & 2385 & 2383 & 2391.7 \\
\hline 8 & $176 / 16$ & 2254 & 2351 & 2304 & 2303.0 & 2233 & 2303 & 2133 & 2223.0 \\
\hline Averages --- & $----->$ & 2054.7 & 2064.7 & 2049.0 & 2114.6 & 2042.2 & 2043.7 & 2034.9 & 2096.5 \\
\hline
\end{tabular}

\begin{tabular}{|lrr|lrrrr|}
\hline All & $\underline{\mathrm{ft} / \mathrm{min}}$ & Dev. from mean & Center 2/3 & & NorthEast & SouthEast & All \\
Mean & 2105.6 & & Mean & 2053.7 & 2077.4 & 2065.5 \\
Min Point & 1652.0 & $-21.5 \%$ & Std. Dev. & 234.6 & 257.2 & 235.0 \\
Max Point & 2391.7 & $13.6 \%$ & COV as \% & 11.4 & 12.4 & $\mathbf{1 1 . 4}$ \\
\hline
\end{tabular}

Flow w/o C-Pt 3721 acfm Vel Avg w/o C-Pt $2106 \mathrm{fpm}$

\begin{tabular}{|c|c|}
\hline Start & Finish \\
\hline 97.0 & 96.0 \\
\hline 83 & 87 \\
\hline 91 & 93 \\
\hline 0.00 & 0.00 \\
\hline 29.42 & 29.40 \\
\hline 996 & 996 \\
\hline $28 \%$ & $23 \%$ \\
\hline
\end{tabular}

Notes:

Stack temp Equipment temp Ambient temp Stack static Ambient pressure Total Stack pressure Ambient humidity

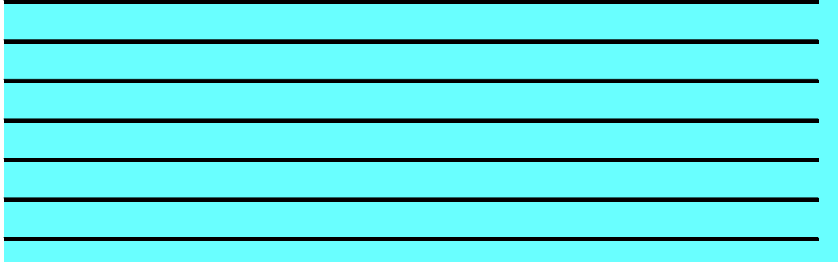

Instuments Used:

Temp >> TSI 8360 SN 209060 Cal due 9/27/06 Vel >> Solomat Zephyr SN 12951472 Cal due 8/29/06 Pitot \# 5 -- 36" Standard Pitot Dwyer

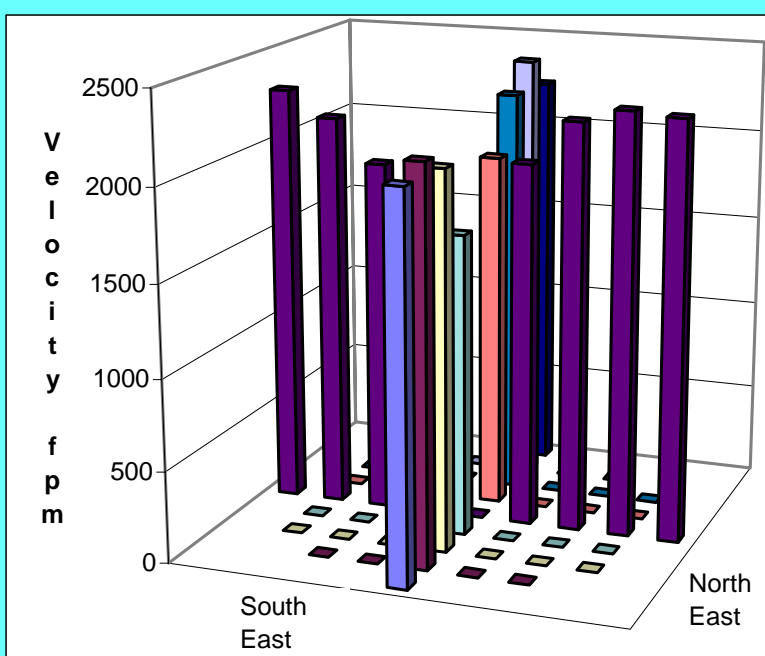

Signature signifies compliance with Procedure EMS-JAG-4 Signature/date
Signature verifying data and calculations:

Signature on file copy 


\section{VELOCITY TRAVERSE DATA FORM}

Site 296-S-21 Model

Date 8/7/2006

Testers BG Fritz

Stack Dia.

Stack X-Area

Elevation N.A.

Distance to disturbance

Velocity units $\mathrm{ft} / \mathrm{min}$
Run No. VT-16

Fan Configuration $3 \& 4$

Fan Setting $\mathbf{5 0 ~ \& ~} \mathbf{5 2}$

Stack Temp

Start/End Time 13:00 to $14: 00$

Center 2/3 from

Points in Center $2 / 3$

Data Files: $\overline{N A}$

\begin{tabular}{|c|c|c|c|c|c|c|c|c|c|}
\hline \multirow{2}{*}{$\begin{array}{l}\text { Traverse--> } \\
\text { Trial ----> }\end{array}$} & & \multicolumn{4}{|c|}{ Port_3_ Northeast } & \multicolumn{4}{|c|}{ Port_3_Southeast } \\
\hline & & 1 & 2 & 3 & Mean & 1 & 2 & 3 & Mean \\
\hline Point & Depth, in. & \multicolumn{4}{|c|}{ Velocity } & \multicolumn{4}{|c|}{ Velocity } \\
\hline 1 & $9 / 16$ & 2136 & 2217 & 2096 & 2149.7 & 2042 & 2054 & 2006 & 2034.0 \\
\hline 2 & $114 / 16$ & 2409 & 2319 & 2364 & 2364.0 & 2046 & 2082 & 2125 & 2084.3 \\
\hline 3 & $38 / 16$ & 2292 & 2281 & 2257 & 2276.7 & 1901 & 1972 & 1918 & 1930.3 \\
\hline 4 & $513 / 16$ & 1992 & 1950 & 1891 & 1944.3 & 1615 & 1649 & 1639 & 1634.3 \\
\hline Center & 9 & 1538 & 1523 & 1566 & 1542.3 & 1541 & 1539 & 1588 & 1556.0 \\
\hline 5 & $12 \quad 3 / 16$ & 1661 & 1644 & 1680 & 1661.7 & 1889 & 1856 & 1947 & 1897.3 \\
\hline 6 & $148 / 16$ & 1946 & 1952 & 1976 & 1958.0 & 2187 & 2148 & 2177 & 2170.7 \\
\hline 7 & $16 \quad 1 / 16$ & 2187 & 2191 & 2179 & 2185.7 & 2336 & 2396 & 2411 & 2381.0 \\
\hline 8 & $176 / 16$ & 2233 & 2293 & 2219 & 2248.3 & 2325 & 2199 & 2244 & 2256.0 \\
\hline Averages ---- & $---->$ & 2043.8 & 2041.1 & 2025.3 & 2036.7 & 1986.9 & 1988.3 & 2006.1 & 1993.8 \\
\hline
\end{tabular}

\begin{tabular}{|llr|lrrrr|}
\hline All & $\underline{\mathrm{ft} / \mathrm{min}}$ & Dev. from mean & Center 2/3 & & NorthEast & SouthEast & All \\
Mean & 2015.3 & & Mean & & 1990.4 & 1950.6 & 1970.5 \\
Min Point & 1542.3 & $-23.5 \%$ & Std. Dev. & 308.7 & 291.7 & 289.3 \\
Max Point & 2381.0 & $18.1 \%$ & COV as \% & 15.5 & 15.0 & $\mathbf{1 4 . 7}$ \\
\hline
\end{tabular}

Flow w/o C-Pt Vel Avg w/o C-Pt

3664 acfm

$2074 \mathrm{fpm}$

Stack temp

Equipment temp

Ambient temp

Stack static

Ambient pressure

Total Stack pressure

Ambient humidity

\begin{tabular}{|c|c|l|}
\multicolumn{1}{c|}{ Start } & Finish & \multicolumn{2}{l}{} \\
\hline 100.0 & 100.0 & $\mathrm{~F}$ \\
\hline 78 & - & $\mathrm{F}$ \\
\hline 94 & 99 & $\mathrm{~F}$ \\
\hline 0.00 & 0.00 & mbars \\
\hline 29.393 & 29.36 & in Hg \\
\hline 995 & 994 & mbars \\
\hline $23 \%$ & $21 \%$ & $\mathrm{RH}$ \\
\hline
\end{tabular}

Notes:

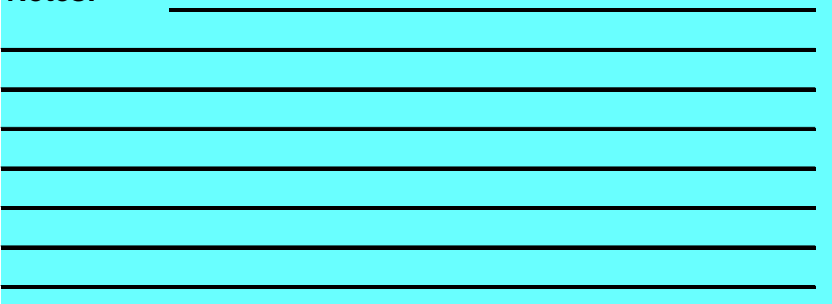

Instuments Used:

Temp >> TSI 8360 SN 209060 Cal due 9/27/06 Vel $>>$ Solomat Zephyr SN 12951472 Cal due 8/29/06 Pitot \# 5 -- 36" Standard Pitot Dwyer

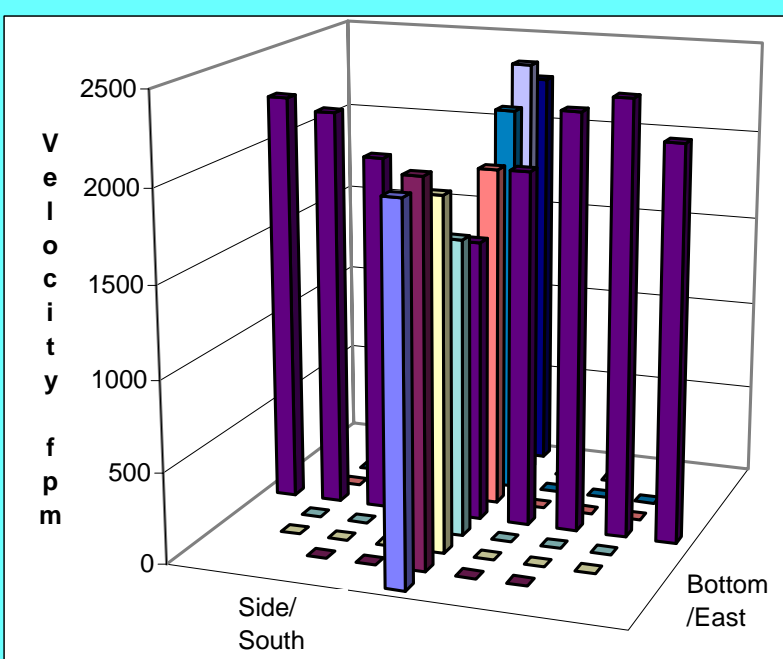

Signature signifies compliance with

Procedure EMS-JAG-4

Signature/date
Signature verifying data and calculations:

Signature on file copy 


\section{VELOCITY TRAVERSE DATA FORM}

Site 296-S-21 Model

Date 8/7/2006

Testers BG Fritz

Stack Dia.

Stack X-Area

Elevation N.A.

Distance to disturbance

Velocity units $\mathrm{ft} / \mathrm{min}$
Run No. VT-16 No centerpoint

Fan Configuration $3 \& 4$ no prefilter

Fan Setting $\mathbf{5 0 ~ \& ~} \mathbf{5 2}$

Stack Temp

Start/End Time 13:00 to $14: 00$

Center 2/3 from

Points in Center $2 / 3$

Data Files: NA

\begin{tabular}{|c|c|c|c|c|c|c|c|c|c|}
\hline \multirow{2}{*}{$\begin{array}{l}\text { Traverse--> } \\
\text { Trial ----> }\end{array}$} & & \multicolumn{4}{|c|}{ Port_3_Northeast } & \multicolumn{4}{|c|}{ Port_3_Southeast } \\
\hline & & 1 & 2 & 3 & Mean & 1 & 2 & 3 & Mean \\
\hline Point & Depth, in. & \multicolumn{4}{|c|}{ Velocity } & \multicolumn{4}{|c|}{ Velocity } \\
\hline 1 & $9 / 16$ & 2136 & 2217 & 2096 & 2149.7 & 2042 & 2054 & 2006 & 2034.0 \\
\hline 2 & $114 / 16$ & 2409 & 2319 & 2364 & 2364.0 & 2046 & 2082 & 2125 & 2084.3 \\
\hline 3 & $38 / 16$ & 2292 & 2281 & 2257 & 2276.7 & 1901 & 1972 & 1918 & 1930.3 \\
\hline 4 & 5 13/16 & 1992 & 1950 & 1891 & 1944.3 & 1615 & 1649 & 1639 & 1634.3 \\
\hline Center & 9 & 1538 & 1523 & 1566 & N.A. & 1541 & 1539 & 1588 & N.A. \\
\hline 5 & $123 / 16$ & 1661 & 1644 & 1680 & 1661.7 & 1889 & 1856 & 1947 & 1897.3 \\
\hline 6 & $148 / 16$ & 1946 & 1952 & 1976 & 1958.0 & 2187 & 2148 & 2177 & 2170.7 \\
\hline 7 & $16 \quad 1 / 16$ & 2187 & 2191 & 2179 & 2185.7 & 2336 & 2396 & 2411 & 2381.0 \\
\hline 8 & $176 / 16$ & 2233 & 2293 & 2219 & 2248.3 & 2325 & 2199 & 2244 & 2256.0 \\
\hline Averages ---- & $-\cdots$ & 2043.8 & 2041.1 & 2025.3 & 2098.5 & 1986.9 & 1988.3 & 2006.1 & 2048.5 \\
\hline
\end{tabular}

\begin{tabular}{|lrr|lrrrr|}
\hline All & $\underline{\mathrm{ft} / \mathrm{min}}$ & Dev. from mean & Center 2/3 & & NorthEast & SouthEast & All \\
Mean & 2073.5 & & Mean & 2065.1 & 2016.3 & 2040.7 \\
Min Point & 1634.3 & $-21.2 \%$ & Std. Dev. & 259.8 & 256.5 & 247.4 \\
Max Point & 2381.0 & $14.8 \%$ & COV as $\%$ & 12.6 & 12.7 & $\mathbf{1 2 . 1}$ \\
\hline
\end{tabular}

Flow w/o C-Pt Vel Avg w/o C-Pt

3664 acfm

$2074 \mathrm{fpm}$

Stack temp

Equipment temp

Ambient temp

Stack static

Ambient pressure

Total Stack pressure

Ambient humidity

\begin{tabular}{|c|c|l|}
\multicolumn{1}{c|}{ Start } & Finish & \multicolumn{2}{l}{} \\
\hline 100.0 & 100.0 & $\mathrm{~F}$ \\
\hline 78 & - & $\mathrm{F}$ \\
\hline 94 & 99 & $\mathrm{~F}$ \\
\hline 0.00 & 0.00 & mbars \\
\hline 29.393 & 29.36 & in Hg \\
\hline 995 & 994 & mbars \\
\hline $23 \%$ & $21 \%$ & $\mathrm{RH}$ \\
\hline
\end{tabular}

Notes:

Instuments Used:

Temp >> TSI 8360 SN 209060 Cal due 9/27/06 Vel >> Solomat Zephyr SN 12951472 Cal due 8/29/06 Pitot \# 5 -- 36" Standard Pitot Dwyer

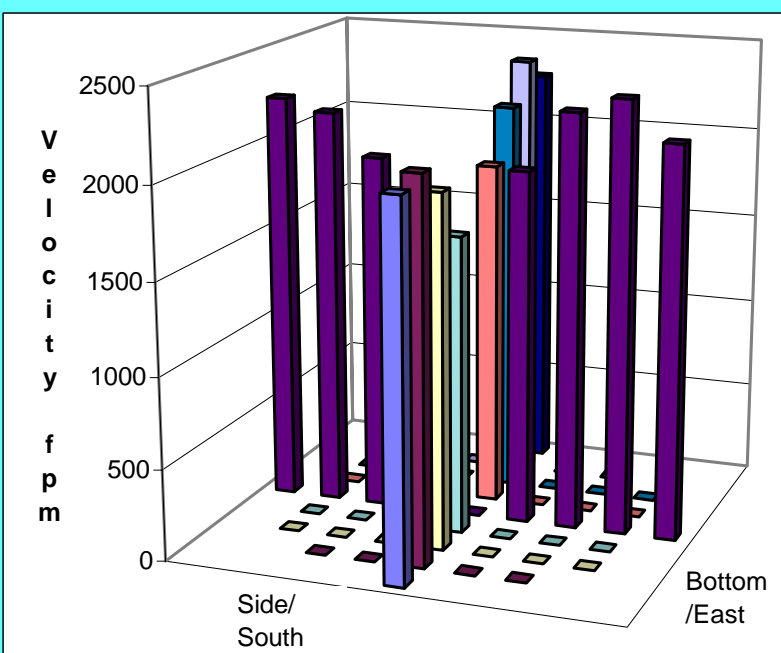

Signature signifies compliance with

Procedure EMS-JAG-4

Signature/date
Signature verifying data and calculations:

Signature on file copy 


\section{VELOCITY TRAVERSE DATA FORM}

Site 296-S-21 Model

Date 8/7/2006

Testers BG Fritz

Stack Dia.

Stack X-Area

$177 / 8$ in.

Elevation N.A.

Distance to disturbance

Velocity units $\mathrm{ft} / \mathrm{min}$
Run No. VT-17

Fan Configuration 1

Fan Setting 56

Stack Temp

Start/End Time 14:30 to 15:30

Center 2/3 from

Points in Center 2/3

Data Files: NA

\begin{tabular}{|c|c|c|c|c|c|c|c|c|c|}
\hline \multirow{2}{*}{\multicolumn{2}{|c|}{$\begin{array}{l}\text { Traverse--> } \\
\text { Trial ----> }\end{array}$}} & \multicolumn{4}{|c|}{ Port_4_Bottom/East } & \multicolumn{4}{|c|}{ Port_4_Side/South } \\
\hline & & 1 & 2 & 3 & Mean & 1 & 2 & 3 & Mean \\
\hline Point & Depth, in. & \multicolumn{4}{|c|}{ Velocity } & \multicolumn{4}{|c|}{ Velocity } \\
\hline 1 & $9 / 16$ & 790 & 813 & 774 & 792.3 & 747 & 740 & 764 & 750.3 \\
\hline 2 & $114 / 16$ & 853 & 899 & 917 & 889.7 & 793 & 849 & 787 & 809.7 \\
\hline 3 & $38 / 16$ & 908 & 862 & 848 & 872.7 & 847 & 855 & 815 & 839.0 \\
\hline 4 & $513 / 16$ & 860 & 804 & 826 & 830.0 & 794 & 743 & 770 & 769.0 \\
\hline Center & 9 & 804 & 766 & 764 & 778.0 & 784 & 808 & 761 & 784.3 \\
\hline 5 & $123 / 16$ & 780 & 780 & 753 & 771.0 & 824 & 805 & 801 & 810.0 \\
\hline 6 & $148 / 16$ & 820 & 788 & 808 & 805.3 & 815 & 834 & 804 & 817.7 \\
\hline 7 & $16 \quad 1 / 16$ & 800 & 803 & 775 & 792.7 & 842 & 841 & 843 & 842.0 \\
\hline 8 & $176 / 16$ & 772 & 772 & 763 & 769.0 & 813 & 810 & 751 & 791.3 \\
\hline Averages --- & $----->>$ & 820.8 & 809.7 & 803.1 & 811.2 & 806.6 & 809.4 & 788.4 & 801.5 \\
\hline
\end{tabular}

\begin{tabular}{|lrr|lrrr|}
\hline All & $\underline{\mathrm{ft} / \mathrm{min}}$ & Dev. from mean & Center 2/3 & $\underline{\text { East }}$ & $\underline{\text { South }}$ & $\underline{\text { All }}$ \\
\cline { 2 - 3 } Mean & 806.3 & & Mean & 819.9 & 810.2 & 815.1 \\
Min Point & 750.3 & $-6.9 \%$ & Std. Dev. & 46.3 & 26.7 & 36.6 \\
Max Point & 889.7 & $10.3 \%$ & COV as \% & 5.6 & 3.3 & $\mathbf{4 . 5}$ \\
\hline
\end{tabular}

Flow w/o C-Pt Vel Avg w/o C-Pt 1411 acfm 809 fpm

Stack temp Equipment temp Ambient temp Stack static Ambient pressure Total Stack pressure Ambient humidity

\begin{tabular}{|c|c|l}
\multicolumn{1}{|c|}{ Start } & Finish & \\
\hline 105.0 & 105.0 & $\mathrm{~F}$ \\
\hline- & - & $\mathrm{F}$ \\
\hline 101 & 99 & $\mathrm{~F}$ \\
\hline 0.00 & 0.00 & mbars \\
\hline 29.36 & 29.33 & in Hg \\
\hline 994 & 993 & $\mathrm{mbars}$ \\
\hline $24 \%$ & $23 \%$ & $\mathrm{RH}$ \\
\hline
\end{tabular}

Instuments Used:

Temp >> TSI 8360 SN $209060 \mathrm{Cal}$ due 9/27/06 Vel $>>$ Solomat Zephyr SN 12951472 Cal due 8/29/06 Pitot \# 5 -- 36" Standard Pitot Dwyer

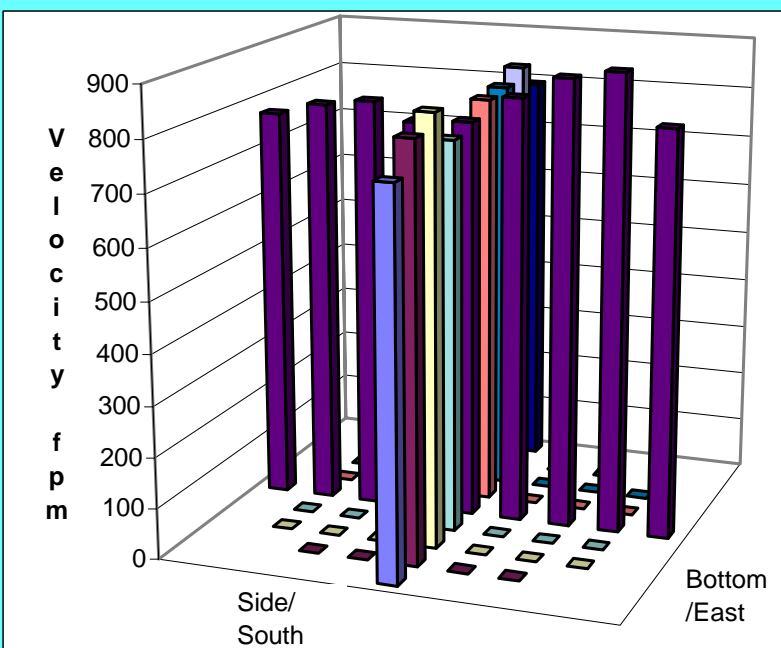

Signature signifies compliance with Procedure EMS-JAG-4 Signature/date
Signature verifying data and calculations:

Signature on file copy 


\section{VELOCITY TRAVERSE DATA FORM}

Site 296-S-21 Model

Date 8/7/2006

Testers BG Fritz

Stack Dia.

Stack X-Area

$177 / 8$ in

Elevation N.A.

Distance to disturbance

Velocity units $\mathrm{ft} / \mathrm{min}$
Run No. VT-17 No centerpoint

Fan Configuration 1 no prefilter

Fan Setting 56

Stack Temp

Start/End Time 14:30 to $15: 30$

Center $2 / 3$ from

Points in Center $2 / 3$

Data Files: $\mathrm{NA}$

\begin{tabular}{|c|c|c|c|c|c|c|c|c|c|}
\hline \multirow{2}{*}{\multicolumn{2}{|c|}{$\begin{array}{l}\text { Traverse--> } \\
\text { Trial ----> }\end{array}$}} & \multicolumn{4}{|c|}{ Port_4_Bottom/East } & \multicolumn{4}{|c|}{ Port_4_Side/South } \\
\hline & & 1 & 2 & 3 & Mean & 1 & 2 & 3 & Mean \\
\hline Point & Depth, in. & \multicolumn{4}{|c|}{ Velocity } & \multicolumn{4}{|c|}{ Velocity } \\
\hline 1 & $9 / 16$ & 790 & 813 & 774 & 792.3 & 747 & 740 & 764 & 750.3 \\
\hline 2 & $114 / 16$ & 853 & 899 & 917 & 889.7 & 793 & 849 & 787 & 809.7 \\
\hline 3 & $38 / 16$ & 908 & 862 & 848 & 872.7 & 847 & 855 & 815 & 839.0 \\
\hline 4 & $513 / 16$ & 860 & 804 & 826 & 830.0 & 794 & 743 & 770 & 769.0 \\
\hline Center & 9 & 804 & 766 & 764 & N.A. & 784 & 808 & 761 & N.A. \\
\hline 5 & $123 / 16$ & 780 & 780 & 753 & 771.0 & 824 & 805 & 801 & 810.0 \\
\hline 6 & $148 / 16$ & 820 & 788 & 808 & 805.3 & 815 & 834 & 804 & 817.7 \\
\hline 7 & $16 \quad 1 / 16$ & 800 & 803 & 775 & 792.7 & 842 & 841 & 843 & 842.0 \\
\hline 8 & $176 / 16$ & 772 & 772 & 763 & 769.0 & 813 & 810 & 751 & 791.3 \\
\hline Averages --- & $----->>$ & 820.8 & 809.7 & 803.1 & 815.3 & 806.6 & 809.4 & 788.4 & 803.6 \\
\hline
\end{tabular}

\begin{tabular}{|lrr|lrrr|}
\hline All & $\underline{\mathrm{ft} / \mathrm{min}}$ & Dev. from mean & Center 2/3 & $\underline{\text { East }}$ & $\underline{\text { South }}$ & $\underline{\text { All }}$ \\
\cline { 2 - 3 } & 809.5 & & Mean & 826.9 & 814.6 & 820.7 \\
Min Point & 750.3 & $-7.3 \%$ & Std. Dev. & 46.5 & 26.4 & 36.6 \\
Max Point & 889.7 & $9.9 \%$ & Cov as \% & 5.6 & 3.2 & $\mathbf{4 . 5}$ \\
\hline
\end{tabular}

Flow w/o C-Pt Vel Avg w/o C-Pt 1411 acfm 809 fpm

Stack temp Equipment temp Ambient temp Stack static Ambient pressure Total Stack pressure Ambient humidity

\begin{tabular}{|c|c|l}
\multicolumn{1}{|c|}{ Start } & Finish & \\
\hline 105.0 & 105.0 & $\mathrm{~F}$ \\
\hline- & - & $\mathrm{F}$ \\
\hline 101 & 99 & $\mathrm{~F}$ \\
\hline 0.00 & 0.00 & mbars \\
\hline 29.36 & 29.33 & in Hg \\
\hline 994 & 993 & $\mathrm{mbars}$ \\
\hline $24 \%$ & $23 \%$ & $\mathrm{RH}$ \\
\hline
\end{tabular}

Instuments Used:

Temp >> TSI 8360 SN $209060 \mathrm{Cal}$ due 9/27/06 Vel $>>$ Solomat Zephyr SN 12951472 Cal due 8/29/06 Pitot \# 5 -- 36" Standard Pitot Dwyer

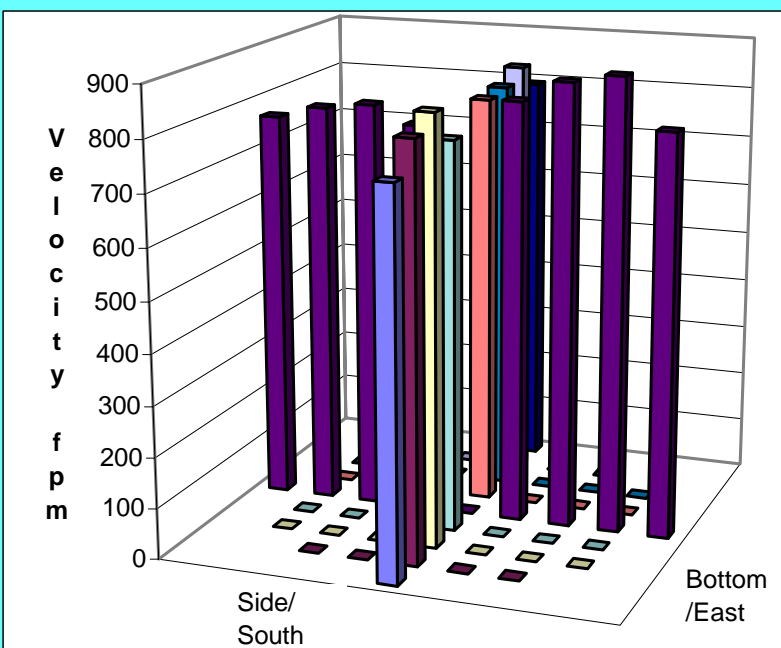

Signature signifies compliance with Procedure EMS-JAG-4 Signature/date
Signature verifying data and calculations:

Signature on file copy 


\section{VELOCITY TRAVERSE DATA FORM}

Site 296-S-21 Model

Date 8/8/2006

Testers BG Fritz

Stack Dia.

Stack X-Area

$177 / 8$ in.

Elevation N.A.

Distance to disturbance

Velocity units $\underline{\mathrm{ft} / \mathrm{min}}$
Run No. VT-18

Fan Configuration 2

Fan Setting 44

Stack Temp

Start/End Time 10:00 to 10:35

Center 2/3 from

Points in Center $2 / 3$

Data Files: NA

\begin{tabular}{|c|c|c|c|c|c|c|c|c|c|}
\hline \multirow{2}{*}{\multicolumn{2}{|c|}{$\begin{array}{l}\text { Traverse--> } \\
\text { Trial ----> }\end{array}$}} & \multicolumn{4}{|c|}{ Port_4_Bottom/East } & \multicolumn{4}{|c|}{ Port_4_Side/South } \\
\hline & & 1 & 2 & tha & Mean & 1 & 2 & & Mean \\
\hline Point & Depth, in. & \multicolumn{4}{|c|}{ Velocity } & \multicolumn{4}{|c|}{ Velocity } \\
\hline 1 & $9 / 16$ & 639 & 680 & & 659.5 & 658 & 689 & & 673.5 \\
\hline 2 & $114 / 16$ & 701 & 677 & & 689.0 & 719 & 748 & & 733.5 \\
\hline 3 & $38 / 16$ & 698 & 704 & & 701.0 & 682 & 710 & & 696.0 \\
\hline 4 & $513 / 16$ & 656 & 675 & & 665.5 & 613 & 663 & & 638.0 \\
\hline Center & 9 & 678 & 659 & & 668.5 & 672 & 700 & & 686.0 \\
\hline 5 & $123 / 16$ & 683 & 687 & & 685.0 & 691 & 702 & & 696.5 \\
\hline 6 & $148 / 16$ & 732 & 729 & & 730.5 & 753 & 715 & & 734.0 \\
\hline 7 & $161 / 16$ & 753 & 742 & & 747.5 & 707 & 730 & & 718.5 \\
\hline 8 & $176 / 16$ & 678 & 717 & & 697.5 & 722 & 705 & & 713.5 \\
\hline Averages ---. & $-\cdots>$ & 690.9 & 696.7 & \#DIV/O! & 693.8 & 690.8 & 706.9 & \#DIV/O! & 698.8 \\
\hline
\end{tabular}

\begin{tabular}{|lrr|lrrr|}
\hline All & $\underline{\mathrm{ft} / \mathrm{min}}$ & Dev. from mean & Center 2/3 & East & $\underline{\text { South }}$ & $\underline{\text { All }}$ \\
Mean & 696.3 & & Mean & 698.1 & 700.4 & 699.3 \\
Min Point & 638.0 & $-8.4 \%$ & Std. Dev. & 30.8 & 33.4 & 30.9 \\
Max Point & 747.5 & $7.4 \%$ & COV as \% & 4.4 & 4.8 & $\mathbf{4 . 4}$ \\
\hline
\end{tabular}

Flow w/o C-Pt Vel Avg w/o C-Pt 1218 acfm $699 \mathrm{fpm}$

Stack temp Equipment temp Ambient temp Stack static Ambient pressure Total Stack pressure Ambient humidity

\begin{tabular}{|c|c|l}
\multicolumn{1}{|c|}{ Start } & Finish & \\
\hline 90.0 & 86.0 & $\mathrm{~F}$ \\
\hline- & - & $\mathrm{F}$ \\
\hline 89 & 84 & $\mathrm{~F}$ \\
\hline 0.00 & 0.00 & mbars \\
\cline { 1 - 2 } 29.492 & 29.509 & in Hg \\
\hline 999 & 999 & mbars \\
\hline $32 \%$ & $37 \%$ & $\mathrm{RH}$ \\
\hline
\end{tabular}

Notes: $\quad$ Based on VT-17, 2 traverses were considered enough for these supplemental tests. There was not a significant change in the velocity profile of VT-17 when analyzed using 2 or 3 traverses.

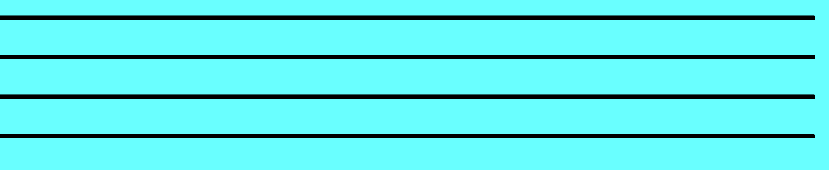

Instuments Used:

Temp >> TSI 8360 SN 209060 Cal due 9/27/06 Vel >> Solomat Zephyr SN 12951472 Cal due 8/29/06 Pitot \# 5 -- 36" Standard Pitot Dwyer

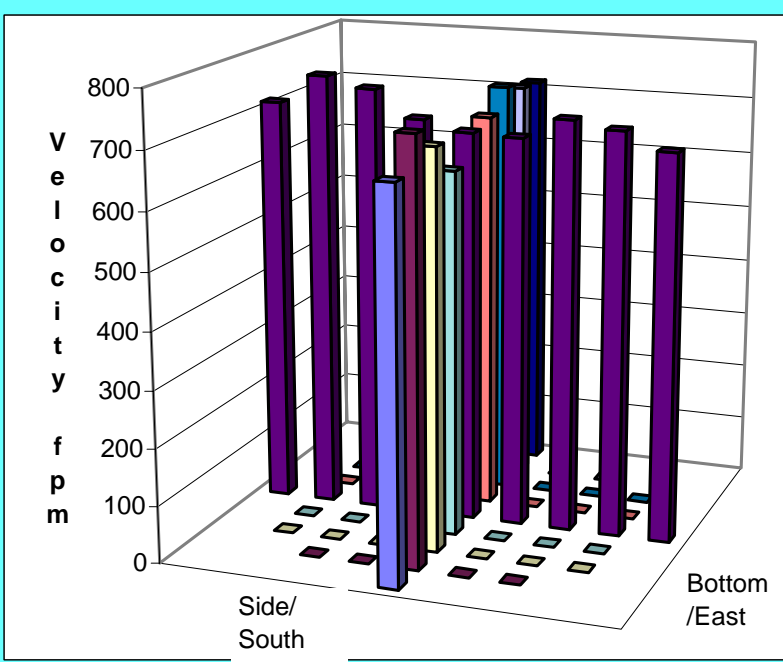

Signature signifies compliance with Procedure EMS-JAG-4 Signature/date
Signature verifying data and calculations:

Signature on file copy 


\section{VELOCITY TRAVERSE DATA FORM}

Site 296-S-21 Model

Date 8/8/2006

Testers BG Fritz

Stack Dia.

Stack X-Area

$177 / 8$ in

Elevation N.A.

Distance to disturbance

Velocity units $\mathrm{ft} / \mathrm{min}$
Run No. VT-18 No centerpoint

Fan Configuration 2 no prefilter

Fan Setting 44

Stack Temp

Start/End Time 10:00 to 10:35

Center $2 / 3$ from

Points in Center 2/3

Data Files: NA

\begin{tabular}{|c|c|c|c|c|c|c|c|c|c|}
\hline \multirow{2}{*}{\multicolumn{2}{|c|}{$\begin{array}{l}\text { Traverse--> } \\
\text { Trial ----> }\end{array}$}} & \multicolumn{4}{|c|}{ Port_4__Bottom/East } & \multicolumn{4}{|c|}{ Port_4_Side/South } \\
\hline & & 1 & 2 & & Mean & 1 & 2 & & Mean \\
\hline Point & Depth, in. & \multicolumn{4}{|c|}{ Velocity } & \multicolumn{4}{|c|}{ Velocity } \\
\hline 1 & $9 / 16$ & 639 & 680 & & 659.5 & 658 & 689 & & 673.5 \\
\hline 2 & $114 / 16$ & 701 & 677 & & 689.0 & 719 & 748 & & 733.5 \\
\hline 3 & $38 / 16$ & 698 & 704 & & 701.0 & 682 & 710 & & 696.0 \\
\hline 4 & $513 / 16$ & 656 & 675 & & 665.5 & 613 & 663 & & 638.0 \\
\hline Center & 9 & 678 & 659 & & N.A. & 672 & 700 & & N.A. \\
\hline 5 & $123 / 16$ & 683 & 687 & & 685.0 & 691 & 702 & & 696.5 \\
\hline 6 & $148 / 16$ & 732 & 729 & & 730.5 & 753 & 715 & & 734.0 \\
\hline 7 & $161 / 16$ & 753 & 742 & & 747.5 & 707 & 730 & & 718.5 \\
\hline 8 & $176 / 16$ & 678 & 717 & & 697.5 & 722 & 705 & & 713.5 \\
\hline Averages --- & $-\cdots>$ & 690.9 & 696.7 & \#DIV/O! & 696.9 & 690.8 & 706.9 & \#DIV/O! & 700.4 \\
\hline
\end{tabular}

\begin{tabular}{|lrr|lrrr|}
\hline All & ft/min & Dev. from mean & Center 2/3 & East & South & All \\
Mean & 698.7 & & Mean & 703.1 & 702.8 & 702.9 \\
Min Point & 638.0 & $-8.7 \%$ & Std. Dev. & 30.6 & 35.9 & 31.8 \\
Max Point & 747.5 & $7.0 \%$ & COV as \% & 4.3 & 5.1 & $\mathbf{4 . 5}$ \\
\hline
\end{tabular}

Flow w/o C-Pt Vel Avg w/o C-Pt $1218 \mathrm{acfm}$ 699 fpm

Stack temp Equipment temp Ambient temp Stack static Ambient pressure Total Stack pressure Ambient humidity

\begin{tabular}{|c|c|l}
\multicolumn{1}{|c|}{ Start } & Finish & \\
\hline 90.0 & 86.0 & $\mathrm{~F}$ \\
\hline- & - & $\mathrm{F}$ \\
\hline 89 & 84 & $\mathrm{~F}$ \\
\hline 0.00 & 0.00 & mbars \\
\cline { 1 - 2 } 29.492 & 29.509 & in Hg \\
\hline 999 & 999 & mbars \\
\hline $32 \%$ & $37 \%$ & $\mathrm{RH}$ \\
\hline
\end{tabular}

Notes: $\quad$ Based on VT-17, 2 traverses were considered enough for these supplemental tests. There was not a significant change in the velocity profile of VT-17 when analyzed using 2 or 3 traverses.

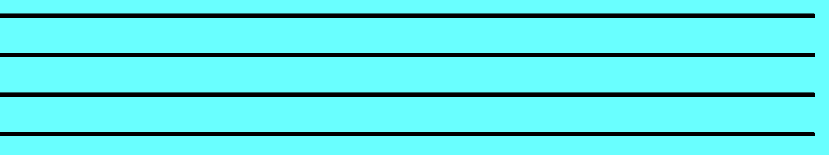

Instuments Used:

Temp >> TSI 8360 SN 209060 Cal due 9/27/06 Vel $>>$ Solomat Zephyr SN 12951472 Cal due 8/29/06 Pitot \# 5 -- 36" Standard Pitot Dwyer

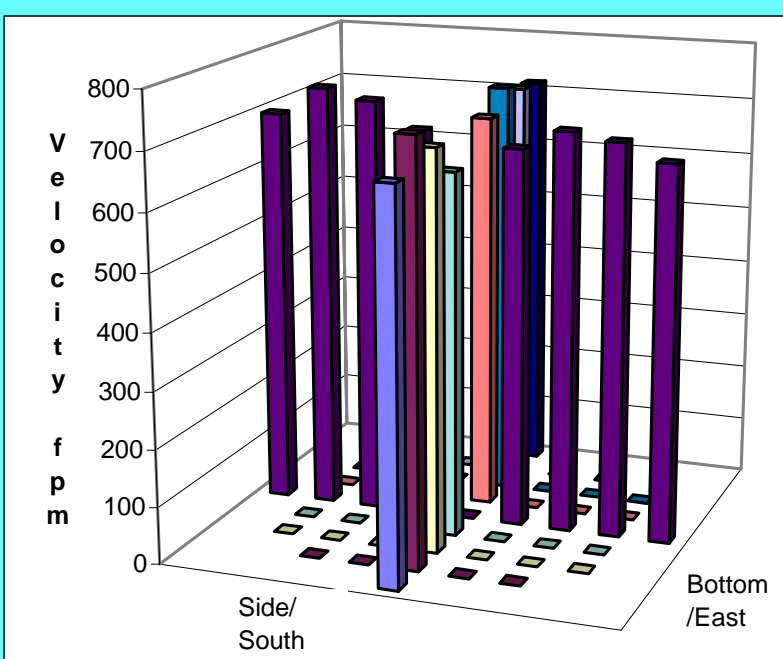

Signature signifies compliance with Procedure EMS-JAG-4 Signature/date
Signature verifying data and calculations:

Signature on file copy 


\section{VELOCITY TRAVERSE DATA FORM}

Site 296-S-21 Model

Date 8/8/2006

Testers BG Fritz

Stack Dia.

Stack X-Area

$177 / 8$ in

Elevation N.A.

Distance to disturbance

Velocity units $\underline{\mathrm{ft} / \mathrm{min}}$
Run No. VT-19

Fan Configuration 3

Fan Setting $\mathbf{5 0}$

Stack Temp

Start/End Time 11:15 to 11:40

Center 2/3 from

Points in Center $2 / 3$

Data Files: NA

\begin{tabular}{|c|c|c|c|c|c|c|c|c|c|}
\hline \multirow{2}{*}{\multicolumn{2}{|c|}{$\begin{array}{l}\text { Traverse--> } \\
\text { Trial ----> }\end{array}$}} & \multicolumn{4}{|c|}{ Port_4_Bottom/East } & \multicolumn{4}{|c|}{ Port_4_Side/South } \\
\hline & & 1 & 2 & tha & Mean & 1 & 2 & & Mean \\
\hline Point & Depth, in. & \multicolumn{4}{|c|}{ Velocity } & \multicolumn{4}{|c|}{ Velocity } \\
\hline 1 & $9 / 16$ & 701 & 702 & & 701.5 & 715 & 720 & & 717.5 \\
\hline 2 & $114 / 16$ & 726 & 737 & & 731.5 & 779 & 796 & & 787.5 \\
\hline 3 & $38 / 16$ & 759 & 755 & & 757.0 & 778 & 803 & & 790.5 \\
\hline 4 & $513 / 16$ & 757 & 716 & & 736.5 & 734 & 780 & & 757.0 \\
\hline Center & 9 & 775 & 763 & & 769.0 & 774 & 758 & & 766.0 \\
\hline 5 & $123 / 16$ & 827 & 821 & & 824.0 & 830 & 808 & & 819.0 \\
\hline 6 & $148 / 16$ & 896 & 869 & & 882.5 & 931 & 899 & & 915.0 \\
\hline 7 & $161 / 16$ & 864 & 875 & & 869.5 & 922 & 889 & & 905.5 \\
\hline 8 & $176 / 16$ & 827 & 822 & & 824.5 & 852 & 829 & & 840.5 \\
\hline Averages -- & $\overline{--->>}$ & 792.4 & 784.4 & \#DIV/O! & 788.4 & 812.8 & 809.1 & \#DIV/O! & 810.9 \\
\hline
\end{tabular}

\begin{tabular}{|lrr|lrrr|}
\hline All & ft/min & Dev. from mean & Center 2/3 & East & South & All \\
\cline { 2 - 3 } Mean & 799.7 & & Mean & 795.7 & 820.1 & 807.9 \\
Min Point & 701.5 & $-12.3 \%$ & Std. Dev. & 62.7 & 64.7 & 62.5 \\
Max Point & 915.0 & $14.4 \%$ & COV as \% & 7.9 & 7.9 & 7.7 \\
\hline
\end{tabular}

Flow w/o C-Pt Vel Avg w/o C-Pt 1401 acfm $804 \mathrm{fpm}$

Stack temp Equipment temp Ambient temp Stack static Ambient pressure Total Stack pressure Ambient humidity

\begin{tabular}{|c|c|l}
\multicolumn{1}{|c|}{ Start } & Finish & \multicolumn{1}{l}{8} \\
\hline 86.0 & 86.0 & $F$ \\
\hline- & - & $F$ \\
\hline 84 & 86 & $F$ \\
\hline 0.00 & 0.00 & mbars \\
\hline 29.509 & 29.51 & in Hg \\
\hline 999 & 999 & mbars \\
\hline $32 \%$ & $35 \%$ & $\mathrm{RH}$ \\
\hline
\end{tabular}

Notes: $\quad$ Only 2 traverses done per port. See note on data sheet VT-18.
Instuments Used:

Temp >> TSI 8360 SN $209060 \mathrm{Cal}$ due 9/27/06 Vel >> Solomat Zephyr SN 12951472 Cal due 8/29/06 Pitot \# 5 -- 36" Standard Pitot Dwyer

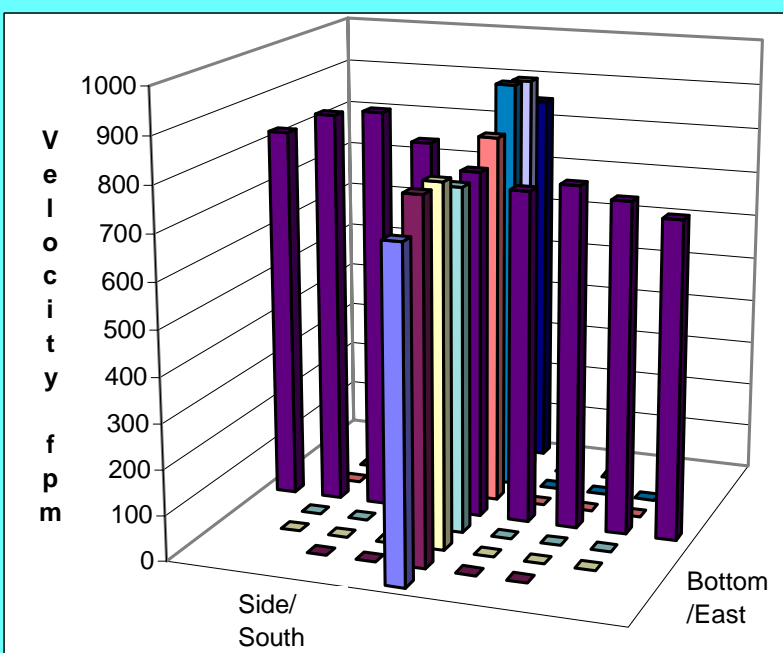

Signature signifies compliance with Procedure EMS-JAG-4 Signature/date
Signature verifying data and calculations:

Signature on file copy 


\section{VELOCITY TRAVERSE DATA FORM}

Site 296-S-21 Model

Date 8/8/2006

Testers BG Fritz

Stack Dia.

Stack X-Area

$177 / 8$ in

Elevation N.A.

Distance to disturbance

Velocity units $\mathrm{ft} / \mathrm{min}$
Run No. VT-19 No centerpoint

Fan Configuration 3 no prefilter

Fan Setting $\mathbf{5 0}$

Stack Temp

Start/End Time 11:15 to $11: 40$

Center $2 / 3$ from

Points in Center $2 / 3$

Data Files: NA

\begin{tabular}{|c|c|c|c|c|c|c|c|c|c|}
\hline \multirow{2}{*}{\multicolumn{2}{|c|}{$\begin{array}{l}\text { Traverse--> } \\
\text { Trial ----> }\end{array}$}} & \multicolumn{4}{|c|}{ Port_4__Bottom/East } & \multicolumn{4}{|c|}{ Port_4_Side/South } \\
\hline & & 1 & 2 & & Mean & 1 & 2 & & Mean \\
\hline Point & Depth, in. & \multicolumn{4}{|c|}{ Velocity } & \multicolumn{4}{|c|}{ Velocity } \\
\hline 1 & $9 / 16$ & 701 & 702 & & 701.5 & 715 & 720 & & 717.5 \\
\hline 2 & $114 / 16$ & 726 & 737 & & 731.5 & 779 & 796 & & 787.5 \\
\hline 3 & $38 / 16$ & 759 & 755 & & 757.0 & 778 & 803 & & 790.5 \\
\hline 4 & $513 / 16$ & 757 & 716 & & 736.5 & 734 & 780 & & 757.0 \\
\hline Center & 9 & 775 & 763 & & N.A. & 774 & 758 & & N.A. \\
\hline 5 & $123 / 16$ & 827 & 821 & & 824.0 & 830 & 808 & & 819.0 \\
\hline 6 & $148 / 16$ & 896 & 869 & & 882.5 & 931 & 899 & & 915.0 \\
\hline 7 & $161 / 16$ & 864 & 875 & & 869.5 & 922 & 889 & & 905.5 \\
\hline 8 & $176 / 16$ & 827 & 822 & & 824.5 & 852 & 829 & & 840.5 \\
\hline Averages --- & $-\cdots>$ & 792.4 & 784.4 & \#DIV/O! & 790.9 & 812.8 & 809.1 & \#DIV/O! & 816.6 \\
\hline
\end{tabular}

\begin{tabular}{|lrr|lrrr|}
\hline All & ft/min & Dev. from mean & Center 2/3 & East & $\underline{\text { South }}$ & $\underline{\text { All }}$ \\
\cline { 2 - 3 } Mean & 803.7 & & Mean & 800.2 & 829.1 & 814.6 \\
Min Point & 701.5 & $-12.7 \%$ & Std. Dev. & 67.5 & 65.9 & 65.4 \\
Max Point & 915.0 & $13.8 \%$ & Cov as \% & 8.4 & 8.0 & $\mathbf{8 . 0}$ \\
\hline
\end{tabular}

Flow w/o C-Pt Vel Avg w/o C-Pt 1401 acfm $804 \mathrm{fpm}$

Stack temp Equipment temp Ambient temp Stack static Ambient pressure Total Stack pressure Ambient humidity

\begin{tabular}{|c|c|c|}
\hline Start & Finish & \\
\hline 86.0 & 86.0 & $F$ \\
\hline - & - & $F$ \\
\hline 84 & 86 & $F$ \\
\hline 0.00 & 0.00 & mbars \\
\hline 29.509 & 29.51 & in $\mathrm{Hg}$ \\
\hline 999 & 999 & mbars \\
\hline $32 \%$ & $35 \%$ & $\mathrm{RH}$ \\
\hline
\end{tabular}

Notes: $\quad$ Only 2 traverses done per port. See note on data sheet VT-18.
Instuments Used:

Temp >> TSI 8360 SN $209060 \mathrm{Cal}$ due 9/27/06 Vel >> Solomat Zephyr SN 12951472 Cal due 8/29/06 Pitot \# 5 -- 36" Standard Pitot Dwyer

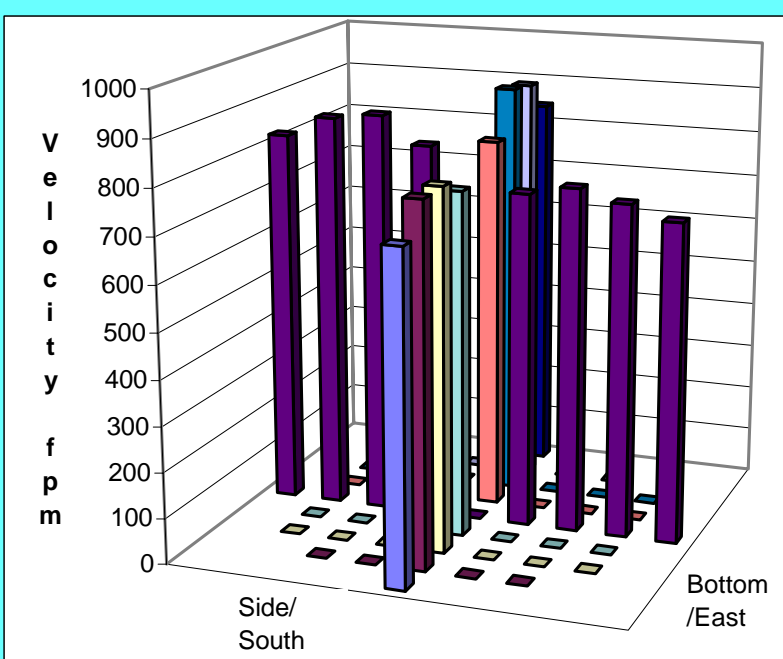

Signature signifies compliance with Procedure EMS-JAG-4 Signature/date
Signature verifying data and calculations:

Signature on file copy 


\section{VELOCITY TRAVERSE DATA FORM}

Site 296-S-21 Model

Date 8/8/2006

Testers BG Fritz

Stack Dia.

Stack X-Area

$177 / 8$ in

Elevation N.A.

Distance to disturbance

Velocity units $\mathrm{ft} / \mathrm{min}$
Run No. VT-20

Fan Configuration 4

Fan Setting $\mathbf{5 2}$

Stack Temp

Start/End Time 11:50 to 12:30

Center $2 / 3$ from

Points in Center 2/3

Data Files: NA

\begin{tabular}{|c|c|c|c|c|c|c|c|c|c|}
\hline \multirow{2}{*}{\multicolumn{2}{|c|}{$\begin{array}{l}\text { Traverse--> } \\
\text { Trial ----> }\end{array}$}} & \multicolumn{4}{|c|}{ Port_4_Bottom/East } & \multicolumn{4}{|c|}{ Port_4_Side/South } \\
\hline & & 1 & 2 & & Mean & 1 & 2 & 3 & Mean \\
\hline Point & Depth, in. & \multicolumn{4}{|c|}{ Velocity } & \multicolumn{4}{|c|}{ Velocity } \\
\hline 1 & $9 / 16$ & 1136 & 1167 & & 1151.5 & 1342 & 1359 & & 1350.5 \\
\hline 2 & $114 / 16$ & 1265 & 1276 & & 1270.5 & 1423 & 1421 & & 1422.0 \\
\hline 3 & $38 / 16$ & 1221 & 1288 & & 1254.5 & 1439 & 1431 & & 1435.0 \\
\hline 4 & 5 13/16 & 1267 & 1226 & & 1246.5 & 1369 & 1371 & & 1370.0 \\
\hline Center & 9 & 1354 & 1378 & & 1366.0 & 1338 & 1365 & & 1351.5 \\
\hline 5 & $123 / 16$ & 1502 & 1573 & & 1537.5 & 1542 & 1509 & & 1525.5 \\
\hline 6 & $148 / 16$ & 1573 & 1599 & & 1586.0 & 1684 & 1697 & & 1690.5 \\
\hline 7 & $161 / 16$ & 1584 & 1601 & & 1592.5 & 1684 & 1715 & & 1699.5 \\
\hline 8 & $176 / 16$ & 1436 & 1415 & & 1425.5 & 1580 & 1658 & & 1619.0 \\
\hline Averages ---- & $-\cdots$ & 1370.9 & 1391.4 & \#DIV/O! & 1381.2 & 1489.0 & 1502.9 & \#DIV/O! & 1495.9 \\
\hline
\end{tabular}

\begin{tabular}{|lrr|lrrr|}
\hline All & $\underline{\mathrm{ft} / \mathrm{min}}$ & Dev. from mean & Center 2/3 & $\underline{\text { East }}$ & $\underline{\text { North }}$ & $\underline{\text { All }}$ \\
Mean & 1438.6 & & Mean & 1407.6 & 1499.1 & 1453.4 \\
Min Point & 1151.5 & $-20.0 \%$ & Std. Dev. & 159.6 & 144.9 & 154.0 \\
Max Point & 1699.5 & $18.1 \%$ & Cov as \% & 11.3 & 9.7 & $\mathbf{1 0 . 6}$ \\
\hline
\end{tabular}

Flow w/o C-Pt

2524 acfm

Vel Avg w/o C-Pt $1449 \mathrm{fpm}$

Stack temp

Equipment temp

Ambient temp

Stack static

Ambient pressure

Total Stack pressure

Ambient humidity

\begin{tabular}{|c|c|l|}
\multicolumn{1}{|c|}{ Start } & Finish & \\
\hline 86.0 & 78.0 & $\mathrm{~F}$ \\
\hline- & - & $\mathrm{F}$ \\
\hline 86 & 78 & $\mathrm{~F}$ \\
\hline 0.00 & 0.00 & mbars \\
\hline 29.509 & 29.51 & in Hg \\
\hline 999 & 999 & mbars \\
\hline $35 \%$ & $53 \%$ & $\mathrm{RH}$ \\
\hline
\end{tabular}

Notes: $\quad$ Only 2 traverses done per port. See note on data sheet VT-18.
Instuments Used:

Temp $\gg$ > TSI 8360 SN $209060 \mathrm{Cal}$ due 9/27/06 Vel >> Solomat Zephyr SN 12951472 Cal due 8/29/06 Pitot \# 5 -- 36" Standard Pitot Dwyer

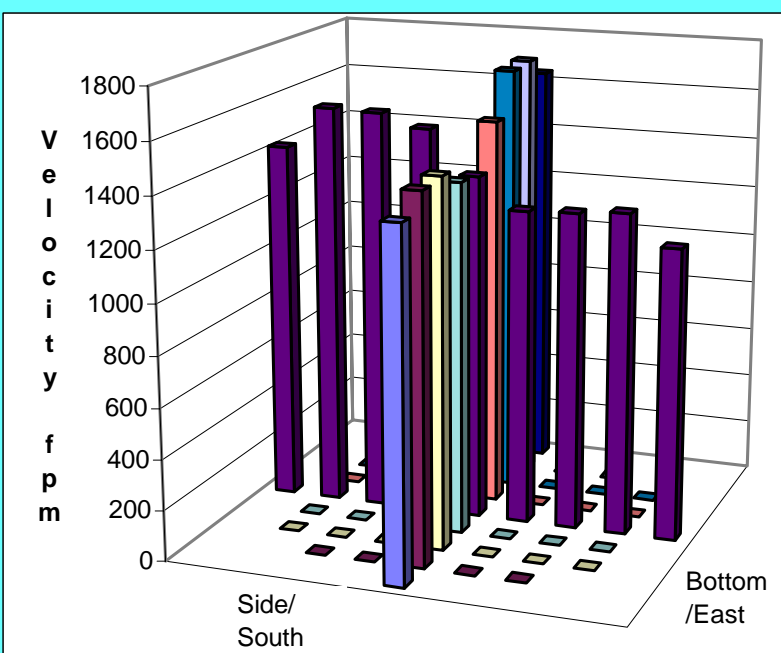

Signature signifies compliance with Procedure EMS-JAG-4 Signature/date
Signature verifying data and calculations:

Signature on file copy 


\section{VELOCITY TRAVERSE DATA FORM}

Site 296-S-21 Model

Date 8/8/2006

Testers BG Fritz

Stack Dia.

Stack X-Area

$177 / 8$ in

Elevation N.A.

Distance to disturbance

Velocity units $\mathrm{ft} / \mathrm{min}$
Run No. VT-20 No centerpoint

Fan Configuration 4

Fan Setting 52

Stack Temp

Start/End Time 11:50 to $12: 30$

Center $2 / 3$ from

Points in Center 2/3

Data Files: NA

no prefilter

$\mathrm{Hz}$

$773 / 4$ inches

(1/

\begin{tabular}{|c|c|c|c|c|c|c|c|c|c|}
\hline \multirow{2}{*}{\multicolumn{2}{|c|}{$\begin{array}{l}\text { Traverse--> } \\
\text { Trial ----> }\end{array}$}} & \multicolumn{4}{|c|}{ Port_4_Bottom/East } & \multicolumn{4}{|c|}{ Port_4_Side/South } \\
\hline & & 1 & 2 & & Mean & 1 & 2 & & Mean \\
\hline Point & Depth, in. & \multicolumn{4}{|c|}{ Velocity } & \multicolumn{4}{|c|}{ Velocity } \\
\hline 1 & $9 / 16$ & 1136 & 1167 & & 1151.5 & 1342 & 1359 & & 1350.5 \\
\hline 2 & $114 / 16$ & 1265 & 1276 & & 1270.5 & 1423 & 1421 & & 1422.0 \\
\hline 3 & $38 / 16$ & 1221 & 1288 & & 1254.5 & 1439 & 1431 & & 1435.0 \\
\hline 4 & $513 / 16$ & 1267 & 1226 & & 1246.5 & 1369 & 1371 & & 1370.0 \\
\hline Center & 9 & 1354 & 1378 & & N.A. & 1338 & 1365 & & N.A. \\
\hline 5 & $123 / 16$ & 1502 & 1573 & & 1537.5 & 1542 & 1509 & & 1525.5 \\
\hline 6 & $148 / 16$ & 1573 & 1599 & & 1586.0 & 1684 & 1697 & & 1690.5 \\
\hline 7 & $16 \quad 1 / 16$ & 1584 & 1601 & & 1592.5 & 1684 & 1715 & & 1699.5 \\
\hline 8 & $176 / 16$ & 1436 & 1415 & & 1425.5 & 1580 & 1658 & & 1619.0 \\
\hline Averages --- & $---->$ & 1370.9 & 1391.4 & \#DIV/O! & 1383.1 & 1489.0 & 1502.9 & \#DIV/0! & 1514.0 \\
\hline
\end{tabular}

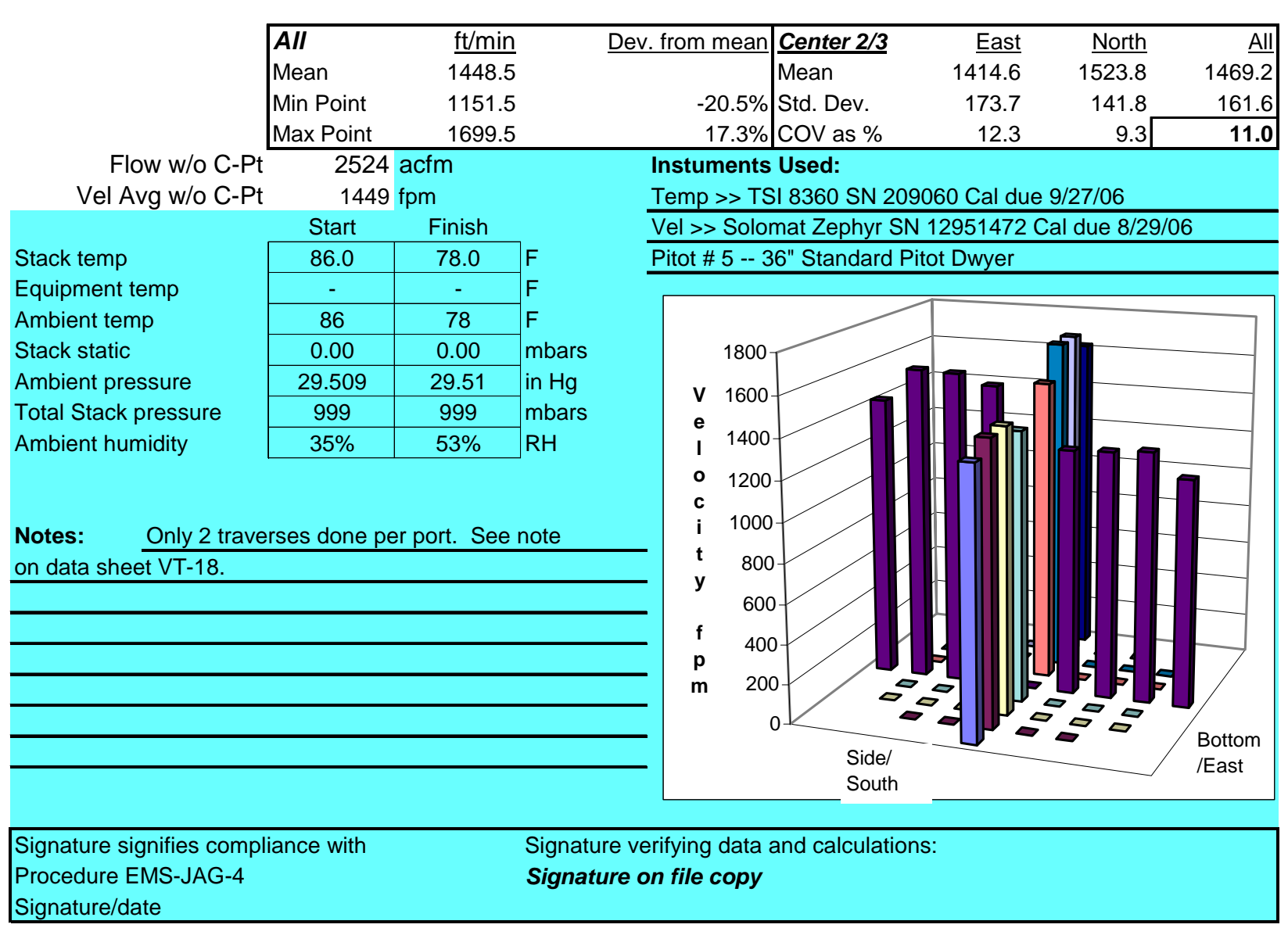


VELOCITY TRAVERSE DATA FORM

Site 296-S-21 Model Date 8/11/2006

Testers BGF

Stack Dia. Stack X-Area

Elevation$$
\text { - }
$$

$\frac{\frac{18 \text { in. }}{254.5 \text { in. } 2}}{\frac{\text { N.A. }}{54.625 \quad \text { inches }}}$

Distance to disturbance 54.625 inches

\begin{abstract}
Order--->
\end{abstract}
Traverse-->

Trial ---->

\begin{tabular}{|c|c|}
\hline Point & Depth, in. \\
\hline 1 & 0.50 \\
\hline 2 & 0.88 \\
\hline 3 & 1.53 \\
\hline 4 & 2.25 \\
\hline 5 & 3.04 \\
\hline 6 & 3.96 \\
\hline 7 & 5.09 \\
\hline 8 & 6.75 \\
\hline 9 & 11.25 \\
\hline 10 & 12.91 \\
\hline 11 & 14.04 \\
\hline 12 & 14.96 \\
\hline 13 & 15.75 \\
\hline 14 & 16.47 \\
\hline 15 & 17.12 \\
\hline 16 & 17.50 \\
\hline
\end{tabular}
$1 \mathrm{st}$
Run No. VT-21

Fan Configuration Fan 1 \& 4

Fan Setting 56,52

Stack Temp

Start/End Time $0930 / 1030$

Center 2/3 from Points in Center 2/3
$77.5 \mathrm{deg} F$

30

$\begin{array}{lll}1.65 & \text { to: } & \frac{16.35}{13} \\ & \text { to: }\end{array}$

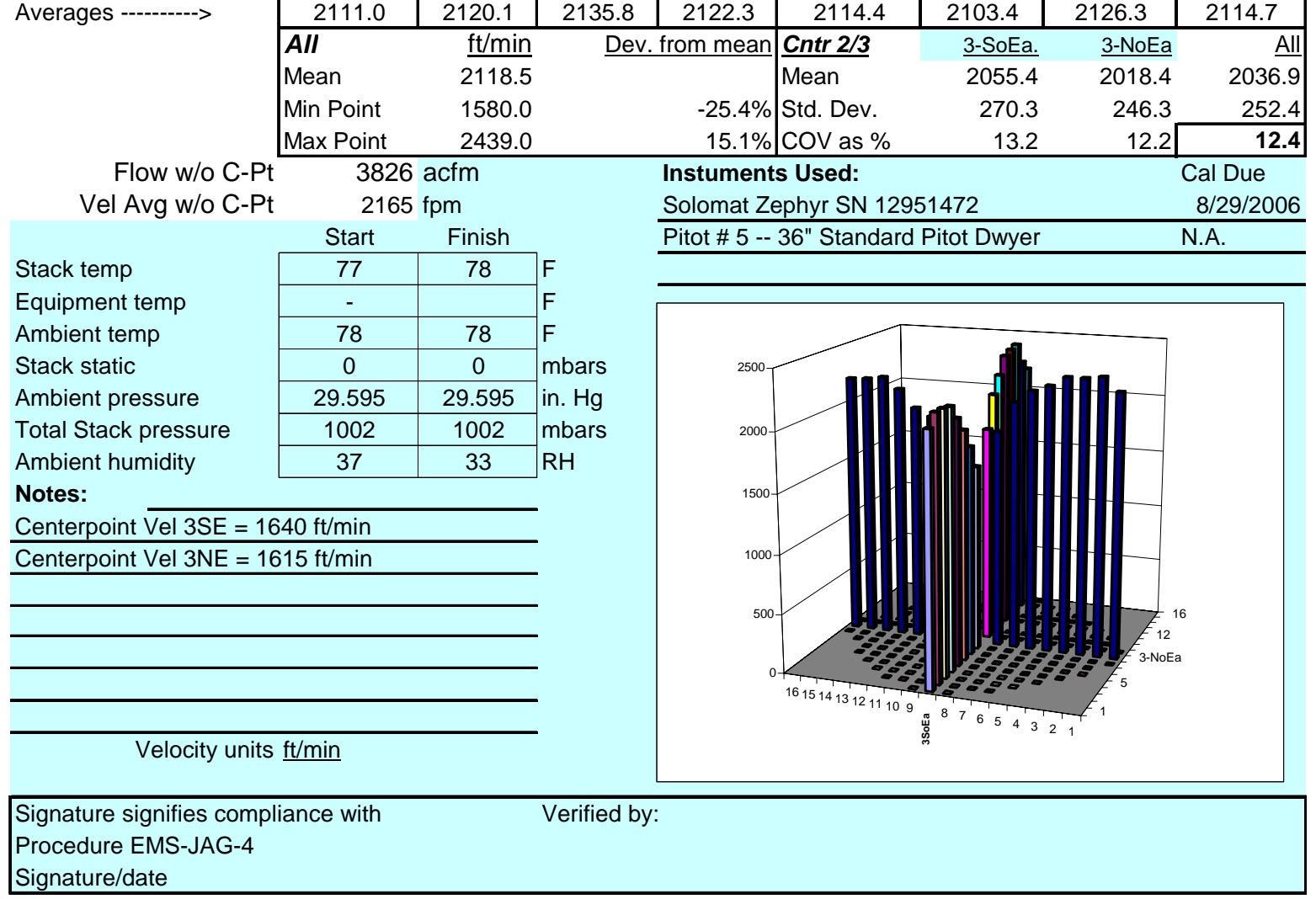


VELOCITY TRAVERSE DATA FORM

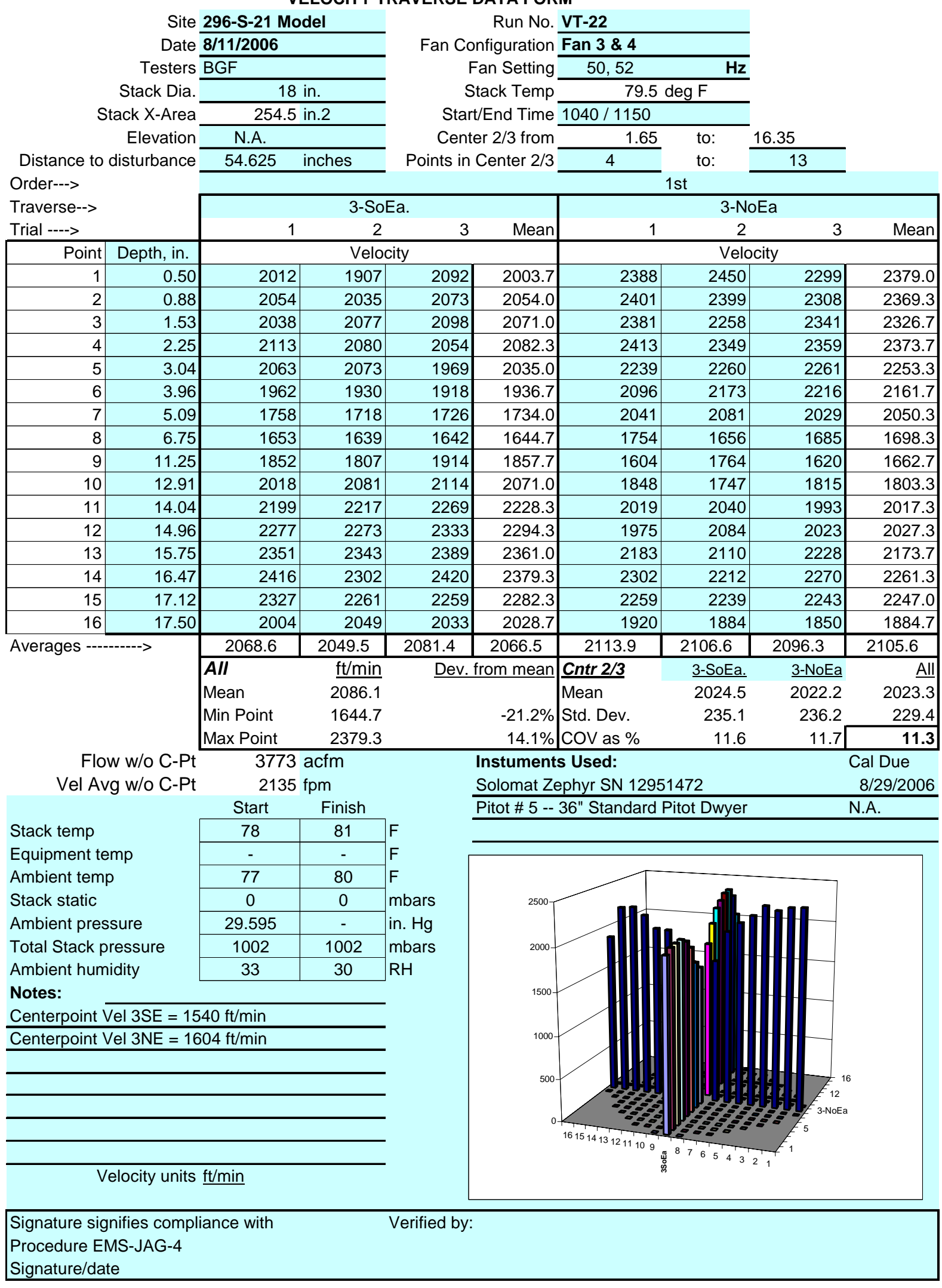




\section{Appendix E}

Flow Angle Procedure 
APPENDIX E: FLOW ANGLE PROCEDURE

\begin{tabular}{|c|c|c|}
\hline \multicolumn{3}{|l|}{ PNNL Operating Procedure } \\
\hline $\begin{array}{l}\text { Title: Test to Determine } \\
\text { Flow Angle }\end{array}$ & $\begin{array}{l}\text { Org. Code: } \\
\text { Procedure No.: } \\
\text { Rev. No.: }\end{array}$ & $\begin{array}{l}\text { D7E74 } \\
\text { EMS-JAG-05 } \\
\qquad 1\end{array}$ \\
\hline Work Location: General & Effective Date: & April 28, 2006 \\
\hline Author: John A. Glissmeyer & Supersedes Date: & November 24, 1998 \\
\hline $\begin{array}{l}\text { Identified Hazards: } \\
\square \text { Radiological } \\
\square \text { Hazardous Materials } \\
\text { 冈 Physical Hazards } \\
\square \text { Hazardous Environment } \\
\square \text { Other: }\end{array}$ & \multicolumn{2}{|c|}{$\begin{array}{l}\text { Identified Use Category: } \\
\square \text { Mandatory Use } \\
\square \text { Continuous Use } \\
\text { 冈 Reference Use } \\
\square \text { Information Use }\end{array}$} \\
\hline
\end{tabular}

\begin{tabular}{|c|c|c|}
\hline \multicolumn{2}{|c|}{ Are One-Time Modifications Allowed? $\square$ Yes Signature } & Date \\
\hline Person Signing & & \\
\hline $\begin{array}{c}\text { Technical review: } \\
\text { J. Matthew Barnett }\end{array}$ & & \\
\hline $\begin{array}{c}\text { Project Manager: } \\
\text { John Glissmeyer }\end{array}$ & & \\
\hline $\begin{array}{c}\text { Line Manager: } \\
\text { James Droppo }\end{array}$ & \\
\hline $\begin{array}{c}\text { Quality Engineer: } \\
\text { Barry L. Sachs }\end{array}$ & \\
\hline
\end{tabular}




\subsection{Purpose}

The performance of new stack sampling systems must be shown to satisfy the requirements of 40 CFR 61, Subpart H, "National Emission Standards for Emissions of Radionuclides Other than Radon from Department of Energy Facilities." This regulation governs portions of the design and implementation of effluent air sampling. The stack sampler performance is adequately characterized when potential contaminants in the effluent are of a uniform concentration at the sampling plane and line losses are within acceptable limits. (The sampling plane is the cross section of the stack or duct where the sampling nozzle inlet is located.) Uniformity of contaminant concentration is highly unlikely where the mean angle of the gas velocity throughout the cross section of the stack or duct is significantly non-zero. This condition would also mean that the air velocity approaches the sampling nozzle at an unacceptable angle, degrading the performance of the nozzle. This procedure provides the means to determine the mean flow angle, and is performed prior to measurements of contaminant uniformity. This procedure is performed after the range of gas flow conditions is established. Other associated procedures generally follow and address uniformity of flow and of gas and aerosol contaminants.

\subsection{Applicability}

This procedure can be used in the field or on modeled stacks and ducts to determine the angle of the air velocity relative to the axis of the duct or stack. The angle measured is the roll angle. This should be determined at the sampling plane. The tests are applicable within the following constraints:

- The operating limits of the air velocity measurement device used are observed.

- The air velocity sensor element does not occupy more than a few percent of the crosssectional area in the plane of the element.

This procedure may need to be repeated if there are changes made in the configuration of the ventilation system during the conduct of the remaining tests in the Test Plan. If the system under test operates within a limited range of airflow that does not change more than $\pm 25 \%$, this procedure is usually conducted once at the middle of the range. If the flow varies more, the procedure is performed at least at the extremes of flow.

\subsection{Prerequisites and Conditions}

Conditions and concerns that must be satisfied prior to performing this procedure are listed below:

- The job-hazards analysis for the work area must be followed.

- Safety glasses, hard toed or substantial shoes may be required in the work areas.

- Special training may be required to access the test ports.

- Means must be provided to achieve the airflow parameters in the Test Instruction.

- Air velocity measurement equipment must be within calibration. 
- The Test Instruction must be read and understood.

- This procedure must be read and understood.

\subsection{Precautions and Limitations}

Access to the test ports may require the use of ladders, scaffolding or manlifts, which may necessitate special training for sampling personnel and any observers. The training requirements will be indicated in the job hazard analysis.

\subsection{Equipment Used for Measurements}

The following are essential items of equipment:

- A Type-S pitot tube with sufficient length to reach across the diameter of the test stack,

- Slant tube or calibrated electronic manometer to indicate when the differential pressure reading of the pitot tube is about zero,

- Device for measuring the pitot tube angle at traverse points (e.g., a commercial protractor level with good angle resolution). (Note: A three dimensional velocity probe capable of measuring both pitch and yaw angles of gas flow is also acceptable provided that modifications in the method outlined below are made),

- Tape or template to mark insertion depths on the pitot tube,

- Velocity sensor to check the stack airflow,

- Platform, ladders, or manlifts as needed to support equipment and to access the test ports,

- Fittings to limit leakage around the pitot tube and to stabilize the tube so that it can be positioned repeatedly in the test stack at the same location.

- To provide information about the test conditions, commercial grade sensors for stack temperature, barometric pressure, static pressure, and humidity provide acceptable information. Likewise, data from a nearby meteorology or facility station is acceptable.

Further details on specific equipment for the job are provided in the Test Instruction. The test method is based on 40 CFR 60, Appendix A, Method 1, Section 2.4, "Verification of the Absence of Cyclonic Flow." The measurement instrumentation may be either the type used in Method 1, or another measurement device designed for measuring the angle of the velocity vector at discrete points. The user should be aware that different devices may give different readings.

\subsection{Work Instructions for Setup, Measurements, and Calculations}

Job specific instructions given in the Test Instruction, illustrated in Exhibit A, will provide details and operating parameters necessary to perform this procedure. Prior to determination of flow angles, measurements should be made to assess whether the stack velocity flow is within normal limits. 


\subsection{Preliminary Steps:}

6.1.1 Verify that the interior dimensions of the stack or duct at the measurement locations agree with those used in calculating the grid of measurement points given in the Test Instruction or data form.

Note. The grid of measurement points is calculated in accordance with 40 CFR 60, Appendix A, Method 1. A centerpoint is included as a common reference and for graphical purposes. The layout design divides the area of the sampling plane so that each point represents approximately an equal-sized area.

6.1.2 Provide essential supplies at the sampling location. (S-Type pitot tube, manometer, tubing, fittings to adapt the sensor to the test ports, marking pens, data forms, writing and sensor supporting platforms).

6.1.3 Verify that the ventilation flow control device is capable of the flow control settings given in the Test Instruction.

6.1.4 Prepare a data form for the measurement traverse. See illustration in Exhibit B. Label the columns of traverse data by the direction of the traverse. For example, if the first reading is closest to the east port, and the last reading is closest to the west port, then label the traverse "eastwest." Print blank copies of the data form.

6.1.5 Record on the data form the test setup parameters characterizing the airflow, configuration and conditions as applicable according to the Test Instruction.

6.1.6 Mark the Type-S pitot tube to indicate the insertion depth for each point in the measurement grid.

6.1.7 Set the stack flow control per the Test Instruction. (Use a velocity or flow sensor to verify that target flow condition has been achieved within \pm $10 \%$.)

Note. Flow verification can be based on a single point velocity reading. The single point can be the same one determined in the stack flow controller calibration in Procedure EMS-JAG-03.

6.1.8 Insert the Type-S pitot tube in the stack or duct, seal the opening around it, and check for smooth operation of the pitot tube.

Note. Good measurements are dependent upon making small repeatable rotations of the pitot tube in the available fittings. 
6.1.9 Establish a convention for representing the angular direction of flow.

Note. If an inclined manometer is used, connect the flexible tubes between the connectors on the pitot tube and the manometer so that rotating the pitot tube assembly clockwise drives the meniscus to the right, i.e., to higher positive numbers.

Attach a circular protractor to the pitot tube near the tubing connectors. Generally the protractor hangs below the pitot tubes. When the parallel tubes are in horizontal position, the protractor should indicate zero degrees. If the tubing assembly is rotated clockwise, the resulting counter-clockwise movement of the angle indicator produces an angle that is read as a positive number. This is consistent with the convention for reading circular angles.

6.1.10 Position the inclined manometer on a stable platform and level the manometer.

Note: Movement on the test platform may affect the manometer level. It should be checked frequently. Adjustments can be made at any time when the pitot tube is moved to the next position, but not during readings at any single point.

6.1.11 Connect the flexible tubes to the inclined manometer but disconnect them from the pitot tube.

6.1.12 Increase or decrease the red oil level in the inclined portion of the manometer to zero the meniscus. (This is done using a finger-adjustable screw at the base of the manometer.)

6.1.13 Reconnect the flexible tubes to the pitot tube.

\subsection{Angular Flow Measurements}

6.2.1 Verify that the directional orientations and the numbered measurement positions are consistent with the data form.

6.2.2 Measure and record, on the data form, the angular reading at each measurement point in succession. If the readout device has an averaging feature, record the average of a series of several readings.

Note: The readings may be erratic for some flow conditions and at some traverse positions. Care should be taken to approach these variable readings from both higher and lower angles to obtain the most accurate equilibrium reading. 
6.2.3 Repeat Step 6.2.2 two more times for a total of three measurements at each grid point.

6.2.4 Review the dataforms for completeness.

6.2.5 Sign and date the data forms attesting to having completed the data collection portion of the procedure.

\subsection{Calculations}

6.3.1 Perform the following calculations using a spreadsheet or hand calculations

6.3.2 Calculate the absolute average air-flow angle for each measurement point.

6.3.3 Calculate the average absolute flow angle for all measurement points.

6.3.4 Have the data transfers and calculations reviewed and verified

Note: The acceptance criterion is that the average flow angle not exceed 20 degrees.

\subsection{Records}

7.1 Transfer the original data forms and calculations to the records custodian as project records 


\section{Exhibits/Attachments}

\section{Exhibit A - Typical Test Instruction}

\begin{tabular}{|c|c|c|}
\hline \multicolumn{3}{|c|}{ Test Instruction } \\
\hline Project: Model Stack & Date: December 25, 2007 & Work Package: K83017 \\
\hline \multicolumn{3}{|c|}{ Tests: Flow Angle at High Flow in the Model Stack } \\
\hline \multicolumn{3}{|l|}{ Staff: David Maughan } \\
\hline \multicolumn{3}{|c|}{$\begin{array}{l}\text { Reference Procedures: } \\
\text { 1. Operating Manual for Solomat Zephyr } \\
\text { 2. Test to Determine Flow Angle at the Elevation of a Sampler Probe, Procedure EMS-JAG- } \\
05\end{array}$} \\
\hline \multicolumn{3}{|c|}{$\begin{array}{l}\text { Equipment: } \\
\text { 1. Full-Scale Model Stack, Fan and Fan Speed Controller located in } 305 \text { Bldg. } \\
\text { 2. S-type Pitot Tube, slant tube or electronic manometer, and Protractor Level }\end{array}$} \\
\hline \multicolumn{3}{|c|}{$\begin{array}{l}\text { Safety Considerations: } \\
\text { Review and observe the applicable Job Hazard Analysis for the project }\end{array}$} \\
\hline \multicolumn{3}{|c|}{$\begin{array}{l}\text { 1. Assemble the equipment for the flow angle test at the ports at the elevation of the } \\
\text { sampling probe. } \\
\text { 2. Layout the measurement points with the following distances from the inside of the stack } \\
\text { wall: } 0.5,0.66,1.23,2.04,3.16,4.28,5.10,5.66,5.83 \text { inches. } \\
\text { 3. Measure the flow angle at each point at the high }(400 \mathrm{cfm}) \text { extreme of stack flow. Repeat } \\
\text { each measurement twice. } \\
\text { 4. Record the data on flow angle data forms. } \\
\text { 5. Diagram mounting fixtures and retain assembly for subsequent tests }\end{array}$} \\
\hline \multicolumn{3}{|c|}{ Desired Completion Date: 12/25/07 } \\
\hline \multicolumn{3}{|l|}{ Approvals: } \\
\hline Test completed by: & & \\
\hline
\end{tabular}


Exhibit B - Typical Flow Angle Data Form

\begin{tabular}{|c|c|c|}
\hline \multicolumn{3}{|c|}{ Site Model } \\
\hline Date & $10 / 19 / 2002$ & \\
\hline \multicolumn{3}{|c|}{ Tester Maughan } \\
\hline Stack Dia. & 23.5 & in \\
\hline Stack X-Area & 433.7 & in2 \\
\hline Elevation & N.A. & $\mathrm{ft}$ \\
\hline Distance to disturbance & 75 & in \\
\hline
\end{tabular}

Run No. FA-1

Fan Setting $50 \mathrm{~Hz}$

Fan configuration 4-fan: EF1, EF4, EF5, EF7, 15 deg port

Approx. stack flow $\sim 5860 \quad \mathrm{cfm}$

Units degrees (clockwise > pos. nos.)

Baro Press $992.4 \mathrm{mb}$

Rel Hum $57 \% \mathrm{RH}$

Stack Temp $55 \mathrm{~F}$

\begin{tabular}{|c|c|c|c|c|c|c|c|c|c|}
\hline \multirow{2}{*}{\multicolumn{2}{|c|}{$\begin{array}{l}\text { Traverse--> } \\
\text { Trial ----> }\end{array}$}} & \multicolumn{4}{|c|}{ West } & \multicolumn{4}{|c|}{ North } \\
\hline & & \multirow{2}{*}{$\frac{1}{\text { deg. } \mathrm{cw}}$} & \multirow{2}{*}{$\frac{2}{\text { deq }}$} & \multicolumn{2}{|l|}{3} & \multirow{2}{*}{$\frac{1}{\text { deg. cw }}$} & 2 & \multicolumn{2}{|l|}{3} \\
\hline Point & Depth, in. & & & deg. CW & Avg. & & deg. $\mathrm{cw}$ & deg. CW & Avg. \\
\hline 1 & 0.75 & 7 & 4 & 6 & 5.7 & 2 & 2 & 4 & 2.7 \\
\hline 2 & 2.47 & 4 & 5 & 4 & 4.3 & -1 & 1 & 0 & 0.0 \\
\hline 3 & 4.56 & -2 & 0 & 1 & -0.3 & -2 & -1 & -2 & -1.7 \\
\hline 4 & 7.59 & -2 & -2 & -3 & -2.3 & -3 & -4 & -3 & -3.3 \\
\hline Center & 11.75 & -1 & 0 & -1 & -0.7 & 0 & -1 & -1 & -0.7 \\
\hline 5 & 15.91 & 0 & -1 & -1 & -0.7 & -1 & -1 & -1 & -1.0 \\
\hline 6 & 18.94 & -1 & -1 & -2 & -1.3 & -3 & -2 & -2 & -2.3 \\
\hline 7 & 21.03 & -2 & -3 & -2 & -2.3 & -3 & -2 & -1 & -2.0 \\
\hline 8 & 22.75 & -2 & -2 & -2 & -2.0 & -2 & -1 & -1 & -1.3 \\
\hline \multirow{2}{*}{\multicolumn{2}{|c|}{$\begin{array}{l}\text { Mean of absolute values } \\
\text { w/o points by wall: }\end{array}$}} & 2.3 & 2.0 & \multirow{2}{*}{\multicolumn{2}{|c|}{$\begin{array}{l}2.4 \\
2.0\end{array}$}} & 1.9 & 1.7 & \multicolumn{2}{|l|}{1.7} \\
\hline & & 1.7 & 1.7 & & & 1.9 & 1.7 & 1.4 & \\
\hline \multicolumn{8}{|c|}{ Instuments Used: } & \multicolumn{2}{|l|}{$\begin{array}{r}\text { al } \\
\text { w/o wall pts }\end{array}$} \\
\hline
\end{tabular}

Instuments Used:

S-type pitot

Stanley protractor level

Manometer

\begin{tabular}{lll} 
Pitot-2 & 36 inch & $\begin{array}{l}\text { Cal. Due } \\
\text { Cert. of compliance }\end{array}$ \\
\hline Prot-1 & N.A. \\
\hline Man-1 & S/N 14591 & \multicolumn{1}{c}{ 4/1/2007 } \\
\hline & Notes:
\end{tabular}

Note:

To assure similar hose connections

between the manometer and pitot tube, rotating

the pitot tube assembly clockwise drives the

meniscus to the right (to higher pos. numbers).
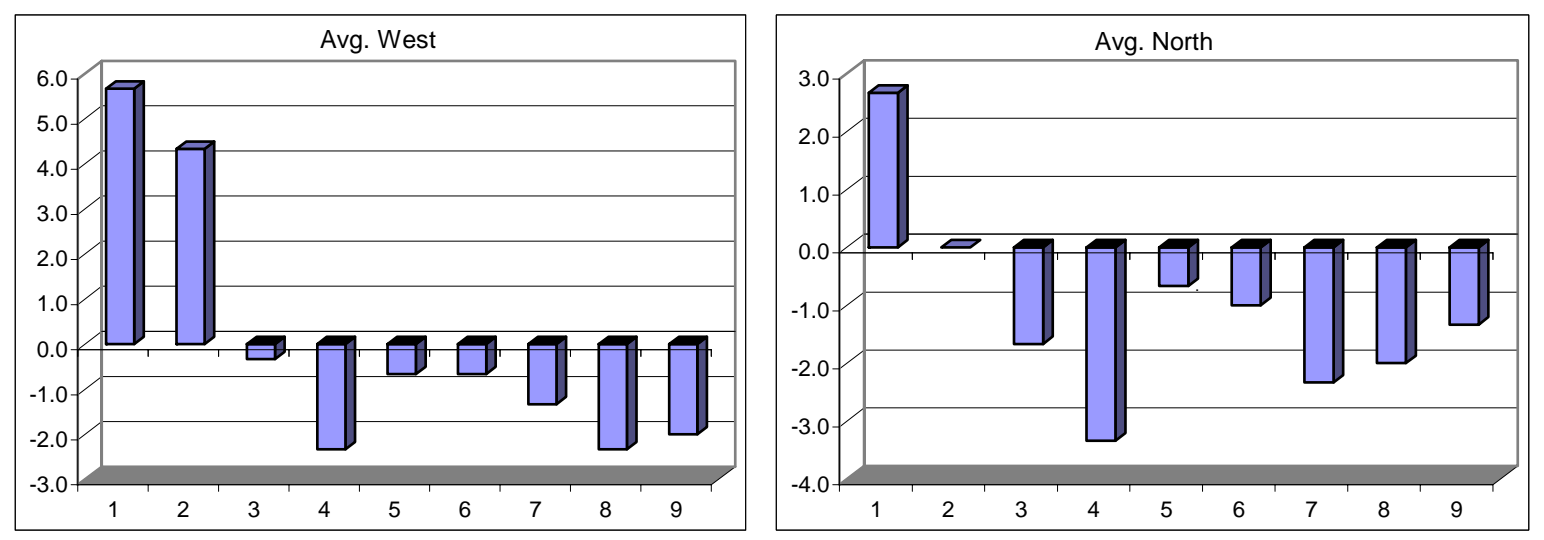

Signature signifies compliance with

Signature verifying data and calculations:

Procedure EMS-JAG-05

Signature/date 


\section{Appendix F}

\section{Flow Angle Data Sheets}




\section{APPENDIX F: FLOW ANGLE DATA SHEETS}

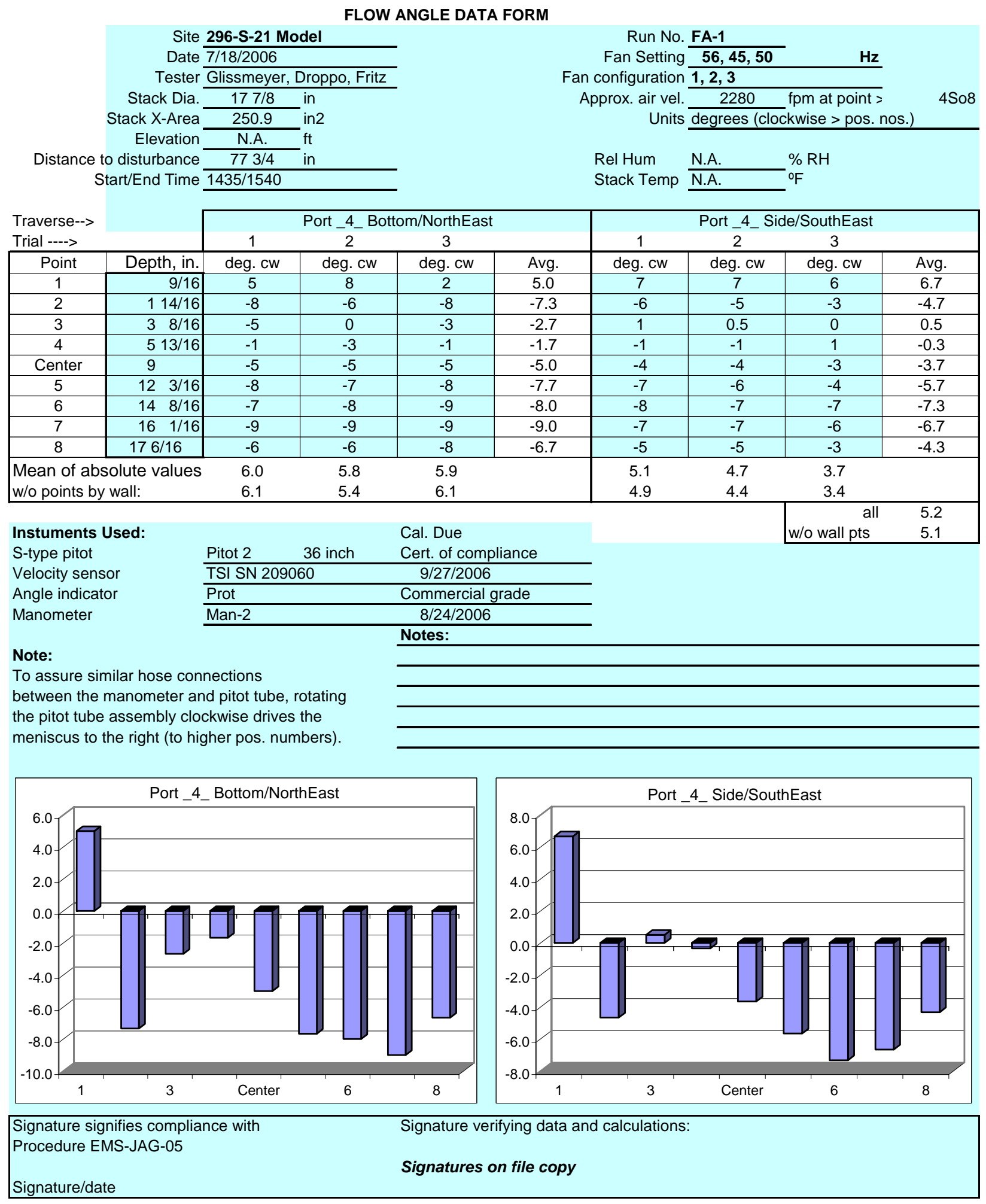


FLOW ANGLE DATA FORM

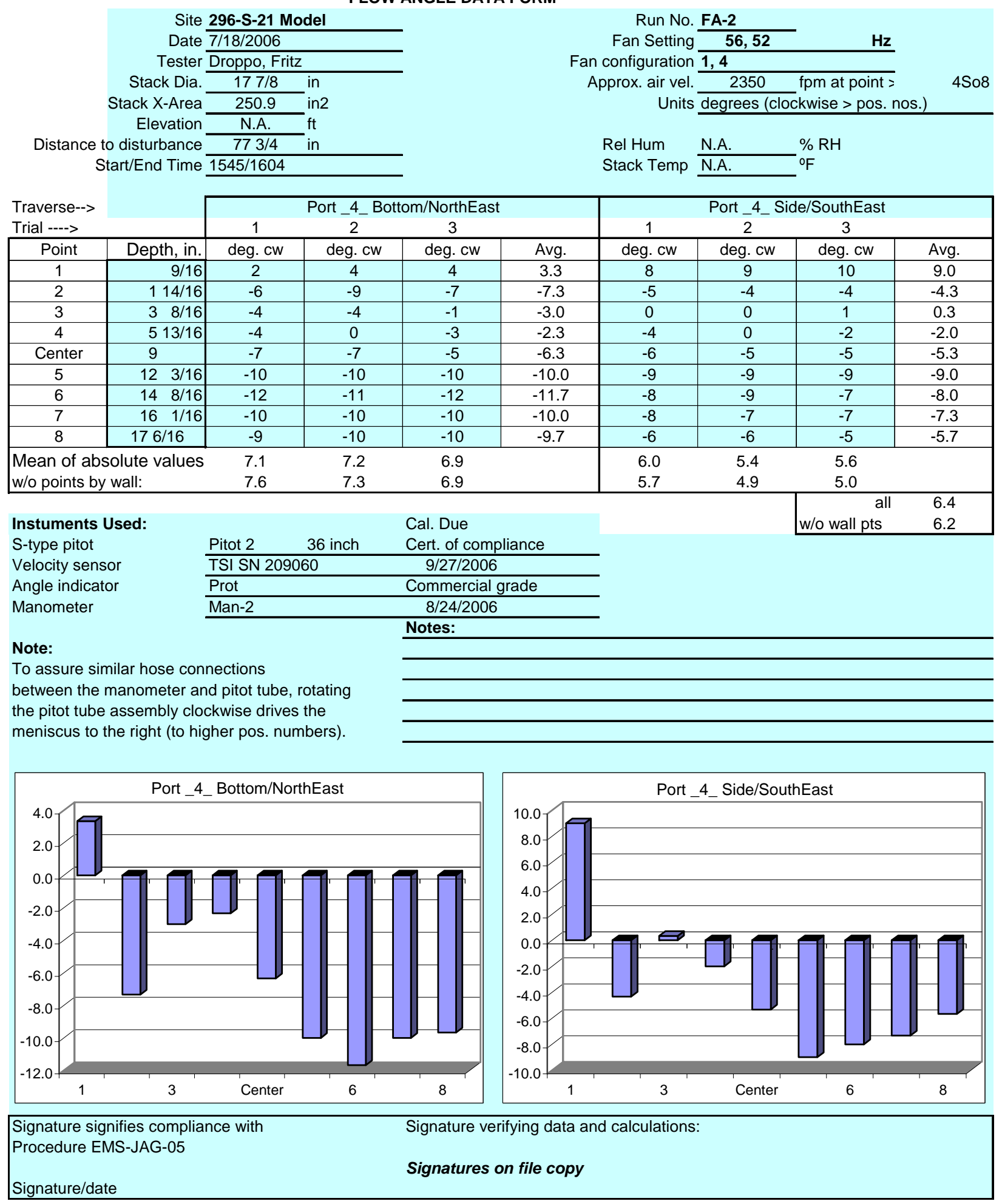


FLOW ANGLE DATA FORM

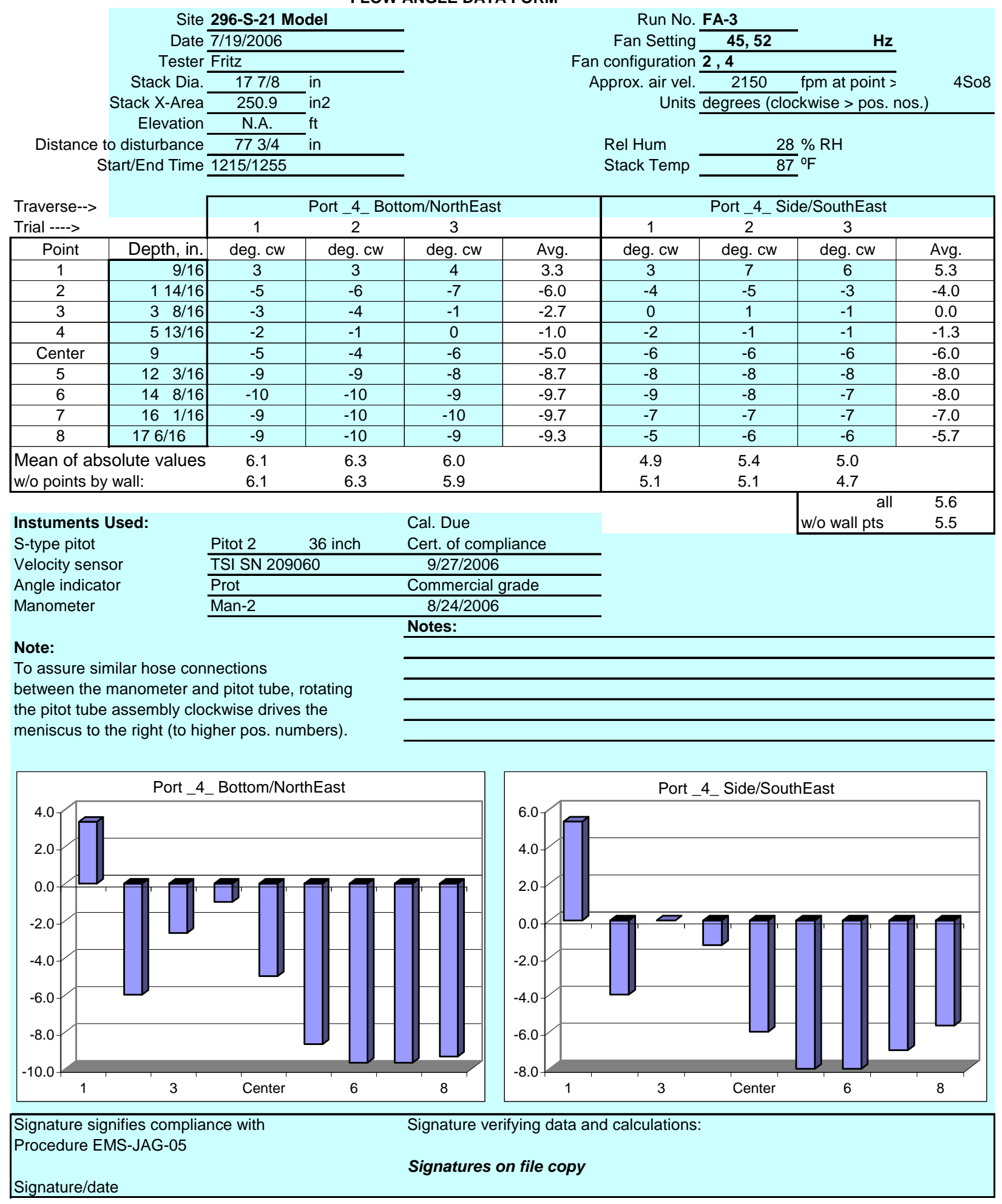


FLOW ANGLE DATA FORM

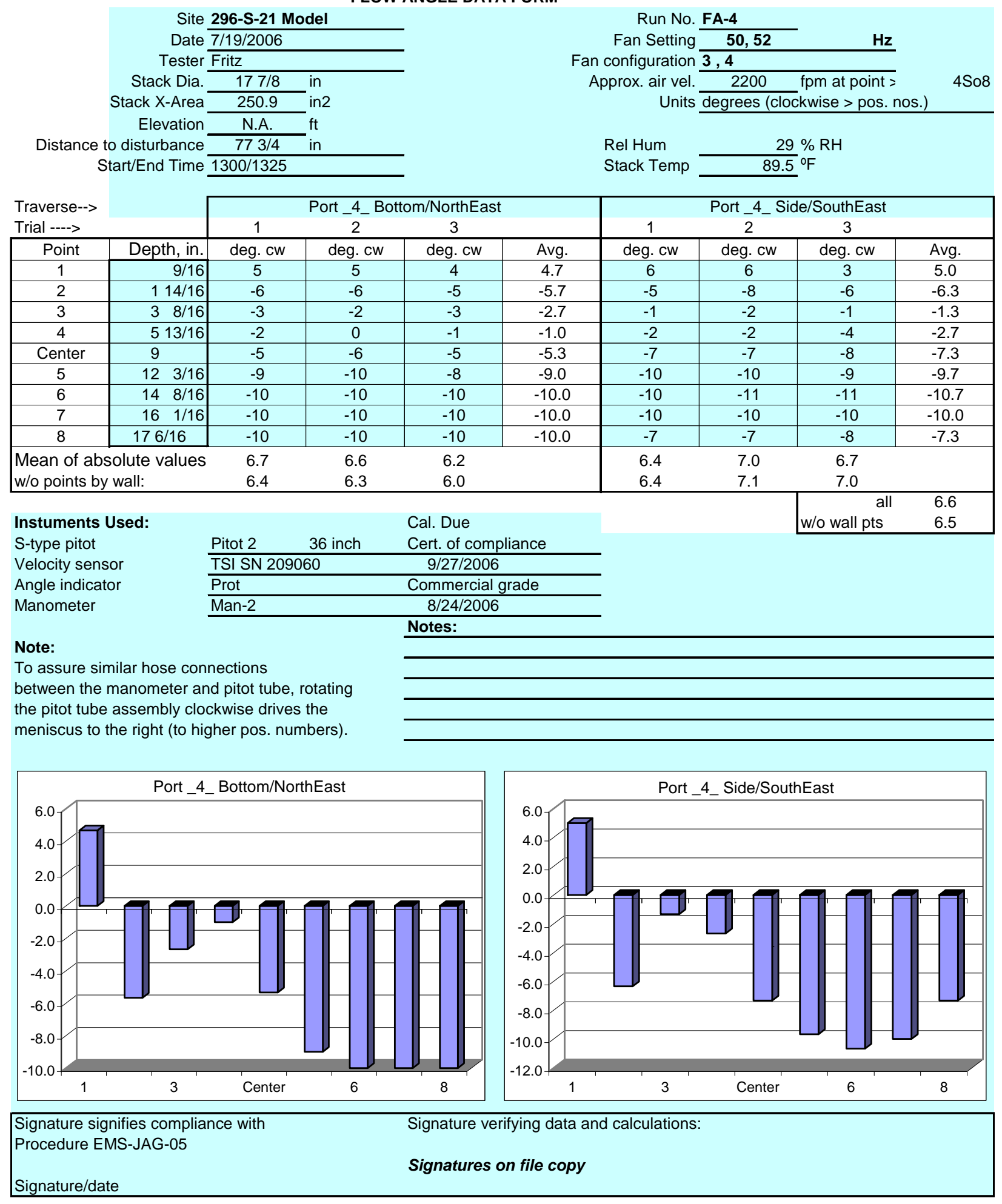




\section{FLOW ANGLE DATA FORM}

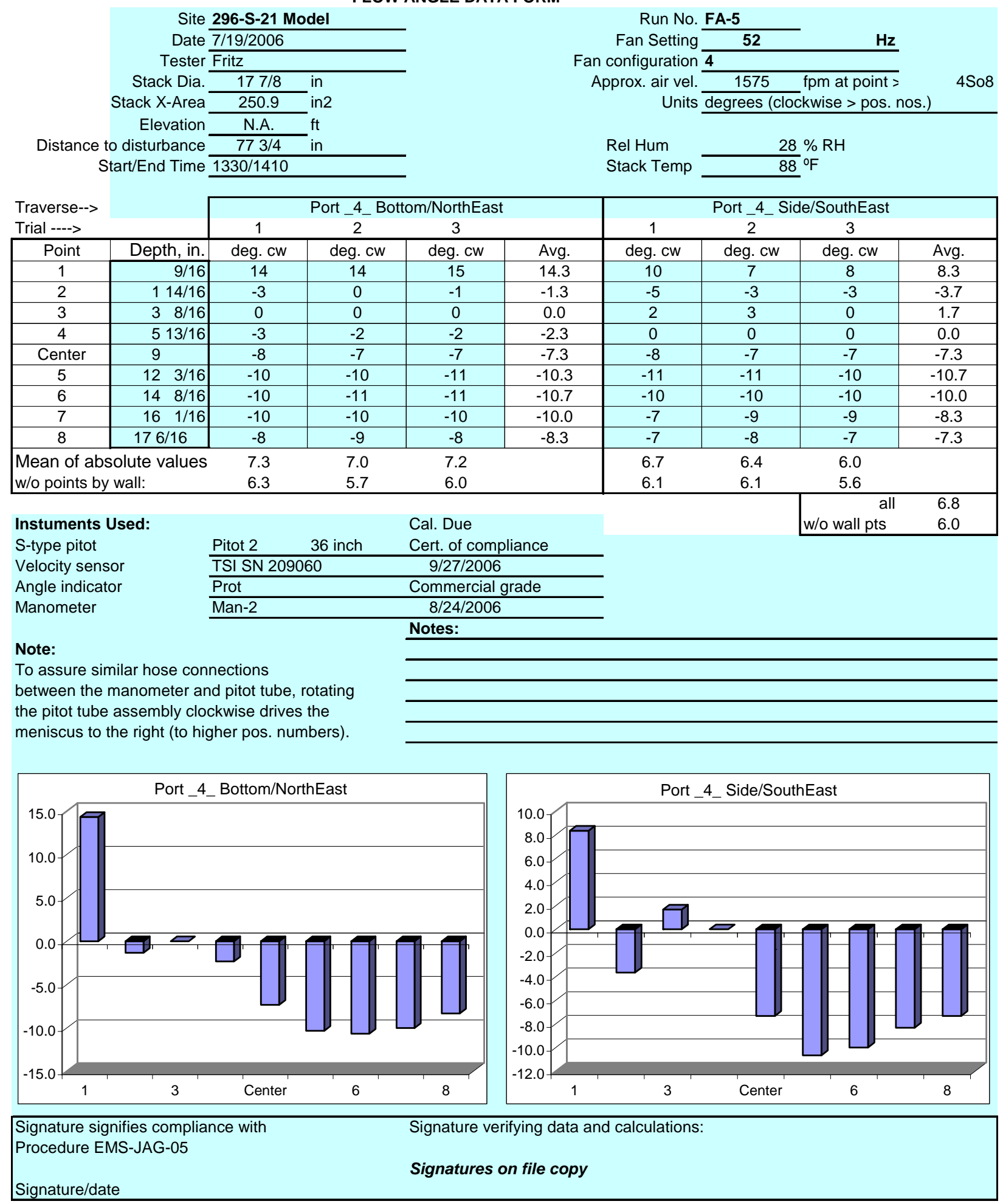




\section{FLOW ANGLE DATA FORM}

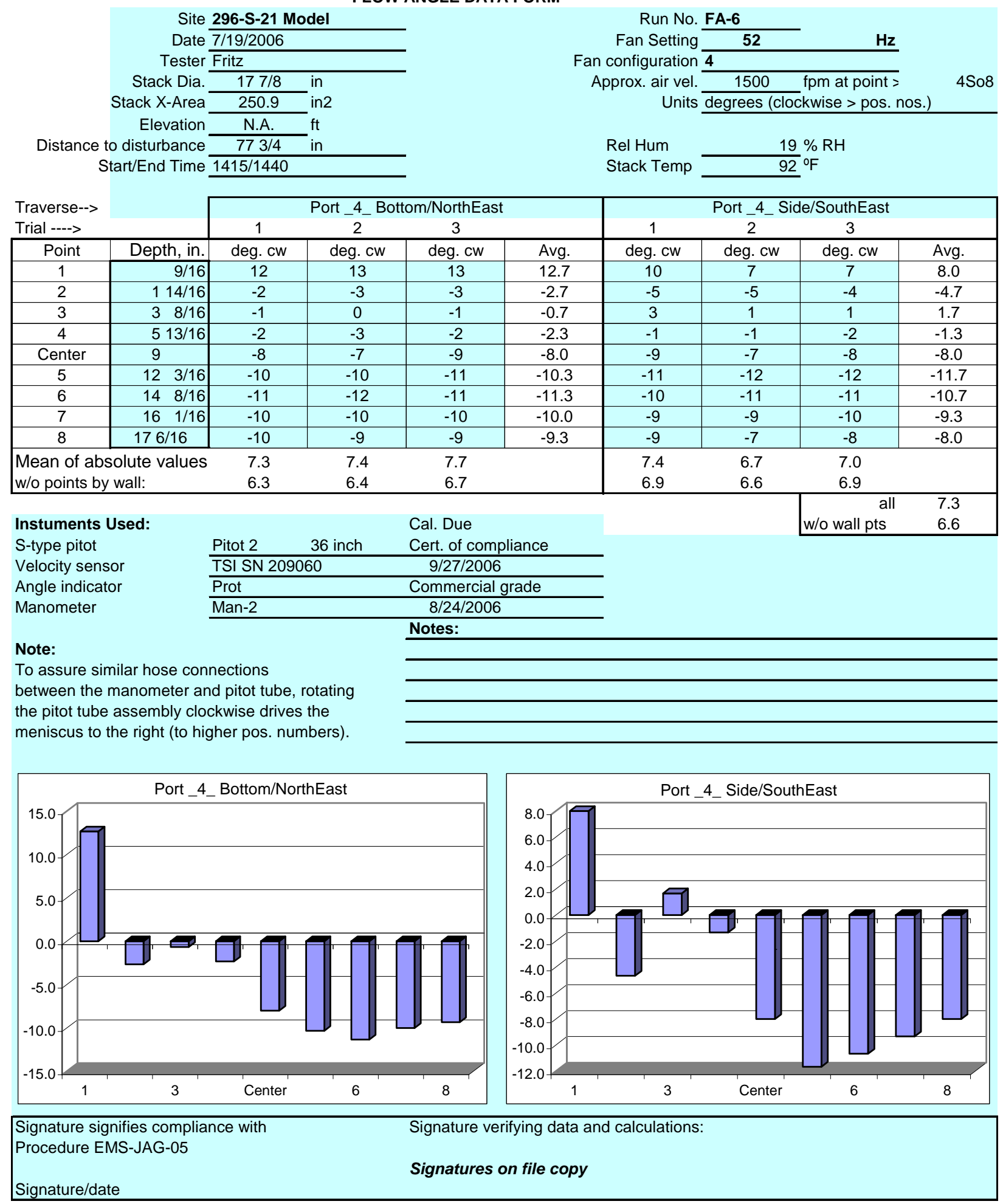




\section{FLOW ANGLE DATA FORM}

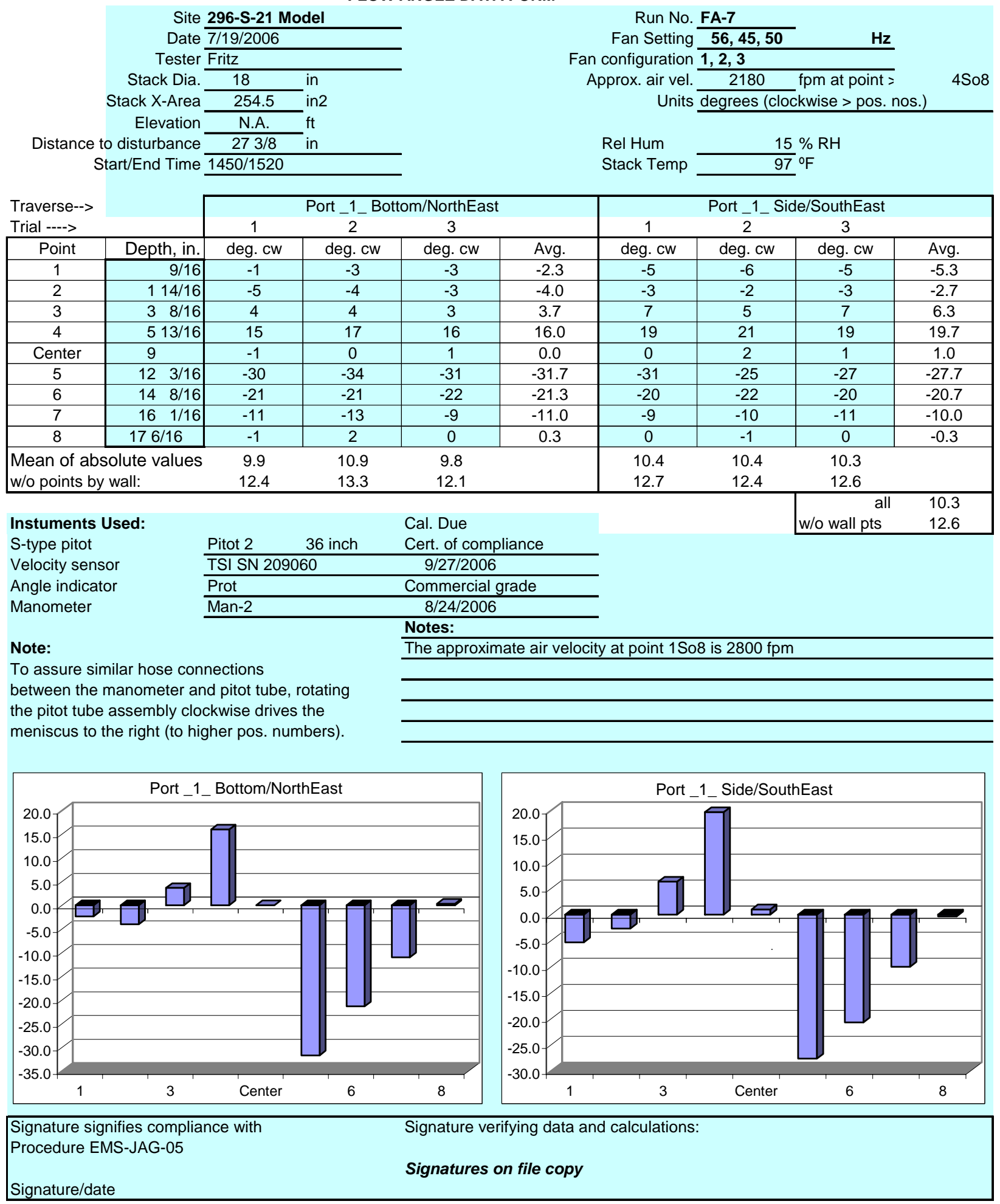




\section{FLOW ANGLE DATA FORM}

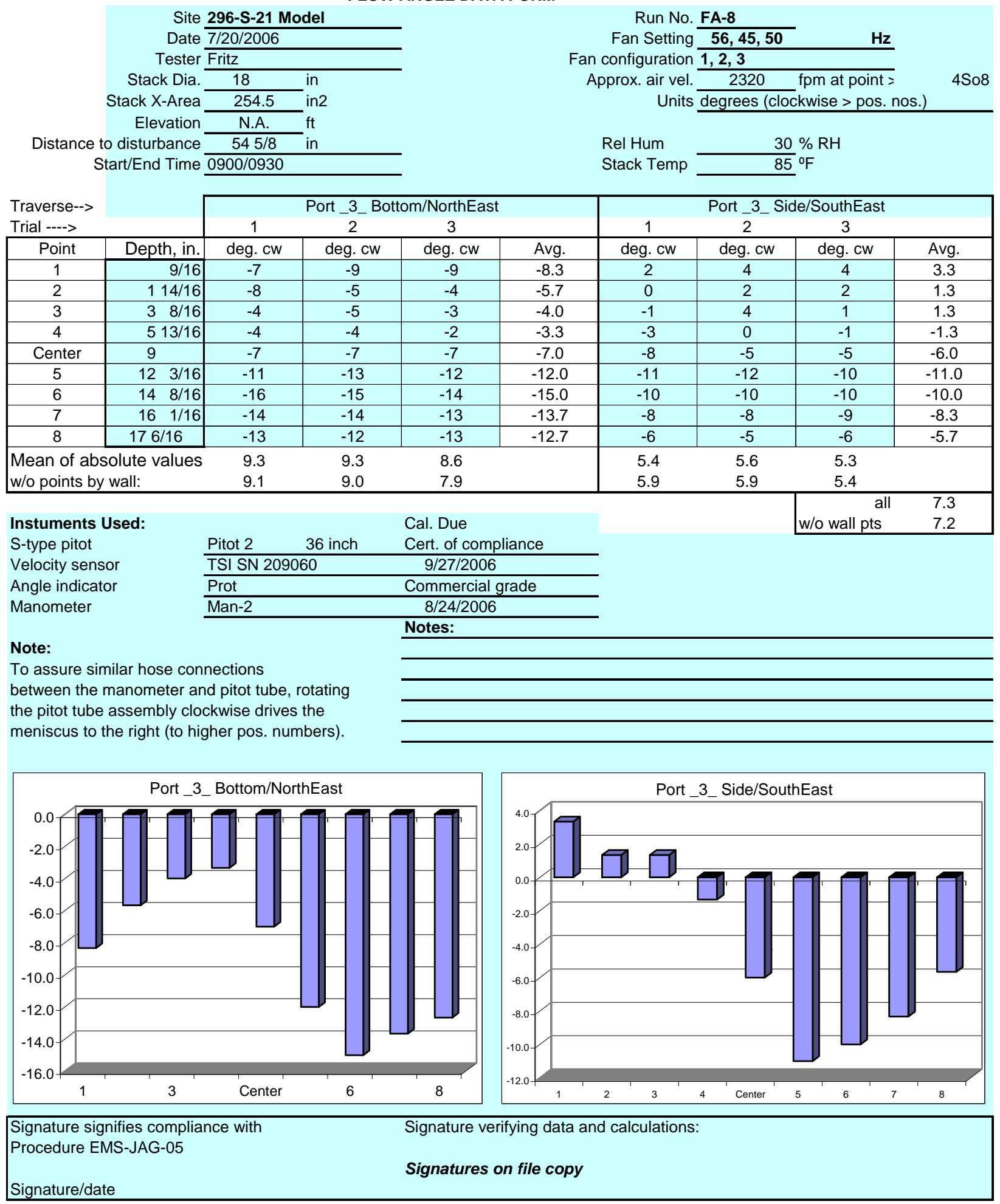




\section{FLOW ANGLE DATA FORM}

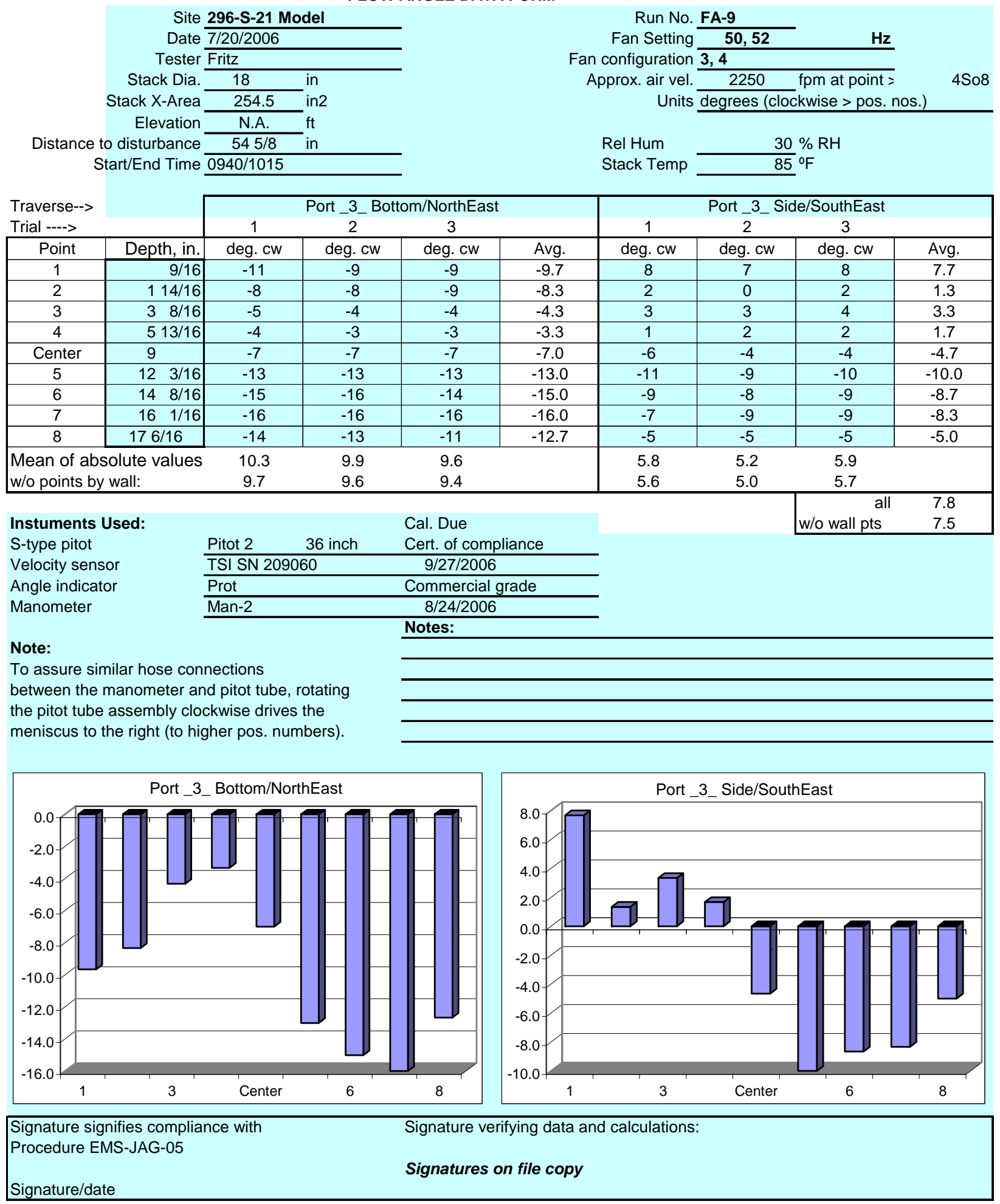




\section{Appendix G}

\section{Tracer Gas Uniformity Procedure}


APPENDIX G: TRACER GAS UNIFORMITY PROCEDURE

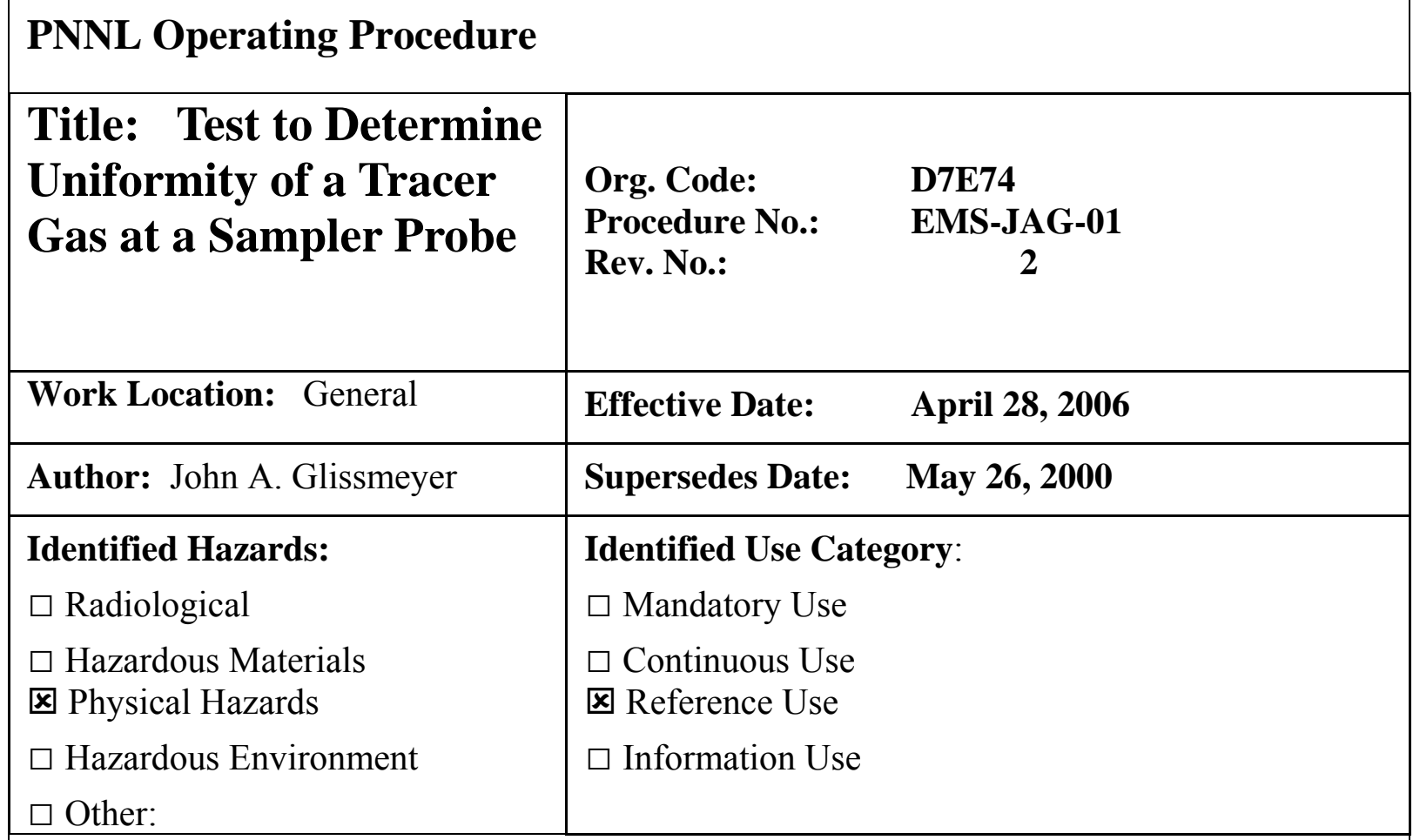

\begin{tabular}{|c|c|c|}
\hline \multicolumn{2}{|c|}{ Are One-Time Modifications Allowed? $\mathbf{y e s}$ 囚No } & Date \\
\hline Person Signing & & \\
\hline $\begin{array}{c}\text { Technical review: } \\
\text { J. Matthew Barnett }\end{array}$ & \\
\hline $\begin{array}{c}\text { Project Manager: } \\
\text { John Glissmeyer }\end{array}$ & & \\
\hline $\begin{array}{c}\text { Line Manager: } \\
\text { James Droppo }\end{array}$ & & \\
\hline $\begin{array}{c}\text { Concurrence: } \\
\text { Quality Engineer: } \\
\text { Barry L. Sachs }\end{array}$ & \\
\hline
\end{tabular}




\subsection{Purpose}

The performance of new stack sampling systems must be shown to satisfy the requirements of 40 CFR 61, Subpart H, "National Emission Standards for Emissions of Radionuclides Other than Radon from Department of Energy Facilities." This regulation governs portions of the design and implementation of effluent air sampling. The stack sampler performance is adequately characterized when potential contaminants in the effluent are of a uniform concentration at the sampling location (plane), and line losses are within acceptable limits. This procedure determines whether the concentration of gaseous contaminants is uniformly distributed in the area of the sampling probe. Other procedures address flow angle, uniformity of gas velocity, and uniformity of particulate contaminants. A contaminant concentration that is uniform at the sampling plane enables the extraction of samples that represent the true emission concentration.

The uniformity is expressed as the variability of the measurements about the mean. This is expressed using the relative coefficient of variance (COV), which is the standard deviation divided by the mean and expressed as a percentage. The lower the COV value, the more uniform the gas concentration. The acceptance criterion is that the COV of the measured gas concentrations be $\bigcirc 20 \%$ across the center two-thirds of the area of the stack. Furthermore, the average concentration measured at any point cannot differ from the mean of all points by more than $30 \%$.

\subsection{Applicability}

This procedure can be used in the field or on scale model stacks to determine whether air-sampling probes can collect representative samples under normal operations. The test procedure is applicable to effluent stacks or ducts within the following constraints:

- The tracer gas tests are generally limited to stacks with flowrates greater than 50 cubic feet per minute range. The upper bound of flowrate is determined by the sensitivity of the gas analyzer, the background reading for the tracer gas, and the availability of the tracer.

- Environmental constraints - the operating temperature range of the gas analyzer must be observed (minimum of $55^{\circ} \mathrm{F}$ ).

\subsection{Prerequisites and Conditions}

Conditions and concerns that must be satisfied before obtaining measurements are listed below:

- Safety glasses, hard toed or substantial shoes may be required in the work areas.

- Properly constructed and inspected work platforms may be needed to access the test ports.

- Special training may be required in some instances to access the sampling ports of the stack. 
- Alcohol (methanol, ethanol, or rubbing) may be used to erase grid point markings on the sampling probes. A flammable equipment storage cabinet is required for flammable chemicals.

- Familiarity with the use and operation of compressed gas delivery systems and the ability to detect concentration build-ups of the gas is essential to avoid exceeding ACGIH concentration for the tracer gas.

- Knowledge of the setup, use of, and operation of flowmeters, gas analyzers, and computers is essential.

- A job-hazards analysis may be required in certain cases.

- Read and understand this procedure (documented required reading)

\subsection{Precautions and Limitations}

Caution: The American Conference of Governmental Industrial Hygienists (ACGIH) 8-hour time-weighted average limit for human exposure to sulfur hexafluoride gas is 1000 ppm $\left(6,000 \mathrm{mg} / \mathrm{m}^{3}\right)$. It is colorless and odorless.

During the tests, sulfur hexafluoride will be injected into the base of the stack or duct at a rate to achieve a target concentration $<5 \mathrm{ppm}$ at the sampling ports. The gas is five times as heavy as air. If a leak occurs in the gas delivery system, the potential is present for a buildup of $\mathrm{SF}_{6}$ in confined spaces and in low areas. Leak tests of the delivery system will be made at least daily to prevent such an occurrence.

Access to the test ports may require the use of scaffolding or manlifts, either of which will necessitate special training for test personnel and observers. The training requirements will be indicated in the job hazard analysis. This will limit access to the sampling ports to trained personnel.

If the purpose of a given run is to investigate the sensitivity of the COV determination to the tracer-injection location, the test may be invalid if the ending ambient concentration is elevated above that at the start of the test. This would indicate poor dispersion away from the test site and recirculation of the tracer to the inlet of the fan if the stack exhaust point is in view of and is reasonably close to the fan inlet. This may result in a false indication of good mixing. The tracer recycle and buildup conditions are unlikely in outdoor tests.

\subsection{Equipment Used for Stack Measurements}

Specific calibration check standards, probe dimensions, measurement grids, flow rates, and other special requirements will be provided in the specific Test Instruction. Exhibit A shows a typical Test Instruction. Exhibits B and C provide a typical layout for the test setup. The following are essential items of equipment:

- Sulfur hexafluoride calibration standards

- Sulfur hexafluoride bulk gas

- Bruel and Kjaer Model 1302 Gas analyzer and associated users manual.

- Gas regulators and flowmeters 
- Gas sampling probe

- Gas injection probe

- Vacuum pump

- Air velocity meter

- Platform, ladders, or manlifts as needed to access the test ports;

- Fittings to limit leakage around the sampling probe and to stabilize it so it can be repositioned repeatedly.

- To provide information about the test conditions, commercial grade sensors for stack temperature, barometric pressure, static pressure, air velocity, and humidity provide acceptable information. Likewise, data from a nearby meteorology or facility station is acceptable.

The absolute calibration of the gas analyzer is not as important as its general response because the concentration data are used in a relative manner in calculating the COV and in plotting the concentrations at the measurement points. Consequently, the analyzer is Category 2 MTE (user calibrated) and will be checked against a calibrated gas mixture before and after the series of tests, and the instrument's response may be checked on a daily basis. Agreement within $10 \%$ of the calibration gas is acceptable.

\subsection{Work Instructions for Setup, Measurements, and Calculations}

The steps taken to setup, configure, and operate the stack fans and test equipment are listed. Based on previous field measurements, the steps are ordered to achieve maximum efficiency in the testing. In addition to these steps, test instructions, which are developed for each test series, provide specific details and operating parameters.

\subsection{Gas Analyzer Calibration Check}

Check the gas analyzer calibration by subjecting the analyzer to sulfur hexafluoride calibration gas. Refer to the analyzer's manual, parts 2 and 4 . Perform this check at least at the beginning and end of the series of test runs, and at least weekly during the test runs. It is also recommended to repeat these steps if the following conditions are observed during the uniformity test runs: concentration drift with time not accounted for by changing flowrates, or a test with results failing to meet the acceptance criteria.

\subsubsection{Prepare a data form such as that shown in Exhibit D.}

6.1.2 Record the ambient barometric pressure, temperature and humidity on the data from.

6.1.3 Record the cylinder number, sulfur hexafluoride concentration and expiration date for each standard being used. 
6.1.4 Program the analyzer's "units of measurement" as in its manual Part 4.2.3. Set the gas analyzer's clock.

6.1.5 Enter the barometric pressure in $\mathrm{mm} \mathrm{Hg}$ pressure, standard temperature (that used by the calibration gas vendor), and the sampling tube length into the analyzer's environmental setup menu (analyzer manual Part 4.2.4). Record the information on the data form.

6.1.6 Program for a continuous monitoring task, with water compensation, and initiate monitoring (manual sections $4.2 .5 \& 4.2 .6$ ).

6.1.7 Monitor the sulfur hexafluoride concentration at the test location, and record the measurements for at least 5 minutes on the data form.

Note: If the test location has a buildup of the gas, a zero air cylinder or clean air supply will be needed. The $\mathrm{SF}_{6}$ concentration in the room should be several orders of magnitude below that of the calibration-gas.

6.1.8 Repeat the previous step with water vapor compensation disabled.

6.1.9 Attach the regulator to the calibration gas with the lowest concentration. To the regulator discharge valve, attach flexible tubing, and a tee with one leg exhausting excess gas through a rotameter and the other leg attached to the inlet of the gas analyzer. The rotameter's valve must be wide open.

6.1.10 Start the flow of calibration gas with the valve at the regulator. The flowrate should be just high enough so the rotameter float is showing a non-zero flow with the analyzer sampling.

8

6.1.11 With the water compensation still disabled, sample the calibration gas for at least five readings or until no observable trend is found. Record the identification of the calibration gas used. Record the concentration readings on the data form.

6.1.12 Repeat Step 6.1.11 with water compensation enabled.

6.1.13 Repeat Steps 6.1.9 to 6.1.12 with the higher concentration of calibration gas. 
Note: Set the calibration gas flow rate just high enough to ensure that the ball in the rotameter does not drop to zero during any of the observed steps of a sample cycle. As the calibration check continues, calibration gas will be released into the room, and the $\mathrm{SF}_{6}$ background concentrations may increase as the analyzer is checked. The analyzer's $\mathrm{SF}_{6}$ reading should be within $10 \%$ of the calibration-gas concentration, and the water content should be much lower than ambient

Note: The readings will generally be recorded from the analyzer's digital display. Optionally, it may be convenient to record the data on a printer or computer, which can be coupled to the analyzer. See the analyzer's Manual Part 12 (especially Part 12.2.5) for connecting to a printer in data log mode.

Note: Gas analyzer readings can be made with or without water-vapor correction. If the air is sufficiently dry ( $<$ than about $60 \%$ relative humidity) where the water vapor contribution is negligible ( $<$ than about $14.5 \mathrm{E}+03$ $\mathrm{ppm}$ ), the readings can be made without water vapor compensation to reduce sample times. The preliminary measurements of Steps 6.1.7 and 6.1.8 will determine whether water compensation will be needed for the uniformity

\subsection{Preparing for Measurements:}

6.2.1 Prepare a spreadsheet for calculating results and plotting data similar to the illustration in Exhibit E. Label the columns of data by the direction to be used in traversing the stack. For example, if the first reading is closest to the east port, and the last reading is closest to the west port, then label the traverse east-west. Print blank copies of the spreadsheet as data forms for hand recording measurements for later transfer to the spreadsheet.

6.2.2 Provide essential supplies at the sampling location. (gas cylinders and regulators, fittings and probe-port couplers, marking pens, data sheets, writing, and probe-supporting platforms).

6.2.3 Setup the test parameters characterizing the stack or duct airflow, configuration and conditions according to the test instruction.

6.2.4 Fill in test information on data form (run number, test crew, instrumentation used, configuration, date, etc.).

6.2.5 Set up the gas analyzer system at the test port according to the illustrations in Exhibits B and C. 
6.2.6 Enter the barometric pressure and the sampling tube length into the analyzer's environmental setup menu (analyzer manual Part 4.2.4). Also record the information on the data form.

\subsubsection{Program the analyzer for:}

- 60-second samples,

- continuous operation,

- moisture compensation if needed as determined in Section 6.1.

6.2.8 Mark the sampling probe with a permanent marker so the inlet can be placed at the successive measurement points.

6.2.9 Assemble the injection equipment according to the Test Instruction and generally as shown in Exhibits B and C. The equipment consists of:

- A pressurized cylinder of sulfur hexafluoride

- a gas regulator,

- valve,

- flowmeter,

- flexible tubing, and a stainless steel injection probe with a $90^{\circ}$ bend at the discharge end

6.2.10 Plug the end of the injection probe. Pressurize the probe with the gas cylinder and check for leaks with soap bubble solution. For the same test setup, any future leak checks can be performed with the flowmeter valve closed instead of plugging the probe.

6.2.11 Secure the injection probe at one of the five injection positions according to the test instruction.

Note: The sampling probe consists of a stainless steel tube ( $1 / 4$ or $3 / 8$-inch) with enough length to reach across the inside of the stack or duct, allowing for fittings. The intake end should have a $90^{\circ}$ bend so that the open end of the tube faces downward or into the flow within the stack. The discharge end of the probe should terminate in a tee. One leg of the tee connects by flexible tubing to a rotameter and vacuum pump. This leg should draw about a 10-1pm flow of air, depending on the volumetric flow in the stack. The other leg of the tee connects via flexible tubing to an in-line filter (47-mm-diameter glass fiber filter) and then to the gas analyzer inlet. To minimize tubing length to the analyzer, locate the gas analyzer near the test ports. 
Note: Sampling plane traverse points Use the grid of measurement points provided with the test instruction and data form. This is usually the same as used for the velocity uniformity test (EMS-JAG-04). A centerpoint, is included as a common reference and for graphical purposes. The layout design divides the area of the sampling plane so that each point represents approximately an equal-sized area.

\subsection{System Startup}

6.3.1 If not already running, start the stack fan(s) and make adjustments to obtain the fan configuration or flow setting called for in the test instruction and recorded on the data form.

6.3.2 Use a velocity or flow sensor to verify that the target flow condition has been achieved within $\pm 10 \%$. Record the value on the data form.

6.3.3 Set the sample probe at the center position.

6.3.4 Start the sampler vacuum pump and set its flowrate at about $10 \mathrm{lpm}$

6.3.5 After the analyzer has stabilized (about 10 minutes), record at least four consecutive background readings on the data form. Do not proceed with the test if the background exceeds $5 \%$ of the anticipated average concentration in the stack.

6.3.6 Start the flow of the tracer gas and adjust its flow rate as called for in the test instruction.

\subsubsection{Record the starting}

- tracer injection flow rate

- time

- pressure in the tracer gas cylinder

- ambient temperature

- stack air temperature

- stack air velocity or flowrate

- sampling flowrate

- ambient pressure

- ambient humidity

- analyzer water vapor correction setting 


\section{Note: Estimation of Sulfur Hexafluoride Injection Rate}

Estimate the $\mathrm{SF}_{6}$ injection rate so the average diluted concentration will be within the range calibration gas standards and according to the following equation:

$$
\text { injection flowrate }=\text { stack flowrate } \times \frac{\text { target ppmv }}{10^{6}}
$$

The rotameter reading should be adjusted for the density of the $\mathrm{SF}_{6}$. The air equivalent reading is about

$$
\text { rotameter reading }=k x \text { actual flowrate }
$$

where $k$ is 2.53 (the square-root of the density) for $\mathrm{SF}_{6}$.

\subsection{Record Measurements}

6.4.1 Position the sample probe at the first measurement point. After the analyzer has drawn the sample, move the probe to the second point. After a few seconds, the analyzer will display the tracer concentration for the sample drawn at the first measurement point. Record the reading on the data form as the reading for the first point and in the first column.

6.4.2 After the analyzer draws the sample at the second measurement point move the probe to the third position. After a few more seconds, the analyzer will display the concentration of tracer for the sample drawn from the second point. Record that value as the second reading.

6.4.3 Proceed in a similar manner through all of the points across the stack, moving the probe after each sample is drawn so that it is in the proper position to draw the next sample. In this manner, while the analyzer is waiting to draw the next sample, the display will change to show the concentration of the previously drawn sample.

6.4.4 After drawing the last sample across the stack, move the probe back to the first position and record the second series of measurements for that traverse direction.

6.4.5 After drawing the sample for the second time at the last position, move the probe back to the first position and record the third series of measurements for that traverse direction.

Note: The calculations use three measurements taken at each measurement point in each traverse direction. The repeats are made during the three separate traverses across and not as three consecutive measurements at each point.

6.4.6 After completing the three measurements for the points in the first traverse 
direction, relocate the probe to the second test port.

6.4.7 In a manner similar to Steps 6.4.1 - 6.4.5, make three tracer concentration measurements at each point along the traversing direction through the second test port.

6.4.8 Record the ending:

- tracer injection flow rate

- time

- pressure in the tracer gas cylinder

- ambient temperature

- stack air temperature

- stack air velocity or flowrate

- sampling flowrate

- ambient pressure

- ambient humidity

- analyzer water vapor correction setting

6.4.9 Shut down the delivery of tracer gas.

6.4.10 Continue operation of the gas analyzer for several minutes to purge any remaining gas through the exhauster and the analyzer.

6.4.11 Record on the data form at least four background tracer gas concentration readings.

6.4.12 Record any deviations from the above procedure on the data form.

6.4.13 Verify that the data form is complete and sign and date in the signature block attesting to performing this procedure to this point.

6.4.14 Repeat steps 6.4.1 - 6.4.13 for each run as indicated in the Test Instruction.

\subsection{Calculations}

6.5.1 For a test run, transfer the hand written data into the electronic spreadsheet.

6.5.2 Calculate the COV for the run and the maximum percent deviation from the mean.

Note: The spreadsheet shown in Exhibit E is set up to calculate the COV for each tracer gas concentration traverse using the average concentration data from all points in the inner two-thirds of the cross section area of the plane (including the center point). The calculation of percent deviation from the mean includes all measurement points. 
6.5.3 Compare the observed COV and percent deviation from the mean to the acceptance criterion.

Note: The test is acceptable if the COV is within $\pm 20 \%$ for the inner two-thirds of the stack diameter and if no point in the entire grid of points differs from the mean by more than $30 \%$. This is determined by inspecting the average concentration at each measurement point. The $\mathrm{COV}$ is 100 times the standard deviation divided by the mean.

6.5.4 Have the data transfers and calculations independently reviewed and verified and the finished spreadsheets signed by the reviewer.

6.5.5 Complete the calculations for each test run in the Test Instruction

\subsection{Records}

7.1 Transfer the original signed data forms and verified spreadsheets to the records custodian as project records. 


\section{Exhibits}

Exhibit A -Typical Test Instruction.

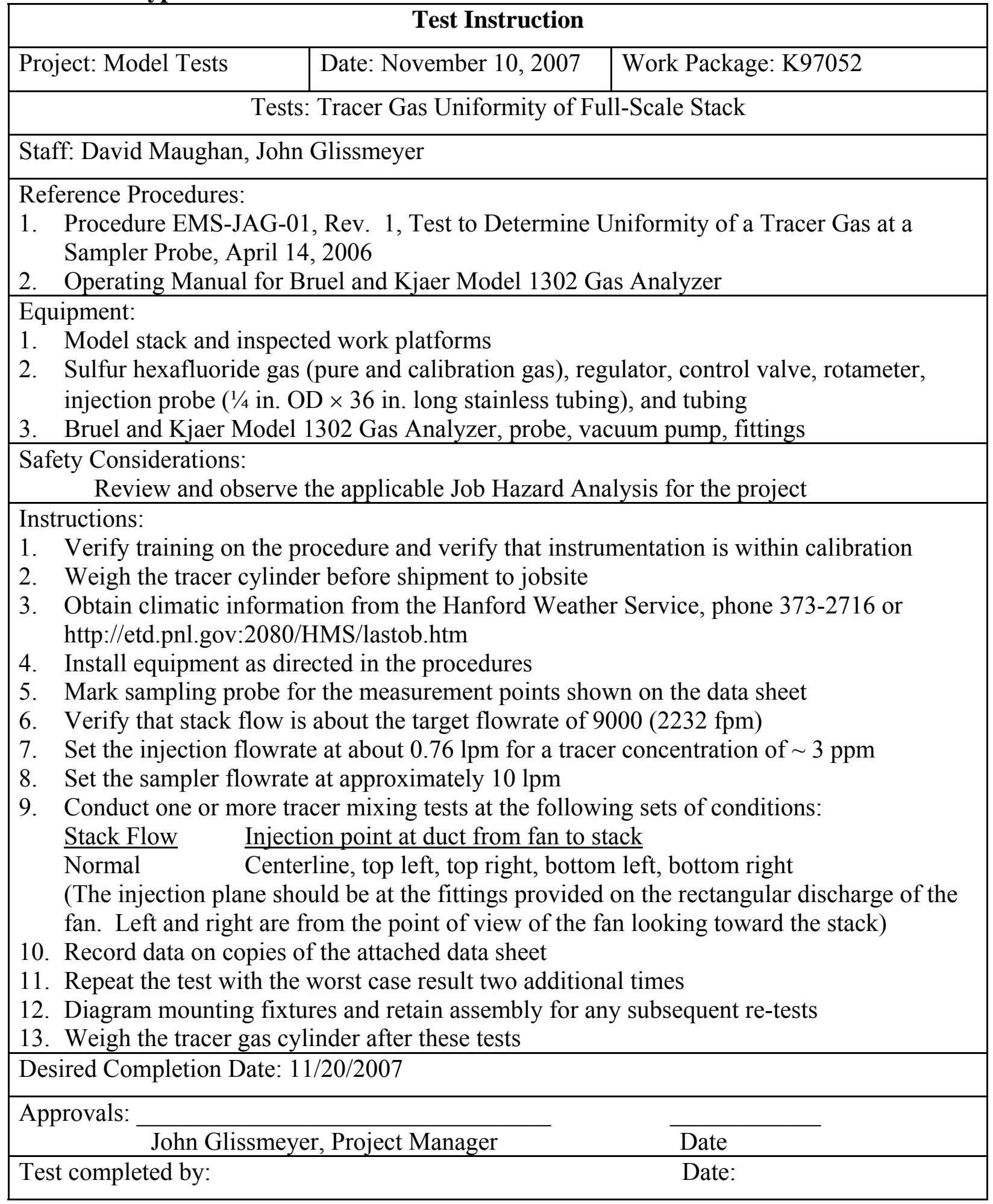




\section{Exhibit B - Overview of Stack and Injection/Sampling Setups}

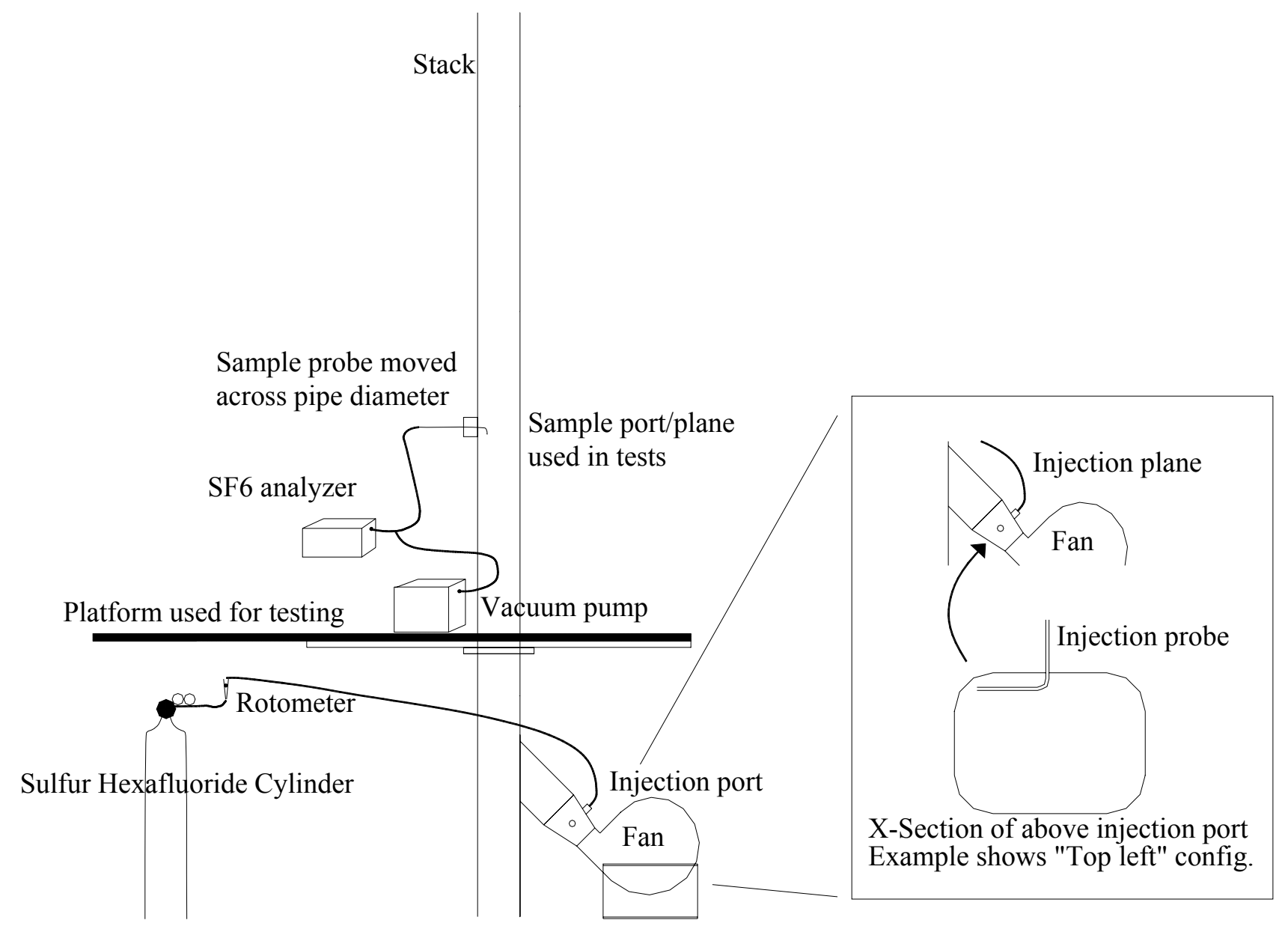


Exhibit C - Details for Stack Sampling Probe and Gas Analyzer Setup

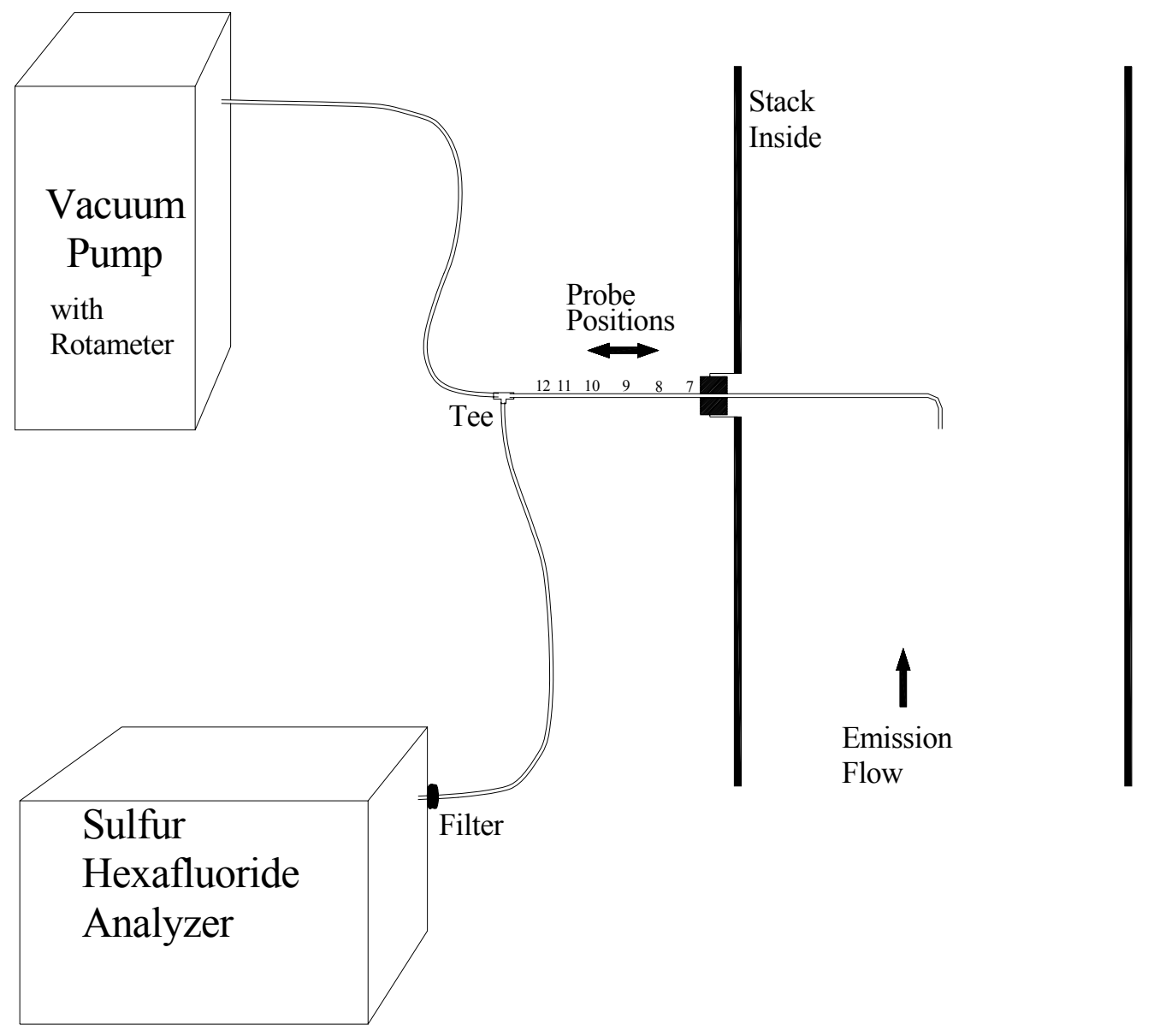


Exhibit D - Typical Calibration Check of Gas Analyzer

Sulfur hexafluoride Gas Calibration performed on B\&K on

Setup details: B\&K sample inlet tube $=6 \mathrm{ft}$

992.7 mbar station pressure, analyzer corrects to $20 \mathrm{deg} C$ 38 deg F ambient temp

87 percent $\mathrm{RH}$

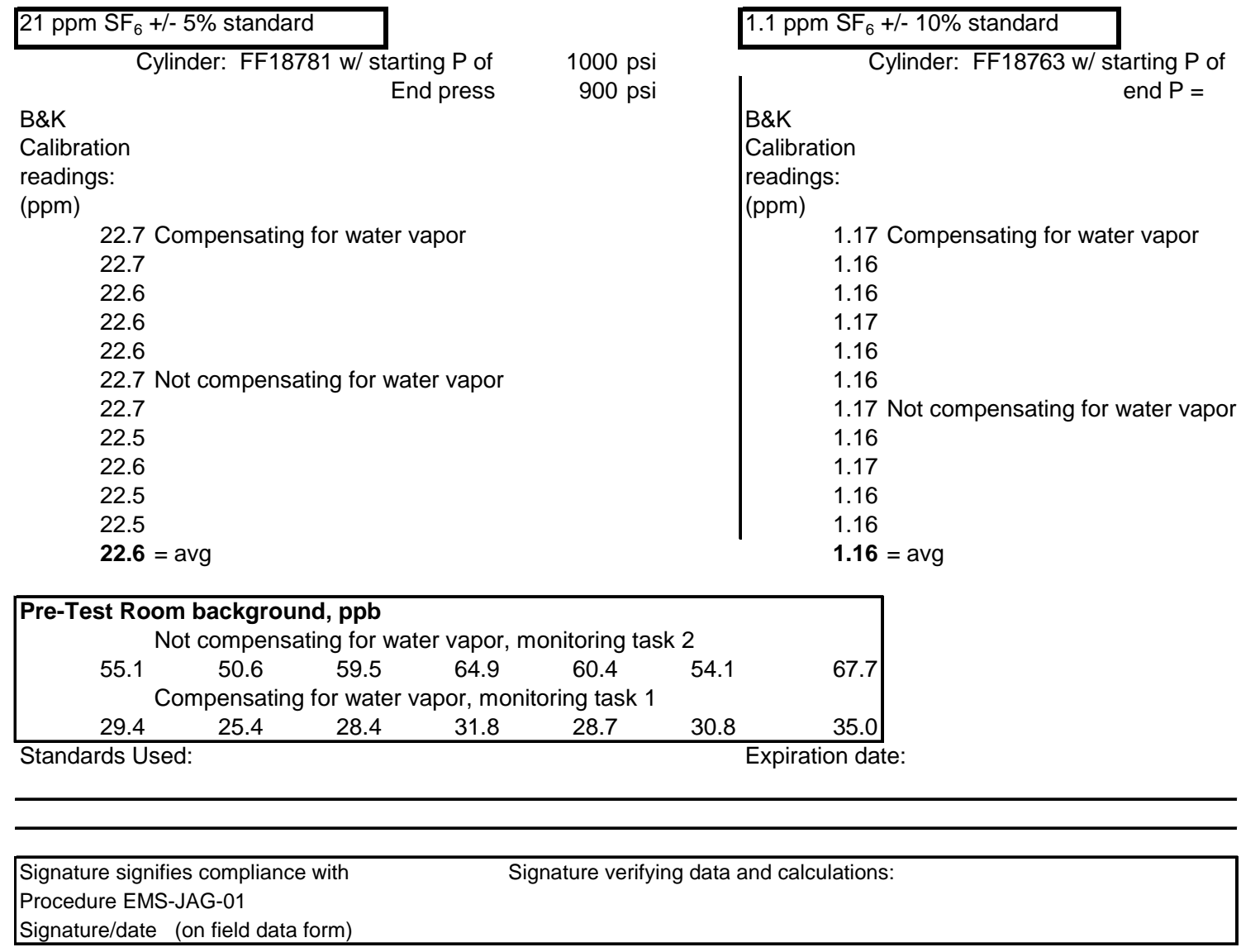


Exhibit E - Typical Data Form

\begin{tabular}{|c|c|c|c|c|c|c|c|c|c|}
\hline & & & RACER GAS & TRAVERSE & DATA FOR & & & & \\
\hline & Site & Model & & & Run No. & GT-23 & & & \\
\hline & Date & $5 / 6 / 2007$ & & Fan Co & nfiguration & Turbine Fan & 30 degre & e east port & \\
\hline & Tester & Glissmeyer & & & Fan Setting & $60 \mathrm{~Hz}$ & & & \\
\hline & Stack Dia. & 23.5 & & & tack Temp & 59 & $g \mathrm{~F}$ & & \\
\hline & tack X-Area & 433.7 & in. 2 & Star & t/End Time & $957 / 1245$ & & & \\
\hline & Elevation & N.A. & & Cent & er $2 / 3$ from & 2.16 & to: & 21.34 & \\
\hline Distance to & disturbance & 75 & inches & Points in & Center $2 / 3$ & 2 & to: & 7 & \\
\hline Measur & ement units & $\frac{\mathrm{ppm} \mathrm{SF6}}{1 \mathrm{st}}$ & & Inje & ction Point & ET8 centerli & & & \\
\hline Traverse--> & & & $\overline{W e}$ & est & & & Nor & rth & \\
\hline Trial ----> & & 1 & 2 & 3 & Mean & 1 & 2 & 3 & Mean \\
\hline Point & Depth, in. & & $\mathrm{pp}$ & $\mathrm{m}$ & & & $\mathrm{pp}$ & $\mathrm{m}$ & \\
\hline 1 & 0.75 & 2.70 & 2.64 & 2.64 & 2.660 & 2.95 & 2.87 & 2.93 & 2.917 \\
\hline 2 & 2.47 & 2.72 & 2.68 & 2.67 & 2.690 & 2.97 & 2.82 & 2.92 & 2.903 \\
\hline 3 & 4.56 & 2.66 & 2.70 & 2.66 & 2.673 & 2.89 & 2.85 & 2.85 & 2.863 \\
\hline 4 & 7.59 & 2.81 & 2.76 & 2.71 & 2.760 & 2.87 & 2.88 & 2.88 & 2.877 \\
\hline Center & 11.75 & 2.82 & 2.78 & 2.81 & 2.803 & 2.80 & 2.82 & 2.79 & 2.803 \\
\hline 5 & 15.91 & 2.94 & 2.93 & 2.92 & 2.930 & 2.80 & 2.76 & 2.79 & 2.783 \\
\hline 6 & 18.94 & 3.03 & 3.02 & 2.97 & 3.007 & 2.76 & 2.79 & 2.82 & 2.790 \\
\hline 7 & 21.03 & 2.96 & 3.03 & 3.09 & 3.027 & 2.78 & 2.78 & 2.75 & 2.770 \\
\hline 8 & 22.75 & 3.07 & 3.03 & 3.02 & 3.040 & 2.80 & 2.79 & 2.82 & 2.803 \\
\hline Averages ---- & $----->$ & 2.857 & 2.841 & 2.832 & 2.843 & 2.847 & 2.818 & 2.839 & 2.834 \\
\hline & & $A I I$ & $\mathrm{ppm}$ & Dev. & from mean & Center 2/3 & West & North & All \\
\hline & & Mean & 2.84 & & & Mean & 2.84 & 2.83 & 2.83 \\
\hline & & Min Point & 2.66 & & $-6.3 \%$ & Std. Dev. & 0.15 & 0.05 & 0.11 \\
\hline & & Max Point & 3.04 & & $7.1 \%$ & Cov as $\%$ & 5.2 & 1.9 & 3.7 \\
\hline Avg. Conc. & 2.843 & ppm & & & as analyze & $\begin{array}{r}\text { er checked: } \\
6 \text {-May-07 } \\
\end{array}$ & & & \\
\hline & & Start & Finish & & & & & & \\
\hline Tracer tank $p$ & ressure & 100 & 170 & psig & & & & & \\
\hline Sample Port & Temp & 58 & 60 & $F^{*}$ & & & & & \\
\hline Centerline ve & & n 1285 & w 1364/1330 & fpm & & & & & \\
\hline Injection flowr & meter & 20 & 20 & ball** & & & & & \\
\hline Stack flow & & & & & 3.5 & & & & \\
\hline Sampling flon & vmeter & 10 & 10 & Ipm Sierra & & & & & \\
\hline Ambient pres & sure & 1001.5 & 1000.1 & mbar & 3 & & & & \\
\hline Ambient hum & idity & 98 & 99 & $\mathrm{RH}$ & & & & & \\
\hline B\&K vapor co & orrection & $Y$ & $Y$ & $\mathrm{Y} / \mathrm{N}$ & 2.5 & & & & \\
\hline Back-Gd gas & $\mathrm{ppb}$ & $21 / 21 / 14 / 20$ & $44 / 49 / 30 / 18 /$ & & & & & & \\
\hline No. Bk-Gd sa & imples & 4 & 4 & $n$ & & & & & \\
\hline Ambient Tem & $p, F$ & 31 & 30 & & $\begin{array}{l}\mathbf{p} \\
\mathbf{m}\end{array}$ & & & & \\
\hline Instuments & Jsed: & & & & 1.5 & & & & \\
\hline B \& K Model & $1302 \# 17652$ & 299 & & & 1 & & & & \\
\hline Sierra Inc. Co & onstant Flow & Air Sampler & & & & & & & \\
\hline Solmat Zephy & /r SN 12951 & 472 Cal. Due & $7 / 26 / 07$ & & 0.5 & & & & \\
\hline Notes: & & & & & & & & & \\
\hline & & & & & & North & & & \\
\hline $\begin{array}{l}\text { Signature sig } \\
\text { Procedure EN } \\
\text { Signature/dat }\end{array}$ & $\begin{array}{l}\text { nifies compli } \\
\text { MS-JAG-01 } \\
\text { e }\end{array}$ & iance with & & Signature $\mathrm{v}$ & fying data $a$ & and calculatio & & & \\
\hline
\end{tabular}




\section{Appendix $\mathbf{H}$}

\section{Tracer Gas Uniformity Data Sheets}




\section{APPENDIX H: TRACER GAS UNIFORMITY DATA SHEETS}

\begin{tabular}{|c|c|c|c|c|c|c|c|c|c|}
\hline & & & ER GAS & RAVERSE & DATA FOR & & & & \\
\hline & Site & 296-S-21 M & & & Run No. & GT-1 & & & \\
\hline & Date & $7 / 28 / 2006$ & & Fan C & nfiguration & $1,2,3$ & & & \\
\hline & Tester & Jag/Jgd & & & Ean Setting & $56,45,50$ & $\mathrm{~Hz}$ & & \\
\hline & Stack Dia. & $177 / 8$ & & & Stack Temp & 100.8 & $\operatorname{deg} F$ & & \\
\hline & tack X-Area & 250.9 & & Sta & t/End Time & $1415 / 1540$ & & & \\
\hline & Elevation & N.A. & & Cen & er $2 / 3$ from & 1.64 & to: & 16.23 & \\
\hline Distance to $c$ & disturbance & 77.75 & hes & Points ir & Center $2 / 3$ & 2 & to: & 7 & \\
\hline Measure & ement units & ppm SF6 & & & ection Point & $\overline{\text { Fan } 1}$ & & & \\
\hline & & & & & & $1 \mathrm{st}$ & & & \\
\hline Traverse--> & & & ort_4_B & om/East & & & Port_4_S & Side/South & \\
\hline Trial ----> & & 1 & 2 & 3 & Mean & 1 & $\begin{array}{r}- \\
\end{array}$ & 3 & Mean \\
\hline Point & Depth, in. & & $\mathrm{pp}$ & & & & & $\mathrm{pm}$ & \\
\hline 1 & 0.58 & 1.78 & 1.82 & 1.86 & 1.820 & 1.88 & 1.82 & 1.88 & 1.860 \\
\hline 2 & 1.89 & 1.79 & 1.81 & 1.81 & 1.803 & 1.84 & 1.84 & 1.86 & 1.847 \\
\hline 3 & 3.49 & 1.79 & 1.84 & 1.83 & 1.820 & 1.86 & 1.87 & 1.87 & 1.867 \\
\hline 4 & 5.81 & 1.80 & 1.90 & 1.87 & 1.857 & 1.89 & 1.89 & 1.85 & 1.877 \\
\hline Center & 9.00 & 1.94 & 1.89 & 1.90 & 1.910 & 1.89 & 1.89 & 1.89 & 1.890 \\
\hline 5 & 12.19 & 2.00 & 2.02 & 2.01 & 2.010 & 1.98 & 1.95 & 1.89 & 1.940 \\
\hline 6 & 14.51 & 2.02 & 2.06 & 2.05 & 2.043 & 2.09 & 2.04 & 2.01 & 2.047 \\
\hline 7 & 16.11 & 2.06 & 2.06 & 2.02 & 2.047 & 2.05 & 2.04 & 2.04 & 2.043 \\
\hline 8 & 17.42 & 2.06 & 2.05 & 2.06 & 2.057 & 2.11 & 2.11 & 2.06 & 2.093 \\
\hline rages & $->$ & 1.916 & 1.939 & .934 & 1.930 & 1.954 & 1.939 & 1.928 & .940 \\
\hline
\end{tabular}

Avg. Conc.

\begin{tabular}{|lr}
\hline All & $\mathrm{ppm}$ \\
Mean & 1.94 \\
Min Point & 1.80 \\
Max Point & 2.09 \\
\hline
\end{tabular}

\section{$1.939 \mathrm{ppm}$}

Tracer tank pressure Stack Temp

Ref. Pt. air vel. Injection flowmeter Stack flow

Sampling flowmeter Ambient pressure Ambient humidity B\&K vapor correction Back-Gd gas ppb No. Bk-Gd samples Ambient Temp, $\mathrm{F}$

Instuments Used:

B \& K Model $1302 \# 1765299$

Sierra Inc. Constant Flow Air Sampler

TSI 8360 SN 209060 Cal due 9/27/06

\section{Notes:}

** black glass ball

Reference point air velocity measured at point $4 \mathrm{So} 8$.

\begin{tabular}{|c|c|c|c|c|}
\hline Dev. from mean & Center $2 / 3$ & East & South & All \\
\hline & Mean & 1.93 & 1.93 & 1.93 \\
\hline$-6.8 \%$ & Std. Dev. & 0.11 & 0.08 & 0.09 \\
\hline $8.2 \%$ & COV as $\%$ & 5.5 & 4.3 & 4.7 \\
\hline
\end{tabular}

Gas analyzer checked:

25-Jul-06

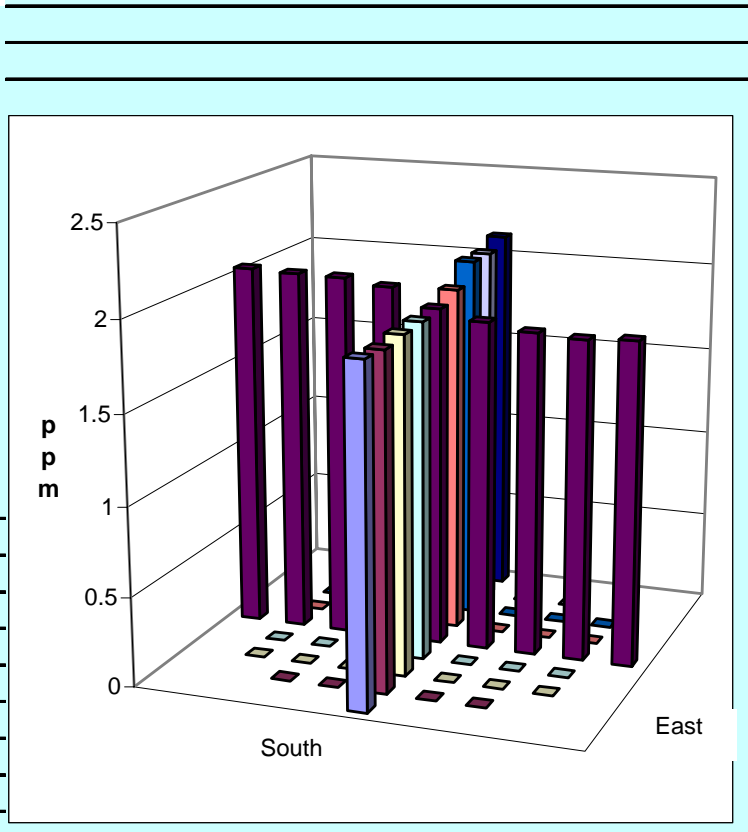

Signature signifies compliance with Procedure EMS-JAG-01

Signature/date

JAG

Signature verifying data and calculations:

Signatures on file copy 


\section{TRACER GAS TRAVERSE DATA FORM}

\begin{tabular}{|c|c|}
\hline \multicolumn{2}{|c|}{ Site 296-S-21 Model } \\
\hline Date & $7 / 28 / 2006$ \\
\hline Tester & $\mathrm{Jag} / \mathrm{Jgd}$ \\
\hline Stack Dia. & $177 / 8$ in. \\
\hline Stack X-Area & 250.9 in. $^{2}$ \\
\hline Elevation & N.A. \\
\hline to disturbance & 77.75 inches \\
\hline
\end{tabular}

Run No. GT-2

Fan Configuration $\mathbf{1 , 4}$

Fan Setting $\mathbf{5 6 , 5 2}$

Stack Temp

Start/End Time 1545/1645

Center $2 / 3$ from

Points in Center 2/3

Injection Point Fan 1

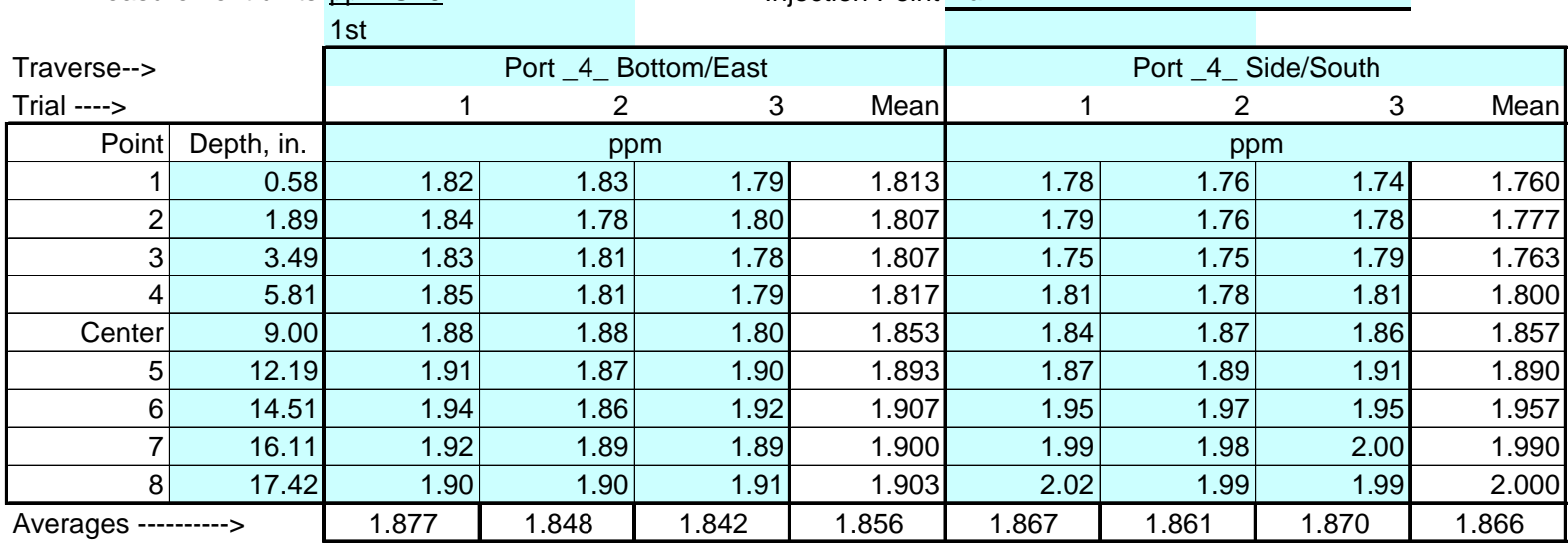

\begin{tabular}{|c|c|c|c|c|c|c|}
\hline$A I I$ & $\mathrm{ppm}$ & Dev. from mean & Center 2/3 & East & South & All \\
\hline Mean & 1.86 & & Mean & 1.85 & 1.86 & 1.86 \\
\hline Min Point & 1.76 & $-5.4 \%$ & Std. Dev. & 0.05 & 0.09 & 0.07 \\
\hline Max Point & 2.00 & $7.5 \%$ & COV as $\%$ & 2.4 & 4.8 & 3.6 \\
\hline
\end{tabular}

Avg. Conc. $\quad 1.861$ ppm

\begin{tabular}{|c|c|c|c|}
\hline \multirow[b]{2}{*}{ Tracer tank pressure } & Start & Finish & \\
\hline & 400 & 450 & \\
\hline \multirow{4}{*}{$\begin{array}{l}\text { Stack Temp } \\
\text { Ref. Pt. air vel. } \\
\text { Injection flowmeter } \\
\text { Stack flow }\end{array}$} & 100.6 & 99.6 & $F^{\circ}$ \\
\hline & 2310 & 2350 & \multirow{3}{*}{$\begin{array}{l}\text { fpm } \\
\text { ball** } \\
\mathrm{cfm}\end{array}$} \\
\hline & 10 & & \\
\hline & & & \\
\hline Sampling flowmeter & 10 & 10 & \multirow{4}{*}{$\begin{array}{l}\text { lpm Sierra } \\
\text { in } \mathrm{Hg} \\
\mathrm{RH} \\
\mathrm{Y} / \mathrm{N}\end{array}$} \\
\hline \multirow{4}{*}{$\begin{array}{l}\text { Ambient pressure } \\
\text { Ambient humidity } \\
\text { B\&K vapor correction } \\
\text { Back-Gd gas ppb }\end{array}$} & 29.3 & 29.3 & \\
\hline & 15 & 15 & \\
\hline & $\mathrm{N}$ & $\mathrm{N}$ & \\
\hline & $23,24,26,28,28$ & $30,30.28,31,33$ & \multirow{4}{*}{ n } \\
\hline \multirow{3}{*}{$\begin{array}{l}\text { No. Bk-Gd samples } \\
\text { Ambient Temp, F } \\
\text { Instuments Used: }\end{array}$} & 5 & 5 & \\
\hline & 96 & 97.1 & \\
\hline & & & \\
\hline \multicolumn{4}{|c|}{ B \& K Model $1302 \# 1765299$} \\
\hline \multicolumn{4}{|c|}{ Sierra Inc. Constant Flow Air Sampler } \\
\hline \multicolumn{4}{|c|}{ TSI 8360 SN 209060 Cal due 9/27/06 } \\
\hline \multicolumn{4}{|l|}{ Notes: } \\
\hline \multicolumn{4}{|l|}{ ** black glass ball } \\
\hline \multicolumn{4}{|c|}{ Reference point air velocity measured at point 4 So 8 . } \\
\hline
\end{tabular}

Gas analyzer checked:

25-Jul-06

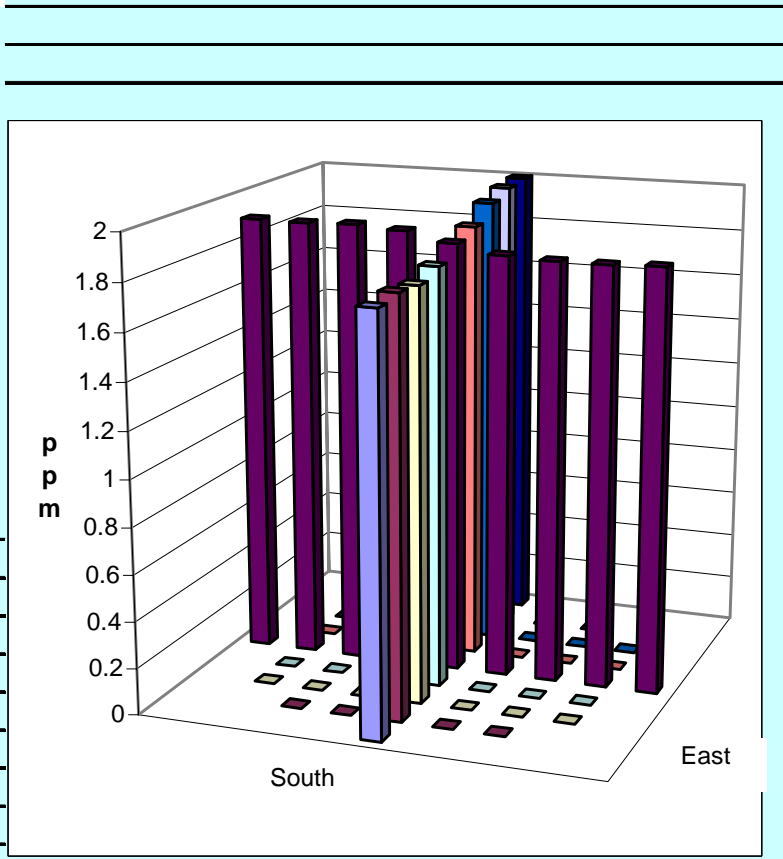

Signature signifies compliance with

Procedure EMS-JAG-01

Signature/date

Signature verifying data and calculations:

JAG

Signatures on file copy 


\section{TRACER GAS TRAVERSE DATA FORM}

\begin{tabular}{|c|c|}
\hline \multicolumn{2}{|c|}{ Site 296-S-21 Model } \\
\hline Date & $7 / 28 / 2006$ \\
\hline Tester & Jag/Jgd \\
\hline Stack Dia. & $177 / 8$ in. \\
\hline Stack X-Area & 250.9 in. $^{2}$ \\
\hline Elevation & N.A. \\
\hline to disturbance & 77.75 inches \\
\hline
\end{tabular}

Run No. GT-3

Fan Configuration 2,4

Fan Setting 45,52

Stack Temp

Start/End Time 1650/1740

Center $2 / 3$ from

Points in Center 2/3

Injection Point 2

$\mathrm{Hz}$

$8.85 \operatorname{deg} \mathrm{F}$

Distance to disturbance

Measurement units ppm SF6

1st

\begin{tabular}{|c|c|c|c|c|c|c|c|c|c|}
\hline \multirow{2}{*}{\multicolumn{2}{|c|}{$\begin{array}{l}\text { Traverse--> } \\
\text { Trial ----> }\end{array}$}} & \multicolumn{4}{|c|}{ Port_4_Bottom/East } & \multicolumn{4}{|c|}{ Port_4_Side/South } \\
\hline & & 1 & 2 & 3 & Mean & 1 & 2 & 3 & Mean \\
\hline Point & Depth, in. & \multicolumn{4}{|c|}{ ppm } & \multicolumn{4}{|c|}{ ppm } \\
\hline 1 & 0.58 & 2.21 & 2.17 & 2.27 & 2.217 & 2.47 & 2.45 & 2.53 & 2.483 \\
\hline 2 & 1.89 & 2.36 & 2.42 & 2.43 & 2.403 & 2.48 & 2.50 & 2.49 & 2.490 \\
\hline 3 & 3.49 & 2.25 & 2.20 & 2.47 & 2.307 & 2.44 & 2.41 & 2.16 & 2.337 \\
\hline 4 & 5.81 & 2.38 & 2.36 & 2.51 & 2.417 & 2.28 & 2.38 & 2.42 & 2.360 \\
\hline Center & 9.00 & 2.30 & 2.50 & 2.41 & 2.403 & 2.21 & 2.22 & 2.37 & 2.267 \\
\hline 5 & 12.19 & 2.51 & 2.33 & 2.31 & 2.383 & 2.46 & 2.33 & 2.28 & 2.357 \\
\hline 6 & 14.51 & 2.35 & 2.34 & 2.37 & 2.353 & 2.45 & 2.30 & 2.36 & 2.370 \\
\hline 7 & 16.11 & 2.52 & 2.25 & 2.31 & 2.360 & 2.52 & 2.18 & 2.18 & 2.293 \\
\hline 8 & 17.42 & 2.34 & 2.44 & 2.32 & 2.367 & 2.31 & 2.20 & 2.23 & 2.247 \\
\hline Averages --.- & ------> & 2.358 & 2.334 & 2.378 & 2.357 & 2.402 & 2.330 & 2.336 & 2.356 \\
\hline
\end{tabular}

\begin{tabular}{|lrr|lrrr|}
\hline All & ppm & Dev. from mean & Center 2/3 & $\underline{\text { East }}$ & South & All \\
Mean & 2.36 & & Mean & 2.38 & 2.35 & 2.36 \\
Min Point & 2.22 & $-5.9 \%$ & Std. Dev. & 0.04 & 0.07 & 0.06 \\
Max Point & 2.49 & $5.7 \%$ & COV as \% & 1.6 & 3.0 & $\mathbf{2 . 4}$ \\
\hline
\end{tabular}

Avg. Conc.

$$
2.359 \mathrm{ppm}
$$

Tracer tank pressure

Stack Temp

Ref. Pt. air vel.

Injection flowmeter

Stack flow

Sampling flowmeter

Ambient pressure

Ambient humidity

B\&K vapor correction

Back-Gd gas ppb

No. Bk-Gd samples

Ambient Temp, F

Instuments Used:

B \& K Model 1302 \#1765299

Sierra Inc. Constant Flow Air Sampler

TSI 8360 SN $209060 \mathrm{Cal}$ due 9/27/06

\section{Notes:}

** black glass ball

Reference point air velocity measured at point $4 \mathrm{So} 8$.

\begin{tabular}{|c|c|c|}
\hline Start & Finish & \\
\hline 450 & 450 & psig \\
\hline 99.6 & 98.1 & $F^{\circ}$ \\
\hline 1920 & 1870 & fpm \\
\hline 10 & 10 & ball ${ }^{\star *}$ \\
\hline- & & $\mathrm{cfm}$ \\
\hline 10 & 10 & Ipm Sierra \\
\hline 29.3 & 29.3 & in $\mathrm{Hg}$ \\
\hline 15 & 17 & $\mathrm{RH}$ \\
\hline $\mathrm{N}$ & $\mathrm{N}$ & $\mathrm{Y} / \mathrm{N}$ \\
\hline $30,30,28,31,33$ & $45,55,26,26$ & \\
\hline & 4 & $n$ \\
\hline 97.1 & 98.6 & \\
\hline
\end{tabular}

Gas analyzer checked:

25-Jul-06

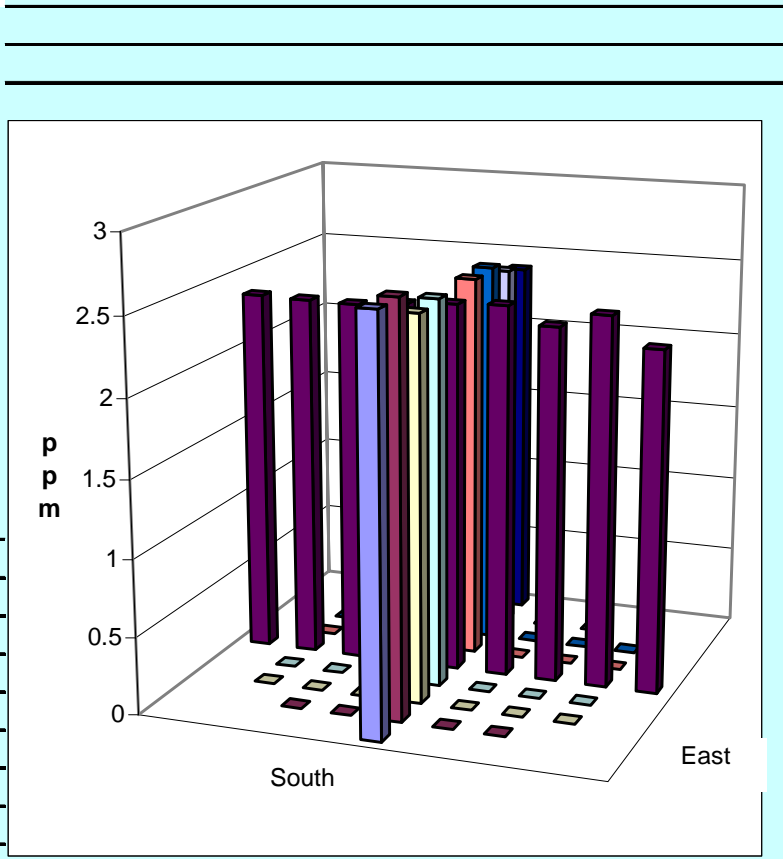

Signature signifies compliance with Procedure EMS-JAG-01

Signature/date

JAG

Signature verifying data and calculations:

$7 / 28 / 2006$

Signatures on file copy 
TRACER GAS TRAVERSE DATA FORM

\begin{aligned} Site & \multicolumn{2}{c}{ 296-S-21 Model } \\ Date & $\frac{7 / 31 / 2006}{\text { Jag/Jgd }} \\$ Tester & $\frac{177 / 8 \text { in. }}{250.9 \text { in. }^{2}} \\$ Stack Dia. & $\frac{\text { N.A. }}{77.75 \text { inches }} \\$ Stack X-Area & Elevation \\ to disturbance & $\frac{77}{\text { ppm SF6 }}\end{aligned}$

Run No. GT-4

Fan Configuration 3.4

Fan Setting $\mathbf{5 0 , 5 2}$

Stack Temp

Start/End Time 0910/1031

Center $2 / 3$ from

Points in Center $2 / 3$

Injection Point Fan 3

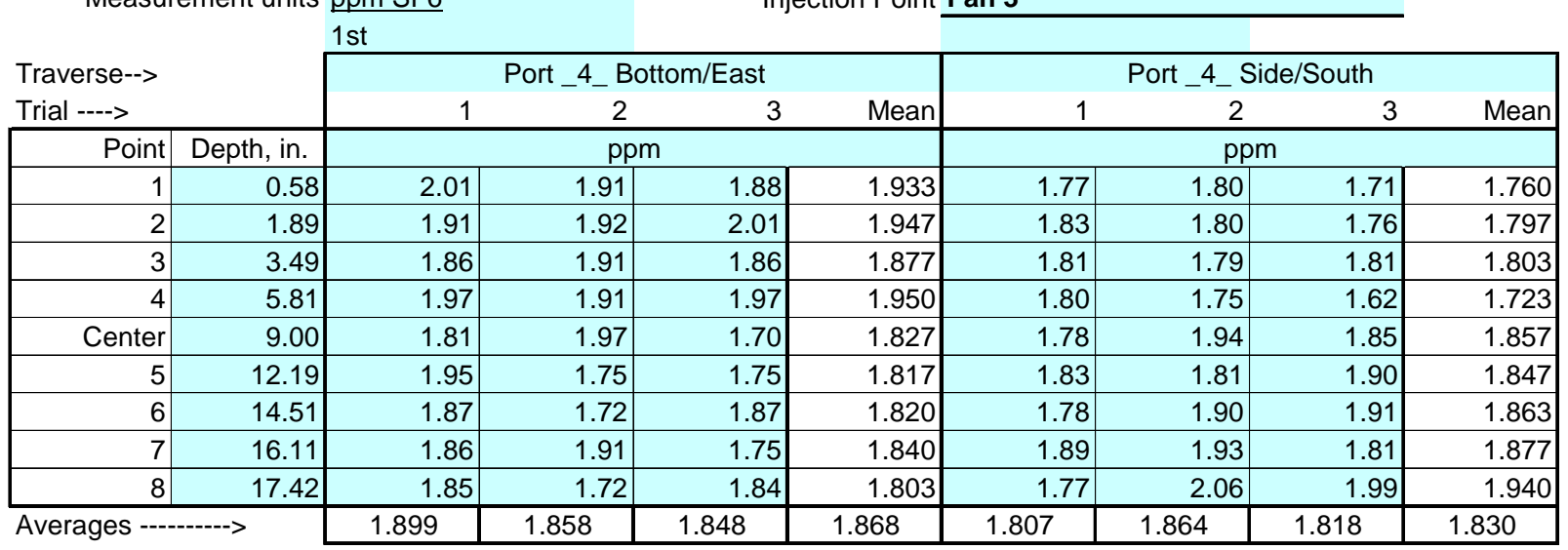

\begin{tabular}{|c|c|c|c|c|c|c|}
\hline$A I I$ & $\mathrm{ppm}$ & Dev. from mean & Center $2 / 3$ & East & South & All \\
\hline Mean & 1.85 & & Mean & $\overline{1.87}$ & 1.82 & 1.85 \\
\hline Min Point & 1.72 & $-6.8 \%$ & Std. Dev. & 0.06 & 0.05 & 0.06 \\
\hline Max Point & 1.95 & $5.5 \%$ & cov as $\%$ & 3.1 & 2.9 & 3.2 \\
\hline
\end{tabular}

Avg. Conc. $\quad 1.850$ ppm

\begin{tabular}{|c|c|c|c|}
\hline \multirow{3}{*}{$\begin{array}{l}\text { Tracer tank pressure } \\
\text { Stack Temp }\end{array}$} & Start & Finish & \multirow{2}{*}{$\begin{array}{l}\text { psig } \\
F^{\circ}\end{array}$} \\
\hline & 300 & 302 & \\
\hline & 74 & 75 & $F^{\circ}$ \\
\hline Ref. Pt. air vel. & 2250 & 2310 & $\mathrm{fpm}$ \\
\hline Injection flowmeter & 10 & 8 & ball ${ }^{\star \star}$ \\
\hline Stack flow & - & & $\mathrm{cfm}$ \\
\hline Sampling flowmeter & 10 & 10 & Ipm Sierra \\
\hline Ambient pressure & 29.6 & 29.5 & in $\mathrm{Hg}$ \\
\hline Ambient humidity & 41 & 36 & $\mathrm{RH}$ \\
\hline B\&K vapor correction & $\mathrm{N}$ & $\mathrm{N}$ & $\mathrm{Y} / \mathrm{N}$ \\
\hline Back-Gd gas ppb & $33,30,28,24$ & $28,28,28,34$ & \\
\hline No. Bk-Gd samples & & 4 & $\mathrm{n}$ \\
\hline nbient Temp, F & 69 & 73 & \\
\hline
\end{tabular}

Instuments Used:

B \& K Model $1302 \# 1765299$

Sierra Inc. Constant Flow Air Sampler

TSI 8360 SN 209060 Cal due 9/27/06

Notes:

** black glass ball

Reference point air velocity measured at point 4 So8.
Gas analyzer checked:

25-Jul-06

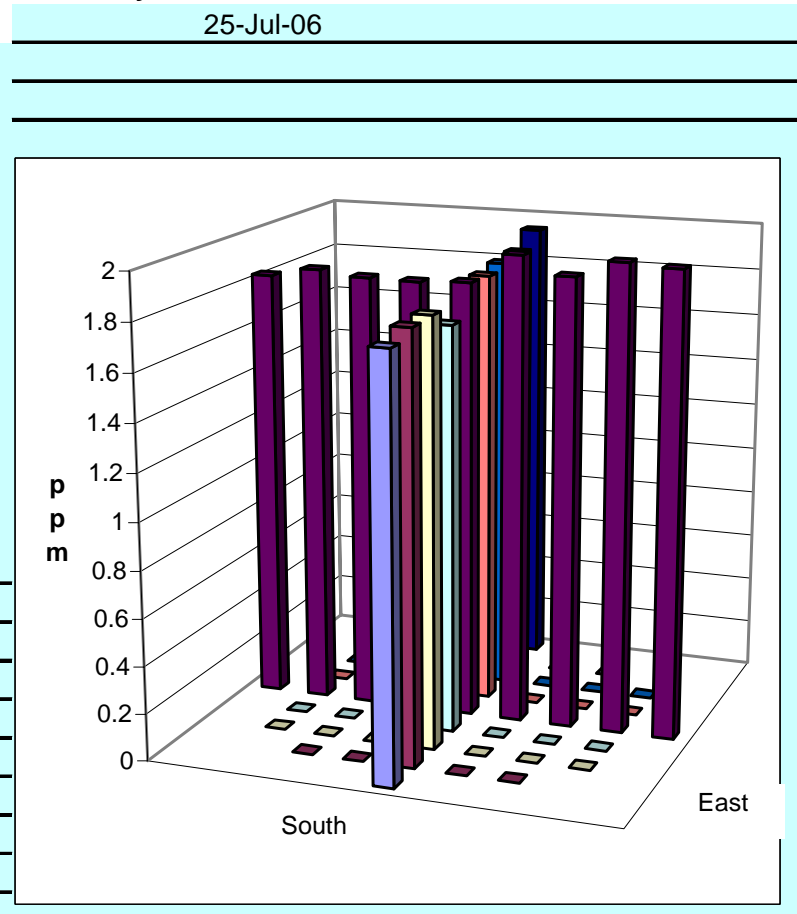

Signature signifies compliance with Procedure EMS-JAG-01

Signature/date

JGD
Signature verifying data and calculations:

$7 / 31 / 2006$

Signatures on file copy 
TRACER GAS TRAVERSE DATA FORM

$\begin{aligned} \text { Site } & \frac{296-S-21 \text { Model }}{7 / 31 / 2006} \\ \text { Date } & \frac{71}{\text { Jag/Jgd }} \\ \text { Tester } & \frac{177 / 8 \text { in. }}{250.9 \text { in. }^{2}} \\ \text { Stack Dia. } & \frac{\text { N.A. }}{77.75 \text { inches }} \\ \text { Stack X-Area } & \text { Elevation } \\ \text { to disturbance } & \frac{77}{\text { ppm SF6 }}\end{aligned}$

Run No. GT-5

Fan Configuration 3,4

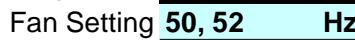

Stack Temp $76.1 \mathrm{deg} F$

Start/End Time 1031/1125

Center $2 / 3$ from

Points in Center $2 / 3$

Injection Point 4

Measurement units ppm SF6

1st

\begin{tabular}{l} 
Traverse--> \\
\cline { 3 - 10 } \\
\cline { 3 - 10 }
\end{tabular}

\begin{tabular}{|c|c|c|c|c|c|c|}
\hline$A I I$ & $\mathrm{ppm}$ & Dev. from mean & Center $2 / 3$ & East & South & All \\
\hline Mean & 2.02 & & Mean & $\overline{2.02}$ & 2.03 & 2.02 \\
\hline Min Point & 1.97 & $-2.5 \%$ & Std. Dev. & 0.04 & 0.04 & 0.04 \\
\hline Max Point & 2.08 & $2.6 \%$ & cov as $\%$ & 1.8 & 2.0 & 1.8 \\
\hline
\end{tabular}

Avg. Conc. 2.024 ppm

Gas analyzer checked:

25-Jul-06

Tracer tank pressure

Stack Temp

Ref. Pt. air vel.

Injection flowmeter

Stack flow

Sampling flowmeter

Ambient pressure

Ambient humidity

B\&K vapor correction

Back-Gd gas ppb

No. Bk-Gd samples

Ambient Temp, $\mathrm{F}$

Instuments Used:

\begin{tabular}{|c|c|c|}
\hline Start & Finish & \\
\hline 302 & 300 & psig \\
\hline 75 & 77.2 & $F^{\circ}$ \\
\hline 2310 & 2230 & $\mathrm{fpm}$ \\
\hline 10 & 10 & ball ${ }^{\star \star}$ \\
\hline - & - & $\mathrm{cfm}$ \\
\hline 10 & 10 & Ipm Sierra \\
\hline 29.5 & 29.5 & in $\mathrm{Hg}$ \\
\hline 36 & 35 & $\mathrm{RH}$ \\
\hline $\mathrm{N}$ & $\mathrm{N}$ & $Y / N$ \\
\hline $28,28,28,34$ & $27,25,27,34$ & \\
\hline 4 & 4 & $n$ \\
\hline 73 & 74 & \\
\hline
\end{tabular}

B \& K Model 1302 \#1765299

Sierra Inc. Constant Flow Air Sampler

TSI 8360 SN 209060 Cal due 9/27/06

Notes:

** black glass ball

Reference point air velocity measured at point 4 So8.

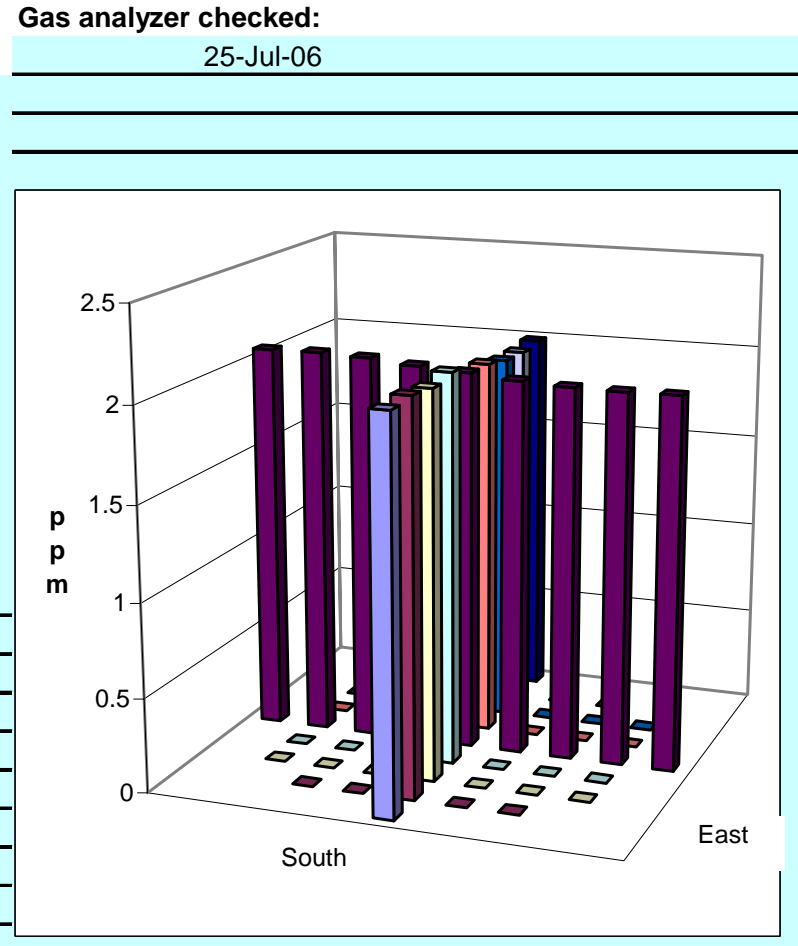

Signature signifies compliance with

Procedure EMS-JAG-01

Signature/date

JGD

$7 / 31 / 2006$

Signature verifying data and calculations:

Signatures on file copy 
TRACER GAS TRAVERSE DATA FORM

\begin{tabular}{|c|c|c|c|c|c|}
\hline \multicolumn{2}{|c|}{ Site 296-S-21 Model } & \multicolumn{3}{|c|}{ Run No. GT-6 } & \\
\hline Date & $7 / 31 / 2006$ & \multicolumn{3}{|c|}{ Fan Configuration $\overline{1,4}$} & \\
\hline \multicolumn{2}{|c|}{ Tester $\overline{\mathrm{Jgd}}$} & Fan Setting & 56,52 & $\mathrm{~Hz}$ & \\
\hline Stack Dia. & $177 / 8$ in. & Stack Temp & & $78.6 \operatorname{deg} \mathrm{F}$ & \\
\hline \multirow{4}{*}{$\begin{array}{r}\text { Stack X-Area } \\
\text { Elevation } \\
\text { to disturbance } \\
\text { surement units }\end{array}$} & 250.9 in. $^{2}$ & Start/End Time & $1130 / 1220$ & & \\
\hline & N.A. & Center $2 / 3$ from & 1.64 & to: & 16.23 \\
\hline & 77.75 inches & Points in Center $2 / 3$ & 2 & to: & 7 \\
\hline & ppm SF6 & Injection Point & Fan 4 & & \\
\hline
\end{tabular}

\begin{tabular}{|c|c|c|c|c|c|c|c|c|c|}
\hline \multirow{3}{*}{$\begin{array}{l}\text { Traverse--> } \\
\text { Trial ----> }\end{array}$} & & & & & & & & & \\
\hline & & \multicolumn{4}{|c|}{ Port_4_Bottom/East } & \multicolumn{4}{|c|}{ Port_4_Side/South } \\
\hline & & \multicolumn{2}{|r|}{2} & 3 & Mean & 1 & $\overline{2}$ & 3 & Mean \\
\hline Point & Depth, in. & \multicolumn{4}{|c|}{ ppm } & \multicolumn{4}{|c|}{ ppm } \\
\hline 1 & 0.58 & 2.12 & 2.11 & 2.05 & 2.093 & 2.12 & 2.09 & 2.11 & 2.107 \\
\hline 2 & 1.89 & 2.22 & 2.09 & 2.07 & 2.127 & 2.10 & 2.08 & 2.12 & 2.100 \\
\hline 3 & 3.49 & 2.07 & 2.09 & 2.06 & 2.073 & 2.10 & 2.07 & 2.10 & 2.090 \\
\hline 4 & 5.81 & 2.08 & 2.12 & 2.07 & 2.090 & 2.10 & 2.08 & 2.10 & 2.093 \\
\hline Center & 9.00 & 2.07 & 2.08 & 2.05 & 2.067 & 2.10 & 2.05 & 2.08 & 2.077 \\
\hline 5 & 12.19 & 2.05 & 2.09 & 2.02 & 2.053 & 2.02 & 1.99 & 2.03 & 2.013 \\
\hline 6 & 14.51 & 2.03 & 2.02 & 2.02 & 2.023 & 1.97 & 1.97 & 2.00 & 1.980 \\
\hline 7 & 16.11 & 2.07 & 1.99 & 2.04 & 2.033 & 2.26 & 1.99 & 1.99 & 2.080 \\
\hline 8 & 17.42 & 2.10 & 2.07 & 2.08 & 2.083 & 1.97 & 1.95 & 2.00 & 1.973 \\
\hline Averages --- & $---->$ & 2.090 & 2.073 & 2.051 & 2.071 & 2.082 & 2.030 & 2.059 & 2.057 \\
\hline
\end{tabular}

\begin{tabular}{|lll|lrrrr|}
\hline All & ppm & Dev. from mean & Center 2/3 & & East & South & All \\
Mean & 2.06 & & & Mean & 2.07 & 2.06 & 2.06 \\
Min Point & 1.97 & $-4.4 \%$ & Std. Dev. & 0.04 & 0.05 & 0.04 \\
\hline Max Point & 2.13 & $3.0 \%$ & Cov as \% & 1.7 & 2.2 & 1.9 \\
\hline
\end{tabular}

Avg. Conc. $2.063 \mathrm{ppm}$

\begin{tabular}{|c|c|c|c|}
\hline \multirow{3}{*}{$\begin{array}{l}\text { Tracer tank pressure } \\
\text { Stack Temp }\end{array}$} & Start & Finish & \multirow{3}{*}{$\begin{array}{l}\text { psig } \\
\mathrm{F}^{\mathrm{o}}\end{array}$} \\
\hline & 300 & 300 & \\
\hline & 77.2 & 80 & \\
\hline \multirow{3}{*}{$\begin{array}{l}\text { Ref. Pt. air vel. } \\
\text { Injection flowmeter } \\
\text { Stack flow }\end{array}$} & na & 2320 & \multirow{7}{*}{$\begin{array}{l}\text { fpm } \\
\text { ball } \\
\text { cfm } \\
\text { Ipm Sierra } \\
\text { in } \mathrm{Hg} \\
\mathrm{RH} \\
\mathrm{Y} / \mathrm{N}\end{array}$} \\
\hline & 10 & 10 & \\
\hline & - & & \\
\hline Sampling flowmeter & 10 & 10 & \\
\hline Ambient pressure & 29.5 & 29.5 & \\
\hline \multirow{2}{*}{$\begin{array}{l}\text { Ambient humidity } \\
\text { B\&K vapor correction }\end{array}$} & 35 & 31 & \\
\hline & $\mathrm{N}$ & $\mathrm{N}$ & \\
\hline \multirow{2}{*}{$\begin{array}{l}\text { Back-Gd gas ppb } \\
\text { No. Bk-Gd samples }\end{array}$} & $27,25,27,34$ & $24,39,24,24$ & \multirow{4}{*}{$n$} \\
\hline & \multicolumn{2}{|c|}{\begin{tabular}{l|l}
4 & 4
\end{tabular}} & \\
\hline Ambient Temp, F & 74 & 79 & \\
\hline \multicolumn{3}{|l|}{ Instuments Used: } & \\
\hline \multicolumn{4}{|c|}{ B \& K Model $1302 \# 1765299$} \\
\hline \multicolumn{4}{|c|}{ Sierra Inc. Constant Flow Air Sampler } \\
\hline \multicolumn{4}{|c|}{ TSI 8360 SN 209060 Cal due 9/27/06 } \\
\hline \multicolumn{4}{|l|}{ Notes: } \\
\hline \multicolumn{4}{|l|}{ ** black glass ball } \\
\hline \multicolumn{4}{|c|}{ Reference point air velocity measured at point 4 So8. } \\
\hline
\end{tabular}

\section{Gas analyzer checked:}

25-Jul-06

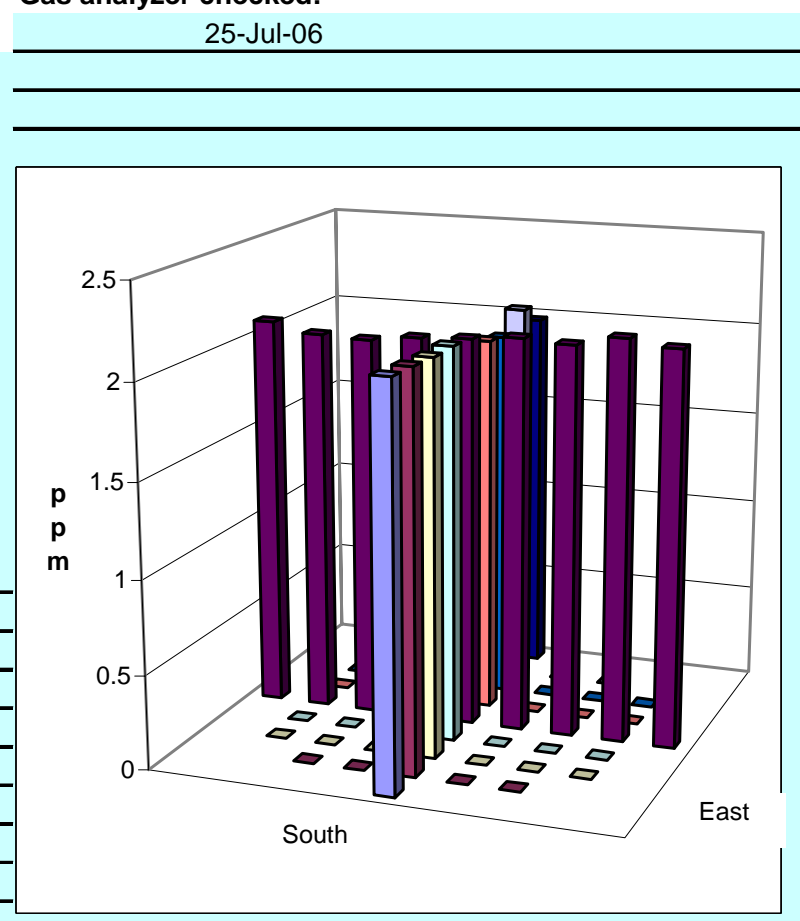

Signature signifies compliance with Procedure EMS-JAG-01

Signature/date

JGD
Signature verifying data and calculations:

$7 / 31 / 2006$

Signatures on file copy 
TRACER GAS TRAVERSE DATA FORM

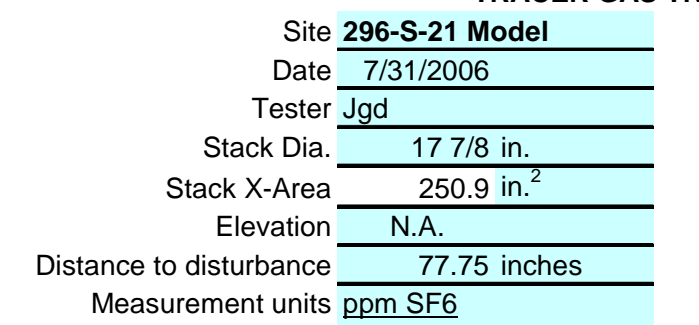

Site 296-S-21 Model

Date 7/31/2006

Tester Jgd

Stack X-Area

Elevation

Mpm SF6
Run No. GT-7

Fan Configuration 2,4

Fan Setting $\mathbf{4 5 , 5 2}$

Stack Temp

Start/End Time 1225/1325

Center 2/3 from

Points in Center $2 / 3$

Injection Point Fan 4

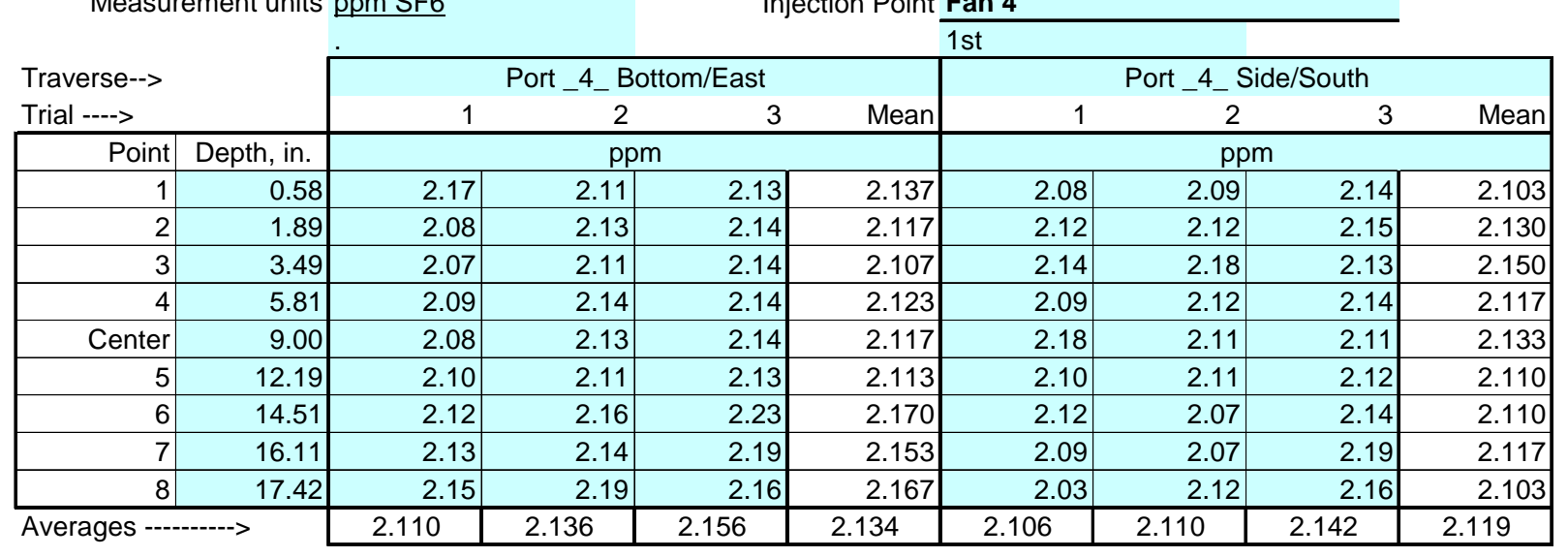

\begin{tabular}{|c|c|c|c|c|c|c|}
\hline$A / I$ & $\mathrm{ppm}$ & Dev. from mean & Center $2 / 3$ & East & South & All \\
\hline Mean & 2.13 & & Mean & 2.13 & 2.12 & 2.13 \\
\hline Min Point & 2.10 & $-1.1 \%$ & Std. Dev. & 0.02 & 0.01 & 0.02 \\
\hline Max Point & 2.17 & $2.0 \%$ & COV as $\%$ & 1.1 & 0.7[ & 0.9 \\
\hline
\end{tabular}

Avg. Conc. $\quad 2.127 \mathrm{ppm}$

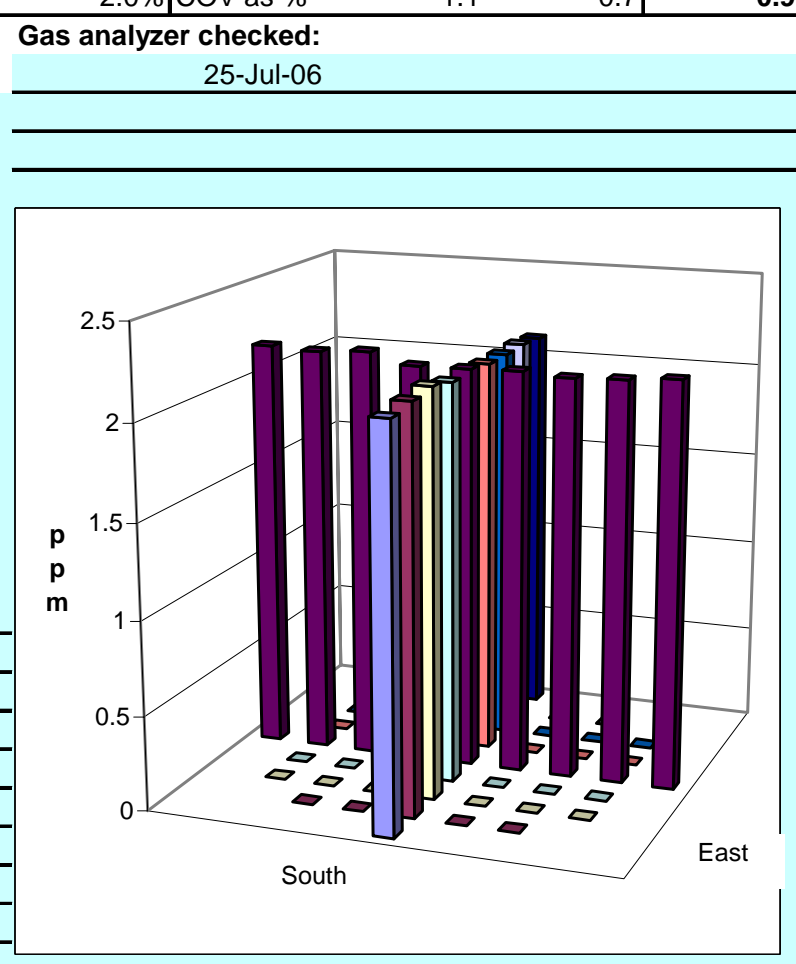

Tracer tank pressure

Stack Temp

Ref. Pt. air vel.

Injection flowmeter

Stack flow

Sampling flowmeter

Ambient pressure

Ambient humidity

B\&K vapor correction

Back-Gd gas ppb

No. Bk-Gd samples

Ambient Temp, F

Instuments Used:

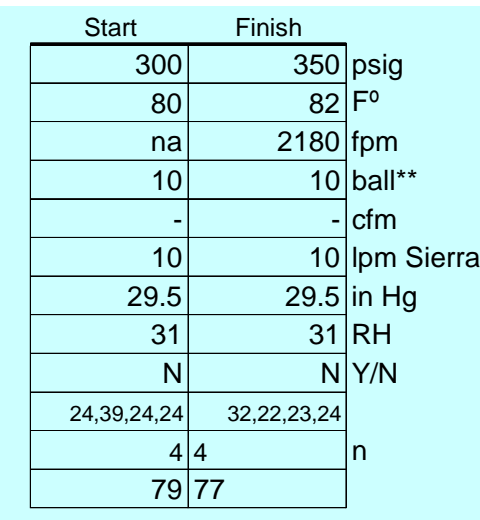

B \& K Model 1302 \#1765299

Sierra Inc. Constant Flow Air Sampler

TSI 8360 SN 209060 Cal due 9/27/06

\begin{tabular}{l} 
Notes: \\
${ }^{\star \star}$ black glass ball \\
\hline Reference point air velocity measured at point 4 So8. \\
\hline
\end{tabular}

Signature signifies compliance with Procedure EMS-JAG-01

Signature/date

JGD

Signature verifying data and calculations:

$7 / 31 / 2006$

Signatures on file copy 
TRACER GAS TRAVERSE DATA FORM

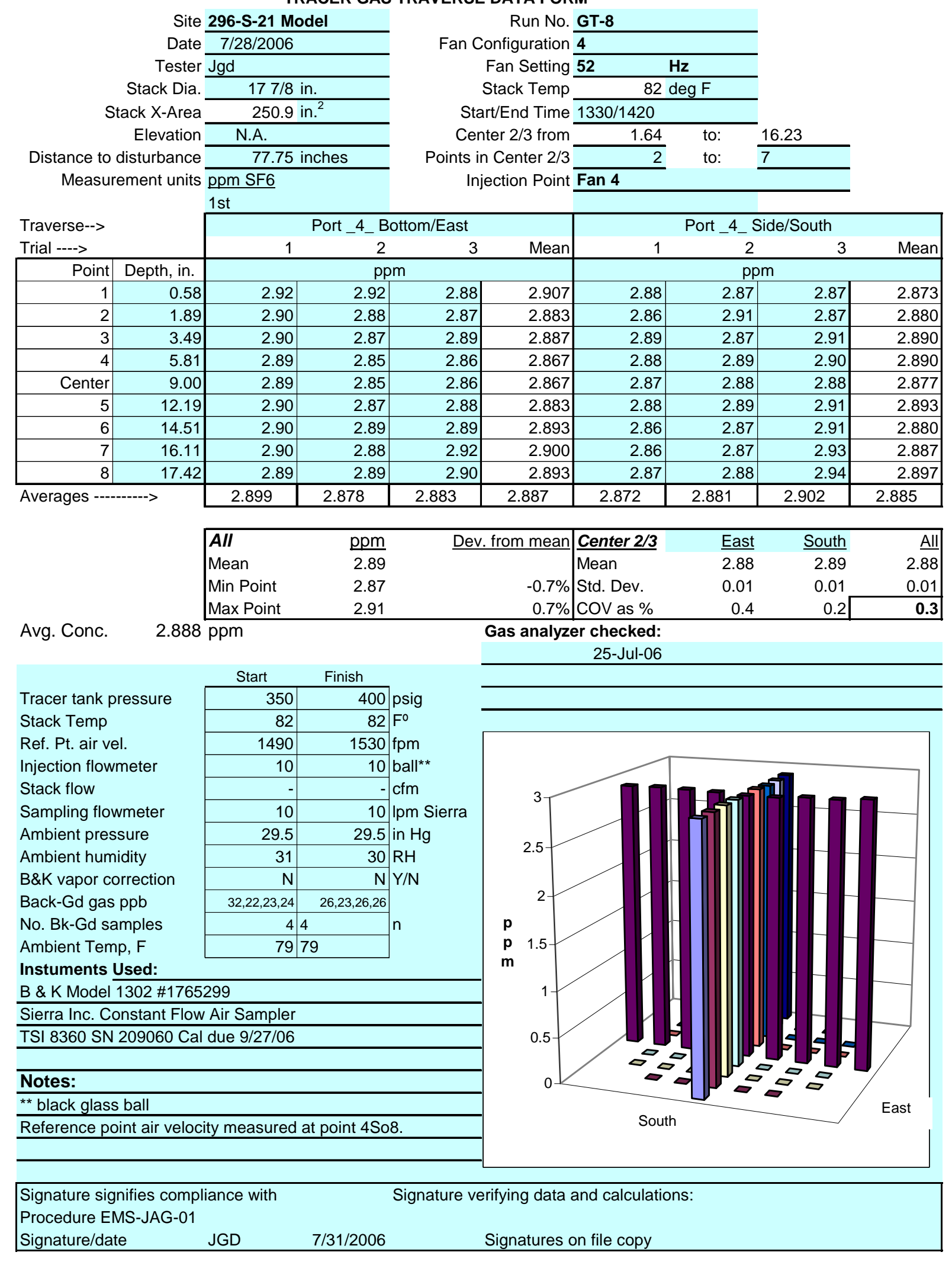


TRACER GAS TRAVERSE DATA FORM

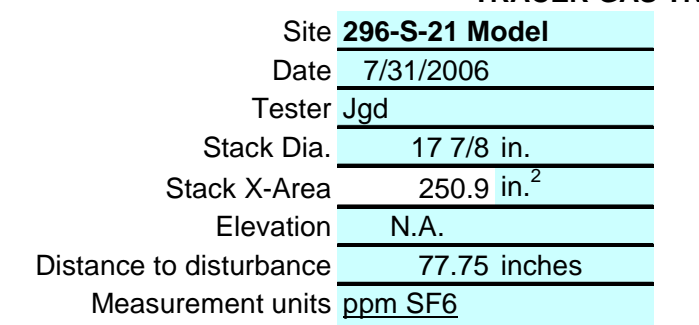

Site 296-S-21 Model

Date 7/31/2006

Tester Jgd

Stack Dia

$177 / 8$ in.

X-Area
Run No. GT-9

Fan Configuration $1,2,3$

Fan Setting $\mathbf{5 6 , 4 5 . 5 0 ~} \mathrm{Hz}$

Stack Temp

Start/End Time $1425 / 1525$

Center $2 / 3$ from

Points in Center $2 / 3$

Injection Point Fan 3

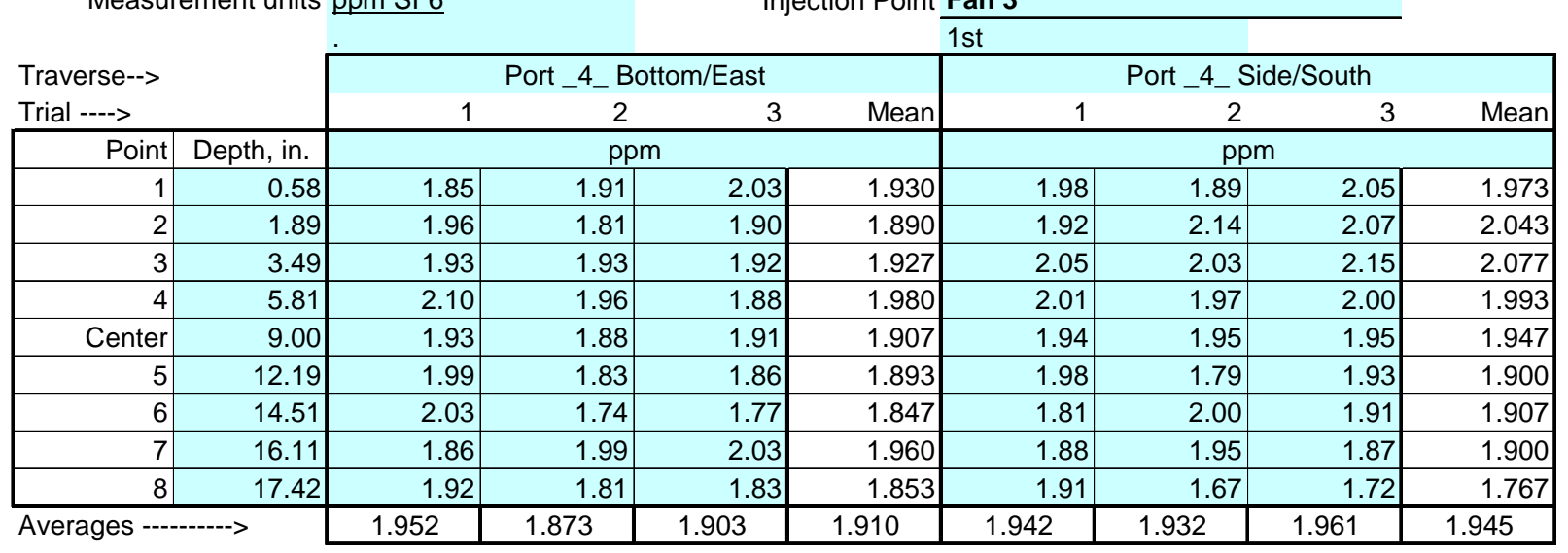

\begin{tabular}{|c|c|c|c|c|c|c|}
\hline$A I I$ & $\mathrm{ppm}$ & Dev. from mean & Center 2/3 & East & South & All \\
\hline Mean & 1.93 & & Mean & 1.91 & 1.97 & 1.94 \\
\hline Min Point & 1.77 & $-8.3 \%$ & Std. Dev. & 0.05 & 0.07 & 0.06 \\
\hline Max Point & 2.08 & $7.7 \%$ & Cov as $\%$ & 2.4 & 3.7[ & 3.3 \\
\hline
\end{tabular}

Avg. Conc. $\quad 1.928 \mathrm{ppm}$

\begin{tabular}{|c|c|c|c|}
\hline \multirow{3}{*}{$\begin{array}{l}\text { Tracer tank pressure } \\
\text { Stack Temp }\end{array}$} & Start & Finish & \multirow{3}{*}{$\begin{array}{l}\text { psig } \\
\mathrm{F}^{\circ}\end{array}$} \\
\hline & 400 & 400 & \\
\hline & 88 & 87 & \\
\hline \multirow{3}{*}{$\begin{array}{l}\text { Ref. Pt. air vel. } \\
\text { Injection flowmeter } \\
\text { Stack flow }\end{array}$} & 2270 & 2340 & \multirow{3}{*}{ 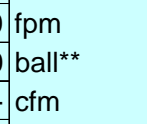 } \\
\hline & 10 & 10 & \\
\hline & - & & \\
\hline \multirow{2}{*}{$\begin{array}{l}\text { Sampling flowmeter } \\
\text { Ambient pressure }\end{array}$} & 10 & 10 & \multirow{4}{*}{$\begin{array}{l}\text { lpm Sierra } \\
\text { in } \mathrm{Hg} \\
\mathrm{RH} \\
\mathrm{Y} / \mathrm{N}\end{array}$} \\
\hline & 29.5 & 29.5 & \\
\hline \multirow{2}{*}{$\begin{array}{l}\text { Ambient humidity } \\
B \& K \text { vapor correction }\end{array}$} & 25 & 25 & \\
\hline & $\mathrm{N}$ & $\mathrm{N}$ & \\
\hline Back-Gd gas ppb & $26,23,26,26$ & $33,49,55,54,49$ & \multirow{4}{*}{$n$} \\
\hline \multicolumn{3}{|l|}{ No. Bk-Gd samples } & \\
\hline Ambient Temp, F & \multicolumn{2}{|c|}{8484} & \\
\hline \multicolumn{3}{|l|}{ Instuments Used: } & \\
\hline \multicolumn{4}{|c|}{ B \& K Model $\overline{1302 \# 1765299}$} \\
\hline \multicolumn{4}{|c|}{ Sierra Inc. Constant Flow Air Sampler } \\
\hline \multicolumn{4}{|c|}{ TSI 8360 SN 209060 Cal due 9/27/06 } \\
\hline \multicolumn{4}{|l|}{ Notes: } \\
\hline \multicolumn{4}{|l|}{ ** black glass ball } \\
\hline \multicolumn{4}{|c|}{ Reference point air velocity measured at point 4 So8. } \\
\hline
\end{tabular}

Gas analyzer checked:

25-Jul-06

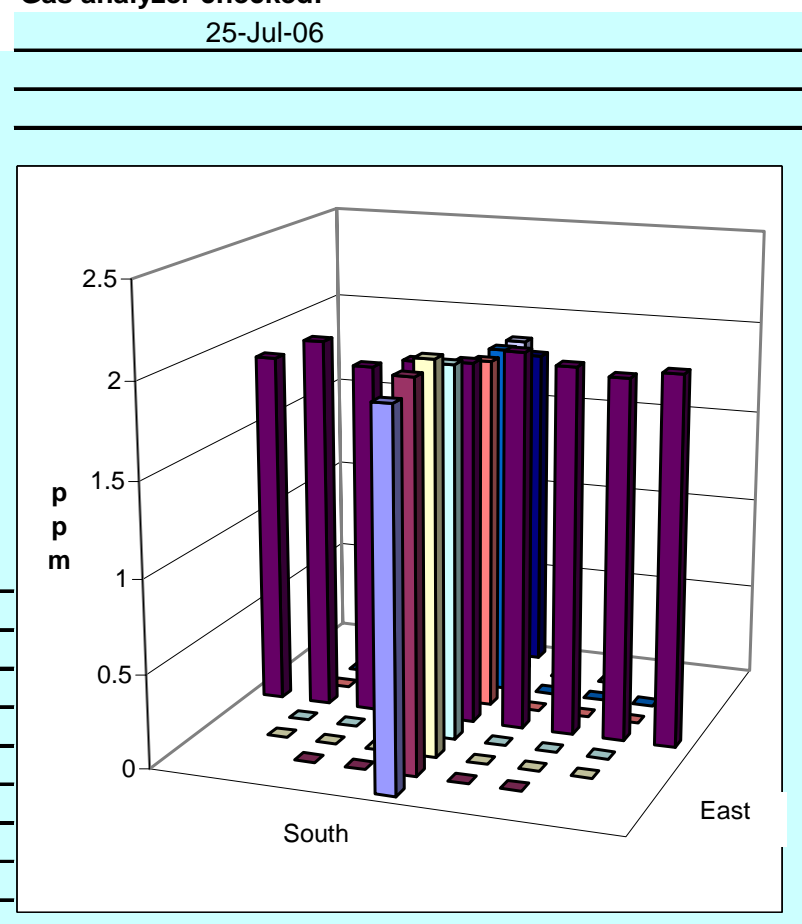

Signature signifies compliance with Procedure EMS-JAG-01

Signature/date
Signature verifying data and calculations:

$7 / 31 / 2006$
Signatures on file copy 


\section{TRACER GAS TRAVERSE DATA FORM}

Site 296-S-21 Model

Date 7/31/2006

Tester Jgd

Stack Dia

Stack X-Area

Elevation

\begin{tabular}{l}
$\frac{\mathrm{Jgd}}{177 / 8 \text { in. }}$ \\
\hline 250.9 in. $^{2}$ \\
\hline N.A. \\
\hline 77.75 inches \\
\hline
\end{tabular}

Distance to disturbance

Measurement units ppm SF6
Run No. GT-10

Fan Configuration $\mathbf{1 , 2 . 3}$

Fan Setting $\mathbf{5 6 , 4 5 , 5 0 \quad \mathrm { Hz }}$

Stack Temp

Start/End Time 1645/1740

Center $2 / 3$ from

Points in Center $2 / 3$

Injection Point Fan 2

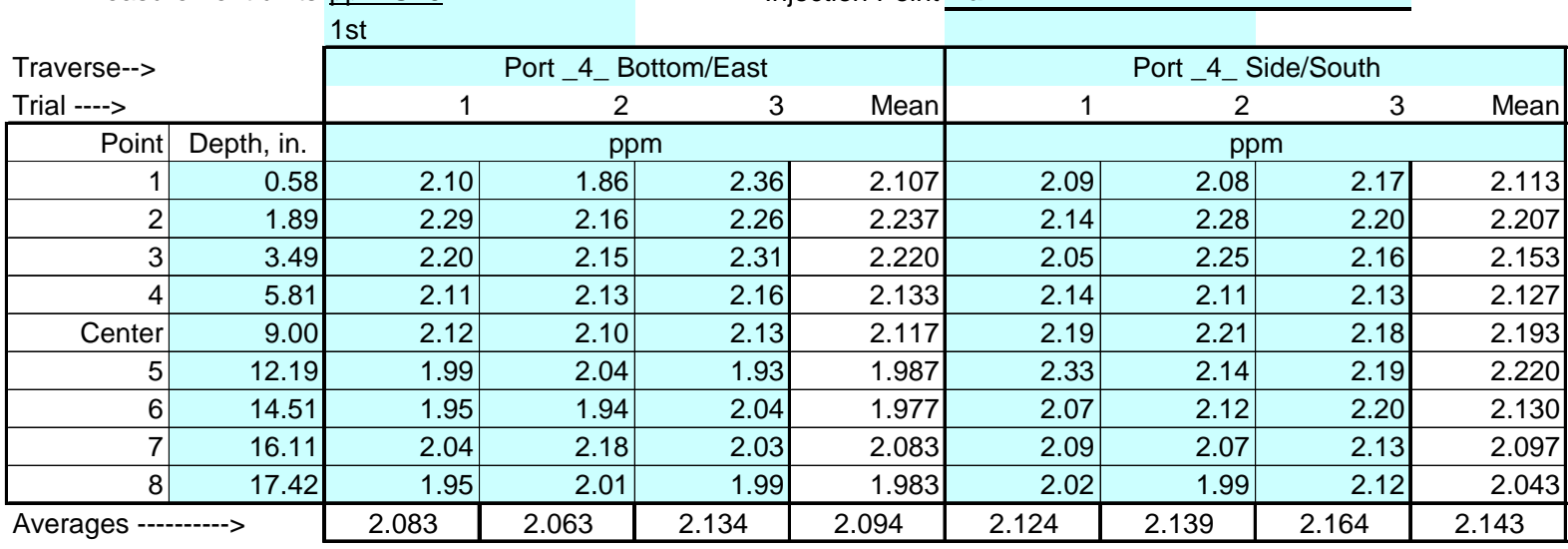

\begin{tabular}{|c|c|c|c|c|c|c|}
\hline$A I I$ & $\mathrm{ppm}$ & Dev. from mean & Center 2/3 & East & South & All \\
\hline Mean & 2.12 & & Mean & 2.11 & 2.16 & 2.13 \\
\hline Min Point & 1.98 & $-6.7 \%$ & Std. Dev. & 0.10 & 0.05 & 0.08 \\
\hline Max Point & 2.24 & $5.6 \%$ & cov as $\%$ & 4.8 & 2.1 & 3.8 \\
\hline
\end{tabular}

Avg. Conc. $\quad 2.114 \mathrm{ppm}$

\begin{tabular}{|c|c|c|c|}
\hline \multirow{3}{*}{$\begin{array}{l}\text { Tracer tank pressure } \\
\text { Stack Temp }\end{array}$} & Start & Finish & \\
\hline & 400 & 400 & \\
\hline & 89 & 85 & $F^{\circ}$ \\
\hline \multirow{3}{*}{$\begin{array}{l}\text { Ref. Pt. air vel. } \\
\text { Injection flowmeter } \\
\text { Stack flow }\end{array}$} & 2240 & 2390 & \multirow{3}{*}{$\begin{array}{l}\text { fpm } \\
\text { ball** } \\
\text { cfm }\end{array}$} \\
\hline & 10 & 10.5 & \\
\hline & - & & \\
\hline Sampling flowmeter & 10 & 10 & Ipm Sierra \\
\hline \multirow{2}{*}{$\begin{array}{l}\text { Ambient pressure } \\
\text { Ambient humidity }\end{array}$} & 29.4 & 29.4 & \multirow{3}{*}{$\begin{array}{l}\text { in } \mathrm{Hg} \\
\mathrm{RH} \\
\mathrm{Y} / \mathrm{N}\end{array}$} \\
\hline & 28 & 27 & \\
\hline \multirow{2}{*}{$\begin{array}{l}\text { B\&K vapor correction } \\
\text { Back-Gd gas ppb }\end{array}$} & $\mathrm{N}$ & $\mathrm{N}$ & \\
\hline & $31,71,52,34$ & $44,52,71,28$ & \multirow{3}{*}{ n } \\
\hline No. Bk-Gd samples & & 4 & \\
\hline Ambient Temp, F & 80 & 83 & \\
\hline
\end{tabular}

Instuments Used:

B \& K Model 1302 \#1765299

Sierra Inc. Constant Flow Air Sampler

TSI 8360 SN 209060 Cal due 9/27/06

\begin{tabular}{l} 
Notes: \\
\hline R* black glass ball \\
\hline Reference point air velocity measured at point 4 So8. \\
\hline
\end{tabular}

Gas analyzer checked:

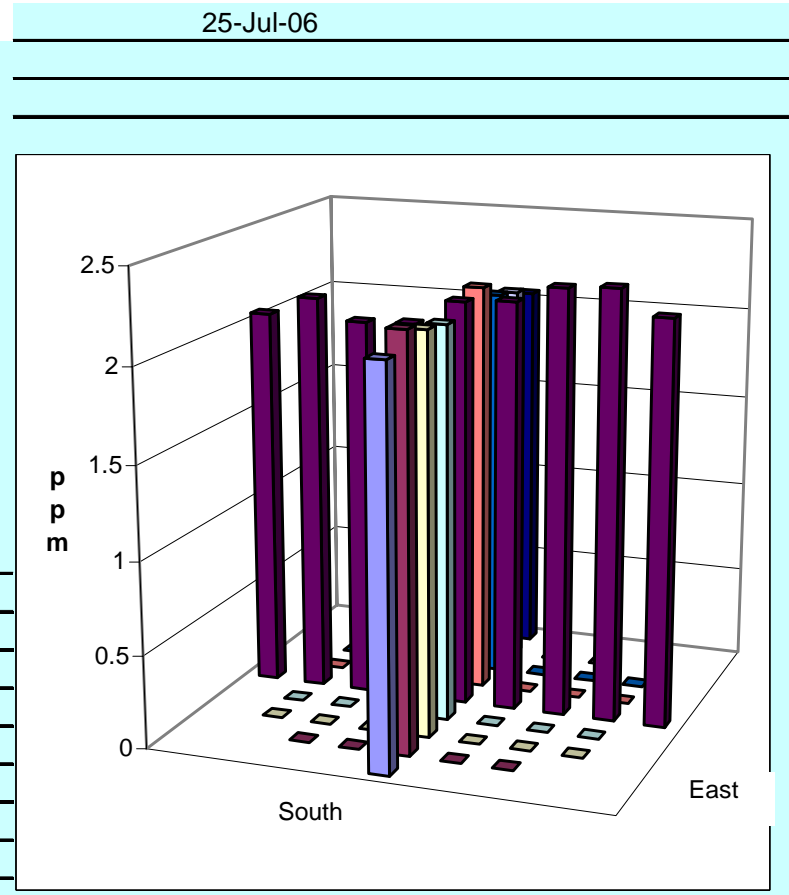

Signature signifies compliance with Procedure EMS-JAG-01

Signature/date

JAG

Signature verifying data and calculations:

$7 / 31 / 2006$
Signatures on file copy 


\section{TRACER GAS TRAVERSE DATA FORM}

Site 296-S-21 Model

Date 8/1/2006

Tester Jgd

Stack Dia.

Stack X-Area

Elevation

Distance to disturbance

Measurement units ppm SF6
Run No. GT-11

Fan Configuration $1,2.3$

Fan Setting $\mathbf{5 6 , 4 5 , 5 0 \quad \mathrm { Hz }}$

Stack Temp

Start/End Time 0950/1040

Center $2 / 3$ from

Points in Center 2/3

Injection Point Fan 1 Center

\begin{tabular}{|c|c|c|c|c|c|c|c|c|c|}
\hline \multirow{3}{*}{$\begin{array}{l}\text { Traverse--> } \\
\text { Trial ----> }\end{array}$} & & & & & & \multicolumn{4}{|l|}{$1 s t$} \\
\hline & & \multicolumn{4}{|c|}{ Port 4 Bottom/East } & \multicolumn{4}{|c|}{ Port 4 Side/South } \\
\hline & & \multicolumn{2}{|c|}{1} & \multirow{2}{*}{\multicolumn{2}{|c|}{ Mean }} & 1 & 2 & 3 & Mean \\
\hline Point & Depth, in. & \multicolumn{2}{|c|}{ ppm } & & & \multicolumn{4}{|c|}{ ppm } \\
\hline 1 & 0.58 & 1.82 & 1.82 & 1.83 & 1.823 & 1.86 & 1.84 & 1.87 & 1.857 \\
\hline 2 & 1.89 & 1.83 & 1.80 & 1.76 & 1.797 & 1.91 & 1.84 & 1.83 & 1.860 \\
\hline 3 & 3.49 & 1.78 & 1.77 & 1.77 & 1.773 & 1.91 & 1.88 & 1.84 & 1.877 \\
\hline 4 & 5.81 & 1.79 & 1.80 & 1.81 & 1.800 & 1.91 & 1.96 & 1.93 & 1.933 \\
\hline Center & 9.00 & 1.89 & 1.92 & 1.83 & 1.880 & 1.92 & 1.97 & 1.95 & 1.947 \\
\hline 5 & 12.19 & 1.96 & 1.95 & 1.96 & 1.957 & 2.02 & 2.04 & 1.98 & 2.013 \\
\hline 6 & 14.51 & 2.01 & 1.97 & 2.02 & 2.000 & 2.04 & 2.01 & 2.02 & 2.023 \\
\hline 7 & 16.11 & 2.02 & 1.99 & 2.01 & 2.007 & 2.10 & 2.10 & 2.14 & 2.113 \\
\hline 8 & 17.42 & 2.00 & 2.06 & 1.99 & 2.017 & 2.09 & 2.09 & 2.07 & 2.083 \\
\hline Averages --- & $-\overline{---->}$ & 1.900 & 1.898 & 1.887 & 1.895 & 1.973 & 1.970 & 1.959 & 1.967 \\
\hline
\end{tabular}

\begin{tabular}{|lr}
\hline All & ppm \\
Mean & 1.93 \\
Min Point & 1.77 \\
Max Point & 2.11 \\
\hline
\end{tabular}

Avg. Conc.

2.11
Dev. from mean $\underline{\text { Center 2/3 }}$
Mean
$-8.2 \%$ Std. Dev.
9.4\% COV as \%

\begin{tabular}{rrr} 
East & South & All \\
1.89 & 1.97 & 1.93 \\
0.10 & 0.09 & 0.10 \\
& 4.5 & $\mathbf{5 . 2}$ \\
\hline
\end{tabular}

Gas analyzer checked:

25-Jul-06

Tracer tank pressure

Stack Temp

Ref. Pt. air vel.

Injection flowmeter

Stack flow

Sampling flowmeter

Ambient pressure

Ambient humidity

B\&K vapor correction

Back-Gd gas ppb

No. Bk-Gd samples

Ambient Temp, $\mathrm{F}$

Instuments Used:

\begin{tabular}{|c|c|c|}
\hline Start & Finish & \\
\hline 290 & 300 & psig \\
\hline 78 & 80 & $F^{\circ}$ \\
\hline 2320 & 2290 & $\mathrm{fpm}$ \\
\hline 10 & 9 & ball ${ }^{\star \star}$ \\
\hline- & - & $\mathrm{cfm}$ \\
\hline 10 & 10 & Ipm Sierra \\
\hline 29.6 & 29.5 & in $\mathrm{Hg}$ \\
\hline 41 & 37 & $\mathrm{RH}$ \\
\hline $\mathrm{N}$ & $\mathrm{N}$ & $\mathrm{Y} / \mathrm{N}$ \\
\hline
\end{tabular}

40, 49, 53, 31, $2975,86,125,104,90$

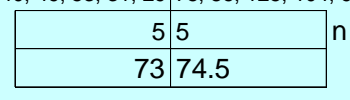

B \& K Model 1302 \#1765299

Sierra Inc. Constant Flow Air Sampler

TSI 8360 SN 209060 Cal due 9/27/06

\section{Notes:}

** black glass ball

Reference point air velocity measured at point $4 \mathrm{So} 8$.

Signature signifies compliance with

Procedure EMS-JAG-01

Signature/date

JGD

Signature verifying data and calculations:

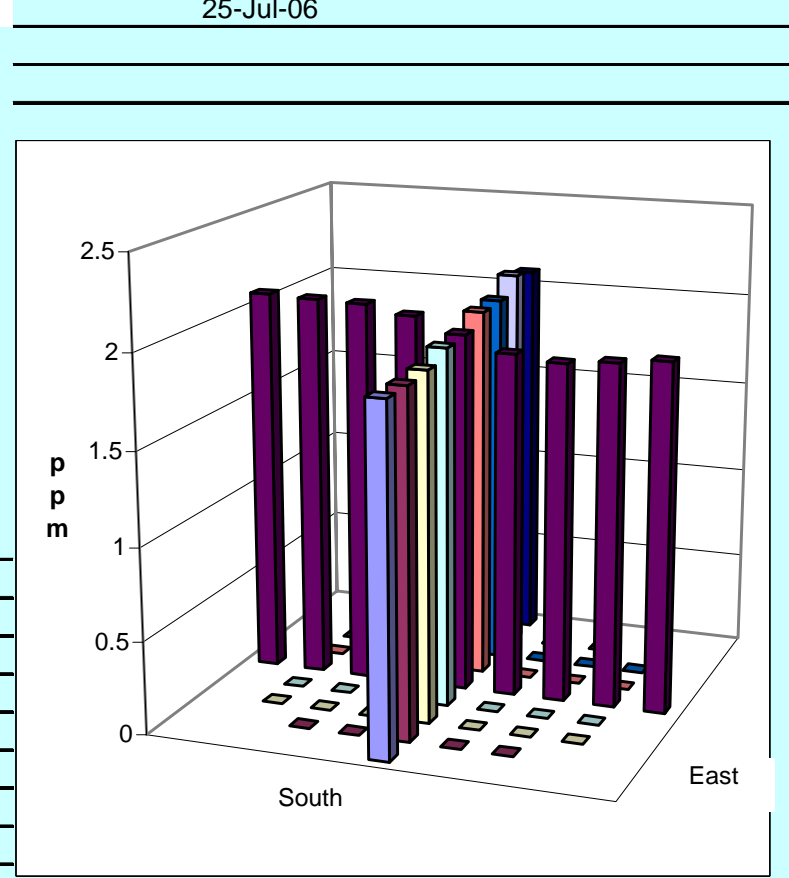

ast

8/1/2006 Signatures on file copy




\section{TRACER GAS TRAVERSE DATA FORM}

Site 296-S-21 Model

Date 8/1/2006

Tester Jgd

Stack Dia.

Stack X-Area

Elevation

$177 / 8$ in

250.9 in. $^{2}$
Distance to disturbance

Measurement units ppm
Run No. GT-12

Fan Configuration $1,2.3$

Fan Setting $\mathbf{5 6 , 4 5 , 5 0 \quad ~} \mathbf{H z}$

Stack Temp

Start/End Time 1110/1300

Center $2 / 3$ from

Points in Center $2 / 3$

Injection Point Fan 1 Bottom N (NE)

\begin{tabular}{|c|c|c|c|c|c|c|c|c|c|}
\hline \multirow{4}{*}{$\begin{array}{l}\text { Traverse--> } \\
\text { Trial ----> }\end{array}$} & & \multirow{2}{*}{\multicolumn{8}{|c|}{$1 \mathrm{st}$}} \\
\hline & & & & & & & & & \\
\hline & & \multicolumn{4}{|c|}{ Port_4_Bottom/East } & \multicolumn{4}{|c|}{ Port_4_Side/South } \\
\hline & & 1 & 2 & 3 & Mean & 1 & 2 & 3 & Mean \\
\hline Point & Depth, in. & \multicolumn{4}{|c|}{ ppm } & \multicolumn{4}{|c|}{ ppm } \\
\hline 1 & 0.58 & 1.84 & 1.69 & 1.92 & 1.817 & 1.93 & 1.95 & 1.99 & 1.957 \\
\hline 2 & 1.89 & 1.90 & 2.04 & 1.80 & 1.913 & 2.04 & 1.97 & 2.06 & 2.023 \\
\hline 3 & 3.49 & 1.76 & 1.73 & 1.91 & 1.800 & 1.98 & 2.06 & 1.97 & 2.003 \\
\hline 4 & 5.81 & 1.83 & 1.86 & 1.80 & 1.830 & 1.96 & 2.19 & 1.92 & 2.023 \\
\hline Center & 9.00 & 2.02 & 1.97 & 2.12 & 2.037 & 2.12 & 2.04 & 2.04 & 2.067 \\
\hline 5 & 12.19 & 1.98 & 1.91 & 1.97 & 1.953 & 2.10 & 2.10 & 1.99 & 2.063 \\
\hline 6 & 14.51 & 2.03 & 1.98 & 2.13 & 2.047 & 2.19 & 2.08 & 2.11 & 2.127 \\
\hline 7 & 16.11 & 1.96 & 2.08 & 2.05 & 2.030 & 2.06 & 2.13 & 2.13 & 2.107 \\
\hline 8 & 17.42 & 2.00 & 2.04 & 2.03 & 2.023 & 2.19 & 2.21 & 2.09 & 2.163 \\
\hline Averages ---- & $-\cdots$ & 1.924 & 1.922 & 1.970 & 1.939 & 2.063 & 2.081 & 2.033 & 2.059 \\
\hline
\end{tabular}

\begin{tabular}{|lr}
\hline All & $\mathrm{ppm}$ \\
Mean & 2.00 \\
Min Point & 1.80 \\
Max Point & 2.16 \\
\hline
\end{tabular}

Avg. Conc.
Dev. from mean Center 2/3
Mean
$-10.0 \%$ Std. Dev.
$8.2 \%$ COV as $\%$

\begin{tabular}{|c|c|c|}
\hline East & South & Al \\
\hline 1.94 & 2.06 & 2.00 \\
\hline 0.10 & 0.05 & 0.10 \\
\hline 5.2 & 2.2 & 4. \\
\hline
\end{tabular}

Gas analyzer checked:

\begin{tabular}{|c|c|c|}
\hline Start & Finish & \\
\hline 300 & 300 & psig \\
\hline 83 & 85.5 & $F^{\circ}$ \\
\hline 2270 & 2260 & fpm \\
\hline 10 & 10 & ball** \\
\hline & - & $\mathrm{cfm}$ \\
\hline 10 & 10 & Ipm Sierra \\
\hline 29.5 & 29.5 & in $\mathrm{Hg}$ \\
\hline 30 & 31 & $\mathrm{RH}$ \\
\hline$Y$ & $Y$ & $\mathrm{Y} / \mathrm{N}$ \\
\hline $0,4,-2,3,0$ & $26,2,24,10$ & \\
\hline 5 & 4 & $n$ \\
\hline 80 & 80.7 & \\
\hline
\end{tabular}

Tracer tank pressure

Stack Temp

Ref. Pt. air vel.

Injection flowmeter

Stack flow

Sampling flowmeter

Ambient pressure

Ambient humidity

B\&K vapor correction

Back-Gd gas ppb

No. Bk-Gd samples

Ambient Temp, F

Instuments Used:

B \& K Model 1302 \#1765299

Sierra Inc. Constant Flow Air Sampler

TSI 8360 SN 209060 Cal due 9/27/06

\section{Notes:}

** black glass ball

Reference point air velocity measured at point 4 So8.

Used 4th traverse data in place of 1st on bottom because

locations may have been wrong in 1st bottom traverse

Signature signifies compliance with Signature verifying data and calculations:

Procedure EMS-JAG-01

Signature/date

JGD

$8 / 1 / 2006$

Signatures on file copy

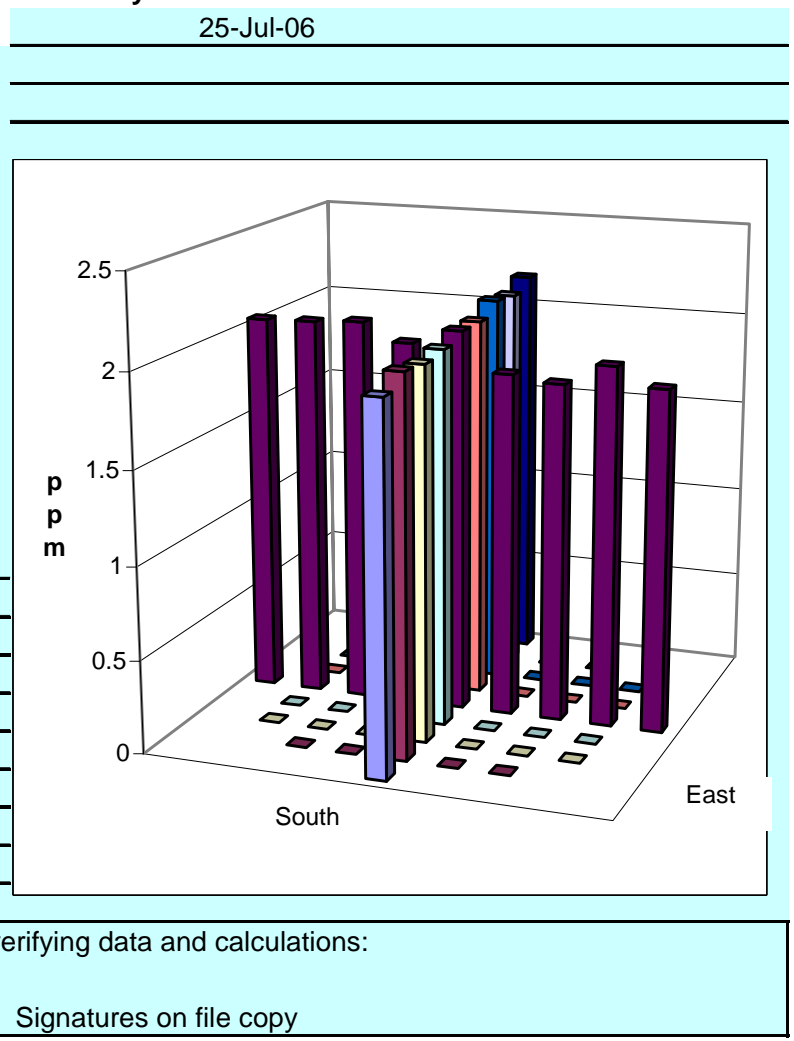




\section{TRACER GAS TRAVERSE DATA FORM}

Site 296-S-21 Model

Date 8/1/2006

Tester Jgd

Stack Dia

Stack X-Area

Elevation

Distance to disturbance

Measurement units ppm SF6
Run No. GT-13

Fan Configuration $\mathbf{1 , 2 . 3}$

Fan Setting $\mathbf{5 6 , 4 5 , 5 0 \quad ~} \mathbf{H z}$

Stack Temp $85.5 \operatorname{deg} F$

Start/End Time 1305/1440

Center $2 / 3$ from

Points in Center $2 / 3$ 1.64 2 to:

to: $\frac{16.23}{7}$

Injection Point Fan 1 Top N (Northwest corner)

\begin{tabular}{|c|c|c|c|c|c|c|c|c|c|}
\hline \multirow{2}{*}{\multicolumn{2}{|c|}{$\begin{array}{l}\text { Traverse--> } \\
\text { Trial ----> }\end{array}$}} & \multicolumn{4}{|c|}{ Port_4_Bottom/East } & \multicolumn{4}{|c|}{ Port_4_Side/South } \\
\hline & & 1 & 2 & 3 & Mean & 1 & 2 & 3 & Mean \\
\hline Point & Depth, in. & \multicolumn{4}{|c|}{$\mathrm{ppm}$} & \multicolumn{4}{|c|}{$\mathrm{ppm}$} \\
\hline 1 & 0.58 & 1.83 & 1.84 & 1.80 & 1.823 & 1.83 & 1.92 & 1.92 & 1.890 \\
\hline 2 & 1.89 & 1.89 & 1.89 & 2.05 & 1.943 & 1.96 & 1.93 & 2.06 & 1.983 \\
\hline 3 & 3.49 & 2.01 & 2.06 & 1.81 & 1.960 & 2.06 & 2.08 & 1.87 & 2.003 \\
\hline 4 & 5.81 & 1.82 & 2.01 & 2.01 & 1.947 & 2.05 & 2.03 & 1.91 & 1.997 \\
\hline Center & 9.00 & 2.16 & 1.95 & 1.93 & 2.013 & 2.04 & 2.07 & 2.00 & 2.037 \\
\hline 5 & 12.19 & 1.96 & 2.13 & 2.21 & 2.100 & 1.93 & 2.04 & 2.01 & 1.993 \\
\hline 6 & 14.51 & 2.21 & 2.20 & 2.19 & 2.200 & 2.02 & 2.03 & 2.02 & 2.023 \\
\hline 7 & 16.11 & 2.11 & 2.16 & 2.27 & 2.180 & 2.22 & 2.07 & 2.16 & 2.150 \\
\hline 8 & 17.42 & 2.17 & 2.15 & 2.08 & 2.133 & 2.23 & 2.10 & 2.17 & 2.167 \\
\hline Averages ---- & - & 2.018 & 2.043 & 2.039 & 2.033 & 2.038 & 2.030 & 2.013 & 2.027 \\
\hline
\end{tabular}

Avg. Conc.

\begin{tabular}{|lr}
\hline AlI & $\mathrm{ppm}$ \\
Mean & 2.03 \\
Min Point & 1.82 \\
Max Point & 2.20 \\
\hline
\end{tabular}

$$
2.031 \mathrm{ppm}
$$

Tracer tank pressure Stack Temp

Ref. Pt. air vel. Injection flowmeter Stack flow

Sampling flowmeter Ambient pressure Ambient humidity B\&K vapor correction Back-Gd gas ppb No. Bk-Gd samples Ambient Temp, $\mathrm{F}$ Instuments Used:

B \& K Model 1302 \#1765299

Sierra Inc. Constant Flow Air Sampler

TSI 8360 SN 209060 Cal due 9/27/06

\section{Notes:}

** black glass ball

Reference point air velocity measured at point 4 So 8 .

\begin{tabular}{|c|c|c|}
\hline Start & Finish & \\
\hline 300 & 300 & \multirow{2}{*}{$\frac{10}{5}$} \\
\hline 85.5 & 85.5 & \\
\hline 2260 & 2340 & \\
\hline 10 & 10 & \\
\hline - & - & \\
\hline 10 & 10 & \\
\hline 29.5 & 29.5 & \\
\hline 31 & 29 & \\
\hline$Y$ & $Y$ & \\
\hline $26,2,24,10,6$ & $7,4,3,1,1$ & \\
\hline 5 & 5 & \\
\hline 80.7 & 82.7 & \\
\hline
\end{tabular}
Procedure EMS-JAG-01 Signature/date

JGD

Signature signifies compliance with
JGD
Dev. from mean Center 2/3
Mean
$-10.2 \%$ Std. Dev.
$8.4 \%$ COV as $\%$

Gas analyzer checked:

25-Jul-06

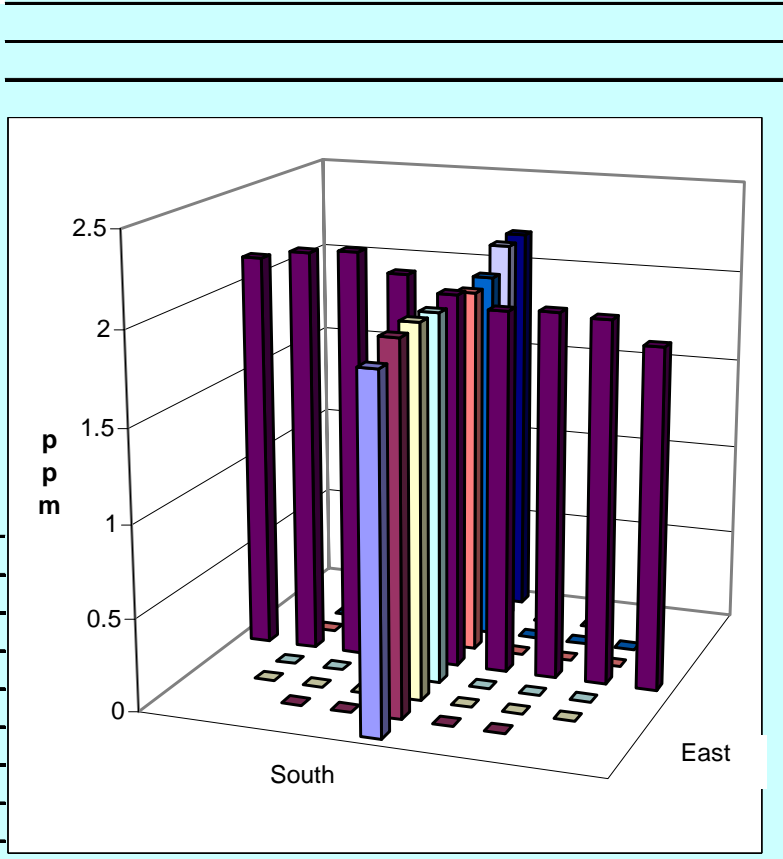

Signature verifying data and calculations:

Signatures on file copy 
TRACER GAS TRAVERSE DATA FORM

\begin{tabular}{|c|c|}
\hline \multicolumn{2}{|c|}{ Site 296-S-21 Model } \\
\hline Date & $8 / 1 / 2006$ \\
\hline Tester & \\
\hline Stack Dia. & $177 / 8$ in. \\
\hline Stack X-Area & 250.9 in. $^{2}$ \\
\hline Elevation & N.A. \\
\hline to disturbance & 77.75 inches \\
\hline
\end{tabular}

Run No. GT-14

Fan Configuration $\mathbf{1 , 2 . 3}$

Fan Setting $\mathbf{5 6 , 4 5 , 5 0 \quad \mathrm { Hz }}$

Stack Temp $89.8 \mathrm{deg} F$

Start/End Time 1620/1740

Center $2 / 3$ from

Points in Center $2 / 3$

Injection Point Fan 1 Southwest corner

Distance to disture
Measurement units ppm SF6

\begin{tabular}{|c|c|c|c|c|c|c|c|c|c|}
\hline \multirow{3}{*}{$\begin{array}{l}\text { Traverse--> } \\
\text { Trial ----> }\end{array}$} & & & & & & & & & \\
\hline & & \multicolumn{4}{|c|}{ Port_4_Bottom/East } & \multicolumn{4}{|c|}{ Port_4_Side/South } \\
\hline & & \multirow[t]{2}{*}{1} & \multirow{2}{*}{\multicolumn{2}{|c|}{$\frac{2}{\mathrm{ppm}}$}} & \multirow[t]{2}{*}{ Mean } & \multirow[t]{2}{*}{1} & 2 & 3 & Mean \\
\hline Point & Depth, in. & & & & & & $\mathrm{pp}$ & & \\
\hline 1 & 0.58 & 1.77 & 1.78 & 1.86 & 1.803 & 1.85 & 1.83 & 1.76 & 1.813 \\
\hline 2 & 1.89 & 1.75 & 1.83 & 1.87 & 1.817 & 1.94 & 1.85 & 1.80 & 1.863 \\
\hline 3 & 3.49 & 1.73 & 1.80 & 1.83 & 1.787 & 2.02 & 1.84 & 1.77 & 1.877 \\
\hline 4 & 5.81 & 1.83 & 1.86 & 1.89 & 1.860 & 1.86 & 1.99 & 1.80 & 1.883 \\
\hline Center & 9.00 & 1.86 & 1.96 & 1.96 & 1.927 & 1.92 & 2.02 & 1.93 & 1.957 \\
\hline 5 & 12.19 & 1.99 & 2.10 & 2.04 & 2.043 & 2.00 & 1.95 & 2.06 & 2.003 \\
\hline 6 & 14.51 & 2.02 & 2.14 & 2.10 & 2.087 & 2.03 & 1.97 & 2.20 & 2.067 \\
\hline 7 & 16.11 & 2.11 & 2.12 & 2.11 & 2.113 & 2.18 & 2.02 & 2.21 & 2.137 \\
\hline 8 & 17.42 & 2.06 & 2.16 & 2.23 & 2.150 & 2.23 & 2.05 & 2.10 & 2.127 \\
\hline Averages --.. & $--->$ & 1.902 & 1.972 & 1.988 & 1.954 & 2.003 & 1.947 & 1.959 & 1.970 \\
\hline
\end{tabular}

\begin{tabular}{|c|c|c|c|c|c|c|}
\hline$A I I$ & $\mathrm{ppm}$ & Dev. from mean & Center 2/3 & East & South & All \\
\hline Mean & 1.96 & & Mean & 1.95 & 1.97 & 1.96 \\
\hline Min Point & 1.79 & $-8.9 \%$ & Std. Dev. & 0.13 & 0.10 & 0.12 \\
\hline Max Point & 2.15 & $9.6 \%$ & cov as $\%$ & 6.9 & 5.3 & 5.9 \\
\hline
\end{tabular}

Avg. Conc. $\quad 1.964$ ppm

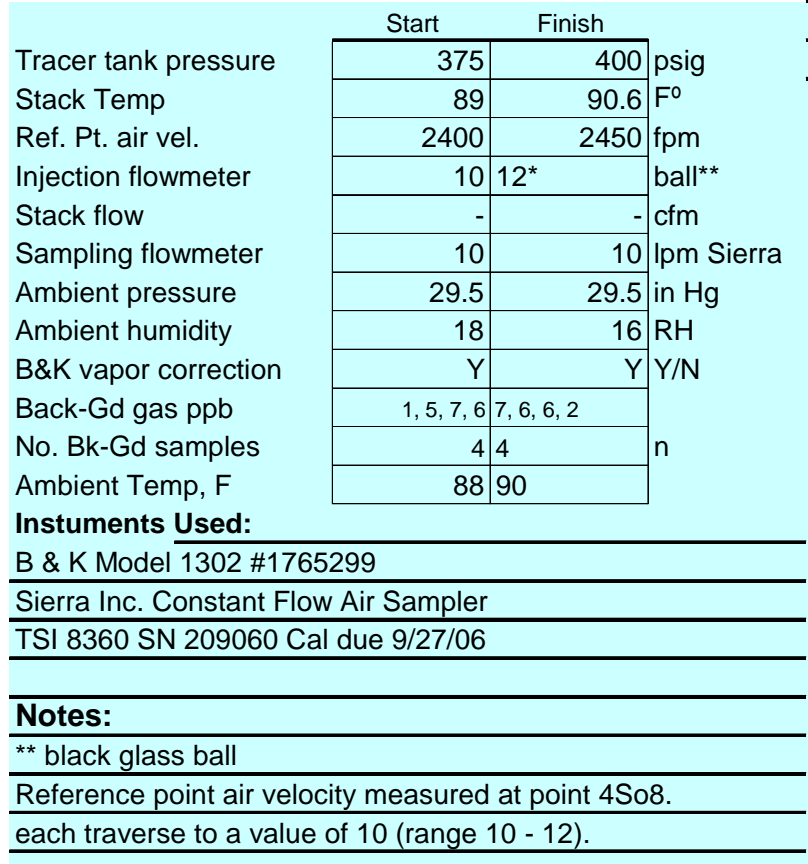

\section{Gas analyzer checked:}

25-Jul-06

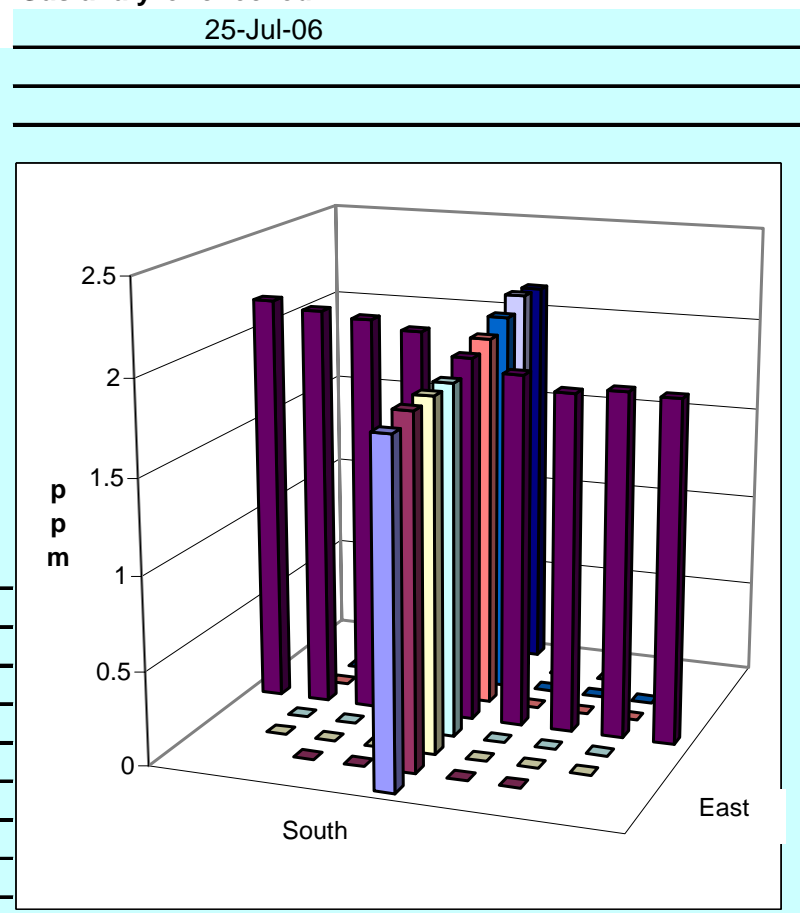

Signature signifies compliance with

Procedure EMS-JAG-01

Signature/date

JGD
Signature verifying data and calculations:
$8 / 1 / 2006$

Signatures on file copy 
TRACER GAS TRAVERSE DATA FORM

\begin{tabular}{|c|c|}
\hline \multicolumn{2}{|c|}{ Site 296-S-21 Model } \\
\hline Date & $8 / 2 / 2006$ \\
\hline Tester & \\
\hline Stack Dia. & $177 / 8$ in. \\
\hline Stack X-Area & 250.9 in. $^{2}$ \\
\hline Elevation & N.A. \\
\hline to disturbance & 77.75 inches \\
\hline
\end{tabular}

Run No. GT-15

Fan Configuration 1, 2, 3

Fan Setting $\frac{\mathbf{5 6 , 4 5 , 5 0} \mathbf{H z}}{20.5 \mathrm{deg}}$

Stack Temp $\frac{76.5 \mathrm{deg} F}{2}$

Start/End Time $0810 / 0948$

Center $2 / 3$ from

Points in Center $2 / 3 \longdiv { 2 }$ to: 7

Injection Point Fan 1 Southeast corner

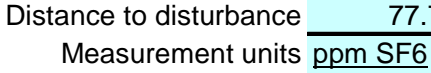

1st

\begin{tabular}{|c|c|c|c|c|c|c|c|c|c|}
\hline \multirow{3}{*}{\multicolumn{2}{|c|}{$\begin{array}{l}\text { Traverse--> } \\
\text { Trial ----> }\end{array}$}} & & & & & \multirow{2}{*}{\multicolumn{4}{|c|}{ Port_4_Side/South }} \\
\hline & & \multicolumn{4}{|c|}{ Port_4_Bottom/East } & & & & \\
\hline & & 1 & 2 & 3 & Mean & 1 & 2 & 3 & Mean \\
\hline Point & Depth, in. & \multicolumn{4}{|c|}{$\mathrm{ppm}$} & \multicolumn{4}{|c|}{ ppm } \\
\hline 1 & 0.58 & 1.70 & 1.75 & 1.96 & 1.803 & 1.67 & 1.74 & 1.82 & 1.743 \\
\hline 2 & 1.89 & 1.69 & 1.77 & 2.01 & 1.823 & 1.73 & 1.70 & 1.77 & 1.733 \\
\hline 3 & 3.49 & 1.76 & 1.73 & 2.02 & 1.837 & 1.72 & 1.77 & 1.77 & 1.753 \\
\hline 4 & 5.81 & 1.70 & 2.08 & 1.97 & 1.917 & 1.74 & 1.78 & 1.76 & 1.760 \\
\hline Center & 9.00 & 1.79 & 1.82 & 2.04 & 1.883 & 1.91 & 1.90 & 1.83 & 1.880 \\
\hline 5 & 12.19 & 1.90 & 2.06 & 1.96 & 1.973 & 1.84 & 1.89 & 1.86 & 1.863 \\
\hline 6 & 14.51 & 1.88 & 2.08 & 1.97 & 1.977 & 2.02 & 2.08 & 1.93 & 2.010 \\
\hline 7 & 16.11 & 1.94 & 2.07 & 1.94 & 1.983 & 1.95 & 1.98 & 1.96 & 1.963 \\
\hline 8 & 17.42 & 1.92 & 2.26 & 1.94 & 2.040 & 1.91 & 1.95 & 1.92 & 1.927 \\
\hline \multicolumn{2}{|c|}{ Averages ---------> } & 1.809 & 1.958 & 1.979 & 1.915 & 1.832 & 1.866 & 1.847 & 1.848 \\
\hline
\end{tabular}

Avg. Conc. $\quad 1.882 \mathrm{ppm}$

\begin{tabular}{|c|c|c|c|c|c|c|}
\hline$A / I$ & $\mathrm{ppm}$ & Dev. from mean & Center 2/3 & East & South & All \\
\hline Mean & 1.88 & & Mean & 1.91 & 1.85 & 1.88 \\
\hline Min Point & 1.73 & $-7.9 \%$ & Std. Dev. & 0.07 & 0.11 & 0.09 \\
\hline Max Point & 2.04 & $8.4 \%$ & cov as $\%$ & 3.5 & 5.9 & 4.9 \\
\hline
\end{tabular}

\begin{tabular}{|c|c|c|c|}
\hline \multirow{3}{*}{$\begin{array}{l}\text { Tracer tank pressure } \\
\text { Stack Temp }\end{array}$} & Start & Finish & \\
\hline & 350 & 375 & \\
\hline & 73 & 80 & $F^{\circ}$ \\
\hline \multirow{3}{*}{$\begin{array}{l}\text { Ref. Pt. air vel. } \\
\text { Injection flowmeter } \\
\text { Stack flow }\end{array}$} & 2350 & 2370 & fpm \\
\hline & 10 & 10 & ball ${ }^{\star \star}$ \\
\hline & - & - & $\mathrm{cfm}$ \\
\hline Sampling flowmeter & 10 & 10 & Ipm Sierra \\
\hline Ambient pressure & 29.7 & 29.7 & in $\mathrm{Hg}$ \\
\hline \multirow{3}{*}{$\begin{array}{l}\text { Ambient humidity } \\
\text { B\&K vapor correction } \\
\text { Back-Gd gas ppb }\end{array}$} & 44 & 36 & \multirow{3}{*}{$\begin{array}{l}\mathrm{RH} \\
\mathrm{Y} / \mathrm{N}\end{array}$} \\
\hline & $\mathrm{Y}$ & $Y$ & \\
\hline & $12,5,3,1,20$ & $19,1,0,-5$ & \\
\hline \multirow{3}{*}{$\begin{array}{l}\text { No. Bk-Gd samples } \\
\text { Ambient Temp, F } \\
\text { Instuments Used: }\end{array}$} & 55 & & \multirow[t]{3}{*}{ n } \\
\hline & 707 & & \\
\hline & & & \\
\hline \multicolumn{4}{|c|}{ B \& K Model 1302 \#1765299 } \\
\hline \multicolumn{4}{|c|}{ Sierra Inc. Constant Flow Air Sampler } \\
\hline \multicolumn{4}{|c|}{ TSI 8360 SN 209060 Cal due 9/27/06 } \\
\hline \multicolumn{4}{|l|}{ Notes: } \\
\hline \multicolumn{4}{|l|}{ ** black glass ball } \\
\hline \multicolumn{4}{|c|}{ Reference point air velocity measured at point 4 So8. } \\
\hline
\end{tabular}

Gas analyzer checked:

25-Jul-06

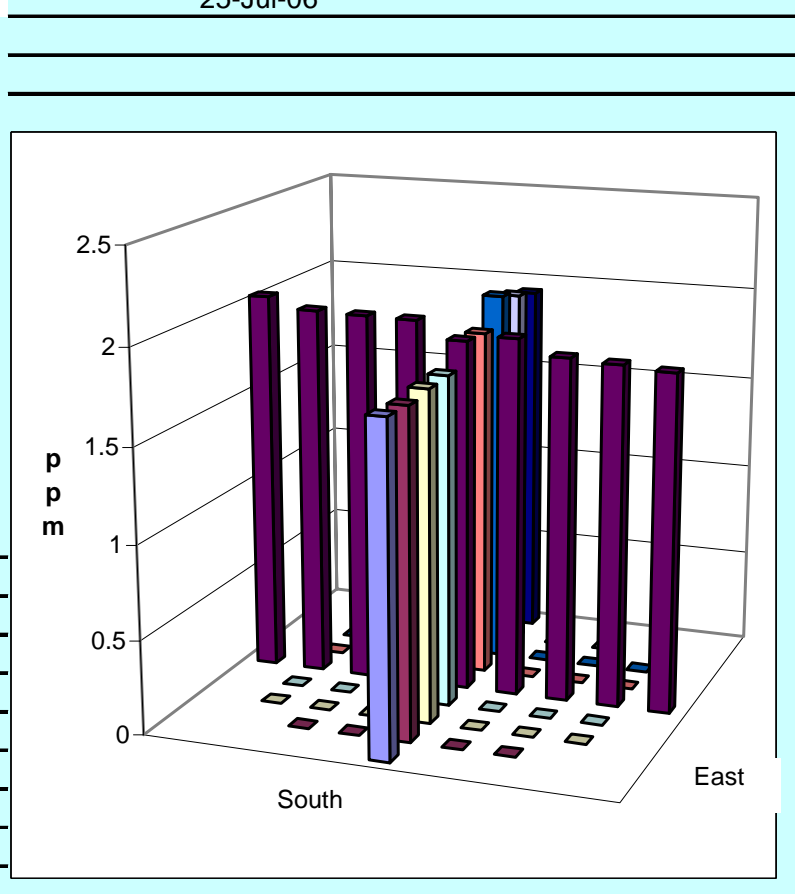

2nd -- 9- 10 .

Signature signifies compliance with

Procedure EMS-JAG-01

Signature/date

JGD

Signature verifying data and calculations:

$8 / 2 / 2006$

Signatures on file copy 
TRACER GAS TRAVERSE DATA FORM

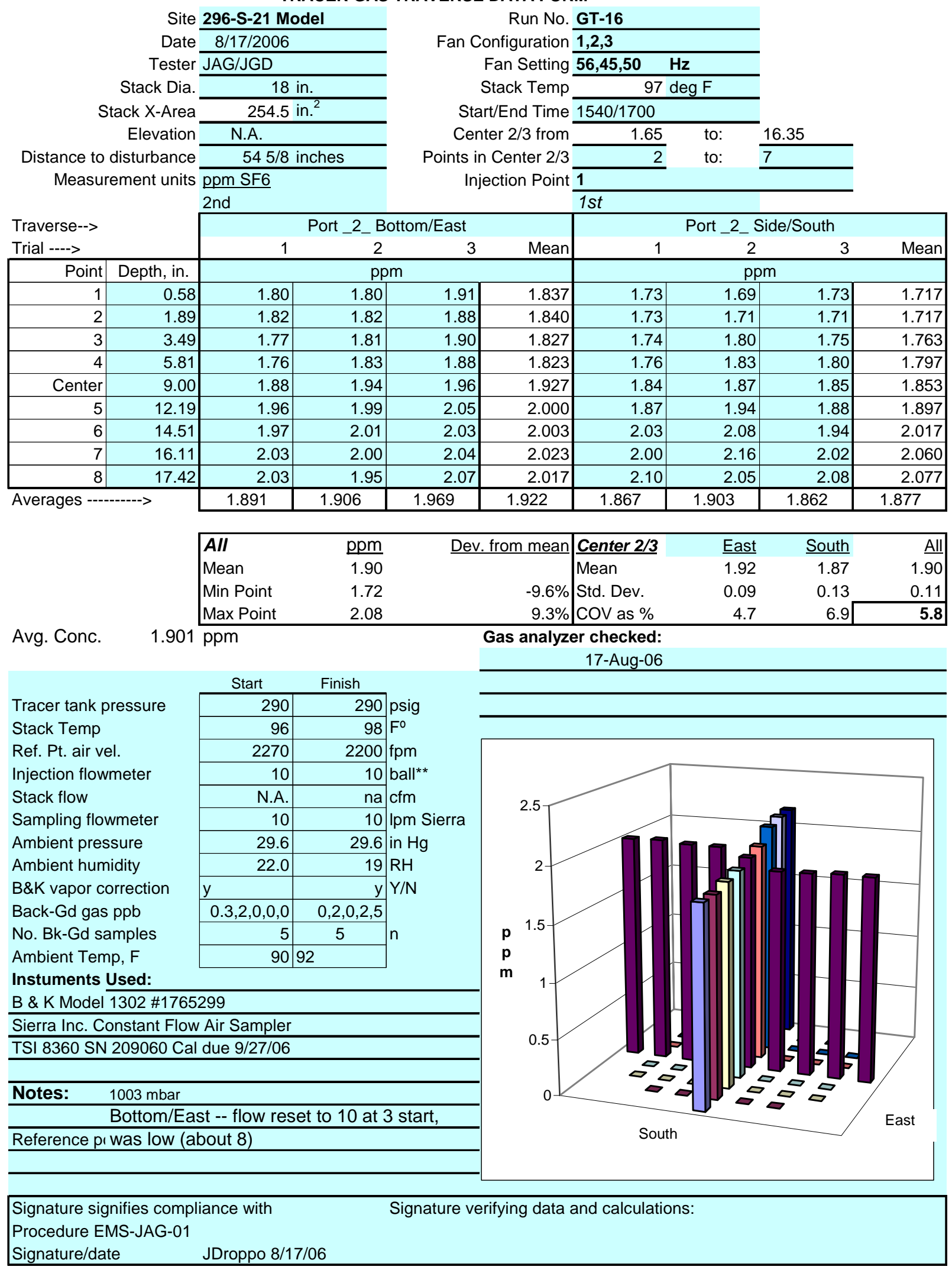


TRACER GAS TRAVERSE DATA FORM

Site 296-S-21 Model

Date 8/17/2006

Tester JGD

Stack Dia.

Stack X-Area

Elevation

Distance to disturbance

Measurement units

$\frac{18 \text { in. }}{\frac{254.5 \text { in. }^{2}}{\text { N.A. }}}$

$273 / 8$ inches

ppm SF6
Run No. GT-17

Fan Configuration $1,2,3$

Fan Setting $\mathbf{5 6 , 4 5 , 5 0 ~} \mathrm{Hz}$

Stack Temp

Start/End Time 1700/1900

Center $2 / 3$ from Points in Center $2 / 3$

Injection Point 1

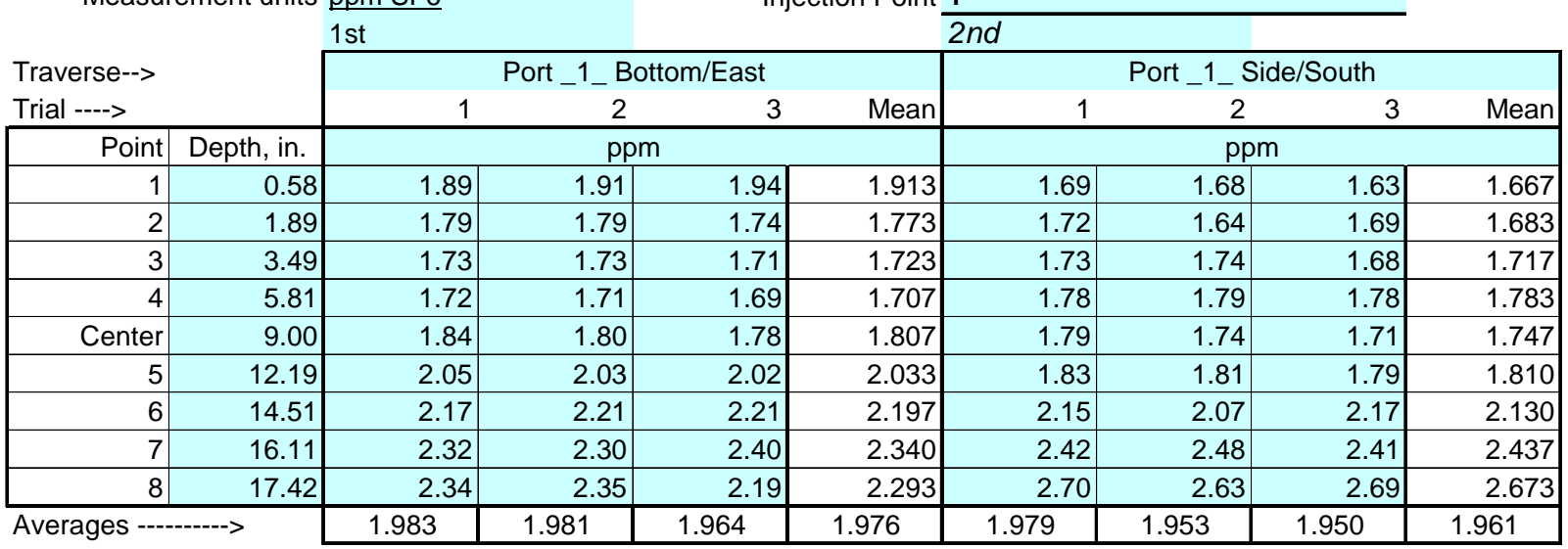

\begin{tabular}{|c|c|c|c|c|c|c|}
\hline$A / I$ & $\mathrm{ppm}$ & Dev. from mean & Center $2 / 3$ & East & South & All \\
\hline Mean & 1.97 & & Mean & $\overline{1.94}$ & 1.90 & $1 . \overline{92}$ \\
\hline Min Point & 1.67 & $-15.3 \%$ & Std. Dev. & 0.25 & 0.28 & 0.26 \\
\hline Max Point & 2.67 & $35.8 \%$ & cov as $\%$ & 13.0 & 14.7 & 13.3 \\
\hline
\end{tabular}

Avg. Conc. $\quad 1.993$ ppm

Tracer tank pressure

Stack Temp

Ref. Pt. air vel.

Injection flowmeter

Stack flow

Sampling flowmeter

Ambient pressure

Ambient humidity

$B \& K$ vapor correction

Back-Gd gas ppb

No. Bk-Gd samples

Ambient Temp, $\mathrm{F}$

Instuments Used:

B \& K Model 1302 \#1765299

Sierra Inc. Constant Flow Air Sampler

TSI 8360 SN 209060 Cal due 9/27/06

Notes:

\begin{tabular}{|c|c|c|}
\hline Start & Finish & \\
\hline 290 & 290 & psig \\
\hline 98 & 90 & $F^{\circ}$ \\
\hline 2200 & 2190.0 & fpm \\
\hline 10 & 10 & ball ${ }^{\star \star}$ \\
\hline na & na & $\mathrm{cfm}$ \\
\hline 10 & 10 & Ipm Sierra \\
\hline 29.610 & 29.6 & in $\mathrm{Hg}$ \\
\hline 19 & 28 & $\mathrm{RH}$ \\
\hline$y$ & $\mathrm{y}$ & $Y / N$ \\
\hline $0,2,0,2,5$ & $1,0,3,7,0$ & \\
\hline 5 & 5 & $n$ \\
\hline 92 & 87 & \\
\hline
\end{tabular}

Gas analyzer checked:

17-Aug-06

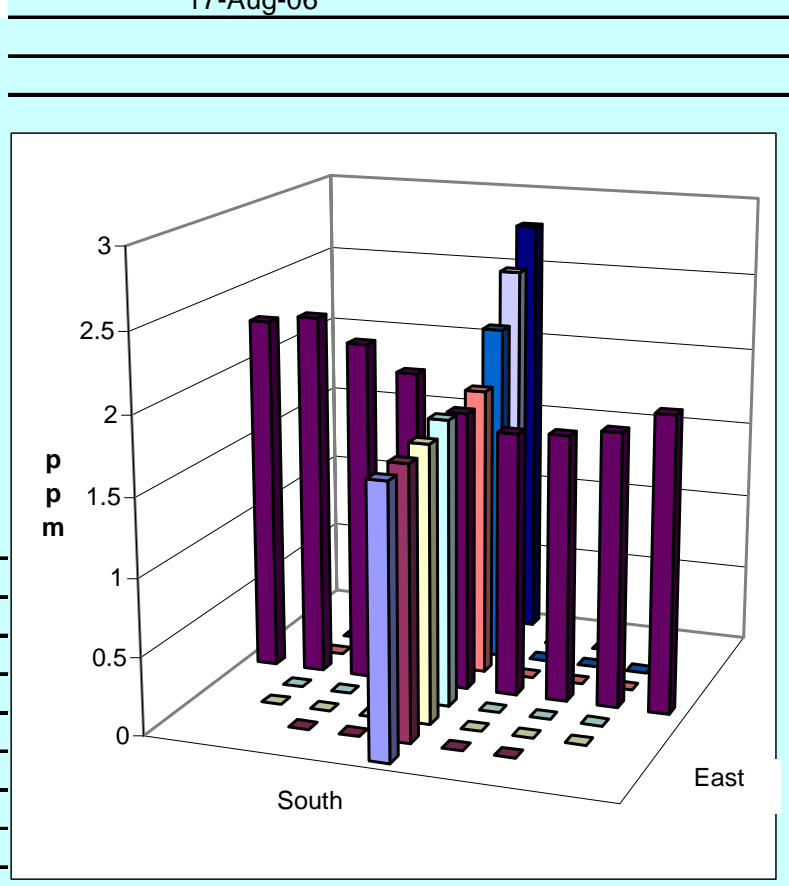

Signature signifies compliance with

Procedure EMS-JAG-01

Signature/date

JDroppo 8/17/06
Signature verifying data and calculations: 


\section{Sulfur hexafluoride Gas Calibration performed on B\& 7/25/2006 by John Glissmeyer}

Setup: $\quad 6.83 \mathrm{ft} \quad$ B\&K sample inlet tube length

993 mbar station pressure

$74 \operatorname{deg} \mathrm{F}$ ambie analyzer corrects to $20 \operatorname{deg} \mathrm{C}$

53 percent $\mathrm{RH}$

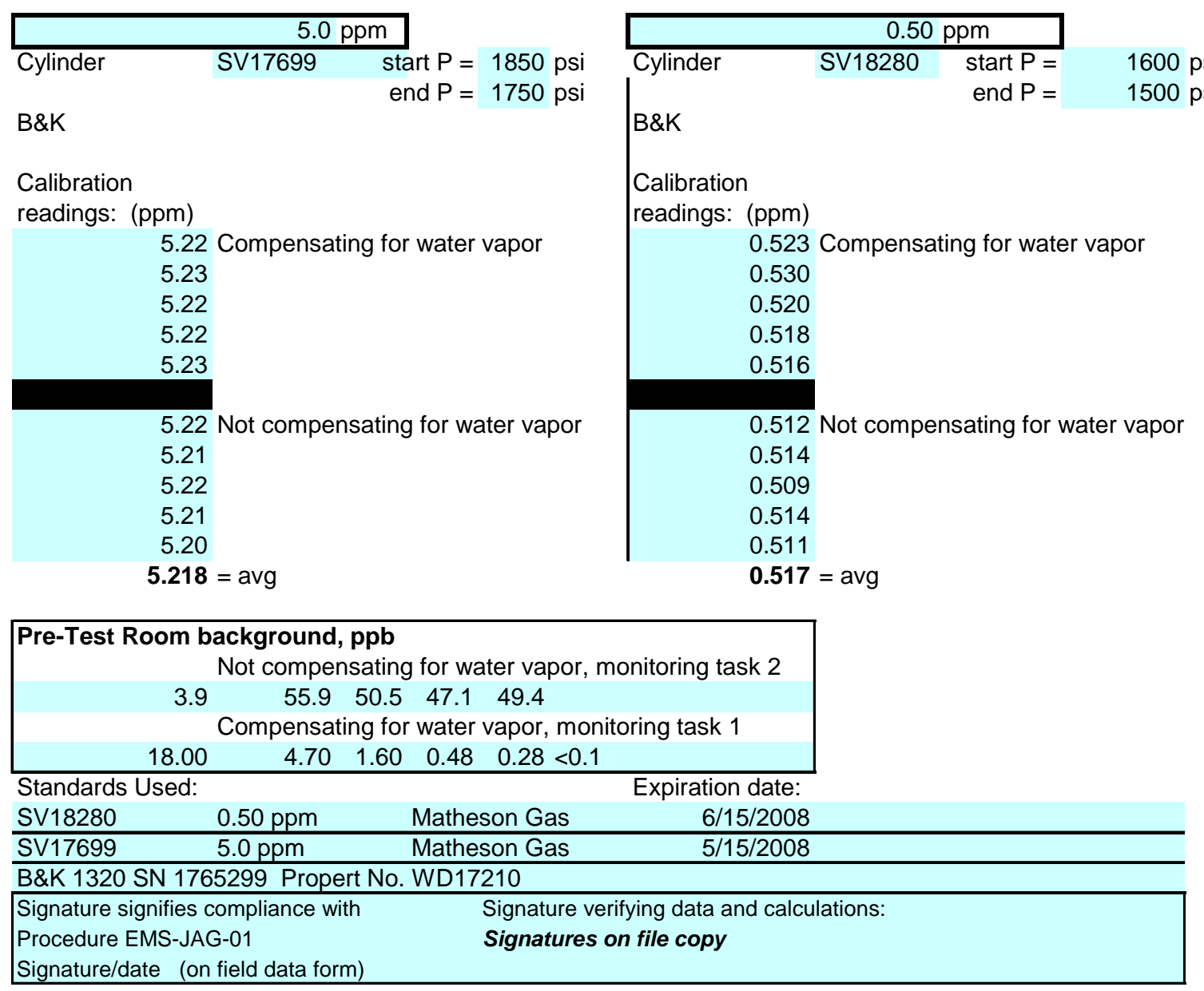


Setup:

$6.83 \mathrm{ft} \quad B \& \mathrm{~K}$ sample inlet tube length

996 mbar station pressure

77 deg F ambie analyzer corrects to $20 \mathrm{deg} C$

46 percent $\mathrm{RH}$

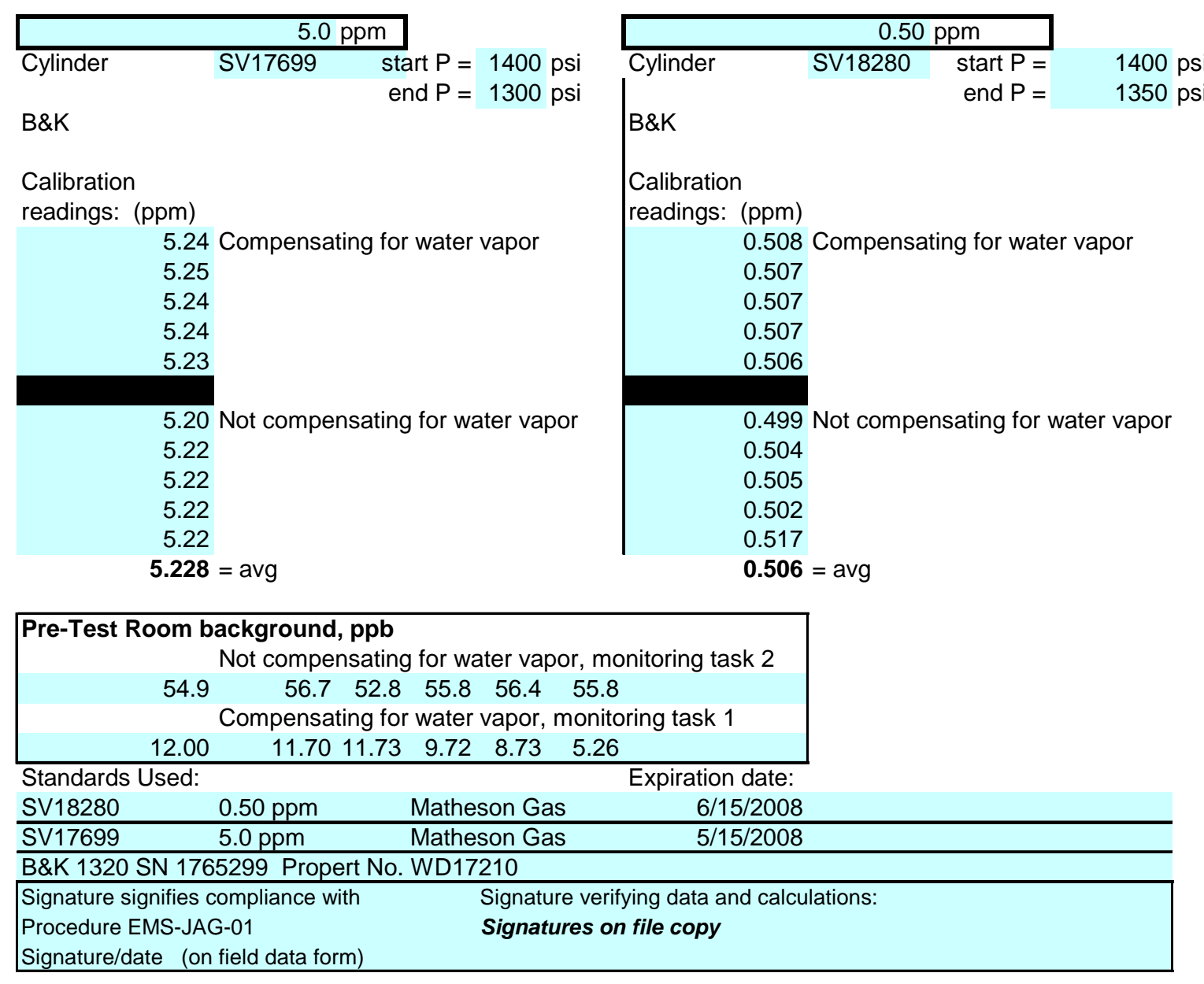




\section{Sulfur hexafluoride Gas Calibration performed on B\& 8/17/2006 by John Glissmeyer}

Setup: $\quad 6.83 \mathrm{ft} \quad$ B\&K sample inlet tube length

1003 mbar station pressure

78 deg F ambie analyzer corrects to 20 deg C

45 percent $\mathrm{RH}$

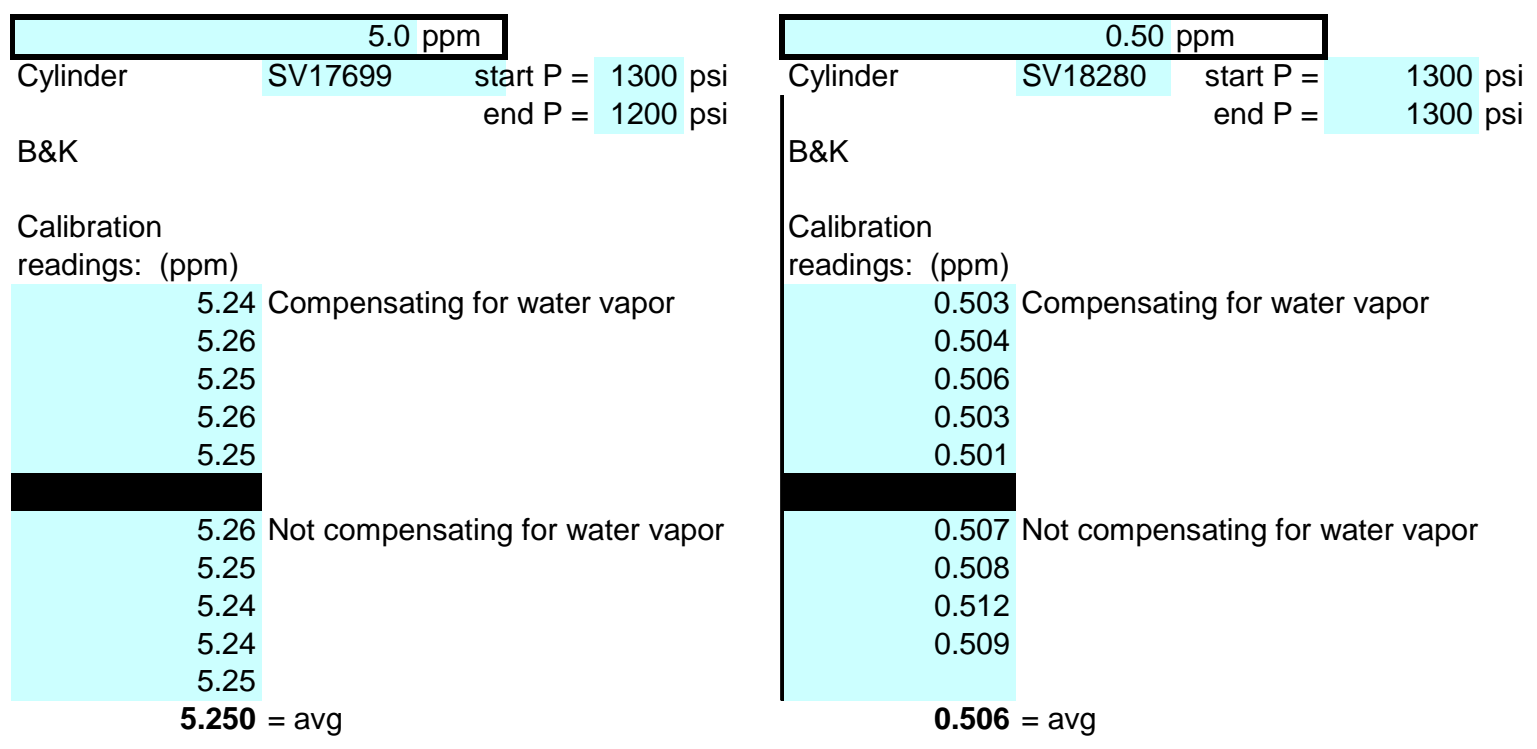

\section{Pre-Test Room background, ppb}

Not compensating for water vapor, monitoring task 2 46.9

Compensating for water vapor, monitoring task 1 3.85 $\begin{array}{lllll}1.46 & 4.32 & 5.67 & 1.07 & 3.82\end{array}$

\begin{tabular}{lllr} 
Standards Used: & & & Expiration date: \\
SV18280 & $0.50 \mathrm{ppm}$ & Matheson Gas & $6 / 15 / 2008$ \\
\hline SV17699 & $5.0 \mathrm{ppm}$ & Matheson Gas & $5 / 15 / 2008$
\end{tabular}

B\&K 1320 SN 1765299 Propert No. WD17210

Signature signifies compliance with Signature verifying data and calculations:

Procedure EMS-JAG-01 Signatures on file copy

Signature/date (on field data form) 
Setup:

$6.83 \mathrm{ft} \quad B \& K$ sample inlet tube length 1000.6 mbar station pressure

75 deg $\mathrm{F}$ ambie analyzer corrects to $20 \mathrm{deg} \mathrm{C}$

37 percent $\mathrm{RH}$

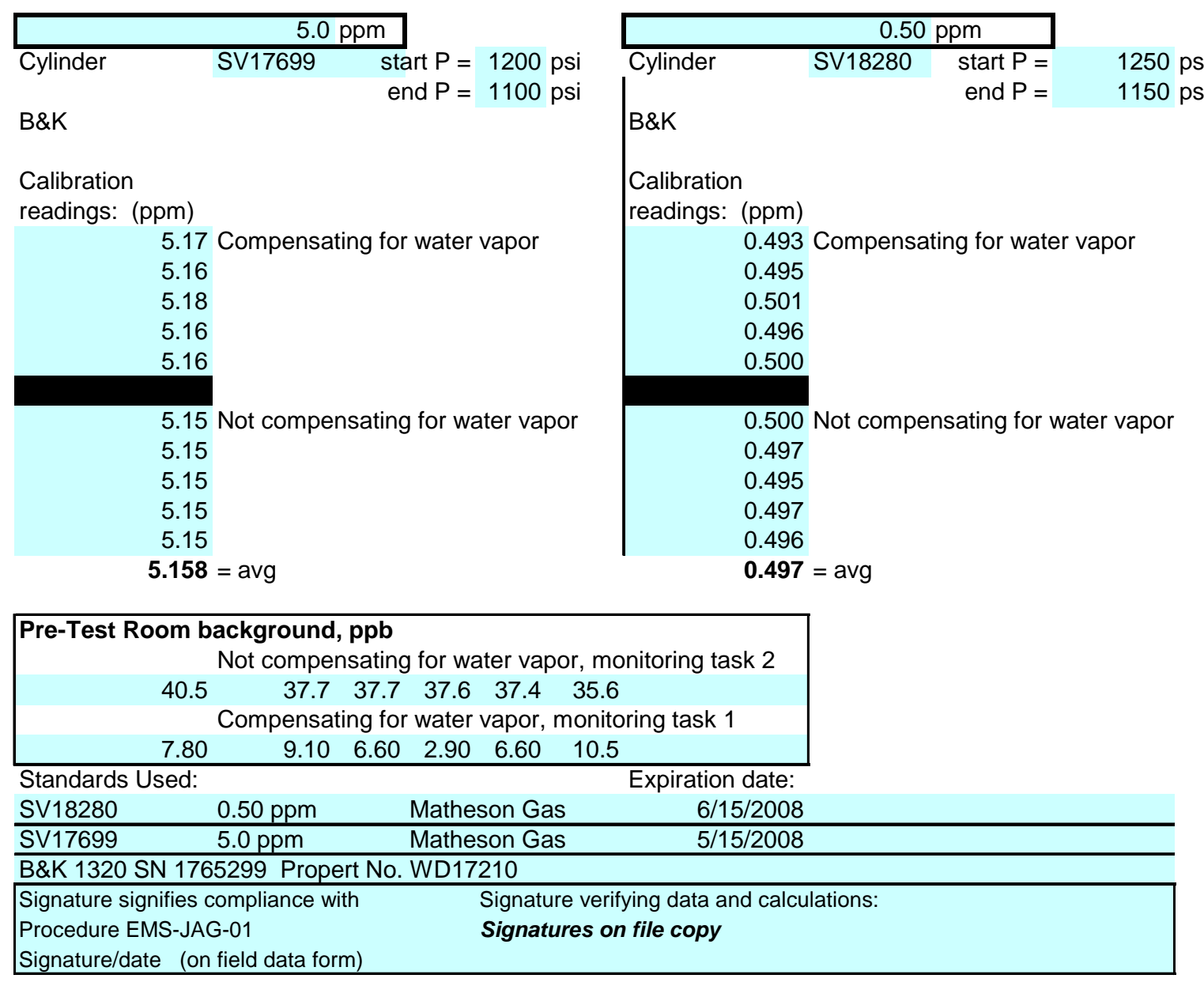




\section{Appendix I}

\section{Particle Tracer Uniformity Procedure}


APPENDIX I: PARTICLE TRACER UNIFORMITY PROCEDURE

\section{PNNL Operating Procedure}

\begin{tabular}{l|lc}
\hline Title: Test to Determine & & \\
Uniformity of a Tracer & Org. Code: & D7E74 \\
Aerosol at a Sampler & Procedure No.: & EMS-JAG-02 \\
Probe & Rev. No.: & 2
\end{tabular}

\begin{tabular}{|c|c|}
\hline Work Location: General & Effective Date: \\
\hline Author: John A. Glissmeyer & Supersedes Date: $\quad$ May 24, 2000 \\
\hline $\begin{array}{l}\text { Identified Hazards: } \\
\square \text { Radiological } \\
\square \text { Hazardous Materials } \\
\text { 冈 Physical Hazards } \\
\square \text { Hazardous Environment } \\
\square \text { Other: }\end{array}$ & $\begin{array}{l}\text { Identified Use Category: } \\
\square \text { Mandatory Use } \\
\square \text { Continuous Use } \\
\text { 冈 Reference Use } \\
\square \text { Information Use }\end{array}$ \\
\hline
\end{tabular}

\begin{tabular}{|c|c|c|}
\hline \multicolumn{2}{|c|}{ Are One-Time Modifications Allowed? $\quad$ Yes So Nonature } & Date \\
\hline Person Signing & & \\
\hline $\begin{array}{c}\text { Technical review: } \\
\text { J. Matthew Barnett }\end{array}$ & \\
\hline $\begin{array}{c}\text { Project Manager: } \\
\text { John Glissmeyer }\end{array}$ & & \\
\hline $\begin{array}{c}\text { Line Manager: } \\
\text { James Droppo }\end{array}$ & & \\
\hline Concurrence: & & \\
\hline $\begin{array}{c}\text { Quality Engineer: } \\
\text { Barry L. Sachs }\end{array}$ & \\
\hline
\end{tabular}




\subsection{Purpose}

The performance of new stack sampling systems must be shown to satisfy the requirements of 40 CFR 61, Subpart H, "National Emission Standards for Emissions of Radionuclides Other than Radon from Department of Energy Facilities." This regulation governs portions of the design and implementation of effluent air sampling. The stack sampler performance is adequately characterized when potential contaminants in the effluent are of a uniform concentration at the sampling location (plane), and line losses are within acceptable limits. This procedure determines whether the concentration of aerosol particulate contaminants is uniformly distributed in the area of the sampling probe. Other procedures address flow angle, uniformity of gas velocity, and uniformity of gas contaminants. A contaminant concentration that is uniform at the sampling plane enables the extraction of samples that represent the true emission concentration.

The uniformity is expressed as the variability of the measurements about the mean. This is expressed using the relative coefficient of variance (COV), which is the standard deviation divided by the mean and expressed as a percentage. The lower the COV value, the more uniform the particle concentration. The acceptance criterion is that the COV of the measured particle concentrations be $<20 \%$ across the center two-thirds of the area of the stack.

\subsection{Applicability}

This procedure can be used for actual or scale model stacks to determine whether airsampling probes can collect representative samples under normal operations. The tests are applicable to effluent stacks or ducts within the following constraints:

- The aerosol particulate tests are generally limited to stacks with flowrates greater than 50 cubic feet per minute range. The upper bound of flowrate is determined by the output capacity of the aerosol generator, the background reading for particulate aerosols, and the operational detection range of the optical particle counters.

- Environmental constraints - the operating temperature range $\left(55^{\circ}-100^{\circ} \mathrm{F}\right)$ of the optical particle counter must be observed.

\subsection{Prerequisites and Conditions}

Conditions and concerns that must be satisfied before sampling are listed below:

- Safety glasses, hard toed or substantial shoes may be required in work areas.

- Test ports for tracer injection and sampling.

- Properly constructed and inspected work platforms may be needed to access the test and injection ports.

- Special training may be required in some instances to access the test ports of the stack.

- Alcohol (methanol, ethanol, or rubbing) may be used to erase grid point markings on the sampling probes. A flammable equipment storage cabinet may be required to hold chemicals. Material Safety Data Forms must be provided. 
- Air pressure (up to about 75 psi) is used to aerosolize oil into fine particles. Observations of any buildup of oil mist outside of the generator are essential to prevent exceeding American Conference of Governmental Industrial Hygienists (ACGIH) levels listed below.

- Knowledge of the setup, use of, and operation of pressurized air lines, flowmeters, and particle counters is essential.

- A job-hazards analysis may be required in certain cases.

\subsection{Precautions and Limitations}

\section{Caution: The ACGIH 8-hour time-weighted average limit for human exposure to mineral oil mist is $5 \mathrm{mg} / \mathrm{m}^{3}$. It is odorless.}

During the tests, oil droplets will be injected into the base of the stack or duct at a rate to achieve a target concentration of $200-400010-\mu \mathrm{m}$ particles $/ \mathrm{ft}^{3}\left(\mathrm{pt} / \mathrm{ft}^{3}\right)$ at the test ports. The potential is present for a buildup of oil mist to occur outside of the aerosol generator that could approach the $5 \mathrm{mg} / \mathrm{m}^{3}$ caution level. The undiluted mist is heavier than air, so it may accumulate in confined spaces and in low areas. Visual inspections of the delivery system will be made at least daily to prevent such an occurrence. Adequate ventilation is an added mitigating precaution.

Access to the test ports may require the use of scaffolding or manlifts, either of which will necessitate special training for sampling personnel and any observers. The training requirements will be indicated in the job hazard analysis.

The test may be invalid if the background $9-11 \mu \mathrm{m}$ aerosol concentration in the stack is more than about $20 \%$ of that observed during the test. This would indicate poor filtration of the air at the fan inlet. This may result in a false indication of good mixing.

\subsection{Equipment Used for Measurements}

Specific probe dimensions, measurement grids, stack flowrate settings, and other special requirements will be provided in a specific Test Instruction. Exhibit A provides a typical layout for the test setup. Exhibit $\mathrm{C}$ shows a typical Test Instruction. The following are essential items of equipment:

- Vacuum pump oil (or other approved oil specified in the Test Instruction)

- Oil mist generator

- Compressed air, compressed air hoses, air filter and air regulators

- Oil mist injection probe

- Aerosol sampling probes

- Mechanism for accurate placement of sampling probe

- Calibrated optical particle counter (OPC)

- OPTIONAL -- Computer linked to optical particle counter to log readings

- Platform, ladders, or manlifts as needed to access the test ports; 
- Fittings to limit leakage around the sampling probe and to stabilize it so it can be repositioned repeatedly.

- To provide information about the test conditions, commercial grade sensors for stack temperature, barometric pressure, static pressure, air velocity, and humidity provide acceptable information. Likewise, data from a nearby meteorology or facility station is acceptable.

The optical particle counter is calibrated annually for particle sizing and internal flowrate. If there is reason to suspect a change in the tracer aerosol concentration with time, an optional second OPC may be used at a fixed location. The measurements from the fixed OPC may be used to determine a correction to the other OPC readings with time.

The aerosol generator siphons oil from a reservoir and forces the air/oil mixture through a spray nozzle to produce polydisperse particles. Non-hazardous oil with a low vapor pressure (such as Fisherbrand 19 vacuum pump oil) should be used in the reservoir. The quantity of aerosol generated is controlled by the compressed air pressure, which should be filtered and controlled by a regulator. The nozzle is mounted in a large diameter, clear-plastic pipe (4-inches diameter or larger) so the output level can be observed. The aerosol generator output should connect to an injection probe with an inside diameter of at least 0.5 inches to minimize collisions with the inner wall of the tubing. Optimal operation depends on uniformly "wetting" the inner surfaces of the generator and injection probes; thus, a warm up period of up to $1 / 2$ hour is recommended for a constant aerosol output.

\subsection{Work instructions for Setup, Measurements, and Calculations}

The steps taken to set up, configure, and operate the stack fans and test equipment are listed. The test instruction (illustrated in Exhibit C) will provide more specific details and operating parameters.

\subsection{Preparing for Measurements:}

6.1.1 Prepare a spreadsheet for calculating results and plotting data similar to the illustration in Exhibit B. Label the columns of data by the direction to be used in traversing the stack. For example, if the first reading is closest to the east port, and the last reading is closest to the west port, then label the traverse east-west. Print blank copies of the spreadsheet as data forms for hand recording measurements for later transfer to the spreadsheet.

6.1.2 Provide essential supplies at the sampling location (aerosol generation equipment, compressed air, regulators, fittings and probe-port fittings, marking pens, data forms, writing and probe-supporting platforms).

6.1.3 Mark the sampling probe with a permanent marker so the inlet can be placed at each successive measurement point. 
Note: Sampling plane traverse points. Use the grid of measurement points provided with the test instruction and dataform. This is usually the same as used for the velocity uniformity test (EMS-JAG-04). A center point is included as a common reference and for graphical purposes. The layout design divides the area of the sampling plane so that each point represents approximately an equal-sized area

6.1.4 Setup the test parameters characterizing the stack or duct airflow, configuration, fan control setting, and conditions according to the test instruction.

6.1.5 Record the test information on the data form (run number, test crew, instrumentation used, configuration, date, etc.).

6.1.6 Using an air velocity or flow sensor, verify that the target flow condition has been achieved within $\pm 10 \%$. Record the value on the data form.

6.1.7 Insert the sampling probe in a test port and connect the other end to the OPC as illustrated in Exhibit A.

Note: The sampling probe typically consists of stainless steel thin-wall tubing with $3 / 4$ outside diameter and with sufficient length to reach across the inside diameter of the stack while allowing for fittings. The sampling probe should have gradual $90^{\circ}$ bends to minimize the inertial impact of particles with inner walls at bends, and the open end of the tube should face into the flow in the stack. The outlet end of the probe should terminate with a connection at the OPC inlet. This connection is typically a modified 1-inch plastic syringe body with the probe inserted into the body with o-rings to fit between the outside of the probe and the inside of the body. The needle end of the body is cut back and the taper has a rubber grommet inserted, which fits over the inlet of the OPC.

Note: The elevation of the sampling probe nozzle should be approximately in the same as the plane as the actual stack sampling probe nozzle. The intake nozzles may be of subisokinetic or of shrouded design to optimize the collection of 10-micron particles.

Minimize tubing length to minimize particle losses.

The OPC should be mounted on a sliding platform to move as a unit, with its attached probe, along the axis of the test port.

6.1.8 On the data form, record the starting

- ambient temperature

- stack air temperature

- stack air velocity or flowrate

- ambient pressure

- ambient humidity

- time 
6.1.9 Turn-on the optical particle counter. Ensure that internal air circulation fans in the OPCs are on and that the sample probe is tightly connected to the OPC inlet.

6.1.10 Program the OPC for

- 60-second samples and a 15-sec hold time between samples

- 9- to 11-micron particle counting

- the current time

- cumulative counting mode.

6.1.11 Set the OPC sample flowrate to $0.98-1.02 \mathrm{cfm}$. Allow it to warm up for 5 minutes.

6.1.12 Locate the sampling probe approximately in the middle of the stack.

6.1.13 Record on the data form at least four consecutive background particle concentration readings in the $9-11 \mu \mathrm{m}$ channel.

\subsection{Aerosol Injection}

The aerosol injection equipment includes an air regulator, an air pressure gauge, and other components described in Section 5. The 1/2-inch (ID) (or larger) injection probe with a $90^{\circ}$ bend (with an approximately 3 -inch radius of turn) will inject aerosol particles in the direction of emission flow. The connections and fittings should be checked to ensure that they are secure and leak free.

\section{Note: Location of the Injection Point}

Injection plane -- The aerosol injection point is usually along the centerline of the duct or stack.

6.2.1 Position the injection probe, according to the test instruction.

6.2.2 Start and run the aerosol generator for approximately 30 minutes to stabilize its output. (For successive runs on the same day, a warm up time of less than 30 minutes may provide stable readings.)

6.2.3 Adjust the aerosol generator output so the particle count is at least five times background and no more than about $4000 \mathrm{pt} / \mathrm{ft}^{3}$ in the $9-11 \mu \mathrm{m}$ channel.

6.2.4 Record on the data form the initial

- injection system dispersion pressure in psi

- compressor or air line pressure in psi 
Note: The control over the aerosol injection rate is limited. The only apparent control is the selection of the spray nozzle and the air pressure to the nozzle. At air pressure readings above about 10 psi for the specific PNNL generator used, a dense oil mist is created in the generator and is available for injection. However, if there is backpressure in the stack, carrier air may be required to reach a concentration above 200 $\mathrm{pt} / \mathrm{ft}^{3}$. In contrast, if there is little back-pressure, most of the generated aerosol, minus that lost from interactions with internal generator system and line walls, becomes available for injection. Here the output and concentration will be high .

Note: The OPC draws air from the stack, via the sample probe, at a fixed rate (one cubic foot per minute). Within the OPC, the air stream with particles passes through a laser beam where the particles are counted and placed in six size categories. In the less than 0.5-micron category, several hundred thousand differential counts are typical; but in the 9- to 11-micron category, oil mists greater than about 3,000 cpm cause a sensor overload condition. There is no adjustment of particle concentration range at the OPC, so the aerosol generator provides the only control for aerosol

\subsection{Measurements}

6.3.1 On the data form, mark which test port was used first with a " 1 st" indication.

6.3.2 Verify that the directional orientations and the numbered sample positions are consistent.

6.3.3 Position the OPC and sample probe at each measurement point in succession, and record the particle concentration reading on the data form.

Note: In each test, the measurement at each point is the average of three readings. The repeats are made as three separate runs and not as three consecutive measurements at each point.

\subsubsection{Perform two additional repetitions of Step 6.3.3.}

6.3.5 Switch to the other test port and repeat Steps 6.3.2 to 6.3.4.

6.3.6 During the making of measurements, periodically check the OPC flowrate and correct as needed.

6.3.7 Record on the data form the ending:

- injection system dispersion pressure in psi

- compressor or air line pressure in psi 
6.3.8 Shut off the air pressure to the aerosol generator.

6.3.9 Continue operation of the OPCs for several minutes while any remaining test aerosol is purged from the stack.

6.3.10 Record on the data form at least four centerline background particulate concentrations on the $9-11 \mu \mathrm{m}$ channel.

6.3.11 On the data form, record the final:

- ambient temperature

- stack air temperature

- stack air velocity or flowrate

- ambient pressure

- ambient humidity

- centerline air velocity

- time

6.3.12 Record any deviations from the above procedure on the data form.

6.3.13 Verify that the data form is complete and sign and date in the signature block attesting to performing this procedure to this point.

6.3.14 Repeat steps 6.1.1 to 6.3.13 for each run as indicated in the test instruction.

\subsection{Calculations}

6.4.1 Transfer the hand written data into the electronic spreadsheet.

6.4.2 Calculate the COV result for the run.

Note: The spreadsheet (Exhibit B is typical) will calculate the COV result using the average concentration data from all points in the inner two-thirds of the cross section area of the plane (including the center point). It also calculates the COV for each test port separately for information. It also attempts to correct the data for a change in aerosol concentration with time. It calculates a normalized overall COV by correcting the data from one test port as needed to equalize the center point averages for both test ports.

\subsubsection{Compare the observed COV for each run to the acceptance criterion.}

Note: The test is acceptable if the COV is $<20 \%$ for the inner two-thirds of the stack diameter. 
6.4.4 Complete the calculations for each test run in the Test Instruction

6.4.5 Have the data transfers and calculations independently reviewed and verified. The reviewer should sign the finished data form.

\subsection{Records}

7.1 Transfer the original signed data forms and verified spreadsheets to the records custodian as project records. 


\section{Exhibits}

\section{Exhibit A - Overview of Stack and Injection Setup and Particle Counters}

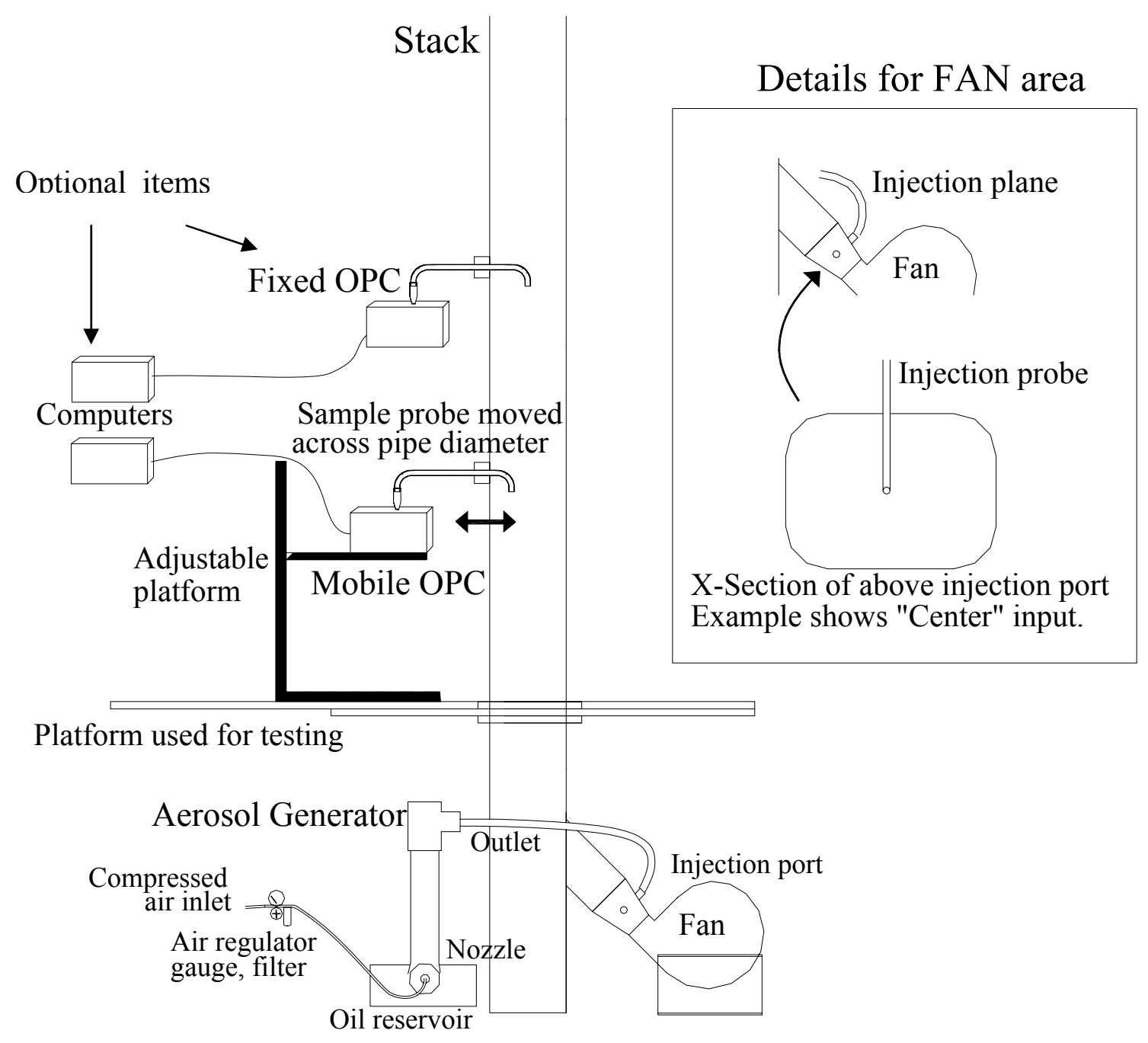




\section{Exhibit B -Typical Data Collection Form \\ PARTICLE TRACER TRAVERSE DATA FORM}

\begin{tabular}{|c|c|}
\hline \multicolumn{2}{|c|}{ Site Model Stack } \\
\hline Date & $9 / 17 / 2004$ \\
\hline \multicolumn{2}{|c|}{ Tester JAG/MYB/JMB } \\
\hline Stack Dia. & 18 in. \\
\hline Stack X-Area & 254.5 in. 2 \\
\hline \multicolumn{2}{|c|}{ Elevation N.A. } \\
\hline Distance to disturbance & 120 inches \\
\hline
\end{tabular}
Fan configuration Near Fan
Fan Setting $37.1 \mathrm{~Hz}$
Stack Temp $66 \mathrm{deg} \mathrm{F}$
Start/End Time 10:50/13:20
Center $2 / 3$ from
Points in Center 2/3

$\frac{1.65}{2}$ to: $\frac{16.35}{7}$
Injection Point $\overline{\text { Near Fan, Centerline downstream }}$

\begin{tabular}{|c|c|c|c|c|c|c|c|c|c|}
\hline \multirow{3}{*}{\multicolumn{2}{|c|}{$\begin{array}{l}\text { Order -----> } \\
\text { Traverse--> } \\
\text { Trial ----> } \\
\end{array}$}} & \multicolumn{4}{|c|}{ 1st } & \multicolumn{3}{|c|}{ 2nd } & \\
\hline & & \multicolumn{4}{|c|}{ East } & \multicolumn{4}{|c|}{ North } \\
\hline & & \multirow[t]{2}{*}{1} & \multirow{2}{*}{\multicolumn{2}{|c|}{$\frac{2}{\text { particles/ft3 }}$}} & \multirow[t]{2}{*}{ an } & \multirow[t]{2}{*}{1} & \multirow{2}{*}{\multicolumn{2}{|c|}{$\frac{2}{\text { particles/ft3 }}$}} & Mean \\
\hline Point & Depth, in. & & & & & & & & \\
\hline 1 & 0.58 & 4987 & 2769 & 4527 & 4094.3 & 2629 & 2880 & 3068 & 2859.0 \\
\hline 2 & 1.89 & 4980 & 2677 & 3534 & 3730.3 & 2772 & 3186 & 2879 & 2945.7 \\
\hline 3 & 3.49 & 3190 & 3812 & 4235 & 3745.7 & 2758 & 3355 & 3548 & 3220.3 \\
\hline 4 & 5.81 & 3499 & 4058 & 4313 & 3956.7 & 2787 & 3185 & 4027 & 3333.0 \\
\hline Center & 9.00 & 4049 & 4810 & 4084 & 4314.3 & 2560 & 2986 & 4222 & 3256.0 \\
\hline 5 & 12.19 & 4318 & 3638 & 3973 & 3976.3 & 2658 & 2651 & 3828 & 3045.7 \\
\hline 6 & 14.51 & 3785 & 4313 & 3856 & 3984.7 & 3913 & 2753 & 3528 & 3398.0 \\
\hline 7 & 16.11 & 3673 & 4102 & 3532 & 3769.0 & 3755 & 2513 & 4860 & 3709.3 \\
\hline 8 & 17.42 & 3103 & 3172 & 2589 & 2954.7 & 2951 & 2999 & 5331 & 3760.3 \\
\hline Averages & $>$ & 3953.8 & 3705.7 & 3849.2 & 3836.2 & 2975.9 & 2945.3 & 3921.2 & 3280.8 \\
\hline
\end{tabular}

\begin{tabular}{|lrr|lrrrr|r|}
\hline All & pt/ft3 & Dev. from mean & Center 2/3 & East & North & All & Normlzd \\
\hline Mean & 3558.5 & & & Mean & 3925.3 & 3272.6 & 3598.93 & 4130.79 \\
Min Point & 2859.0 & $-19.7 \%$ & Std. Dev. & 205.4 & 248.8 & 403.43 & 339.31 \\
Max Point & 4314.3 & & $21.2 \%$ & COV as $\%$ & 5.2 & 7.6 & $\mathbf{1 1 . 2 1}$ & $\mathbf{8 . 2 1}$ \\
\hline
\end{tabular}

Avg Conc $3530 \mathrm{pt} / \mathrm{ft} 3$

\begin{tabular}{|c|c|c|c|}
\hline \multirow[b]{2}{*}{ Generator Inlet Press } & Start & Finish & \multirow[b]{2}{*}{ psig } \\
\hline & 4 & & \\
\hline Stack Temp & 61 & 71 & $\mathrm{~F}$ \\
\hline \multirow{2}{*}{$\begin{array}{l}\text { Centerline vel. } \\
\text { Ambient pressure }\end{array}$} & 855 & 795.0 & $\mathrm{fpm}$ \\
\hline & 29.3 & 29.258 & $\mathrm{inHg}$ \\
\hline Ambient humidity & $74 \%$ & $53 \%$ & $\mathrm{RH}$ \\
\hline Ambient temp & 58 & 66 & $\mathrm{~F}$ \\
\hline \multirow{2}{*}{$\begin{array}{l}\text { Back-Gd aerosol } \\
\text { No. Bk-Gd samples }\end{array}$} & $3,3,2,3,4,5$ & $1,3,4,3,0,3$ & $\mathrm{pt} / \mathrm{ft} 3$ \\
\hline & 6 & 6 & \\
\hline $\begin{array}{l}\text { Compressor output reg } \\
\text { Optical Particle Counte }\end{array}$ & 110 & 115 & psig \\
\hline Met One A2408 & S/N 96258 & 675 & Cal 8/5/04 \\
\hline
\end{tabular}

Wind 12 mph steady

Oil Used: FisherBrand 19

Probe has a 4.5-in throw so is that much closer to the stack than the port.

\section{Instuments Used:}

TSI Velocity Calc Plus S/N $209060 \quad$ Calib 8/25/04

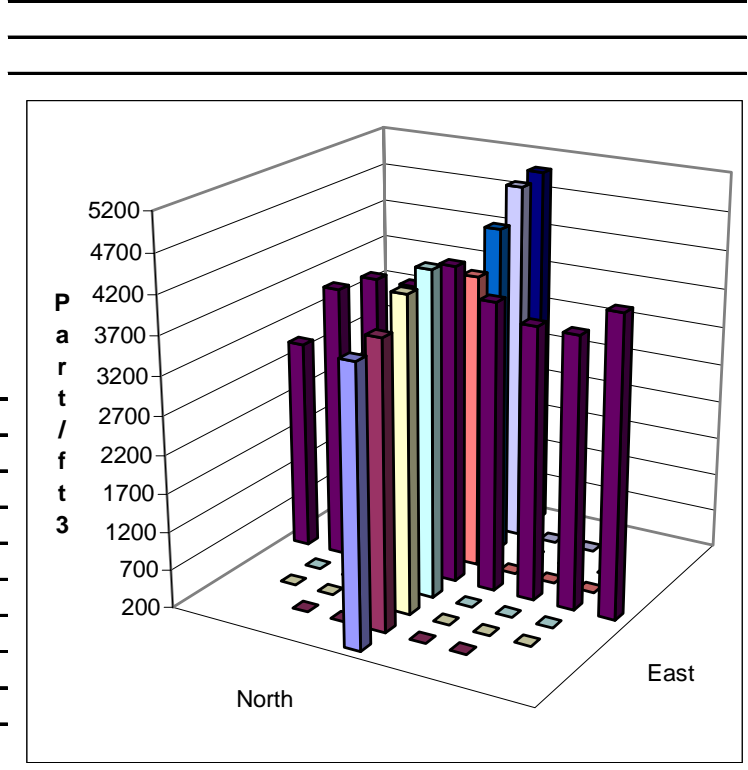




\section{Exhibit C - Typical Test Instruction}

\begin{tabular}{|c|c|c|}
\hline \multicolumn{3}{|c|}{ Test Instruction } \\
\hline $\begin{array}{l}\text { Project: Model Stack } \\
\text { Qualification }\end{array}$ & Date: November 10, 2008 & Work Package: K97052 \\
\hline \multicolumn{3}{|c|}{ Tests: Particle Tracer Uniformity of Full-Scale Stack } \\
\hline \multicolumn{3}{|c|}{ Staff: David Maughan, John Glissmeyer } \\
\hline \multicolumn{3}{|c|}{$\begin{array}{l}\text { Reference Procedures: } \\
\text { 1. Procedure EMS-JAG-02, Rev. 2, Test to Determine Uniformity of a Particulate } \\
\text { Aerosol at a Sampler } \\
\text { 2. Operating Manual for Met-One Optical Particle Counter (OPC), Model A2408 }\end{array}$} \\
\hline \multicolumn{3}{|c|}{$\begin{array}{l}\text { Equipment: } \\
\text { 1. Model Stack and inspected work platforms } \\
\text { 2. Vacuum pump oil, oil mist generator, air lines, regulator, precision pressure gauge } \\
\text { 3. Oil mist injection probe, OPC sample probes, probe/stack couplers } \\
\text { 4. OPCs with computers and links } \\
\text { 5. Velocity measurement device }\end{array}$} \\
\hline \multicolumn{3}{|c|}{$\begin{array}{l}\text { Safety Considerations: } \\
\text { Review and observe the applicable Job Hazard Analysis for the project }\end{array}$} \\
\hline $\begin{array}{ll}\text { Instructions: } \\
\text { 1. Verify training or } \\
\text { 2. Obtain Fisherbrar } \\
\text { 3. Obtain climatic ir } \\
\text { http://etd.pnl.gov } \\
\text { 4. Install equipment } \\
\text { 5. Mark sampling p } \\
\text { 6. Verify that stack } \\
\text { 7. Initially set the in } \\
\text { 8ampling ports th } \\
\text { 8. Set the sampler } \mathrm{fl} \\
\text { 9. Conduct one or } \mathrm{m} \\
\\
\frac{\text { Stack Flow }}{\text { Norma1 }}\end{array}$ & $\begin{array}{l}\text { rocedure and that instr } \\
\text { Mechanical Pump Flui } \\
\text { tion from the Hanford } \\
\text { HMS/lastob.htm } \\
\text { ected in the procedure } \\
\text { the measurement po } \\
\text { about the target flow } \\
\text { system input psi at } 5 \\
\text { about } 10 \text { times backgr } \\
\text { at approximately } 10 \\
\text { cer mixing tests at the } \mathrm{f} \\
\text { ion point at duct from } \\
\text { rline }\end{array}$ & $\begin{array}{l}\text { ion is within calibration } \\
\text { Service, phone } 373-2716 \text { or } \\
\text { wn on the data form } \\
(2232 \mathrm{fpm}) \\
\text { to obtain particle counts at the } \\
10 \text {-micron particles. } \\
\text { sets of conditions: } \\
\text { ack }\end{array}$ \\
\hline \multicolumn{3}{|c|}{ 12. Diagram mounting fixtures and retain assembly for any subsequent re-tests } \\
\hline \multicolumn{3}{|l|}{ Approvals: } \\
\hline Test completed by: & & Date: \\
\hline
\end{tabular}




\section{Appendix $\mathbf{J}$}

\section{Particle Tracer Uniformity Data Sheets}




\section{APPENDIX J: PARTICLE TRACER UNIFORMITY DATA SHEETS}

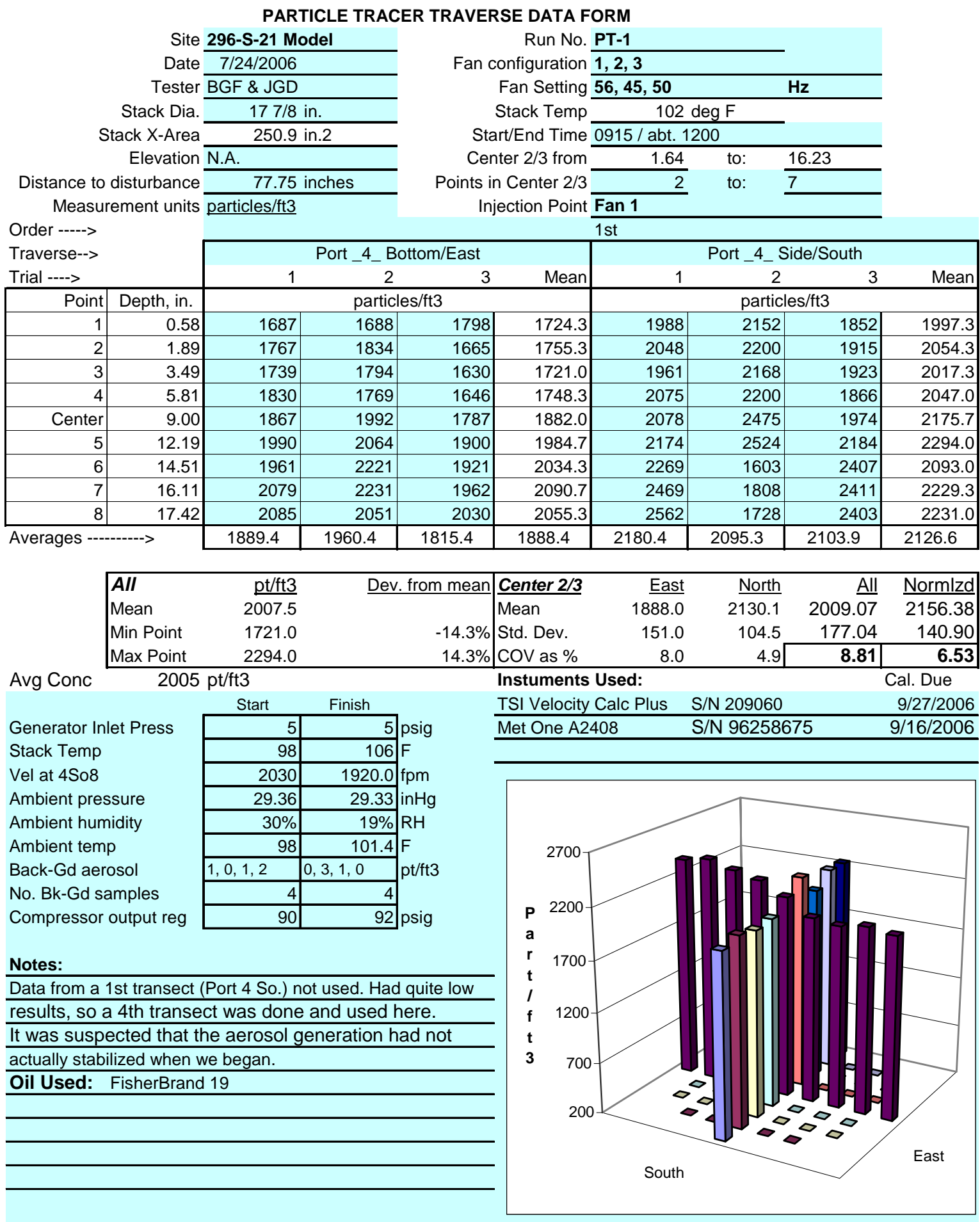

Signature signifies compliance with
Procedure EMS-JAG-02
Signature/date
Signature verifying data and calculations:

Signatures on file copies 
PARTICLE TRACER TRAVERSE DATA FORM

Site 296-S-21 Model

Date 7/24/2006

Tester JGD \& BGF

Stack Dia. $177 / 8$ in

Stack X-Area 250.9 in.2

Elevation N.A.

Distance to disturbance

Measurement units particles/ft3

77.75 inches
Run No. PT-2

Fan configuration 1,4

Fan Setting 56, 52

Stack Temp

1300/1450

Center 2/3 from

Points in Center 2/3

Injection Point Fan 1

Order ----->
Traverse-->

Trial ---->

\begin{tabular}{|r|r|}
\hline Point & Depth, in \\
\hline 1 & 0.58 \\
\hline 2 & 1.8 \\
\hline 3 & 3.4 \\
\hline 4 & 5.8 \\
\hline Center & 9.00 \\
\hline 5 & 12.1 \\
\hline 6 & 14.5 \\
\hline 7 & 16.1 \\
\hline 8 & \multicolumn{2}{|c|}{17.4} \\
\hline Averages --------->
\end{tabular}

1ST

\begin{tabular}{|ccc}
\hline & Port_4_Bottom/East \\
2 & 3
\end{tabular}

\begin{tabular}{|r|r|r|r|r|}
\hline & \multicolumn{4}{|c|}{ Port_4_Side/South } \\
\hline & 1 & \multicolumn{4}{|c|}{ particles/ft3 } \\
\hline 1443.7 & 1159 & 1512 & 1138 & 1269.7 \\
\hline 1476.3 & 1386 & 1554 & 1170 & 1370.0 \\
\hline 1485.7 & 1447 & 1426 & 1076 & 1316.3 \\
\hline 1545.7 & 1429 & 1418 & 1067 & 1304.7 \\
\hline 1585.7 & 1393 & 1465 & 1094 & 1317.3 \\
\hline 1651.7 & 1358 & 1507 & 1165 & 1343.3 \\
\hline 1703.3 & 1404 & 1662 & 1379 & 1481.7 \\
\hline 1744.7 & 1442 & 1885 & 1367 & 1564.7 \\
\hline 1681.7 & 1409 & 1559 & 1366 & 1444.7 \\
\hline 1590.9 & 1380.8 & 1554.2 & 1202.4 & 1379.1 \\
\hline
\end{tabular}

\begin{tabular}{|lrr|lrrrr|r|}
\hline All & $\mathrm{pt} / \mathrm{ft} 3$ & Dev. from mean & Center 2/3 & East & South & All & Normlzd \\
Mean & 1485.0 & & Mean & 1599.0 & 1385.4 & 1492.21 & 1633.32 \\
Min Point & 1269.7 & $-14.5 \%$ & Std. Dev. & 104.7 & 99.4 & 148.00 & 113.76 \\
Max Point & 1744.7 & $17.5 \%$ & COV as $\%$ & 6.5 & 7.2 & $\mathbf{9 . 9 2}$ & $\mathbf{6 . 9 7}$ \\
\hline
\end{tabular}

Avg Conc $1489 \mathrm{pt} / \mathrm{ft} 3$

Generator Inlet Press Stack Temp

Vel at $4 \mathrm{So8}$

Ambient pressure Ambient humidity Ambient temp

Back-Gd aerosol

No. Bk-Gd samples

Compressor output reg

\begin{tabular}{|c|c|c|}
\hline Start & Finish & \\
\hline 5 & & $5 \mathrm{ps}$ \\
\hline 107.5 & 108 & $8 \mathrm{~F}$ \\
\hline 2450 & 2440.0 & \\
\hline 29.3 & 29.27 & 7 \\
\hline $15 \%$ & $17 \%$ & $\mathrm{RH}$ \\
\hline 106 & 104 & \\
\hline $4,4,5,3$ & $1,2,1,0$ & per \\
\hline 4 & 4 & 4 \\
\hline 90 & 90 & 0 \\
\hline
\end{tabular}

Notes:

OPC flow adjusted to 1.00 at the start of each traverse

Oil Used: $\quad$ FisherBrand 19
Instuments Used:

\begin{tabular}{llr} 
TSI Velocity Calc Plus & S/N 209060 & 9/27/2006 \\
\hline Met One A2408 & S/N 96258675 & 9/16/2006
\end{tabular}

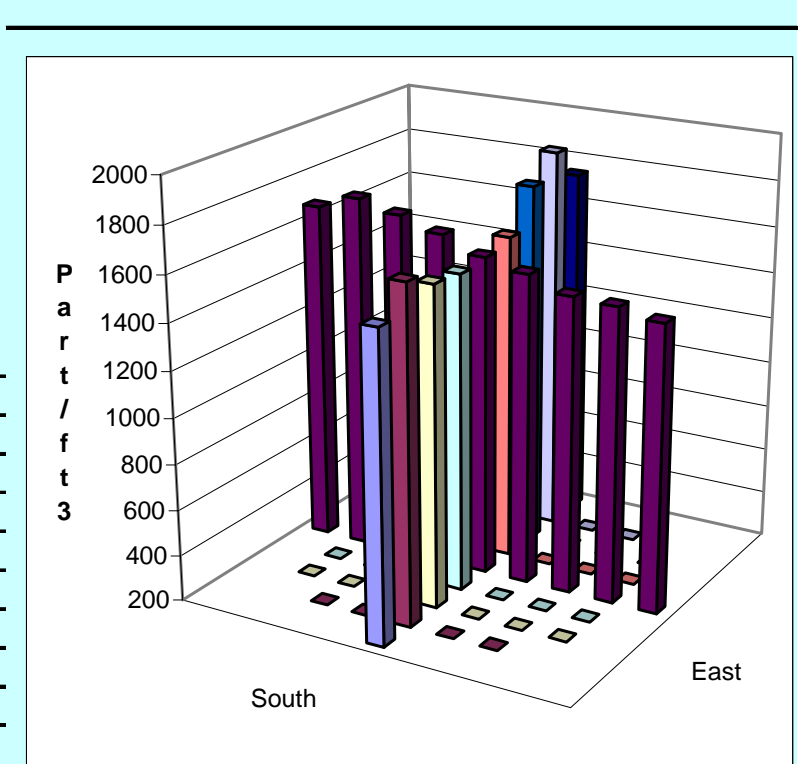

Signature signifies compliance with Procedure EMS-JAG-02

Signature/date
Signature verifying data and calculations:

Signatures on file copies 
PARTICLE TRACER TRAVERSE DATA FORM

Site 296-S-21 Model

Date 7/24/2006

Tester JAG \& BGF

Stack Dia. $177 / 8$ in.

Stack X-Area 250.9 in.2

Elevation N.A.

Distance to disturbance

Measurement units particles/ft3

77.75 inches
Run No. PT-3

Fan configuration $1,2,3$

Fan Setting $\mathbf{5 6 , 4 5 , 5 0}$

Stack Temp

$112.5 \operatorname{deg} \mathrm{F}$

Start/End Time 1500/1645

Center 2/3 from

Points in Center $2 / 3$

Injection Point Fan 2

Order ----->

Traverse-->

Trial ---->

\begin{tabular}{|r|r|}
\hline Point & Depth, in. \\
\hline 1 & 0.58 \\
\hline 2 & 1.89 \\
\hline 3 & 3.49 \\
\hline 4 & 5.81 \\
\hline Center & 9.00 \\
\hline 5 & 12.19 \\
\hline 6 & 14.51 \\
\hline 7 & 16.11 \\
\hline 8 & 17.42 \\
\hline
\end{tabular}

Averages ---------->

17.42

\begin{tabular}{|r|r|r|}
\hline \multicolumn{3}{|c|}{ Port_4_Bottom/East } \\
\hline 1 & \multicolumn{3}{|c|}{ particles/ft3 } \\
\hline 1159 & 1334 & 1473 \\
\hline 1124 & 1365 & 1553 \\
\hline 1154 & 1378 & 1442 \\
\hline 1195 & 1309 & 149 \\
\hline 1092 & 1211 & 140 \\
\hline 1061 & 1225 & 1238 \\
\hline 1017 & 1156 & 128 \\
\hline 1029 & 1234 & 1270 \\
\hline 996 & 1171 & 1196 \\
\hline 1091.9 & 1264.8 & 1371.7 \\
\hline
\end{tabular}

1ST

$\begin{array}{lll}\frac{1.64}{2} & \text { to: } & 16.23 \\ 2 & \text { to: } & \end{array}$

\begin{tabular}{|r|r|r|r|r|}
\cline { 2 - 5 } Mean & \multicolumn{4}{|c|}{ Port_4_Side/South } \\
& 1 & \multicolumn{3}{c|}{ p } \\
\hline 1322.0 & 1424 & 1115 & 1040 & 1193.0 \\
\hline 1347.3 & 1368 & 1034 & 1070 & 1157.3 \\
\hline 1324.7 & 1438 & 1037 & 1042 & 1172.3 \\
\hline 1331.7 & 1419 & 961 & 1140 & 1173.3 \\
\hline 1234.7 & 1423 & 1006 & 1163 & 1197.3 \\
\hline 1174.7 & 1310 & 1204 & 1151 & 1221.7 \\
\hline 1151.3 & 1382 & 1153 & 1138 & 1224.3 \\
\hline 1177.7 & 1324 & 1058 & 1114 & 1165.3 \\
\hline 1121.0 & 1154 & 897 & 789 & 946.7 \\
\hline 1242.8 & 1360.2 & 1051.7 & 1071.9 & 1161.3 \\
\hline
\end{tabular}

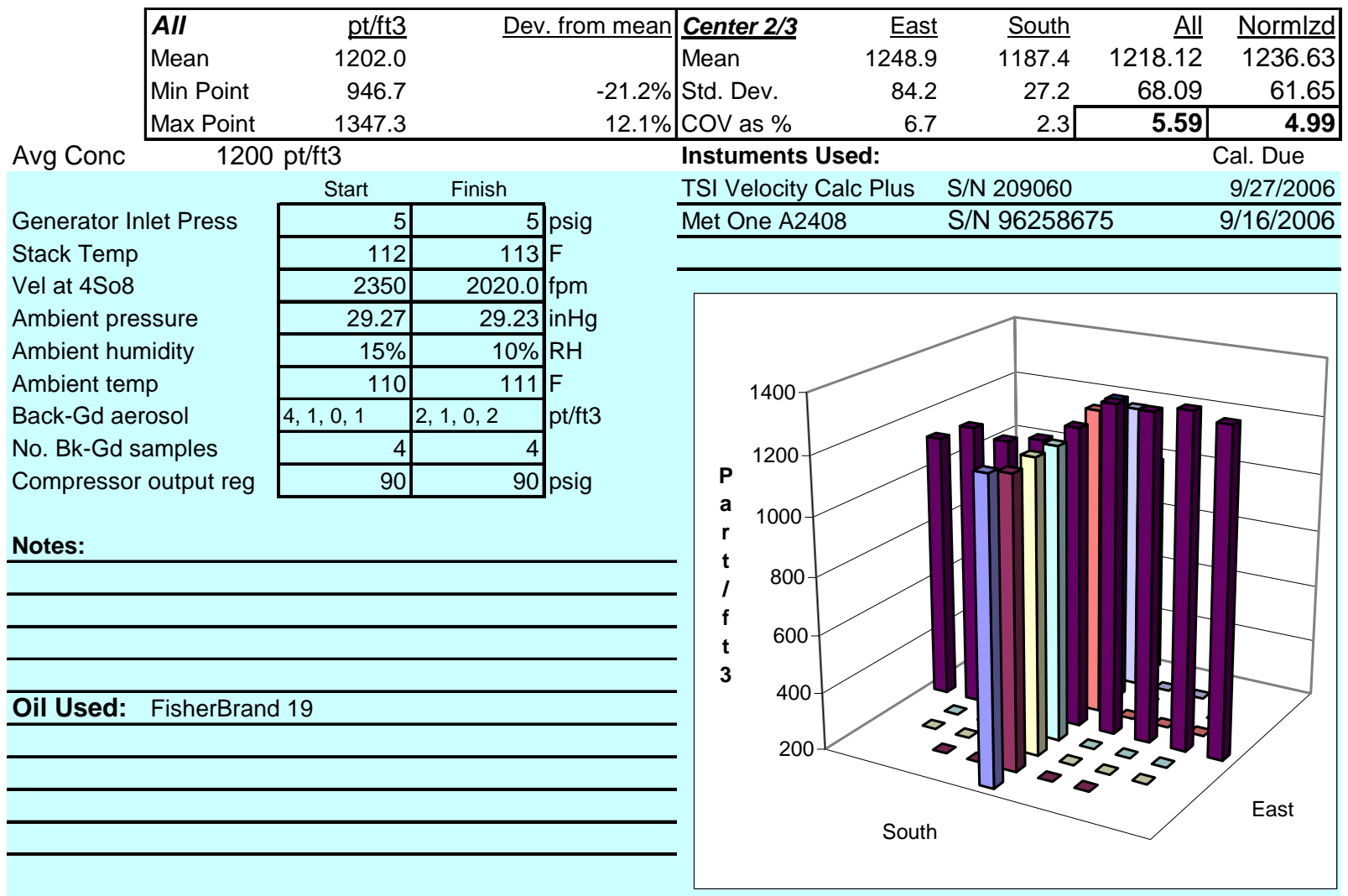

Signature signifies compliance with Procedure EMS-JAG-02

Signature/date
Signature verifying data and calculations:

Signatures on file copies 


\section{PARTICLE TRACER TRAVERSE DATA FORM}

Site 296-S-21 Model

Date 7/25/2006

Tester JAG \& JGD

Stack Dia. $177 / 8$ in.

Stack X-Area 250.9 in.2

Elevation N.A.

Distance to disturbance

Measurement units particles/ft3
Run No. PT-4

Fan configuration $1,2,3$

Fan Setting $\mathbf{5 6 , \mathbf { 4 5 } , \mathbf { 5 0 }}$

Stack Temp

Start/End Time 0750/1025

Center $2 / 3$ from

Points in Center 2/3

Injection Point Fan 3

Order ----->

Traverse-->

Trial ---->

1ST

\begin{tabular}{|c|c|}
\hline Point & Depth, in. \\
\hline 1 & 0.58 \\
\hline 2 & 1.89 \\
\hline 3 & 3.49 \\
\hline 4 & 5.81 \\
\hline Center & 9.00 \\
\hline 5 & 12.19 \\
\hline 6 & 14.51 \\
\hline 7 & 16.11 \\
\hline 8 & 17.42 \\
\hline
\end{tabular}

Averages ---------->

\begin{tabular}{|c|c|c|}
\hline \multicolumn{3}{|c|}{ Port_4_Bottom/East } \\
\hline 1 & 2 & \\
\hline \multicolumn{3}{|c|}{ particles/ft3 } \\
\hline 1132 & 1043 & 96 \\
\hline 1146 & 1089 & 105 \\
\hline 1197 & 1077 & 101 \\
\hline 1033 & 1082 & 101 \\
\hline 1073 & 1072 & 103 \\
\hline 1100 & 1042 & 103 \\
\hline 1105 & 1066 & 112 \\
\hline 1135 & 1192 & 116 \\
\hline 1125 & 1107 & 98 \\
\hline 1116.2 & 1085.6 & 1042.3 \\
\hline
\end{tabular}

$90 \operatorname{deg} \mathrm{F}$

$\mathrm{Hz}$

25

1.64 to: 16.23

to: $\frac{16}{7}$

\begin{tabular}{|c|c|c|c|c|c|c|c|}
\hline$A I I$ & $\mathrm{pt} / \mathrm{ft} 3$ & Dev. from mean & Center 2/3 & East & South & All & $\underline{\text { Normlzd }}$ \\
\hline Mean & 1019.3 & & Mean & 1088.0 & 967.9 & 1027.93 & 1076.74 \\
\hline Min Point & 858.7 & $-15.8 \%$ & Std. Dev. & 40.2 & 27.3 & 70.49 & 36.02 \\
\hline Max Point & 1164.3 & $14.2 \%$ & COV as $\%$ & 3.7 & 2.8 & 6.86 & 3.35 \\
\hline
\end{tabular}

Avg Conc 1020 pt/ft3

\begin{tabular}{|c|c|c|c|}
\hline \multirow[b]{2}{*}{ Generator Inlet Press } & Start & Finish & \\
\hline & 5 & 5 & psig \\
\hline Stack Temp & 86 & 94 & $F$ \\
\hline \multirow{2}{*}{$\begin{array}{l}\text { Vel at } 4 \mathrm{So} 8 \\
\text { Ambient pressure }\end{array}$} & 2070 & 2020.0 & $\mathrm{fpm}$ \\
\hline & 29.367 & 29.36 & $\mathrm{inHg}$ \\
\hline \multirow{2}{*}{$\begin{array}{l}\text { Ambient humidity } \\
\text { Ambient temp }\end{array}$} & $42 \%$ & $25 \%$ & $\mathrm{RH}$ \\
\hline & 81 & 91 & $F$ \\
\hline \multirow{3}{*}{$\begin{array}{l}\text { Back-Gd aerosol } \\
\text { No. Bk-Gd samples } \\
\text { Compressor output reg }\end{array}$} & $0,0,0,0$ & $3,11,2,4$ & $\mathrm{pt} / \mathrm{ft} 3$ \\
\hline & 4 & 4 & \\
\hline & 90 & 90 & psig \\
\hline
\end{tabular}

Notes:

Added 1/3 L oil to aerosol generator after 1st traverse of Port4South. Took an extra column of data so 1st column can be deleted.

Oil Used: FisherBrand 19

\section{Instuments Used:}

TSI Velocity Calc Plus S/N 209060 Cal. Due

Met One A2408 S/N 96258675 $\quad 9 / 16 / 2006$

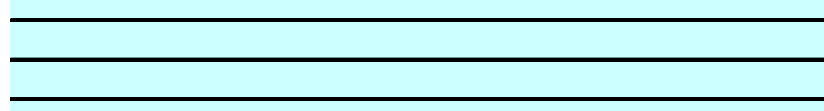

3- Port_4_Side/South

\begin{tabular}{|r|r|r|r|r|r|}
\hline \multicolumn{2}{|c|}{} & \multicolumn{4}{|c|}{ particles/ft3 } \\
\hline 62 & 1045.7 & 987 & 1018 & 939 & 981.3 \\
\hline 10 & 1095.0 & 1077 & 995 & 945 & 1005.7 \\
\hline 14 & 1094.7 & 1053 & 1004 & 905 & 987.3 \\
\hline 31 & 1043.0 & 990 & 972 & 873 & 945.0 \\
\hline 39 & 1060.3 & 1011 & 974 & 945 & 976.7 \\
\hline 66 & 1099.7 & 1065 & 975 & 886 & 975.3 \\
\hline 81 & 1164.3 & 969 & 930 & 872 & 923.7 \\
\hline & 1071.0 & 971 & 736 & 869 & 858.7 \\
\hline
\end{tabular}




\section{PARTICLE TRACER TRAVERSE DATA FORM}

Site 296-S-21 Model

Date 7/25/2006

Tester BGF

Stack Dia.

Stack X-Area

Elevation N.A.

Distance to disturbance

Measurement units particles/ft3
Run No. PT-5

Fan configuration 3,4

Fan Setting $\mathbf{5 0 , 5 2}$

Stack Temp

Start/End Time 1050/

Center $2 / 3$ from

Points in Center 2/3

Injection Point Fan 3

Order ----->

Traverse-->

Trial ---->

\begin{tabular}{|r|r|}
\hline Point & Depth, in. \\
\hline 1 & 0.58 \\
\hline 2 & 1.89 \\
\hline 3 & 3.49 \\
\hline 4 & 5.81 \\
\hline Center & 9.00 \\
\hline 5 & 12.19 \\
\hline 6 & 14.51 \\
\hline 7 & 16.11 \\
\hline 8 & 17.42 \\
\hline
\end{tabular}

Averages ---------->

250.9 in. 2

7.75 inches

3

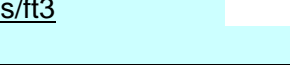

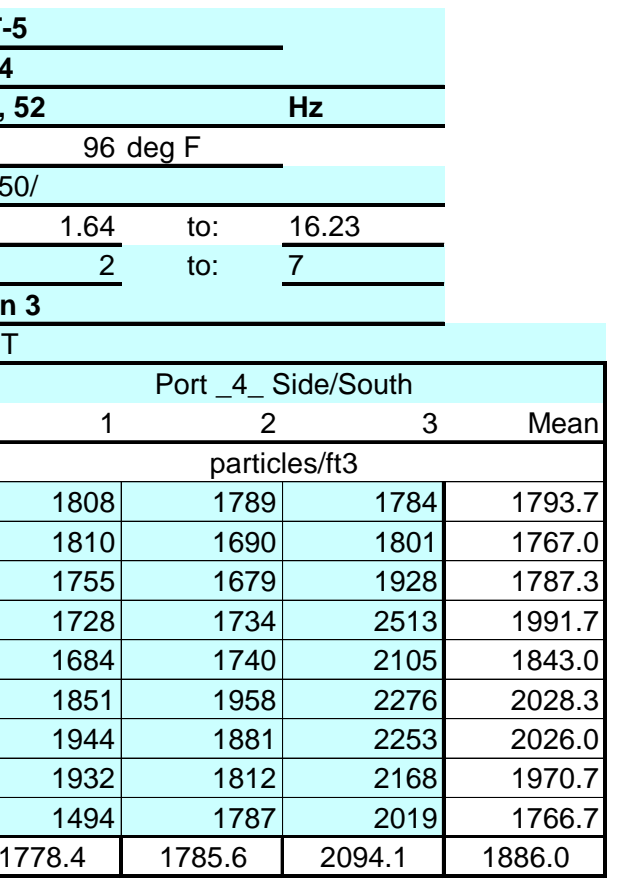

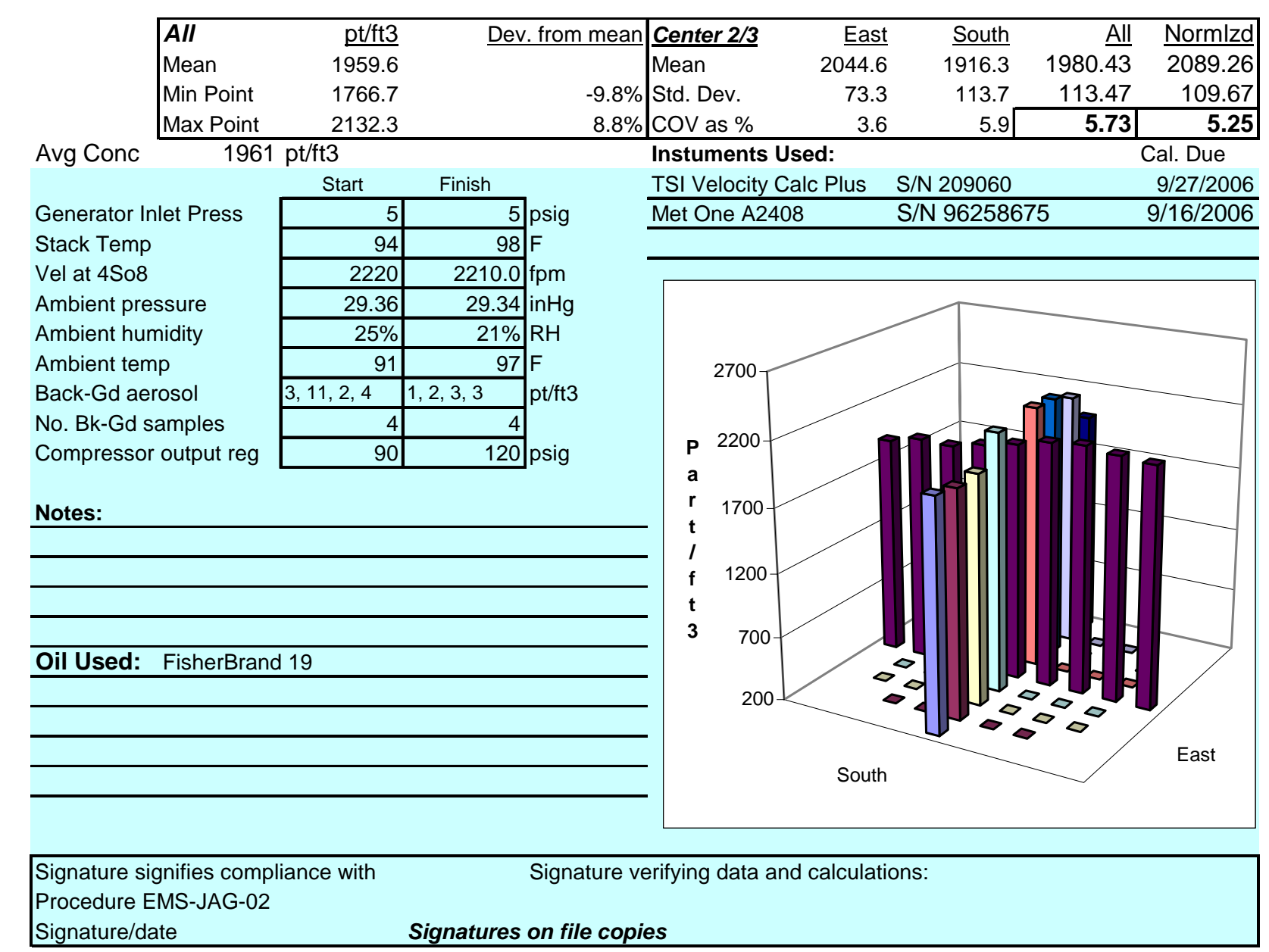




\section{PARTICLE TRACER TRAVERSE DATA FORM}

Site 296-S-21 Model

Date 7/25/2006

Tester BGF

Stack Dia.

Stack X-Area

Elevation N.A.

Distance to disturbance

Measurement units particles/ft3
Run No. PT-6

Fan configuration 2,4

Fan Setting 45, 52

Stack Temp

Start/End Time 1300/1425

Center $2 / 3$ from

Points in Center $2 / 3$

Injection Point Fan 2

Order ----->

Traverse-->

Trial ---->

\begin{tabular}{|r|r|} 
Trial ----> \\
\hline Point & Depth, in. \\
\hline 1 & 0.58 \\
\hline 2 & 1.89 \\
\hline 3 & 3.49 \\
\hline 4 & 5.81 \\
\hline Center & 9.00 \\
\hline 5 & 12.19 \\
\hline 6 & 14.51 \\
\hline 7 & 16.11 \\
\hline 8 & 17.42 \\
\hline
\end{tabular}

1ST

Averages ---------->

\begin{tabular}{lr}
\hline All & $\mathrm{pt} / \mathrm{ft}$ \\
Mean & 1890. \\
Min Point & 1534. \\
Max Point & 2354. \\
\hline
\end{tabular}

Avg Conc

$$
1887 \mathrm{pt} / \mathrm{ft} 3
$$

Generator Inlet Press

Stack Temp

Vel at $4 \mathrm{So} 8$

Ambient pressure

Ambient humidity

Ambient temp

Back-Gd aerosol

No. Bk-Gd samples

Compressor output reg

\begin{tabular}{|c|c|c|}
\hline Start & Finish & \\
\hline 5 & \multicolumn{2}{|l|}{5} \\
\hline 104 & 106 & \\
\hline 2150 & 2200.0 & \\
\hline 29.33 & 29.3 & \\
\hline $16 \%$ & $17 \%$ & \\
\hline 103 & 104 & \\
\hline $2,0,3,0$ & $2,0,0,0$ & \\
\hline 4 & 4 & \\
\hline 90 & 90 & \\
\hline
\end{tabular}

Notes:

Oil Used: $\quad$ FisherBrand 19

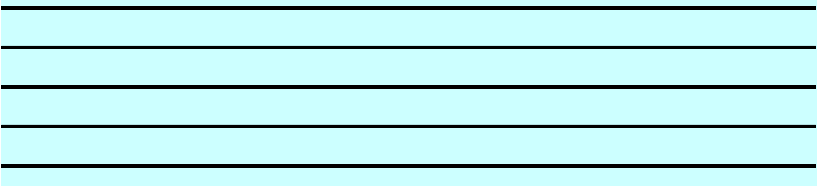

Signature signifies compliance with Procedure EMS-JAG-02

Signature/date
Signature verifying data and calculations:

Signatures on file copies 
PARTICLE TRACER TRAVERSE DATA FORM

Site 296-S-21 Model

Date 7/25/2006

Tester BGF

Stack Dia.

Stack X-Area

Elevation N.A.

Distance to disturbance

Measurement units particles/ft3
Run No. PT-7

Fan configuration $\mathbf{1 , 4}$

Fan Setting $\mathbf{5 6 , 5 2}$

Stack Temp

Start/End Time 1440/1600

Center $2 / 3$ from

Points in Center 2/3

Injection Point Fan 4

Order ----->

Traverse-->

Trial ---->

\begin{tabular}{|r|r|}
\hline Point & Depth, in \\
\hline 1 & 0.58 \\
\hline 2 & 1.89 \\
\hline 3 & 3.49 \\
\hline 4 & 5.81 \\
\hline Center & 9.00 \\
\hline 5 & 12.19 \\
\hline 6 & 14.51 \\
\hline 7 & 16.11 \\
\hline 8 & 17.42 \\
\hline
\end{tabular}

Averages ---------->

17.42

\begin{tabular}{r|r}
\cline { 2 - 2 } & \\
\hline & \\
\hline 0.58 & 153 \\
1.89 & 1 \\
\hline 3.49 & 135 \\
\hline 5.81 & 1 \\
\hline 9.00 & 1 \\
\hline 2.19 & 1 \\
\hline 4.51 & 128 \\
\hline 6.11 & 127 \\
\hline 7.42 & 120 \\
& 1310.8 \\
\hline
\end{tabular}

Port 4

$106.5 \operatorname{deg} \mathrm{F}$

1600

to: 16.23

2 to: 7

1ST

\begin{tabular}{|c|c|c|c|c|c|c|c|}
\hline$A I I$ & $\mathrm{pt} / \mathrm{ft} 3$ & Dev. from mean & Center 2/3 & East & South & All & Normlzd \\
\hline Mean & 1749.5 & & Mean & 1294.5 & 2209.6 & 1752.05 & 2246.93 \\
\hline Min Point & 1161.0 & $-33.6 \%$ & Std. Dev. & 37.7 & 78.7 & 478.48 & 80.01 \\
\hline Max Point & 2340.7 & $33.8 \%$ & cov as $\%$ & 2.9 & 3.6 & 27.31 & 3.56 \\
\hline
\end{tabular}

Avg Conc $1751 \mathrm{pt} / \mathrm{ft} 3$

1 Port_4_Bottom/East

3 Mean $\quad$ Port_4_Side/South

\begin{tabular}{|l|l|l|}
\hline \multicolumn{2}{|l|}{ particles/ft3 } & \multicolumn{3}{c}{1} \\
\hline
\end{tabular}

\begin{tabular}{|l|l|l|l|r|r|r|r|}
\hline 532 & 1380 & 1256 & 1389.3 & 2169 & 2294 & 2252 & 2238.3 \\
\hline 289 & 1321 & 1238 & 1282.7 & 2060 & 2279 & 2200 & 2179.7 \\
\hline 354 & 1408 & 1313 & 1358.3 & 2118 & 2223 & 2096 & 2145.7 \\
\hline 307 & 1417 & 1275 & 1333.0 & 2079 & 2190 & 2092 & 2120.3 \\
\hline 233 & 1324 & 1215 & 1257.3 & 2012 & 2292 & 2352 & 2218.7 \\
\hline 280 & 1323 & 1237 & 1294.0 & 2030 & 2483 & 2015 & 2176.0 \\
\hline 276 & 1190 & 1320 & 1263.3 & 2058 & 2473 & 2327 & 2286.0 \\
\hline 8 & 1253 & 1290 & 1273.0 & 2080 & 2655 & 2287 & 2340.7 \\
\hline
\end{tabular}

Generator Inlet Press

Stack Temp

Vel at $4 \mathrm{So} 8$

Ambient pressure

Ambient humidity

Ambient temp

Back-Gd aerosol

No. Bk-Gd samples

Compressor output reg

\begin{tabular}{|c|c|c|}
\hline Start & Finish & \\
\hline 5 & 5 & 5 psig \\
\hline 106 & 107 & $7 \mathrm{~F}$ \\
\hline 2240 & 2200.0 & $0 \mathrm{fpm}$ \\
\hline 29.3 & 29.27 & $7 \mathrm{inHg}$ \\
\hline $17 \%$ & $11 \%$ & $\mathrm{RH}$ \\
\hline 104 & 105 & $5 \mid F$ \\
\hline $1,3,0,3$ & $1,0,1,0$ & $\mathrm{pt} / \mathrm{ft} 3$ \\
\hline 4 & 4 & 4 \\
\hline 100 & 90 & 0 psig \\
\hline
\end{tabular}

Instuments Used:

\begin{tabular}{llr} 
TSI Velocity Calc Plus & S/N 209060 & 9/27/2006 \\
\hline Met One A2408 & S/N 96258675 & 9/16/2006 \\
\hline
\end{tabular}

Notes:

4th traverse done on bottom port because the

concentrations from the 2nd and 3rd traverses

were markedly lower than the concentrations

during the first traverse. The 4th traverse data were used.

Oil Used: FisherBrand 19

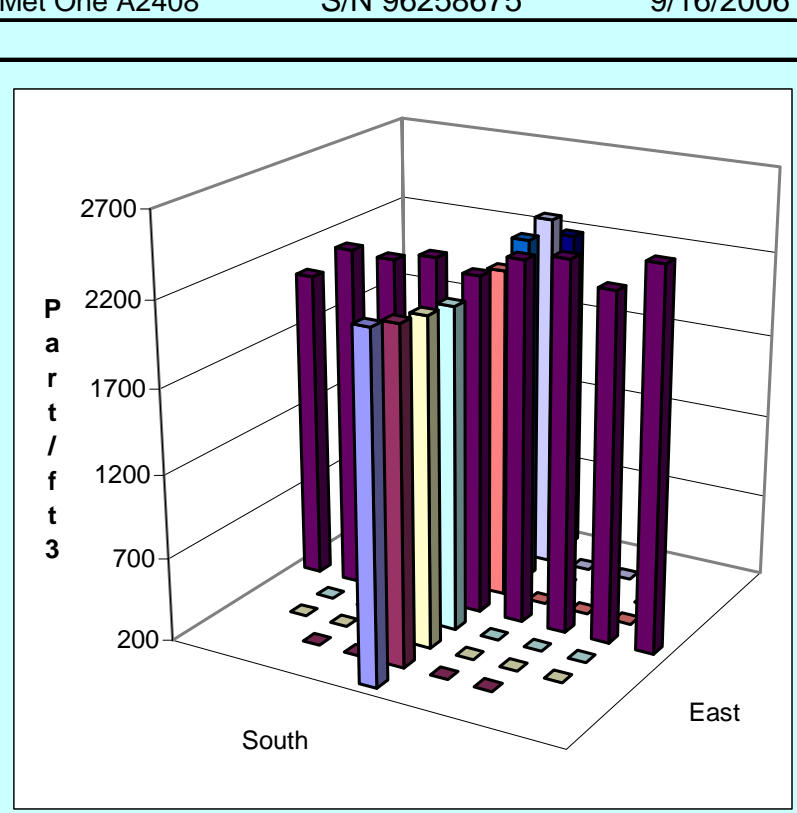

Signature signifies compliance with

Procedure EMS-JAG-02

Signature/date
Signature verifying data and calculations:

Signatures on file copies 
PARTICLE TRACER TRAVERSE DATA FORM

Site 296-S-21 Model

Date 7/25/2006

Tester BGF

Stack Dia.

Stack X-Area

Elevation N.A

Distance to disturbance

Measurement units particles/ft3
Run No. PT-8

Fan configuration 2,4

Fan Setting 45, 52

Stack Temp

1405/1535

Center 2/3 from

Points in Center 2/3

\begin{tabular}{lll}
\hline 1.64 & to: & 16.23 \\
\hline Fan 4 & to: & \\
\hline
\end{tabular}

Injection Point Fan 4

Order ----->

Trial ---->

\begin{tabular}{|r|r|}
\hline Point & Depth, in \\
\hline 1 & 0.58 \\
\hline 2 & 1.89 \\
\hline 3 & 3.49 \\
\hline 4 & 5.81 \\
\hline Center & 9.00 \\
\hline 5 & 12.1 \\
\hline 6 & 14.51 \\
\hline 7 & 16.11 \\
\hline 8 & \multicolumn{2}{|c|}{17.4} \\
\hline Averages -------->
\end{tabular}

1ST

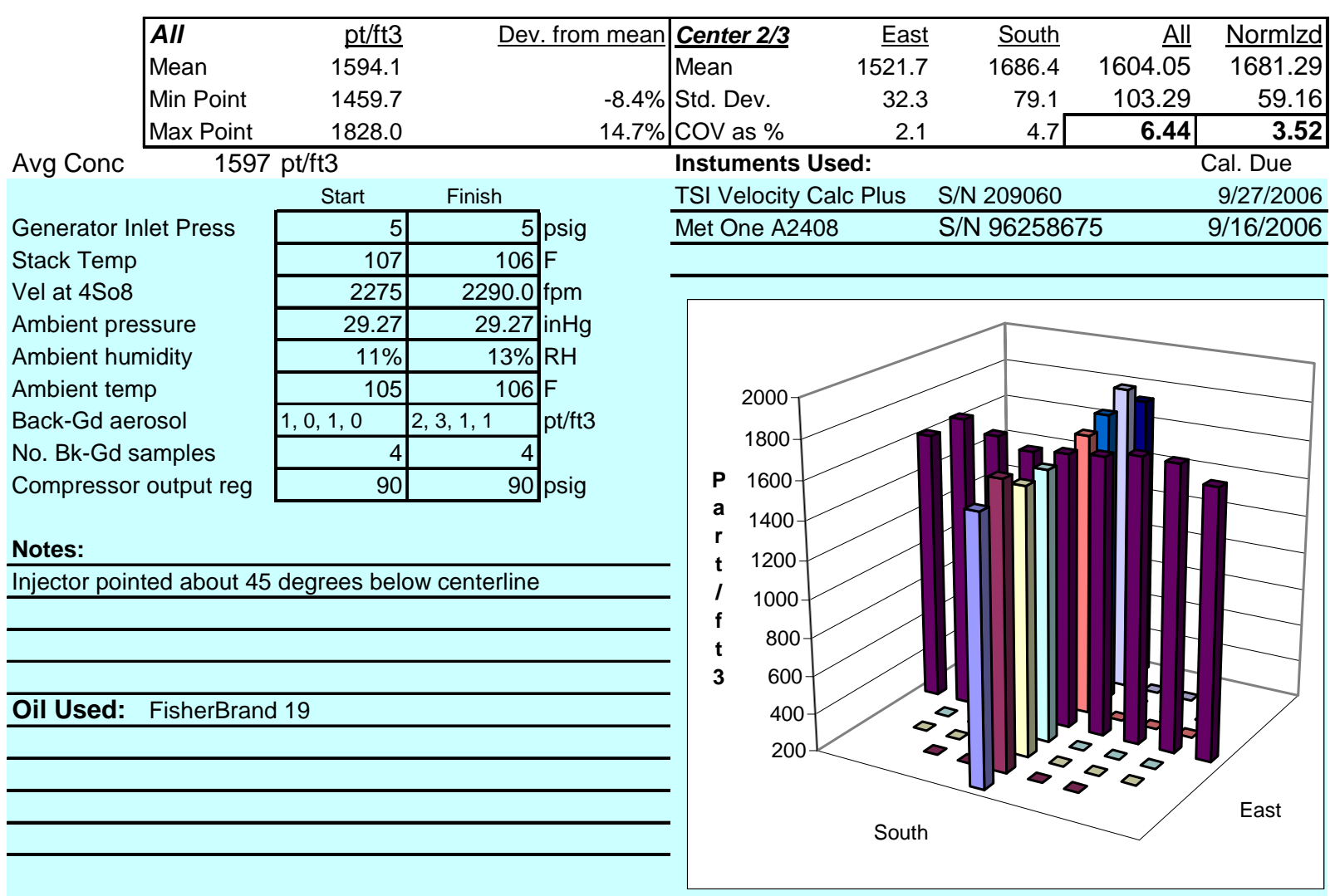

Signature signifies compliance with Procedure EMS-JAG-02

Signature/date
Signature verifying data and calculations:

Signatures on file copies 
PARTICLE TRACER TRAVERSE DATA FORM

Site 296-S-21 Model

Date 7/26/2006

Tester BGF JAG JGD

Stack Dia. $177 / 8$ in.

Stack X-Area 250.9 in.2

Elevation N.A.

Distance to disturbance

Measurement units particles/ft3
Run No. PT-9

Fan configuration 3,4

Fan Setting $\mathbf{5 0 , 5 2}$

Stack Temp

Start/End Time 0900/1020

Center 2/3 from

Points in Center 2/3

Injection Point Fan 4

Order ----->

Traverse-->

Trial ---->

\begin{tabular}{|r|r|}
\hline Point & Depth, in \\
\hline 1 & 0.58 \\
\hline 2 & 1.89 \\
\hline 3 & 3.49 \\
\hline 4 & 5.81 \\
\hline Center & 9.00 \\
\hline 5 & 12.1 \\
\hline 6 & 14.5 \\
\hline 7 & 16.1 \\
\hline 8 & \multicolumn{2}{|c}{17.4} \\
\hline Averages -------->
\end{tabular}

77.75 inches

Port 4 Bd

\author{
Inje
}

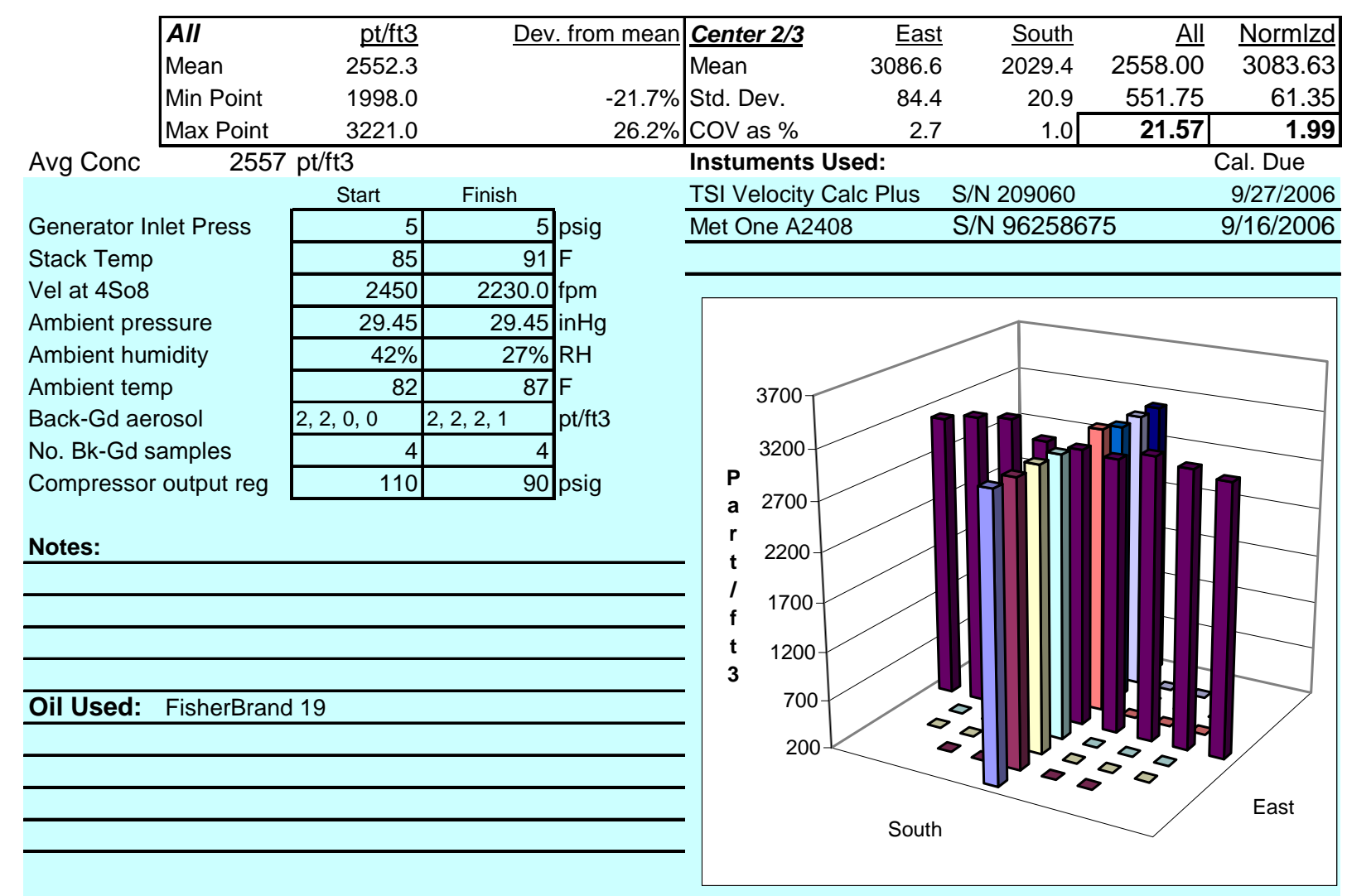

Signature signifies compliance with Procedure EMS-JAG-02

Signature/date

Signature verifying data and calculations:

Signatures on file copies 


\section{PARTICLE TRACER TRAVERSE DATA FORM}

Site 296-S-21 Model

Date 7/26/2006

Tester BGF

Stack Dia.

Stack X-Area

Elevation N.A.

Distance to disturbance

Measurement units particles/ft3
Run No. PT-10

Fan configuration 4

Fan Setting $\mathbf{5 2}$

Stack Temp

Start/End Time 1020/1145

Center $2 / 3$ from

Points in Center $2 / 3$

Injection Point Fan 4

Order ----->

Traverse-->

Trial ---->

\begin{tabular}{|r|r|}
\hline Point & Depth, in. \\
\hline 1 & 0.58 \\
\hline 2 & 1.89 \\
\hline 3 & 3.49 \\
\hline 4 & 5.81 \\
\hline Center & 9.00 \\
\hline 5 & 12.19 \\
\hline 6 & 14.51 \\
\hline 7 & 16.11 \\
\hline 8 & 17.42 \\
\hline
\end{tabular}

1ST

Averages ---------->

\begin{tabular}{|c|c|c|c|}
\hline$A I I$ & $\mathrm{pt} / \mathrm{ft} 3$ & $\underline{\mathrm{Dev}}$ & v. from \\
\hline Mean & 1111.6 & & \\
\hline Min Point & 1009.3 & & \\
\hline Max Point & 1198.7 & & \\
\hline \multicolumn{4}{|c|}{$1119 \mathrm{pt} / \mathrm{ft} 3$} \\
\hline & Start & Finish & \\
\hline \multirow[t]{3}{*}{ alet Press } & 2 & 2 & psig \\
\hline & 91 & 93 & $F$ \\
\hline & 1560 & 1570.0 & $\mathrm{fpm}$ \\
\hline \multirow{3}{*}{$\begin{array}{l}\text { sure } \\
\text { iidity }\end{array}$} & 29.45 & 29.4 & in $\mathrm{Hg}$ \\
\hline & $27 \%$ & $20 \%$ & $\mathrm{RH}$ \\
\hline & 87 & 90 & $F$ \\
\hline \multirow{3}{*}{ sol } & $1,3,2,1$ & $4,3,0,1$ & $\mathrm{pt} / \mathrm{ft} 3$ \\
\hline & 4 & 4 & \\
\hline & 90 & 90 & psig \\
\hline
\end{tabular}

Avg Conc

Generator Inlet Press

Stack Temp

Vel at $4 \mathrm{So} 8$

Ambient pressure

Ambient humidity

Ambient temp

Back-Gd aerosol

No. Bk-Gd samples

Compressor output reg

Notes:

5 psi on generator inlet resulted in concentrations $>4000 \mathrm{pt} / \mathrm{ft} 3$. Regulator was adjusted down to $\sim 2 \mathrm{psig}$

Oil Used: $\quad$ FisherBrand 19

Signature signifies compliance with Procedure EMS-JAG-02

Signature/date
Signature verifying data and calculations:

Signatures on file copies 


\section{PARTICLE TRACER TRAVERSE DATA FORM}

Site 296-S-21 Model

Date 7/26/2006

Tester BGF

Stack Dia.

Stack X-Area

Elevation N.A.

Distance to disturbance

Measurement units particles/ft3
Run No. PT-11

Fan configuration 1,4

Fan Setting $\mathbf{5 6 , 5 2}$

Stack Temp

Start/End Time 1530/1710

Center $2 / 3$ from

Points in Center 2/3

Injection Point Fan 4
Repeat of PT-7

Order ----->

Traverse-->

Trial ---->

\begin{tabular}{|r|r|}
\hline Point & Depth, in. \\
\hline 1 & 0.58 \\
\hline 2 & 1.89 \\
\hline 3 & 3.49 \\
\hline 4 & 5.81 \\
\hline Center & 9.00 \\
\hline 5 & 12.19 \\
\hline 6 & 14.51 \\
\hline 7 & 16.11 \\
\hline 8 & 17.42 \\
\hline
\end{tabular}

1ST

\begin{tabular}{|c|c|c|}
\hline \multicolumn{3}{|c|}{ Port_4_Bottom/Eas } \\
\hline 1 & 2 & \\
\hline \multicolumn{3}{|c|}{ particles/ft3 } \\
\hline 2058 & 1862 & 166 \\
\hline 2127 & 1943 & 168 \\
\hline 2109 & 1882 & 171 \\
\hline 2028 & 1788 & 165 \\
\hline 1975 & 1773 & 165 \\
\hline 1980 & 1668 & 164 \\
\hline 1838 & 1758 & 156 \\
\hline 1818 & 1732 & 147 \\
\hline 1706 & 1528 & 147 \\
\hline 1959.9 & 1770.4 & 1614.8 \\
\hline
\end{tabular}

Averages --------->

$\mathrm{Hz}$

710

10

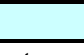

2

to: $\quad 16.23$

\begin{tabular}{|c|c|c|c|c|}
\hline \multirow[b]{2}{*}{ Mean } & \multicolumn{4}{|c|}{ Port_4_Side/South } \\
\hline & 1 & 2 & 3 & Mean \\
\hline & \multicolumn{4}{|c|}{ particles/ft3 } \\
\hline 1863.0 & 1605 & 1785 & 1532 & 1640.7 \\
\hline 1918.0 & 1650 & 1876 & 1616 & 1714.0 \\
\hline 1900.7 & 1682 & 1700 & 1478 & 1620.0 \\
\hline 1823.3 & 1549 & 1713 & 1433 & 1565.0 \\
\hline 1800.0 & 1797 & 1772 & 1430 & 1666.3 \\
\hline 1764.3 & 1464 & 1789 & 1576 & 1609.7 \\
\hline 1721.0 & 1425 & 1811 & 1716 & 1650.7 \\
\hline 1676.3 & 1402 & 1704 & 1567 & 1557.7 \\
\hline 1568.7 & 1663 & 1576 & 1376 & 1538.3 \\
\hline 1781.7 & 1581.9 & 1747.3 & 1524.9 & 1618.0 \\
\hline
\end{tabular}

Avg Conc $1696 \mathrm{pt} / \mathrm{ft} 3$

\begin{tabular}{|lr}
\hline All & $\mathrm{pt} / \mathrm{ft} 3$ \\
Mean & 1699.9 \\
Min Point & 1538.3 \\
Max Point & 1918.0 \\
\hline \multicolumn{2}{r}{$1696 \mathrm{pt} / \mathrm{ft} 3$}
\end{tabular}

Generator Inlet Press

Stack Temp

Vel at $4 \mathrm{So} 8$

Ambient pressure

Ambient humidity

Ambient temp

Back-Gd aerosol

No. Bk-Gd samples

Compressor output reg

\begin{tabular}{|c|c|c|}
\hline Start & Finish & \\
\hline 4 & & 4 psig \\
\hline 101 & 102 & $2 \mathrm{~F}$ \\
\hline 2230 & 2250.0 & $0 \mathrm{fpm}$ \\
\hline - & - & $\mathrm{inHg}$ \\
\hline $19 \%$ & $20 \%$ & $\mathrm{RH}$ \\
\hline 100 & 97 & $7 \mathrm{~F}$ \\
\hline $4,4,5,2$ & $0,2,0,1$ & $\mathrm{pt} / \mathrm{ft} 3$ \\
\hline 4 & 4 & 4 \\
\hline 90 & 60 & 0 psig \\
\hline
\end{tabular}

Notes:

Called met station at beginning of test, but forgot to record. Did not bother tocall for end pressure. Retrieve from HMS later.

Oil Used: $\quad$ FisherBrand 19
Signature verifying data and calculations:

Signatures on file copies
Procedure EMS-JAG-02

Signature/date

\begin{tabular}{|c|c|c|c|}
\hline East & South & All & Normlzd \\
\hline 1800.5 & $\overline{1626.2}$ & $1713 . \overline{36}$ & $\overline{1778.58}$ \\
\hline 88.9 & 55.8 & 115.18 & 76.43 \\
\hline 4.9 & 3.4 & 6.72 & 4.30 \\
\hline
\end{tabular}

Instuments Used: Cal. Due

TSI Velocity Calc Plus S/N $209060 \quad$ 9/27/2006

Met One A2408 $\quad$ S/N 96258675 $\quad$ 9/16/2006

South 


\section{PARTICLE TRACER TRAVERSE DATA FORM}

Site 296-S-21 Model

Date 7/27/2006

Tester JAG \& JGD

Stack Dia. $177 / 8$ in

Stack X-Area 250.9 in.2

Elevation N.A.

Distance to disturbance

Measurement units particles/ft3
Run No. PT-12

Fan configuration 3,4

Fan Setting $\mathbf{5 0 , 5 2}$

Stack Temp

0840/1050

Center 2/3 from

Points in Center 2/3
Injection Point Fan 4

Repeat of PT-9

Order ----->

Traverse-->

Trial ---->
77.75 inches

Pot 4 Bo

$\begin{array}{lll}\frac{1.64}{2} & \text { to: } & 16.23 \\ 4 & \text { to: } & \end{array}$

Port 4 Side/South

\begin{tabular}{|c|c|c|c|c|c|c|c|c|c|}
\hline \multirow{2}{*}{\multicolumn{2}{|c|}{ Trial ----> }} & \multirow{2}{*}{\multicolumn{4}{|c|}{ A }} & \\
\hline & & & & 3 & Mean & 1 & 2 & 3 & Mean \\
\hline Point & Depth, in. & \multicolumn{4}{|c|}{ particles/ft3 } & \multicolumn{4}{|c|}{ particles/ft3 } \\
\hline 1 & 0.58 & 1019 & 1170 & 1042 & 1077.0 & 1217 & 1137 & 1201 & 1185.0 \\
\hline 2 & 1.89 & 1186 & 1204 & 1088 & 1159.3 & 1204 & 1167 & 1094 & 1155.0 \\
\hline 3 & 3.49 & 1205 & 1163 & 1113 & 1160.3 & 1224 & 1190 & 1099 & 1171.0 \\
\hline 4 & 5.81 & 1240 & 1176 & 1127 & 1181.0 & 1256 & 1198 & 1180 & 1211.3 \\
\hline Center & 9.00 & 1225 & 1080 & 1006 & 1103.7 & 1233 & 1200 & 1112 & 1181.7 \\
\hline 5 & 12.19 & 1218 & 1113 & 1105 & 1145.3 & 1336 & 1227 & 1179 & 1247.3 \\
\hline 6 & 14.51 & 1211 & 1208 & 1149 & 1189.3 & 1319 & 1260 & 1262 & 1280.3 \\
\hline 7 & 16.11 & 1208 & 1128 & 1143 & 1159.7 & 1282 & 1227 & 1235 & 1248.0 \\
\hline 8 & 17.42 & 1210 & 1103 & 1147 & 1153.3 & 1183 & 1161 & 1206 & 1183.3 \\
\hline Averages --- & $-->$ & 1191.3 & 1149.4 & 1102.2 & 1147.7 & 1250.4 & 1196.3 & 1174.2 & 1207.0 \\
\hline
\end{tabular}

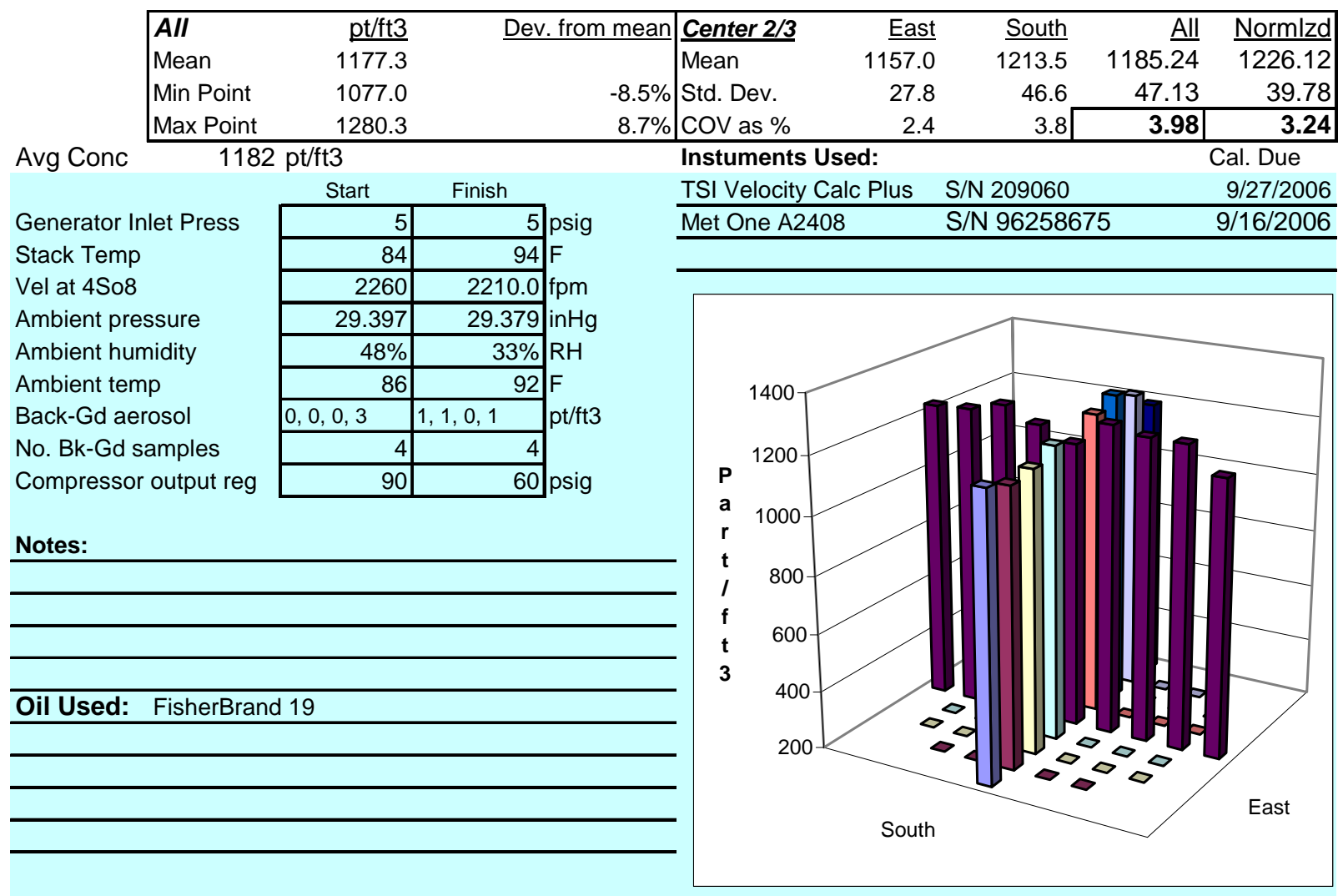

Signature signifies compliance with

Signature verifying data and calculations:

Procedure EMS-JAG-02

Signature/date

Signatures on file copies 


\section{PARTICLE TRACER TRAVERSE DATA FORM}

Site 296-S-21 Model

Date 7/27/2006

Tester JAG

Stack Dia.

Stack X-Area

Elevation N.A.

Distance to disturbance

Measurement units particles/ft3
Run No. PT-13

Fan configuration 3,4

Fan Setting $\mathbf{5 0 , 5 2}$

Stack Temp

Start/End Time 1055/1215

Center 2/3 from

Points in Center $2 / 3$

Injection Point Fan 4

Repeat of PT-9

Order ----->

Traverse-->

Trial ---->

\begin{tabular}{|r|r|}
\hline Point & Depth, in. \\
\hline 1 & 0.58 \\
\hline 2 & 1.89 \\
\hline 3 & 3.49 \\
\hline 4 & 5.81 \\
\hline Center & 9.00 \\
\hline 5 & 12.19 \\
\hline 6 & 14.51 \\
\hline 7 & 16.11 \\
\hline 8 & 17.42 \\
\hline
\end{tabular}

1ST

\begin{tabular}{|r|r|r|r|r|r|r|r|}
\hline \multicolumn{7}{|c|}{ Port_4_Bottom/East } & \multicolumn{5}{|c|}{ Port_4_Side/South } \\
1 & \multicolumn{3}{|c|}{ p } & \multicolumn{3}{|c|}{ particles/ft3 } \\
\hline 1148 & 1118 & 1125 & 1130.3 & 1112 & 1179 & 1097 & 1129.3 \\
\hline 1209 & 1126 & 1201 & 1178.7 & 1105 & 1188 & 1128 & 1140.3 \\
\hline 1292 & 1123 & 1252 & 1222.3 & 1139 & 1174 & 1125 & 1146.0 \\
\hline 1185 & 1126 & 1270 & 1193.7 & 1122 & 1076 & 1142 & 1113.3 \\
\hline 1161 & 1082 & 1269 & 1170.7 & 1134 & 1133 & 1198 & 1155.0 \\
\hline 1179 & 1193 & 1266 & 1212.7 & 1208 & 1173 & 1229 & 1203.3 \\
\hline 1211 & 1208 & 1288 & 1235.7 & 1238 & 1264 & 1204 & 1235.3 \\
\hline 1247 & 1163 & 1343 & 1251.0 & 1214 & 1258 & 1261 & 1244.3 \\
\hline 1177 & 1212 & 1262 & 1217.0 & 1128 & 1137 & 1113 & 1126.0 \\
\hline 1201.0 & 1150.1 & 1252.9 & 1201.3 & 1155.6 & 1175.8 & 1166.3 & 1165.9 \\
\hline
\end{tabular}

Averages ---------->

\begin{tabular}{lr}
\hline All & $\mathrm{pt} / \mathrm{ft} 3$ \\
Mean & 1183. \\
Min Point & 1113.3 \\
Max Point & 1251. \\
\hline
\end{tabular}

Avg Conc

$$
1186 \mathrm{pt} / \mathrm{ft} 3
$$

Generator Inlet Press

Stack Temp

Vel at $4 \mathrm{So} 8$

Ambient pressure

Ambient humidity

Ambient temp

Back-Gd aerosol

No. Bk-Gd samples

Compressor output reg

\begin{tabular}{|c|c|c|}
\hline Start & Finish & \\
\hline 5 & 4 & psig \\
\hline 94 & 97 & $F$ \\
\hline 2210 & 2220.0 & $\mathrm{fpm}$ \\
\hline 29.379 & 29.359 & $\mathrm{inHg}$ \\
\hline $33 \%$ & $33 \%$ & $\mathrm{RH}$ \\
\hline 92 & 95 & $F$ \\
\hline $1,1,0,1$ & $2,0,1,2$ & $\mathrm{pt} / \mathrm{ft} 3$ \\
\hline 4 & 4 & \\
\hline 90 & 105 & \\
\hline
\end{tabular}

Notes:

Repeated some measurements on 3rd Bottom traverse. Probe had partly twisted off particle counter.

Oil Used: $\quad$ FisherBrand 19

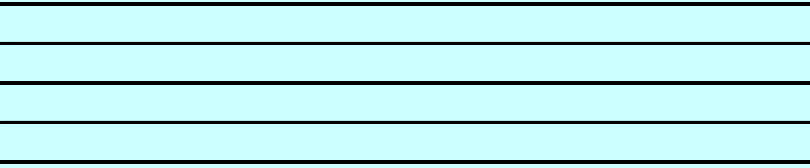

Signature signifies compliance with Procedure EMS-JAG-02

Signature/date
Signature verifying data and calculations:

Signatures on file copies 


\section{PARTICLE TRACER TRAVERSE DATA FORM}

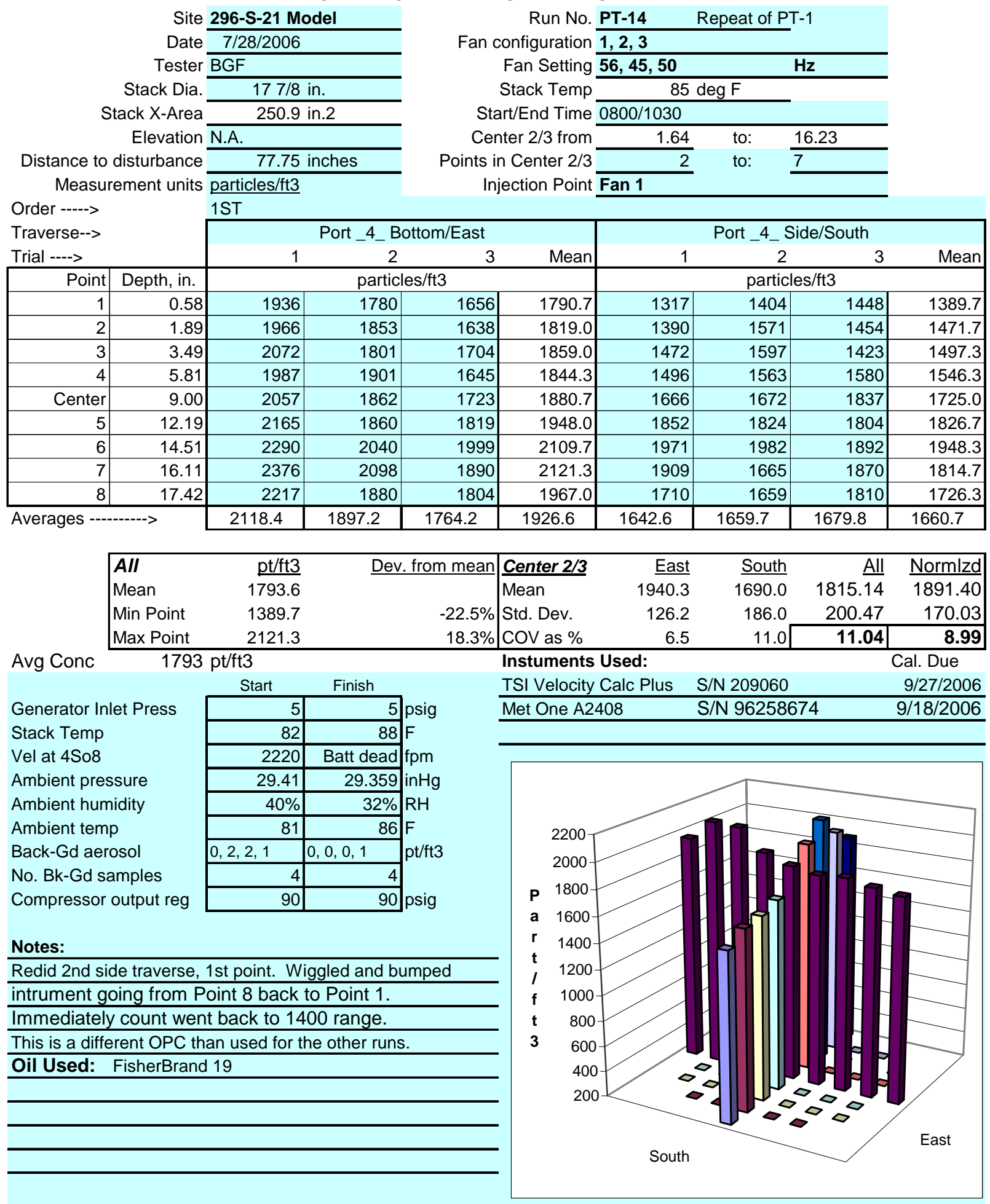




\section{Appendix K}

Flow Angle and Velocity Uniformity Data for the Actual Stack

(Calculated from Data Provided by CH2MHill) 


\section{APPENDIX K: FLOW ANGLE AND VELOCITY UNIFORMITY DATA FOR THE ACTUAL STACK \\ (Calculated from Data Provided by CH2MHill)}

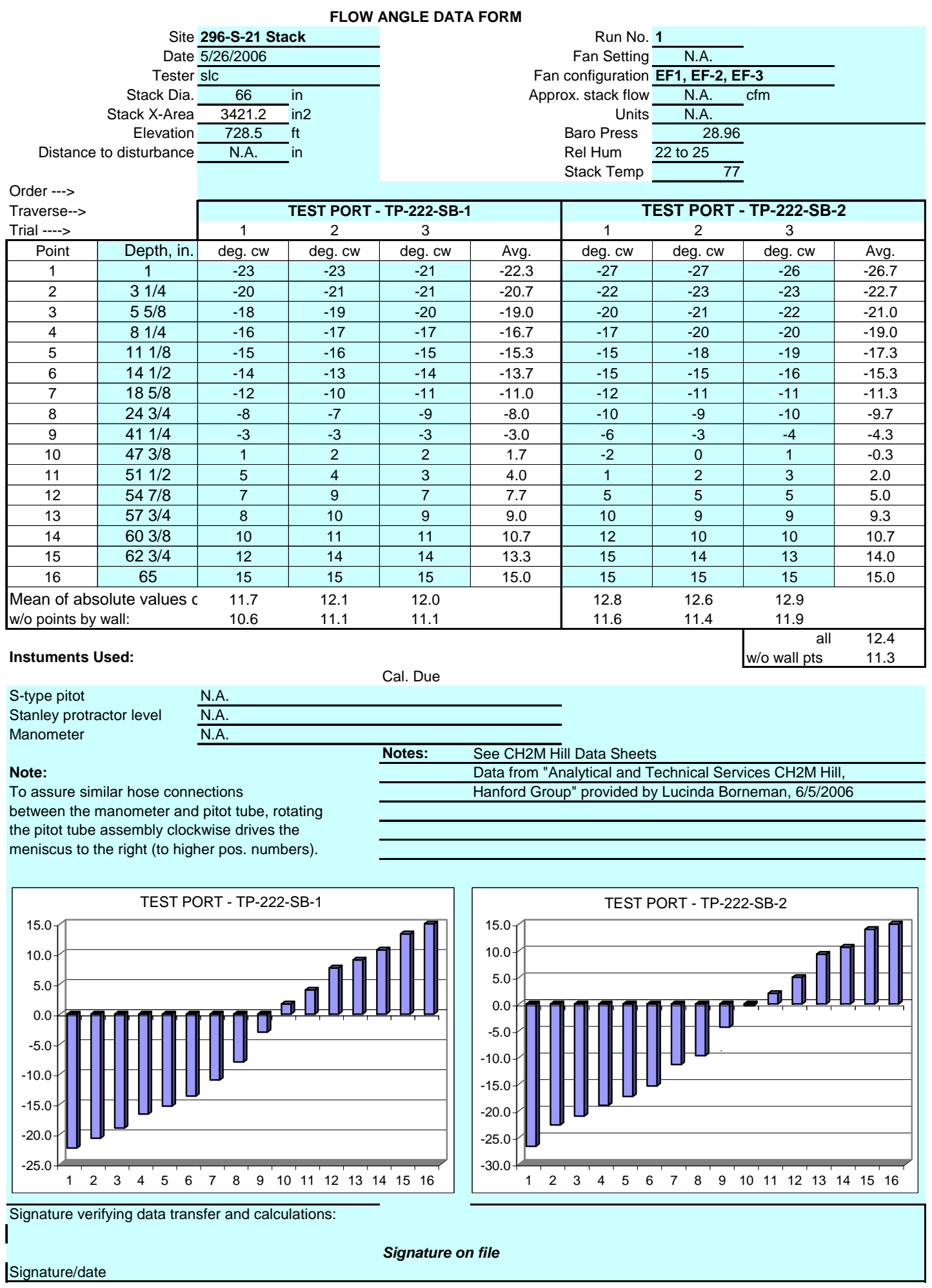


INPUT

Dataln

Site 296-S-21

Date $5 / 26 / 2006$

Testers Vent \& Balance

Stack Dia. 66 in.

Stack X-Area 3421.2 in. 2

Elevation $728.5 \mathrm{ft}$

Distance to disturbance N.A. inches

Velocity units $\underline{\mathrm{ft} / \mathrm{min}}$
VELOCITY TRAVERSE DATA FORM

Run No. 1

Fan Configuration EF1, EF-2, EF-3

Fan Setting N.A.

Stack Temp $77.0 \mathrm{deg} F$

Start/End Time -1410, -1445

Center 2/3 from 6.06 to: 59.94

Points in Center $2 / 3 \overline{\text { N.A. }}$ to: N.A. Data Files: NA

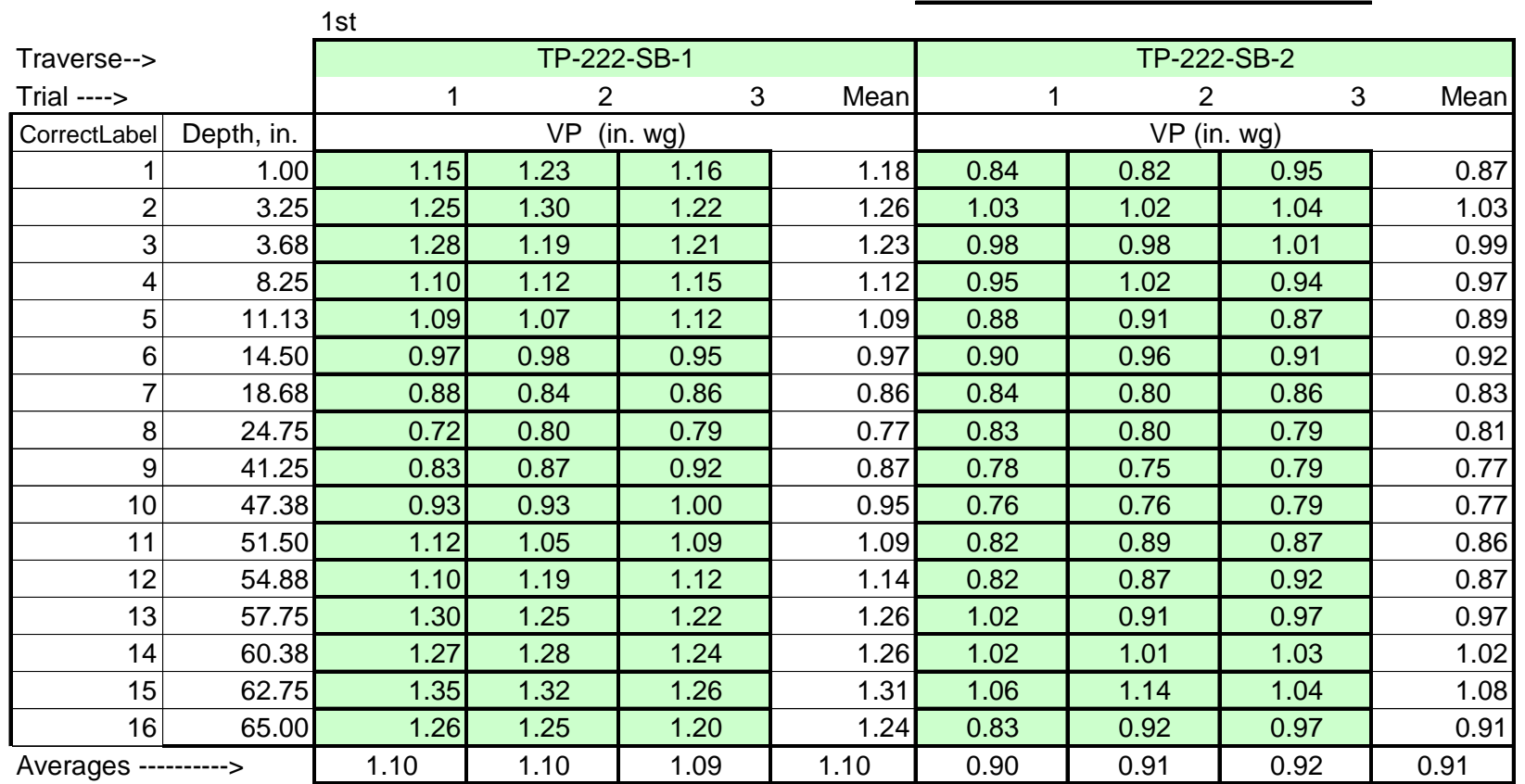

Notes: Data from "Analytical and Technical Services $\mathrm{CH} 2 \mathrm{M}$ Hill, Hanford Group" provided by Lucinda Borneman, 6/5/2006 
OUTPUT

\begin{tabular}{|c|c|}
\hline \multicolumn{2}{|c|}{ Site 296-S-21 } \\
\hline Date & $5 / 26 / 2006$ \\
\hline \multicolumn{2}{|c|}{ Testers Vent \& Balance } \\
\hline Stack Dia. & 66 in. \\
\hline Stack X-Area & 3421.2 in.2 \\
\hline Elevation & $728.5 \mathrm{ft}$ \\
\hline
\end{tabular}

Distance to disturbance N.A. inches

Velocity units $\underline{\mathrm{ft} / \mathrm{min}}$

Site

296-S-21

\section{VELOCITY TRAVERSE DATA FORM}

Run No. 1

Fan Configuration EF1, EF-2, EF-3

Fan Setting N.A.

Stack Temp $77.0 \mathrm{deg} F$

Start/End Time $-1410,-1445$

Center $2 / 3$ from $\frac{6.06}{n}$ to: 59.94 Points in Center 2/3 Data Files: NA Run No. 1

Traverse-->

Trial ----> \begin{tabular}{|cc}
\hline & TP-222-SB-1 \\
1 & 2
\end{tabular}

\begin{tabular}{|c|c|c|c|c|c|c|c|c|c|}
\hline \multirow{3}{*}{$\begin{array}{l}\text { Trial ----> } \\
\begin{array}{|r}\text { CorrectLabel } \\
1 \\
\end{array}\end{array}$} & & 1 & 2 & & Mean & 1 & $\leqslant$ & 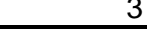 & Me \\
\hline & \multirow{2}{*}{$\begin{array}{r}\text { Depth, in. } \\
1.00\end{array}$} & \multicolumn{4}{|c|}{ Velocity } & \multicolumn{4}{|c|}{ Velocity } \\
\hline & & 3608 & 3731 & 3623 & 3654.0 & 3083 & 3046 & 3279 & 3136.3 \\
\hline 2 & 3.25 & 3761 & 3836 & 3716 & 3771.0 & 3414 & 3398 & 3431 & 3414.3 \\
\hline 3 & 3.68 & 3806 & 3670 & 3701 & 3725.6 & 3330 & 3330 & 3381 & 3347. \\
\hline 4 & 8.25 & 3528 & 3560 & 3608 & 3565.5 & 3279 & 3398 & 3262 & 3312.8 \\
\hline 5 & 11.13 & 3512 & 3480 & 3560 & 3517.5 & 3156 & 3209 & 3138 & 3167. \\
\hline 6 & 14.50 & 3313 & 3330 & 3279 & 3307.6 & 3192 & 3296 & 3209 & 3232. \\
\hline 7 & 18.68 & 3156 & 3083 & 3120 & 3119.7 & 3083 & 3009 & 3120 & 3070.7 \\
\hline 8 & 24.75 & 2855 & 3009 & 2990 & 2951.3 & 3065 & 3009 & 2990 & 3021 \\
\hline 9 & 41.25 & 3065 & 3138 & 3227 & 3143.2 & 2971 & 2913 & 2990 & 2958 \\
\hline 10 & 47.38 & 3244 & 3244 & 3364 & 3284.3 & 2933 & 2933 & 2990 & 2951 \\
\hline 11 & 51.50 & 3560 & 3447 & 3512 & 3506.6 & 3046 & 3174 & 3138 & 3119 \\
\hline 12 & 54.88 & 3528 & 3670 & 3560 & 3586.2 & 3046 & 3138 & 3227 & 3137 \\
\hline 13 & 57.75 & 3836 & 3761 & 3716 & 3771.0 & 3398 & 3209 & 3313 & 3306 \\
\hline 14 & 60.38 & 3791 & 3806 & 3746 & 3781.2 & 3398 & 3381 & 3414 & 3397 \\
\hline 15 & 62.75 & 3909 & 3865 & 3776 & 3850.1 & 3464 & 3592 & 3431 & 3495 \\
\hline 16 & 65.00 & 3776 & 3761 & 3685 & 3741.0 & 3065 & 3227 & 3313 & 3201 \\
\hline erages & 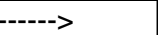 & 5.6 & 3524.6 & 11.5 & 3517.2 & 82.7 & 03.9 & 26.7 & 204.4 \\
\hline
\end{tabular}

Averages

\begin{tabular}{|c|c|c|c|c|c|c|}
\hline$A / I$ & $\mathrm{ft} / \mathrm{min}$ & Dev. from mean & Cntr 2/3 & TP-222-SB-1 & TP-222-SB-2 & All \\
\hline Mean & $\overline{3360.8}$ & & Mean & 3375.3 & 3127.8 & 3251.6 \\
\hline Min Point & 2951.3 & $-12.2 \%$ & Std. Dev. & 255.3 & 130.5 & 234.6 \\
\hline Max Point & 3850.1 & $14.6 \%$ & Cov as $\%$ & 7.6 & 4.2\lceil & 7.2 \\
\hline
\end{tabular}

Flow w/o C-Pt $81331 \mathrm{acfm}$ Vel Avg w/o C-Pt 3423 fpm

Stack temp

Equipment temp Ambient temp

Stack static

Ambient pressure

Total Stack pressure

Ambient humidity

Notes:

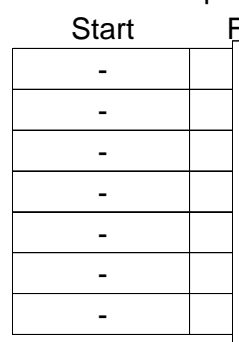
Finish

Instuments Used:

See $\mathrm{CH} 2 \mathrm{M}$ Hill Data Sheets

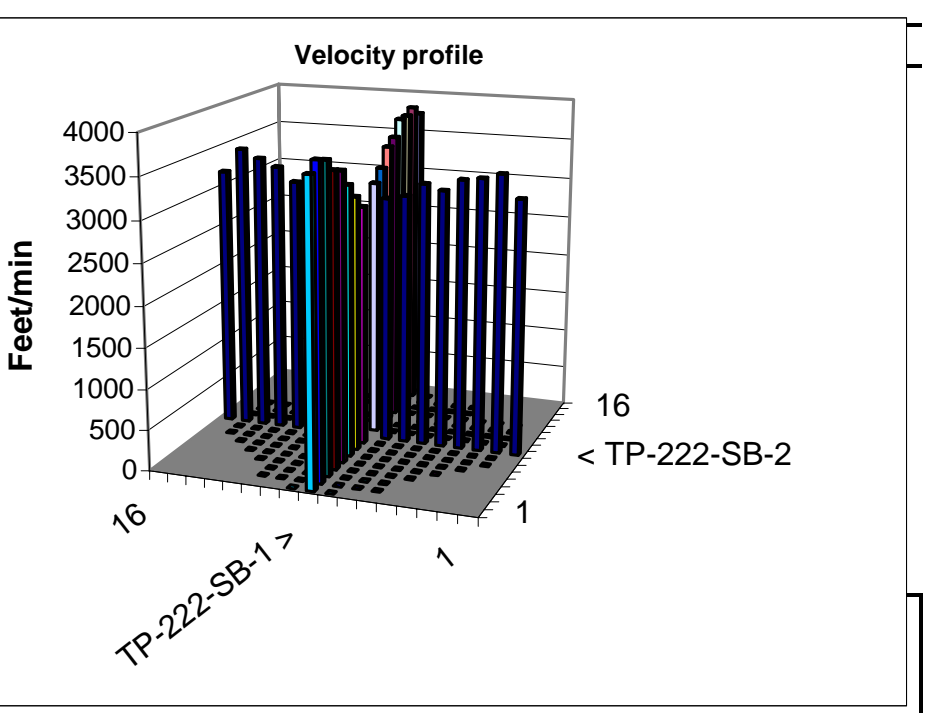




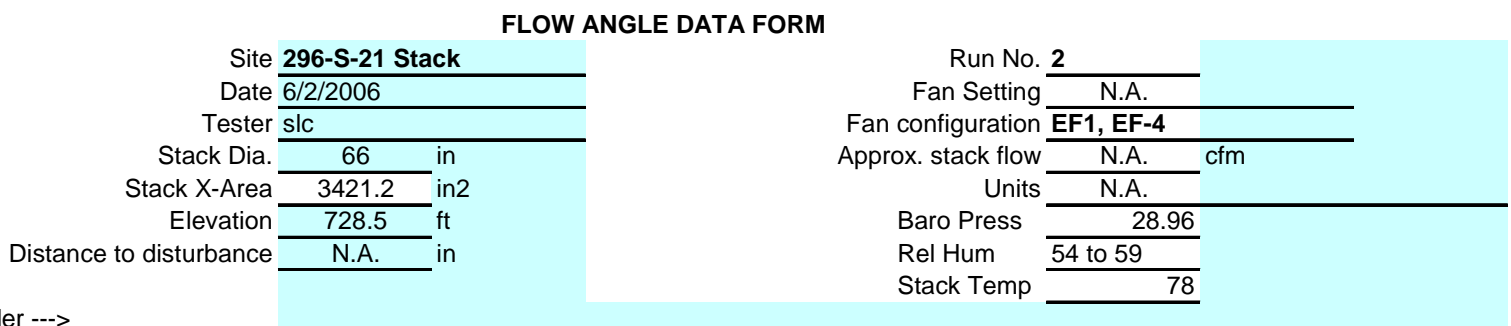

Order --->

Traverse-->

Trial ---->

\begin{tabular}{|c|c|c|c|c|c|c|c|c|c|}
\hline Point & Depth, in. & deg. cw & deg. cw & deg. cw & Avg. & deg. cw & deg. cw & deg. cw & Avg. \\
\hline 1 & 1 & -20 & -21 & -20 & -20.3 & -27 & -26 & -28 & -27.0 \\
\hline 2 & $31 / 4$ & -18 & -20 & -18 & -18.7 & -21 & -26 & -25 & -24.0 \\
\hline 3 & $55 / 8$ & -16 & -16 & -15 & -15.7 & -22 & -25 & -25 & -24.0 \\
\hline 4 & $81 / 4$ & -12 & -14 & -14 & -13.3 & -21 & -20 & -20 & -20.3 \\
\hline 5 & $111 / 8$ & -11 & -11 & -12 & -11.3 & -17 & -13 & -17 & -15.7 \\
\hline 6 & $141 / 2$ & -10 & -10 & -11 & -10.3 & -13 & -10 & -12 & -11.7 \\
\hline 7 & $185 / 8$ & -9 & -7 & -8 & -8.0 & -9 & -9 & -8 & -8.7 \\
\hline 8 & $243 / 4$ & -8 & -6 & -5 & -6.3 & -5 & -6 & -6 & -5.7 \\
\hline 9 & $411 / 4$ & -2 & -2 & -2 & -2.0 & -1 & -3 & -4 & -2.7 \\
\hline 10 & $473 / 8$ & 2 & 1 & 0 & 1.0 & 0 & 0 & -1 & -0.3 \\
\hline 11 & $511 / 2$ & 3 & 4 & 4 & 3.7 & 2 & 2 & 0 & 1.3 \\
\hline 12 & $547 / 8$ & 5 & 6 & 4 & 5.0 & 5 & 3 & 3 & 3.7 \\
\hline 13 & $573 / 4$ & 7 & 9 & 8 & 8.0 & 6 & 5 & 5 & 5.3 \\
\hline 14 & $603 / 8$ & 8 & 9 & 10 & 9.0 & 9 & 8 & 6 & 7.7 \\
\hline 15 & $623 / 4$ & 11 & 12 & 11 & 11.3 & 10 & 9 & 10 & 9.7 \\
\hline 16 & 65 & 14 & 15 & 14 & 14.3 & 11 & 11 & 10 & 10.7 \\
\hline
\end{tabular}

Instuments Used:

S-type pitot

Stanley protractor level

Manometer

Cal. Due

Run No. 2

Fan configuration EF1, EF-4

Approx stack flow N.A. cfm

Baro Press 28.96

Stack Temp

Note:

To assure similar hose connections

between the manometer and pitot tube, rotating

the pitot tube assembly clockwise drives the

meniscus to the right (to higher pos. numbers).
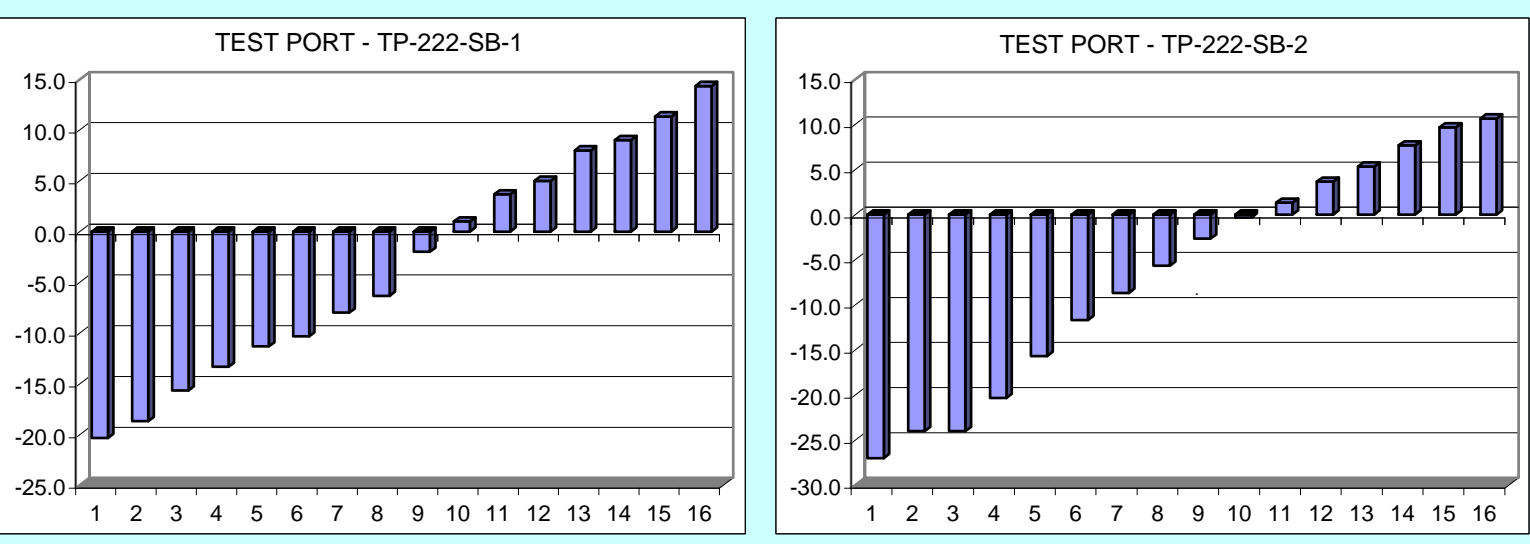

Signature verifying data transfer and calculations:

Signature/date

Signature on file 
Site 296-S-21

Date $6 / 2 / 2006$

Testers Vent \& Balance

Stack Dia. 66 in.

Stack X-Area 3421.2 in.2

Elevation $728.5 \mathrm{ft}$

Distance to disturbance N.A. inches

Velocity units $\underline{\mathrm{ft} / \mathrm{min}}$
Run No. 2

Fan Configuration EF1, EF-4

Fan Setting

Stack Temp

Start/End Time $-1354,1509$

Center 2/3 from

Points in Center 2/3

Data Files: NA

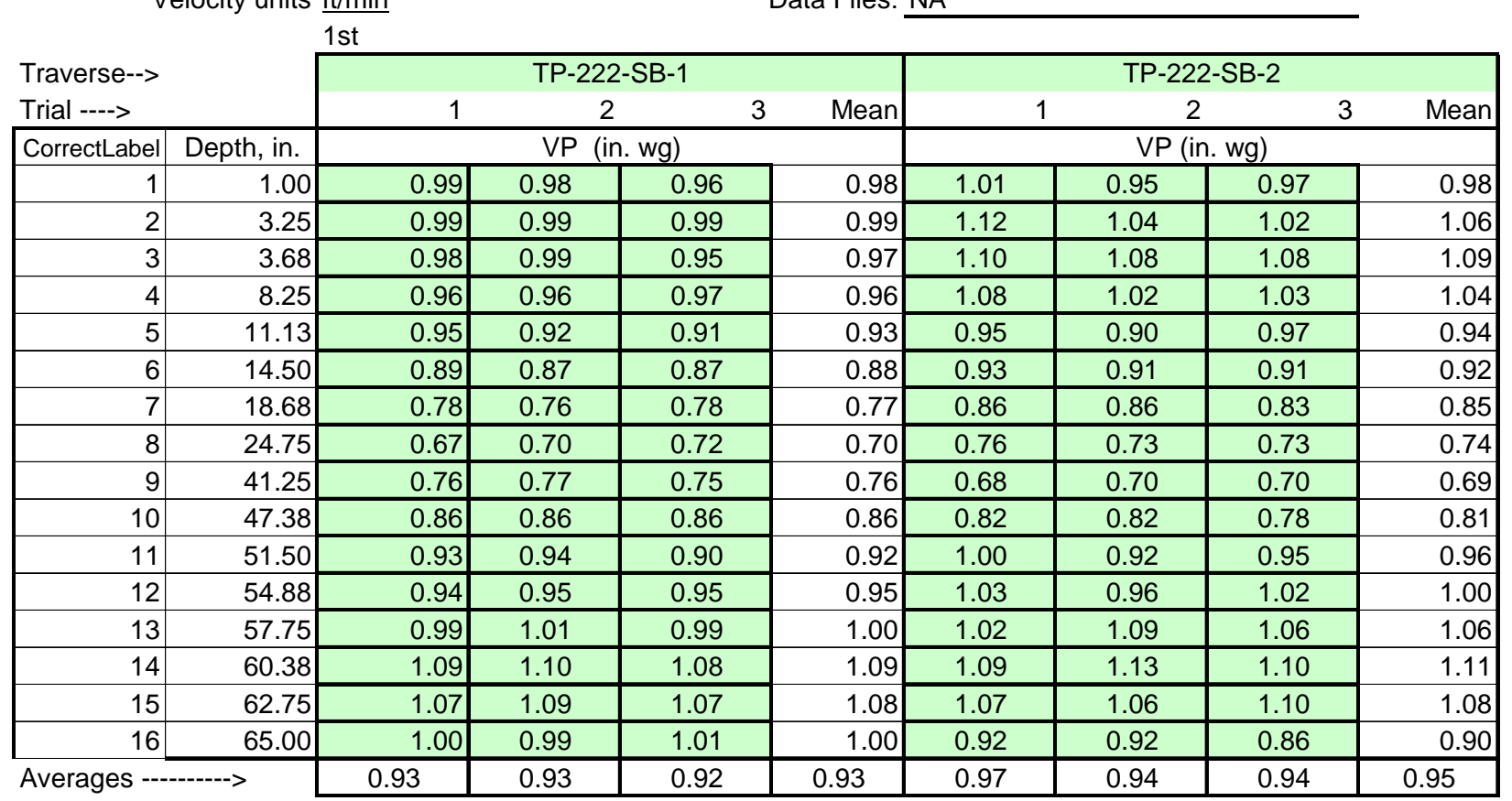

Notes: Data from "Analytical and Technical Services $\mathrm{CH} 2 \mathrm{M}$ Hill, Hanford Group" provided by Lucinda Borneman, 6/5/2006 
VELOCITY TRAVERSE DATA FORM

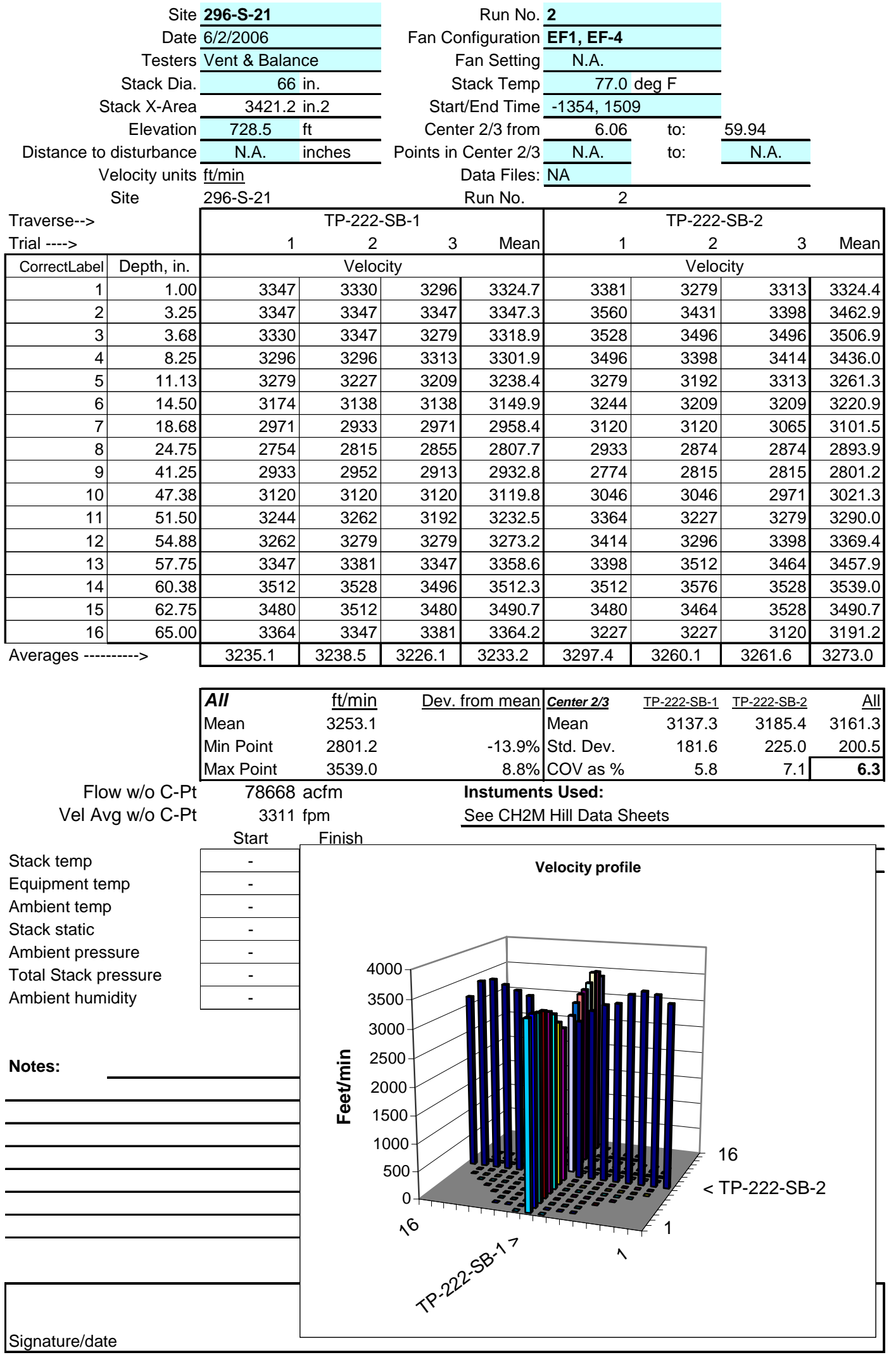


FLOW ANGLE DATA FORM

\begin{tabular}{|c|c|c|}
\hline Site 296-S-21 Stack & \multicolumn{2}{|c|}{ Date $6 / 2 / 2006$} \\
\hline \multicolumn{3}{|c|}{ Tester slc } \\
\hline Stack Dia. & 66 & in \\
\hline Stack X-Area & 3421.2 & in2 \\
\hline Elevation & 728.5 & $\mathrm{ft}$ \\
\hline Distance to disturbance & N.A. & in \\
\hline
\end{tabular}

Run No. 3

Fan Setting N.A.

Fan configuration EF2, EF-4

Approx. stack flow N.A. cfm

Units $\frac{\text { N.A. }}{28.96}$

Rel Hum $\frac{28.96}{48.9 \text { to } 56}$

Stack Temp

Order --->

Traverse-->

Trial ---->

Point

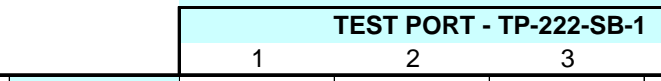

TEST PORT - TP-222-SB-1

TEST PORT - TP-222-SB-2

\begin{tabular}{|c|c|c|c|c|c|c|c|c|c|}
\hline \multicolumn{2}{|l|}{ Trial ----> } & \multicolumn{4}{|c|}{3} & \multicolumn{2}{|c|}{$1 \quad 2$} & 3 & \multirow[b]{2}{*}{ Avg. } \\
\hline Point & Depth, in. & deg. cW & deg. cW & deg. cw & Avg. & deg. cW & deg. CW & deg. CW & \\
\hline 1 & 1 & -20 & -20 & -19 & -19.7 & -23 & -26 & -26 & -25.0 \\
\hline 2 & $31 / 4$ & -18 & -19 & -19 & -18.7 & -24 & -25 & -26 & -25.0 \\
\hline 3 & $55 / 8$ & -17 & -18 & -18 & -17.7 & -22 & -21 & -23 & -22.0 \\
\hline 4 & $81 / 4$ & -15 & -15 & -16 & -15.3 & -21 & -19 & -20 & -20.0 \\
\hline 5 & $111 / 8$ & -14 & -14 & -15 & -14.3 & -17 & -16 & -17 & -16.7 \\
\hline 6 & $141 / 2$ & -12 & -11 & -14 & -12.3 & -14 & -12 & -11 & -12.3 \\
\hline 7 & $185 / 8$ & -10 & -9 & -10 & -9.7 & -10 & -9 & -9 & -9.3 \\
\hline 8 & $243 / 4$ & -8 & -8 & -8 & -8.0 & -5 & -5 & -5 & -5.0 \\
\hline 9 & $411 / 4$ & -2 & -2 & -2 & -2.0 & 0 & 1 & 1 & 0.7 \\
\hline 10 & $473 / 8$ & -1 & 0 & 0 & -0.3 & 2 & 2 & 1 & 1.7 \\
\hline 11 & 51 1/2 & 4 & 3 & 2 & 3.0 & 4 & 3 & 3 & 3.3 \\
\hline 12 & $547 / 8$ & 5 & 5 & 4 & 4.7 & 5 & 5 & 5 & 5.0 \\
\hline 13 & $573 / 4$ & 7 & 7 & 6 & 6.7 & 6 & 5 & 5 & 5.3 \\
\hline 14 & $603 / 8$ & 10 & 9 & 9 & 9.3 & 7 & 7 & 8 & 7.3 \\
\hline 15 & $623 / 4$ & 14 & 13 & 14 & 13.7 & 7 & 8 & 9 & 8.0 \\
\hline 16 & 65 & 16 & 15 & 15 & 15.3 & 9 & 9 & 10 & 9.3 \\
\hline \multicolumn{2}{|c|}{ Mean of absolute values c } & 10.8 & 10.5 & 10.7 & & 11.0 & 10.8 & 11.2 & \\
\hline \multicolumn{2}{|c|}{ w/o points by wall: } & 9.8 & 9.5 & 9.8 & & 10.3 & 9.9 & 10.2 & \\
\hline \multicolumn{2}{|c|}{ Instuments Used: } & & & & & & & $\begin{array}{r}\mathrm{a} \\
\text { w/o wall pts }\end{array}$ & $\begin{array}{c}10.8 \\
9.9\end{array}$ \\
\hline
\end{tabular}

S-type pitot

Stanley protractor level

Manometer

$\frac{\text { N.A. }}{\text { N.A. }}$

Cal. Due

Note:

To assure similar hose connections

between the manometer and pitot tube, rotating

the pitot tube assembly clockwise drives the

meniscus to the right (to higher pos. numbers).
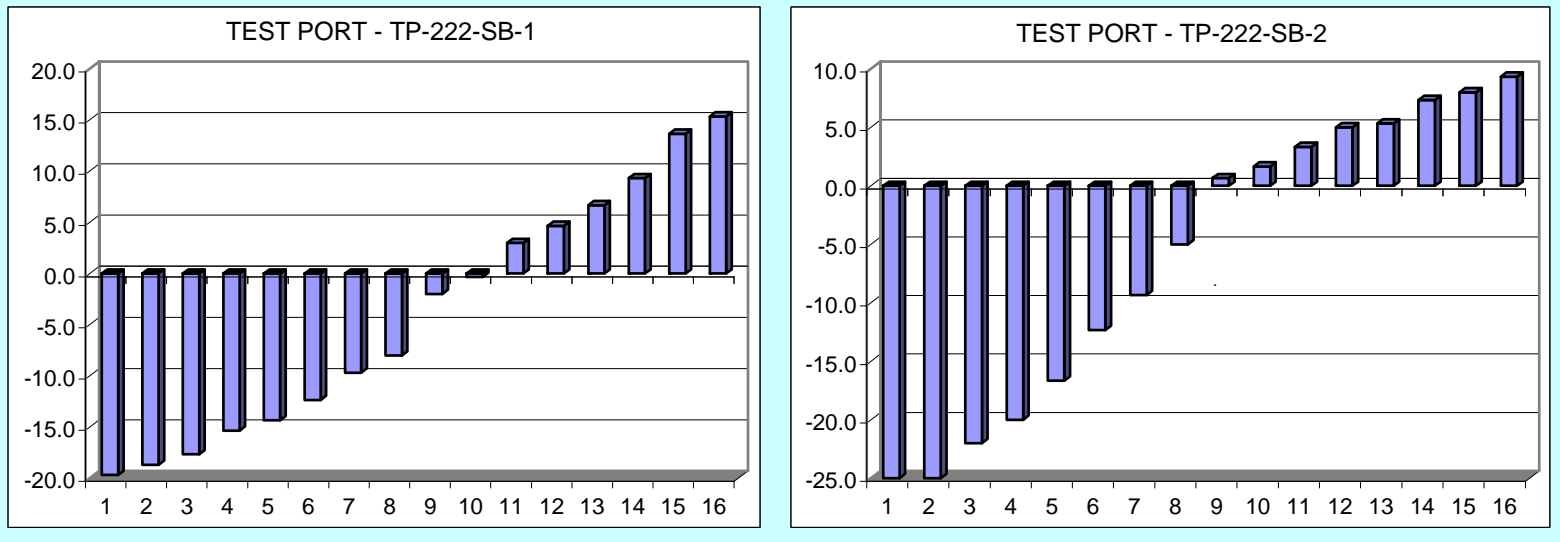

Signature verifying data transfer and calculations:

Signature/date

Signature on file

K.7 
INPUT

\section{Dataln}

Site 296-S-21

Date $6 / 2 / 2006$

Testers Vent \& Balance

Stack Dia.

Elevation $728.5 \mathrm{ft}$

Distance to disturbance N.A. inches

Velocity units $\underline{\mathrm{ft} / \mathrm{min}}$

\section{VELOCITY TRAVERSE DATA FORM}

Run No.

Fan Configuration EF2, EF-4

Fan Setting N.A.

Stack Temp 77.0 deg F

Start/End Time $-1625,1736$

Center 2/3 from 6.06 to: 59.94

Points in Center $2 / 3 \longdiv { \text { N.A. } }$ to: N.A.

Data Files: NA

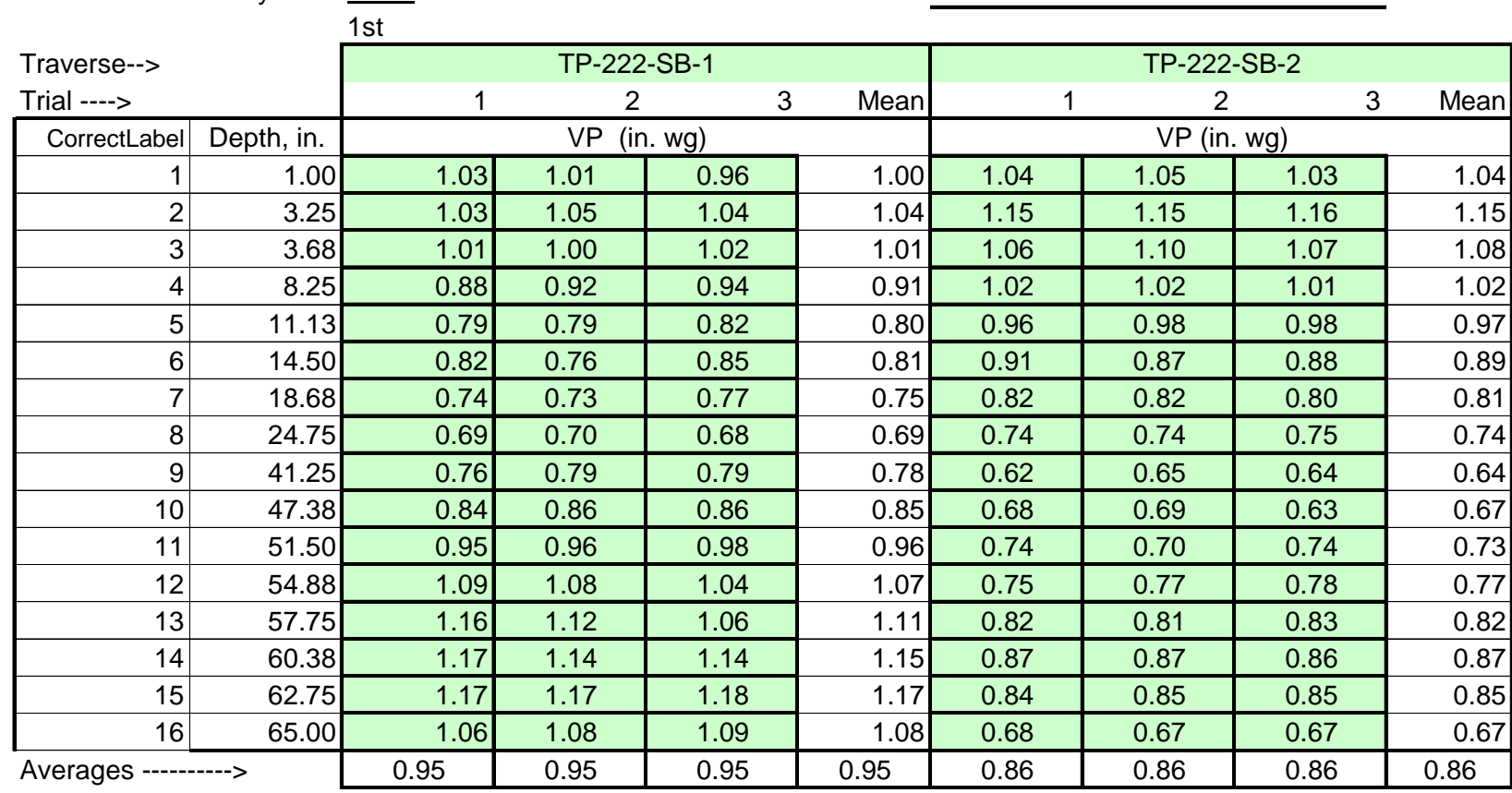

Notes: $\quad$ Data from "Analytical and Technical Services $\mathrm{CH} 2 \mathrm{M}$ Hill, Hanford Group" provided by Lucinda Borneman, 6/5/2006 


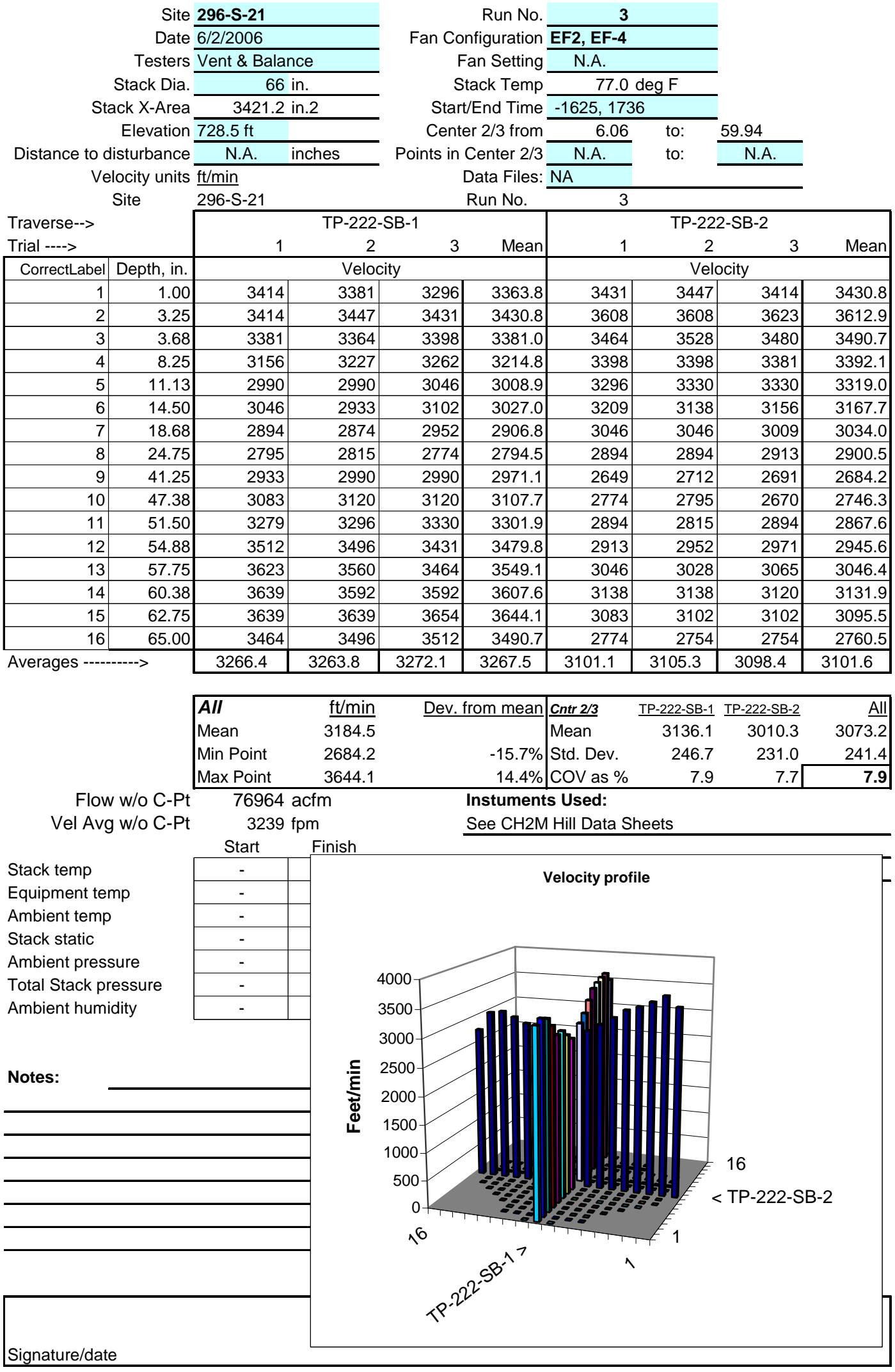




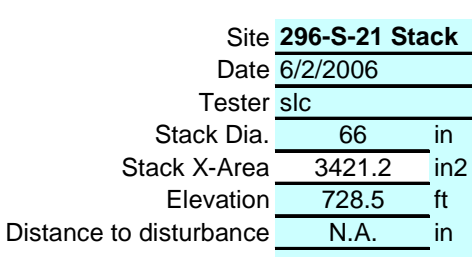

FLOW ANGLE DATA FORM

order --->

Traverse-->

Trial ---->

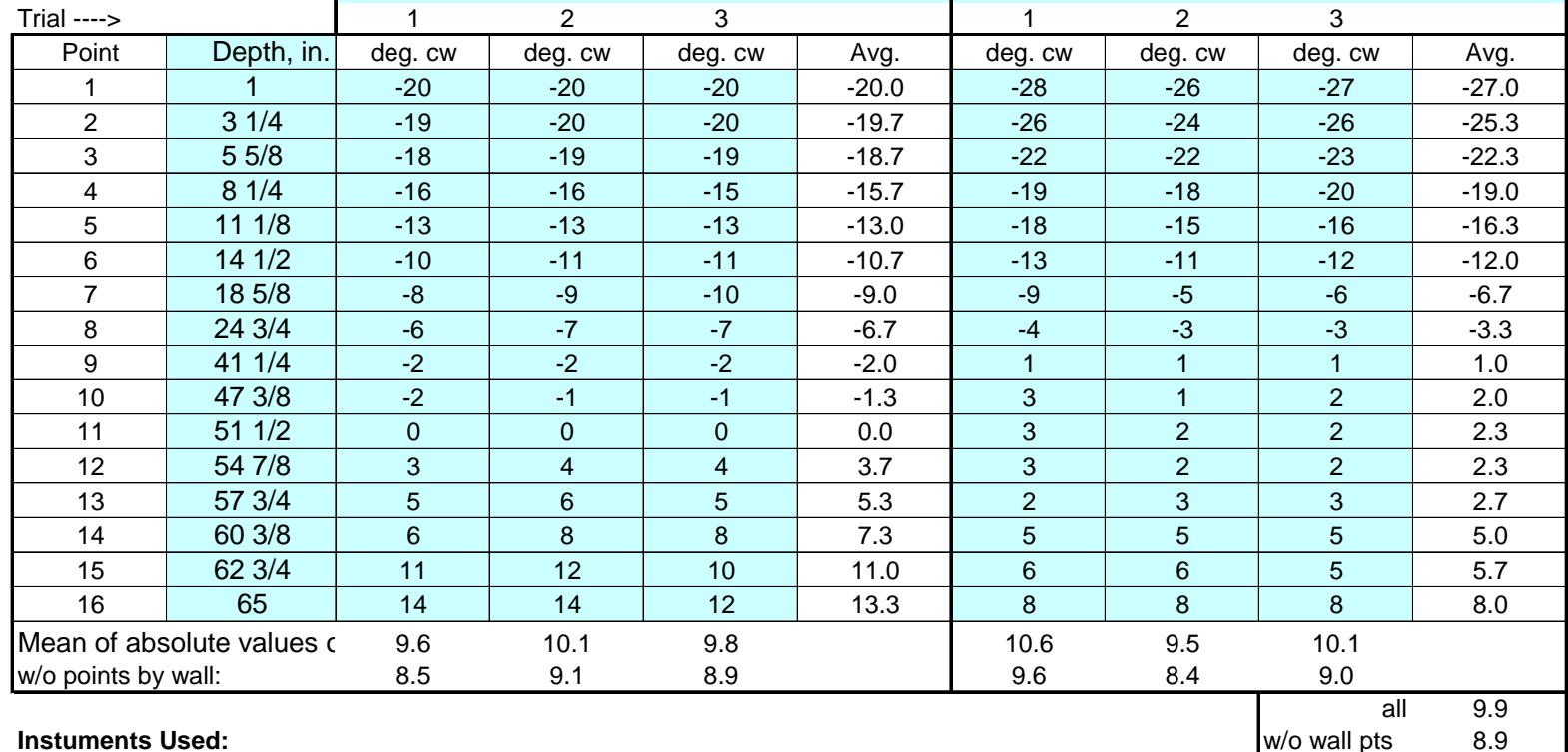

\section{Instuments Used:}

S-type pitot

Stanley protractor level

Manometer

$\frac{\text { N.A. }}{\text { N.A. }}$

Cal. Due

Note:

To assure similar hose connections between the manometer and pitot tube, rotating the pitot tube assembly clockwise drives the meniscus to the right (to higher pos. numbers).
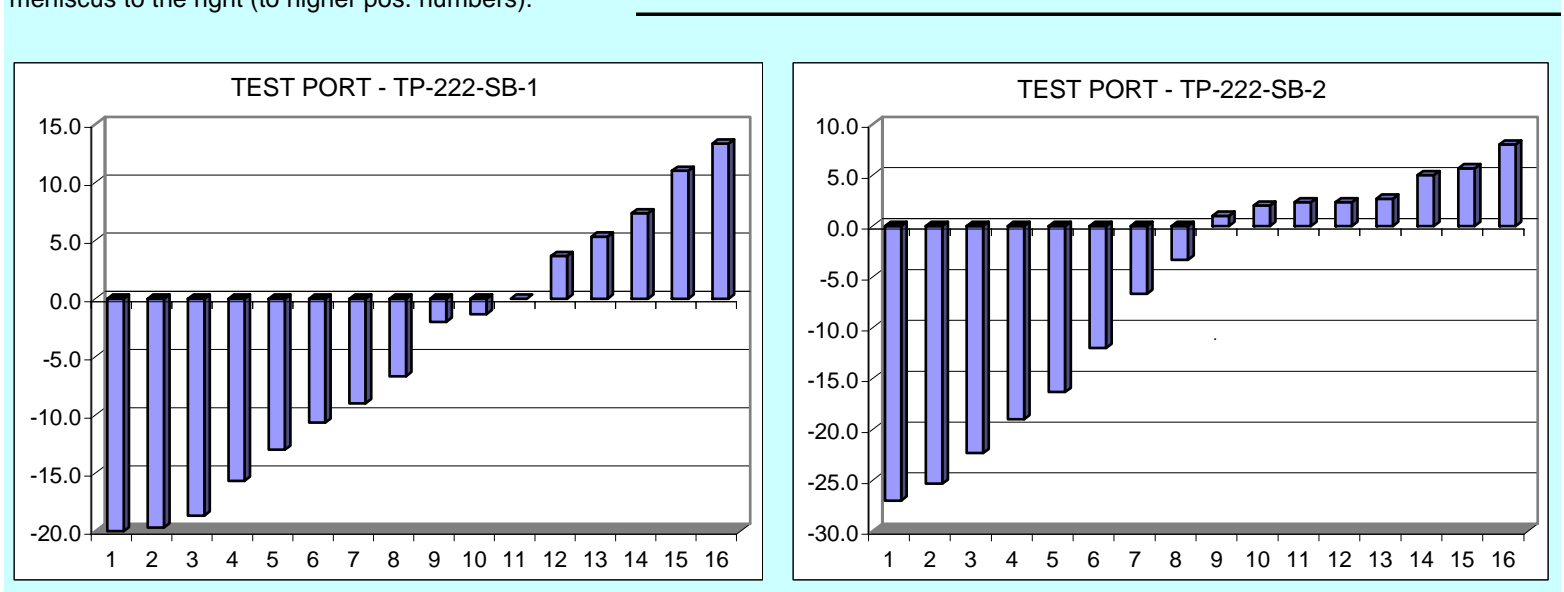

Signature verifying data transfer and calculations:

I

Signature/date 
Site 296-S-21

Date 6/2/2006

Testers Vent \& Balance

Stack Dia. 66 in.

Stack X-Area 3421.2 in.2

Elevation $728.5 \mathrm{ft}$

Distance to disturbance N.A. inches

Velocity units $\underline{\mathrm{ft} / \mathrm{min}}$
Run No. 4

Fan Configuration EF3, EF-4

Fan Setting N.A.

Stack Temp

Start/End Time

Center $2 / 3$ from 77.0 deg $F$

$-1830,-1921$

Points in Center 2/3

Data Files: NA

\begin{tabular}{|c|c|c|c|c|c|c|c|c|c|}
\hline \multirow{3}{*}{\multicolumn{2}{|c|}{$\begin{array}{l}\text { Traverse--> } \\
\text { Trial ----> }\end{array}$}} & & & & & & & & \\
\hline & & \multicolumn{4}{|c|}{ TP-222-SB-1 } & \multicolumn{4}{|c|}{ TP-222-SB-2 } \\
\hline & & \multicolumn{2}{|l|}{1} & 2 & Mean & \multicolumn{2}{|c|}{1} & 2 & Mean \\
\hline CorrectLabel & Depth, in. & \multicolumn{4}{|c|}{ VP (in. wg) } & \multicolumn{4}{|c|}{ VP (in. wg) } \\
\hline 1 & 1.00 & 0.97 & 0.96 & 0.95 & 0.96 & 1.14 & 1.09 & 1.07 & 1.10 \\
\hline 2 & 3.25 & 1.04 & 1.06 & 1.04 & 1.05 & 1.12 & 1.09 & 1.12 & 1.11 \\
\hline 3 & 3.68 & 1.06 & 1.06 & 1.06 & 1.06 & 1.03 & 1.03 & 1.04 & 1.03 \\
\hline 4 & 8.25 & 0.86 & 0.90 & 0.89 & 0.88 & 0.99 & 0.96 & 1.02 & 0.99 \\
\hline 5 & 11.13 & 0.82 & 0.82 & 0.84 & 0.83 & 0.92 & 0.95 & 0.89 & 0.92 \\
\hline 6 & 14.50 & 0.76 & 0.77 & 0.76 & 0.76 & 0.90 & 0.91 & 0.90 & 0.90 \\
\hline 7 & 18.68 & 0.65 & 0.66 & 0.70 & 0.67 & 0.85 & 0.82 & 0.83 & 0.83 \\
\hline 8 & 24.75 & 0.63 & 0.67 & 0.64 & 0.65 & 0.75 & 0.79 & 0.76 & 0.77 \\
\hline 9 & 41.25 & 0.81 & 0.80 & 0.80 & 0.80 & 0.62 & 0.69 & 0.62 & 0.64 \\
\hline 10 & 47.38 & 0.82 & 0.84 & 0.82 & 0.83 & 0.73 & 0.74 & 0.71 & 0.73 \\
\hline 11 & 51.50 & 0.92 & 0.93 & 0.96 & 0.94 & 0.78 & 0.75 & 0.79 & 0.77 \\
\hline 12 & 54.88 & 1.01 & 0.99 & 1.00 & 1.00 & 0.86 & 0.83 & 0.86 & 0.85 \\
\hline 13 & 57.75 & 1.00 & 0.99 & 0.99 & 0.99 & 0.83 & 0.86 & 0.90 & 0.86 \\
\hline 14 & 60.38 & 1.04 & 1.07 & 1.08 & 1.06 & 0.98 & 0.92 & 0.93 & 0.94 \\
\hline 15 & 62.75 & 1.05 & 1.05 & 1.05 & 1.05 & 0.92 & 0.96 & 0.94 & 0.94 \\
\hline 16 & 65.00 & 1.02 & 1.04 & 1.03 & 1.03 & 0.74 & 0.76 & 0.74 & 0.75 \\
\hline Averages --- & - & 0.90 & 0.91 & 0.91 & 0.91 & 0.89 & 0.88 & 0.88 & 0.88 \\
\hline
\end{tabular}

Notes: Data from "Analytical and Technical Services $\mathrm{CH} 2 \mathrm{M}$ Hill, Hanford Group" provided by Lucinda Borneman, 6/5/2006 
VELOCITY TRAVERSE DATA FORM

Site 296-S-21

Date 6/2/2006

Testers Vent \& Balance

Stack Dia.

Stack X-Area

Elevation $728.5 \mathrm{ft}$

Distance to disturbance N.A. inches

Velocity units $\mathrm{ft} / \mathrm{min}$

Site

296-S-21
Run No. 4

Fan Configuration EF3, EF-4

Fan Setting N.A.

Start/End Time $-1830,-1921$

Center $2 / 3$ from

Points in Center $2 / 3$ N.A. to: N.A.

Data Files: NA

Run No. 4

\begin{tabular}{|c|c|c|c|c|c|c|c|c|c|}
\hline \multirow{3}{*}{\multicolumn{2}{|c|}{ Trav }} & \multirow{2}{*}{\multicolumn{4}{|c|}{ TP-222-SB-1 }} & \multirow{2}{*}{\multicolumn{4}{|c|}{ TP-222-SB-2 }} \\
\hline & & & & & & & & & \\
\hline & & \multicolumn{2}{|l|}{1} & 3 & Mean & 1 & 2 & 3 & Mean \\
\hline CorrectLabel & Depth, in. & \multicolumn{4}{|c|}{ Velocity } & \multicolumn{4}{|c|}{ Velocity } \\
\hline 1 & 1.00 & 3313 & 3296 & 3279 & 3296.2 & 3592 & 3512 & 3480 & 3528.1 \\
\hline 2 & 3.25 & 3431 & 3464 & 3431 & 3441.8 & 3560 & 3512 & 3560 & 3544.3 \\
\hline 3 & 3.68 & 3464 & 3464 & 3464 & 3463.7 & 3414 & 3414 & 3431 & 3419.8 \\
\hline 4 & 8.25 & 3120 & 3192 & 3174 & 3161.7 & 3347 & 3296 & 3398 & 3347.1 \\
\hline 5 & 11.13 & 3046 & 3046 & 3083 & 3058.7 & 3227 & 3279 & 3174 & 3226.5 \\
\hline 6 & 14.50 & 2933 & 2952 & 2933 & 2939.3 & 3192 & 3209 & 3192 & 3197.5 \\
\hline 7 & 18.68 & 2712 & 2733 & 2815 & 2753.4 & 3102 & 3046 & 3065 & 3071.0 \\
\hline 8 & 24.75 & 2670 & 2754 & 2691 & 2705.1 & 2913 & 2990 & 2933 & 2945.5 \\
\hline 9 & 41.25 & 3028 & 3009 & 3009 & 3015.3 & 2649 & 2795 & 2649 & 2697.5 \\
\hline 10 & 47.38 & 3046 & 3083 & 3046 & 3058.7 & 2874 & 2894 & 2835 & 2867.7 \\
\hline 11 & 51.50 & 3227 & 3244 & 3296 & 3255.8 & 2971 & 2913 & 2990 & 2958.3 \\
\hline 12 & 54.88 & 3381 & 3347 & 3364 & 3364.2 & 3120 & 3065 & 3120 & 3101.5 \\
\hline 13 & 57.75 & 3364 & 3347 & 3347 & 3353.0 & 3065 & 3120 & 3192 & 3125.4 \\
\hline 14 & 60.38 & 3431 & 3480 & 3496 & 3469.0 & 3330 & 3227 & 3244 & 3267.2 \\
\hline 15 & 62.75 & 3447 & 3447 & 3447 & 3447.3 & 3227 & 3296 & 3262 & 3261.6 \\
\hline 16 & 65.00 & 3398 & 3431 & 3414 & 3414.3 & 2894 & 2933 & 2894 & 2906.9 \\
\hline Averages --- & & 3188.2 & 3205.6 & 3205.7 & 3199.8 & 3154.9 & 3156.4 & 3151.1 & 3154.1 \\
\hline
\end{tabular}

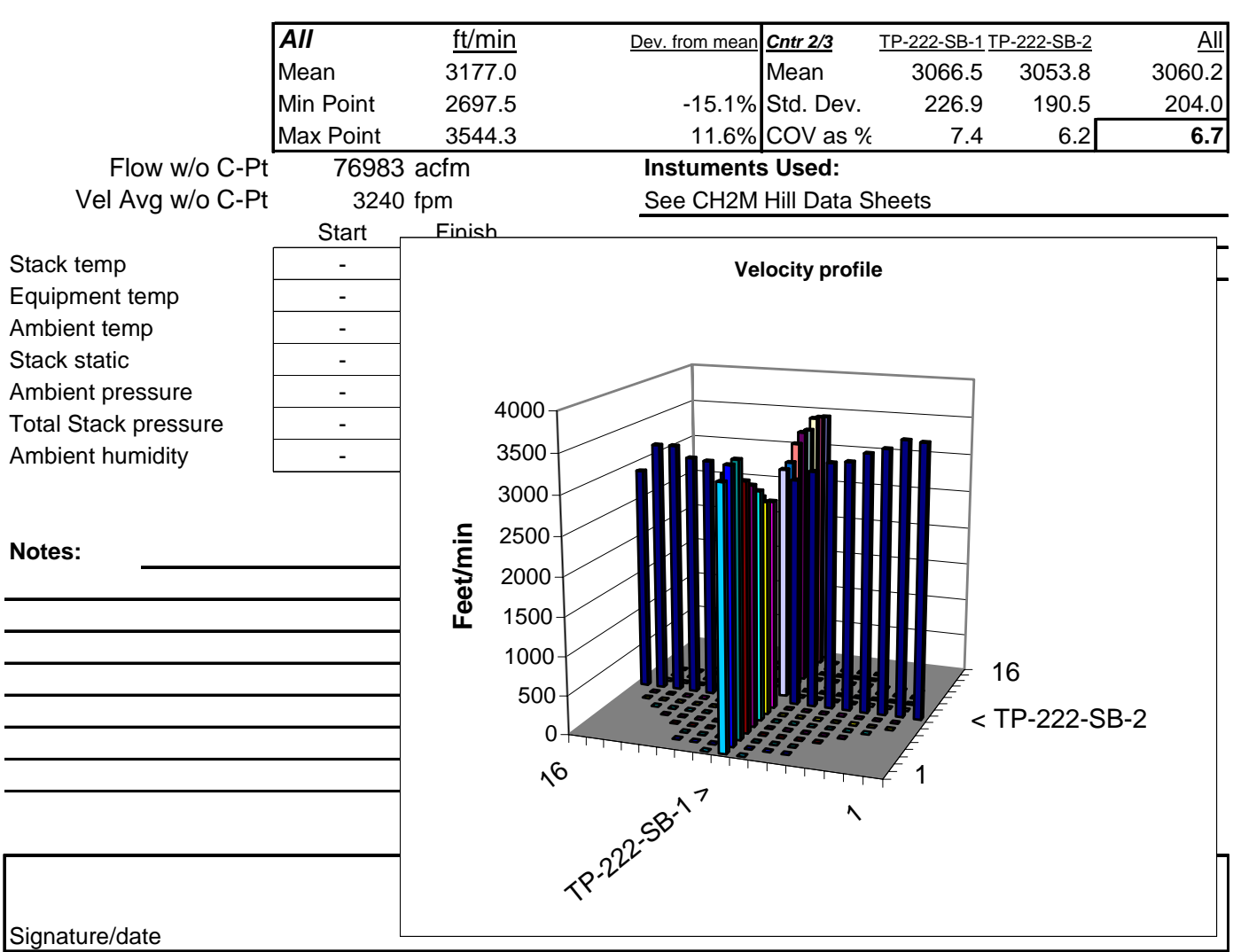




\section{Distribution}

No. of

Copies

ONSITE

2 U.S. Department of Energy Office of $\underline{\text { River Protection }}$

D. W. Bowser H6-60

A. J. Stevens H6-60

10 CH2MHill

L. E. Borneman T6-03

S. L. Brey T6-04

B. H. Ruealas T6-04

L. L. Weaver T6-04

L. L. Penn H6-03

P. Miller R1-51

J. D. Guberski R1-51

D. B. Hardy T6-14

D. L. Renberger H6-07

B. R. Hill T6-03

8 Pacific Northwest National Laboratory
J. A. Glissmeyer (5)
K3-54
K. Salisbury-Miller
K9-34
B. G. Fritz
K6-75
J. M. Barnett
J2-25

2 Hanford Technical Library Technical Report Files

P8-55

Distr. 1 